\title{
Lebenszeit-Mortalität von Männern mit normalen und subnormalen Spermienkonzentrationen
}

\author{
Dissertation \\ zur \\ Erlangung des Doktorgrades \\ der Naturwissenschaften \\ (Dr. rer. nat.) \\ dem \\ Fachbereich Biologie \\ der Philipps-Universität Marburg \\ vorgelegt von \\ Sabine Groos \\ aus Biedenkopf
}


Vom Fachbereich Biologie der Philipps-Universität Marburg als Dissertation am 01.12.2005 angenommen

Erstgutachter: Prof. Dr. R. Brandl

Zweitgutachter: Prof. Dr. Dr. U.O. Mueller

Tag der mündlichen Prüfung: 24.01.2006 


\section{Inhaltsverzeichnis}

1 Einleitung 1

1.1 Hintergrund und Ziel der Studie 1

1.2 Stand der Forschung 3

1.2.1 Mortalität 3

1.2.1.1 Lebenserwartung und Lebensspanne 3

1.2.1.2 Determinanten der Lebensspanne 4

1.2.1.2.1 Vererbbarkeit der Lebensspanne $\quad 5$

1.2.1.2.2 Lebensstil und Lebensspanne 5

1.2.1.2.3 Sozioökonomische und soziale Einflussfaktoren 6

1.2.1.3 Geschlechtstypische Mortalität 8

1.2.1.4 Todesursachen von Männern 9

$\begin{array}{ll}\text { 1.2.2 Fertilität } & 10\end{array}$

1.2.2.1 Männliche Reproduktionsbiologie $\quad 10$

1.2.2.1.1 Generelle Organisation des Hodens $\quad 10$

$\begin{array}{ll}\text { 1.2.2.1.2 Ontogenese } & 11\end{array}$

$\begin{array}{ll}\text { 1.2.2.1.3 Spermatogenese } & 11\end{array}$

1.2.2.1.4 Hormonelle Steuerung der Spermatogenese 13

1.2.2.1.5 Weitere Entwicklung der Spermien und Ejakulat-Zusammensetzung 13

$\begin{array}{ll}\text { 1.2.2.2 Ejakulatanalyse und Spermiogramm } & 14\end{array}$

$\begin{array}{ll}\text { 1.2.2.3 Ejakulatqualität als zeitliches und räumliches Phänomen } & 16\end{array}$

$\begin{array}{ll}\text { 1.2.2.3.1 Intraindividuelle Variabilität der Ejakulatqualität } & 16\end{array}$

$\begin{array}{ll}\text { 1.2.2.3.2 Globale Variabilität der Ejakulatqualität } & 18\end{array}$

$\begin{array}{ll}1.2 .3 \text { Infertilität } & 19\end{array}$

$\begin{array}{ll}\text { 1.2.3.1 Männliche Infertilität } & 19\end{array}$

1.2.3.2 Ätiologie männlicher Infertilität $\quad 20$

1.2.3.2.1 Störungen im Bereich der Testes $\quad 20$

1.2.3.2.2 Störungen im Bereich der ableitenden Samenwege und der
akzessorischen Geschlechtsdrüsen

1.2.3.2.3 Störungen der Samendeposition $\quad 23$

1.2.3.2.4 Störungen im Bereich des Hypothalamus und der Hypophyse 24

1.2.3.2.5 Störungen im Bereich der Androgenzielorgane 25

1.2.3.2.6 Genetische Störungen $\quad 25$

$\begin{array}{ll}\text { 1.2.3.2.7 Autoimmunreaktionen } & 26\end{array}$

$\begin{array}{ll}\text { 1.2.3.2.8 Systemische Erkrankungen } & 26\end{array}$

$\begin{array}{ll}\text { 1.2.3.2.9 Psychogene Faktoren } & 27\end{array}$

$\begin{array}{ll}\text { 1.2.3.2.10 Exogene Noxen } & 27\end{array}$

1.2.4 Mortalität und Reproduktion 29

2 Daten und Methoden 32

2.1 Forschungsleitende Hypothesen 32

2.2 Daten 32

2.2.1 Projektbeschreibung 32

2.2.2 Datengrundlage $\quad 32$

2.2.3 Patientenakten 33

2.2.4 Medizinische Parameter und Fertilitätsstatus 33 
2.2.5 Ausschluss von Fällen 34

2.2.6 Recherche 35

2.2.7 Analysierte Stichprobe 36

2.2.8 Beruf, Sozioökonomischer Index des beruflichen Status (ISEI) und $\begin{array}{ll}\text { Sozioökonomischer Status (SES) } & 37\end{array}$

2.2.9 Lebenserwartung bei Geburt 38

2.3 Datenauswertung 38

2.3.1 Verfahren zur Analyse von Ereignisdaten 38

$\begin{array}{ll}\text { 2.3.1.1 Grundbegriffe } & 39\end{array}$

2.3.1.2 Nicht-parametrische Verfahren $\quad 40$

2.3.1.3 Semi-parametrische Verfahren 41

2.3.1.4 Parametrische Verfahren 42

2.3.2 Weitere statistische Verfahren 43

2.3.3 Statistik-Software $\quad 44$

2.3.4 Irrtumswahrscheinlichkeit $p \quad 44$

3 Ergebnisse $\quad 45$

3.1 Beschreibung des Datensatzes $\quad 45$

3.1.1 Spermienkonzentration und sexuelle Karenz 51

3.1.2 Zusammenhang der Spermienkonzentration mit weiteren Faktoren 55

3.2 Lebenszeit-Mortalität und Fertilitätsstatus $\quad 58$

3.2.1 Ergebnisse der nicht-parametrischen Verfahren $\quad 58$

3.2.1.1 Fertile und Subfertile $\quad 59$

3.2.1.2 Normozoosperme, Oligozoosperme und Azoosperme 61

3.2.2 Ergebnisse der semi-parametrischen Verfahren (Cox-Regression) 65

3.2.2.1 Fertile und Subfertile $\quad 65$

3.2.2.2 Normozoosperme, Oligozoosperme und Azoosperme 66

3.2.2.3 Alternativ-Modell 1: Alter bei Untersuchung als zeitabhängige Variable 68

3.2.2.4 Alternativ-Modell 2: Sexuelle Karenz als Kontrollvariable 69

$\begin{array}{ll}\text { 3.2.2.5 Betrachtung der verstorbenen Fälle } & 70\end{array}$

3.2.3 Ergebnisse der parametrischen Regressionsmodelle $\quad 77$

$\begin{array}{ll}\text { 3.2.3.1 Exponential-Modell } & 77\end{array}$

$\begin{array}{ll}\text { 3.2.3.2 Gompertz-Makeham-Modell } & 80\end{array}$

3.2.3.3 Weibull-Modell 82

3.2.4 Validität der Trennung zwischen subfertilen und fertilen Fällen 84

3.2.4.1 Sukzessive Verschiebung der Klassifikationsgrenze 85

3.2.4.2 Klassifikation nach Guzick et al. (2001) 86

3.3 Lebenszeit-Mortalität und Spermienkonzentration 92

3.3.1 Einfluss der Spermienkonzentration auf die Lebensspanne der normo- und
oligozoospermen Fälle

3.3.2 Einfluss der Spermienkonzentration auf die Lebensspanne der oligozoospermen Fälle 93

3.3.3 Einfluss der Spermienkonzentration auf die Lebensspanne der normo-
zoospermen Fälle

3.4 Selektivität der Daten $\quad \mathbf{9 4}$

3.4.1 Vergleich von Fällen mit bekanntem und unbekannten Vitalstatus 94

3.4.2 Vergleich von Marburgern und Nicht-Marburgern 96 
$\begin{array}{ll}4 \text { Diskussion } & 98\end{array}$

$\begin{array}{lr}\text { 4.1 Datenqualität } & 98\end{array}$

4.1.1 Selektivität nach Wohnort $\quad 98$

4.1.2 Selektivität nach „Auffindbarkeit“ 99

4.1.3 Selektivität nach Sozioökonomischem Status (SES) und Ehestand 100

$\begin{array}{lr}4.2 \text { Befunddokumentation } & 101\end{array}$

4.2.1 Spermienkonzentration 102

4.2.2 Verteilung der Fertilitätsklassen 103

4.2.3 Konfundierung der Spermienkonzentration und des Fertilitätsstatus 105

4.2.4 Spermienkonzentration, Fertilitätsstatus und sexuelle Karenz 107

$\begin{array}{lr}\text { 4.3 Diskussion der Ergebnisse } & 109\end{array}$

4.3.1 Lebenszeit-Mortalität und Fertilitätsstatus 110

4.3.1.1 Ergebnisse der nicht-parametrischen Verfahren $\quad 110$

4.3.1.2 Ergebnisse der semi-parametrischen Verfahren $\quad 110$

4.3.1.2.1 Sexuelle Karenz als Kontrollvariable 111

4.3.1.2.2 Analyse der Verstorbenen 112

4.3.1.3 Ergebnisse der parametrischen Verfahren 112

$\begin{array}{ll}\text { 4.3.1.4 Abgrenzung der Subfertilen } & 114\end{array}$

4.3.2 Lebenszeit-Mortalität und Spermienkonzentrationen 116

4.3.3 Unterschiede zwischen frühen und späten Geburtsjahrgängen 117

4.4 Erklärungsansätze zum Zusammenhang zwischen männlicher Fertilität und Lebenszeit-Mortalität

4.4.1 Lebensumstände und Verhalten im Erwachsenenalter: Subfertilität bzw.

Kinderlosigkeit und Gesundheit

$\begin{array}{ll}\text { 4.4.1.1 Kinderlosigkeit und Partnerschaft } & 121\end{array}$

4.4.1.2 Kinderlosigkeit und psychisches Wohlbefinden 123

4.4.1.3 Kinderlosigkeit und Lebenszeit-Mortalität 125

4.4.2 Konditionen in utero und in der frühen Kindheit $\quad 129$

4.4.3 Genetische Ursachen 132

5 Fazit und Ausblick 136

6 Zusammenfassung 139

7 Literaturverzeichnis $\quad 143$

8 Anhang 159 


\section{Einleitung}

\subsection{Hintergrund und Ziel der Studie}

Im Gegensatz zur Frauengesundheit ist das Forschungsfeld der Morbidität und Mortalität von Männern erstaunlich wenig untersucht. Als Erklärung für dieses Phänomen wird häufig das Fehlen eines der Frauenbewegung entsprechenden Pendant in Form einer „Männerbewegung" als die Forschung voran treibende Kraft angeführt. Auch die Tatsache, dass über lange Zeiträume hinweg der männliche Organismus als Standard in der Gesundheitsforschung galt, führte nicht zu einer Wissenschaft, welche die spezifischen Gesundheitsprobleme von Männern beachtet (Kolip \& Hurrelmann, 2002). Dies spiegelt die Tatsache wider, dass sich wenige Studien gezielt mit gesundheitsbezogenen Themen bei Männern beschäftigen (Brähler et al., 2001a). In einigen Ansätzen ist in den letzten Jahren eine Wende erkennbar. So erschien in der Reihe "Jahrbuch der Medizinischen Psychologie" ein Band zum Thema „Mann und Medizin" (Brähler \& Kupfer, 2001). Im Jahr 2004 wurde der 1. Österreichische Männergesundheitsbericht veröffentlicht, mit dem Ziel, zum einen Daten zum Gesundheitszustand der männlichen Bevölkerung darzustellen, und zum anderen aber auch Erklärungen für diese zu finden (Habl et al., 2004). Ein entsprechender Bericht wird auch für Deutschland gefordert (siehe http://www.petitiononline.com/manndat5/petition.html). Des Weiteren wird zunehmend auch in anderen Werken der Gesundheit von Männern und deren spezifischen Aspekten Aufmerksamkeit zuteil (z.B. Altgeld, 2004; Kolip \& Hurrelmann, 2002). Die neueste Entwicklung dieses Trends lässt sich in Leeds, England, in Form der ersten Professur für Männergesundheit finden.

Gerade im Hinblick auf den Forschungsbereich der reproduktiven Gesundheit von Männern gibt es einigen Nachholbedarf. Das Fachgebiet der Männerheilkunde oder Andrologie als „Lehre von den Fortpflanzungsfunktionen des Mannes und ihren Störungen im engeren Sinne" (Nieschlag, 2000a; S. 1) ist eine relativ neue Disziplin. Die Ausbildung zum Andrologen kann in Deutschland bislang nur als Zusatz-Weiterbildung von Fachärzten für Haut- und Geschlechtskrankheiten oder Urologie bzw. Ärzten mit Schwerpunkt-anerkennung für Innere Medizin, Endokrinologie und Diabetologie absolviert werden (Bundesärztekammer, 2005). Traditionell ist die Andrologie in der Mehrzahl der Universitäts-kliniken Deutschlands der Dermatologie zugeordnet. Dies ergibt sich historisch daraus, dass Infertilität häufig durch Gonorrhoe verursacht wurde, und Geschlechtskrankheiten wiederum in den Behandlungsbereich der Dermatologen fallen.

Nach Vorgabe der Weltgesundheitsorganisation WHO (1994) zeichnet sich reproduktive Gesundheit dadurch aus, ,... that people are able to have a responsible, satisfying and safe sex life and that they have the capability to reproduce and the freedom to decide if, when and 
how often to do so". Helfferich \& Fichtner (2001) vermuten, dass ca. 1 bis $4 \%$ aller Männer in Deutschland von Fertilitätsstörungen betroffen ist. Generell kann der Stand der Forschung hinsichtlich der Fruchtbarkeitsstörungen bei Männern bislang als unbefriedigend betrachtet werden. Die Ätiologien für männliche Infertilität sind vielfältig, daher sind weite Bereiche nicht umfassend bzw. noch gar nicht erforscht. Ein Drittel aller Fälle gilt als idiopathisch, hier kann mit gegenwärtiger Technik keine Ursache für die Beeinträchtigung der Fruchtbarkeit nachgewiesen werden. Fertilitätsstörungen können sich auf pretestikulärer, testikulärer oder posttestikulärer Ebene auswirken. Denkbare Ursachen sind anatomische Anomalien und Pathologien, Obstruktionen und Infektionen sowie Tumore. Auch sexuelle Funktionsstörungen, hypothalamisch-hypophysäre Störungen, Androgenresistenzen, Autoimmunreaktionen, systemische Erkrankungen und psychogene Faktoren können zur Infertilität beitragen. Z.T. liegt die Unfruchtbarkeit auch in genetischen Defekten und Erkrankungen begründet. Eine wichtige Rolle spielen außerdem exogene Noxen wie z.B. Genussmittelkonsum und arbeitsbedingte Einflüsse.

Die Spermatogenese in ihrer zentralen Rolle hinsichtlich der männlichen Fruchtbarkeit wird im Rahmen der meisten der genannten Ätiologien negativ beeinflusst. Sie ist ein komplexer Prozess und stellt eines der wenigen Beispiele kontinuierlicher Proliferation von hochdifferenzierten und hochspezialisierten Zellen im menschlichen Organismus dar. Sie reagiert auf Grund ihrer raschen Reproduktion und dem hohen Differenzierungsgrad ihrer Zellen empfindlich auf Noxen und Stressoren. Der vorliegenden Studie liegt nun die Überlegung zu Grunde, dass die Spermatogenese bzw. Störungen der Spermatogenese ein Anzeichen für die Belastung des männlichen Organismus mit Noxen darstellen könnten. Werden gewisse Störungen und Vorerkrankungen ausgeschlossen, so könnte eine Beeinträchtigung der Spermatogenese, geäußert durch subnormale Spermienparameter, als Indikator für den Gesundheitszustand des männlichen Organismus angesehen werden. Daraus ergibt sich weiterhin die Konsequenz, dass Männer mit Spermatogenesestörungen aufgrund ihres schlechteren allgemeinen Gesundheitszustandes früher versterben sollten als Männer ohne solche Störungen. Es sollte sich also in diesem Fall ein Zusammenhang zwischen den Ejakulatparametern bzw. einer auf diesen beruhenden Fertilitätsklassifikation und der Lebensdauer eines Individuums, also der Lebenszeit-Mortalität, finden lassen. Eine Untersuchung dieses Zusammenhangs ist Ziel dieser Studie.

Bislang wurden Untersuchungen ähnlicher Art nach Kenntnis der Verfasserin noch nicht durchgeführt. Dies ist sicherlich nicht zuletzt der Tatsache zuzuschreiben, dass nur selten geeignete Daten vorliegen. Die Betrachtung männlicher Mortalität gerade im Zusammenhang mit Störungen, welche nicht erst im höheren Alter, sondern ebenfalls bereits in jungen Erwachsenenjahren auftreten, ist idealerweise dann möglich, wenn entsprechende 
Unterlagen bzw. valide Informationen vorliegen. Gut geeignet sind Aufzeichnungen medizinischer Untersuchungen, da diese „objektive“ Gesundheitsdaten enthalten, welche in einen Zusammenhang mit der jeweiligen Lebensdauer eines Individuums gebracht werden können. Solche Patientenakten, welche detaillierte Informationen über den Gesundheitszustand geben könnten, dürfen jedoch in Deutschland bereits nach 10 Jahren vernichtet werden und stehen somit in den seltensten Fällen für Mortalitäts-Untersuchungen zur Verfügung.

Datengrundlage der vorliegenden Arbeit sind Patientenakten von Infertilitätspatienten der andrologischen Sprechstunde des Universitätsklinikums Marburg. Es handelt sich um Spermiogramm-Analysen ab dem Untersuchungsjahr 1949. Da alle betrachteten Männer vor 1938 geboren wurden (vgl. 2.2.2 zum Einschlussverfahren), erlauben die Daten eine sinnvolle Untersuchung eines potentiellen Zusammenhangs zwischen den Ergebnissen des Spermiogramms und dem heutigen Vitalstatus („lebend“ - „verstorben“) bzw. der Lebensdauer. So kann untersucht werden, ob sich die Männer hinsichtlich ihrer LebenszeitMortalität in Abhängigkeit von ihren Spermaparametern unterscheiden.

\subsection{Stand der Forschung}

In den sich anschließenden Abschnitten soll zum einen der momentane Kenntnisstand der Forschung zur Lebenserwartung und Mortalität (vgl. 1.2.1) sowie zum anderen Forschungsergebnisse zum Thema männlicher Reproduktionsphysiologie (vgl. 1.2.2) und Infertilität (vgl. 1.2.3) vorgestellt werden. Anschließend erfolgt die Darstellung des - leider geringen - Wissensstandes hinsichtlich der Synthese der Themenkomplexe Mortalität und Fertilität (vgl. 1.2.4).

\subsubsection{Mortalität}

Seitdem im Jahr 1750 in Skandinavien die ersten nationalen Bevölkerungsdaten erhoben wurden, stehen für viele Länder bevölkerungsbezogene Informationen über die Mortalität zur Verfügung (Wilmoth, 2000). Das menschliche Mortalitätsrisiko in seinem zeitlichen Verlauf ist ein mittlerweile deskriptiv ausgiebig untersuchtes Phänomen.

\subsubsection{Lebenserwartung und Lebensspanne}

Die Lebenserwartung eines Neugeborenen in einer bestimmten Population errechnet sich als Durchschnitt aus den faktischen Lebensspannen der Angehörigen dieser Population (Wilmoth, 2000). Dank der sinkenden Kindersterblichkeit sowie der Abnahme von infektiösen 
Erkrankungen zum einen und dem medizinischen Fortschritt, z.B. bei der Behandlung von Herz-Kreislauf-Erkrankungen zum anderen, hat sich die Lebenserwartung bei Geburt in Deutschland im Laufe des letzten Jahrhunderts erheblich erhöht (Birg \& Flöthmann, 2002). Betrug sie im Jahre 1910 noch knapp 45 Jahre für einen neugeborenen Jungen, so wird einem im Jahre 2004 geborenen Jungen eine Lebenserwartung von rund 75 Jahren prognostiziert (Statistisches Bundesamt, 2004a).

Dennoch kann man bei Extrapolationen der potentiell erreichbaren Lebensspanne nicht von einer unbegrenzten Fortsetzung dieses Trends ausgehen, denn die gesundheitliche Belastung eines Individuums korreliert eindeutig mit seinem Alter. In der heutigen Zeit dominieren intrinsische Sterbeursachen wie Herzerkrankungen und bösartige Neubildungen (siehe auch 1.2.1.4), die als Anzeichen für den altersabhängigen Verlust der Funktionsfähigkeit der Zellen gesehen werden können. Auch die molekularen ReparaturMechanismen, welche die Unversehrtheit der Zellen gewährleisten, werden mit zunehmendem Alter schwächer (Carnes et al., 2003). Zu den biologischen Ursachen für das Phänomen des Alterns bzw. des Todes gibt es vielfältige Theorien. Die Überlegungen gehen z.B. von zunehmenden fehlerhaften Translations- bzw. Transkriptionsvorgängen über nachlassende Immunantworten und neuroendokrinologische Prozesse bis hin zur Begrenzung der Teilungsfähigkeit der Zellen. Die Mehrzahl der Autoren vermutet gegenwärtig, dass es sich beim Altern um multifaktorielle Prozesse handelt (Übersichten bei Troen, 2003; Weinert \& Timiras, 2003). Bisher wurden alle Vorhersagen extrem langer durchschnittlicher Lebenserwartungen von 100 Jahren und mehr von der Realität widerlegt (Carnes et al., 2003).

\subsubsection{Determinanten der Lebensspanne}

Altern und Langlebigkeit scheinen auf komplexe Art und Weise von der Fähigkeit, mit einer Vielzahl von Stressoren umzugehen, abzuhängen. Wirbeltiere haben ein immunoneuro-endokrines System entwickelt, welches trotz kontinuierlicher, intrinsischer sowie extrinsischer Störungen, deren Effekte sich im Laufe der Zeit akkumulieren, die Aufrechterhaltung der vielschichtigen Körperfunktionen erlaubt (de Benedictis et al., 2001).

Es bleibt jedoch die Frage, warum einige Individuen älter werden als andere und wodurch die Lebensdauer bestimmt wird. Zur Erklärung können individuelle Faktoren wie Lebensstil, Verhalten, sozioökonomischer Hintergrund und genetische Ausstattung herangezogen werden: Die Lebensspanne ist eine multifaktorielle quantitative Eigenschaft, welche durch genetische und Umweltfaktoren beeinflusst wird (de Benedictis et al., 2001). 


\subsection{Vererbbarkeit der Lebensspanne}

In einer Studie an dänischen Zwillingen konnte gezeigt werden, dass Langlebigkeit nur mäßig vererbbar ist: Bei Männern kann 26\% der Unterschiede in den Lebensspannen der genetischen Variation zugeschrieben werden (Herskind et al., 1996). Die übrigen Unterschiede in der Lebensdauer von Männern müssen somit durch „extrinsische“ Faktoren erklärt werden. Die „Umwelt“ selbst spielt eine große Rolle beim Überleben. Es ist allerdings auch bekannt, dass Gen-Umwelt-Interaktionen die Lebensspannen in großem Maße modulieren können (Finch \& Tanzi, 1997; Tan et al., 2001). Als Beispiel kann hier die Phenylketonurie genannt werden, eine genetisch bedingte Stoffwechselerkrankung, die ohne Behandlung zu psychomotorischer Retardierung führt. Bei entsprechender phenylalaninarmer Diät kann jedoch eine normale Lebensspanne erreicht werden (Christensen \& Vaupel, 1996). Vaupel und Kollegen (1998) konstatieren, dass die Variation der Lebensspanne des Menschen abgesehen von den genetischen Faktoren etwa zu einem Viertel durch die Bedingungen, welche pränatal bzw. in der frühen Kindheit herrschen, bestimmt wird. Die verbleibende Hälfte der Variation könne den Umständen und Umweltbedingungen im Erwachsenenalter zugeschrieben werden.

\subsection{Lebensstil und Lebensspanne}

Als Lebensstil-Faktoren, welche differentiell die Sterblichkeit beeinflussen, kommen zunächst Rauchen, Alkohol sowie die Ernährung in Betracht. In einem Mortalitäts-Follow-up des Nationalen Befragungssurveys der Deutschen Herz-Kreislauf-Präventionsstudie (DHP) wurde die Bedeutung des Rauchens als Risikofaktor für die Mortalitätsentwicklung verdeutlicht. Bei 50-Jährigen betrug die mittlere fernere Lebenserwartung der Nichtraucher 33 Jahre, während sie bei starken Rauchern um etwa 10 Jahre reduziert war. Des Weiteren wurden als Ursachen für die unterschiedliche Sterblichkeit der Männer noch der Bluthochdruck sowie die Anzahl chronischer Erkrankungen ermittelt (Helmert et al., 2002).

Auch der Einfluss anderer Konsumgewohnheiten lässt sich deutlich darstellen. Sowohl das absolute Körpergewicht als auch der Body-Mass-Index korrelieren - in moderatem Ausmaß - negativ mit der Lebenserwartung (Fontaine et al., 2003; Samaras et al., 2002; Stevens et al., 2002). Hier sind vor allem kardiovaskuläre Erkrankungen für die Mortalität bedeutsam. Eine Studie an älteren Personen bestätigte einen positiven Zusammenhang zwischen einer gesunden Ernährungsweise (z.B. häufiger Konsum von Vitamin $C$ und Milchprodukten) und der Lebenserwartung (Fortes et al., 2000). Ebenso erhöht Alkoholmissbrauch in allen Lebensaltern das Mortalitätsrisiko und führt zu höheren Risiken für Tod durch Unfall, Suizid, kardiovaskuläre Erkrankungen und bösartige Neubildungen (Banks et al., 2000; Rossow \& Amundsen, 1997; Thomas \& Rockwood, 2001). Alkoholmissbrauch ist zusätzlich oft assoziiert mit psychischen Erkrankungen (Petrakis et al., 2002). 
Es wird ebenfalls diskutiert, ob intrauterine und perinatale Bedingungen sich auf die Mortalität im höheren Lebensalter und somit auf die Lebensspanne auswirken (Christensen \& Vaupel, 1996). Eine interessante Beobachtung ist in diesem Zusammenhang die Abhängigkeit der Lebensdauer vom Geburtsmonat. Die Betrachtung einer großen nordrheinwestfälischen Stichprobe von in den Jahren 1984 und 1999 verstorbenen Männern ergab, dass jene Männer, welche von Mai bis Juli geboren waren, ein geringeres Sterbealter aufwiesen als solche, deren Geburtstag in die Monate Oktober, November und Dezember datierte (Lerchl, 2004). Die Unterschiede umfassten Zeiträume von bis zu einem Jahr. Solche jahreszeitlichen Effekte wurden auch für andere Länder, wie z.B. Österreich, Dänemark, USA und Australien, bestätigt (Doblhammer, 2004). Die zu Grunde liegenden Mechanismen sind noch unklar, jedoch vermutet die Autorin, dass die Bedingungen in utero entscheidend sind. Der Gesundheitszustand der Mutter sei essentiell; deren Fehlernährung und infektiöse Erkrankungen wirkten sich negativ aus. Für im Frühling geborene Kinder falle diese Phase in eine Zeit unzureichender mütterlicher Ernährung, während die Wachstumsphase der im Herbst geborenen Kinder durch eine Zeit mit ausreichender Versorgung gekennzeichnet sei. Ferner sei bekannt, dass die Umweltbedingungen in der frühen Lebensphase die spätere Anfälligkeit für infektiöse wie chronische Erkrankungen - und somit auch die Mortalität - beeinflussen (Doblhammer, 2004; Doblhammer \& Vaupel, 2001). Es ist davon auszugehen, dass auch die heute geborenen Kohorten von diesen unterschiedlichen Lebensspannen in Abhängigkeit vom Geburtsmonat betroffen sein werden (Doblhammer, 2004).

Allgemein bleibt $\mathrm{zu}$ beachten, dass gesundheitsrelevantes Verhalten meist mit dem sozioökonomischen Status variiert. Die mit diesen Lebensstil-Variablen in Zusammenhang gebrachten Unterschiede in der Mortalität sind wohl in einem noch höheren Ausmaß auf Einkommens-, Berufs- und Ausbildungsunterschiede, d.h. also auf "harte" soziostrukturelle Variablen zurückzuführen (Mielck, 2000; Mueller \& Heinzel-Gutenbrunner, 2001)

\subsection{Sozioökonomische und soziale Einflussfaktoren}

Verschiedene Studien weisen auf einen Einfluss sozialer Faktoren auf die Gesundheit (z.B. Mueller \& Heinzel-Gutenbrunner, 2001) sowie auch auf die Mortalität hin (z.B. WittwerBackofen, 1999; Übersicht über internationale Studien in Hradil, 1997). Zuerst sei hier die berufliche Stellung zu nennen. Es lässt sich ein deutlich erhöhtes Sterberisiko für Männer mit niedriger beruflicher Position zeigen (Geyer \& Peter, 1999; Klein, 1993b). Die Beziehung zwischen beruflichem Status und Mortalität wird als Konsequenz unterschiedlicher Arbeitsbedingungen, verbunden mit psychosozialen Belastungen und Gesundheitsrisiken am Arbeitsplatz, diskutiert (Geyer \& Peter, 1999; Peter, 2001), aber auch mit Wohlstandsunterschieden zwischen den diversen Berufspositionen in Zusammenhang gebracht (Luy, 
2002a; Klein, 1993a; b). Aufschlussreich sind in dieser Hinsicht auch die Ergebnisse von Studien an US-amerikanischen Armee-Angehörigen. In dieser Population sind die sozioökonomischen Unterschiede vernachlässigbar, so dass der ermittelte positive Zusammenhang zwischen der Lebenserwartung und dem erreichten militärischen Rang auf die psychosozialen Faktoren des beruflichen Erfolges zurückgeführt wird (Biegel, 2003; Walter, 2004).

Das Einkommen stellt ebenfalls einen wichtigen Prädiktor für die Mortalität dar (Geyer \& Peter, 2000). Dass einkommensschwächere Personen früher versterben, konnte nicht nur für Deutschland gezeigt werden (Klein \& Unger, 2001; Voges, 1996; Voges \& Schmidt, 1996). Analysen der Daten des Sozioökonomischen Panels (SOEP) weisen darauf hin, dass der Grundstein für den Zusammenhang zwischen Einkommen und Gesundheit bereits im Kindesalter gelegt wird. Hier schlagen sich materielle Einschränkungen sowie psychosoziale Belastungen nieder (Heinzel-Gutenbrunner, 2001).

Auch ein Einfluss der Bildung (Schulbildung/berufliche Ausbildung) auf die Mortalität lässt sich zeigen. In einer Follow-up-Studie des Forschungsprogramms „Monitoring Trends and Determinants in Cardiovascular Disease" (MONICA) der WHO fand man, dass sich bei Männern das Sterberisiko mit jedem Ausbildungsjahr um 7,6\% verminderte. Dieser Zusammenhang wird darauf zurückgeführt, dass das gesundheitsrelevante Verhalten mit dem Bildungsniveau korreliert: Höher Gebildete nehmen häufiger ärztliche Vorsorge- und Versorgungsleistungen in Anspruch, Rauchen weniger, betreiben mehr Sport und ernähren sich gesünder (Klein et al., 2001). Ein Zusammenhang kann hier auch mit den höheren körperlichen Belastungen und den stärker gesundheitsgefährdenden Umständen der schlechter (Berufs)Ausgebildeten bei der Erwerbsarbeit hergestellt werden (Stolpe, 2001).

In der bereits genannten Analyse der SOEP-Daten von Klein (1993a; b) fand sich ebenfalls ein Zusammenhang zwischen Sterblichkeit und dem Familienstand. Das Sterberisiko bei geschiedenen Männern ist 2,1fach höher, das bei verwitweten Männern 1,5fach erhöht im Vergleich zu verheirateten Männern. Dieser Zusammenhang konnte in anderen Studien, z.B. auch an männlichen Krankenversicherten, bestätigt werden (Baumann et al., 1998; Helmert \& Shea, 1998; Helmert et al., 2002).

Abschließend bleibt zu bemerken, dass noch immer wenig über die Determinanten der Lebensdauer bekannt ist. Auch wenn auf Populationsebene einige mit der Mortalität assoziierte Faktoren wie Rauchen, sozioökonomische Gegebenheiten oder genetische Ausstattung identifiziert werden konnten, so sind diese Zusammenhänge jedoch zu schwach, um auf Individualebene reliable Prädiktoren der Lebensdauer eines Menschen darzustellen (Christensen \& Vaupel, 1996). 


\subsubsection{Geschlechtstypische Mortalität}

Die durchschnittliche Lebenserwartung für ein neugeborenes Mädchen war im Jahre 2004 mit 81,1 Jahren im Vergleich zu einem neugeborenen Jungen um 6 Jahre erhöht (Statistisches Bundesamt, 2004a). Dieser Unterschied ergibt sich zum einen aus der geringeren Säuglingssterblichkeit von Mädchen $(5,8 \%$ bei weiblichen Neugeborenen gegen $7,7 \%$ bei männlichen Neugeborenen; Kolip, 1998). Zum anderen ist in allen Lebensaltern die Mortalität der Männer gegenüber jener der Frauen erhöht (Merbach et al., 2002; Schmidt, 2002). Historisch betrachtet hat sich die höhere Sterblichkeit der Männer allerdings erst rezent entwickelt (Wittwer-Backofen, 1999).

Für die geringere Lebenserwartung der Männer werden verschiedene Ursachen diskutiert (Übersichten bei Kolip, 1998; Luy, 2002a; 2002b; Wittwer-Backofen, 1999): Erstens werden biologische bzw. genetische Faktoren ins Feld geführt, welche den Frauen einen Überlebensvorteil verschaffen sollen (z.B. Christensen et al., 2000). Wie schon lange bekannt, unterscheiden sich die beiden Geschlechter in ihren hormonellen und immunologischen Eigenschaften. Männer besitzen nur ein X-Chromosom und sind somit vermutlich anfälliger für rezessiv vererbte Krankheiten und allgemein weniger resistent gegenüber infektiösen Erkrankungen, da das X-Chromosom einige immunregulatorische Gene (z.B. für die IgM-Produktion) trägt. Ebenso schützt das weibliche Sexualhormon Östrogen $\left(E_{2}\right)$ Frauen vor koronaren Herzerkrankungen - tendenziell zeigen Frauen ein günstigeres LDL/HDLVerhältnis und leiden seltener an Arteriosklerose - während erhöhte $E_{2}$-Level bei Männern das Risiko für koronare Herzerkrankungen sogar noch steigern (Gyllenborg et al., 2001; Hazzard, 1986; Waldron, 1983). Eine besondere Rolle scheinen die männlichen Sexualhormone zu spielen, welche vermutlich für eine Beschleunigung der Stoffwechselprozesse, eine höhere Infektionsanfälligkeit und für einen verstärkten Alterungsprozess verantwortlich gemacht werden können (Wittwer-Backofen, 1999). Die hohen AndrogenLevel von Männern werden zusätzlich ins Feld geführt, um zu erklären, warum Männer häufiger Opfer von tödlichen Unfällen bzw. gewaltsamem Tod werden: Die höheren Testosteron-Level begünstigen offenbar riskante Verhaltensweisen (risk taking) und führen zu häufigeren Unfällen (Waldron, 1983; 1995). Bereits seit längerem wurde vermutet, dass die biologischen Ursachen für eine Lebenserwartungsdifferenz von 1 bis 2 Jahren zugunsten der Frauen verantwortlich sein könnten (Pressat, 1973). Vergleichbare Ergebnisse erbrachte eine jüngere Studie, welche die geschlechtsspezifischen Sterblichkeitsunterschiede in Allgemein- und Klosterbevölkerung verglich (Dinkel \& Luy, 1999; Luy, 2002a).

Zweitens weisen verhaltens- bzw. umweltorientierte Erklärungsansätze darauf hin, dass Frauen sich gesundheitsbewusster verhalten als Männer und weniger umweltspezifischen Risiken ausgesetzt sind. Vor allem der Lebensstil kommt hier zum Tragen. Männer trinken 
mehr Alkohol und rauchen häufiger als Frauen. Ebenso nehmen sie seltener an Vorsorgeuntersuchungen teil (Brähler et al., 2001a). Hier sollte die Bedeutung des Rauchens als Beitrag zur Entwicklung von Lungenkrebs sowie koronaren Herzerkrankungen betont werden (Case \& Paxson, 2005; Hazzard, 1986; Waldron, 1993; 1995). Auch üben Männer häufiger gefährliche und gesundheitsgefährdende Berufe aus (Bründel \& Hurrelmann, 1999).

Bei beiden Geschlechtern zeigt sich ein sozialer Gradient in der Mortalität, abhängig von Variablen wie Bildung, Einkommen oder Beruf. Jedoch sind die Ergebnisse hinsichtlich Stärke des Zusammenhangs ist, nicht eindeutig, und es bleibt unsicher, in welchem Ausmaß sich dieser Gradient auf geschlechtsspezifische Mortalitätsunterschiede auswirkt (Babitsch, 2001).

\subsubsection{Todesursachen von Männern}

Schließlich bleibt im Rahmen der männlichen Mortalität noch darzustellen, welche Krankheiten letztendlich für die Todesfälle verantwortlich gemacht werden können. Betrachtet man die Todesursachen, so heben sich zwei Kategorien quantitativ deutlich ab. Erstens spielen mit zunehmendem Individualalter Kreislauf-Erkrankungen eine immer bedeutendere Rolle. Nach Angaben des Statistischen Bundesamts (2004b) konnten im Jahr 2002 über 41\% der Todesfälle auf Krankheiten des Herz-Kreislauf-Systems zurückgeführt werden (siehe Tab. 1.1).

Tab. 1.1 Ausgewählte Ursachen für männliche Todesfälle nach der ICD-10 im Jahr 2002 (Statistisches Bundesamt, 2004b; eigene Überarbeitung)

\begin{tabular}{|c|c|c|}
\hline Todesursachen nach ICD-10 & $\begin{array}{c}\text { Anzahl } \\
\text { Todes- } \\
\text { fälle }\end{array}$ & $\begin{array}{l}\text { Anteil an } \\
\text { Gesamtzahl } \\
\text { d. } \\
\text { Todesfälle }\end{array}$ \\
\hline Alle Krankheiten + Folgen äußerer Ursachen (A00-T98) & 389.116 & $100 \%$ \\
\hline \multicolumn{3}{|l|}{ davon: } \\
\hline $\begin{array}{l}\text { Krankheiten des Kreislaufsystems (I00-I99) } \\
\text { z.B. Ischämische Herzkrankheiten (I20-I25) } \\
\text { z.B. Sonstige Formen der Herzkrankheit (I30-I52) } \\
\text { z.B. Zerebrovaskuläre Krankheiten (I60-I69) }\end{array}$ & $\begin{array}{r}160.629 \\
77.395 \\
33.099 \\
28.331\end{array}$ & $\begin{array}{r}41,3 \% \\
19,9 \% \\
8,5 \% \\
7,3 \%\end{array}$ \\
\hline $\begin{array}{l}\text { Neubildungen (C00-D48) } \\
\text { z.B. Bösartige Neubildung d. Verdauungsorgane (C15-C26) } \\
\text { z.B. Bösartige Neubildung d. Atmungsorgane + sonstiger } \\
\text { intrathorakaler Organe (C30-C39) } \\
\text { z.B. Bösartige Neubildung d. männlichen Genitalorgane (C60-C63) }\end{array}$ & $\begin{array}{r}112.245 \\
35.878 \\
30.420 \\
11.762\end{array}$ & $\begin{array}{r}\mathbf{2 8 , 8} \% \\
9,2 \% \\
7,8 \% \\
3,0 \%\end{array}$ \\
\hline Krankheiten des Atmungssystems (J00-J99) & 27.562 & $7,1 \%$ \\
\hline $\begin{array}{l}\text { Verletzungen, Vergiftungen + bestimmte andere Folgen äußerer } \\
\text { Ursachen (S00-T98) }\end{array}$ & 21.437 & $5,5 \%$ \\
\hline Krankheiten des Verdauungssystems (K00-K93) & 21.177 & $5,4 \%$ \\
\hline
\end{tabular}


Die zweite große Rolle haben Neubildungen inne; auf sie sind fast $29 \%$ aller Sterbefälle zurückzuführen. Hier zeichnen sich vor allem bösartige Neubildungen sowohl der Verdauungs- als auch der Atmungsorgane als wesentlich ab. Eine besondere Bedeutung kommt dem Lungenkrebs zu: Im Jahre 2000 konnten z.B. ca. 27\% der männlichen KrebsTodesfälle auf Lungenkrebs zurückgeführt werden (Arbeitsgemeinschaft Bevölkerungsbezogener Krebsregister in Deutschland, 2004). Immerhin 3\% aller Männer versterben aufgrund von bösartigen Neubildungen der Genitalorgane - mit hervor ragender Bedeutung des Prostata-Krebs.

\subsubsection{Fertilität}

Spermatogenese und Ejakulatqualität sind von zentraler Bedeutung für die männliche Fertilität. Um Infertilität als Phänomen zu betrachten, ist es zunächst unerlässlich, erstens die physiologischen und ontogenetischen Vorgänge zu verstehen, zweitens die Erstellung eines Spermiogramms mit der sich daraus ergebenden Fertilitätsklassifikation darzustellen sowie drittens die Variabilität der Spermaqualität zu diskutieren.

\subsubsection{Männliche Reproduktionsbiologie}

Die folgende Darstellung der wesentlichen physiologischen und ontogenetischen Abläufe orientiert sich - sofern nicht anders angegeben - an den Ausführungen von Bergmann (1998) und Weinbauer et al. (2000).

\subsection{Generelle Organisation des Hodens}

Der Hoden (Testes) erfüllt 2 wesentliche Aufgaben: In inm findet die Spermatogenese statt, während derer die männlichen Gameten entstehen, und er produziert den weitaus größten Teil der männlichen Geschlechtshormone (Steroidogenese). Er besteht aus mehreren Läppchen (Lobuli testis), welche die Samenkanälchen (Tubuli seminiferi) enthalten. Im Interstitium zwischen den Samenkanälchen finden sich Blut- und Lymphgefäße, Nervenfasern sowie die Testosteron-produzierenden Leydig-Zellen. In den Kanälchen selbst befindet sich das Keimepithel, welches aus Keimzellen in unterschiedlichen Entwicklungsstadien sowie den somatischen Sertoli-Zellen besteht. Letztere sind im adulten Stadium teilungsinaktiv und erfüllen mehrere Aufgaben. Nicht zuletzt läuft u.a. entlang der SertoliZellen die Entwicklung der Spermien ab. Das Keimepithel wird von einer Basallamina umschlossen. 


\subsection{Ontogenese}

Für die männliche Geschlechtsbestimmung ist das SRY-Gen (sex-determining region - $Y$ chromosome) auf dem Y-Chromosom essentiell. Wird es aktiviert, beginnt die männliche Geschlechtsentwicklung: Die inneren männlichen Geschlechtsorgane entwickeln sich aus den zunächst undifferenzierten embryonalen Keimdrüsen sowie den Wolffschen Gängen (Urnieren-Gänge). Erstere werden zu Hoden, aus letzteren entstehen Samenbläschen, Nebenhoden und Vas deferens. Die Hodenanlage wandert im Laufe der Embryonalentwicklung von ihrer ursprünglichen Position hinter dem Bauchfell in den Skrotalsack ab. Dieser Descensus testis ist im Regelfall bis zum 8. Schwangerschaftsmonat abgeschlossen. Die äußeren Genitale entwickeln sich aus dem ebenfalls noch undifferenzierten Genitalhöcker (Frohneberg, 2001; Martínez Portillo et al., 2001; Parker et al., 1999).

Die Geschlechtsdifferenzierung des männlichen Embryos wird weitestgehend durch den Einfluss der Androgene bestimmt. Testosteron aus den Leydig-Zellen wird in Dehydrotestosteron (DHT) umgewandelt und sorgt in dieser Form ab der 8. Schwangerschaftswoche für die Entwicklung des männlichen Phänotyps. Auch die Sertoli-Zellen sind entscheidend für die männliche Geschlechtsentwicklung. Sie produzieren im fötalen Hoden das Anti-MüllerHormon (AMH), welches für die Degeneration der Müllerschen Gänge essentiell ist (Frohneberg, 2001; Parker et al., 1999). Die Anzahl der Sertoli-Zellen nimmt bis zum 15. Lebensjahr zu und bestimmt die Hodengröße und die Anzahl der beim Erwachsenen produzierten Spermien.

Während der Pubertät entwickeln sich die sekundären Geschlechtsmerkmale, die Hoden nehmen an Volumen zu und ein verstärktes Längenwachstum setzt ein. Zu Beginn der Pubertät kommt es, vorwiegend nachts, zu einer Reaktivierung der pulsatilen $\mathrm{GnRH}-$ Ausschüttungen, welche schon während der Fötalphase und im frühen Säuglingsalter vorhanden waren (Roth \& Partsch, 1999). Der Zeitpunkt des Pubertätsbeginns ist interindividuell sehr variabel. Man geht jedoch von einer Entwicklungsverzögerung aus, wenn sich bis zum Alter von 14 Jahren noch keinerlei Pubertätszeichen finden lassen (Behre et al., 2000b; Traggiai \& Stanhope, 2002).

\subsection{Spermatogenese}

Die erste Phase der Spermatogenese beginnt mit den Stammzellformen der Keimzellen, den Spermatogonien, an der Basallamina. Man unterscheidet hier zunächst 2 verschiedene Formen: Spermatogonien des Typs A „dark" zeigen wenig bis keine mitotische Aktivität und verbleiben als Stammzellen. Spermatogonien des Typs A „pale" hingegen teilen sich mitotisch und werden somit zu Spermatogonien des Typs B (siehe Abb. 1.1). 
In einer zweiten Phase verlassen die B-Spermatogonien die Basallamina und durchlaufen die Prophase einer Meiose, welche durch eine DNA-Reduplikation gekennzeichnet ist. So entstehen Spermatozyten I. Ordnung (primäre Spermatozyten). Während des weiteren Verlaufs der Meiose entstehen durch die 1. Reifeteilung aus einer Spermatozyte I. Ordnung zwei Tochterzellen: die Spermatozyten II. Ordnung (sekundäre Spermatozyten) Anschließend entstehen in der 2. Reifeteilung die Spermatiden. Aus ursprünglich einer Stamm-Keimzelle haben sich vier Spermatiden entwickelt. Diese haben eine runde Form und teilen sich nicht mehr.

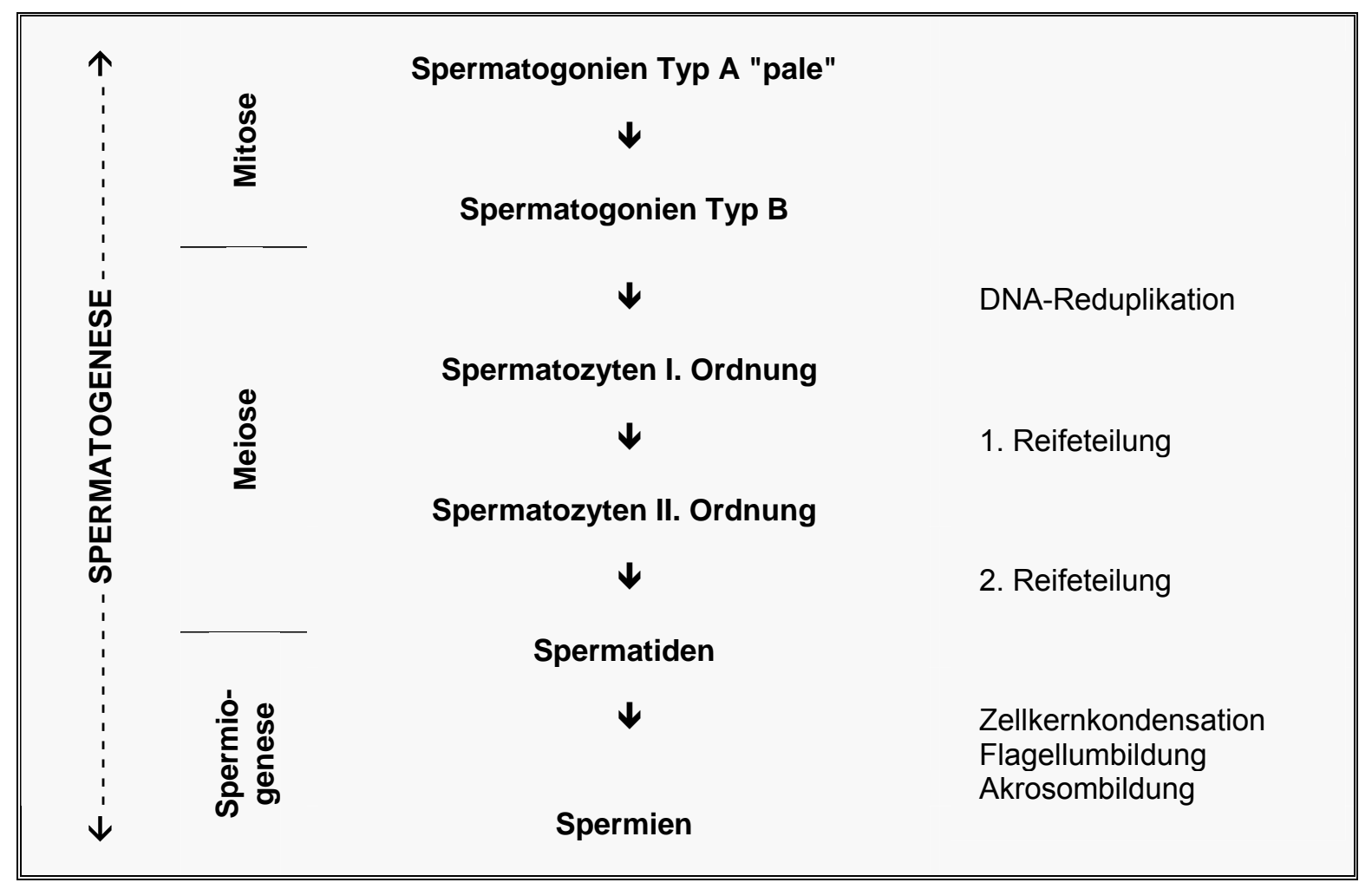

\section{Abb. 1.1 Spermatogenese mit unterschiedlichen Keimzellstadien}

Die dritte Phase wird als Spermiogenese bezeichnet und ist durch verschiedene Differenzierungsprozesse gekennzeichnet, welche sich größtenteils gleichzeitig vollziehen. Zum einen kondensiert der Zellkern, zum anderen bilden sich ein Akrosom und eine Geißel (Flagellum) aus. Aus den runden Spermatiden entstehen längliche Spermien oder Spermatozoen, welche aus Kopf und Flagellum bestehen. Am Spermienkopf findet sich das für die Befruchtung der Eizellen essentielle Akrosom; außerdem enthält er die kondensierte DNA. Das Flagellum wird von einem Axonem mit einer typischen „9+2-Struktur" der Tubuli durchzogen und dient der Fortbewegung der Spermien. Der gesamte Ablauf der Spermatogenese benötigt mindestens 64 Tage. 


\subsection{Hormonelle Steuerung der Spermatogenese}

Die Spermatogenese wird über die Hypothalamus-Hypophysen-Gonaden-Achse wie folgt reguliert: Der Hypothalamus setzt pulsatil das Hormon GnRH (Gonadotropin Releasing Hormon) in das Pfortadersystem frei, welches auf die Adenohypophyse wirkt und dort wiederum Synthese und Freisetzung der beiden Gonadotropine Luteinisierendes Hormon (LH) und Follikel-stimulierendes Hormon (FSH) induziert. FSH dient der Anregung der Spermatogenese über die Sertoli-Zellen, während LH die Leydig-Zellen zur TestosteronProduktion stimuliert.

Spermienproduktion und Androgen-Synthese werden zum einen durch einen endokrinen Regelkreis beeinflusst: Testosteron kann die LH- und FSH-Freisetzung hemmen. Zusätzlich kann die FSH-Sekretion über eine negative Rückkopplung durch Inhibin B beeinflusst werden. Zum anderen werden die Funktionen des Hodens zusätzlich lokal, z.B. über verschiedene Wachstums- oder immunologische Faktoren, reguliert. Testosteron ist jedoch auch hier der wichtigste lokale Regulator, welcher auf die Keimzellreifung in den Samenkanälchen wirkt (Holdcraft \& Braun, 2004; Wennemuth \& Aumüller, 1998).

\subsection{Weitere Entwicklung der Spermien und Ejakulat-Zusammensetzung}

Die Spermien verlassen den Hoden und gelangen, zusammen mit den Sekreten der Sertoli-Zellen, in den Nebenhoden (Epididymis). Dort durchlaufen sie Reifungsprozesse, welche für ihren weiteren Weg sowohl im männlichen als auch im weiblichen Reproduktionstrakt bis zum Erreichen der Eizelle essentiell sind. Durch den Kontakt mit dem Nebenhodensekret entwickeln die Spermien ihr Potenzial zur Motilität, sie erhalten einen Schutz gegen Proteolyse und Angriffe des weiblichen Immunsystems und werden fertilisationsfähig. Eine besondere Rolle spielen hier verschiedene sezernierte Glykoproteine, welche an die Spermien-Oberfläche binden und die Spermien-Ei-Interaktion sicherstellen (Cooper, 2000; Cooper \& Yeung, 2000; Seitz, 1998).

Für die Nebenhodenpassage benötigen die Spermien ungefähr weitere 12 Tage. Sie verbleiben im Nebenhoden, bis sie ejakuliert werden. Auf ihrem anschließenden Weg werden sie dann durch den Ductus deferens und den Ductus ejaculatorius in die Samenharnröhre transportiert. Sie sind in einem Sekret, dem Seminalplasma, suspensiert, dessen Gesamtvolumen zu 60-70\% von den Bläschendrüsen und zu 30-40\% von der Prostata sezerniert wird und verschiedene Aufgaben wie Transport, „Ernährung“ und Schutz der Spermien erfüllt. Hierzu tragen unterschiedliche Sekretionsprodukte bei: Die Bläschendrüsen produzieren Fruktose, Prostaglandine und Seminogelin. Aus der Prostata stammen Zink, saure Phosphatase, Zitronensäure sowie Prostata-spezifisches Antigen (PSA), die 
Nebenhoden produzieren Carnitin, Glycerophosphocholin und $\alpha$-Glucosidase (Chughtai et al., 2005; Cooper \& Yeung, 2000; Henkel, 1998).

\subsubsection{Ejakulatanalyse und Spermiogramm}

Im Rahmen der Infertilitätsberatung werden verschiedene Ejakulat- und Hormonparameter ermittelt (Übersicht bei Behre et al., 2000b; Haidl \& Krause, 1998). Erst vor wenigen Jahren wurden einheitliche Laborrichtlinien entwickelt (WHO, 1992; 1999), welche eine standardisierte Spermiogramm-Analyse gewährleisten, und so die Evaluation und den Vergleich der Untersuchungen zwischen verschiedenen Labors ermöglichen.

Zum „Routine-Spermiogramm", dessen konventionelle Analyse durch mikroskopische Untersuchungen erfolgt, gehören zunächst die drei Standardparameter Spermienkonzentration, -morphologie und -motilität (Krause, 1993). Die Spermienkonzentration, auch Spermiendichte genannt, wird mittels Zählkammermethode nach einer Verdünnung des Ejakulates zur Immobilisierung der Spermien bestimmt und ergibt sich als Anzahl der Samenzellen pro Volumeneinheit Ejakulat (Köhn \& Haidl, 2002). Mit Hilfe des Ejakulatvolumens lässt sich auch die gesamte Spermienanzahl näherungsweise berechnen. Die Motilität hingegen wird im Nativpräparat beurteilt. Sie wird als Anteil der motilen Samenzellen sowie durch einige Qualitäten ihrer Bewegung erfasst (Haidl \& Krause, 1998). Man unterscheidet hier nach den WHO-Laborrichtlinien (1999) vier verschiedene Kategorien: a) schnelle, progressive Bewegungen, b) langsame, progressive Bewegungen, c) nichtprogressive Bewegungen und d) Immotilität. Die Analyse der Motilität beinhaltet das Problem der Subjektivität der Beurteilung und hängt von der Erfahrung der Untersucher ab (Keck \& Wetterauer, 1998). Zur Betrachtung der Morphologie werden die Spermien angefärbt und jeweils die Spermatozoenköpfe, Hälse und Mittelstücke sowie die Spermienschwänze auf Defekte hin untersucht, und der Anteil der morphologisch anormalen und normalen Spermatozoen ermittelt (Keck \& Wetterauer, 1998; Köhn \& Haidl, 2002). Auch bei der Beurteilung der Morphologie treten große inter- und intraindividuelle Varianzen auf (Eustache \& Auger, 2003; Franken et al., 2000). Heutzutage wird mittels Computer-assistierter Samenanalyse (CASA) versucht, die Erfassung der Ejakulat-Parameter zu objektivieren.

Weitere Analysen werden in Form von Spermienfunktionstests durchgeführt. Sie geben Aufschluss über Integrität und Funktionalität der Spermatozoen sowie über ihre Fähigkeit zur Bindung an und Interaktion mit der Eizelle. So kann z.B. die Integrität der Spermienmembran, die tubuläre Mikrostruktur des Spermienschwanzes oder die funktionelle Kapazität des Akrosoms untersucht werden (Keck \& Wetterauer, 1998). 
$\mathrm{Zu}$ untersuchende Ejakulateigenschaften sind Volumen, pH-Wert, Verflüssigungszeit und Konsistenz. Sie können Hinweise auf funktionelle Störungen der akzessorischen Geschlechtsdrüsen, auf Entzündungen der Prostata oder der Bläschendrüsen und auf Obstruktionen der ableitenden Samenwege geben (Keck \& Wetterauer, 1998; Köhn \& Haidl, 2002). Auch das Auftreten und die Quantität von Spermatogenesezellen, Leukozyten, Makrophagen, Erythrozyten, Epithelzellen und Entzündungsmarkersubstanzen wie z.B. Interleukin 6 oder 8 und Reaktiven Sauerstoffspezies (ROS) kann untersucht werden (Köhn \& Haidl, 2002).

Immunologische Untersuchungen befassen sich mit dem Auftreten von Agglutinationen der Spermien, sowie dem Nachweis von Spermatozoen-Antikörpern auf der SpermatozoenOberfläche. Mit Hilfe endokrinologischer Untersuchungen kann die Produktion von Testosteron, GnRH sowie den Gonadotropinen LH und FSH bestimmt und somit Hinweise auf Störungen der Hypothalamus-Hypophysen-Gonaden-Achse gewonnen werden. Ebenfalls können Konzentrationen von Prolaktin, Inhibin und Sexualhormon-bindendem Globulin (SHBG) von Interesse sein (Köhn \& Haidl, 2002).

Tab. 1.2 Normalwerte einiger Ejakulatparameter nach den Richtlinien der WHO (1999)

\begin{tabular}{|l|l|}
\hline Ejakulatvolumen & $\geq 2 \mathrm{ml}$ \\
\hline $\mathrm{pH}$ & $\geq 7,2$ \\
\hline Spermienkonzentration & $\geq 20 \times 10^{6}$ Spermatozoen / ml \\
\hline gesamte Spermienzahl & $\geq 40 \times 10^{6}$ Spermatozoen / Ejakulat \\
\hline Motilität & $\begin{array}{l}\geq 50 \% \text { Spermatozoen mit Vorwärtsbeweglichkeit (Kategorien } \\
\text { a }+\mathrm{b} \text { oder } \\
\end{array}$ \\
& $\begin{array}{l}\geq 25 \% \text { Spermatozoen mit schneller progressiver Motilität } \\
\text { (Kategorie a) }\end{array}$ \\
\hline Morphologie & $\geq 30 \%$ normal geformte Spermatozoen ${ }^{a}$ \\
\hline
\end{tabular}

${ }^{a}$ Normwerte für Morphologie in den WHO-Richtlinien von 1999 nicht mehr enthalten; Angaben aus den WHOLaborrichtlinien von 1992 entnommen

Tab. 1.3 Nomenklatur für normale und pathologische Befunde der Spermiogramm-Analyse nach den Richtlinien der WHO (1999)

\begin{tabular}{|l|l|}
\hline Normozoospermie & normale Ejakulatbefunde (siehe Tab. 1.2) \\
\hline Oligozoospermie & $<20 \times 10^{6}$ Spermatozoen / ml \\
\hline Asthenozoospermie & $\begin{array}{l}<50 \% \text { Spermatozoen mit Vorwärtsbeweglichkeit (Kategorien } \\
\text { a }+\mathrm{b} \text { ) oder } \\
<25 \% \text { Spermatozoen mit schneller progressiver Motilität } \\
\text { (Kategorie a) }\end{array}$ \\
\hline Teratozoospermie & $<30 \%$ normal geformte Spermatozoen ${ }^{\text {a }}$ \\
\hline Oligoasthenoteratozoospermie & Kombination aus den 3 zuvor genannten Defekten \\
\hline Azoospermie & keine Spermatozoen im Ejakulat vorhanden \\
\hline Aspermie & kein Ejakulat \\
\hline Parvisemie & Volumen $<2$ ml \\
\hline $\begin{array}{l}\text { a Normwerte für Morphologie in den WHO-Richtlinien von 1999 nicht mehr enthalten; Angaben aus den WHO- } \\
\text { Richtlinien von 1992 entnommen }\end{array}$
\end{tabular}


Einige heute gängige klinische Normwerte bzw. pathologische Nomenklaturen laut WHO (1999) werden in den Tabellen 1.2 und 1.3 dargestellt. Die Festlegung der Normalwerte und die Bedeutung der Spermienparameter als prognostische Faktoren für die männliche Fertilität werden zurzeit noch diskutiert (Chia et al., 1998; Krause, 1993; Menkveld et al., 2001; Ombelet et al., 1997). Speziell für die in vivo-Situation fehlen genauere Befunde zu Normal- und Minimalwerten hinsichtlich der Konzentration, Morphologie und Motilität der Spermien (Menkveld et al., 2001; Ombelet et al., 1997). Jedoch wurde bereits sehr früh darauf hingewiesen und in rezenten Studien bestätigt, dass sich Unterschiede zwischen fertilen und subfertilen Populationen häufig erst dann zeigen, wenn zwei oder alle drei Spermienparameter von erniedrigten Werten betroffen sind (Falk \& Kaufman, 1950; Ombelet et al., 1997). Für eine erfolgreiche Schwangerschaft scheinen niedrigere Ejakulatwerte nötig zu sein, als bisher von der WHO angenommen. So geben Menkveld und Kollegen (2001) zur Unterscheidung von fertilen und subfertilen Männern bezüglich des Anteils morphologisch normaler Zellen einen Grenzwert von nur 3\% an. Des Weiteren sehen die Autoren den Grenzwert der Motilität bei einem Anteil beweglicher Zellen von lediglich 20\%. Hinsichtlich der Spermienkonzentration wurde ein Grenzwert von 13,5 Mio./ml zur Abgrenzung der subfertilen Fälle ermittelt (Guzick et al., 2001).

Der zeitliche Abstand zwischen zwei Ejakulationen kann verschiedene Ejakulatparameter beeinflussen. Nach mehreren kurz aufeinander folgenden Ejakulationen lässt sich auch bei fertilen Männern eine reduzierte Spermienkonzentration in Form einer Oligozoospermie feststellen (Keck \& Wetterauer, 1997). So werden als sexuelle Karenzzeit vor Messung 2-7 Tage empfohlen (WHO, 1999). Jedoch auch innerhalb dieser empfohlenen Periode variieren die Spermienparameter Motilität und Morphologie in Anhängigkeit von den abstinenten Tagen (Elzanaty et al., 2005). Neueste Analysen empfehlen für oligozoosperme Patienten sogar eine sexuelle Abstinenz von nur einem Tag, da so verbesserte Werte hinsichtlich der Motilität und Morphologie der Spermien erreicht werden könnten (Levitas et al., 2005).

\subsubsection{Ejakulatqualität als zeitliches und räumliches Phänomen}

Die Qualität des Ejakulates ist keine konstante Eigenschaft, sondern kann sowohl intraindividuell als auch global gesehen Schwankungen und Veränderungen unterliegen.

\subsection{Intraindividuelle Variabilität der Ejakulatqualität}

Die Ejakulatqualität kann intraindividuell nicht unbeträchtlich variieren (Köhn \& Haidl, 2002). Erstens zeigen sich Unterschiede im Tagesverlauf mit höheren Spermienkonzentrationen und besserer Beweglichkeit am Nachmittag im Vergleich zum Vormittag (Cagnacci et al., 1999). Eine mögliche Erklärung wird nicht in der Spermatogenese selbst gesehen, sondern in einer funktionalen Schwankung der neuro-muskulären Mechanismen, 
welche die Ejakulation steuern und damit auch für unterschiedliche Spermienkonzentrationen im Ejakulat verantwortlich sein könnten. Die unterschiedliche Beweglichkeit ergibt sich vermutlich durch Einflüsse von Komponenten des Spermas wie z.B. Hormone (Cagnacci et al., 1999).

Zweitens ist die Ejakulatqualität eines Mannes auch im Jahresverlauf vermutlich nicht konstant, sondern weist saisonale Unterschiede auf. Henkel und Kollegen (1998) konnten in einer Studie mit deutschen Patienten der andrologischen Sprechstunde zeigen, dass sowohl die Spermatozoenkonzentration als auch die Chromatinkondensation jahreszeitlich schwankt. Die Spermatozoendichte ist in den Frühlingsmonaten am höchsten und in den Herbstmonaten am niedrigsten, während die Chromatinkondensation in positivem Zusammenhang zu den jahreszeitlichen Temperaturen steht. Für weitere Parameter wie Motilität, Akrosinaktivität und Akrosomreaktion wurden keine Schwankungen festgestellt. Diese Befunde konnten in US-amerikanischen, spanischen, dänischen und israelischen Studien bestätigt werden (Andolz et al., 2001; Fisch et al., 1997; Gyllenborg et al., 1999; Yogev et al., 2004).

Offen bleibt die Frage, ob zwischen diesen Befunden und den jahreszeitlichen Geburtenschwankungen ein Zusammenhang bestehen könnte. Während die Geburtenschwankungen der nördlichen Hemisphäre mit den höchsten Geburtenzahlen in den Frühlingsmonaten und den niedrigsten Raten in den Monaten Oktober bis Dezember (Lam \& Miron, 1996; Levine, 1999; Rojansky et al., 1992) nicht zu den Schwankungen der Spermienkonzentrationen zu passen scheinen, konnte für den US-Bundesstaat Minnesota eine Korrelation zwischen der Spermienkonzentration und der jahreszeitlichen Geburtenrate aufgezeigt werden (Fisch et al., 1997). Als mögliche Ursachen für die jahreszeitabhängige verminderte Spermaqualität wird die größere Hitze während der Sommermonate diskutiert; eine Spermatogenese kann nur dann optimal ablaufen, wenn eine gewisse, 2 bis $4{ }^{\circ} \mathrm{C}$ unterhalb der übrigen Körpertemperatur liegende Skrotaltemperatur gewährleistet wird (vgl. 1.2.3.2.10). Eine größere Bedeutung wird jedoch der Dauer der Photoperiode zugesprochen. Für niedere Säugetiere ist eine jahreszeitliche Schwankung der Melatoninsekretion in Abhängigkeit von der Dauer des Tageslichtes bekannt. Dies kann vermutlich wiederum die Sekretion von $\mathrm{LH}$ und $\mathrm{FSH}$ und somit die Spermatogenese in Abhängigkeit von der Jahreszeit beeinträchtigen (Fisch et al., 1997; Gyllenborg et al., 1999). Vermutlich spielt ein solcher Mechanismus auch bezüglich der menschlichen Reproduktion eine gewisse Rolle (Kennaway, 2005). Allerdings gibt es ebenfalls Befunde, die auf eine starke Konfundierung der Saisonalität der Geburten durch soziodemographische Faktoren hindeuten (Bobak \& Gjonca, 2001). 
Drittens ist an eine Abnahme der Ejakulatqualität mit dem Alter zu denken. Während für Frauen eine Abnahme der Fertilität mit steigendem Alter bekannt ist, bleibt beim alternden Mann dagegen die Zeugungsfähigkeit grundsätzlich erhalten (Rolf \& Nieschlag, 2000). Jedoch kann auch die männliche Spermaqualität einige Veränderungen erfahren (Übersichten bei ESHRE Capri Workshop Group, 2005; Kühnert \& Nieschlag, 2004): Mit steigendem Alter lassen sich Abnahmen des Ejakulatvolumens, der Spermien-Motilität sowie des Anteils der normalgeformten Spermien feststellen. Kein eindeutiger Einfluss des Alters zeigt sich hinsichtlich der Spermienkonzentration (Eskenazi et al., 2003; Jung et al., 2002; Kidd et al., 2001). Mögliche Gründe sind zum einen zelluläre oder physiologische Veränderungen des urogenitalen Traktes mit zunehmenden Lebensjahren, zum anderen aber auch die mit dem Alter steigende Wahrscheinlichkeit der reproduktiven Schädigung durch exogene Noxen und Erkrankungen (Eskenazi et al., 2003).

\subsection{Globale Variabilität der Ejakulatqualität}

In ihrer viel beachteten Metaanalyse von Studien, welche Spermiogramme von Männern ohne anamnestisch bekannte Fertilitätsstörung zum Inhalt hatten, kommen Carlsen und ihre Kollegen (1992) zu dem alarmierenden Schluss, dass sich im Zeitraum von 1938 bis 1990 die durchschnittliche Spermienkonzentration und das Ejakulatvolumen signifikant reduziert haben. Als mögliche Ursachen für die Verschlechterung werden in die Umwelt gelangte Östrogene und östrogenartig wirksame Verbindungen zur Diskussion gestellt, welche sich auf die fötale Entwicklung während der Schwangerschaft auswirken könnten (Sharpe \& Skakkebæk, 1993).

Zahlreiche Einwände hinsichtlich der Probenauswahl sowie der statistischen Auswertung der dargestellten Studie wurden gemacht (Haidl, 1999; Köhn \& Schill, 1996; Nieschlag, 1998; Olsen et al., 1995), so dass die Ergebnisse angezweifelt werden können. Zudem sind die Aussagen verschiedener anderer Studien, welche sich empirisch mit der Veränderung der Spermienqualität über die Zeit befassen, nicht einheitlich (Irvine, 2000). Einige Studien bestätigen eine vermeintliche Abnahme der Ejakulatqualität, während andere keine Verschlechterung bzw. sogar eine Verbesserung der Spermien-Parameter feststellen konnten (Auger et al., 1995; Berling \& Wolner-Hanssen, 1997; Fisch et al., 1996; Gyllenborg et al., 1999; Paulsen et al., 1996).

In einer erneuten Analyse konnten Fisch und Gobuloff (1996) zeigen, dass sich die von Carlsen et al. (1992) gefundenen Ergebnisse auch dadurch erklären lassen, dass die herangezogenen Daten aus unterschiedlichen Ländern stammten. Auch einige weitere Studien deuten auf geographische Unterschiede hin. So wurde für dänische Männer eine deutlich schlechtere Spermienqualität festgestellt als für Männer aus Finnland. Französische 
und schottische Männer bewegten sich im Mittelfeld (Andersen et al., 2000; Jørgensen et al., 2001; Richthoff et al., 2002). Sogar ein europäischer Ost-West-Gradient mit besserer Spermienqualität in Finnland und Estland und schlechteren Werten in Dänemark und Norwegen wird postuliert (Jørgensen et al., 2002). Auch regionale Variationen in der Spermaqualität lassen sich aufzeigen: Bei Männern aus verschiedenen französischen Städten zeigen sich signifikant unterschiedliche Werte der Standard-Parameter (Auger \& Jouannet, 1997). Als mögliche Ursachen für solche Unterschiede werden Lebensstil- und Umweltfaktoren sowie endokrine Disruptoren (vgl. 1.2.3.2.10) diskutiert, wobei jedoch auch genetische Differenzen nicht ausgeschlossen werden können (Irvine, 2000; Jørgensen et al., 2001).

Interessanterweise zeigen sich beim Auftreten von Hodenkrebs ähnliche geographische Variationen, mit der höchsten Inzidenz in Dänemark und der niedrigsten Inzidenz in Litauen; insgesamt zeichnen sich die osteuropäischen Länder durch niedrigere Hodenkrebs-Raten aus (Adami et al, 1994). Diese synchronen Trends in Europa bezüglich Ejakulatqualität und Hodenkrebs könnten auf gemeinsame Risikofaktoren, wie z.B. die Östrogen-Exposition in utero, hinweisen (Joffe, 2001; Møller \& Shakkebæk, 1999).

\subsubsection{Infertilität}

Ein Paar wird als infertil bezeichnet, wenn bei regelmäßigem, ungeschütztem Geschlechtsverkehr nach einem Jahr keine Schwangerschaft eingetreten ist. Die Prävalenz ungewollter Kinderlosigkeit kann bislang nur eingeschränkt erfasst werden (Strauß \& Beyer, 2004). Schätzungen zufolge bleiben in Deutschland $15-20 \%$ der Paare im reproduktionsfähigen Alter ungewollt kinderlos, jedoch wird diese Zahl als zu hoch angesehen: Die Raten für Westdeutschland bzw. Ostdeutschland liegen eher unter 10\% bzw. unter 5\% (Brähler et al., 2001b). Nach Angaben der WHO (1987) können die Ursachen dieser Kinderlosigkeit zu $38 \%$ Fertilitätsstörungen der Frau und zu 20\% Fertilitätsstörungen des Mannes zugeschrieben werden. Bei $27 \%$ der Paare liegt die Ursache bei beiden Partnern und bei $15 \%$ kann überhaupt keine Störung diagnostiziert werden.

\subsubsection{Männliche Infertilität}

Es sei bemerkt, dass in den verschiedenen Publikationen und auch im deutschen Sprachgebrauch der Begriff der Infertilität synonym sowohl für tatsächliche Unfähigkeit, ein Kind zu zeugen, als auch für Subfertilität, bei welcher die Zeugungsfähigkeit generell erhalten bleibt, verwendet wird. Die beiden Begriffe werden also häufig nicht klar voneinander getrennt. Generell muss man zwischen primärer und sekundärer Infertilität unterscheiden, wobei im 
letzteren Falle die Zeugungsfähigkeit erst nach einer bzw. mehreren Schwangerschaft/en der Partnerin/nen auftritt. Die sekundäre Infertilität ist dahingehend von Bedeutung, dass angeborene Störungen hier eher unwahrscheinlich sind (Köhn \& Haidl, 2002).

Nach Internationalem Menschenrecht, dem auch die Rechtssprechung der meisten Staaten folgt, wird die männliche (wie auch die weibliche) Reproduktion als ein Grundrecht erachtet (Universal Declaration of Human Rights, Art. 16; http://www.unhchr.ch/udhr/ lang/eng/htm). Folgt man der WHO, welche Gesundheit als vollständiges körperliches, psychisches und soziales Wohlbefinden definiert, so kann männliche Infertilität durchaus als Krankheit betrachtet werden. Zum einen lassen sich objektivierbare, körperliche Symptome feststellen, zum anderen kann ungewollte Kinderlosigkeit das seelische Wohlbefinden beeinträchtigen und wird zudem häufig als soziale Benachteiligung erlebt (Köhn \& Schill, 2000).

\subsubsection{2 Ätiologie männlicher Infertilität}

Generell kann der Stand der Forschung hinsichtlich der Fruchtbarkeitsstörungen bei Männern als bislang unbefriedigend betrachtet werden, und die Ursachen männlicher Infertilität sind, wie zu Beginn des einleitenden Kapitels bereits erwähnt, noch nicht hinreichend untersucht. Lediglich bei $70 \%$ aller Infertilitäts-Patienten liegt eine definitive Diagnose vor, bei den übrigen 30\% kann keine nachweisbare Ursache festgestellt werden; man spricht hier von idiopathischer Infertilität. In der Hauptzahl dieser Fälle erscheinen jedoch - noch nicht erkannte - genetische Aberrationen als zugrunde liegende Ursache plausibel (Huynh et al., 2002; Krausz \& Forti, 2000).

Im Folgenden werden einige der möglichen Ursachen männlicher Infertilität dargestellt. Dies ist zum einen wichtig, um gewisse Vorgehensweisen in der vorliegenden Studie und ihren Methoden verstehen zu können. Zum anderen wird dieses Kapitel aber auch verdeutlichen, wie empfindlich die Vorgänge der Reproduktionsbiologie sind und auf welch vielfältige Weise die Spermatogenese und die Ejakulatqualität beeinträchtigt werden können. Aufgrund des großen Umfangs potentieller Kausalitäten bleibt die Beschreibung auf ausgewählte Krankheitsbilder und Noxen beschränkt. Generell kann unterschieden werden, ob die Infertilität in pretestikulär, testikulär oder posttestikulär wirksamen Ursachen begründet liegt. Die hier gewählte Einteilung der physiologischen und genetischen Ursachen basiert auf der Klassifikation nach Nieschlag \& Behre (2000).

\subsection{Störungen im Bereich der Testes}

Testikuläre Störungen treten häufig in Form von Varikozelen, also varikösen Veränderungen der Venen des Plexus pampiniformis im Skrotum, auf. Ihre Prävalenz beträgt 
$5 \%$ bis $20 \%$ (Nieschlag et al., 2000a). Als Auswirkungen der Varikozelen auf die Fertilität werden verschiedene Mechanismen diskutiert wie eine verminderte Durchblutung des Hodens aufgrund des hohen venösen Drucks mit daraus folgender Hodenatrophie, Erhöhung der Skrotaltemperatur (vgl. 1.2.3.2.10) oder unzureichender Abtransport oder Reflux von toxischen Verbindungen aus der Niere (Benoff \& Gilbert, 2001; Nieschlag, 2000b). Allerdings lässt sich kein für Varikozele-Patienten typisches, einheitliches Spermiogramm-Muster finden, und nicht alle Männer mit Varikozelen sind von einer reduzierten Fertilität und schlechten Sperma-Parametern betroffen. Varikozelen schließen somit Zeugungsfähigkeit nicht aus (Hauser et al., 2001; Redmon et al., 2002; Schreiber \& Wilmer, 1998). Des Weiteren haben die meisten Studien an subfertilen Patienten bislang auch bei Behandlung der Varikozelen keine Verbesserung der Sperma-Parameter nachweisen können (Krause et al., 2002), so dass die Varikozele nicht zwangsläufig als Ursache der reduzierten Fertilität angesehen werden muss.

Eine weitere Rolle im Zusammenhang mit Infertiliät spielen Lageanomalien der Testes. Die Wanderung der Hoden in den Skrotalsack während der letzten zwei Schwangerschaftsmonate (Descensus testis) sollte bei der Geburt abgeschlossen sein. Der Descensus kann jedoch in verschiedenen Stadien zum Stillstand kommen; die Lageanomalien dieses Maldescensus testis (auch Hodendystopie) werden je nach Position des Hodens unterschieden. Die Ursache für die Lageanomalien ist noch ungeklärt, jedoch zeigen sie sich mit einer Prävalenz von zwei bis drei Prozent aller termingerechten Geburten. In ca. einem Drittel dieser Fälle erfolgt ein spontaner Abstieg in den ersten drei Lebensmonaten. Ein Maldescensus testis tritt häufig bei endokrinen Störungen oder im Zusammenhang mit anderen Diagnosen wie Klinefelter- oder Noonan-Syndrom auf (Martínez-Portillo et al., 2001; Nieschlag et al., 2000a). Bei Betroffenen mit Hodenfehllagen sind im Erwachsenenalter die Risiken sowohl für Hodentumore als auch für Hodentorsionen massiv erhöht. Außerdem führt eine Hoden-Fehllage zur Schädigung des Keimepithels. Auch bei einer nur unilateralen Lageanomalie kann die Störung oft nicht ausgeglichen werden: 50 bis $70 \%$ der Betroffenen mit einseitigem Kryptorchismus sind oligozoo- bzw. azoosperm (Hermanns \& Ringert, 1998; Martínez-Portillo et al., 2001; Nieschlag et al., 2000a).

Schließlich seien Hodentumore erwähnt, welche sich ebenfalls negativ auf die Fruchtbarkeit auswirken. Während der letzten vierzig bis fünfzig Jahre ist die Inzidenz von Hodentumoren aus bislang ungeklärten Gründen global um das drei- bis vierfache gestiegen, wobei starke geographische und ethnische Unterschiede festzustellen sind (vgl. 1.2.2.3.2, Adami et al., 1994; Nieschlag et al., 2000a). Die einzigen gesicherten Risikofaktoren für einen Hodentumor sind der Maldescensus testis sowie ein bereits vorliegender kontralateraler Hodentumor (Albers, 2000). Insgesamt treten Hodentumore bei Infertilitäts- 
patienten mit einer Prävalenz von 1:200 auf (Nieschlag et al., 2000). Etwa 50\% der Hodentumor-Patienten weisen bereits vor der Tumordiagnose ein pathologisches Spermiogramm auf (Hobisch et al., 1998). Zum einen kann ein ätiologischer Zusammenhang z.B. in Form von Expositionen gegenüber endokrinen Disruptoren in der fötalen Phase bestehen (Petersen et al., 1998), und auch ein genetischer Zusammenhang zwischen der Infertilität und dem Auftreten des Hodentumors wird vermutet (Albers, 2000). Zum anderen kann sich der Tumor direkt auf die Fertilität auswirken. So können Tumoren zu einer verminderten $\mathrm{LH}$ - und $\mathrm{FSH}-$ Produktion führen, sie können durch ihre Wucherung das keimzellproduzierende Gewebe verdrängen oder behindern, sie können für eine testikuläre Temperaturerhöhung verantwortlich gemacht werden, und im Falle einer Öffnung der BlutHoden-Schranke kann es zur Ausbildung von Anti-Spermien-Antikörpern kommen (Albers, 2000). Therapiebedingte Infertilität hingegen kann durch chirurgische Eingriffe, Chemotherapie und Radiatio auftreten und sich in einem Ejakulationsverlust bzw. einer Schädigung des Keimepithels äußern (siehe auch 1.2.3.2.10, Albers, 1998; Hobisch et al., 1998).

\subsection{Störungen im Bereich der ableitenden Samenwege und der akzessorischen Geschlechtsdrüsen}

Im Bereich der ableitenden Samenwege und der akzessorischen Geschlechtsdrüsen spielen vor allem urogenitale Infektionen eine wichtige Rolle. Solche Infektionen können die Urethra (in Form einer Urethritis), die Prostata (Prostatitis), die Samenbläschen (Vesikulitis) sowie Hoden (Orchitis) und Nebenhoden (Epididymitis) betreffen, oder auch als Kombinationen, z.B. als Epididymoorchitis, auftreten (Haidl \& Weidner, 2002). Sie können von verschiedenen Erregern, z.B. Chlamydia trachomatis und Neisseria gonorrhoeae, verursacht werden (Diemer et al., 2000; Gonzales et al., 2004; Paavonen \& Eggert-Kruse, 1999). Die häufigste Ursache einer Hoden-/ Nebenhodenentzündung ist eine Infektion mit Mumps-Viren; ca. ein Viertel der Infizierten entwickelt eine Orchitis (Jarow, 2003). Auch Tuberkulose kann in ihrer urogenitalen Form eine Epididymoorchitis verursachen (Haidl \& Weidner, 2002).

Eine Beeinflussung der männlichen Fertilität im Falle einer Entzündung kann z.B. durch Zytokine stattfinden, welche die testikulären Zellen, speziell Leydig-Zellen und das Keimzellepithel, sowie die Funktionen der Spermatozoen beeinflussen (Diemer et al., 2000). Ebenfalls kommt es bei einer Entzündung zu oxidativem Stress durch pathologische Mengen von reaktiven Sauerstoffverbindungen, welche zu Beeinträchtigungen von Spermatozoenfunktionen wie Motilität und Akrosomreaktion und zu oxidativen Schädigungen der SpermienDNA führen (Haidl \& Weidner, 2002; Ochsendorf, 1999). Die Entzündungsreaktion kann zusätzlich zu Obstruktionen der ableitenden Samenwege (siehe unten) und zur Bildung von Anti-Spermien-Antikörpern führen (Diemer et al., 2000). In Einzelfällen kann es bei einer 
Epididymoorchitis zur Zerstörung der Samenkanälchen sowie einer Hodennekrose kommen (Diemer et al., 2000). Im Falle einer Prostatitis kann neben Spermatogenese- und Spermienfunktionsstörungen auch eine sekretorische Dysfunktion induziert werden, welche eine Veränderung der Drüsensekrete des Ejakulates bewirkt (Weidner et al., 1999; Diemer et al., 2000). In Folge dessen kann die Liquifizierungsfähigkeit des Spermas reduziert und der pH-Wert erhöht sein (Comhaire et al., 1999).

Ungefähr 5\% der Infertilitätspatienten leiden unter einem Verschluss der ableitenden Samenwege (Popken et al., 1998). Es können Nebenhoden, Ductus deferens sowie Ductus ejaculatorius betroffen sein. Beidseitige vollständige Verschlüsse der ableitenden Samenwege führen zur Azoospermie, eine einseitige Obstruktion kann vollständig kompensiert werden, und ein partieller Verschluss führt zu unterschiedlichen Ausprägungen einer Oligozoospermie (Behre et al., 2000a). Eine Obstruktion kann kongenital auftreten, z.B. als kongenitales beidseitiges Fehlen des Vas deferens, bei welchem es sich um eine genitale Form der Cystischen Fibrose handelt (Stuhrmann, 1998). Sekundär ursächlich sind häufig akute oder chronische Entzündungen der Nebenhoden, der Prostata und der Samenbläschen, Zysten oder auch iatrogene Maßnahmen wie z.B. Herniotomien (besonders im Kindesalter) oder chirurgische Eingriffe im Bereich des Ductus ejaculatorius (Knuth \& Schulze, 2001; Popken et al., 1998).

\subsection{Störungen der Samendeposition}

Bei der Betrachtung von sexuellen Funktionsstörungen muss zwischen Störungen in Form von Penisveränderungen, Erektions- und Ejakulationsstörungen unterschieden werden. Angeborene anatomische Penisveränderungen können in Form von Hypospadien bzw. Epispadien vorliegen, bei welchen die Harnröhre nicht an der Spitze, sondern auf der Unterseite bzw. Oberseite des Penis ausmündet (van Ahlen \& Hertle, 2000). Die Ätiologie von Hypospadien kann nur in einem Drittel der Fälle geklärt werden; die Anomalie wird u.a. mit Hormoneinnahmen während der Schwangerschaft, komplexen genetischen Syndromen oder abnormalen Karyotypen in Zusammenhang gebracht; Hypospadie-Fälle treten jedoch familiär gehäuft auf (Boehmer et al., 2001). Ebenfalls kann es zu Phimosen und Deviationen kommen. Letztere können auch sekundär erworben werden (van Ahlen \& Hertle, 2000).

Erektile Dysfunktionen wurden lange Zeit hauptsächlich psychogenen Gründen zugeschrieben. Heute geht man in $50 \%$ bis $80 \%$ der Fälle von einer organischen Ursache aus (van Ahlen \& Hertle, 2000). Ätiologisch lassen sich vaskuläre Störungen, z.B. in Form von Arteriosklerose und ischämischen Herzerkrankungen, neurogene Ursachen wie Verletzungen der Wirbelsäule und zerebrale Erkrankungen sowie endokrin bedingte Störungen wie z.B. Hyperprolaktinämie unterscheiden. Ebenfalls können sich Medikamente 
negativ auf die Erektionsfähigkeit auswirken. Zusätzlich sind einige systemische Erkrankungen wie Diabetes mellitus mit erektiler Dysfunktion assoziiert (Fazio \& Brock, 2004). Auch Rauchen stellt einen wichtigen negativen Einflussfaktor dar (Natali et al., 2005). Schließlich nimmt die Häufigkeit der Erektionsstörungen mit dem Alter zu (Madersbacher et al., 2003).

$\mathrm{Zu}$ den Ejakulationsstörungen werden retrograde sowie fehlende Ejakulationen (Anejakulation oder Aspermie) gezählt. Während bei Anejakulation der Samenerguss vollständig ausbleibt, wird bei retrograden Ejakulationen das Ejakulat nicht antegrad ausgestoßen, sondern teilweise bis vollständig in die Blase entleert (Denil, 1998; Kamischke \& Nieschlag, 1999). Nach idiopathischen Störungen sowie Störungen durch Verletzungen der Wirbelsäule folgt Diabetes mellitus als dritthäufigste Ursache (Jarow, 2003). Funktionelle Ejakulationsstörungen können sich als frühzeitige Ejaculatio praecox sowie verzögerte Ejaculatio tarda zeigen (Denil, 1998).

\subsection{Störungen im Bereich des Hypothalamus und der Hypophyse}

Infertilität kann ebenfalls durch hypothalamisch-hypophysäre Störungen hervorgerufen werden, welche sich in Form eines Hypogonadismus, also als Unterfunktion der Gonaden, auswirken. Dies bedeutet bei Männern eine endokrine oder auch exokrine Störung der Hodenfunktion mit folgendem Testosteronmangel bzw. Beeinträchtigung der Spermatogenese.

Der hypogonadotrope (zentrale) Hypogonadismus zeigt sich als Störung der Gonadotropin-Sekretion, er kann erworben oder angeboren sein. Erworbene Formen können u.a. durch Tumore, systemische Erkrankungen (z.B. Leberzirrhose, chronische Niereninsuffizienz oder AIDS) sowie medikamentös hervorgerufen werden (Übersicht in Bliesener et al., 2001). Die häufigsten angeborenen Formen des hypogonadotropen Hypogonadismus sind der idiopathische hypogonadotrope Hypogonadismus $(\mathrm{HH})$ und das eng verwandte Kallmann-Syndrom (Bliesener et al., 2001). Der IHH zeigt sich in einer Störung der GnRHSekretion des Hypothalamus mit daraus folgender mangelnder hypophysärer FSH- und LHProduktion, ohne dass anatomische Ursachen erkennbar sind. Sowohl die Reifung der Keimzellen als auch eine ausreichende gonadale Testosteron-Produktion sind verhindert (Behre et al., 2000b).

Hypothalamisch-hypophysäre Störungen können sich ebenfalls in einer verzögerten Pubertät (Pubertas tarda) auswirken. Von einer Pubertas tarda spricht man, wenn die zur Pubertätsentwicklung gehörende Genitalentwicklung bzw. das Hodenwachstum bei einem Alter von 14 Jahren noch nicht begonnen hat (Traggiai \& Stanhope, 2002). Es bleibt 
ungeklärt, wodurch genau die Pubertätsentwicklung ausgelöst wird, jedoch ist gesichert, dass in verzögerten Fällen die pulsatile GnRH-Sekretion (vgl. 1.2.2.1.4) verspätet einsetzt (Behre et al., 2000b).

\subsection{Störungen im Bereich der Androgenzielorgane}

Die Wirkung der Androgene wird über Androgenrezeptoren (AR) vermittelt, welche in den Zielgeweben sowohl im Zytoplasma als auch in den Zellkernen vorkommen (Ochsenkühn \& de Kretser, 2003). Verschiedene Mutationen der AR-codierenden Gensequenz auf dem XChromosom führen zu Rezeptor-Defekten und somit zu einer Androgenresistenz (Hiort \& Holterhus, 2003; Ochsenkühn \& de Kretser, 2003; Yong et al., 2003). Die Konsequenz ist erstens eine verminderte Virilisierung während der embryonalen Entwicklung. Rudimente weiblicher innerer Genitale können erhalten und das äußere Genitale kann männlich oder weiblich sein (Hiort \& Holterhus, 2003; Hiort et al., 1999; Meschede et al., 2000). Zweitens kann im Erwachsenenalter eine Androgenresistenz für männliche Infertilität verantwortlich gemacht werden, da Androgene, vor allem Testosteron, für die Funktion der Sertoli-Zellen sowie für die Spermienreifung unabdingbar sind (Hiort \& Holterhus, 2003; Yong et al., 2003).

\subsection{Genetische Störungen}

Bei infertilen Männern werden 12mal häufiger Chromosomenaberrationen gefunden als im Durchschnitt bei Neugeborenen (6\% vs. 0,5\%; van Assche et al., 1996). Dabei sind die Geschlechtschromosomen häufiger betroffen als die Autosomen (Brugh III et al., 2003). Es lassen sich strukturelle und numerische Aberrationen unterscheiden. Y-chromosomale Mikrodeletionen werden als die häufigste genetische Ursache für Azoospermie bzw. Oligozoospermie angesehen; sie wurden in verschiedenen internationalen Studien bei ca. $3 \%$ bis $18 \%$ der infertilen Männer gefunden (Übersicht in Meschede \& Horst, 1997). Sie führen zu Beeinträchtigungen der Spermatogenese vom Spermatogenesearrest bis zum Sertoli-Cell-Only-Syndrom (auch Germinalzellaplasie genannt), also dem vollständigen Fehlen von Keimzellen (Ferlin et al., 2004; Huynh et al., 2002; Kim et al., 1999). Die endokrine Funktion der Hoden wird durch die Y-Mikrodeletionen nicht beeinflusst (Nieschlag et al., 2000a). Neuere Untersuchungen haben gezeigt, dass zusätzlich zu den Deletionen auch weitere strukturelle Veränderungen wie Inversionen und Duplikationen zur Verminderung der Fertilität beitragen (Vogt, 2005).

Bei ca. 3,5\% der infertilen Männer lässt sich das Klinefelter-Syndrom finden (van Assche et al., 1996), welches sich durch einen 47,XXY-Karyotyp auszeichnet (Nieschlag et al, 2000a). Die Betroffenen leiden unter Hypotestosteronämien unterschiedlichen Ausmaßes mit ernsten Beeinträchtigungen der Spermatogenese, meist in Form einer Azoospermie (Amory et al., 2000). Eine weitere numerische Chromosomenaberration stellt dass XX-Mann- 
Syndrom mit dem weiblichen Karyotyp 46,XX dar. Ungefähr $80 \%$ dieser Fälle können durch die Translokation des Testis-determinierenden Gens SRY (vgl. 1.2.2.1.2) vom Y-Chromosom auf ein X-Chromosom erklärt werden (Krausz \& Forti, 2000). Jedoch ist es wahrscheinlich, dass auch noch andere der am Prozess der Geschlechtsdifferenzierung beteiligten Gene involviert sind (Birnbacher \& Frisch, 1995). Trotz des weiblichen Karyotyps sind die Betroffenen phänotypisch männlich mit männlichen Geschlechtsorganen (Nieschlag et al., 2000a), zeichnen sich jedoch durch Gynäkomastie, kleine Testes sowie Azoospermie aus (Krausz \& Forti, 2000).

\subsection{Autoimmunreaktionen}

Die männlichen Keimzellen exprimieren Oberflächenantigene, welche vom Immunsystem als körperfremd erkannt werden. Der Kontakt zwischen Spermien und immunkompetenten Zellen wird normalerweise durch die so genannte Blut-Hoden-Schranke, welche die SertoliZellen in den Hodentubuli bilden, verhindert (Bohring \& Krause, 2003; Mazumdar \& Levine, 1998; Weinbauer et al., 2000). Aufgrund von Entzündungen wie Orchitis und akuter Epididymitis, Traumata, testikulären Biopsien, Tumoren oder steigendem hydrostatischen Druck infolge von Obstruktionen kann es zu einer Störung dieser Blut-Hoden-Schranke kommen (McLachlan, 2002). Kommen die Spermien mit immunkompetenten Zellen in Berührung, werden Anti-Spermien-Antikörper gebildet, welche für eine reduzierte SpermienVitalität und -Motilität, eine verschlechterte Fähigkeit der Spermien zur Mukus-Penetration, gestörte Ei-Spermium-Interaktionen sowie eine Beeinträchtigung der Ei-Einnistung und der embryonalen Entwicklung verantwortlich gemacht werden und somit die Fertiliät beeinträchtigen können (Bohring \& Krause, 2000; 2003; Mazumdar \& Levine, 1998; McLachlan, 2002).

\subsection{Systemische Erkrankungen}

Einer Infertilität können zudem systemische Erkrankungen zugrunde liegen. So können z.B. von akutem bzw. chronischem Versagen von Organen wie Nieren oder Leber betroffene Männer temporär bzw. dauerhaft unter testikulärer Atrophie, reduzierter Spermatogenese, Infertilität, sexueller Dysfunktion und Gynäkomastie leiden. Auch Erkrankungen maligner, neurologischer, gastrointestinaler, hämatologischer, endokriner, immunologischer, kardiovaskulärer und dermatologischer Art, chronische Erkrankungen der Atemwege wie z.B. Cystische Fibrose (vgl. 1.2.3.2.2) sowie Infektionskrankheiten können in mehr oder weniger großem Ausmaß zu reduzierter Fertilität führen (Übersicht in Handelsman, 2000). Die Zeugungsfähigkeit kann direkt durch die Erkrankung selbst, z.B. als Folge von erhöhter Temperatur, aber auch durch therapeutische Maßnahmen, z.B. als Folge von Bestrahlungen oder Medikamenten, beeinflusst werden. 


\subsection{Psychogene Faktoren}

Vor dem Hintergrund der Stresstheorie und psychobiologischer Ansätze entstand die Annahme, dass sich eine subjektiv belastende Stresssituation auf die körperliche Verfassung auswirken und z.B. über nervöse und endokrinologische Störungen eine Infertilität hervorrufen kann (Hurst \& Dye, 2000; Oberpenning et al., 2000). Es muss beachtet werden, dass Infertilität nicht nur durch (chronische) Stressbelastungen bedingt sein, sondern selbst auch als Stressor fungieren kann (Henning \& Strauß, 2000). Infertilität kann als emotional belastend empfunden werden, was wiederum zu einer Reduktion des Selbstwertgefühls sowie der Lebensqualität führen kann (Hurst \& Dye, 2000; Strauß \& Beyer, 2004). Generell kann man von einem interaktiven Zusammenhang zwischen Fertilitätsstörungen und psychischen Belastungen ausgehen (Henning \& Strauß, 2000).

Bislang wurden Störungen und Ätiologien vorgestellt, welche ihren Ursprung im Organismus selbst hatten. Jedoch kann die Fruchtbarkeit auch durch von außen einwirkende Faktoren beeinflusst werden, wie im Folgenden vorgestellt wird.

\subsection{Exogene Noxen}

Als exogene Noxen kommen vielfältige Einflussfaktoren in Frage (Übersicht in Sharpe, 2000; Sharpe \& Franks, 2002). Zunächst ist der Genussmittelkonsum von großer Bedeutung. Hier ist erstens der Alkoholkonsum zu nennen. Starker chronischer Alkohol-Missbrauch kann sich direkt toxisch auf den Hoden auswirken, geäußert in Spermiogenesestörungen bis hin zum Sertoli-Cell-Only-Syndrom sowie Störungen der Motilität und Morphologie von Spermien in vitro (Donelly et al., 1999; Haider et al, 1985; Parajinen \& Karhunen, 1994).

Zweitens weisen einige Studien ebenfalls auf einen Einfluss des Tabakkonsums hin (Künzle et al., 2003; Saleh et al., 2002; Wallock et al., 2001; siehe auch The Practice Commitee of the American Society for Reproductive Medicine, 2004). Bislang ist ungeklärt, über welche Wirkungsmechanismen sich das Rauchen von Zigaretten auf die Ejakulatqualität auswirken könnte. Die gefundenen Beeinträchtigungen der Spermiendichte, -Motilität und -Morphologie lassen eine direkte Wirkung auf Hodenfunktion und Spermatogenese vermuten (Künzle et al., 2003; Vine et al., 1996; Vine et al., 1994). Bedeutsam scheint auch eine Verminderung der Spermatozoenfunktionen, in Form einer erniedrigten Akrosinaktivität (Gerhard \& Runnebaum, 1992) oder Schädigungen der axonemalen Strukturen des Spermatozoenflagellums (Zavos et al., 1998), zu sein. Metabolite der Bestandteile des Zigarettenrauches können Entzündungsreaktionen im männlichen Genitaltrakt hervorrufen, was oxidative Schädigungen der Spermien durch reaktive Sauerstoffradikale nach sich ziehen kann (Agarwal \& Said, 2003; Belcheva et al., 2004; Trummer et al., 2002). Die negativen Wirkungen des Rauchens und des Alkoholkonsums addieren sich gemeinsam zu 
einem synergetischen Effekt, welcher sich in verminderten Ejakulatwerten äußert (Martini, 2004).

Und schließlich wird drittens auch die Ernährungszusammensetzung als Risikofaktor für verminderte Fertilität diskutiert. So wird ein zu geringer Konsum von Obst und Gemüse mit Oligozoospermie in Verbindung gebracht (Wong et al., 2003).

Einen weiteren wichtigen Einflussbereich stellen Berufs- und umweltbedingte Noxen dar. Bei einigen Umweltchemikalien handelt es sich um so genannte endokrine Disruptoren (endocrine disruptors): endokrin wirksame Stoffe mit antiöstrogener, östrogener, antigestagener oder antiandrogener Wirksamkeit, welche ebenfalls die männliche Fertilität hemmen können (Übersicht bei Pflieger-Bruss \& Schill, 2002; Pflieger-Bruss et al., 2004). Endokrine Disruptoren können die plazentale Barriere direkt passieren und sich so ebenfalls in utero auswirken, in dem sie die Teilung und Vermehrung der fötalen Sertoli-Zellen beeinträchtigen und somit für eine Oligozoospermie verantwortlich gemacht werden können (Comhaire et al., 1998).

Eine besondere Rolle wird für die Wirkung von Östrogenen diskutiert. Es wird im Rahmen dieser "Östrogen-Hypothese“ postuliert, dass die - z.T. umstrittene - Zunahme von Hodenkrebs, Kryptorchismus, Hypospadien und verminderten Spermienkonzentrationen in den letzten Jahren durch steigende Östrogen-Exposition in der fötalen bzw. neonatalen Phase bedingt sein könnte (Pflieger-Bruss et al., 2004). Für Östrogen werden in diesem Zusammenhang verschiedene Wirkungswege angenommen: Erstens die Hemmung der Produktion von Androgenen, Gonadotropinen (speziell FSH) und/oder von InsL3 (insulin-like factor-3), welcher von den fötalen Leydig-Zellen produziert wird, zweitens die Hemmung der Ausbildung von Androgen-Rezeptoren sowie drittens die Störung der Entwicklung der Leydig-Zellen (Sharpe, 2003).

Arbeitsplatzbedingte Expositionen bzw. Umweltnoxen, welche den HypothalamusHypophysen-Gonaden-Regelkreis oder die Hoden-Funktionen beeinflussen können, stellen Schwermetalle, hier vor allem Blei, aber auch Kobalt, Nickel und Platin, Lösungsmittel oder komplexe chlororganische Verbindungen (z.B. Dioxine) sowie Pestizide (z.B. Herbizide oder Insektizide) dar (Brinkworth \& Handelsman, 2000; Dallinga et al., 2002; De Rosa et al., 2003; Monsees et al., 1998; Oliva et al., 2001; Wong et al., 2003). Eine weitere arbeitsbedingte Noxe ist die Temperatur, denn eine normale testikuläre Funktion kann nur dann aufrecht erhalten werden, wenn die Hodentemperatur von zwei Kühlsystemen niedrig gehalten wird und um 2 bis $4{ }^{\circ} \mathrm{C}$ unter der Körpertemperatur liegt (Jung et al., 2003). Arbeitsplatzbedingte Hitzeeinwirkungen (z.B. bei Stahlarbeitern) und langes Sitzen (z.B. bei Berufsfahrern) können für eine Erhöhung der Skrotaltemperatur sorgen und haben sich somit als eventuell 
fertilitätsmindernd aufgrund reduzierter Spermienkonzentrationen herausgestellt ( $\mathrm{Hjøllund} \mathrm{et}$ al., 2000; Übersicht in Thonneau et al., 1998). Auch Schweißen wird mit einem erhöhten Risiko für abnormale Spermien-Parametern in Verbindung gebracht. So ist das Oligozoospermie-Risiko bei Schweißern um den Faktor 2,8 erhöht (Wong et al., 2003).

Auch iatrogene Faktoren können einen beeinträchtigenden Einfluss auf die Fruchtbarkeit darstellen: Pharmaka können zum einen über endokrine Regulationsmechanismen wirken, so z.B. Anabolika oder Arzneimittel mit östrogen-, gestagen- oder androgenartiger Wirkung. Zum anderen zeigen einige Substanzen wie Zytostatika, Antibiotika oder Immunsuppressiva eine direkte gonadotoxische Wirkung in Form von Hemmungen der Spermatogenese. Schließlich wird im Zusammenhang mit einer Vielzahl von Pharmaka (z.B. Tranquilizern und Antidepressiva, Antihypertensiva, Antiepileptika sowie Kardiaka) auch von verminderter Libido sowie reduzierter Erektionsfähigkeit, z.T. mit einhergehender verminderter Ejakulationsfähigkeit, berichtet (Hinsch, 2000; Köhn \& Schill, 1998). Weitere iatrogene Faktoren sind im Rahmen von Tumorbehandlungen zu nennen. Sowohl Chemotherapie als auch Radiatio verursachen eine dosisabhängige Schädigung der Gonaden, welche z.T. reversibel ist (Albers, 1998; Nieschlag et al., 2000a). Ebenso kommt es nach tumorchirurgischen Eingriffen (z.B. nach Prostata-, Harnblasen- oder kolorektalen Eingriffen, im Falle der retroperitonealen Lypmhadenektomie bei Hodentumoren, aber auch nach Aorten-Bypass-Operationen) häufig zu sexuellen Funktionsstörungen durch zerebrale, neuronale oder arterielle Schädigungen (Miller et al., 1998; Popken et al., 1999).

\subsubsection{Mortalität und Reproduktion}

Im Zentrum der vorliegenden Untersuchung steht die Frage, ob die Spermatogenese und die Lebensspanne von Männern in einer Beziehung stehen könnten. Über einen solchen potentiellen Zusammenhang liegen nach Wissen der Verfasserin bislang keine Veröffentlichungen vor. Ebenso lassen sich in der Medline-Datenbank (siehe http://www.ncbi.nlm.nih.gov/entrez/query.fcgi?db=PubMed) unter verschiedenen Kombinationen der Stichworte fertility, infertility, mortality, disease und health keine entsprechenden Publikationen finden. So bleibt nur eine indirekte Betrachtung über die Fertilität bzw. den Reproduktionserfolg in Form der Kinderanzahl als Indikator einer hinreichend ungestörten Spermatogenese. Jedoch wurden auch Themenbereiche der Mortalität bzw. Lebenserwartung auf der einen und der männlichen Fertilität auf der anderen Seite bislang selten in einen Kontext gebracht. Wenn überhaupt, so lassen sich eher Studien zum Zusammenhang weiblicher Mortalität und Fertilität bzw. Reproduktionsgeschichte finden. Im 
Folgenden wird ein Überblick über den quantitativ noch spärlichen Wissensstand über Frauen und Männer in dieser Hinsicht gegeben.

Eine führende biologische Theorie über die Evolution des Alterns, die disposable soma theory, geht davon aus, dass Individuen bei der Verwendung der innen zur Verfügung stehenden Ressourcen abwägen müssen, ob diese entweder somatischen oder aber reproduktiven Funktionen dienen sollen (Kirkwood, 1977; Kirkwood \& Rose, 1991). Die zentrale Frage ist also "... which of the many different allocation strategies is optimal, i.e. maximizes fitness for an organism subject to natural selection under a given set of ecological constraints" (Kirkwood \& Rose, 1991).

$\mathrm{Ob}$ sich diese Theorie tatsächlich in der menschlichen Reproduktion bzw. in der Langlebigkeit bestätigen lässt, bleibt zu diskutieren. Die Ergebnisse der Untersuchungen von verschiedenen historischen Populationen hierzu sind inkongruent. Einige Studien an europäischen Adelsfamilien, der finnischen Landbevölkerung und einer französischkanadischen Kohorte weisen zunächst auf einen positiven Zusammenhang zwischen der Lebensdauer und der Anzahl der Kinder pro Frau hin (Doblhammer \& Oeppen, 2003; Korpelainen, 2000; Müller et al., 2002; Westendorp \& Kirkwood, 1998). Jedoch ergab sich in einigen der untersuchten Populationen, dass Frauen, welche 80 Jahre und älter wurden, im Schnitt wiederum weniger Kinder hatten als jene, die früher verstarben (Korpelainen, 2000; Westendorp \& Kirkwood, 1998). Zudem ergab die Untersuchung einer deutschen historischen Population (Krummhörn, Ostfriesland) einen negativen Zusammenhang zwischen Kinderzahl und Lebensdauer, welcher mit zunehmender finanzieller Deprivation, also in den ärmsten sozialen Schichten, stärker wurde (Lycett et al., 2000). Ungeklärt bleibt der Einfluss einer späten Geburt auf die Lebensspanne. Während einige Studien einen lebensverlängernden Effekt einer späten Geburt bei Frauen ermittelten (Müller et al., 2002; Grundy \& Tomassini, 2005; Yi \& Vaupel, 2004), ließ sich bei Betrachtung des europäischen Adels weder für Frauen noch für Männer ein solcher Effekt bestätigen (Doblhammer \& Oeppen, 2003; Mueller, 2004).

Die Ergebnisse deuten also darauf hin, dass jene Frauen, welche am längsten leben, eine reduzierte Fertilität besitzen. Es scheint ein 'trade-off' zwischen Reproduktion und Lebensspanne zu existieren, welcher für die disposable soma theory sprechen würde. In einer globalen Untersuchung von Daten aus 153 Ländern fand sich ein weiterer Hinweis. Solche Länder, in welchen Frauen mehr Kinder haben, zeichnen sich ebenfalls durch eine durchschnittlich kürzere Lebensdauer aus (Thomas et al., 2000). Allerdings bleibt bei dieser Studie zu bedenken, dass soziokulturelle und ökonomische Einflussfaktoren unberücksichtigt 
und somit z.B. Unterschiede in der finanziellen und medizinischen Versorgung unbeachtet bleiben.

Über einen Wirkungsmechanismus, wie ein möglicher Zusammenhang zwischen Kinderzahl und Lebensspanne bei Frauen Erklärung finden könnte, können nur Vermutungen angestellt werden. Einen immunogenetischen Ansatz mit besonderer Betonung des ZytokinProfils bieten Westendorp und Kollegen (2001) an. Für das erfolgreiche Voranschreiten einer Schwangerschaft ist ein bestimmtes Zytokin-Profil vorteilhaft. Dieses Zytokin-Profil sorgt jedoch andererseits für schlechtere immunologische Eigenschaften der Frauen. Für die betrachteten britischen Adelsfamilien wird nun Folgendes vermutet: Frauen, welche das entsprechende Profil nicht besaßen, und somit über einen besseren immunologischen Schutz verfügten, hatten größere Chancen, Infektionen, z.B. Cholera und Tuberkulose, zu überleben. Dafür konnten aber aufgrund des Zytokin-Profils weniger erfolgreiche Schwangerschaften zustande kommen.

Die Befundlage im Hinblick auf den Zusammenhang zwischen männlichem Reproduktionserfolg und Mortalität ist wesentlich dürftiger und erscheint ebenso unstimmig. Da die Frage der Allokation verfügbarer Energien aufgrund des geringeren energetischen Investments des Vaters in seinen Nachwuchs bei Männern eine wesentlich geringere Rolle spielt als bei Frauen, sollte sich hier ein positiver Zusammenhang zwischen Kinderanzahl und Lebensdauer vermuten lassen. Und tatsächlich zeigt sich die Tendenz, dass bei Männern der reproduktive Erfolg mit steigender Lebensdauer stetig zunimmt (Korpelainen, 2000). Allerdings wurden für die männlichen Adligen die gleichen Effekte festgestellt wie für die adligen Frauen: Nach zunächst ansteigender Kinderzahl bei zunehmendem Lebensalter, hatten Männer, welche in hohem Alter verstarben hatten, weniger Nachkommen als Männer, die ein niedrigeres Sterbealter aufwiesen (Westendorp \& Kirkwood, 1998).

Es bleibt also zu betonen, dass die Befundlage hinsichtlich eines Zusammenhangs zwischen dem Reproduktionserfolg eines Mannes und seiner Lebenszeit-Mortalität unbefriedigend ist. Zu einer potentiellen Beziehung zwischen der Spermatogenese und der Lebenszeit-Mortalität liegen keinerlei Untersuchungen vor. 


\section{Daten und Methoden}

\subsection{Forschungsleitende Hypothesen}

Die Auswertungen fanden vor dem Hintergrund der folgenden Fragestellungen statt:

- Besteht ein Zusammenhang zwischen der Fertilität und der Lebenszeit-Mortalität von Männern?

- Wie sieht dieser Zusammenhang aus?

- Wird dieser Zusammenhang durch den sozioökonomischen Status bzw. andere, die Lebensdauer potentiell bestimmende Faktoren beeinträchtigt?

- Besteht ein Zusammenhang zwischen der Spermienkonzentration und der Lebenszeit-Mortalität im Sinne einer Dosis-Wirkungs-Beziehung?

\subsection{Daten}

\subsubsection{Projektbeschreibung}

Die vorliegende Untersuchung ist Teil eines noch laufenden Projektes, welches sich mit Mortalität, langfristigen Erkrankungsrisiken und dem Lebensverlauf von ehemaligen männlichen Infertilitätspatienten befasst. Im Rahmen des Projektes wurden alte Patientenakten ausgewertet und der heutige Vitalstatus der ehemaligen Patienten recherchiert.

\subsubsection{Datengrundlage}

Datengrundlage waren Patientenakten der Fertilitätssprechstunde der Abteilung für Andrologie des Universitätsklinikums Marburg (Hessen) aus den Untersuchungsjahren 1949 bis 1985. Die Akten des Untersuchungsjahres 1954 sind aus heute unbekannten Gründen nicht mehr vorhanden. Die Männer suchten hauptsächlich aufgrund eines unerfüllten Kinderwunsches die Infertilitätsberatung auf und ließen zur weiteren Abklärung eine EjakulatUntersuchung vornehmen. Aus den vorliegenden Patientenakten wurden die Fälle rekrutiert.

In die vorliegende Studie wurden zunächst Patienten eingeschlossen, welche vor dem 01.01.1938 geboren waren. Ein weiteres Kriterium betraf die Nationalität bzw. die zu vermutende Ethnizität. Zwischen dem allgemeinen Gesundheitszustand der Angehörigen verschiedener Nationen lassen sich Unterschiede nicht ausschließen (Weilandt et al., 2000), und ausländische Patienten können sich durch Ausbildung und Beruf (mit den dazu gehörigen spezifischen Noxen) sowie durch Lebensbedingungen in der Kindheit von der deutschen Bevölkerung stark unterscheiden. Daher wurden sie generell von der Studie ausgeschlossen und zur besseren Vergleichbarkeit nur deutsche Patienten berücksichtigt. Insgesamt lagen somit die Patientenakten von 1467 Patienten vor. 


\subsubsection{Patientenakten}

Die Gestaltung der Patientenakten wandelte sich im Laufe der fast 4 Jahrzehnte umfassenden Untersuchungsperiode; es wurden insgesamt 6 verschiedene Formbögen genutzt. Entsprechende Beispiele für jeden der Vordrucke werden im Anhang aufgeführt. Das Formblatt III wurde in der interessierenden Untersuchungsperiode mit Abstand am häufigsten verwendet. Fast $70 \%$ der Akten liegen in Form dieses Vordruckes vor.

Interessant ist die inhaltliche Veränderung der Befunddokumentation seit Ende der 1940er Jahre. In den ersten betrachteten Untersuchungsjahren wurden noch verstärkt umfassendere Anamnesen erstellt. So wurde im Rahmen einer Sexualanamnese das Sexualverhalten des Patienten sowie im Rahmen einer Familienanamnese auch die reproduktive Situation der Ehefrau erfasst. Auch zum Konsum von Zigaretten und Alkohol und zum Erlebnis von belastenden (Kriegs)Ereignissen fanden sich vereinzelt Angaben. Mit fortgeschrittenen technischen Möglichkeiten konzentrierten sich die Befunde zunehmend auf die „reinen" Spermiogramm-Analysen (siehe unten). Neue Angaben wie z.B. die Konzentration der aus den Bläschendrüsen stammenden Fruktose kamen hinzu.

Es bleibt zu berücksichtigen, dass nicht bei jedem Patienten alle vorgesehenen Parameter vollständig dokumentiert wurden. Insgesamt ergab sich nur für wenige Variablen eine relativ konstante und konsistente Erfassung. Es waren dies die persönlichen Angaben wie Name, Vorname und Geburtsdatum bzw. Alter zum einen, und die Ejakulat-Parameter Volumen, pH-Wert, Spermien-Motilität und -Morphologie zum anderen. Vor allem die Spermienkonzentration wurde für fast alle Fälle angegeben. Auch die Fertilität beeinflussende Faktoren wie Geschlechtskrankheiten, Mumps und Orchitis oder Operationen wurden ausreichend dokumentiert.

\subsubsection{Medizinische Parameter und Fertilitätsstatus}

Eine Beschreibung zur Methodik der Spermiogramm-Analyse wurde bereits im Abschnitt 1.2.2.2 der Einleitung gegeben. Die Frage, ob alle bzw. welche der drei „klassischen“ Ejakulatparameter - Spermienkonzentration, -morphologie und -motilität - in die Untersuchung einfließen sollten, wurde von folgender Überlegung begleitet: Hinsichtlich der Spermienkonzentration lag die außergewöhnliche Situation vor, dass die Vorgehensweise zu ihrer Bestimmung, nämlich die Auszählung mittels Zählkammer unter dem Mikroskop, während des Zeitraumes, in welchem die Untersuchungen stattfanden, konstant blieb. Indessen wandelte sich die Beurteilung der Morphologie und die Klassifikation der Motilität im Laufe der Zeit (vgl. Falk \& Kaufman, 1950; WHO, 1992; 1999). Zusätzlich bestand bezüglich dieser Parameter das Problem der intra- sowie interpersonell stark variierenden Beurteilungen. Diese Kriterien führten zu der Entscheidung, in der vorliegenden 
Studie nur die Spermienkonzentration Berücksichtigung finden zu lassen. Lagen von einem Patienten mehrere aufeinander folgende Untersuchungen mit den entsprechenden Angaben vor, so wurden stets die Ergebnisse der ersten Analyse ausgewertet.

Bei der Auswertung wurden fertile und subfertile bzw. normo-, oligo- und azoosperme Fälle entsprechend ihrer Spermatozoen-Konzentration unterschieden. Dabei wurde, den Laborrichtlinien der WHO (1999) gemäß, das folgende Klassifikationsschema angewendet:

Tab. 2.1 Definition des Fertilitätsstatus in Abhängigkeit von der Spermienkonzentration (WHO, 1999)

\begin{tabular}{|l|l|l|}
\hline \multicolumn{2}{|l|}{ Spermienkonzentration } & Fertilitätsstatus \\
\hline$\geq 20$ Mio./ml & fertil & normozoosperm $^{\text {a }}$ \\
\hline$<20$ Mio./ml & subfertil & oligozoosperm $^{\text {a }}$ \\
\hline 0 Mio./ml & & azoosperm \\
\hline $\begin{array}{l}\text { a nach WHO-Richtlinien (1999) werden solche Fälle als normozoosperm bezeichnet, bei welchen Spermien- } \\
\text { konzentration, -Morphologie und -Motilität im Normalbereich liegen }\end{array}$
\end{tabular}

Bezüglich der Angaben zur sexuellen Karenz wurde wie folgt verfahren: Angaben, welche einen Zeitraum von 14 Tagen überschritten, wurden als nicht wahrscheinlich ausgeschlossen und wie fehlende Angaben behandelt, da Spermien nicht langfristig im Nebenhoden gespeichert werden können (Cooper \& Yeung, 2000) und sich ca. zwei Wochen nach der letzten Ejakulation im Urin finden lassen (Barratt \& Cooke, 1988). Selbst wenn also die Patienten eine sexuelle Abstinenz von beispielsweise zwei Monaten angaben, entspricht dies nicht dem Alter der dann ejakulierten Spermien.

\subsubsection{Ausschluss von Fällen}

Nach Durchsicht der Angaben zur Behandlungs- und Krankheitsgeschichte der Fälle wurden 179 Patienten wegen bekannter Vorerkrankungen oder anderer definierter Diagnosen aus der Analyse ausgeschlossen. Der Ausschluss erfolgte praktisch bei folgenden Angaben:

- beidseitige Vasoresektion

- aktuelle oder vergangene Orchitis, Epididymitis und Epididymoorchitis

- aktuelle oder vergangene Mumpserkrankung; kann zu einer Orchitis führen

- Gonorrhoe

- Erkrankung mit Marburg-Virus; kann zu einer Orchitis führen

- urogenitale Form der Tuberkulose; kann Orchitis, Epididymitis und Vesikulitis beinhalten

- Hodenfehllagen: Maldescensus testis, Kryptorchismus, Leistenhoden sowie mit diesen Diagnosen verbundene Orchidopexie 
- Hydrozelen

- vorangegangene Testosteron-Behandlung

- aktuelle oder vergangene Hoden- oder Nebenhodenschwellungen

- Hodenhypoplasie und Hodenatrophie

Bei allen Angaben war davon auszugehen, dass die Ejakulatqualität bzw. die Spermatogenese erheblich beeinträchtigt werden, ohne dass dies auf besondere Belastungen durch Noxen und Stressoren zurückzuführen wäre. Insgesamt umfasste das Projekt nach Anwendung der beschriebenen Auswahl- und Ausschlusskriterien 1283 Fälle.

\subsubsection{Recherche}

Ziel der am 01. September 2002 begonnenen Recherche war die Feststellung des Vitalstatus der ehemaligen Patienten. Es wurde also recherchiert, ob die Fälle zu diesem Zeitpunkt noch am Leben oder bereits verstorben bzw. wann sie verstorben waren.

In Kooperation mit dem Einwohnermeldeamt der Stadt Marburg wurde überprüft, ob die Patienten dort (jemals) gemeldet waren. Die Personen konnten über Namen und Geburtsdatum identifiziert werden. $\mathrm{Zu}$ beachten ist, dass die Registrierung der Einwohner im Einwohnermeldeamt Marburg sukzessive zu Beginn der 1980er Jahre von papierschriftlicher Form auf elektronische Datenverarbeitungsmethoden umgestellt wurde. Vor diesem Zeitpunkt Verstorbene bzw. Verzogene wurden also nicht mit in die PC-Dateien übernommen. Daher wurde zusätzlich zur computergestützten Recherche auch im Archiv des Einwohnermeldeamtes geforscht.

Waren die Männer im Einwohnermeldeamt Marburg gemeldet, so wurden Informationen über den Vitalstatus festgehalten. War ein Fall verstorben, so wurden ebenfalls das Sterbedatum, der Sterbeort sowie das aktenkundliche Zeichen vermerkt. Im Falle eines Fortzuges von Marburg wurde die neue Adresse notiert und die entsprechende Gemeinde angeschrieben, um die Vitaldaten dort zu erfragen. Zum Teil mussten bei mehrfachen Umzügen eines Falls verschiedene Einwohnermeldeämter kontaktiert werden.

Im Verlauf der Studie ergab sich ein weiterer Zugang zu den Vitaldaten über die Zusammenarbeit mit einer gesetzlichen Krankenkasse. Anhand eines hessischen Zentralregisters konnte erfragt werden, ob die ehemaligen Patienten bei dieser Krankenkasse versichert waren. Traf dies zu, so konnte hier Auskunft über den Vitalstatus und gegebenenfalls das Sterbedatum sowie den (zuletzt ausgeführten) Beruf nach der Berufsklassifikation des Statistischen Bundesamtes (KIdB'75) erteilt werden (vgl. 2.2.8). 
Auch die Krankenkassen-Daten wurden schrittweise seit Beginn der 1980er Jahren in elektronische Datenbanken übertragen. Versicherte, welche vor diesem Datum verstorben sind, lassen sich in der elektronischen Zentraldatei nicht mehr finden. Die Papierakten für das Bundesland Hessen werden in einem Archiv aufbewahrt, die ehemaligen Versicherten sind hier jedoch nicht in einem zentralen Register zusammengefasst. Es wurde vielmehr die Struktur der vormals unabhängigen Krankenkassen beibehalten, so dass die Fälle in 21 verschiedenen Listen zu suchen gewesen wären. Das Archiv konnte diese aufwändigen Auskünfte nicht bearbeiten.

Hinsichtlich der unterschiedlichen Informationsquellen ergab sich folgendes Bild: Für 83 Fälle $(13,8 \%)$ konnten die Informationen über die Einwohnermeldeämter erfragt werden, während 302 Personen $(50,2 \%)$ als ehemalige oder aktuelle Krankenkassenmitglieder identifiziert wurden. Die Informationen über die übrigen $35,9 \%$ der Fälle $(n=216)$ wurden sowohl über die Einwohnermeldeämter als auch die Krankenkasse ermittelt.

\subsubsection{Analysierte Stichprobe}

Von den in das Projekt aufgenommenen 1283 ehemaligen Patienten konnte bislang für 601 Fälle der Vitalstatus festgestellt werden, d.h. es ist bekannt, ob sie zum Stichtag am 01.09.02 noch am Leben oder bereits verstorben waren. Dieses Kollektiv der Fälle mit bekanntem Vitalstatus wurde bei allen Analysen herangezogen. Als problematisch war der große Anteil noch nicht verstorbener Fälle zu antizipieren, da mit einer großen Zahl an zensierten Daten (zur Erläuterung vgl. 2.3.1) gerechnet werden musste. Um dem entgegenzuwirken, wurden sämtliche Auswertungen zusätzlich zur Gesamtstichprobe an den beiden nach dem Median des Geburtstags (05.08.1931) getrennten Substichproben, welche als „frühe Geburtsjahrgänge“ und „späte Geburtsjahrgänge“ bezeichnet wurden, durchgeführt. Die Aufteilung nach diesem Median gewährleistet zwei vergleichbar große Gruppen. Eine Aufteilung der Stichprobe in verschiedene Geburtskohorten hätte aufgrund der Zusammensetzung der Stichprobe zu Substichproben mit sehr unterschiedlichem Umfang geführt und die Vergleichbarkeit erschwert. Des Weiteren wäre eine Unterteilung z.B. nach Quartilen des Geburtsdatums problematisch gewesen, da dies weniger umfangreiche Substichproben und somit geringe Fallzahlen zur Folge gehabt hätte. 


\subsubsection{Beruf, Sozioökonomischer Index des beruflichen Status (ISEI) und Sozioökonomischer Status (SES)}

Die Angaben zum Beruf, welche anhand der Krankenkassen-Daten ermittelt wurden, entsprachen der deutschen „Klassifizierung der Berufsbenennungen“, Version von 1975 (KldB '75). Es handelt sich hierbei um ein vom Statistischen Bundesamt herausgegebenes, systematisches Verzeichnis der Berufsbenennungen, welches auch bei der Bundesanstalt für Arbeit Anwendung findet (Statistisches Bundesamt, 1992). Hier wird jeder Berufsbenennung ein 3-stelliger Zahlenschlüssel zugewiesen.

Um von der Berufsbenennung auf den Sozioökonomischen Status (SES) einer Person schließen zu können, waren zwei Schritte notwendig. Die deutsche Berufsbenennung nach KIdB '75 wurde in die internationale Klassifikation, die „International Standard Classification of Occupation", Version von 1968 (ISCO-68), übertragen, welche ebenfalls auf einem 3stelligen Zahlencode basiert. Hierzu existiert ein vom Statistischen Bundesamt entwickelter Umsteigeschlüssel (Statistisches Bundesamt, 1971). Die ISCO-68 stellte nun die Ausgangsdaten zur Generierung des „Internationalen Sozioökonomischen Index des beruflichen Status“ (International socio-economic index of occupational status; ISEI) dar. Diesem liegt die Idee zu Grunde, dass ein enger Zusammenhang zwischen der Berufsposition eines Individuums auf der einen und seiner Bildung und seinem Einkommen auf der anderen Seite besteht. Jede berufliche Tätigkeit erfordert einen gewissen Bildungsgrad und ist mit einem bestimmten Lohnniveau verbunden; so wird der Beruf als intervenierende Variable zwischen Bildung und Einkommen gesehen (Hoffmeyer-Zlotnik \& Geis, 2003). Als Ausgangsmaterial für diese von Ganzeboom und Kollegen (1992) entwickelte Skala dienten Informationen über Bildung, Beruf und Einkommen von rund 74.000 vollzeitbeschäftigten Männern im Alter zwischen 21 und 64 Jahren aus 16 verschiedenen Ländern. Der ISEI berechnet sich als gewichtete Summe der durchschnittlichen Bildung und des durchschnittlichen Einkommens der jeweiligen Berufsgruppe, unter Berücksichtigung des Alters, und stellt somit eine Approximation des SES dar (Ganzeboom et al., 1992; Ganzeboom \& Treiman, 2003). Es ergaben sich ISEI-Werte von 20,0 bis 186,8. Dabei entsprechen niedrige Werte einem niedrigen SES, während hohe Werte einen hohen Status zum Ausdruck bringen. So wird einem Lager- oder Transportarbeiter der ISEI-Wert 22,0 zugeschrieben, während eine Bankfachkraft den Wert 65,5 erhält. Die höchsten Werte erhalten Professoren, Juristen und Ärzte.

Die beschriebene Umsetzung der Berufsbenennungen wurde von der Vercodungsabteilung des Zentrums für Umfragen, Methoden und Analysen (ZUMA), Mannheim, vorgenommen. Für KIdB '75-Kategorien, für die mehr als ein ISCO-68-Code existierte, wurde das arithmetische Mittel der in Frage kommenden Werte angegeben. Eine 
Übersicht der ISEI-Werte der verschiedenen Berufsgruppen der Fälle ist im Anhang in Tab. A-1 zu finden.

Ein Vorteil der Betrachtung des ISEI liegt darin, dass anhand des Berufsstatus auf den SES geschlossen werden kann, ohne dass zusätzliche Angaben über Bildung und Einkommen einer Person vorliegen müssen. Ferner verzichtet der ISEI im Gegensatz zu anderen Skalen auf die Verwendung subjektiver Einschätzungen, wie sie z.B. bei der Ermittlung so genannter Berufsprestige-Werte genutzt werden (Ganzeboom et al., 1992). Die Deutsche Arbeitsgemeinschaft Epidemiologie (DAE) empfiehlt die Verwendung der ISCOKlassifikation und die anschließende Betrachtung der zum internationalen Vergleich entwickelten ISEI-Skala (Ahrens et al., 1998). Wird im Folgenden vom SES gesprochen, so handelt es sich um den Status, welcher sich aus den anhand der Berufsangaben ermittelten ISEI-Werten ergibt.

\subsubsection{Lebenserwartung bei Geburt}

Da die Lebenserwartung im Laufe des letzten Jahrhunderts sehr stark angestiegen ist, muss dieser Parameter in einer Mortalitätsanalyse von Individuen aus verschiedenen Geburtskohorten ebenfalls notwendigerweise berücksichtigt werden. Nach Angaben des Statistischen Bundesamtes (1972) liegen für die einzelnen Kohorten die Lebenserwartungen nur für jene Zeiträume vor, in denen eine Volkszählung stattgefunden hat. Die Lebenserwartung eines neugeborenen Jungen zu unterschiedlichen Zeiten ist in Tab. 2.2 dargestellt. Für diejenigen Jahrgänge, für welche keine konkreten Angaben vorlagen, wurde unter Annahme einer linearen Entwicklung die entsprechende Lebenserwartung für jedes Geburtsjahr interpoliert.

Tab. 2.2 Lebenserwartung für männliche Neugeborene (Statistisches Bundesamt, 1972)

\begin{tabular}{|l|c|c|c|c|c|c|}
\hline & $1881 / 90$ & $1901 / 10$ & $1924 / 26$ & $1932 / 34$ & $1946 / 47$ \\
\hline $\begin{array}{l}\text { Lebenserwartung } \\
\text { bei Geburt [y] }\end{array}$ & 37,17 & 44,82 & 55,97 & 59,86 & 57,72 \\
\hline
\end{tabular}

\subsection{Datenauswertung}

\subsubsection{Verfahren zur Analyse von Ereignisdaten}

Ein Ereignis wird als der Übergang von einem Zustand in einen anderen Zustand definiert. Bei Ereignisdaten handelt es sich um Daten, welche den Zeitraum bis zum Eintreten eines Ereignisses beschreiben. In der vorliegenden Untersuchung bestand das Ereignis im Tod der betrachteten Fälle. Zum Beginn der Recherche mit dem Stichtag des 01 . September 2002 waren jedoch noch nicht alle Fälle verstorben und somit lagen die tatsächlichen 
Lebensspannen dieser überlebenden Fälle nicht vor. Man spricht im Falle dieser unvollständigen Beobachtungen von (rechts)zensierten Daten. Hier bleibt das Ende der letzten Episode im Beobachtungszeitraum offen. Um auch diese in der Analyse berücksichtigen zu können, waren spezielle Verfahren, welche die Schätzungen der Überlebenszeiten der Fälle mit zensierten Daten vornehmen, erforderlich. Mit Hilfe der Ereignisanalyse war es nun möglich, zum einen die Lebensdauer jedes Falls zu erfassen bzw. zu modellieren und zum anderen den Einfluss erklärender Variablen (Kovariaten) auf die Lebensdauer zu überprüfen. Die Ausführungen in diesem Abschnitt basieren, soweit nicht anders angegeben, auf den Publikationen von Blossfeld et al. (1987), Courgeau \& Lelièvre (1992), Diekmann \& Mitter (1984), Hosmer \& Lemeshow (1999), Hougaard (2000) und Wood et al. (2001).

\subsubsection{Grundbegriffe}

Zum weiteren Verständnis der Ereignisanalyse werden einige Grundbegriffe erläutert. Es handelt sich hierbei um die Definitionen der Überlebensfunktion, der Hazard-Rate sowie der Wahrscheinlichkeitsdichteverteilung. Dies sind 3 verschiedene Möglichkeiten, das gleiche Phänomen zu beschreiben.

\section{Hazard-Rate}

Die Hazard-Rate stellt die Übergangsrate dar, also die „momentane Neigung“ der noch lebenden Fälle innerhalb einer Zeiteinheit zu versterben. Sie kann formuliert werden als

$$
h(t)=\lim _{\Lambda t \rightarrow 0} \frac{P(t \leq T<t+\Lambda t / T \geq t)}{\Lambda t}
$$

Die Werte sind immer nicht-negativ, können jedoch auch größer als 1 sein, so dass es sich bei der Übergangsrate nicht um eine Wahrscheinlichkeitsangabe handelt.

\section{Überlebensfunktion}

Die Überlebens- oder Survivorfunktion gibt an, wie groß die Wahrscheinlichkeit ist, dass sich ein Fall zum Zeitpunkt $t$ noch im Zustand 0 befindet, dass er also noch nicht verstorben ist:

$$
\mathrm{S}(\mathrm{t})=\mathrm{P}(\mathrm{T}>\mathrm{t})
$$

Es handelt sich um die Wahrscheinlichkeit, dass die Lebensdauer $T$ größer als die Zeiteinheit $t$ ist. Mit Hilfe der Überlebensfunktion lässt sich darstellen, wie groß der Anteil der Fälle ist, die eine bestimmte Zeiteinheit $t$ überleben. In der vorliegenden Untersuchung wurden zur graphischen Veranschaulichung die Überlebenskurven dargestellt. 


\section{Wahrscheinlichkeitsdichteverteilung}

Diese Funktion gibt die absolute Wahrscheinlichkeit an, dass innerhalb eines Zeitraums $t$ das Ereignis „Tod“ eintritt:

$$
f(t)=\frac{-d S(t)}{d t}
$$

Die vorgestellten Funktionen lassen sich auseinander ableiten und stehen in folgendem Verhältnis zueinander:

$$
h(t)=\frac{f(t)}{S(t)}
$$

Generell ist zu beachten, dass empfohlen wird, einen Stichprobenumfang von 30 Untersuchungseinheiten nicht zu unterschreiten, um zuverlässige Schätzungen zu erhalten.

\subsubsection{Nicht-parametrische Verfahren}

Nicht-parametrische Verfahren gehen von keinen bzw. von möglichst allgemein gehaltenen Modell-Annahmen über die Hazard-Raten aus. Dadurch erlaubt diese Methode eine möglichst wahrheitsgetreue Darstellung der Überlebensdaten und wird nicht von dem Problem der im Laufe eines Menschenleben unterschiedlichen Sterberisiken beeinträchtigt: Das Sterberisiko ist unmittelbar nach der Geburt und während des ersten Lebensjahres sehr hoch, reduziert sich anschließend stark und steigt dann ab dem adoleszenten bzw. jungen Erwachsenenalter wieder an, um schließlich in sehr hohem Alter nochmals etwas zu sinken (Preston et al., 2001).

Im Bereich der nicht-parametrischen Verfahren findet die Sterbetafel-Methode die häufigste Anwendung zur Analyse von Verweildauern und Lebenszeiten. Sie wurde auch in der vorliegenden Studie verwendet. Die Berechnung von Sterbetafeln erlaubt die Darstellung der Überlebensfunktionen einer oder mehrerer Gruppen von Individuen. Dieser Methode liegt die Idee zu Grunde, den Beobachtungszeitraum in gleich große Intervalle (1-JahresIntervalle in der vorliegenden Studie), zu zerlegen und die Hazard-Rate für das Eintreten des Ereignisses, also den Tod, zu berechnen. Anhand des Wilcoxon(Gehan)-Tests kann dann auf statistisch signifikante Unterschiede zwischen den Überlebensfunktionen der verschiedenen Gruppen überprüft werden. Dabei werden sowohl unzensierte (=verstorbene) als auch zensierte (d.h. noch nicht verstorbene) Fälle berücksichtigt. Zur graphischen Darstellung der Überlebenskurven wird die Schätzmethode nach Kaplan-Meier angewendet. Hier werden immer dann, wenn für einen Fall das Ereignis ,Tod' eintritt, die Überlebenswahrscheinlichkeiten der übrigen, überlebenden Fälle berechnet. 


\subsubsection{Semi-parametrische Verfahren}

Auch der semi-parametrische Ansatz geht zunächst von einem allgemeinen, unspezifizierten - und somit nicht-parametrischen - Modell der Hazard-Raten aus. Diese können jedoch durch den Einfluss von unabhängigen Variablen (Kovariaten) modifiziert werden; die Effekte dieser Variablen können auf parametrische Weise modelliert werden. Das bedeutet, dass mit Hilfe dieser Verfahren der Einfluss von Kovariaten geschätzt werden kann, ohne genaue Annahmen über die mathematische Funktion der Verweildauerabhängigkeit aufzustellen. Letzteres ist generell bei parametrischen Verfahren nötig.

Innerhalb der semi-parametrischen Verfahren ist die Proportional-Hazard-Regression nach Cox am weitesten verbreitet. Analog zu den oben vorgestellten Grundideen wird untersucht, inwiefern die Überlebenswahrscheinlichkeit von den Kovariaten beeinflusst wird. Sie ist der multiplen bzw. logistischen Regression verwandt, jedoch fließen auch hier die zensierten Fälle in die Auswertung mit ein. Es liegt die Annahme zu Grunde, dass die Hazard-Rate aus dem unspezifizierten baseline hazard besteht, der zunächst für alle Individuen gleich ist, dann aber durch die Kovariaten unterschiedlich modifiziert wird. Es lässt sich darstellen, dass der baseline hazard, also die "momentane Neigung“ zu versterben, durch eine exponentielle Funktion einer Kovariaten $X$, beeinflusst werden kann:

$$
h(t, X)=h_{0}(t) * e^{\sum_{i=1}^{r} \beta_{i} X_{i}}
$$

Da für alle Fälle der gleiche baseline hazard angenommen wird, lässt sich nun auch ohne dessen genauere Kenntnis darstellen, welchen Einfluss die Kovariaten auf die Übergangsrate und somit auf die Überlebenszeit haben. Das Modell geht von einem proportional hazard aus: Bei einem Vergleich der Hazard-Raten zweier Fälle bleibt das Verhältnis der beiden Raten über die Zeit konstant. Damit ist dieses Verhältnis nur von den Kovariaten, nicht aber von der Zeit abhängig.

Mit Hilfe der Cox-Regression lassen sich bei der Betrachtung kategorialer Variablen, in der vorliegenden Untersuchung die Unterteilung in fertile und subfertile Männer, verschiedene Gruppen miteinander vergleichen. Das so genannte Odds Ratio (OR) gibt nun an, ob die "Chance“, exponiert (also beispielsweise subfertil) gewesen zu sein, bei den Verstorbenen höher war als bei den Nicht-Verstorbenen. Im deutschen Sprachgebrauch existiert keine adäquate Übersetzung und in der vorliegenden Studie wurde des einfacheren Verständnisses halber der Begriff des Risikos bzw. Sterberisikos verwendet, auch wenn es sich streng definitorisch nicht um ein solches handelt. 


\subsubsection{Parametrische Verfahren}

Im Unterschied zu den bereits vorgestellten Verfahren werden bei den parametrischen Regressionsanalysen explizit Annahmen über die zu Grunde liegende Hazard-Rate gemacht. Das Eintreten eines Ereignisses, in unserem Fall ist dies das Versterben der ehemaligen Patienten, wird als von der Zeit sowie von weiteren erklärenden Kovariaten abhängig angesehen. Es fanden 3 unterschiedliche Modelle Anwendung: Das Exponential-, das Weibull- sowie das Gompertz-Makeham-Modell. Sie unterscheiden sich in ihren Annahmen hinsichtlich des Verlaufs der Hazard-Rate. Alle Modelle ermöglichen die Berechnung des Relativen Risikos (RR). Dieses drückt das Verhältnis zweier Inzidenzraten aus und erlaubt so z.B. den Vergleich der Sterberisiken von fertilen und subfertilen Fällen.

\section{Exponential-Modell}

In einem ersten Schritt wurde die Exponential-Verteilung modelliert. Das ExponentialModell ist das einfachste aller parametrischen Modelle und geht von einer konstanten Hazard-Rate und somit von einem konstanten Ereignisrisiko aus. Es beinhaltet nur einen Parameter a, welcher alle positiven Werte annehmen kann:

$$
\mathrm{h}(\mathrm{t})=\mathrm{a}
$$

Der Erwartungswert, also die durchschnittliche Lebensdauer, ergibt sich als 1/a. Dieses Modell erscheint zwar weniger geeignet, um die entsprechenden Lebensdauern zu modellieren, da das Sterberisiko im Verlauf eines Menschenlebens nicht als konstant angenommen werden kann. Es wurde dennoch als erste Annäherung zur Analyse herangezogen.

\section{Weibull-Modell}

Das Weibull-Modell ist bezüglich der angenommenen Hazard-Rate sehr flexibel und beinhaltet 2 positive Parameter $a$ und $b$ :

$$
h(t)=b * a *(a * t) b-1
$$

Die Hazardfunktion ist monoton steigend bei $b>1$ und monoton abnehmend bei $b<1$. Das Weibull-Modell entspricht dem Exponential-Modell mit konstanter Hazard-Rate, wenn $b=1$ ist.

Dieses Modell wird vor allem in der Industrie herangezogen, um zu modellieren, wie Schädigungen eines Produktes sich akkumulieren und schließlich zu dessen Unbrauchbarkeit führen. In einer analogen Betrachtung könnte jedoch auch der menschliche Alterungsprozess als eine Art Abnutzungsprozess verstanden werden, weshalb dieses Modell bei den folgenden Analysen Berücksichtigung fand. 


\section{Gompertz-Makeham-Modell}

Das Gompertz-Makeham-Modell ist sehr gut geeignet für die Analyse von Sterblichkeit, hier vor allem für die Altersstufen von 30 bis 85 Jahren. Es entstand in 2 Schritten: Zunächst wurde ein erstes Modell entwickelt, um die ab dem mittleren Erwachsenenalter regelmäßig, fast exponentiell ansteigenden Mortalitätsraten zu beschreiben:

$$
h(t)=b * e^{c t}
$$

Dieses Modell umfasst den positiven Parameter $b$, welcher die allgemeine Erwachsenensterblichkeit beschreibt und einen ebenfalls positiven Parameter $c$, der den Anstieg des Sterberisikos mit zunehmendem Alter angibt. Es wird angenommen, dass dieser beobachtete Anstieg der Mortalität aus dem Rückgang der physiologischen Kapazitäten resultiert.

Um auch andere Sterbeursachen wie Unfälle oder sexuell übertragbare Krankheiten, zu berücksichtigen, wurde schließlich ein weiterer Parameter $a$ in die Gleichung aufgenommen:

$$
h(t)=a+b * e^{c t}
$$

Der Parameter a stellt also die konstante altersunabhängige Mortalitätskomponente dar, während der Ausdruck $b^{\star} e^{c t}$ die altersabhängige Komponente beschreibt.

\subsubsection{Weitere statistische Verfahren}

In der vorliegenden Studie kamen des Weiteren folgende Verfahren der Statistik zur Anwendung: Zur Darstellung des Zusammenhangs zwischen zwei nicht-metrischen Variablen wurden Kreuztabellen genutzt; der $\chi^{2}$-Test nach Pearson überprüfte hier, ob sich die beobachteten Werte signifikant von den erwarteten Werten unterschieden. Sämtliche intervallskalierten Parameter wurden mit dem Kolmogorov-Smirnov-Test auf ihre Verteilungsform hin überprüft, mit einer signifikanten Abweichung von der Normalverteilung bei $p>0,05$. Im Bereich der nicht-parametrischen Verfahren erlaubte der U-Test nach Mann und Whitney den Vergleich der zentralen Tendenz zweier unabhängiger Stichproben. Als Korrelationsmaße kamen Kendalls $\tau$-b im Falle ordinaler oder intervallskalierter, aber nicht normalverteilter Variablen sowie Spearmans $\rho$ als Rangkorrelationstest bei dichotomen Variablen zur Anwendung. Die verbale Interpretation des Korrelationskoeffizienten nach Bühl \& Zöfel (2002) wird in der Tab. 2.3 dargestellt. Grundlegende Einführungen zu den Verfahren der deskriptiven Statistik sind Sachs (2004) zu entnehmen.

Tab. 2.3 Interpretation des Korrelationskoeffizienten (Bühl \& Zöfel, 2002)

\begin{tabular}{|c|c|c|c|c|c|c|}
\hline & $\begin{array}{c}\text { sehr geringe } \\
\text { Korrelation }\end{array}$ & $\begin{array}{c}\text { geringe } \\
\text { Korrelation }\end{array}$ & $\begin{array}{c}\text { mittlere } \\
\text { Korrelation }\end{array}$ & $\begin{array}{c}\text { hohe } \\
\text { Korrelation }\end{array}$ & $\begin{array}{c}\text { sehr hohe } \\
\text { Korrelation }\end{array}$ \\
\hline Korrelationskoeffizient & bis 0,2 & bis 0,5 & bis 0,7 & bis 0,9 & über 0,9 \\
\hline
\end{tabular}




\subsubsection{Statistik-Software}

Die deskriptiven, nicht-parametrischen und semi-parametrischen Analysen wurden mit der 12. Version des Programmsystems SPSS (Statistical Package for the Social Science) durchgeführt. Auch für die Erstellung der Graphiken kam dieses System zur Anwendung. Die Berechnung der parametrischen Modelle erfolgte mittels des Programms TDA (Transitional Data Analysis). Ein download und Erläuterungen lassen sich unter http://www.stat.ruhr-unibochum.de/tda.html finden.

\subsubsection{Irrtumswahrscheinlichkeit $p$}

Hinsichtlich der Nomenklatur der Signifikanz von Unterschieden und Zusammenhängen wurde in der vorliegenden Studie folgende Festlegung getroffen: Ein Ergebnis wurde dann als signifikant bezeichnet, wenn die Irrtumswahrscheinlichkeit bei $p \leq 0,05$ lag. Jedoch sollte auch ein Ergebnis mit einer Irrtumswahrscheinlichkeit von $p \leq 0,10$ Berücksichtigung finden; hier wurde von einer Tendenz oder einem Trend gesprochen.

Zur besseren Orientierung wurden in den Tabellen im Ergebnisteil die folgenden Symbole verwendet:

${ }^{* *}$ signifikant bei $p \leq 0,01$

* signifikant bei $p \leq 0,05$

(*) Trend bei $p \leq 0,1$ 


\section{Ergebnisse}

\subsection{Beschreibung des Datensatzes}

Im Folgenden werden sowohl die Angaben zu den Vitaldaten und zum ISEI als auch zu den medizinischen Parametern der in der Analyse berücksichtigten Fälle vorgestellt. Zusätzlich soll der Elternstatus der Patienten zum Untersuchungszeitpunkt darstellt werden.

Tab. 3.1 Vitaldaten und ISEI der Fälle

\begin{tabular}{|c|c|}
\hline $\mathbf{n}$ & $601(100 \%)$ \\
\hline Geburtsdatum & $18.01 .1892-14.12 .1937$ \\
\hline $\begin{array}{l}\text { Geburtsjahr } \\
\text { mean } \\
\text { 1.Quartil } \\
\text { 2.Quartil } \\
\text { 3.Quartil }\end{array}$ & $\begin{array}{r}1929,7(\mathrm{SD} 6,7) \\
1926 \\
1931 \\
1935 \\
\end{array}$ \\
\hline $\begin{array}{l}\text { Vitalstatus am 01.09.2002 } \\
\text { lebend } \\
\text { verstorben }\end{array}$ & $\begin{array}{ll}420 & (69,9 \%) \\
181 & (30,1 \%)\end{array}$ \\
\hline & $07.07 .1960-04.08 .2002$ \\
\hline $\begin{array}{l}\text { Sterbejahr } \\
\text { mean } \\
\text { 1.Quartil } \\
\text { 2.Quartil } \\
\text { 3.Quartil }\end{array}$ & $\begin{array}{r}1991,7(\mathrm{SD} 8,2) \\
1988 \\
1993 \\
1998\end{array}$ \\
\hline $\begin{array}{l}\text { Lebensdauer der Verstorbenen }[y](n=181) \\
\text { mean } \\
\text { 1.Quartil } \\
\text { 2. Quartil } \\
\text { 3.Quartil }\end{array}$ & $\begin{array}{r}43,2-85,5 \\
66,3(\mathrm{SD} 9,4) \\
60,5 \\
66,2 \\
73,5\end{array}$ \\
\hline $\begin{array}{l}\text { Sozioökonomischer Index des beruflichen Status (ISEI) }(n=467) \\
\text { mean } \\
\text { 1.Quartil } \\
\text { 2. Quartil } \\
\text { 3.Quartil }\end{array}$ & $\begin{array}{r}20-153,7 \\
49,8(\text { SD } 21,2) \\
39,4 \\
43,9 \\
59,6\end{array}$ \\
\hline
\end{tabular}

Der älteste Patient wurde gegen Ende des vorletzten Jahrhunderts geboren, während der jüngste Patient Ende 1937 zur Welt kam. Fast ein Drittel der Patienten ist bereits verstorben. Diese erreichten ein Lebensalter von 43 bis fast 86 Jahren, wobei der früheste Todesfall auf das Jahr 1960 datiert (Tab. 3.1).

Die Verteilung der Geburten über die Monate ist relativ gleichmäßig; etwas mehr der Patienten kamen im Januar und Februar zur Welt, während weniger der Geburten in die Monate Oktober bis Dezember fielen (Abb. 3.2). Bei der Betrachtung der Geburtsjahre fällt die Häufung in den 1930er Jahren auf: 60,4\% der Fälle wurden 1930 oder später geboren (Abb. 3.1). Entsprechend verstarben die Männer zunehmend ab den 1980er Jahren (Abb. 
3.3). Zwei Drittel aller Todesfälle ereignete sich ab dem Jahr 1990, dabei erreichte ein großer Teil der Verstorbenen ein Lebensalter von 60 bis 80 Jahren (71,8\%; Abb. 3.4).

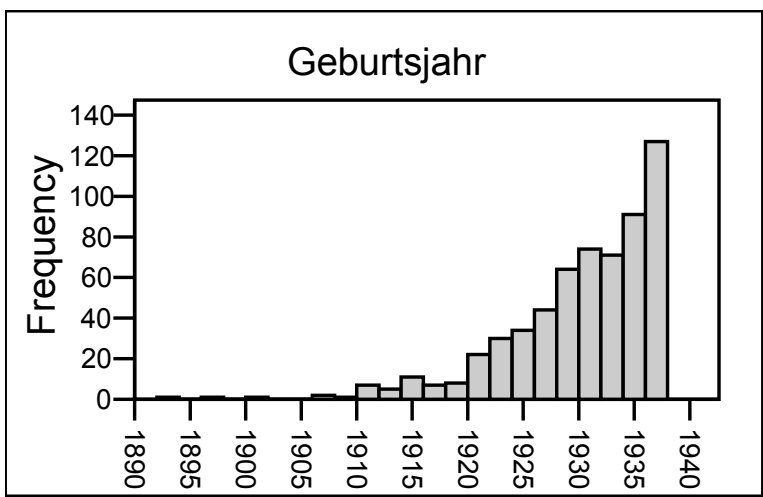

Abb. 3.1 Histogramm: Geburtsjahr der Fälle

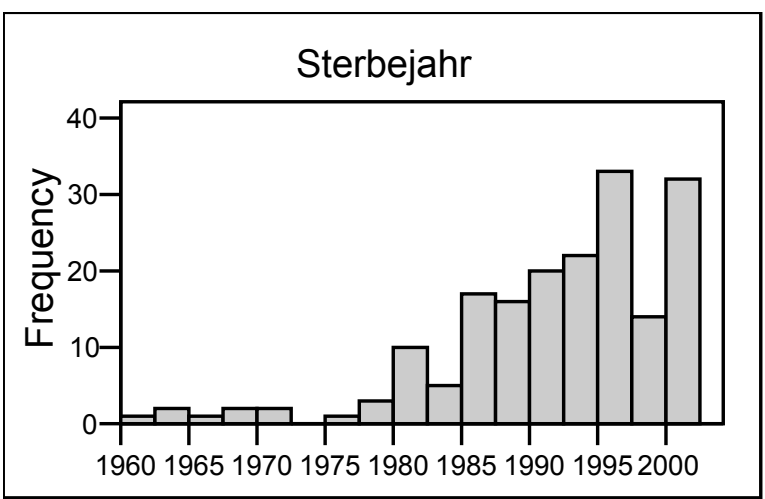

Abb. 3.3 Histogramm: Sterbejahr der Fälle

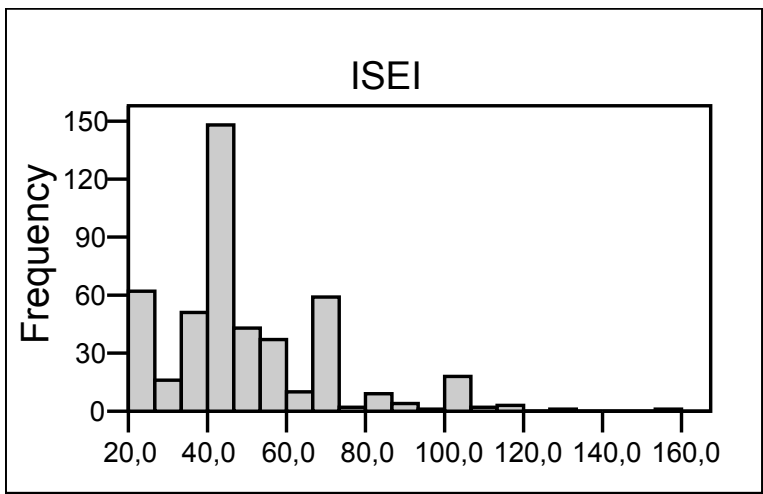

Abb. 3.5 Histogramm: Sozioökonomischer Index des beruflichen Status (ISEI)

Die ISEI-Werte sind für 467 der ehemaligen Patienten bekannt. Die Verteilung konzentriert sich eher auf Werte aus den unteren Bereichen. So beträgt das arithmetische Mittel 49,8 (SD 21,2; Abb. 3.5).

Tabelle 3.2 enthält eine Zusammenfassung der medizinischen Daten und weiterer Angaben aus den Patientenakten der Männer. Es ist zu beachten, dass nicht in jeder Akte 
alle vorgesehenen Werte auch tatsächlich festgehalten wurden bzw. lesbar sind. Aus diesem Grund wird für jede Variable die Anzahl der Fälle aufgeführt, für welche die Angaben vorliegen.

Tab. 3.2 Medizinische Daten und Angaben aus den Patientenakten der Fälle

\begin{tabular}{|c|c|}
\hline Datum der Untersuchung $(n=600)$ & $18.01 .1949-14.05 .1985$ \\
\hline $\begin{array}{l}\text { Jahr der Untersuchung } \\
\text { mean } \\
\text { 1.Quartil } \\
\text { 2.Quartil } \\
\text { 3.Quartil }\end{array}$ & $\begin{array}{r}1964,8(S D 6,3) \\
1960 \\
1965 \\
1969 \\
\end{array}$ \\
\hline $\begin{array}{l}\text { Alter bei Untersuchung }[y](n=600) \\
\text { mean } \\
\text { 1.Quartil } \\
\text { 2. Quartil } \\
\text { 3. Quartil }\end{array}$ & $\begin{array}{r}21,3-63,5 \\
35,1(\text { SD } 6,9) \\
30,4 \\
33,8 \\
38,5 \\
\end{array}$ \\
\hline $\begin{array}{l}\text { Grund der Untersuchung }(\mathrm{n}=297) \\
\text { Kinderwunsch } \\
\text { Libido-, Potenz-, Erektions- o. Ejakulationsstörung } \\
\text { Nachweis Zeugungsfähigkeit } \\
\text { Folgeuntersuchung } \\
\text { weitere }\end{array}$ & $\begin{array}{rr}237 & (79,8 \%) \\
31 & (10,4 \%) \\
5 & (1,7 \%) \\
5 & (1,7 \%) \\
19 & (6,4 \%)\end{array}$ \\
\hline $\begin{array}{l}\text { Abstinenz }[d](n=494) \\
\text { mean } \\
\text { 1.Quartil } \\
\text { 2. Quartil } \\
\text { 3. Quartil }\end{array}$ & $\begin{array}{r}1-14 \\
6,0(\mathrm{SD} 2,7) \\
5 \\
5 \\
7\end{array}$ \\
\hline $\begin{array}{l}\text { Uhrzeit der Ejakulation }(n=560) \\
\text { mean } \\
\text { 1.Quartil } \\
\text { 2. Quartil } \\
\text { 3.Quartil }\end{array}$ & $\begin{array}{r}\text { 5:00 bis 16:30 Uhr } \\
\text { 9:39 Uhr (SD 1,1 h) } \\
9: 30 \mathrm{hr} \\
10: 00 \mathrm{Uhr} \\
10: 45 \mathrm{Uhr}\end{array}$ \\
\hline $\begin{array}{l}\text { Spermienkonzentration [Mio./ml] } \\
\text { Klassifikation der WHO ( } \mathrm{n}=592) \\
\text { normozoosperm } \\
\text { oligozoosperm } \\
\text { azoosperm }\end{array}$ & $\begin{array}{rr}391 & (66,0 \%) \\
117 & (19,8 \%) \\
84 & (14,2 \%)\end{array}$ \\
\hline $\begin{array}{l}\text { normozoosperme Fälle } \\
\text { mean } \\
\text { 1.Quartil } \\
\text { 2.Quartil } \\
\text { 3.Quartil }\end{array}$ & $\begin{array}{r}20-550 \\
106,9(S D 80,1) \\
51 \\
87 \\
136\end{array}$ \\
\hline $\begin{array}{l}\text { oligozoosperme Fälle } \\
\text { mean } \\
\text { 1.Quartil } \\
\text { 2.Quartil } \\
\text { 3.Quartil }\end{array}$ & $\begin{array}{r}0,3-19,0 \\
8,6(S D 6,1) \\
2,3 \\
9,0 \\
14,0\end{array}$ \\
\hline
\end{tabular}

Wie aus der Tabelle ersichtlich, erstreckte sich der Zeitraum der Untersuchungen von 1949 bis 1985 (siehe auch Abb. 3.6). Betrachtet man die Altersspanne der Patienten bei ihrer Untersuchung, so erscheint diese mit Angaben von 20 bis 63 Jahren zunächst sehr groß und die Grenzen eher untypisch (Abb. 3.7). Daher wurden die jüngsten und ältesten 
Fälle individuell nach dem Grund der Untersuchung betrachtet. Neun Fälle waren zum Untersuchungszeitpunkt zwischen 21 und 23 Jahre alt. Bei einem der Fälle wurden Erektionsstörungen als Grund für das Aufsuchen der Sprechstunde angegeben, für die übrigen acht Fälle wurde keine Angabe gemacht. Hingegen waren sieben Fälle zum Zeitpunkt der Spermiogramm-Analyse 55 Jahre oder älter. Einer dieser Patienten litt unter Potenzschwäche, zwei andere suchten die Sprechstunde aufgrund einer unspezifischen Hämospermie auf. Für die übrigen vier Patienten wurde kein expliziter Untersuchungsgrund angegeben. Das durchschnittliche Alter der Patienten bei ihrer Untersuchung von 35 Jahren scheint der Lebensphase zu entsprechen, in welcher Männer sich mit dem Thema Familienplanung beschäftigen. So suchten auch fast $80 \%$ der Männer aufgrund eines Kinderwunsches die andrologische Sprechstunde auf.
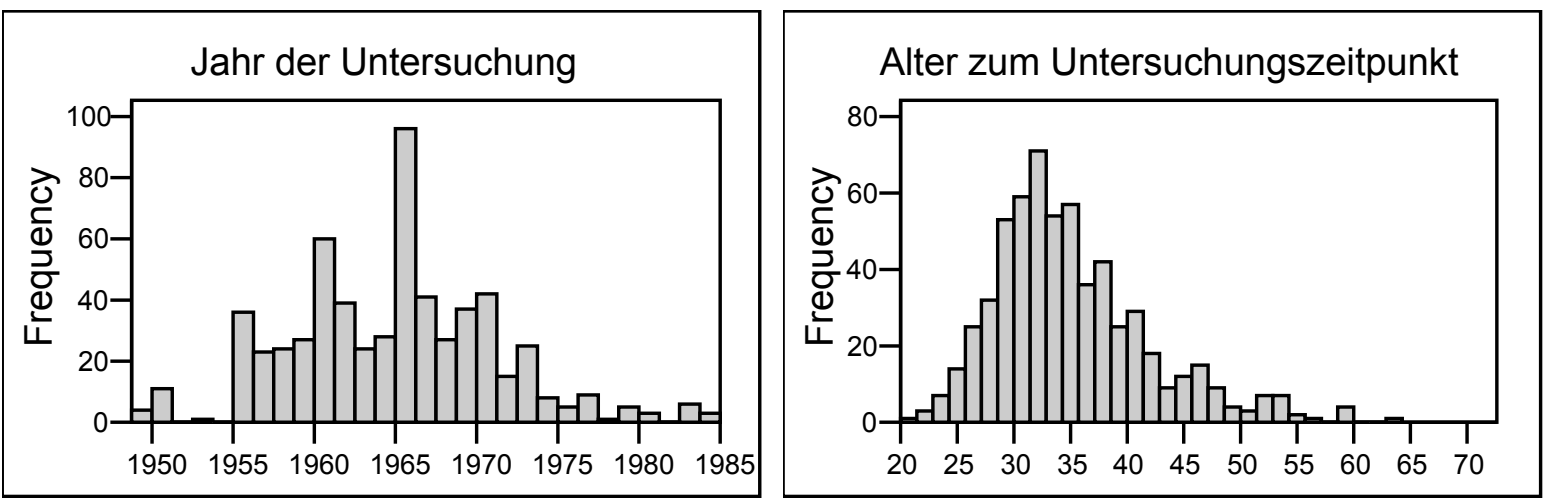

Abb. 3.6 Histogramm: Jahr der Untersuchung

Abb. 3.7 Histogramm: Alter der Patienten zum Untersuchungszeitpunkt

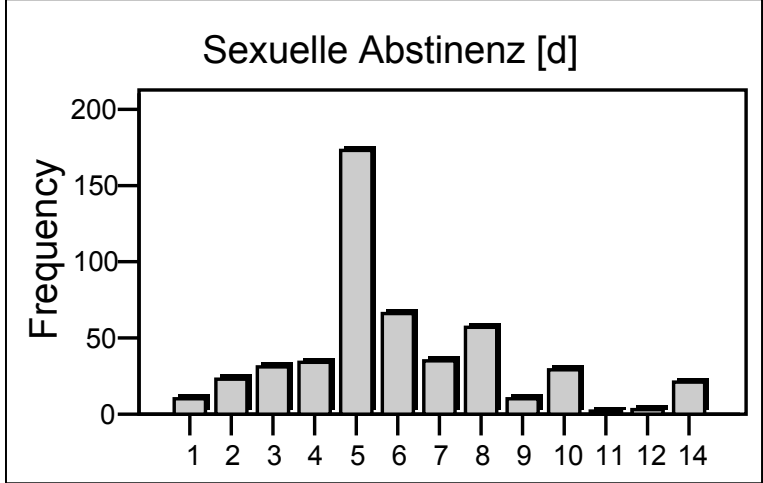

Abb. 3.8 Sexuelle Abstinenz der Patienten

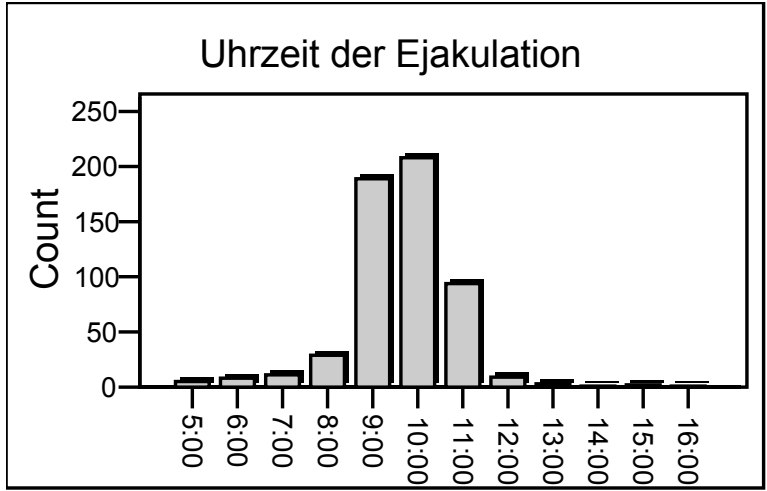

Abb. 3.9 Uhrzeit der Ejakulat-Gewinnung

Die sexuelle Karenz der Patienten vor Gewinn der Ejakulatprobe umfasste bis zu zwei Wochen. Immerhin 73,3\% der Männer hielten die von der WHO (1999) empfohlene Abstinenz von zwei bis sieben Tagen ein (Abb. 3.8). Mit 92,9\% wurde der größte Teil der Proben vormittags zwischen acht Uhr und zwölf Uhr gewonnen (Abb. 3.9). 


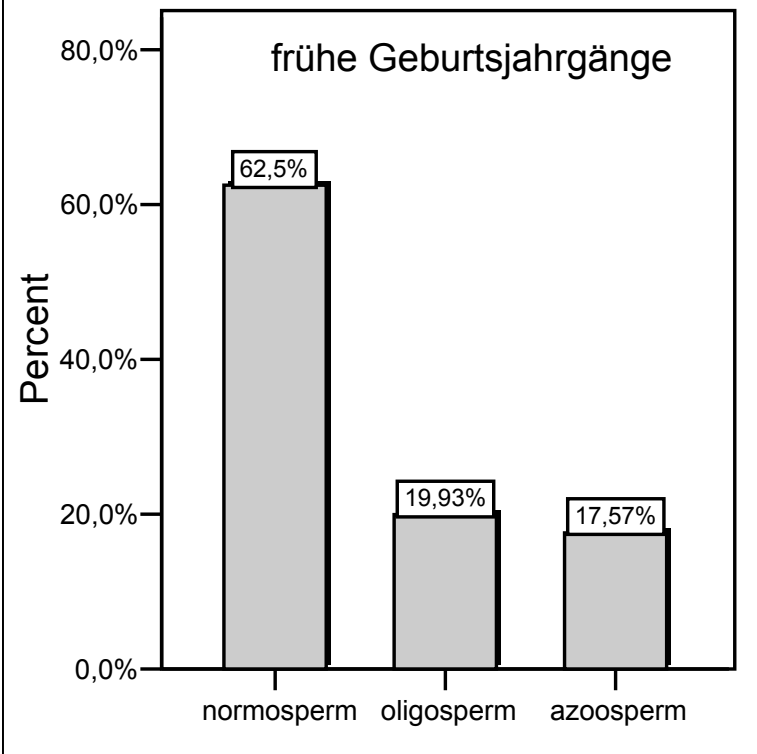

Abb. 3.10 Fertilitätsstatus der Fälle (WHO, 1999); frühe Geburtsjahrgänge

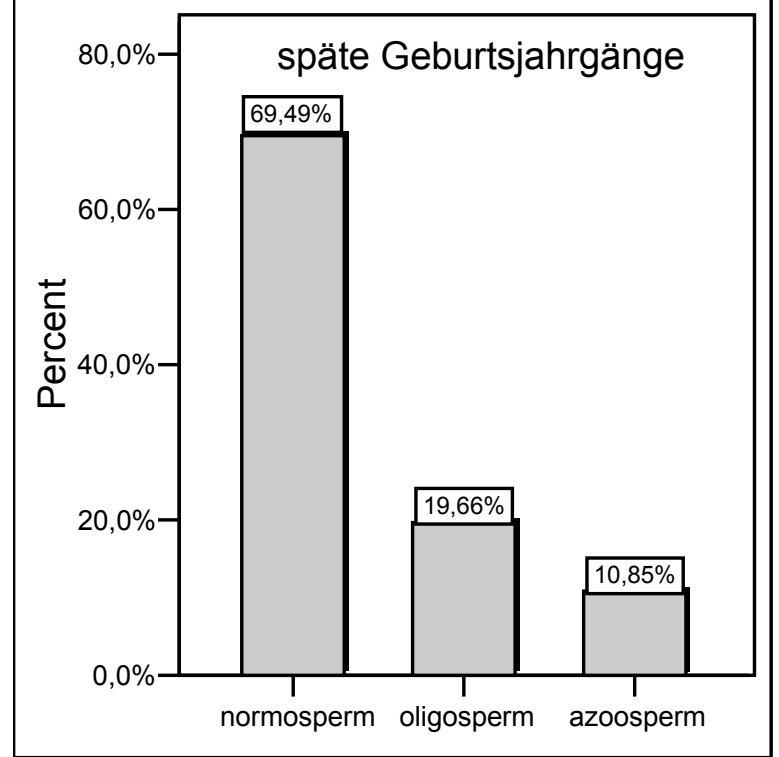

Abb. 3.11 Fertilitätsstatus der Fälle (WHO, 1999); späte Geburtsjahrgänge

Wesentlich für die vorliegende Untersuchung ist die Betrachtung der Spermienkonzentrationen und des daraus hervorgehenden Fertilitätsstatus. Rund zwei Drittel der Patienten war entsprechend der WHO-Klassifikation (WHO, 1999) mit einer Spermiendichte von mindestens 20 Mio./ml als normozoosperm einzustufen. Fast $20 \%$ der Männer konnte mit einer Spermienkonzentration unterhalb dieser Grenze als oligozoosperm klassifiziert werden, während sich im Ejakulat von 14,2\% der Fälle keine Spermien finden ließen (Tab. 3.2).

Betrachtet man die Stichprobe getrennt nach frühen und späten Geburtsjahrgängen, so fällt auf, dass der Anteil der azoospermen Fälle bei den frühen Geburtsjahrgängen noch wesentlich höher liegt als bei den späten Geburtsjahrgängen. Umgekehrt verhält es sich mit dem Anteil der normozoospermen Männer, während sich der Prozentsatz der Oligozoospermen nur unbedeutend ändert (Abb. 3.10 und 3.11).

Die Spermienkonzentration ist der in den vorliegenden Patientenakten am häufigsten dokumentierte Wert; sie ist für 592 Patienten bekannt. Die Spanne der Spermienkonzentration reicht bis zu 550 Millionen Spermien pro Milliliter Ejakulat (Abb. 3.12). Hohe Angaben mit mehr als 300 Mio./ml werden allerdings nur für zwölf ehemalige Patienten gefunden. Bei der Darstellung der Distribution der Spermienkonzentrationen aller Fälle wird deutlich, dass sie stark durch solche Fälle geprägt ist, die subnormale Konzentrationen aufweisen. Daher werden die Verteilungen für normozoosperme und oligozoosperme Männer nochmals getrennt dargestellt (Abb. 3.13 und 3.14). Für die normozoospermen Fälle wird eine durchschnittliche Spermienkonzentration von 107 (SD 80,1) Mio./ml errechnet. $56,8 \%$ weisen eine Konzentration unter 100 Mio./ml auf. 


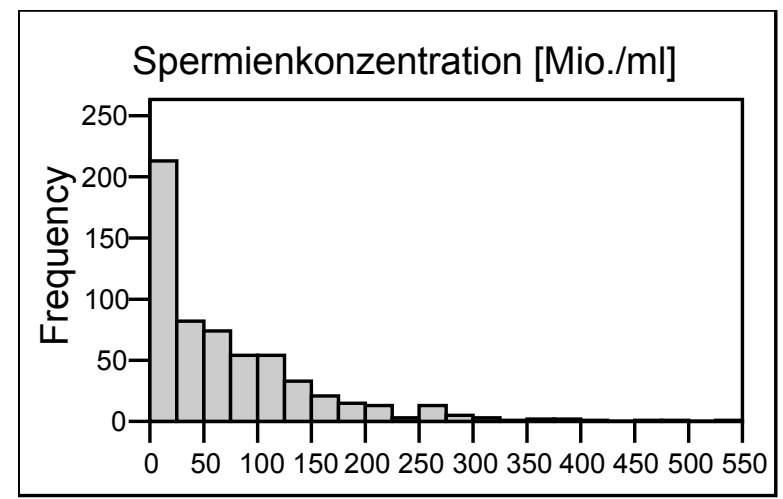

Abb. 3.12 Histogramm: Spermienkonzentration aller Fälle

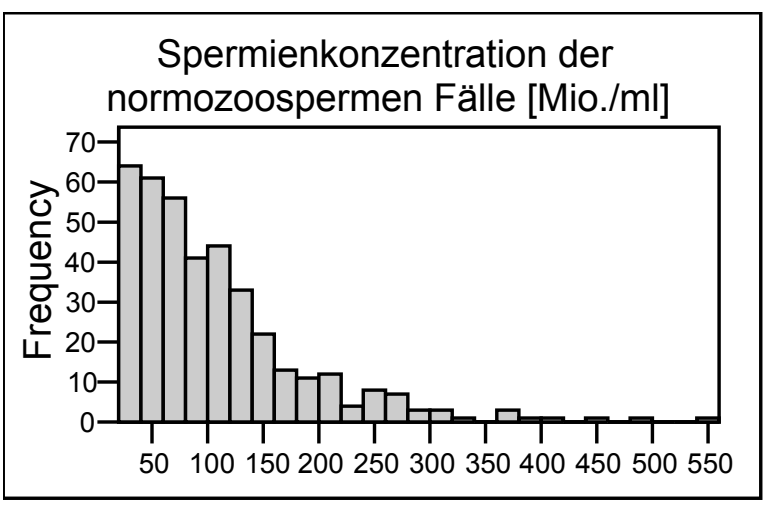

Abb. 3.13 Histogramm: Spermienkonzentration der normozoospermen Fälle

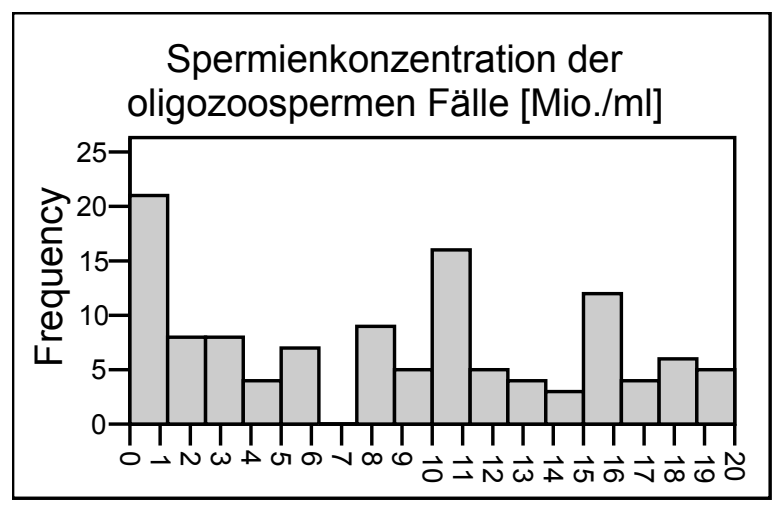

Abb. 3.14 Histogramm: Spermienkonzentration der oligozoospermen Fälle

Das Histogramm zur Darstellung der Spermienkonzentrationen der oligozoospermen Fälle vermittelt den Eindruck einer Verteilung, welche bis auf einige der Klassen relativ gleichmäßig ist. Über ein Fünftel $(21,4 \%)$ der oligozoospermen Männer weist eine Spermienkonzentration unter 2 Mio./ml auf. Das arithmetische Mittel beträgt 8,6 (SD 6,1) Mio./ml.

Nun sei eine Übersicht über den Vitalstatus der Normo-, Oligo- und Azoospermen gegeben (Tab. 3.3). Diese verdeutlicht, dass der Anteil der Verstorbenen bei den Oligozoospermen höher liegt als bei den normozoospermen Männern (35,9\% vs. 26,6\%), während er mit $38,1 \%$ bei den azoospermen Fällen am höchsten ist. Der $\chi^{2}$-Test nach Pearson ergibt einen signifikanten Unterschied zwischen den Subgruppen $\left(\chi^{2}=6,703 ; p=0,035\right)$.

Tab. 3.3 Fertilitätsstatus und Vitalstatus der Fälle

\begin{tabular}{|c|c|c|c|c|c|c|}
\hline \multirow[b]{3}{*}{ normozoosperme Fälle } & \multicolumn{4}{|c|}{ Fall verstorben? } & \multirow{2}{*}{\multicolumn{2}{|c|}{ gesamt }} \\
\hline & ja & & nein & & & \\
\hline & 104 & $(26,6 \%)$ & 287 & $(73,4 \%)$ & 391 & $(100 \%)$ \\
\hline oligozoosperme Fälle & 42 & $(35,9 \%)$ & 75 & $(64,1 \%)$ & 117 & $(100 \%)$ \\
\hline azoosperme Fälle & 32 & $(38,1 \%)$ & 52 & $(61,9 \%)$ & 84 & $(100 \%)$ \\
\hline
\end{tabular}


Tab. 3.4 Fertilitätsstatus und Elternstatus der Fälle

\begin{tabular}{|l|c|c|c|}
\hline & normozoosperme Fälle & oligozoosperme Fälle & azoosperme Fälle \\
\hline kein Kind & 192 & 56 & 37 \\
\hline 1 Kind & 19 & 7 & 3 \\
\hline 2 Kinder & 5 & 3 & 1 \\
\hline 3 Kinder & 3 & 0 & 0 \\
\hline 4 Kinder & 2 & 0 & 1 \\
\hline 5 Kinder & 1 & 0 & 0 \\
\hline Total & 222 & 66 & 42 \\
\hline
\end{tabular}

Zuletzt erfolgt die Betrachtung des Elternstatus der ehemaligen Patienten zum Zeitpunkt ihrer Untersuchung. Leider wurde dieser nur für 335 Fälle dokumentiert. Für die restlichen Fälle kann auch bei fehlender Angabe und explizitem Kinderwunsch nicht ausgeschlossen werden, dass sie bereits Väter waren. Bei der Angabe der Anzahl der Kinder wird nicht zwischen ehelichen und unehelichen bzw. lebendigen und verstorbenen Kindern unterschieden.

Der weitaus größte Teil der ehemaligen Patienten hatte zum Zeitpunkt der Untersuchung noch keine eigenen Kinder. Betrachtet man Tab. 3.4, so ist auffällig, dass sich unter den azoospermen Fällen immerhin 5 Väter finden lassen. In diesen Fällen ist von einer sekundären Infertilität auszugehen. So gab z.B. der 4-fache, nun azoosperme Vater an, die Kinder mit seiner ersten Ehefrau gezeugt zu haben, während seine zweite Ehe dann steril verlief. Unter den oligozoospermen Fällen befinden sich insgesamt 10 Väter. Eine genauere Betrachtung ergibt, dass nur bei drei dieser Fälle eine ernste Oligozoospermie mit Spermienkonzentrationen von 3 bis $5 \mathrm{Mio} . / \mathrm{ml}$ vorliegt. Alle übrigen weisen Konzentrationen von mindestens $10 \mathrm{Mio} . / \mathrm{ml}$ auf bzw. liegen knapp unterhalb der Klassifikationsgrenze von 20 Mio./ml. Da eine Kindeszeugung bei niedrigen Spermienkonzentrationen zwar mit einer geringeren Wahrscheinlichkeit eintritt, jedoch nicht unmöglich ist, kann bei diesen oligozoospermen Fällen eine primäre von einer sekundären Subfertilität nicht unterschieden werden.

\subsubsection{Spermienkonzentration und sexuelle Karenz}

Da vor allem bezüglich der sexuellen Karenzzeit ein Einfluss auf die Spermienkonzentration diskutiert wird, soll diese Variable genauer betrachtet werden. Es werden nur normozoosperme und oligozoosperme Fälle betrachtet, da nicht davon auszugehen ist, dass eine Azoospermie, also das vollständige Fehlen von Spermien im Ejakulat, durch die Abstinenz wesentlich beeinflussbar ist. Da es sich weder bei der Karenzzeit noch bei der Spermienkonzentration um normalverteilte Daten handelt, werden der Mann-Whitney-Test und das Korrelationsmaß Kendalls $\tau$-b verwendet. 
Die Abb. 3.15 gibt die Verteilung der durchschnittlichen Spermiendichten in Abhängigkeit von der eingehaltenen sexuellen Karenz wider. Es ist auffällig, dass die Konzentration mit zunehmender Abstinenz zunächst anzusteigen scheint, bis sie schließlich ab einer Karenz von mehr als 10 Tagen wieder abfällt. Hier sollte jedoch beachtet werden, dass nur drei Personen eine Karenzzeit von 11 bis 12 Tagen angaben. Die häufige Angabe einer 14tätigen Abstinenz $(n=19)$ ist wohl der Tatsache zuzuschreiben, dass ungenaue Angaben wie „ungefähr zwei Wochen“ ebenfalls in der numerischen Angabe „14 Tage“ subsumiert sind.

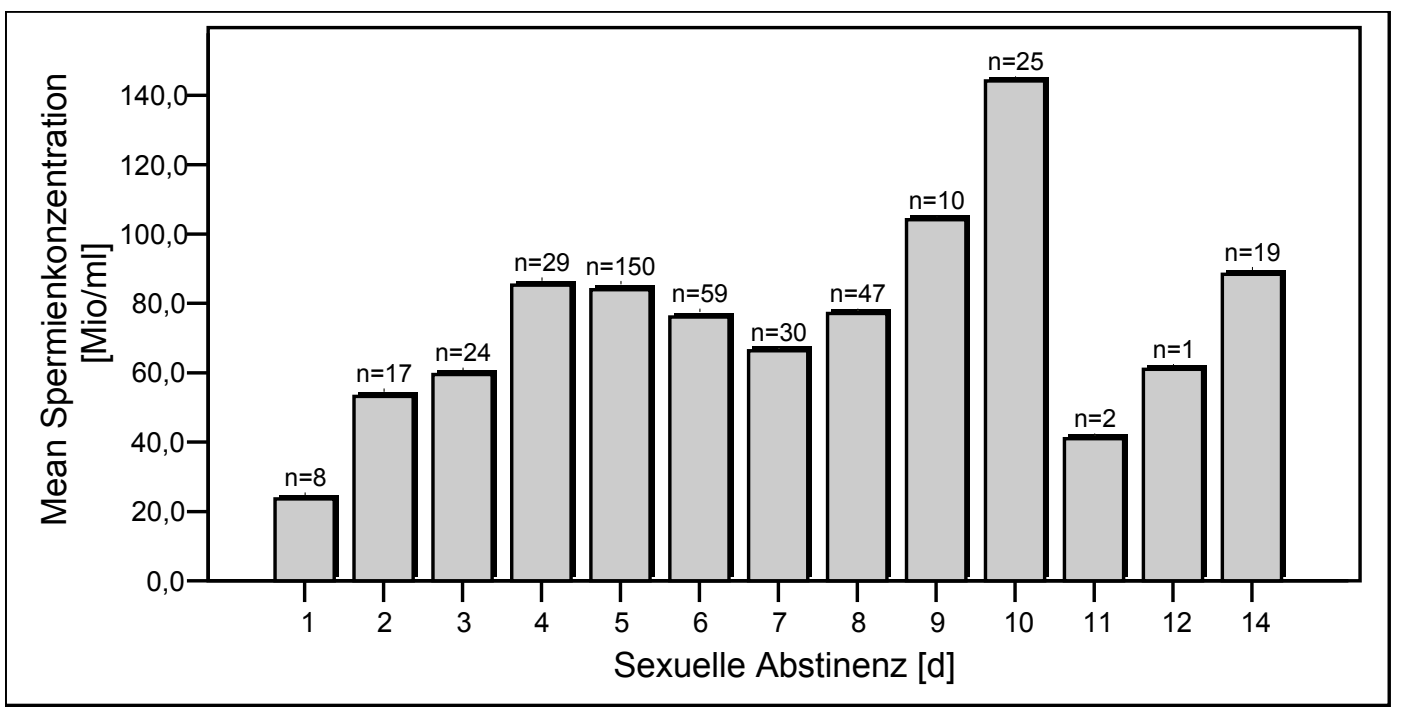

Abb. 3.15 Mittlere Spermienkonzentration in Abhängigkeit von der sexuellen Abstinenz

Von den 421 Männern haben 309 (73,4\%) die empfohlene Karenzzeit von 2 bis 7 Tagen eingehalten, während bei 26,6\% der Fälle die Abstinenz einen Tag $(n=8)$ bzw. 8 bis 14 Tage ( $n=104)$ umfasste. Im Durchschnitt ergibt sich für die erstgenannten Patienten eine durchschnittliche Spermienkonzentration von 77,3 Mio./ml, während Fälle, deren letzte Ejakulation außerhalb der empfohlenen Karenzspanne lag, im Mittel eine Konzentration von 91,8 Mio./ml aufweisen. Der Mann-Whitney-Test ermittelt keinen Unterschied zwischen den beiden Gruppen hinsichtlich ihrer Spermatozoen-Konzentration $(Z=-0,116 ; p=0,907)$.

Eine getrennte Analyse der normozoospermen Fälle ergibt eine durchschnittliche Spermienkonzentration von 98,6 (SD 65,6) Mio./ml für jene Fälle, welche die empfohlene Karenzzeit einhielten ( $n=235)$, und eine mittlere Konzentration von 117,5 (SD 102,2) Mio./ml für jene Patienten, die sich nicht an die empfohlene Abstinenzzeit hielten $(n=81)$. Auch hinsichtlich der normozoospermen Fälle ergibt der Mann-Whitney-Test jedoch keinen Unterschied zwischen der Spermiendichte dieser beiden Gruppen $(Z=-1,020 ; p=0,308)$.

Bei den oligozoospermen Fällen hingegen lässt sich ein solcher Unterschied finden. Diejenigen Fälle, welche die Empfehlungen zur Karenzzeit befolgten, weisen eine mittlere Spermiendichte von 9,5 (SD 5,7) Mio./ml auf ( $\mathrm{n}=74)$, während sich die oligozoospermen 
Fälle mit Abstinenzzeiten außerhalb der empfohlenen Periode durch eine mittlere Konzentration von 6,8 (SD=6,5) Mio./ml auszeichnen ( $\mathrm{n}=23)$. Mittels Mann-Whitney-Test wird ein signifikanter Unterschied in der Spermienkonzentration ermittelt $(Z=-2,000 ; p=0,046)$.

Die Streudiagramme verdeutlichen grafisch den Zusammenhang zwischen der Spermienkonzentration und der Karenzzeit (Abb. 3.16 bis 3.19). Es ist bereits optisch ersichtlich, dass bei keiner der betrachteten Gruppen ein deutlicher Zusammenhang besteht. Dies bestätigen auch die Bestimmtheitsmaße $\mathrm{R}^{2}$ der quadratischen Regressionsgraden. Auch eine analytische Überprüfung eines potentiellen Zusammenhangs ergibt keine Korrelation zwischen der Karenzzeit und der Spermienkonzentration. Dies trifft sowohl für die gemeinsame Analyse der normo- und oligozoospermen Männer zu (Kendalls $\tau-b=0,064 ; p=0,118$ ), als auch für die getrennte Auswertung der normozoospermen (Kendalls $\tau-b=0,047 ; p=0,182$ ) und oligozoospermen Fälle (Kendalls $\tau-b=-0,082 ; p=0,273$ ).
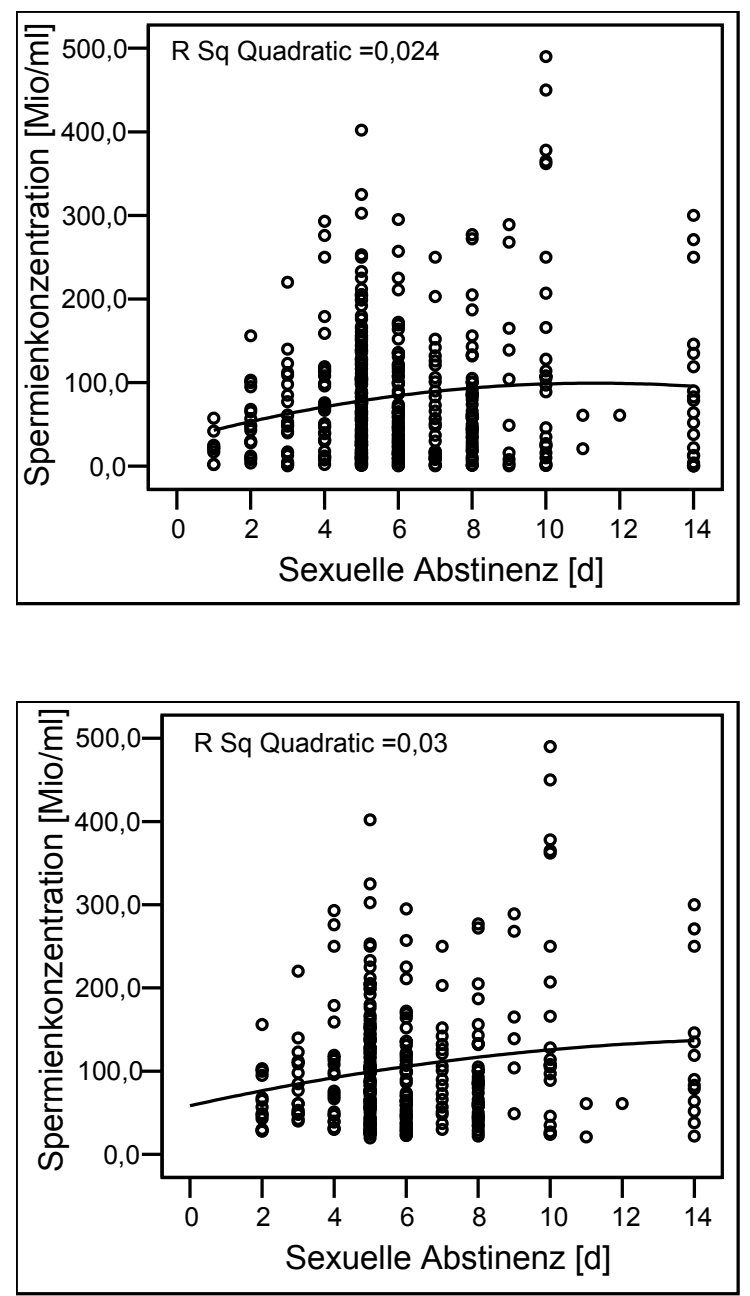

Abb. 3.16 Zusammenhang zwischen Spermienkonzentration und Abstinenz (Normo- und Oligozoosperme)

Abb. 3.17 Zusammenhang zwischen Spermienkonzentration und Abstinenz (Normozoosperme) 


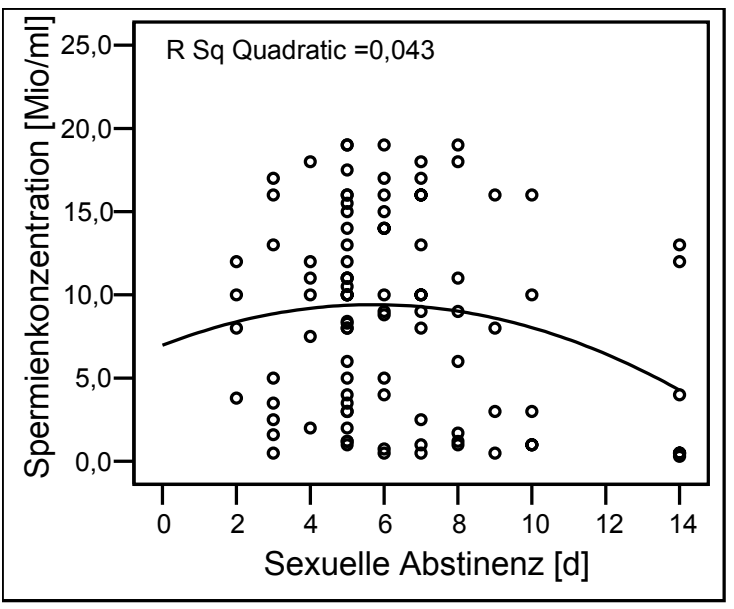

\section{Abb. 3.18 Zusammenhang zwischen Spermienkonzentration und Abstinenz (Oligozoosperme)}

Des Weiteren ist von Interesse, ob zwischen der Karenzzeit der Patienten und ihrem Fertilitätsstatus ein Zusammenhang bestehen könnte. Die grafische Auswertung in Abb. 3.21 verdeutlicht, dass die Verteilung der Karenzzeit in der Gruppe der Oligozoospermen ungefähr jener der Normozoospermen entspricht.

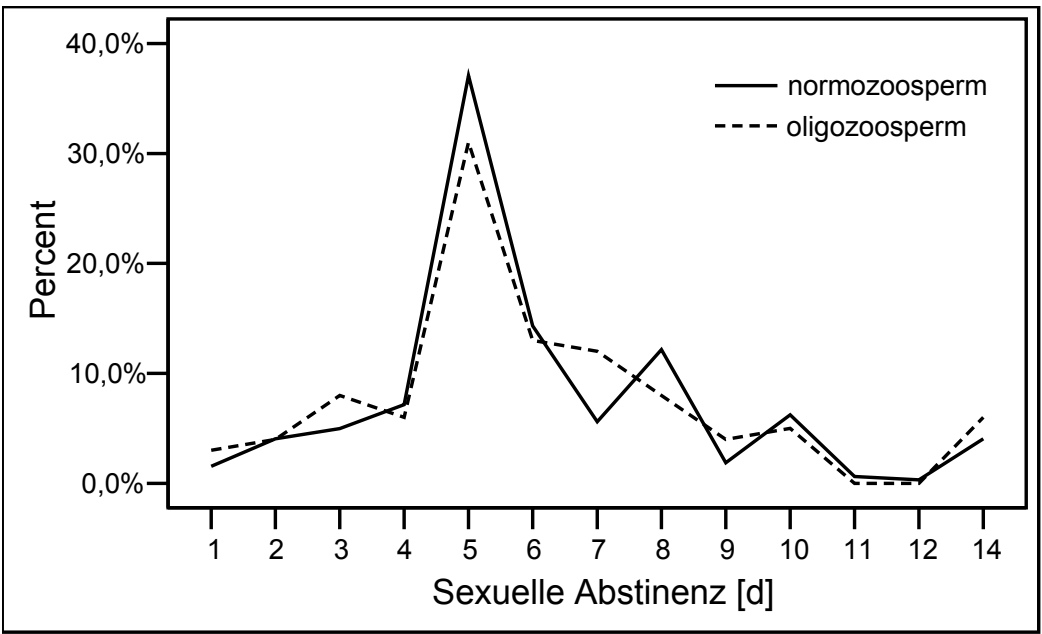

Abb. 3.21 Sexuelle Karenzzeit der normo- und oligozoospermen Fälle

Auch die analytische Untersuchung, der dichotomen Natur des Fertilitätsstatus gemäß eine Rangkorrelation nach Spearman, ermittelt keine Korrelation zwischen diesem und der sexuellen Abstinenz (Spearmans $\rho=-0,001 ; p=0,984$ ). Dieses Ergebnis bleibt konstant, wenn ausschließlich diejenigen Fälle betrachtet werden, welche die empfohlene Abstinenz von 2 bis 7 Tagen einhielten (Spearmans $\rho=-0,039 ; p=0,492$ ). Auch bei der Auswertung der Patienten, deren Karenzzeit außerhalb des empfohlenen Zeitraums liegen, ergibt sich keine Korrelation mit dem Fertilitätsstatus (Spearmans $\rho=-0,035 ; p=0,717$ ). Entsprechend kann ein solcher Zusammenhang auch bei Ausschluss der Fälle mit einer Karenz von nur 1 Tag nicht gefunden werden (Spearmans $\rho=-0,094 ; p=0,343$ ). 
Schließlich soll ausgeschlossen werden, dass die Angaben zur Abstinenz mit dem ISEI zusammenhängen. So wäre z.B. denkbar, dass Personen mit niedrigerem sozioökonomischen Status eher eine sozial erwünschte Antwort geben. Dies könnte dazu geführt haben, dass Patienten mit niedrigerem Status eher Karenzzeiten angaben, welche innerhalb der empfohlenen Periode von 2 bis 7 Tagen liegen. Eine solche Verzerrung kann jedoch ausgeschlossen werden, da erstens keine Korrelation zwischen der sexuellen Karenz und dem ISEI gefunden werden kann (Spearmans $\rho=0,046 ; n=394 ; p=0,208$ ), und sich zweitens die Gruppe, welche die empfohlene Abstinenz eingehalten hat, hinsichtlich ihres ISEI nicht von der Gruppe, welche die Abstinenz-Empfehlungen nicht befolgte, unterscheidet (MannWhitney-Test; $Z=-0,057 ; n=394 ; p=0,954)$. Bei diesen Analysen wurden normo-, oligo- und azoosperme Fälle berücksichtigt.

\subsubsection{Zusammenhang der Spermienkonzentration mit weiteren Faktoren}

Wie im einleitenden Kapitel vorgestellt, kann die Spermienkonzentration zusätzlich zur sexuellen Abstinenz in Abhängigkeit von verschiedenen anderen Faktoren variieren. Es wurden hier die vermutete Abnahme der Spermienqualität in den letzten Jahrzehnten sowie Tageszeit-abhängige, saisonale und altersbedingte Schwankungen vorgestellt. Zunächst soll daher der Zusammenhang zwischen der durchschnittlichen Spermienkonzentration und dem Untersuchungsmonat sowie dem Alter zum Untersuchungszeitpunkt, dem Geburtsjahr und dem Untersuchungsjahr dargestellt werden.

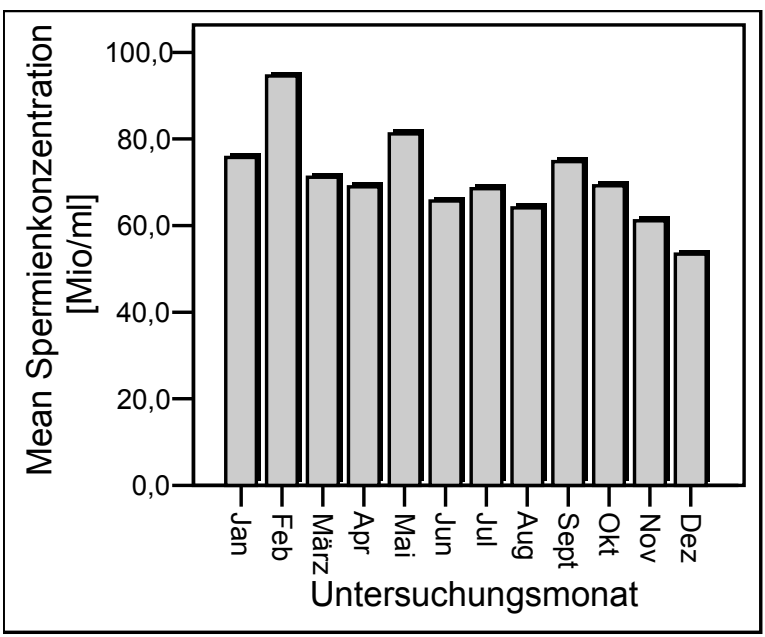

Abb. 3.20 Zusammenhang zwischen der durchschnittlichen Spermienkonzentration und dem Untersuchungsmonat

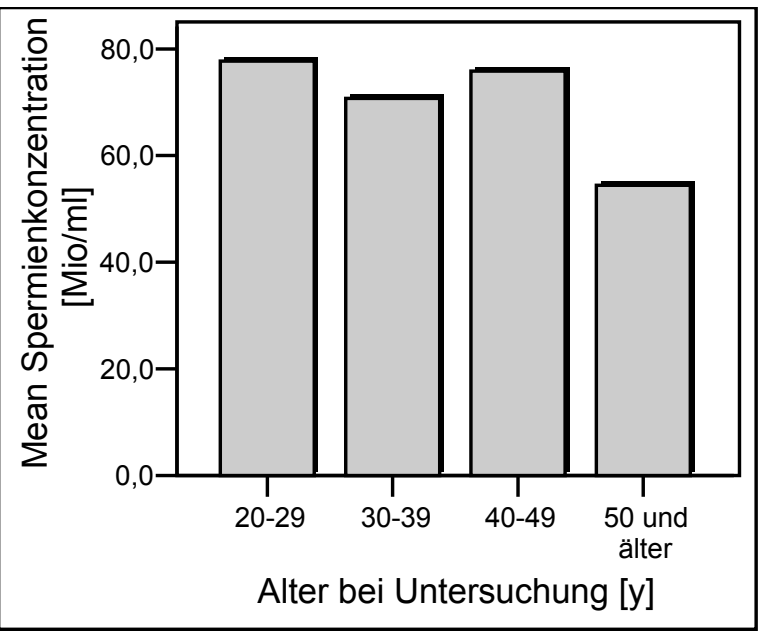

Abb. 3.21 Zusammenhang zwischen der durchschnittlichen Spermienkonzentration und dem Alter zum Untersuchungszeitpunkt 


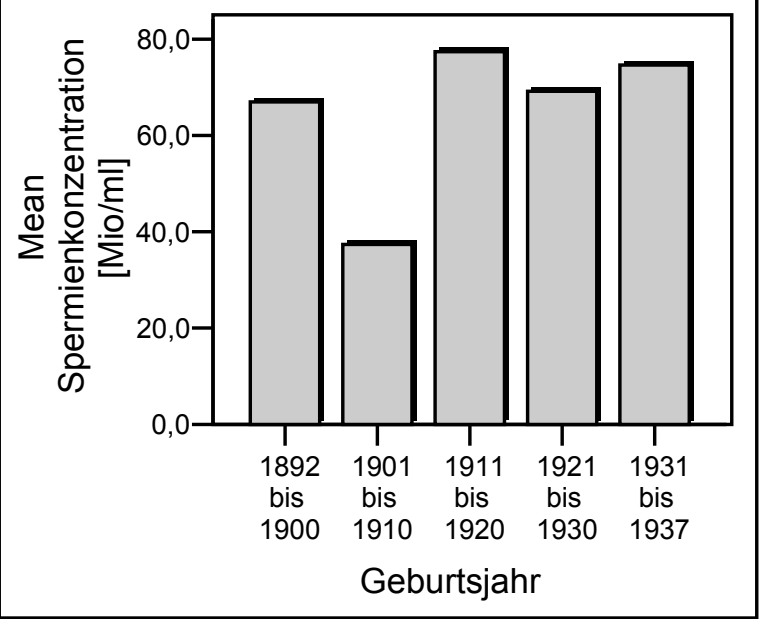

Abb. 3.22 Zusammenhang zwischen der durchschnittlichen Spermienkonzentration und dem Geburtsjahr

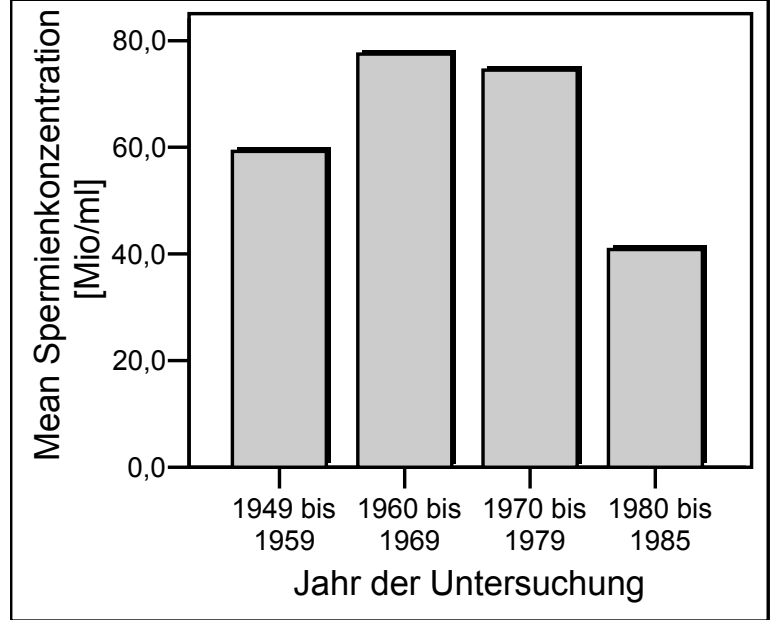

Abb. 3.23 Zusammenhang zwischen der durchschnittlichen Spermienkonzentration und dem Untersuchungsjahr

Die höchsten durchschnittlichen Spermienkonzentrationen lassen sich im Februar und Mai finden, während die mittleren Konzentrationen im November und Dezember am niedrigsten sind (Abb. 3.20). Betrachtet man die durchschnittliche Spermienkonzentration der ehemaligen Patienten in den unterschiedlichen Altersklassen, so fällt auf, dass die niedrigste mittlere Spermiendichte für Männer ab 50 Jahren $(n=25)$ gefunden wird (Abb. 3.21). Wird sie hingegen für die unterschiedlichen Geburtsjahrklassen aufgetragen, so erweist sich die durchschnittliche Spermienkonzentration für die von 1901 bis 1910 geborenen Kohorten als am niedrigsten ist. Es handelt sich allerdings um lediglich 7 Fälle. Für die übrigen Klassen zeigt sie sich relativ homogen (Abb. 3.22). Bei einer Unterteilung der Stichprobe in Klassen nach dem Untersuchungsjahr kann beobachtet werden, dass in den letzten Untersuchungsjahren von 1980 bis 1985 die durchschnittliche Spermiendichte am geringsten war $(n=11$; Abb. 3.23).

Tab. 3.5 Korrelationsmaß Kendalls $\tau$-b zum Zusammenhang zwischen der Spermienkonzentration und weiteren Variablen

\begin{tabular}{|c|c|c|c|}
\hline Korrelation der Spermienkonzentration mit & n & Koeffizient $\tau$ & p \\
\hline $\begin{array}{l}\text { Geburtsjahr } \\
\text { nur normo- und oligozoosperme Fälle } \\
\text { nur normozoosperme Fälle } \\
\text { nur oligozoosperme Fälle }\end{array}$ & $\begin{array}{r}592 \\
508 \\
391 \\
117\end{array}$ & $\begin{array}{l}0,082^{* *} \\
0,036 \\
-0,010 \\
0,112^{(*)}\end{array}$ & $\begin{array}{r}0,004 \\
0,239 \\
0,786 \\
0,086\end{array}$ \\
\hline Untersuchungsmonat & 592 & $-0,034$ & 0,238 \\
\hline $\begin{array}{l}\text { Untersuchungsjahr } \\
\text { nur normo- und oligozoosperme Fälle } \\
\text { nur normozoosperme Fälle } \\
\text { nur oligozoosperme Fälle }\end{array}$ & $\begin{array}{r}591 \\
507 \\
391 \\
116 \\
\end{array}$ & $\begin{array}{l}0,115^{* *} \\
0,052^{(*)} \\
-0,011 \\
0,207^{* *}\end{array}$ & $\begin{array}{r}0,000^{a} \\
0,087 \\
0,742 \\
0,002\end{array}$ \\
\hline Alter bei Untersuchung & 591 & 0,023 & 0,411 \\
\hline Uhrzeit der Ejakulation & 553 & $-0,039$ & 0,229 \\
\hline ISEI & 461 & $0,063^{*}$ & 0,050 \\
\hline
\end{tabular}

a signifikant bei $p<0,001$ 
Zum anderen wird überprüft, ob die individuelle Spermienkonzentration mit dem Jahr der Geburt, dem Monat bzw. dem Jahr der Untersuchung, dem Alter zum Untersuchungszeitpunkt oder der Uhrzeit der Ejakulation im Zusammenhang steht. Zusätzlich wird auch analysiert, ob ein Zusammenhang zwischen der Spermienkonzentration und dem ISEI besteht. Da die Variablen nicht normalverteilt sind, kommt als Korrelationsmaß Kendalls $\tau$-b zur Anwendung. Eine Zusammenfassung der Ergebnisse ist der Tab. 3.5 zu entnehmen.

Die Ergebnisse zeigen, dass die Spermienkonzentration zwar sowohl mit dem Geburtsjahr (Kendalls $\tau$ - $b=0,082 ; p=0,004$ ) als auch mit dem Untersuchungsjahr (Kendalls $\tau$ $b=0,115 ; p<0,001)$ positiv korreliert, dass diese Effekte jedoch zum einen ebenfalls wieder der bereits dargestellten Abnahme der azoospermen Fälle zuzuschreiben sind. Schließt man diese aus der Analyse aus, so ergibt sich kein signifikanter Zusammenhang mehr mit dem Geburtsjahr, und für das Untersuchungsjahr lässt sich nur noch der Trend einer Korrelation feststellen (Kendalls $\tau-b=0,052 ; p=0,087$ ). Zum anderen lassen sich aber für die oligozoospermen Männer eine positive Korrelation zwischen der Spermienkonzentration und dem Untersuchungsjahr (Kendalls $\tau-b=0,207 ; p=0,002$ ) sowie ein Trend eines positiven Zusammenhangs zwischen der Konzentration und dem Geburtsjahr (Kendalls $\tau-b=0,112$; $p=0,086)$ erkennen. Es ist zu berücksichtigen, dass es sich ausschließlich um sehr geringe Korrelationen handelt. Für die normozoospermen Fälle alleine lässt sich wiederum kein Zusammenhang zwischen ihrer Spermiendichte und ihrem Geburts- bzw. dem Untersuchungsjahr erkennen. Zwischen der Spermienkonzentration und dem ISEI wird zwar eine Korrelation ermittelt, es handelt sich jedoch nur um einen sehr geringen Zusammenhang (Kendalls $\tau-b=0,063 ; p=0,050$ ). Alle anderen Variablen weisen keine Korrelation mit der Spermatozoen-Konzentration auf.

Des Weiteren wird überprüft, ob der Fertilitätsstatus der Patienten, also die Klassifikation in normozoosperme, oligozoosperme und azoosperme Fälle, mit anderen Variablen zusammenhängen könnte (Tab. 3.6). Erneut erweisen sich auch hier das Geburtsjahr (Kendalls $\tau-b=-0,114 ; p=0,001$ ) sowie das Untersuchungsjahr (Kendalls $\tau-b=-0,157 ; p<0,001$ ) als mit dem Fertilitätsstatus korrelierende Variablen. Der negative Koeffizient ergibt sich aus der Codierungsrichtung der Variablen des Fertilitätsstatus (normozoosperm $=0$, oligozoosperm $=1$ und azoosperm =2). Bei Ausschluss der azoospermen Fälle - hier wird aufgrund der nunmehr dichotomen Natur der Variable des Fertilitätsstatus eine Rangkorrelation nach Spearman berechnet - verschwindet der Effekt des Geburtsjahres, jener des Untersuchungsjahres bleibt jedoch signifikant (Spearmans $\rho=-0,097 ; p=0,029$ ). 
Tab. 3.6 Korrelationsmaß Kendalls $\tau$-b zur Überprüfung des Zusammenhangs zwischen dem Fertilitätsstatus (normo-l oligo-l azoosperm) und weiteren Variablen

\begin{tabular}{|l|l|l|l|}
\hline Korrelation des Fertilitätsstatus mit & $\mathbf{n}$ & Koeffizient $\boldsymbol{\tau}$ & $\mathbf{p}$ \\
\hline $\begin{array}{l}\text { Geburtsjahr } \\
\text { nur normo- und oligozoosperme Fälle }\end{array}$ & $\begin{array}{c}592 \\
\mathbf{b}\end{array}$ & $\begin{array}{c}-0,114^{* *} \\
-0,071 \text { (Spearmans } \rho \text { ) }\end{array}$ & $\begin{array}{c}0,001 \\
0,108\end{array}$ \\
\hline Untersuchungsmonat & 592 & 0,011 & 0,737 \\
\hline $\begin{array}{l}\text { Untersuchungsjahr } \\
\text { nur normo- und oligozoosperme Fälle }\end{array}$ & 591 & $-0,157^{* *}$ & $0,000^{\text {a }}$ \\
\hline Alter bei Untersuchung & 507 & $-0,097^{*}$ (Spearmans $\left.\rho\right)$ & 0,029 \\
\hline Uhrzeit der Ejakulation & 591 & $-0,025$ & 0,433 \\
\hline \hline ISEI & 453 & 0,037 & 0,321 \\
\hline
\end{tabular}

a signifikant bei $p<0,001$

${ }^{\mathrm{b}}$ Berechnung der Rangkorrelation nach Spearman

Eine abschließende Betrachtung der Angaben zum ISEI ergibt, dass sich die subfertilen Fälle in ihren ISEI-Werten nicht von den fertilen Fällen unterscheiden (Mann-Whitney-Test, $Z=-1,117 ; p=0,264)$. Auch zwischen den oligozoospermen und den normozoospermen Fällen kann kein Unterschied hinsichtlich der ISEI-Werte gefunden werden (Mann-Whitney-Test, $Z=-0,791 ; p=0,429)$.

\subsection{Lebenszeit-Mortalität und Fertilitätsstatus}

\subsubsection{Ergebnisse der nicht-parametrischen Verfahren}

Als Resultate der nicht-parametrischen Analysen werden zum einen die anhand des Kaplan-Meier-Schätzers ermittelten Überlebensfunktionen der ehemaligen Patienten abgebildet. So können sowohl die Überlebenskurven der einzelnen Subgruppen graphisch dargestellt als auch der Anteil der in einem bestimmten Lebensalter bereits Verstorbenen abgelesen werden. Zum anderen kann neben dieser optischen Beurteilung der Überlebenskurven der Wilcoxon(Gehan)-Test Aufschluss darüber geben, ob sich die Überlebensfunktionen der Subgruppen tatsächlich voneinander unterscheiden Es sind bei den nachstehenden Analysen sowohl die tatsächlichen Überlebenszeiten für die Verstorbenen als auch die geschätzten Lebenserwartungen berücksichtigt. Wird im Folgenden von, "Lebensdauer", „Lebenserwartung“, „Überlebensraten“, „Mortalität“ etc. gesprochen, so bezieht sich dies immer auf unzensierte („uncensored“) wie zensierte („,censored“) Fälle, d.h. auf die tatsächlichen wie die geschätzten Daten. Die Begriffe werden synonym verwendet. Die in diesem Abschnitt analysierte Stichprobe setzt sich aus 391 normozoospermen, 117 oligozoospermen und 84 azoospermen Patienten zusammen. 


\subsubsection{Fertile und Subfertile}

\section{Betrachtung aller Fälle}

Die Überlebenskurven der fertilen und subfertilen Fälle sind der Abb. 3.24 zu entnehmen. Zunächst ist auffällig, dass sich die Kurven erst ab einem Sterbealter von ca. 64 Jahren auseinander entwickeln. Danach liegt die Überlebenskurve der subfertilen Fälle konstant unterhalb jener der fertilen Fälle; die Sterblichkeit der Subfertilen scheint erhöht. Schließlich nähern sich die Kurven der Subfertilen und Fertilen ab einem Sterbealter von 85 Jahren wieder etwas an.

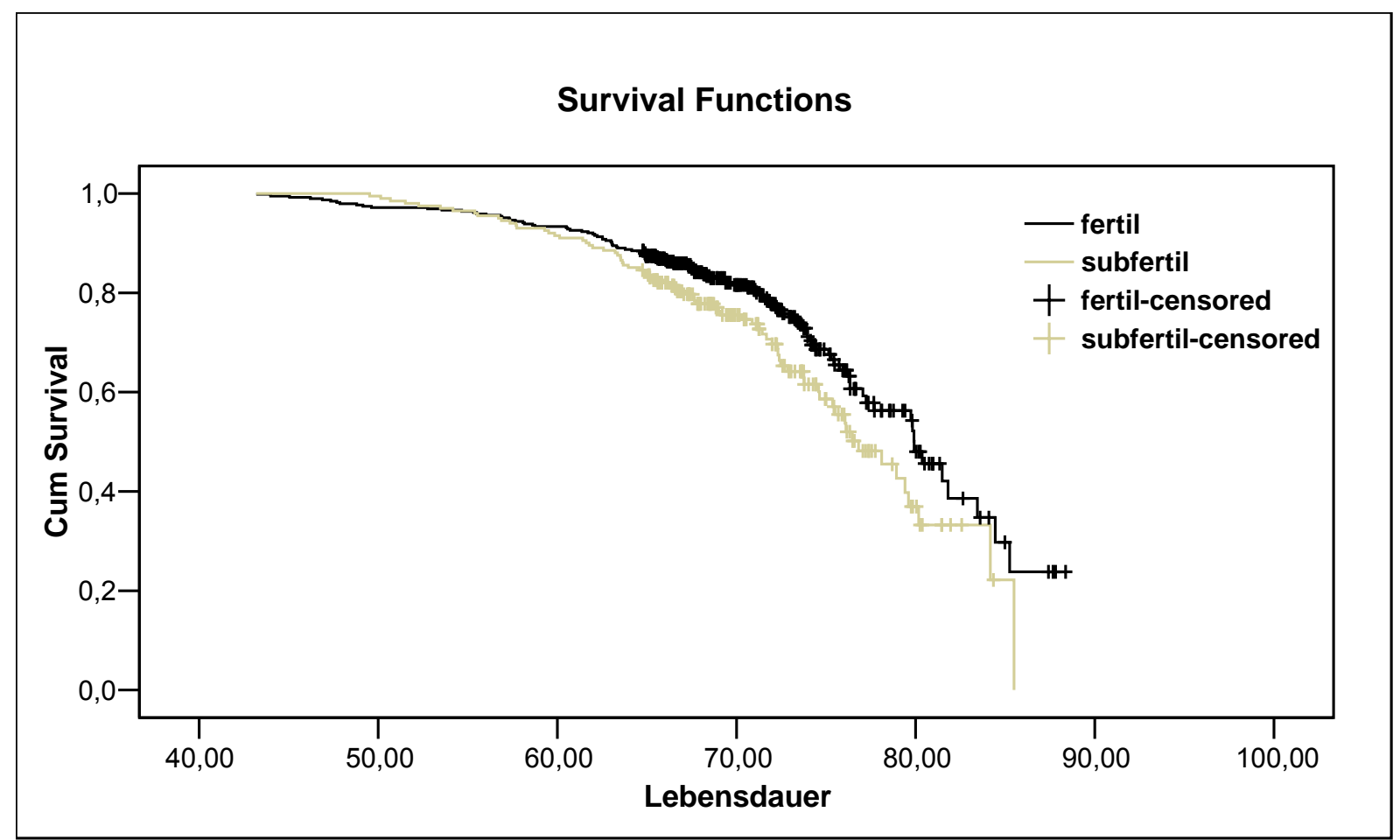

Abb. 3.24 Überlebensfunktionen der fertilen und subfertilen Fälle

Der Wilcoxon(Gehan)-Test zum Vergleich der Überlebensraten der Subfertilen und Fertilen ergibt zwar keinen signifikanten Unterschied zwischen beiden Gruppen, es lässt sich aber zumindest der Trend erkennen, dass die Sterblichkeit der Subfertilen erhöht ist (Wilcoxon $($ Gehan $)=3,430 ; p=0,064 ;$ Tab. 3.7).

Tab. 3.7 Wilcoxon(Gehan)-Test zum Vergleich der Überlebensfunktionen von fertilen und subfertilen Fällen

\begin{tabular}{|l|l|l|l|l|l|}
\hline Vergleich & $\mathbf{n}$ & df & $\begin{array}{l}\text { Wilcoxon } \\
\text { (Gehan) }\end{array}$ & $\mathbf{p}$ \\
\hline fertil-subfertil & unzensiert & zensiert & & \\
\hline
\end{tabular}




\section{Betrachtung der frühen Geburtsjahrgänge}

Bei der Betrachtung der jungen Geburtsjahrgänge wird deutlich, dass sich die Überlebenskurven bereits ab einem Sterbealter von ca. 55 Jahren auseinander entwickeln und sich in der Folge deutlicher voneinander unterscheiden (Abb. 3.25). Die früher abfallende Kurve der Subfertilen zeigt deren höhere Sterblichkeit an. Diese bleibt über alle Altersstufen hinweg erhöht.

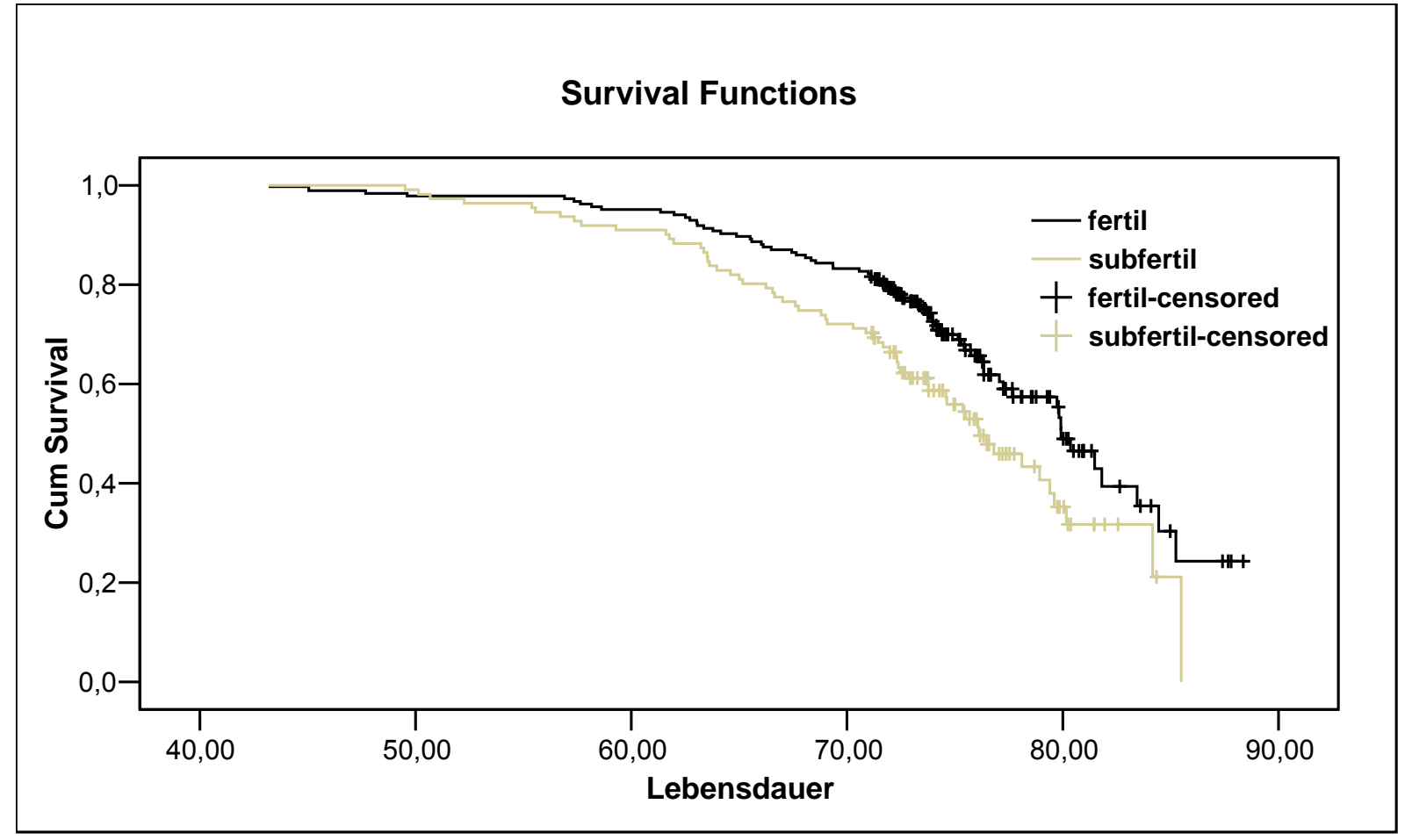

Abb. 3.25 Überlebensfunktionen der fertilen und subfertilen Fälle; frühe Geburtsjahrgänge

Der statistische Vergleich der Mortalität der beiden Fertilitätsgruppen durch den Wilcoxon(Gehan)-Test bestätigt, dass fertile Fälle innerhalb des Kollektivs der frühen Geburtsjahrgänge eine niedrigere Sterblichkeit als subfertile Fälle haben. Der Test ergibt einen deutlichen Unterschied zwischen den beiden Gruppen (Wilcoxon(Gehan)=7,091; $p=0,008$; Tab. 3.8).

Tab. 3.8 Wilcoxon(Gehan)-Test zum Vergleich der Überlebensfunktionen von fertilen und subfertilen Fällen; frühe Geburtsjahrgänge

\begin{tabular}{|l|l|l|l|l|l|}
\hline Vergleich & $\mathbf{n}$ & df & $\begin{array}{l}\text { Wilcoxon } \\
\text { (Gehan) }\end{array}$ & $\mathbf{p}$ \\
\hline fertil-subfertil & unzensiert & zensiert & & \\
\hline
\end{tabular}




\section{Betrachtung der späten Geburtsjahrgänge}

Die separate Betrachtung der späten Jahrgänge hingegen verdeutlicht, dass sich die Überlebenskurven der Fertilen und Subfertilen bereits rein optisch kaum voneinander unterscheiden (Abb. 3.26). Auch die statistische Überprüfung mit Hilfe des Wilcoxon(Gehan)Tests zeigt, dass zwischen den Lebensdauern der beiden Gruppen kein Unterschied besteht (Tab. 3.9).

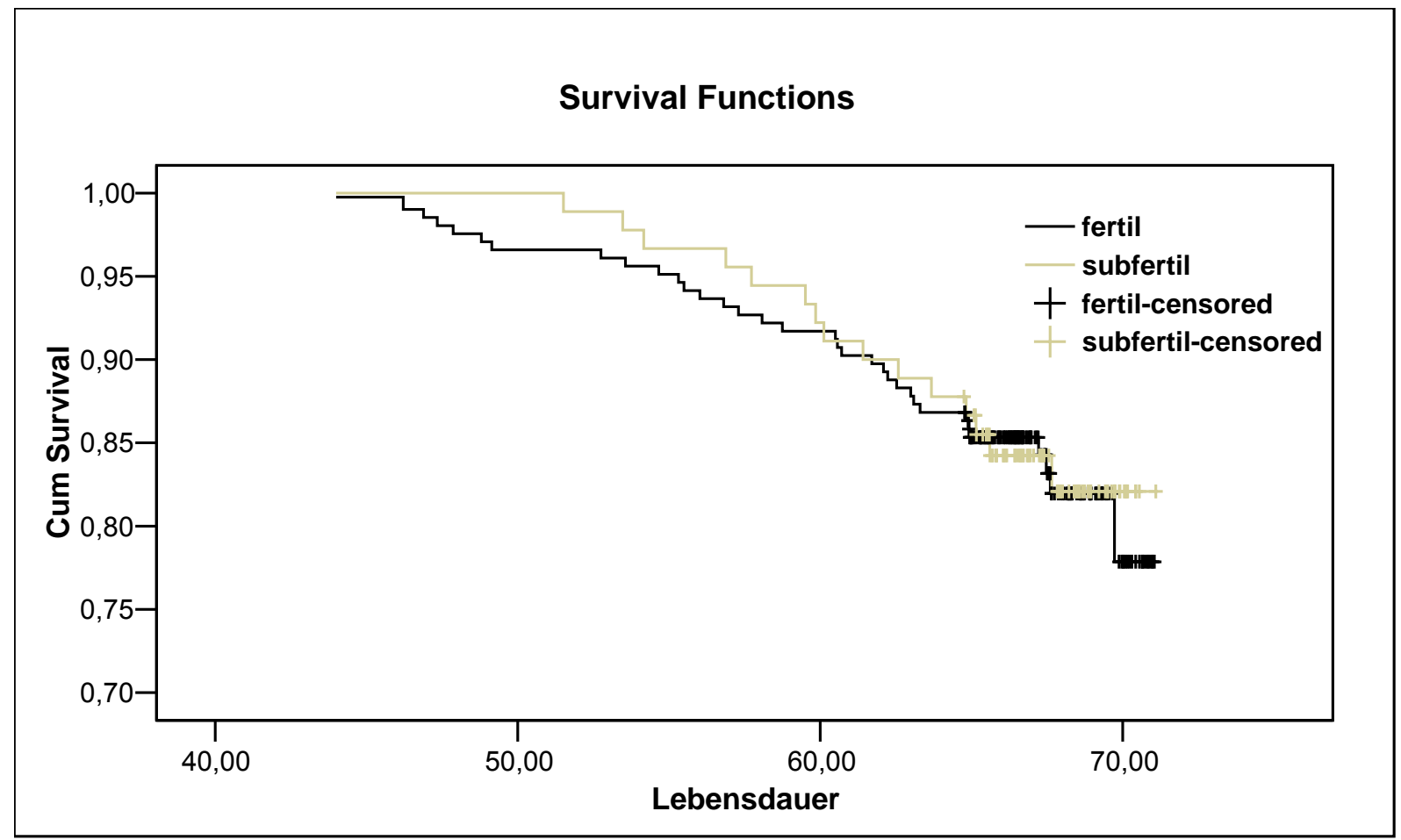

Abb. 3.26 Überlebensfunktionen der fertilen und subfertilen Fälle; späte Geburtsjahrgänge

Tab. 3.9 Wilcoxon(Gehan)-Test zum Vergleich der Überlebensfunktionen von fertilen und subfertilen Fällen; späte Geburtsjahrgänge

\begin{tabular}{|l|l|l|l|l|l|}
\hline \multirow{2}{*}{ Vergleich } & $\mathbf{n}$ & df & $\begin{array}{l}\text { Wilcoxon } \\
\text { (Gehan) }\end{array}$ & $\mathbf{p}$ \\
\hline fertil-subfertil & unzensiert & zensiert & & \\
\hline
\end{tabular}

\subsubsection{Normozoosperme, Oligozoosperme und Azoosperme}

Betrachtung aller Fälle

Zur weiteren Verdeutlichung wird die Gruppe der Subfertilen in azoosperme und oligozoosperme Fälle unterteilt. Diese werden miteinander sowie mit den fertilen Fällen, hier als normozoosperm bezeichnet, verglichen. Bei der Betrachtung der Abb. 3.27 ist die späte Auseinanderentwicklung der Überlebenskurven der normozoospermen und oligozoospermen Fälle ab einem erreichten Lebensalter von ca. 68 Jahren auffällig. Diese könnte dafür 
verantwortlich sein, dass zwischen den Überlebenskurven dieser beiden Gruppen kein statistisch signifikanter Unterschied besteht (Tab. 3.10). Auch die weiteren Subgruppenvergleiche ergeben keine deutliche Differenz; jedoch lässt sich die Tendenz eines Unterschiedes zwischen den Überlebenskurven der normozoospermen und der azoospermen Männer erkennen (Wilcoxon(Gehan)=2,830; $p=0,0925)$.

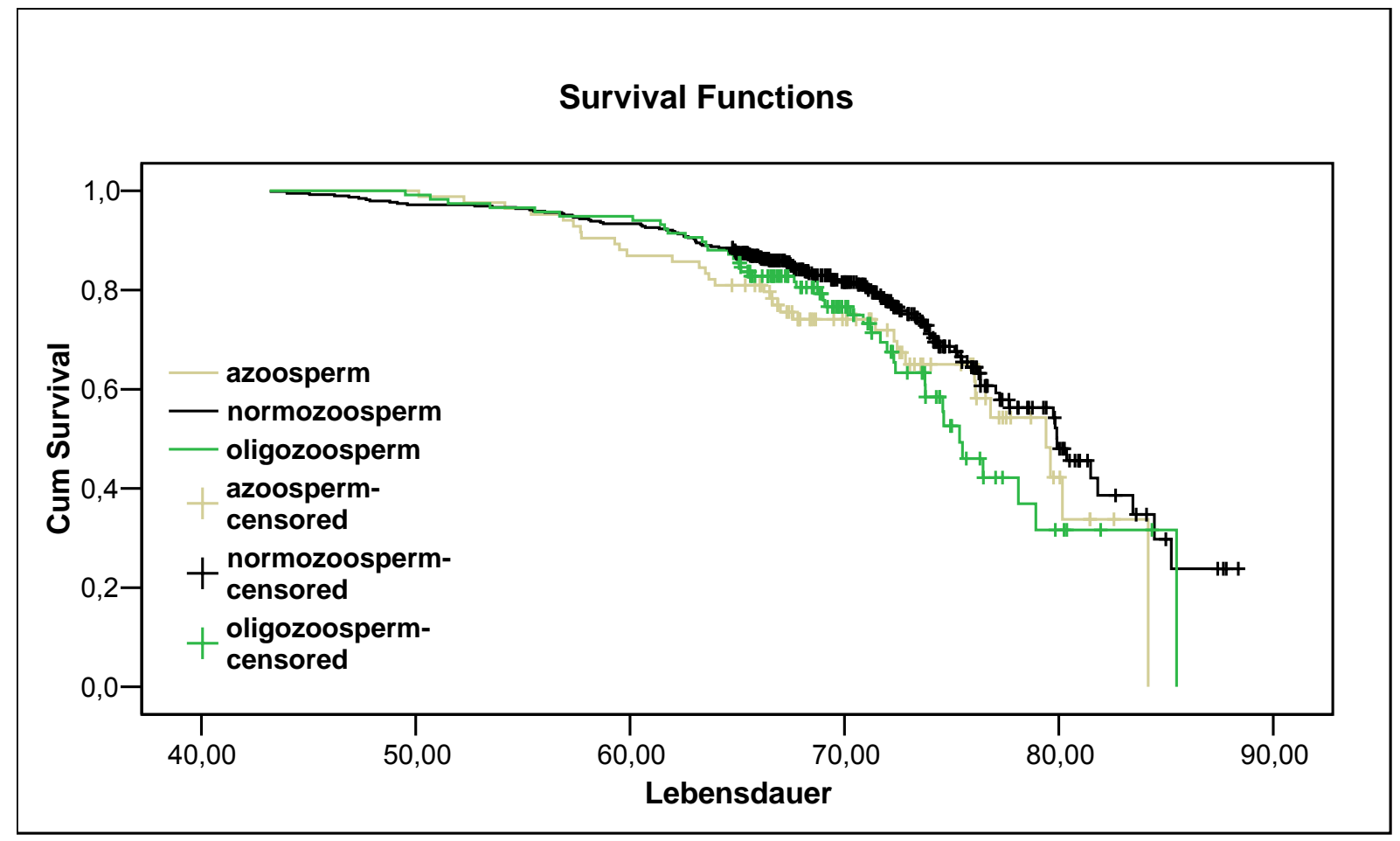

Abb. 3.27 Überlebensfunktionen der normo-, oligo- und azoospermen Fälle

Tab. 3.10 Wilcoxon(Gehan)-Test zum Vergleich der Überlebensfunktionen von normo-, oligound azoospermen Fällen

\begin{tabular}{|l|l|l|l|l|l|}
\hline \multirow{2}{*}{ Vergleich } & \multicolumn{2}{|l|}{$\mathbf{n}$} & df & $\begin{array}{l}\text { Wilcoxon } \\
\text { (Gehan) }\end{array}$ & p \\
\hline unzensiert & zensiert & & \\
\hline overall & 178 & 414 & 2 & 3,638 & 0,162 \\
\hline normo-/oligozoosperm & 146 & 362 & 1 & 1,683 & 0,195 \\
\hline normo-/azoosperm & 136 & 339 & 1 & $2,830^{(*)}$ & 0,093 \\
\hline oligo-/azoosperm & 74 & 127 & 1 & 0,161 & 0,688 \\
\hline
\end{tabular}

\section{Betrachtung der frühen Geburtsjahrgänge}

Hingegen sind die Ergebnisse bei einer ausschließlichen Betrachtung der frühen Geburtsjahrgänge eindeutiger, wie sich aus Abb. 3.28 ersehen lässt. Die Auseinanderentwicklung der Kurven der normozoospermen und oligozoospermen Fälle beginnt zu einem früheren Zeitpunkt, jedoch findet auch hier ab einem hohen erreichten Lebensalter von ca. 85 Jahren eine erneute Annäherung statt. Die Sterblichkeit der oligozoospermen liegt stets über jener der normozoospermen Männer. 
Zudem fällt die Überlebenskurve der azoospermen Patienten ins Auge, da sie eine eher intermediäre Position einnimmt: Sie scheint zunächst der Kurve der oligozoospermen Männer zu gleichen, bis sie sich ab einem Sterbealter von ca. 75 Jahren der Überlebenskurve der normozoospermen Fälle annähert.

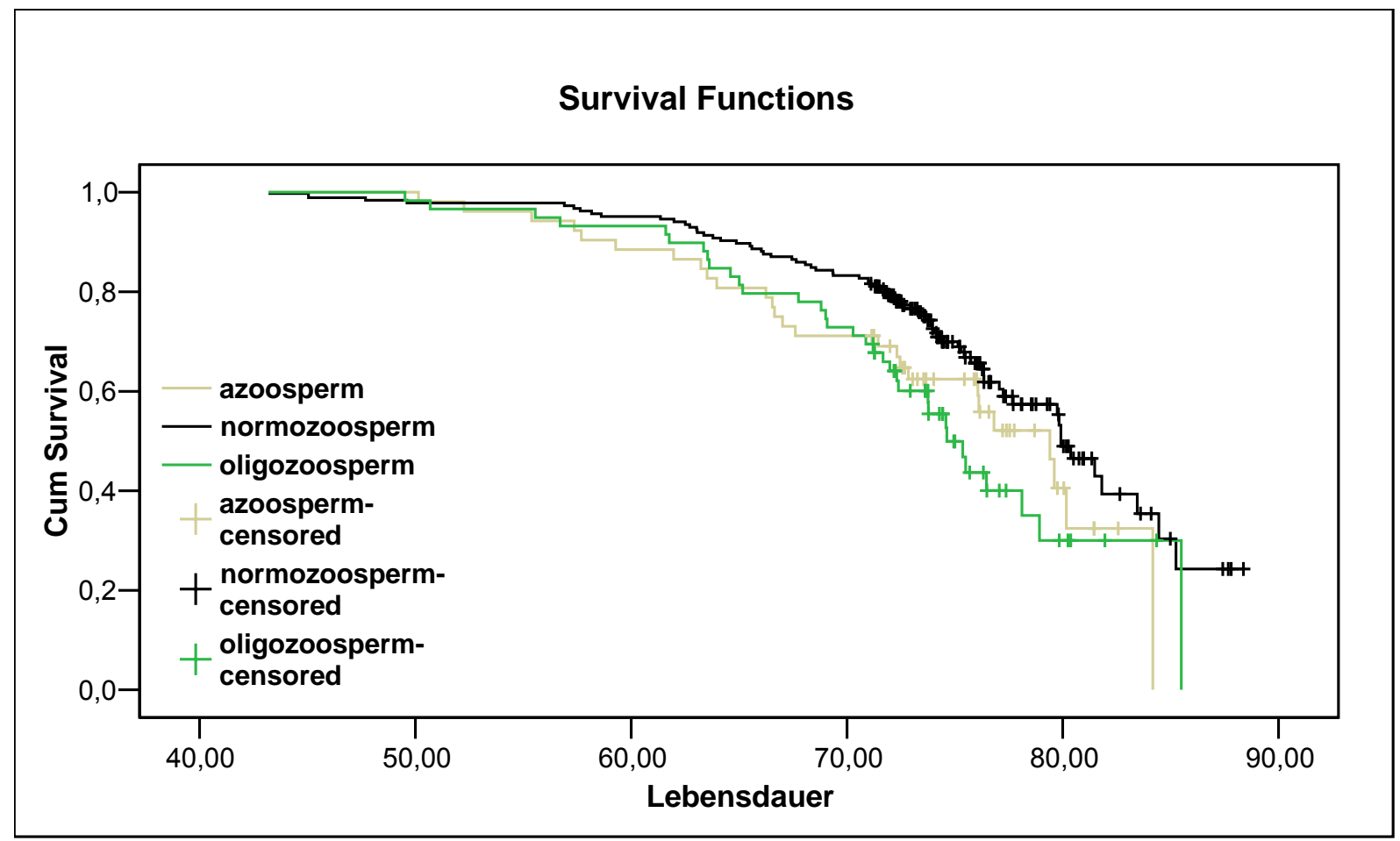

Abb. 3.28 Überlebensfunktionen der normo-, oligo- und azoospermen Fälle; frühe Geburtsjahrgänge

Tab. 3.11 Wilcoxon(Gehan)-Test zum Vergleich der Überlebensfunktionen von normo-, oligound azoospermen Fällen; frühe Geburtsjahrgänge

\begin{tabular}{|l|l|l|l|l|l|}
\hline \multirow{2}{*}{ Vergleich } & $\mathbf{n}$ & df & $\begin{array}{l}\text { Wilcoxon } \\
\text { (Gehan) }\end{array}$ & p \\
\cline { 2 - 5 } & unzensiert & zensiert & & \\
\hline overall & 129 & 167 & 2 & $7,405^{*}$ & 0,025 \\
\hline normo-/oligozoosperm & 103 & 141 & 1 & $6,400^{*}$ & 0,011 \\
\hline normo-/azoosperm & 96 & 141 & 1 & $2,942^{(*)}$ & 0,086 \\
\hline oligo-/azoosperm & 59 & 52 & 1 & 0,222 & 0,638 \\
\hline
\end{tabular}

Diese optischen Eindrücke werden durch die Ergebnisse des Wilcoxon(Gehan)-Tests unterstützt. Der Vergleich des Gesamtmodells ergibt, dass sich die Überlebenskurven der 3 Gruppen voneinander unterscheiden (Wilcoxon(Gehan)=7,405; $p=0,0247 ;$ Tab. 3.11). Bei der paarweisen Betrachtung zeigt sich ein eindeutiger Unterschied zwischen den Überlebensfunktionen der normozoospermen und oligozoospermen Patienten (Wilcoxon (Gehan) $=6,400 ; p=0,0114)$. Für den Vergleich der Kurven der normozoospermen und azoospermen Fälle kann nur eine Tendenz festgestellt werden (Wilcoxon(Gehan)=2,942; 
$p=0,0863$ ), während zwischen den Überlebensraten der oligozoospermen und der azoospermen Männer kein Unterschied besteht.

\section{Betrachtung der späten Geburtsjahrgänge}

Die Beurteilung des Kollektivs der späten Geburtsjahrgänge wird durch die große Anzahl an zensierten Fällen erschwert. In der Abb. 3.29 lässt sich bereits erkennen, dass die Kurven einen ungefähr ähnlichen Verlauf nehmen. Entsprechend lässt sich kein Unterschied zwischen den 3 Subgruppen feststellen (Tab. 3.12).

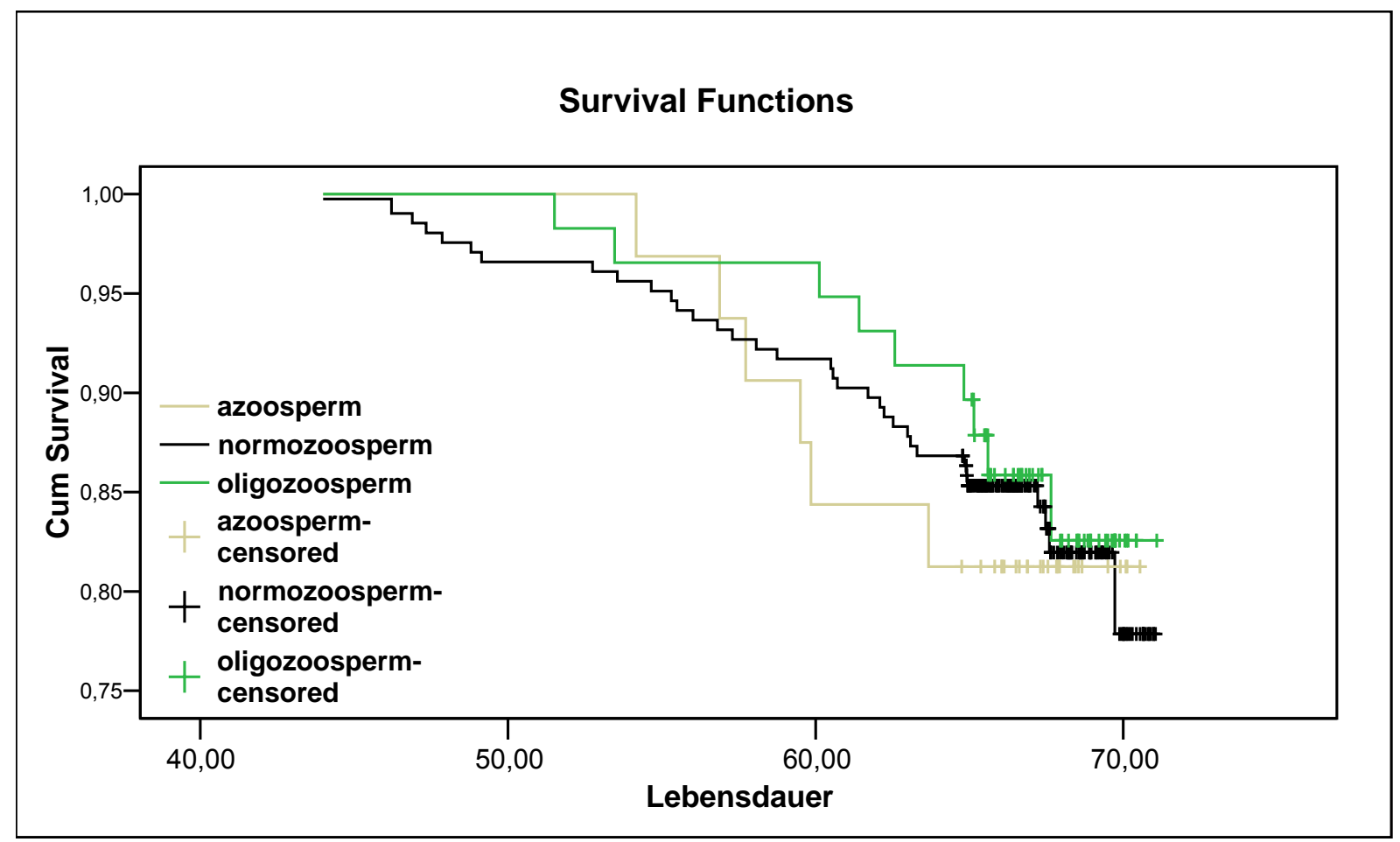

Abb. 3.29 Überlebensfunktionen der normo-, oligo- und azoospermen Fälle; späte Geburtsjahrgänge

Tab. 3.12 Wilcoxon(Gehan)-Test zum Vergleich der Überlebensfunktionen von normo-, oligound azoospermen Fällen; späte Geburtsjahrgänge

\begin{tabular}{|l|l|l|l|l|l||}
\hline \multirow{2}{*}{ Vergleich } & n & df & $\begin{array}{l}\text { Wilcoxon } \\
\text { (Gehan) }\end{array}$ & p \\
\cline { 2 - 5 } & unzensiert & zensiert & & \\
\hline overall & 49 & 246 & 2 & 0,477 & 0,788 \\
\hline normo-/oligozoosperm & 43 & 220 & 1 & 0,183 & 0,668 \\
\hline normo-/azoosperm & 40 & 197 & 1 & 0,211 & 0,646 \\
\hline oligo-/azoosperm & 15 & 75 & 1 & 0,457 & 0,499 \\
\hline
\end{tabular}




\subsubsection{Ergebnisse der semi-parametrischen Verfahren (Cox-Regression)}

Mit Hilfe der Cox-Regression wird der Einfluss des Fertilitätsstatus auf die Lebensdauer bestimmt. Als Kovariaten werden solche Variablen berücksichtigt, welche direkt die Lebensdauer beeinflussen bzw. den Effekt des Fertilitätsstatus auf die Lebensdauer konfundieren könnten. Es werden „Lebenserwartung bei Geburt“, „Geburtsmonat", „Alter bei Untersuchung“, „Jahr der Untersuchung“ und „ISEl“ als Kontrollvariablen in allen Analysen berechnet und ihr Einfluss als von der Zeit unabhängig angenommen. Es handelt sich hierbei um nicht-normalverteilte Daten. Da jedoch auch durch eine Transformation (Logarithmierung zur Basis 10, natürliches Logarithmieren und Bildung der Quadratwurzel) keine einer Normalverteilung entsprechende Distribution erreicht werden kann, muss hier die Normalverteilungsannahme des Verfahrens zu Gunsten seines hohen Aussagegehaltes verletzt werden. Die Cox-Regression erlaubt bei stetigen Variablen die Einschätzung, ob es sich um eine die Lebensdauer vorhersagende Variable handelt. Bei kategorialen Variablen können die Odds Ratios (OR), also die Relation der „Chancen“ einzelner Subgruppen zu versterben, bestimmt werden. Wie schon bereits bei der Darstellung der nicht-parametrischen Analysen erfolgt hier die Betrachtung der Fertilitätsvariablen „fertil/subfertil“ bzw. „normo-/oligo-/ azoosperm“. Auch hier werden unzensierte und zensierte Fälle gemeinsam ausgewertet. Insgesamt werden 308 Normozoosperme, 91 Oligozoosperme und 62 Azoosperme analysiert.

\subsubsection{Fertile und Subfertile}

Werden alle Fälle betrachtet, so ermitteln die Ergebnisse der Cox-Regression das gleiche Mortalitätsrisiko für Fertile wie für Subfertile. Auch der ISEI, das Alter zum Untersuchungszeitpunkt, das Untersuchungsjahr, der Geburtsmonat und die Lebenserwartung bei Geburt können nicht als die Lebensdauer determinierende Variablen angesehen werden (Tab. 3.13).

Tab. 3.13 Cox-Regression zum Einfluss des Fertilitätsstatus (fertil/subfertil) auf die Lebensdauer ${ }^{a}$

\begin{tabular}{|c|c|c|c|c|c|c|c|c|}
\hline & \multirow{2}{*}{ B } & \multirow{2}{*}{ SE } & \multirow{2}{*}{ Wald } & \multirow{2}{*}{ df } & \multirow{2}{*}{$\mathbf{p}$} & \multirow{2}{*}{$\begin{array}{l}\text { Odds } \\
\text { Ratio }\end{array}$} & \multicolumn{2}{|c|}{ 95\% Cl for OR } \\
\hline & & & & & & & Lower & Upper \\
\hline subfertil vs. fertil & 0,284 & 0,186 & 2,330 & 1 & 0,127 & 1,328 & 0,923 & 1,912 \\
\hline $\begin{array}{l}\text { Lebenserwartung } \\
\text { bei Geburt }\end{array}$ & 0,065 & 0,110 & 0,351 & 1 & 0,553 & 1,067 & 0,860 & 1,325 \\
\hline Geburtsmonat & 0,005 & 0,026 & 0,032 & 1 & 0,859 & 1,005 & 0,954 & 1,058 \\
\hline $\begin{array}{l}\text { Alter bei } \\
\text { Untersuchung }\end{array}$ & 0,022 & 0,057 & 0,146 & 1 & 0,702 & 1,022 & 0,914 & 1,144 \\
\hline Untersuchungsjahr & $-0,012$ & 0,057 & 0,048 & 1 & 0,826 & 0,988 & 0,884 & 1,103 \\
\hline ISEI & $-0,003$ & 0,005 & 0,374 & 1 & 0,541 & 0,997 & 0,988 & 1,006 \\
\hline
\end{tabular}

${ }^{a}$ unzensierte Fälle $n=125$; zensierte Fälle $n=336$ 
Anders hingegen zeigen sich die Ergebnisse bei der separaten Betrachtung der frühen Geburtsjahrgänge: Für die subfertilen Männer ist das Risiko zu versterben im Vergleich zu den fertilen Fällen um den Faktor 1,6 erhöht (95\%-Cl 1,1-2,6; Tab. 3.14).

Tab. 3.14 Cox-Regression zum Einfluss des Fertilitätsstatus (fertil/subfertil) auf die Lebensdauer ${ }^{a}$, frühe Geburtsjahrgänge

\begin{tabular}{|l|l|l|l|l|l|l|l|l|}
\hline & B & SE & Wald & df & p & $\begin{array}{l}\text { Odds } \\
\text { Ratio }\end{array}$ & \multicolumn{2}{|l|}{$\begin{array}{l}\text { 95\% Cl for OR } \\
\text { Lower }\end{array}$} \\
\hline subfertil vs. fertil & 0,495 & 0,226 & 4,790 & 1 & 0,029 & $1,640 *$ & 1,053 & 2,554 \\
\hline $\begin{array}{l}\text { Lebenserwartung } \\
\text { bei Geburt }\end{array}$ & 0,169 & 0,226 & 0,560 & 1 & 0,454 & 1,185 & 0,760 & 1,846 \\
\hline Geburtsmonat & 0,004 & 0,033 & 0,013 & 1 & 0,910 & 1,004 & 0,940 & 1,072 \\
\hline $\begin{array}{l}\text { Alter bei } \\
\text { Untersuchung }\end{array}$ & 0,096 & 0,139 & 0,478 & 1 & 0,489 & 1,101 & 0,838 & 1,446 \\
\hline Untersuchungsjahr & $-0,082$ & 0,140 & 0,341 & 1 & 0,559 & 0,921 & 0,700 & 1,213 \\
\hline ISEI & 0,009 & 0,006 & 2,426 & 1 & 0,119 & 1,009 & 0,998 & 1,020 \\
\hline
\end{tabular}

a unzensierte Fälle $n=84$; zensierte Fälle $n=136$

Für die späten Geburtsjahrgänge ergibt sich kein Unterschied im Mortalitätsrisiko zwischen den beiden Subgruppen. Jedoch erweist sich hier der ISEI als erklärende Variable für die Lebensspanne. Mit steigendem ISEI nimmt das Sterblichkeitsrisiko ab (Wald=7,004; $p=0,008 ;$ Tab. 3.15).

Tab. 3.15 Cox-Regression zum Einfluss des Fertilitätsstatus (fertil/subfertil) auf die Lebensdauer ${ }^{a}$, späte Geburtsjahrgänge

\begin{tabular}{|l|l|l|l|l|l|l|l|l|}
\hline & B & SE & Wald & df & p & \multicolumn{2}{|l|}{$\begin{array}{l}\text { Odds } \\
\text { Ratio }\end{array}$} & \multicolumn{2}{|l|}{$\begin{array}{l}\text { L5\% Cl for OR } \\
\text { Lower }\end{array}$} \\
\hline subfertil vs. fertil & 0,021 & 0,346 & 0,004 & 1 & 0,952 & 1,021 & 0,518 & 2,010 \\
\hline $\begin{array}{l}\text { Lebenserwartung } \\
\text { bei Geburt }\end{array}$ & $-0,119$ & 0,842 & 0,020 & 1 & 0,888 & 0,888 & 0,171 & 4,626 \\
\hline Geburtsmonat & 0,015 & 0,046 & 0,099 & 1 & 0,753 & 1,015 & 0,927 & 1,111 \\
\hline $\begin{array}{l}\text { Alter bei } \\
\text { Untersuchung }\end{array}$ & 0,054 & 0,109 & 0,247 & 1 & 0,619 & 1,056 & 0,852 & 1,307 \\
\hline Untersuchungsjahr & $-0,057$ & 0,110 & 0,269 & 1 & 0,604 & 0,945 & 0,762 & 1,171 \\
\hline ISEI & $-0,025$ & 0,009 & 7,004 & 1 & 0,008 & $0,975^{* *}$ & 0,957 & 0,994 \\
\hline
\end{tabular}

a unzensierte Fälle $n=41$; zensierte Fälle $n=199$

\subsubsection{Normozoosperme, Oligozoosperme und Azoosperme}

Das zuvor gefundene Ergebnis des gleichen Mortalitätsrisikos für fertile wie subfertile Patienten bleibt auch bei einer gesonderten Betrachtung der subfertilen Fälle konstant. Weder ist das Sterberisiko der oligozoospermen bzw. azoospermen Männer im Vergleich zu den normozoospermen Fällen signifikant erhöht, noch unterscheiden sich die beiden 
Gruppen in ihrem Mortalitätsrisiko voneinander (Tab. 3.16). Es lässt sich nur der Trend einer größeren Risikos der Oligozoospermen im Vergleich mit den Normozoospermen erkennen $(\mathrm{OR}=1,432 ; 95 \%-\mathrm{Cl}$ 0,9-2,2). Wiederum wird weder der Lebenserwartung bei Geburt noch dem Geburtsmonat, dem Alter der Patienten bei der Untersuchung, dem Untersuchungsjahr oder dem ISEI ein Einfluss auf die Lebensdauer zugeschrieben.

Tab. 3.16 Cox-Regression zum Einfluss des Fertilitätsstatus (normo-loligo-lazoosperm) auf die Lebensdauer ${ }^{\text {a }}$

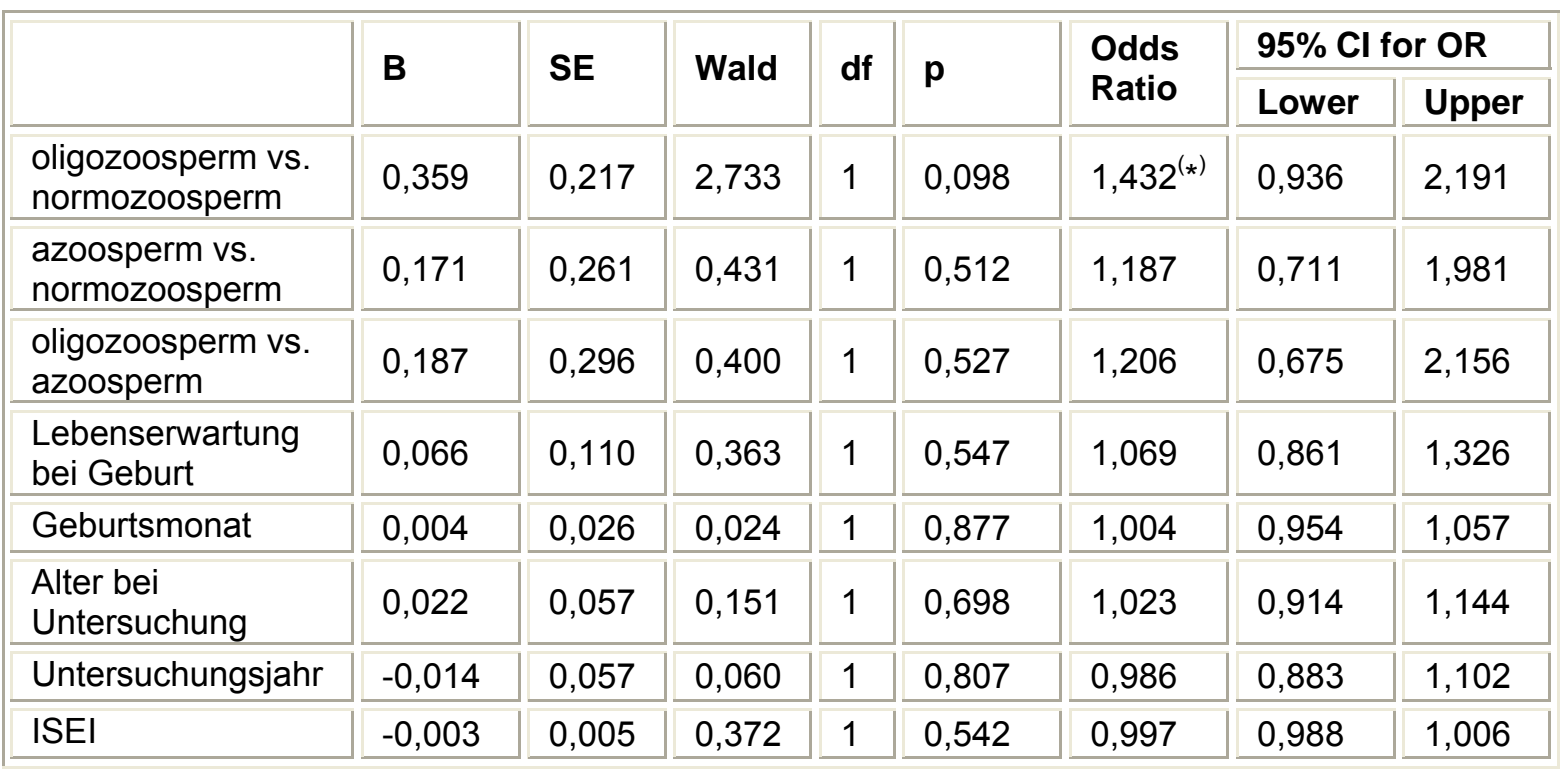

a unzensierte Fälle $n=125$; zensierte Fälle $n=336$

Tab. 3.17 Cox-Regression zum Einfluss des Fertilitätsstatus (normo-loligo-lazoosperm) auf die Lebensdauer ${ }^{a}$; frühe Geburtsjahrgänge

\begin{tabular}{|c|c|c|c|c|c|c|c|c|}
\hline & \multirow{2}{*}{ B } & \multirow{2}{*}{ SE } & \multirow{2}{*}{ Wald } & \multirow{2}{*}{ df } & \multirow{2}{*}{ p } & \multirow{2}{*}{$\begin{array}{l}\text { Odds } \\
\text { Ratio }\end{array}$} & \multicolumn{2}{|c|}{ 95\% Cl for OR } \\
\hline & & & & & & & Lower & Upper \\
\hline $\begin{array}{l}\text { oligozoosperm vs. } \\
\text { normozoosperm }\end{array}$ & 0,722 & 0,260 & 7,722 & 1 & 0,005 & $2,058^{* *}$ & 1,237 & 3,424 \\
\hline $\begin{array}{l}\text { azoosperm vs. } \\
\text { normozoosperm }\end{array}$ & 0,185 & 0,319 & 0,337 & 1 & 0,561 & 1,203 & 0,644 & 2,247 \\
\hline $\begin{array}{l}\text { oligozoosperm vs. } \\
\text { azoosperm }\end{array}$ & 0,537 & 0,352 & 2,320 & 1 & 0,128 & 1,710 & 0,857 & 3,412 \\
\hline $\begin{array}{l}\text { Lebenserwartung } \\
\text { bei Geburt }\end{array}$ & 0,190 & 0,228 & 0,697 & 1 & 0,404 & 1,210 & 0,774 & 1,891 \\
\hline Geburtsmonat & 0,003 & 0,034 & 0,006 & 1 & 0,938 & 1,003 & 0,939 & 1,071 \\
\hline $\begin{array}{l}\text { Alter bei } \\
\text { Untersuchung }\end{array}$ & 0,108 & 0,140 & 0,593 & 1 & 0,441 & 1,114 & 0,847 & 1,465 \\
\hline Untersuchungsjahr & $-0,096$ & 0,141 & 0,467 & 1 & 0,494 & 0,908 & 0,689 & 1,197 \\
\hline ISEI & 0,010 & 0,006 & 2,727 & 1 & 0,099 & $1,010^{(*)}$ & 0,998 & 1,021 \\
\hline
\end{tabular}

a unzensierte Fälle $n=84$; zensierte Fälle $n=136$

Auch hier ergeben sich erst bei der Betrachtung der frühen Jahrgänge eindeutige signifikante Ergebnisse. Wie aus Tab. $3.17 \mathrm{zu}$ entnehmen, ist das Risiko der Oligozoo- 
spermen zu versterben im Vergleich zu den Normozoospermen um mehr als das Doppelte erhöht; dieser Unterschied ist signifikant (OR=2,058; 95\%-Cl 1,2-3,4). Die Analyse der späten Geburtsjahrgänge ergibt analog zu den bereits in Tab. 3.9 vorgestellten Ergebnissen keine Unterschiede in den Sterberisiken der drei Fertilitätsgruppen. Dafür zeigt sich jedoch der ISEI als erklärende Variable, wiederum mit sinkendem Risiko bei steigendem ISEI (Wald=6,868; $p=0,009 ;$ Tab. 3.18).

Tab. 3.18 Cox-Regression zum Einfluss des Fertilitätsstatus (normo-loligo-lazoosperm) auf die Lebensdauer ${ }^{\text {a}}$; späte Geburtsjahrgänge

\begin{tabular}{|c|c|c|c|c|c|c|c|c|}
\hline & \multirow{2}{*}{ B } & \multirow{2}{*}{ SE } & \multirow{2}{*}{ Wald } & \multirow{2}{*}{ df } & \multirow{2}{*}{$\mathbf{p}$} & \multirow{2}{*}{$\begin{array}{l}\text { Odds } \\
\text { Ratio }\end{array}$} & \multicolumn{2}{|c|}{$95 \% \mathrm{Cl}$ for OR } \\
\hline & & & & & & & Lower & Upper \\
\hline $\begin{array}{l}\text { oligozoosperm vs. } \\
\text { normozoosperm }\end{array}$ & $-0,154$ & 0,433 & 0,127 & 1 & 0,722 & 0,857 & 0,367 & 2,002 \\
\hline $\begin{array}{l}\text { azoosperm vs. } \\
\text { normozoosperm }\end{array}$ & 0,269 & 0,459 & 0,342 & 1 & 0,559 & 1,308 & 0,532 & 3,219 \\
\hline $\begin{array}{l}\text { oligozoosperm vs. } \\
\text { azoosperm }\end{array}$ & $-0,423$ & 0,564 & 0,562 & 1 & 0,453 & 0,655 & 0,217 & 1,979 \\
\hline $\begin{array}{l}\text { Lebenserwartung } \\
\text { bei Geburt }\end{array}$ & $-0,098$ & 0,836 & 0,014 & 1 & 0,907 & 0,907 & 0,176 & 4,669 \\
\hline Geburtsmonat & 0,016 & 0,046 & 0,118 & 1 & 0,731 & 1,016 & 0,928 & 1,112 \\
\hline $\begin{array}{l}\text { Alter bei } \\
\text { Untersuchung }\end{array}$ & 0,061 & 0,109 & 0,316 & 1 & 0,574 & 1,063 & 0,859 & 1,315 \\
\hline Untersuchungsjahr & $-0,061$ & 0,108 & 0,317 & 1 & 0,574 & 0,941 & 0,761 & 1,164 \\
\hline ISEI & $-0,025$ & 0,010 & 6,868 & 1 & 0,009 & $0,975^{* *}$ & 0,957 & 0,994 \\
\hline
\end{tabular}

a unzensierte Fälle $n=41$; zensierte Fälle $n=199$

\subsubsection{Alternativ-Modell 1: Alter bei Untersuchung als zeitabhängige Variable}

Bei den bisherigen Berechnungen wurde das Alter zum Untersuchungszeitpunkt als zeitunabhängige Variable angenommen. Alternativ wird dieser Parameter bei der Analyse mittels Cox-Regression ebenfalls als zeitabhängige Kovariate modelliert. So wäre z.B. denkbar, dass die Diagnose einer Subfertilität Verhaltensänderungen in Form einer gesünderen Lebensweise verursachten, was wiederum zu einer höheren Lebensdauer führte. Als weitere Kovariablen werden - wie bei dem bisherigen Modell - die Lebenserwartung bei Geburt, der Geburtsmonat, das Jahr der Untersuchung und der ISEI berechnet. Die Darstellung der Befunde ist im Anhang aufgeführt (Tab. A-2 bis A-7).

Generell ist auffällig, dass sich bei Betrachtung des Alters der Männer zum Zeitpunkt der Spermiogramm-Analyse als zeitabhängiger Kovariate die Ergebnisse nicht wesentlich ändern und die Effekte fast exakt gleich bleiben. Bei Analyse der fertilen und infertilen Fälle ergibt sich ein nicht-signifikantes OR von 1,329 (95\%-Cl 0,9-1,9) und somit für beide Subgruppen das gleiche Risiko zu versterben. Werden ausschließlich die frühen Geburts- 
jahrgänge betrachtet, so ergibt sich ein um den Faktor 1,665 erhöhtes Sterberisiko für die Subfertilen (95\%-Cl 1,1-2,6). Für die späten Geburtsjahrgänge hingegen wird kein Unterschied in der Sterblichkeit festgestellt (OR=1,009; 95\%-Cl 0,5-2,0).

Auch bei Unterscheidung in normo-, oligo- und azoosperme Fälle ergeben sich kaum veränderte Ergebnisse. Bei der Auswertung der gesamten Stichprobe zeigt sich für keine der Subgruppen ein erhöhtes Mortalitätsrisiko $\left(\mathrm{OR}_{\text {oligo-normo }}=1,430 ; 95 \%-\mathrm{Cl} \quad 0,9-2,2 ; \mathrm{OR}_{\text {azoo- }}\right.$ normo $=1,192 ; 95 \%-\mathrm{Cl}$ 0,7-2,0; $\mathrm{OR}_{\text {oligo-azoo }}=1,200 ; 95 \%-\mathrm{Cl}$ 0,7-2,1). Für die frühen Geburtsjahrgänge hingegen ergibt sich für die Oligozoospermen ein mehr als doppelt so hohes Risiko zu versterben wie für die Normozoospermen (OR=2,086; 95\%-Cl 1,3-3,5). Für die Azoospermen zeigt sich kein signifikant größeres Sterberisiko (OR=1,698; 95\%-Cl 0,9-3,4). Die Analyse der späten Geburtsjahrgänge ermittelt wiederum die gleichen Mortalitätsrisiken für die 3 Fertilitäts-Subgruppen $\left(\mathrm{OR}_{\text {oligo-normo }}=0,840 ; 95 \%-\mathrm{Cl} 0,4-2,0 ; \mathrm{OR}_{\text {azoo-normo }}=1,312 ; 95 \%\right.$ $\mathrm{Cl}$ 0,5-3,2; $\left.\mathrm{OR}_{\text {oligo-azoo }}=0,641 ; 95 \%-\mathrm{Cl} 0,2-1,9\right)$.

\subsubsection{Alternativ-Modell 2: Sexuelle Karenz als Kontrollvariable}

Es wurde bereits dargestellt, dass die sexuelle Karenz weder mit der Spermienkonzentration noch mit dem Fertilitätsstatus korreliert (vgl. 3.1.1). Trotzdem soll an dieser Stelle ein Alternativmodell zum Zusammenhang zwischen Fertilitätsstatus und Lebensdauer berechnet werden, welches die sexuelle Abstinenz als mögliche ConfounderVariable berücksichtigt. Des Weiteren fließen die Lebenserwartung bei Geburt, der Geburtsmonat, das Alter zum Untersuchungszeitpunkt, das Untersuchungsjahr und der ISEI in das Modell mit ein. Die tabellarische Aufführung der Ergebnisse erfolgt im Anhang in den Tab. A-8 bis A-13.

Die Modellierung des Einflusses des Fertilitäts-Status auf die Mortalität bei einer Unterscheidung der fertilen und subfertilen Fälle erbringt bei Betrachtung der gesamten Stichprobe das gleiche Sterberisiko für beide Gruppen (OR=1,264; 95\%-Cl 0,8-1,9). Auch keine der anderen Variablen wird als Prädiktorvariable ermittelt. Die bei der Betrachtung der frühen Jahrgänge zuvor gefundene signifikant höhere Risiko der Subfertilen $(O R=1,640$; 95\%-Cl 1,1-2,6), lässt sich bei Einbeziehung der Abstinenzzeit in das Modell zwar nur noch als Trend, jedoch mit einer ähnlichen Effektgröße, wieder finden (OR=1,597; 95\%-Cl 1,0-2,6). Für die späten Geburtsjahrgänge wird keine unterschiedliche Sterblichkeit ermittelt $(\mathrm{OR}=0,931 ; 95 \%-\mathrm{Cl}$ 0,4-1,9). Interessanterweise wird hier die Karenzzeit als Prädiktorvariable für die Lebensdauer ermittelt (Wald=6,094; $p=0,014$ ). Der Einfluss des ISEI zeigt sich nur noch als Trend (Wald=2,877; $p=0,090$ ). 
Der Vergleich aller normo-, oligo- und azoospermen Fälle unter Berücksichtigung der Karenzzeit ergibt die gleichen Sterberisiken für alle 3 Subgruppen $\left(\mathrm{OR}_{\text {oligo-normo }}=1,394 ; 95 \%\right.$ $\mathrm{Cl}$ 0,9-2,2; OR $\mathrm{Ozzo-normo}_{\text {a }}=1,070 ; 95 \%-\mathrm{Cl}$ 0,6-1,9; $\mathrm{OR}_{\text {oligo-azoo }}=1,303 ; 95 \%-\mathrm{Cl}$ 0,7-2,5). Wiederum kommt keine der anderen Variablen als Prädiktorvariable in Betracht. Bei der Auswertung der frühen Geburtsjahrgänge lässt sich bei der Kontrolle der Abstinenz ein um den Faktor 2,1 erhöhtes Sterberisiko der Oligozoospermen im Vergleich zu den Normozoospermen finden $(\mathrm{OR}=2,101 ; 95 \%-\mathrm{Cl}$ 1,2-3,7). Dieses Ergebnis entspricht fast exakt den Befunden bei Berechnung des Modells ohne die Karenzzeit (OR=2,058; 95\%-Cl 1,2-3,4). Das Risiko der azoospermen und normozoospermen Fälle unterscheidet sich nicht $(\mathrm{OR}=1,032 ; 95 \%-\mathrm{Cl}$ 0,52,2), jedoch lässt sich hier der Trend einer höheren Sterblichkeit der Oligozoospermen im Vergleich zu den Azoospermen finden (OR=2,036; 95\%-Cl 0,9-4,6). Innerhalb der späten Geburtsjahrgänge werden für alle 3 Fertilitäts-Gruppen die gleiche Mortalitätsrisiken ermittelt $\left(\mathrm{OR}_{\text {oligo-normo }}=0,779 ; 95 \%-\mathrm{Cl} 0,3-1,9 ; \quad \mathrm{OR}_{\text {azoo-normo }}=1,224 ; 95 \%-\mathrm{Cl} \quad 0,5-3,3 ; \mathrm{OR}_{\text {oligo-azoo }}=0,636\right.$; $95 \%-\mathrm{Cl}$ 0,2-2,1). Hier ergibt sich erneut die sexuelle Karenz als eine die Lebensdauer erklärende Variable $(\mathrm{OR}=1,155 ; 95 \%-\mathrm{Cl} 1,0-1,3)$.

Zusammenfassend lässt sich festhalten, dass sich auch bei Einbeziehung der sexuellen Karenzzeit als Kontrollvariable die Ergebnisse nicht wesentlich ändern. Die Effekte bleiben entweder signifikant oder besitzen zumindest die gleiche Effektgröße. Auch die Effektrichtungen werden beibehalten. Ein überraschendes Ergebnis ist der Befund, dass zumindest bei Betrachtung der späten Geburtsjahrgänge - die sexuelle Abstinenz die Lebensdauer beeinflussen soll.

\subsubsection{Betrachtung der verstorbenen Fälle}

An dieser Stelle erfolgt eine gesonderte Analyse der verstorbenen Fälle, um zu überprüfen, ob die gefundenen Effekte anhand der unzensierten Daten bestätigt werden können. Es bleibt jedoch generell bei diesen Auswertungen zu beachten, dass die Fallzahl bereits gering ist bzw. bei der Betrachtung der Unterstichproben der frühen und späten Geburtsjahrgänge noch einmal reduziert wird. Dieses Fallzahlproblem kann zu nicht-signifikanten Ergebnissen führen. Deshalb wird in diesem Abschnitt das spezielle Augenmerk auf die Effektrichtung und -größe gerichtet.

\section{Betrachtung aller Verstorbenen}

Aus der Abb. 3.30 lässt sich bereits erkennen, dass die Überlebenskurven der fertilen und subfertilen Verstorbenen nahezu identisch verlaufen. Und so ermittelt auch der Wilcoxon(Gehan)-Test keinen Unterschied zwischen den Überlebensfunktionen der beiden Gruppen (Tab. 3.19). Die für die unzensierten und zensierten Daten ermittelte Tendenz eines 
Unterschieds der Kurven der Fertilen und Subfertilen kann anhand der Analyse der Verstorbenen-Daten nicht bestätigt werden.

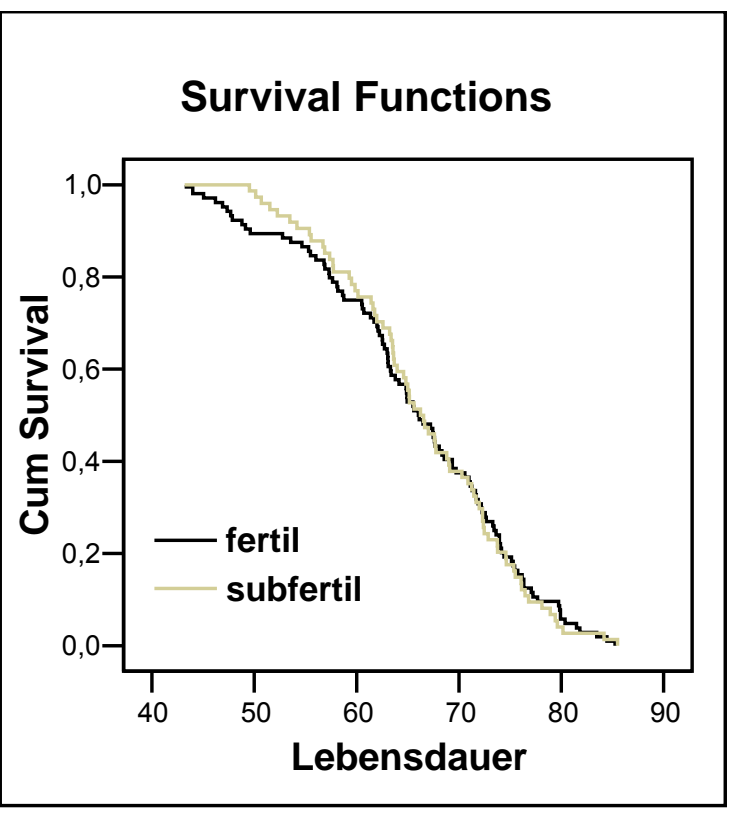

Abb. 3.30 Überlebensfunktionen der fertilen und subfertilen Fälle (Verstorbene)

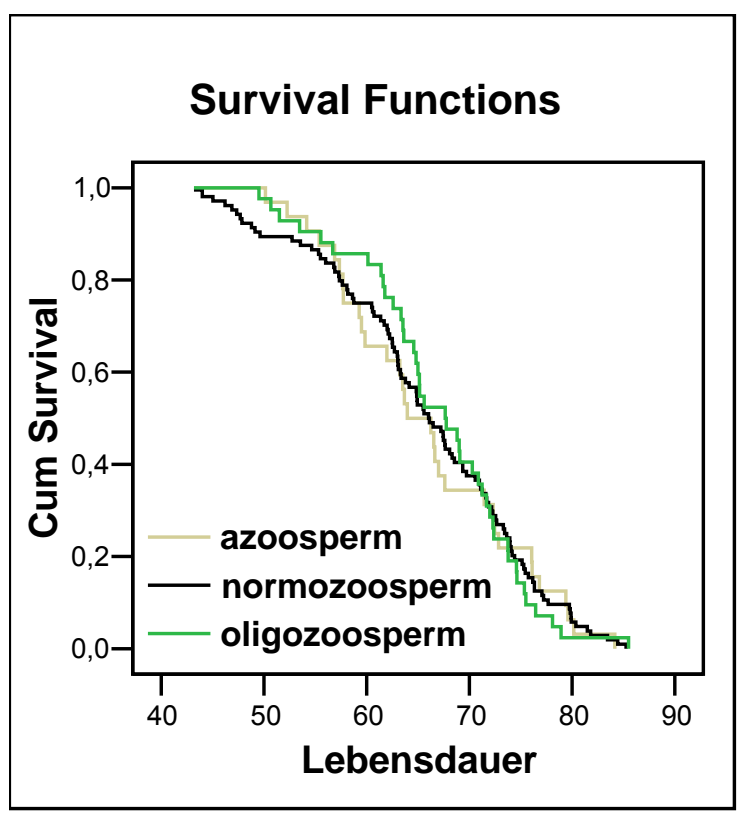

Abb. 3.31 Überlebensfunktionen der normo-, oligo- und azoospermen Fälle (Verstorbene)

Auch bei der Betrachtung der Überlebensfunktionen der Fälle bei einer Einteilung nach normo-, oligo- und azoospermen Verstorbenen fallen die Parallelität der Kurvenverläufe und die häufigen Überschneidungen auf. Wie zu erwarten ist, ermittelt der Wilcoxon(Gehan)-Test keine Unterschiede in den Überlebenskurven der 3 Subgruppen (Abb. 3.31; Tab.3.20). Dies entspricht den Resultaten der Auswertungen der unzensierten und zensierten Daten.

Tab. 3.19 Wilcoxon(Gehan)-Test zum Vergleich der Überlebensfunktion von fertilen und subfertilen Fällen (Verstorbene)

\begin{tabular}{|l|l|l|l|l|}
\hline Vergleich & $\mathbf{n}$ & df & $\begin{array}{l}\text { Wilcoxon } \\
\text { (Gehan) }\end{array}$ & p \\
\hline fertil-subfertil & 178 & 1 & 0,099 & 0,753 \\
\hline
\end{tabular}

Tab. 3.20 Wilcoxon(Gehan)-Test zum Vergleich der Überlebensfunktion von normo-, oligound azoospermen Fällen (Verstorbene)

\begin{tabular}{|l|l|l|l|l|}
\hline Vergleich & $\mathbf{n}$ & $\mathbf{d f}$ & $\begin{array}{l}\text { Wilcoxon } \\
\text { (Gehan) }\end{array}$ & $\mathbf{p}$ \\
\hline overall & 178 & 2 & 0,458 & 0,796 \\
\hline normo-/oligozoosperm & 146 & 1 & 0,351 & 0,554 \\
\hline normo-/azoosperm & 136 & 1 & 0,024 & 0,876 \\
\hline oligo-/azoosperm & 74 & 1 & 0,360 & 0,548 \\
\hline
\end{tabular}


Tab. 3.21 Cox-Regression zum Einfluss des Fertilitätsstatus (fertil/subfertil) auf die Lebensdauer ${ }^{a}$ (Verstorbene)

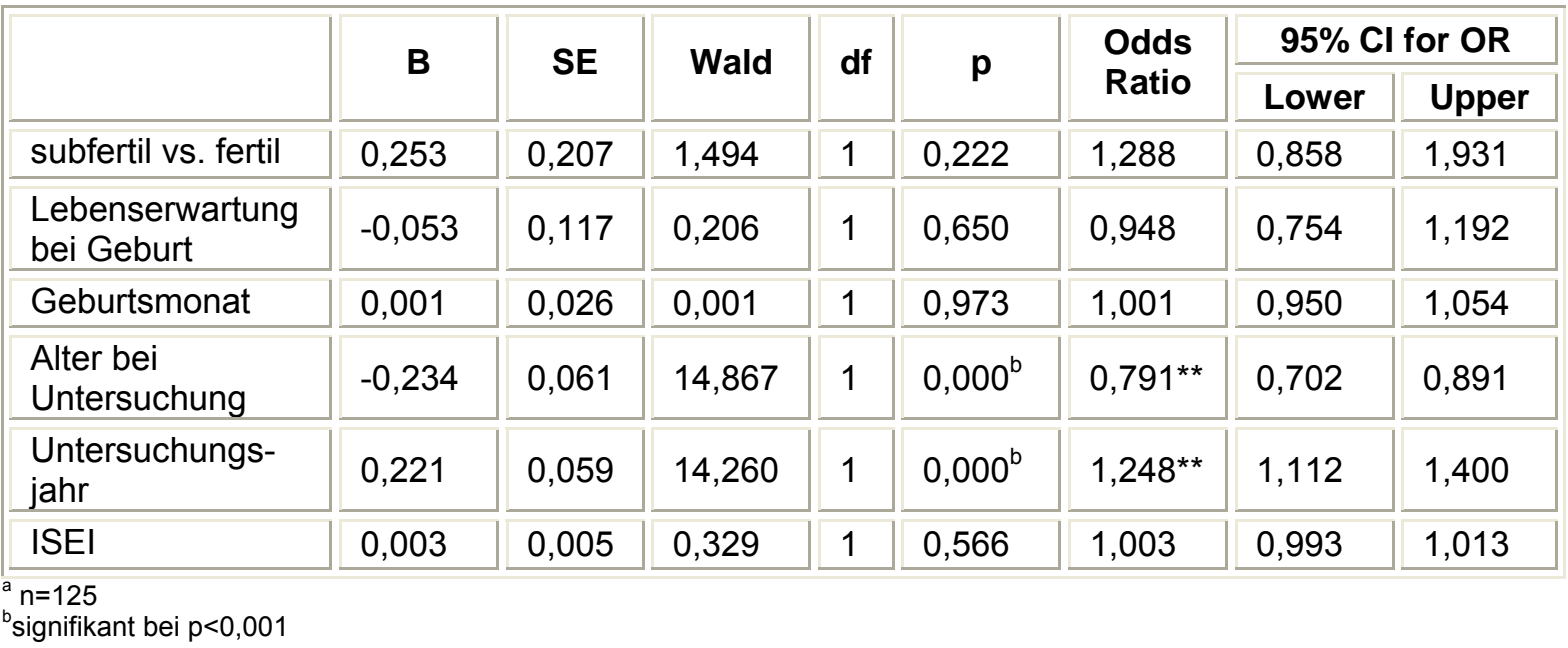

Tab. 3.22 Cox-Regression zum Einfluss des Fertilitätsstatus (normo-/oligo-lazoosperm) auf die Lebensdauer ${ }^{\text {a }}$ (Verstorbene)

\begin{tabular}{|c|c|c|c|c|c|c|c|c|}
\hline & \multirow{2}{*}{ B } & \multirow{2}{*}{ SE } & \multirow{2}{*}{ Wald } & \multirow{2}{*}{ df } & \multirow{2}{*}{$\mathbf{p}$} & \multirow{2}{*}{$\begin{array}{l}\text { Odds } \\
\text { Ratio }\end{array}$} & \multicolumn{2}{|c|}{ 95\% Cl for OR } \\
\hline & & & & & & & Lower & Upper \\
\hline $\begin{array}{l}\text { oligozoosperm vs. } \\
\text { normozoosperm }\end{array}$ & 0,300 & 0,239 & 1,579 & 1 & 0,209 & 1,350 & 0,845 & 2,157 \\
\hline $\begin{array}{l}\text { azoosperm vs. } \\
\text { normozoosperm }\end{array}$ & 0,180 & 0,282 & 0,411 & 1 & 0,522 & 1,198 & 0,690 & 2,080 \\
\hline $\begin{array}{l}\text { oligozoosperm vs. } \\
\text { azoosperm }\end{array}$ & 0,120 & 0,311 & 0,149 & 1 & 0,699 & 1,127 & 0,613 & 2,073 \\
\hline $\begin{array}{l}\text { Lebenserwartung } \\
\text { bei Geburt }\end{array}$ & $-0,054$ & 0,117 & 0,216 & 1 & 0,642 & 0,947 & 0,753 & 1,191 \\
\hline Geburtsmonat & 0,002 & 0,027 & 0,005 & 1 & 0,943 & 1,002 & 0,951 & 1,055 \\
\hline $\begin{array}{l}\text { Alter bei } \\
\text { Untersuchung }\end{array}$ & $-0,236$ & 0,061 & 14,974 & 1 & $0,000^{b}$ & $0,790^{* *}$ & 0,701 & 0,890 \\
\hline $\begin{array}{l}\text { Untersuchungs- } \\
\text { jahr }\end{array}$ & 0,222 & 0,059 & 14,287 & 1 & $0,000^{b}$ & $1,248^{* *}$ & 1,113 & 1,401 \\
\hline ISEI & 0,003 & 0,005 & 0,401 & 1 & 0,527 & 1,003 & 0,993 & 1,013 \\
\hline
\end{tabular}

Die Berechnung der Cox-Regression ergibt keine unterschiedlichen Sterberisiken in Abhängigkeit von der Fertilitätsklasse; die OR lassen keine Unterschiede zwischen den entsprechenden Subgruppen erkennen (Tab 3.21 und 3.22). Dieses Ergebnis entspricht dem für die unzensierten und zensierten Daten gefundenen Resultat. Die Effektrichtungen bleiben erhalten und die OR liegen etwas niedriger. Für die Verstorbenen werden die Parameter des Alters zum Untersuchungszeitpunkt (Wald=14,867 bzw. 14,974; $p<0,001$ ) sowie des Jahres der Untersuchung (Wald=14,260 bzw. 14,287; $p<0,001$ ) als erklärende Variablen modelliert. Das Sterblichkeitsrisiko sinkt bei zunehmendem Alter zum Untersuchungszeitpunkt und bei abnehmendem Untersuchungsjahr. 
Betrachtung der frühen Geburtsjahrgänge der Verstorbenen

Die Darstellung der Überlebenskurven der frühen Geburtsjahrgänge der fertilen und subfertilen Verstorbenen erinnert insgesamt an die Überlebensfunktionen der entsprechenden unzensierten und zensierten Fälle (Abb. 3.32; vgl. Abb. 3.25). Die Trennung der beiden Kurven erfolgt zu einem späteren Zeitpunkt, jedoch nähern sie sich auch hier ab einem erreichten Lebensalter von ca. 84 Jahren wieder an, und die Kurve der Subfertilen fällt schneller ab. Der für unzensierte und zensierte Fälle gefundene statistisch signifikante Unterschied zwischen fertilen und subfertilen Männern lässt sich bei den verstorbenen Fällen noch als Trend wieder finden (Wilcoxon(Gehan)=3,606; $p=0,058 ;$ Tab. 3.23).

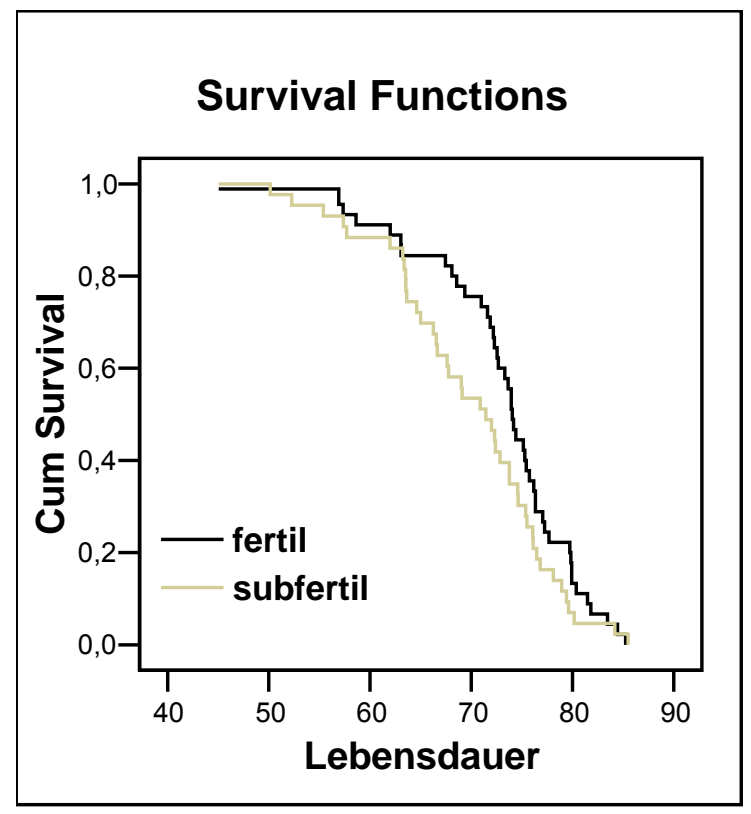

Abb. 3.32 Überlebensfunktionen der

fertilen und subfertilen Fälle (Verstorbene); frühe Geburtsjahrgänge

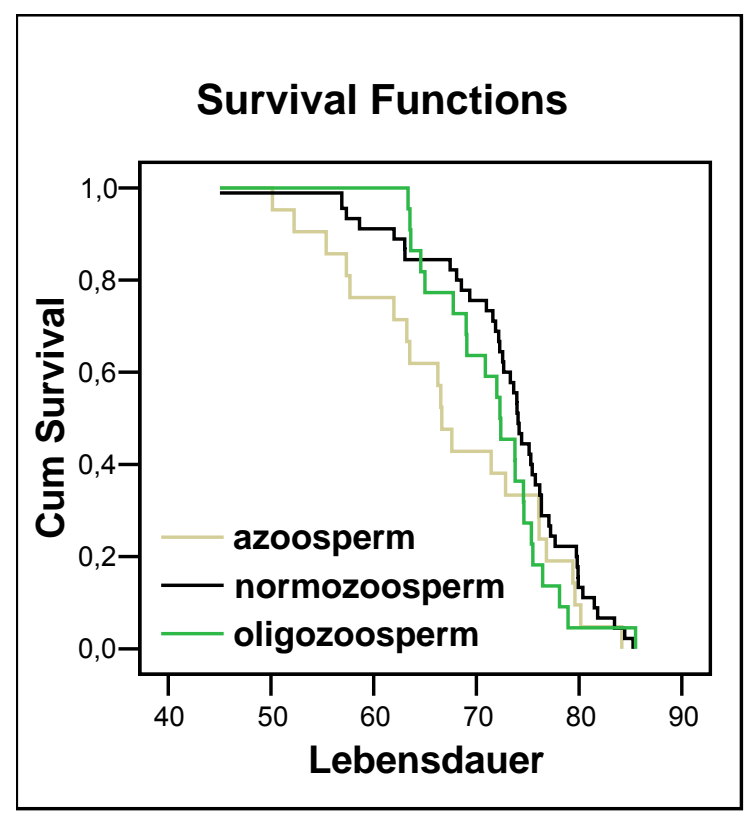

Abb. 3.33 Überlebensfunktionen der normo-, oligo- und azoospermen Fälle (Verstorbene); frühe Geburtsjahrgänge

Aus Abb. 3.33 sind die Überlebenskurven der frühen Jahrgänge der drei Subgruppen der Normo-, Oligo- und Azoospermen ersichtlich. Im Vergleich zu den Überlebenskurven der frühen Geburtsjahrgänge der unzensierten und zensierten Fälle lässt sich ein deutlich früherer Abfall der Kurve der azoospermen Verstorbenen ablesen. Nun nimmt die Kurve der Oligozoospermen eine eher intermediäre Position ein. Dieser optische Eindruck wird durch den Wilcoxon(Gehan)-Test statistisch durch den Unterschied zwischen den Überlebenskurven der normozoospermen und azoospermen Verstorbenen bekräftigt (Wilcoxon (Gehan) $=3,957 ; p=0,047 ;$ Tab. 3.24). Der Unterschied zwischen Normozoospermen und Oligozoospermen, welcher bei der Analyse der unzensierten und zensierten Daten ermittelt wurde, kann für die Verstorbenen nicht belegt werden. 
Tab. 3.23 Wilcoxon(Gehan)-Test zum Vergleich der Überlebensfunktion von fertilen und subfertilen Fällen (Verstorbene); frühe Geburtsjahrgänge

\begin{tabular}{|l|l|l|l|l|}
\hline Vergleich & $\mathbf{n}$ & df & $\begin{array}{l}\text { Wilcoxon } \\
\text { (Gehan) }\end{array}$ & p \\
\hline fertil-subfertil & 88 & 1 & $3,606^{(*)}$ & 0,058 \\
\hline
\end{tabular}

Tab. 3.24 Wilcoxon(Gehan)-Test zum Vergleich der Überlebensfunktion von normo-, oligound azoospermen Fällen (Verstorbene); frühe Geburtsjahrgänge

\begin{tabular}{|l|l|l|l|l|}
\hline Vergleich & $\mathbf{n}$ & df & $\begin{array}{l}\text { Wilcoxon } \\
\text { (Gehan) }\end{array}$ & p \\
\hline overall & 88 & 2 & $4,598^{(*)}$ & 0,100 \\
\hline normo-/oligozoosperm & 67 & 1 & 1,228 & 0,268 \\
\hline normo-/azoosperm & 66 & 1 & $3,957^{*}$ & 0,047 \\
\hline oligo-/azoosperm & 43 & 1 & 1,476 & 0,224 \\
\hline
\end{tabular}

Der Befund der Cox-Regression für die frühen Jahrgänge mit unzensierten und zensierten Daten, nämlich einem höheren Sterberisiko für die Subfertilen, kann bei der Analyse der Verstorbenen nicht repliziert werden. Jedoch bleiben Richtung und Größe des Effektes mit einem OR von 1,5 vergleichbar, auch wenn es sich um ein nicht-signifikantes Ergebnis handelt (Tab. 3.25).

Tab. 3.25 Cox-Regression zum Einfluss des Fertilitätsstatus (fertil/subfertil) auf die Lebensdauer ${ }^{\text {a }}$ (Verstorbene); frühe Geburtsjahrgänge

\begin{tabular}{|l|r|r|r|r|r|r|r|r|}
\hline & B & SE & Wald & df & p & $\begin{array}{l}\text { Odds } \\
\text { Ratio }\end{array}$ & \multicolumn{2}{|c|}{ 95\% Cl for OR } \\
\hline subfertil vs. fertil & 0,402 & 0,308 & 1,701 & 1 & 0,192 & 1,495 & 0,817 & 2,734 \\
\hline $\begin{array}{l}\text { Lebenserwartung } \\
\text { bei Geburt }\end{array}$ & $-0,066$ & 0,300 & 0,048 & 1 & 0,826 & 0,936 & 0,520 & 1,685 \\
\hline Geburtsmonat & $-0,016$ & 0,044 & 0,132 & 1 & 0,716 & 0,984 & 0,903 & 1,072 \\
\hline $\begin{array}{l}\text { Alter bei } \\
\text { Untersuchung }\end{array}$ & $-0,220$ & 0,216 & 1,032 & 1 & 0,310 & 0,803 & 0,526 & 1,226 \\
\hline $\begin{array}{l}\text { Untersuchungs- } \\
\text { jahr }\end{array}$ & 0,203 & 0,218 & 0,865 & 1 & 0,352 & 1,225 & 0,799 & 1,877 \\
\hline ISEI & 0,011 & 0,007 & 2,552 & 1 & 0,110 & 1,011 & 0,997 & 1,025 \\
\hline
\end{tabular}

Auch innerhalb der normozoospermen, oligozoospermen und azoospermen Verstorbenen sind für die frühen Geburtsjahrgänge die Risiken zu versterben gleich groß (Tab. 3.26). Das erhöhte Sterblichkeitsrisiko für oligozoosperme im Vergleich zu normozoospermen Männern, welche für die unzensierten und zensierten Daten ermittelt wurde $(O R=2,1)$, schlägt sich hier in einem Trend mit einem vergleichbarem OR von 1,9 nieder (95\%-Cl 0,9-3,9). Ebenfalls lässt sich der Trend eines Einflusses des ISEI erkennen (Wald=3,586; $p=0,058$ ), jedoch sollte hier beachtet werden, dass es sich lediglich um ein OR von 1,014 handelt. 
Tab. 3.26 Cox-Regression zum Einfluss des Fertilitätsstatus (normo-loligo-lazoosperm) auf die Lebensdauer ${ }^{a}$ (Verstorbene); frühe Geburtsjahrgänge

\begin{tabular}{|c|c|c|c|c|c|c|c|c|}
\hline & \multirow{2}{*}{ B } & \multirow{2}{*}{ SE } & \multirow{2}{*}{ Wald } & \multirow{2}{*}{ df } & \multirow{2}{*}{ p } & \multirow{2}{*}{$\begin{array}{l}\text { Odds } \\
\text { Ratio }\end{array}$} & \multicolumn{2}{|c|}{$95 \% \mathrm{Cl}$ for OR } \\
\hline & & & & & & & Lower & Upper \\
\hline $\begin{array}{l}\text { oligozoosperm vs. } \\
\text { normozoosperm }\end{array}$ & 0,641 & 0,371 & 2,994 & 1 & 0,084 & $1,899^{(*)}$ & 0,918 & 3,927 \\
\hline $\begin{array}{l}\text { azoosperm vs. } \\
\text { normozoosperm }\end{array}$ & 0,117 & 0,424 & 0,076 & 1 & 0,782 & 1,124 & 0,490 & 2,582 \\
\hline $\begin{array}{l}\text { oligozoosperm vs. } \\
\text { azoosperm }\end{array}$ & 0,524 & 0,494 & 1,127 & 1 & 0,288 & 1,689 & 0,642 & 4,445 \\
\hline $\begin{array}{l}\text { Lebenserwartung } \\
\text { bei Geburt }\end{array}$ & $-0,048$ & 0,300 & 0,025 & 1 & 0,874 & 0,954 & 0,530 & 1,717 \\
\hline Geburtsmonat & $-0,018$ & 0,044 & 0,170 & 1 & 0,680 & 0,982 & 0,901 & 1,070 \\
\hline $\begin{array}{l}\text { Alter bei } \\
\text { Untersuchung }\end{array}$ & $-0,213$ & 0,214 & 0,983 & 1 & 0,321 & 0,809 & 0,531 & 1,231 \\
\hline $\begin{array}{l}\text { Untersuchungs- } \\
\text { jahr }\end{array}$ & 0,191 & 0,217 & 0,772 & 1 & 0,380 & 1,210 & 0,791 & 1,852 \\
\hline ISEI & 0,014 & 0,007 & 3,586 & 1 & 0,058 & $1,014^{(*)}$ & 1,000 & 1,029 \\
\hline
\end{tabular}

Betrachtung der späten Geburtsjahrgänge der Verstorbenen

Die Betrachtung der späten Geburtsjahrgänge der Verstorbenen spiegelt die Befunde für unzensierte und zensierte Fälle wider. Die Überlebenskurven verlaufen weitestgehend parallel bzw. überschneiden sich mehrfach, und es lässt sich anhand des Wilcoxon(Gehan)Tests kein Unterschied zwischen ihnen bestimmen (Abb. 3.34 und 3.35; Tab. 3.27 und 3.28).

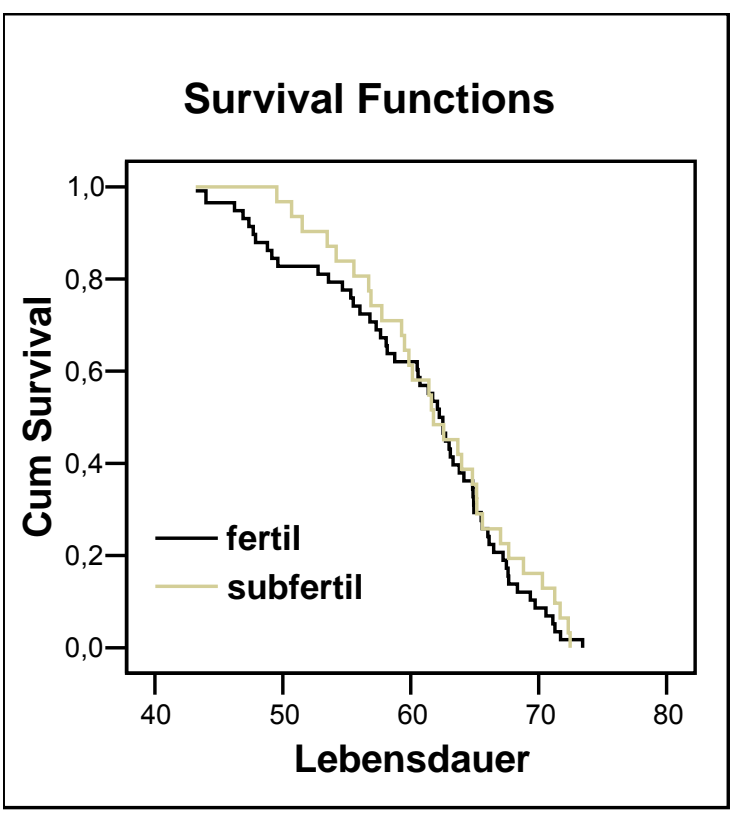

Abb. 3.34 Überlebensfunktionen der fertilen und subfertilen Fälle (Verstorbene); späte Geburtsjahrgänge

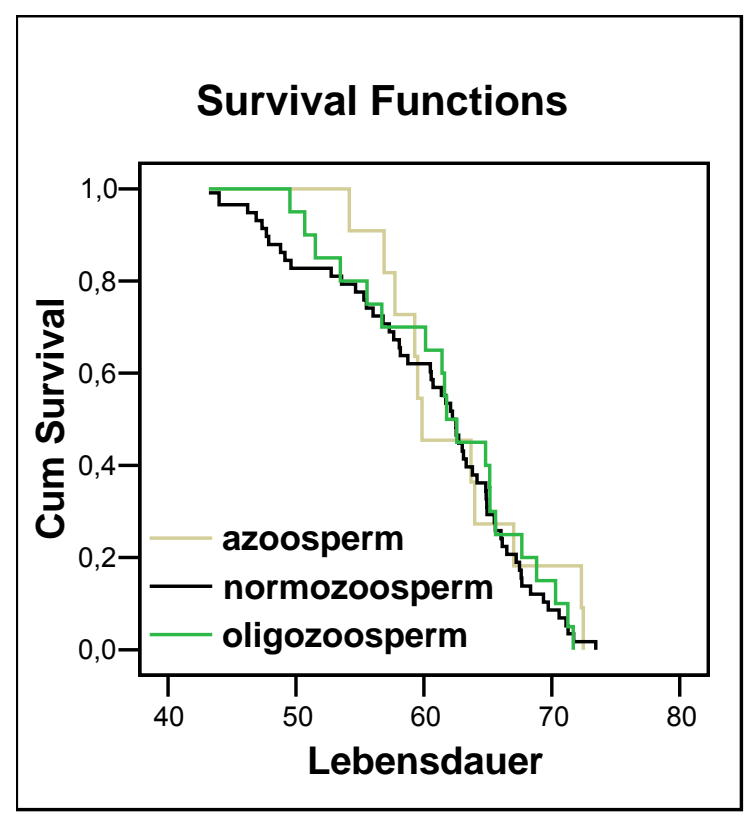

Abb. 3.35 Überlebensfunktionen der normo-, oligo- und azoospermen Fälle (Verstorbene); späte Geburtsjahrgänge 
Tab. 3.27 Wilcoxon(Gehan)-Test zum Vergleich der Überlebensfunktion von fertilen und subfertilen Fällen (Verstorbene); späte Geburtsjahrgänge

\begin{tabular}{|l|l|l|l|l|}
\hline Vergleich & $\mathbf{n}$ & df & $\begin{array}{l}\text { Wilcoxon } \\
\text { (Gehan) }\end{array}$ & p \\
\hline fertil-subfertil & 89 & 1 & 0,428 & 0,513 \\
\hline
\end{tabular}

Tab. 3.28 Wilcoxon(Gehan)-Test zum Vergleich der Überlebensfunktion von normo-, oligound azoospermen Fällen (Verstorbene); späte Geburtsjahrgänge

\begin{tabular}{|l|l|l|l|l|}
\hline Vergleich & $\mathbf{n}$ & $\mathbf{d f}$ & $\begin{array}{l}\text { Wilcoxon } \\
\text { (Gehan) }\end{array}$ & $\mathbf{p}$ \\
\hline overall & 89 & 2 & 0,428 & 0,807 \\
\hline normo-/oligozoosperm & 78 & 1 & 0,327 & 0,567 \\
\hline normo-/azoosperm & 69 & 1 & 0,182 & 0,670 \\
\hline oligo-/azoosperm & 31 & 1 & 0,002 & 0,967 \\
\hline
\end{tabular}

Analog werden auch bei der Cox-Regression vergleichbare Ergebnisse ermittelt. Die Cox-Regression berechnet für die späten Geburtsjahrgänge keine Unterschiede in den Sterberisiken für fertile und subfertile bzw. normo-, oligo- und azoosperme Verstorbene. Das Mortalitätsrisiko ist bei keiner der Subgruppen erhöht (Tab. 3.29 und 3.30). Dies entspricht dem Befund für die unzensierten und zensierten Fälle. Der Befund des ISEI als Prädiktorvariable kann hingegen nicht repliziert werden. Jedoch wird das Untersuchungsjahr als die Lebensdauer erklärende Variable modelliert (Wald=4,114 bzw. 4,144; $p=0,043$ bzw. 0,042). Auch lässt sich der Trend eines Einflusses des Alters der Patienten zum Zeitpunkt der Spermiogramm-Analyse erkennen (Wald=2,978 bzw. 3,001; $p=0,084$ bzw. 0,083).

Tab. 3.29 Cox-Regression zum Einfluss des Fertilitätsstatus (fertil/subfertil) auf die Lebensdauer ${ }^{a}$ (Verstorbene); späte Jahrgänge

\begin{tabular}{|c|c|c|c|c|c|c|c|c|}
\hline & \multirow{2}{*}{ B } & \multirow{2}{*}{ SE } & \multirow{2}{*}{ Wald } & \multirow{2}{*}{ df } & \multirow{2}{*}{$\mathbf{p}$} & \multirow{2}{*}{$\begin{array}{l}\text { Odds } \\
\text { Ratio }\end{array}$} & \multicolumn{2}{|c|}{$95 \% \mathrm{Cl}$ for OR } \\
\hline & & & & & & & Lower & Upper \\
\hline subfertil vs. fertil & $-0,076$ & 0,307 & 0,062 & 1 & 0,803 & 0,926 & 0,508 & 1,690 \\
\hline $\begin{array}{l}\text { Lebenserwartung } \\
\text { bei Geburt }\end{array}$ & 0,252 & 0,211 & 1,426 & 1 & 0,232 & 1,286 & 0,851 & 1,944 \\
\hline Geburtsmonat & 0,046 & 0,040 & 1,332 & 1 & 0,248 & 1,047 & 0,968 & 1,133 \\
\hline $\begin{array}{l}\text { Alter bei } \\
\text { Untersuchung }\end{array}$ & $-0,142$ & 0,082 & 2,978 & 1 & 0,084 & $0,868^{(*)}$ & 0,739 & 1,019 \\
\hline $\begin{array}{l}\text { Untersuchungs- } \\
\text { jahr }\end{array}$ & 0,154 & 0,076 & 4,114 & 1 & 0,043 & $1,166^{*}$ & 1,005 & 1,353 \\
\hline ISEI & $-0,008$ & 0,008 & 1,159 & 1 & 0,282 & 0,992 & 0,976 & 1,007 \\
\hline
\end{tabular}


Tab. 3.30 Cox-Regression zum Einfluss des Fertilitätsstatus (normo-loligo-lazoosperm) auf die Lebensdauer ${ }^{\text {a }}$ (Verstorbene); späte Geburtsjahrgänge

\begin{tabular}{|c|c|c|c|c|c|c|c|c|}
\hline & \multirow{2}{*}{ B } & \multirow{2}{*}{ SE } & \multirow{2}{*}{ Wald } & \multirow{2}{*}{ df } & \multirow{2}{*}{$\mathbf{p}$} & \multirow{2}{*}{$\begin{array}{l}\text { Odds } \\
\text { Ratio }\end{array}$} & \multicolumn{2}{|c|}{$95 \% \mathrm{Cl}$ for OR } \\
\hline & & & & & & & Lower & Upper \\
\hline $\begin{array}{l}\text { oligozoosperm vs. } \\
\text { normozoosperm }\end{array}$ & $-0,052$ & 0,335 & 0,024 & 1 & 0,876 & 0,949 & 0,492 & 1,829 \\
\hline $\begin{array}{l}\text { azoosperm vs. } \\
\text { normozoosperm }\end{array}$ & $-0,131$ & 0,438 & 0,090 & 1 & 0,765 & 0,877 & 0,372 & 2,069 \\
\hline $\begin{array}{l}\text { oligozoosperm vs. } \\
\text { azoosperm }\end{array}$ & 0,079 & 0,448 & 0,031 & 1 & 0,860 & 1,082 & 0,450 & 2,602 \\
\hline $\begin{array}{l}\text { Lebenserwartung } \\
\text { bei Geburt }\end{array}$ & 0,249 & 0,211 & 1,392 & 1 & 0,238 & 1,283 & 0,848 & 1,939 \\
\hline Geburtsmonat & 0,048 & 0,041 & 1,354 & 1 & 0,245 & 1,049 & 0,968 & 1,138 \\
\hline $\begin{array}{l}\text { Alter bei } \\
\text { Untersuchung }\end{array}$ & $-0,144$ & 0,083 & 3,001 & 1 & 0,083 & $0,866^{(*)}$ & 0,736 & 1,019 \\
\hline $\begin{array}{l}\text { Untersuchungs- } \\
\text { jahr }\end{array}$ & 0,155 & 0,076 & 4,144 & 1 & 0,042 & $1,168^{*}$ & 1,006 & 1,357 \\
\hline ISEI & $-0,008$ & 0,008 & 1,156 & 1 & 0,282 & 0,992 & 0,976 & 1,007 \\
\hline
\end{tabular}

\subsubsection{Ergebnisse der parametrischen Regressionsmodelle}

Zur vollständigen Untersuchung des Zusammenhangs zwischen Fertilitätsstatus und Lebensspanne werden zusätzlich parametrische Modelle zur Berechnung herangezogen. Es kommen das Exponential-Modell, des Gompertz-Makeham- Modell sowie das Weibull-Modell zur Anwendung. Allgemeine Vorgehensweise ist die Betrachtung des Gesamt-Modells, jeweils unter Einbeziehung des ISEI, des Untersuchungsjahres, des Alters zum Untersuchungszeitpunkt, des Geburtsmonats und der Lebenserwartung bei Geburt als Kontrollvariablen. Wie bereits bei der Berechnung des Regressionsmodells nach Cox wird die Normalverteilungsannahme hinsichtlich der unabhängigen Variablen vernachlässigt. Es werden wiederum sowohl die tatsächlichen Lebensdauern der Verstorbenen als auch die geschätzten Lebensspannen der zensierten Fälle modelliert $\left(n_{\text {normo }}=308, n_{\text {oligo }}=91, n_{\text {azoo }}=62\right)$.

\subsubsection{Exponential-Modell}

\section{Betrachtung aller Fälle}

Im Exponential-Modell wird kein Unterschied in der Lebensdauer in Abhängigkeit vom Fertilitätsstatus deutlich (Tab. 3.31 und 3.32). Auch der ISEI, der Geburtsmonat und die Lebenserwartung bei Geburt stellen keine Prädiktorvariablen für die Mortalität dar. Die Kovariaten „Alter zum Untersuchungszeitpunkt“ und „Untersuchungsjahr" hingegen zeigen 
einen deutlichen Einfluss auf die Sterblichkeit (Alter bei Untersuchung: C/Error=2,5 bzw. 2,6; $p=0,011$ bzw. 0,010 und Untersuchungsjahr: C/Error=-2,4; $p=0,015$ bzw. 0,014). Die Betrachtung der Relativen Risiken (RR) bestätigt die erwartete Effektrichtung: Das Mortalitätsrisiko sinkt im Laufe der Untersuchungsjahre, während es mit zunehmendem Alter zum Untersuchungszeitpunkt ansteigt.

Tab. 3.31 Exponential-Modell zum Einfluss des Fertilitätsstatus (fertil/subfertil) auf die Lebensdauer $^{a}$

\begin{tabular}{|l|l|l|l|l|l|l|}
\hline Variable & Coeff & Error & C/Error & Signif & p & $\begin{array}{l}\text { Rel. } \\
\text { Risiko }\end{array}$ \\
\hline Fertilitätsstatus: subf./fertil & 0,1958 & 0,1854 & 1,0556 & 0,7089 & 0,291 & 1,2162 \\
\hline Lebenserwartung bei Geburt & 0,1094 & 0,1030 & 1,0617 & 0,7116 & 0,288 & 1,1156 \\
\hline Geburtsmonat & 0,0078 & 0,0265 & 0,2939 & 0,2311 & 0,769 & 1,0078 \\
\hline Alter bei Untersuchung & 0,1374 & 0,0539 & 2,5487 & 0,9892 & 0,011 & $1,1473^{*}$ \\
\hline Untersuchungsjahr & $-0,1292$ & 0,0532 & $-2,4276$ & 0,9848 & 0,015 & $0,8788^{*}$ \\
\hline ISEI & $-0,0039$ & 0,0045 & $-0,8698$ & 0,6156 & 0,384 & 0,9961 \\
\hline
\end{tabular}

${ }^{a}$ unzensierte Fälle $n=125$; zensierte Fälle $n=336$

Tab. 3.32 Exponential-Modell zum Einfluss des Fertilitätsstatus (normo-/oligo-lazoosperm) auf die Lebensdauer ${ }^{\mathrm{a}}$

\begin{tabular}{|l|l|l|l|l|l|l|}
\hline Variable & Coeff & Error & C/Error & Signif & p & $\begin{array}{l}\text { Rel. } \\
\text { Risiko }\end{array}$ \\
\hline $\begin{array}{l}\text { Fertilitätsstatus: } \\
\text { normo-/oligo-/azoosperm }\end{array}$ & 0,0967 & 0,1191 & 0,8124 & 0,5835 & 0,417 & 1,1016 \\
\hline Lebenserwartung bei Geburt & 0,1114 & 0,1029 & 1,0824 & 0,7209 & 0,279 & 1,1179 \\
\hline \hline Geburtsmonat & 0,0085 & 0,0265 & 0,3216 & 0,2523 & 0,748 & 1,0086 \\
\hline Alter bei Untersuchung & 0,1384 & 0,0538 & 2,5708 & 0,9899 & 0,010 & $1,1484^{* *}$ \\
\hline \hline Untersuchungsjahr & $-0,1302$ & 0,0532 & $-2,4491$ & 0,9857 & 0,014 & $0,8779^{*}$ \\
\hline ISEI & $-0,0041$ & 0,0045 & $-0,9045$ & 0,6343 & 0,366 & 0,9959 \\
\hline
\end{tabular}

a unzensierte Fälle $n=125$; zensierte Fälle $n=336$

Betrachtung der frühen Geburtsjahrgänge

Tab. 3.33 Exponential-Modell zum Einfluss des Fertilitätsstatus (fertil/subfertil) auf die Lebensdauer $^{\mathrm{a}}$; frühe Geburtsjahrgänge

\begin{tabular}{|l|l|l|l|l|l|l|}
\hline Variable & Coeff & Error & C/Error & Signif & p & $\begin{array}{l}\text { Rel. } \\
\text { Risiko }\end{array}$ \\
\hline Fertilitätsstatus: subf./fertil & 0,3527 & 0,2246 & 1,5701 & 0,8836 & 0,116 & 1,4229 \\
\hline Lebenserwartung bei Geburt & 0,2257 & 0,2180 & 1,0351 & 0,6994 & 0,301 & 1,2532 \\
\hline Geburtsmonat & 0,0130 & 0,0337 & 0,3871 & 0,3013 & 0,699 & 1,0131 \\
\hline Alter bei Untersuchung & 0,2092 & 0,1334 & 1,5674 & 0,8830 & 0,117 & 1,2326 \\
\hline Untersuchungsjahr & $-0,1993$ & 0,1339 & $-1,4890$ & 0,8635 & 0,137 & 0,8193 \\
\hline $\begin{array}{l}\text { ISEI } \\
\text { a unzensierte Fälle } \mathrm{n}=84 ; \text { zensierte Fälle } \mathrm{n}=136\end{array}$ & 0,0043 & 0,0053 & 0,8104 & 0,5823 & 0,418 & 1,0043 \\
\hline
\end{tabular}


Tab. 3.34 Exponential-Modell zum Einfluss des Fertilitätsstatus (normo-/oligo-lazoosperm) auf die Lebensdauer ${ }^{a}$; frühe Geburtsjahrgänge

\begin{tabular}{|l|l|l|l|l|l|l|}
\hline Variable & Coeff & Error & C/Error & Signif & p & $\begin{array}{l}\text { Rel. } \\
\text { Risiko }\end{array}$ \\
\hline $\begin{array}{l}\text { Fertilitätsstatus: } \\
\text { normo-/oligo-/azoosperm }\end{array}$ & 0,1379 & 0,1402 & 0,9834 & 0,6746 & 0,325 & 1,1479 \\
\hline Lebenserwartung bei Geburt & 0,2269 & 0,2177 & 1,0422 & 0,7027 & 0,297 & 1,2547 \\
\hline Geburtsmonat & 0,0142 & 0,0336 & 0,4235 & 0,3281 & 0,672 & 1,0143 \\
\hline Alter bei Untersuchung & 0,2096 & 0,1332 & 1,5741 & 0,8845 & 0,116 & 1,2332 \\
\hline Untersuchungsjahr & $-0,2004$ & 0,1338 & $-1,4980$ & 0,8659 & 0,134 & 0,8184 \\
\hline ISEI & 0,0036 & 0,0053 & 0,6799 & 0,5034 & 0,497 & 1,0036 \\
\hline
\end{tabular}

a unzensierte Fälle $n=84$; zensierte Fälle $n=136$

Betrachtung der späten Geburtsjahrgänge

Analog zu den frühen Jahrgängen besteht in diesem Modell auch bei den späten Geburtsjahrgängen kein Einfluss des Fertilitätsstatus auf die Sterblichkeit. Hier zeigt sich jedoch der ISEI als signifikante, die Mortalität vorhersagende Variable: Das Sterberisiko sinkt mit steigendem ISEI (C/Error=-2,4; p=0,017 bzw. 0,016; Tab. 3.35 und 3.36).

Tab. 3.35 Exponential-Modell zum Einfluss des Fertilitätsstatus (fertil/subfertil) auf die Lebensdauer $^{\text {a }}$; späte Geburtsjahrgänge

\begin{tabular}{|l|l|l|l|l|l|l|}
\hline Variable & Coeff & Error & C/Error & Signif & p & $\begin{array}{l}\text { Rel. } \\
\text { Risiko }\end{array}$ \\
\hline Fertilitätsstatus: subf./fertil & $-0,0003$ & 0,3452 & $-0,0009$ & 0,0007 & 0,999 & 0,9997 \\
\hline \hline Lebenserwartung bei Geburt & $-0,0884$ & 0,8435 & $-0,1048$ & 0,0835 & 0,917 & 0,9154 \\
\hline \hline Geburtsmonat & 0,0096 & 0,0469 & 0,2043 & 0,1619 & 0,838 & 1,0096 \\
\hline Alter bei Untersuchung & 0,1198 & 0,1052 & 1,1394 & 0,7455 & 0,255 & 1,1273 \\
\hline Untersuchungsjahr & $-0,1185$ & 0,1057 & $-1,1210$ & 0,7377 & 0,262 & 0,8882 \\
\hline \hline ISEl & $-0,0224$ & 0,0094 & $-2,3909$ & 0,9832 & 0,017 & $0,9779^{*}$ \\
\hline
\end{tabular}

Tab. 3.36 Exponential-Modell zum Einfluss des Fertilitätsstatus (normo-/oligo-lazoosperm) auf die Lebensdauer ${ }^{a}$; späte Geburtsjahrgänge

\begin{tabular}{|l|l|l|l|l|l|l|}
\hline Variable & Coeff & Error & C/Error & Signif & p & $\begin{array}{l}\text { Rel. } \\
\text { Risiko }\end{array}$ \\
\hline $\begin{array}{l}\text { Fertilitätsstatus: } \\
\text { normo-/oligo-/azoosperm }\end{array}$ & 0,0535 & 0,2238 & 0,2389 & 0,1888 & 0,811 & 1,0549 \\
\hline Lebenserwartung bei Geburt & $-0,0907$ & 0,8430 & $-0,1075$ & 0,0856 & 0,914 & 0,9133 \\
\hline Geburtsmonat & 0,0093 & 0,0468 & 0,1982 & 0,1571 & 0,843 & 1,0093 \\
\hline Alter bei Untersuchung & 0,1180 & 0,1049 & 1,1248 & 0,7393 & 0,261 & 1,1252 \\
\hline Untersuchungsjahr & $-0,1155$ & 0,1055 & $-1,0951$ & 0,7265 & 0,274 & 0,8909 \\
\hline ISEl & $-0,0224$ & 0,0094 & $-2,3996$ & 0,9836 & 0,016 & $0,9778^{*}$ \\
\hline
\end{tabular}




\subsubsection{Gompertz-Makeham-Modell}

\section{Betrachtung aller Fälle}

Unter Berücksichtigung aller Fälle modelliert das Gompertz-Makeham-Modell keinen Einfluss des Fertilitätsstatus auf die Mortalität (Tab. 3.37 und 3.38). Auch kann weder für die Variablen der Lebenserwartung bei Geburt und des Geburtsmonats noch für die des ISEI ein Einfluss festgestellt werden. Dagegen stellt das Alter der Patienten sowie das Untersuchungsjahres eine Prädiktorvariable dar (Alter bei Untersuchung: C/Error=2,7 bzw. 2,8; $p=0,006$ bzw. 0,008 und Untersuchungsjahr: C/Error=-2,6 bzw. $-2,7 ; p=0,006$ ).

Tab. 3.37 Gompertz-Makeham-Modell zum Einfluss des Fertilitätsstatus (fertil/subfertil) auf die Lebensdauer ${ }^{a}$

\begin{tabular}{|l|l|l|l|l|l|l|}
\hline Variable & Coeff & Error & C/Error & Signif & p & $\begin{array}{l}\text { Rel. } \\
\text { Risiko }\end{array}$ \\
\hline Fertilitätsstatus: subf./fertil & 0,2983 & 0,1859 & 1,6048 & 0,8915 & 0,109 & 1,3476 \\
\hline Lebenserwartung bei Geburt & 0,0850 & 0,1045 & 0,8132 & 0,5839 & 0,416 & 1,0887 \\
\hline Geburtsmonat & 0,0061 & 0,0262 & 0,2331 & 0,1843 & 0,816 & 1,0061 \\
\hline Alter bei Untersuchung & 0,1500 & 0,0546 & 2,7470 & 0,9940 & 0,006 & $1,1618^{* *}$ \\
\hline Untersuchungsjahr & $-0,1418$ & 0,0535 & $-2,6486$ & 0,9919 & 0,008 & $0,8678^{* *}$ \\
\hline ISEI & $-0,0025$ & 0,0047 & $-0,5325$ & 0,4056 & 0,594 & 0,9975 \\
\hline
\end{tabular}

${ }^{a}$ unzensierte Fälle $n=125$; zensierte Fälle $n=336$

Tab. 3.38 Gompertz-Makeham-Modell zum Einfluss des Fertilitätsstatus (normo-/oligo-I azoosperm) auf die Lebensdauer ${ }^{\mathrm{a}}$

\begin{tabular}{|l|l|l|l|l|l|l|}
\hline Variable & Coeff & Error & C/Error & Signif & $\mathbf{p}$ & $\begin{array}{l}\text { Rel. } \\
\text { Risiko }\end{array}$ \\
\hline $\begin{array}{l}\text { Fertiliätsstatus: } \\
\text { normo-/oligo-/azoosperm }\end{array}$ & 0,1428 & 0,1183 & 1,2076 & 0,7728 & 0,227 & 1,1535 \\
\hline \hline Lebenserwartung bei Geburt & 0,0871 & 0,1042 & 0,8356 & 0,5966 & 0,403 & 1,0910 \\
\hline Geburtsmonat & 0,0073 & 0,0261 & 0,2790 & 0,2197 & 0,780 & 1,0073 \\
\hline \hline Alter bei Untersuchung & 0,1505 & 0,0544 & 2,7663 & 0,9943 & 0,006 & $1,1625^{\star *}$ \\
\hline Untersuchungsjahr & $-0,1425$ & 0,0534 & $-2,6664$ & 0,9923 & 0,008 & $0,8672^{* *}$ \\
\hline ISEI & $-0,0026$ & 0,0047 & $-0,5664$ & 0,4289 & 0,571 & 0,9974 \\
\hline
\end{tabular}

a unzensierte Fälle $n=125$; zensierte Fälle $n=336$

Betrachtung der frühen Geburtsjahrgänge

Bei der Analyse der frühen Geburtsjahrgänge mittels Gompertz-Makeham-Modell kann nun dem Fertilitätsstatus (fertil/subfertil) ein Einfluss auf die Sterblichkeit zugeschrieben werden. Subfertile haben ein 1,7mal höheres Risiko zu versterben als Fertile. Dieser Effekt bleibt auch bei Kontrolle der Lebenserwartung bei Geburt, des Geburtsmonats, des Alters bei Untersuchung, des Untersuchungsjahres sowie des ISEI statistisch signifikant (C/Error= 2,2; p=0,023; Tab. 3.39 und 3.40). Im Gegensatz zur Cox-Regression ermitteln die parametrischen Verfahren das Relative Risiko des Fertilitätsstatus im Gesamtmodell und nicht im paarweisen Vergleich, wodurch wohl zu erklären ist, dass sich bei einer Einteilung in normo-, 
oligo- und azoosperme Fälle kein Einfluss der Fertilität auf die Mortalität feststellen lässt. Interessant ist die Betrachtung der Effektrichtung des ISEI. Es lässt sich ein positiver Trend erkennen: Mit sinkendem ISEI sinkt auch die Sterblichkeit (C/Error=1,7; $p=0,095)$.

Tab. 3.39 Gompertz-Makeham-Modell zum Einfluss des Fertilitätsstatus (fertil/subfertil) auf die Lebensdauer ${ }^{a}$; frühe Geburtsjahrgänge

\begin{tabular}{|l|l|l|l|l|l|l|}
\hline Variable & Coeff & Error & C/Error & Signif & p & $\begin{array}{l}\text { Rel. } \\
\text { Risiko }\end{array}$ \\
\hline Fertilitätsstatus: subf./fertil & 0,5136 & 0,2261 & 2,2716 & 0,9769 & 0,023 & $1,6713^{*}$ \\
\hline Lebenserwartung bei Geburt & 0,2249 & 0,2176 & 1,0338 & 0,6987 & 0,301 & 1,2522 \\
\hline Geburtsmonat & 0,0075 & 0,0331 & 0,2278 & 0,1802 & 0,820 & 1,0076 \\
\hline Alter bei Untersuchung & 0,2484 & 0,1337 & 1,8587 & 0,9369 & 0,063 & $1,2820^{(*)}$ \\
\hline Untersuchungsjahr & $-0,2361$ & 0,1343 & $-1,7579$ & 0,9212 & 0,079 & $0,7897^{(*)}$ \\
\hline ISEI & 0,0094 & 0,0056 & 1,6702 & 0,9051 & 0,095 & $1,0094^{(*)}$ \\
\hline
\end{tabular}

${ }^{a}$ unzensierte Fälle $n=84$; zensierte Fälle $n=136$

Tab. 3.40 Gompertz-Makeham-Modell zum Einfluss des Fertilitätsstatus (normo-/oligo-I azoosperm) auf die Lebensdauer ${ }^{a}$; frühe Geburtsjahrgänge

\begin{tabular}{|l|l|l|l|l|l|l|}
\hline Variable & Coeff & Error & C/Error & Signif & p & $\begin{array}{l}\text { Rel. } \\
\text { Risiko }\end{array}$ \\
\hline $\begin{array}{l}\text { Fertilitätsstatus: } \\
\text { normo-/oligo-/azoosperm }\end{array}$ & 0,1948 & 0,1387 & 1,4044 & 0,8398 & 0,160 & 1,2150 \\
\hline Lebenserwartung bei Geburt & 0,2228 & 0,2158 & 1,0326 & 0,6982 & 0,302 & 1,2496 \\
\hline Geburtsmonat & 0,0092 & 0,0329 & 0,2806 & 0,2210 & 0,779 & 1,0093 \\
\hline Alter bei Untersuchung & 0,2460 & 0,1326 & 1,8542 & 0,9363 & 0,064 & $1,2789^{(*)}$ \\
\hline Untersuchungsjahr & $-0,2354$ & 0,1336 & $-1,7622$ & 0,9220 & 0,078 & $0,7902^{(*)}$ \\
\hline ISEI & 0,0084 & 0,0056 & 1,5121 & 0,8695 & 0,131 & 1,0085 \\
\hline
\end{tabular}

a unzensierte Fälle $n=84$; zensierte Fälle $n=136$

\section{Betrachtung der späten Geburtsjahrgänge}

Die Betrachtung der Ergebnisse der späten Jahrgänge in den Tab. 3.41 und 3.42 ergibt ein anderes Bild: Hier wirkt sich der Fertilitätsstatus nicht auf die Lebensspanne aus. Hingegen zeigt sich der ISEI als Prädiktorvariable, nun allerdings mit umgekehrter Effektrichtung, d.h. mit sinkendem Mortalitätsrisiko bei steigendem Status (C/Error=-2,5; $p=0,013$ ).

Tab. 3.41 Gompertz-Makeham-Modell zum Einfluss des Fertilitätsstatus (fertil/subfertil) auf die Lebensdauer ${ }^{a}$; späte Geburtsjahrgänge

\begin{tabular}{|l|l|l|l|l|l|l|}
\hline Variable & Coeff & Error & ClError & Signif & p & $\begin{array}{l}\text { Rel. } \\
\text { Risiko }\end{array}$ \\
\hline Fertilitätsstatus: subf./fertil & 0,0011 & 0,0072 & 0,1582 & 0,1257 & 0,874 & 1,0564 \\
\hline Lebenserwartung bei Geburt & 0,0174 & 0,0235 & 0,7399 & 0,5406 & 0,459 & 2,3105 \\
\hline Geburtsmonat & 0,0004 & 0,0009 & 0,4292 & 0,3322 & 0,668 & 1,0198 \\
\hline Alter bei Untersuchung & $-0,0002$ & 0,0029 & $-0,0773$ & 0,0616 & 0,938 & 0,9892 \\
\hline Untersuchungsjahr & 0,0003 & 0,0029 & 0,1167 & 0,0929 & 0,907 & 1,0166 \\
\hline ISEI & $-0,0005$ & 0,0002 & $-2,4768$ & 0,9867 & 0,013 & $0,9752^{*}$ \\
\hline
\end{tabular}

${ }^{a}$ unzensierte Fälle $n=41$; zensierte Fälle $n=199$ 
Tab. 3.42 Gompertz-Makeham-Modell zum Einfluss des Fertilitätsstatus (normo-/oligo-I azoosperm) auf die Lebensdauer ${ }^{a}$; späte Geburtsjahrgänge

\begin{tabular}{|l|l|l|l|l|l|l|}
\hline Variable & Coeff & Error & C/Error & Signif & p & $\begin{array}{l}\text { Rel. } \\
\text { Risiko }\end{array}$ \\
\hline $\begin{array}{l}\text { Fertilitätsstatus: } \\
\text { normo-/oligo-/azoosperm }\end{array}$ & 0,0020 & 0,0047 & 0,4233 & 0,3279 & 0,672 & 1,0999 \\
\hline Lebenserwartung bei Geburt & 0,0170 & 0,0235 & 0,7235 & 0,5307 & 0,469 & 2,2670 \\
\hline Geburtsmonat & 0,0004 & 0,0009 & 0,4310 & 0,3335 & 0,667 & 1,0199 \\
\hline Alter bei Untersuchung & $-0,0002$ & 0,0029 & $-0,0711$ & 0,0567 & 0,943 & 0,9901 \\
\hline Untersuchungsjahr & 0,0004 & 0,0029 & 0,1214 & 0,0966 & 0,903 & 1,0172 \\
\hline ISEI & $-0,0005$ & 0,0002 & $-2,4864$ & 0,9871 & 0,013 & $0,9750^{*}$ \\
\hline
\end{tabular}

a unzensierte Fälle $n=41$; zensierte Fälle $n=199$

\subsubsection{Weibull-Modell}

\section{Betrachtung aller Fälle}

Wie bereits beim Gompertz-Makeham-Modell zeigt sich kein statistisch signifikanter Einfluss des Fertilitätsstatus auf die Mortalität (Tab. 3.43 und 3.44); jedoch lässt das WeibullModell hier einen Trend beobachten. Subfertile Männer haben ein 1,4fach erhöhtes Sterberisiko im Vergleich zu den Fertilen (C/Error=1,9; $p=0,057)$. Die Lebenserwartung bei Geburt stellt hier eine die Mortalität vorhersagende Variable dar (C/Error=-2,3 bzw. $-2,4 ; p=0,021$ bzw. 0,018). Es muss jedoch berücksichtigt werden, dass das Modell bei der Auswertung der gesamten Stichprobe nicht konvergierte und somit die Ergebnisse keine validen Resultate darstellen.

Tab. 3.43 Weibull-Modell zum Einfluss des Fertilitätsstatus (fertil/subfertil) auf die Lebensdauer $^{a, b}$

\begin{tabular}{|l|l|l|l|l|l|l|}
\hline Variable & Coeff & Error & C/Error & Signif & p & $\begin{array}{l}\text { Rel. } \\
\text { Risiko }\end{array}$ \\
\hline Fertilitätsstatus: subf./fertil & 0,0068 & 0,0036 & 1,9030 & 0,9430 & 0,057 & $1,4314^{(*)}$ \\
\hline Lebenserwartung bei Geburt & $-0,0045$ & 0,0019 & $-2,3173$ & 0,9795 & 0,021 & $0,7900^{*}$ \\
\hline Geburtsmonat & 0,0000 & 0,0005 & $-0,0395$ & 0,0315 & 0,969 & 0,9990 \\
\hline Alter bei Untersuchung & $-0,0004$ & 0,0010 & $-0,4209$ & 0,3262 & 0,674 & 0,9792 \\
\hline Untersuchungsjahr & 0,0006 & 0,0009 & 0,6874 & 0,5081 & 0,492 & 1,0342 \\
\hline ISEI & $-0,0001$ & 0,0001 & $-0,7393$ & 0,5403 & 0,460 & 0,9966 \\
\hline
\end{tabular}

anzensierte Fälle $n=125$; zensierte Fälle $n=336$

${ }^{\mathrm{b}}$ Convergence not reached in 500000 iterations; Number of function evaluations: 1000002 
Tab. 3.44 Weibull-Modell zum Einfluss des Fertilitätsstatus (normo-/oligo-/azoosperm) auf die Lebensdauer ${ }^{a, b}$

\begin{tabular}{|l|l|l|l|l|l|l|}
\hline Variable & Coeff & Error & C/Error & Signif & p & $\begin{array}{l}\text { Rel. } \\
\text { Risiko }\end{array}$ \\
\hline $\begin{array}{l}\text { Fertilitätsstatus: } \\
\text { normo-/oligo-/azoosperm }\end{array}$ & 0,0036 & 0,0023 & 1,5697 & 0,8835 & 0,117 & 1,2071 \\
\hline Lebenserwartung bei Geburt & $-0,0046$ & 0,0020 & $-2,3667$ & 0,9821 & 0,018 & $0,7859^{*}$ \\
\hline Geburtsmonat & 0,0000 & 0,0005 & $-0,0281$ & 0,0224 & 0,978 & 0,9993 \\
\hline Alter bei Untersuchung & $-0,0005$ & 0,0010 & $-0,4842$ & 0,3718 & 0,628 & 0,9762 \\
\hline Untersuchungsjahr & 0,0007 & 0,0009 & 0,7599 & 0,5527 & 0,447 & 1,0379 \\
\hline ISEI & $-0,0001$ & 0,0001 & $-0,7548$ & 0,5496 & 0,450 & 0,9965 \\
\hline
\end{tabular}

a unzensierte Fälle $n=125$; zensierte Fälle $n=336$

b Convergence not reached in 500000 iterations; Number of function evaluations: 1000002

\section{Betrachtung der frühen Geburtsjahrgänge}

Bei der Auswertung der frühen Geburtsjahrgänge zeigt sich ein deutlicher Effekt des Fertilitätsstatus (fertil/subfertil) auf die Sterblichkeit $(C /$ Error $=2,3 ; p=0,020)$. Wie bereits im Gompertz-Makeham-Modell ermittelt, ergibt sich für die Subfertilen ein um den Faktor 1,7 erhöhtes Sterberisiko. Zusätzlich lässt sich der Trend des ISEI als Prädiktorvariable erkennen, wobei - wiederum überraschenderweise - die Sterblichkeit mit abnehmendem Status sinkt (C/Error=1,9 bzw. 1,8; $p=0,052$ bzw. 0,076; Tab. 3.45 und 3.46).

Tab. 3.45 Weibull-Modell zum Einfluss des Fertilitätsstatus (fertil/subfertil) auf die Lebensdauer $^{a}$; frühe Geburtsjahrgänge

\begin{tabular}{|l|l|l|l|l|l|l|}
\hline Variable & Coeff & Error & C/Error & Signif & p & $\begin{array}{l}\text { Rel. } \\
\text { Risiko }\end{array}$ \\
\hline Fertilitätsstatus: subf./fertil & 0,0096 & 0,0041 & 2,3356 & 0,9805 & 0,020 & $1,7137^{*}$ \\
\hline Lebenserwartung bei Geburt & $-0,0035$ & 0,0041 & $-0,8514$ & 0,6054 & 0,395 & 0,8230 \\
\hline Geburtsmonat & $-0,0002$ & 0,0006 & $-0,2999$ & 0,2358 & 0,764 & 0,9900 \\
\hline Alter bei Untersuchung & $-0,0003$ & 0,0025 & $-0,1107$ & 0,0882 & 0,912 & 0,9845 \\
\hline Untersuchungsjahr & 0,0006 & 0,0025 & 0,2214 & 0,1752 & 0,825 & 1,0319 \\
\hline ISEI & 0,0002 & 0,0001 & 1,9417 & 0,9478 & 0,052 & $1,0110^{(*)}$ \\
\hline
\end{tabular}

${ }^{a}$ unzensierte Fälle $n=84$; zensierte Fälle $n=136$

Tab. 3.46 Weibull-Modell zum Einfluss des Fertilitätsstatus (normo-/oligo-lazoosperm) auf die Lebensdauer $^{\mathrm{a}}$; frühe Geburtsjahrgänge

\begin{tabular}{|l|l|l|l|l|l|l|}
\hline Variable & Coeff & Error & C/Error & Signif & $\mathbf{p}$ & $\begin{array}{l}\text { Rel. } \\
\text { Risiko }\end{array}$ \\
\hline $\begin{array}{l}\text { Fertiliätsstatus: } \\
\text { normo-/oligo-/azoosperm }\end{array}$ & 0,0039 & 0,0025 & 1,5623 & 0,8818 & 0,118 & 1,2462 \\
\hline \hline Lebenserwartung bei Geburt & $-0,0035$ & 0,0041 & $-0,8551$ & 0,6075 & 0,393 & 0,8233 \\
\hline Geburtsmonat & $-0,0001$ & 0,0006 & $-0,2500$ & 0,1974 & 0,803 & 0,9917 \\
\hline Alter bei Untersuchung & $-0,0003$ & 0,0025 & $-0,1189$ & 0,0947 & 0,905 & 0,9835 \\
\hline Untersuchungsjahr & 0,0006 & 0,0025 & 0,2216 & 0,1753 & 0,825 & 1,0318 \\
\hline ISEI & 0,0002 & 0,0001 & 1,7761 & 0,9243 & 0,076 & $1,0100^{(*)}$ \\
\hline
\end{tabular}

a unzensierte Fälle $n=84$; zensierte Fälle $n=136$ 
Betrachtung der späten Geburtsjahrgänge

Das Weibull-Modell ermittelt für die späten Jahrgänge lediglich den ISEI als Prädiktorvariable - mit niedrigerer Mortalität bei höherem ISEI (C/Error=-2,5; $p=0,013$; Tab. 3.47 und 3.48).

Tab. 3.47 Weibull-Modell zum Einfluss des Fertilitätsstatus (fertil/subfertil) auf die Lebensdauer ${ }^{a}$; späte Geburtsjahrgänge

\begin{tabular}{|l|l|l|l|l|l|l|}
\hline Variable & Coeff & Error & C/Error & Signif & p & $\begin{array}{l}\text { Rel. } \\
\text { Risiko }\end{array}$ \\
\hline Fertilitätsstatus: subf./fertil & 0,0011 & 0,0072 & 0,1582 & 0,1257 & 0,874 & 1,0564 \\
\hline Lebenserwartung bei Geburt & 0,0174 & 0,0235 & 0,7399 & 0,5406 & 0,459 & 2,3105 \\
\hline Geburtsmonat & 0,0004 & 0,0009 & 0,4292 & 0,3322 & 0,668 & 1,0198 \\
\hline Alter bei Untersuchung & $-0,0002$ & 0,0029 & $-0,0773$ & 0,0616 & 0,938 & 0,9892 \\
\hline Untersuchungsjahr & 0,0003 & 0,0029 & 0,1167 & 0,0929 & 0,907 & 1,0166 \\
\hline ISEI & $-0,0005$ & 0,0002 & $-2,4768$ & 0,9867 & 0,013 & $0,9752^{*}$ \\
\hline
\end{tabular}

a unzensierte Fälle $n=41$; zensierte Fälle $n=199$

Tab. 3.48 Weibull-Modell zum Einfluss des Fertilitätsstatus (normo-/oligo-lazoosperm) auf die Lebensdauer ${ }^{\text {a }}$; späte Geburtsjahrgänge

\begin{tabular}{|l|l|l|l|l|l|l|}
\hline Variable & Coeff & Error & C/Error & Signif & p & $\begin{array}{l}\text { Rel. } \\
\text { Risiko }\end{array}$ \\
\hline $\begin{array}{l}\text { Fertilitätsstatus: } \\
\text { normo-/oligo-/azoosperm }\end{array}$ & 0,0020 & 0,0047 & 0,4233 & 0,3279 & 0,672 & 1,0999 \\
\hline Lebenserwartung bei Geburt & 0,0170 & 0,0235 & 0,7235 & 0,5307 & 0,469 & 2,2670 \\
\hline Geburtsmonat & 0,0004 & 0,0009 & 0,4310 & 0,3335 & 0,667 & 1,0199 \\
\hline Alter bei Untersuchung & $-0,0002$ & 0,0029 & $-0,0711$ & 0,0567 & 0,943 & 0,9901 \\
\hline Untersuchungsjahr & 0,0004 & 0,0029 & 0,1214 & 0,0966 & 0,903 & 1,0172 \\
\hline ISEI & $-0,0005$ & 0,0002 & $-2,4864$ & 0,9871 & 0,013 & $0,9750^{*}$ \\
\hline
\end{tabular}

\subsubsection{Validität der Trennung zwischen subfertilen und fertilen Fällen}

Die bisherige Abgrenzung der oligozoospermen Fälle erfolgte nach der Klassifikation des Laborhandbuches der WHO zur Untersuchung des menschlichen Ejakulates (WHO, 1999). Nach dieser werden solche Fälle als oligozoosperm bezeichnet, welche eine Spermienkonzentration von weniger als 20 Millionen Spermien pro Milliliter Ejakulat aufweisen. Weiterhin sind azoosperme Fälle abzugrenzen, für welche keinerlei Spermien im Ejakulat nachweisbar sind. Diese Unterscheidung findet weltweite Anwendung. Die Klassifikation der Oligozoospermie der WHO folgt nicht physiologisch begründeten Normwerten, sondern es handelt sich um Abgrenzungen im Sinne einer Prognose für die Eintretenswahrscheinlichkeiten einer Schwangerschaft. Es bleibt zu überprüfen, ob die ermittelten Ergebnisse mit einer Trennung von Normozoospermen und Oligozoospermen bei 
einer Grenze von 20 Millionen Spermien/ml auch bei der Wahl einer anderen Grenze repliziert werden.

\subsubsection{Sukzessive Verschiebung der Klassifikationsgrenze}

Zur Überprüfung, ob sich die Ergebnisse auch bei Wahl anderer Klassifikationsgrenzen für die oligozoospermen Fälle wiederholen lassen, wird in 5-Millionen-Schritten die Abgrenzung von 5 Mio./ml bis auf 100 Mio./ml sukzessive erhöht und für jede Grenze sowohl die nicht-parametrischen als auch die semi-parametrischen Verfahren erneut berechnet. Bei der Analyse mittels Cox-Regression werden als Kontrollvariablen die Lebenserwartung bei Geburt, der Geburtsmonat, das Alter bei der Untersuchung, das Untersuchungsjahr und der ISEI berücksichtigt.

Tab. 3.49 Ergebnisse des Wilcoxon(Gehan)-Tests und der Cox-Regression bei sukzessiver Erhöhung der Grenze zur Klassifikation der Oligozoospermen

\begin{tabular}{|c|c|c|}
\hline & & $\begin{array}{l}\text { Ergebnis bei sukzessiver Erhöhung der Grenze im } \\
\text { Vergleich mit Ergebnis bei 20-Millionen-Grenze }\end{array}$ \\
\hline \multirow{6}{*}{ 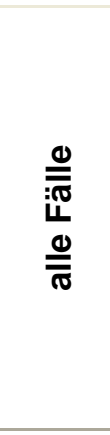 } & \multicolumn{2}{|l|}{ Wilcoxon(Gehan)-Test } \\
\hline & fertil/subfertil & 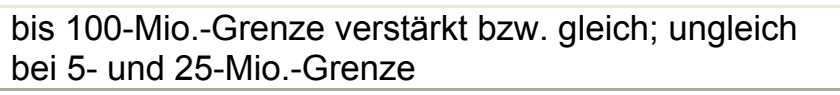 \\
\hline & normo-/oligo-/azoosperm & $\begin{array}{l}\text { bis } 100-\text { Mio.-Grenze gleich bzw. verstärkt; ungleich } \\
\text { bei 5-, 10- und 25-Mio.-Grenze }\end{array}$ \\
\hline & \multicolumn{2}{|l|}{ Cox-Regression } \\
\hline & fertil/subfertil & $\begin{array}{l}\text { bis } 100-\text { Mio.-Grenze gleich; ungleich bei } 60 \text { - und } 85- \\
\text { Mio.-Grenze }\end{array}$ \\
\hline & normo-/oligo-/azoosperm & gleich bei $60-$ und $80-$ Mio.-Grenze \\
\hline \multirow{6}{*}{ 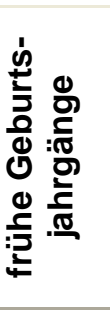 } & \multicolumn{2}{|l|}{ Wilcoxon(Gehan)-Test } \\
\hline & fertil/subfertil & bis 60-Mio.-Grenze gleich; ungleich bei 5-Mio.Grenze \\
\hline & normo-/oligo-/azoosperm & bis 60-Mio.-Grenze gleich; ungleich bei 5-Mio.Grenze \\
\hline & \multicolumn{2}{|l|}{ Cox-Regression } \\
\hline & fertil/subfertil & abgeschwächt bei 25-, 30-, 40- und 50-Mio.-Grenze \\
\hline & normo-/oligo-/azoosperm & gleich bzw. abgeschwächt bei 25- bis 60-Mio.-Grenze \\
\hline \multirow{6}{*}{ 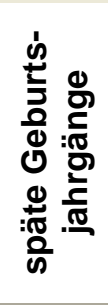 } & \multicolumn{2}{|l|}{ Wilcoxon(Gehan)-Test } \\
\hline & fertil/subfertil & bis 75-Mio.-Grenze gleich \\
\hline & normo-/oligo-/azoosperm & bis 75-Mio.Grenze gleich \\
\hline & \multicolumn{2}{|l|}{ Cox-Regression } \\
\hline & fertil/subfertil & bis 70-Mio.-Grenze gleich \\
\hline & normo-/oligo-/azoosperm & bis 70-Mio.-Grenze gleich \\
\hline
\end{tabular}

Die Ergebnisse finden sich im Anhang (Tab. A-50 bis A-61). An dieser Stelle soll vielmehr ein Überblick über die Befunde gegeben werden, welcher auch der Tab. 3.49 entnommen werden kann. Hinsichtlich der Vergleichbarkeit der Ergebnisse werden folgende Annahmen gemacht: Ein Ergebnis wird als "gleich“ bezeichnet, wenn der Effekt des Fertilitätsstatus, der für die Daten mit 20-Millionen-Grenze ermittelt wurde, auch bei einer schrittweisen Erhöhung der Grenze identisch bleibt: Ein signifikanter Effekt bleibt signifikant und ein nicht- 
signifikantes Resultat drückt sich weiterhin als nicht-signifikant aus. Ein „verstärktes" Ergebnis liegt dann vor, wenn für die 20-Millionen-Grenze ein Trend gefunden wird, welcher sich bei einer anderen Grenze als signifikanter Unterschied zeigt. Hingegen wird ein Ergebnis als „abgeschwächt“ bezeichnet, wenn ein signifikanter Effekt nur noch als Trend zu beobachten ist. Schließlich wird ein Ergebnis dann als „ungleich“ bewertet, wenn sich ein bei der 20-Millionen-Grenze festgestellter Unterschied oder Trend bei einer anderen Grenze nicht mehr finden lässt, das Ergebnis also nicht-signifikant ist. Gleiches gilt für den umgekehrten Fall: Unterscheiden sich Subfertile und Fertile bei der 20-Millionen-Grenze nicht voneinander, lassen sich aber bei einer anderen Grenze Trends oder Unterschiede finden, so wird dies als ungleich bewertet. Hinsichtlich der Überlebenskurven wird stets auf die Beibehaltung deren relativen Position zueinander geachtet (Abb. werden nicht dargestellt). Zusätzlich werden Stärke und Richtung der Effekte berücksichtigt.

Generell kann die Aussage gemacht werden, dass auch bei einer Verschiebung der Grenzkonzentration die Effekte zumeist gleich bzw. vergleichbar bleiben. Eine Ausnahme bildet die Berechnung einer Cox-Regression für die frühen Geburtsjahrgänge. Wie bereits vorgestellt wurde, kann in dieser Substichprobe bei einer Abgrenzung der Oligozoospermen bei einer Spermienkonzentration von weniger als $20 \mathrm{Mio} . / \mathrm{ml}$ ein signifikantes OR berechnet werden; Subfertile haben eine höheres Risiko zu versterben als Fertile. Dieser Befund lässt sich nur bei einigen anderen Klassifikationsgrenzen, und dann auch lediglich als Trend, wieder finden. Dennoch bleibt zu beachten, dass Effektstärke und -richtung vergleichbar bleiben.

Abgesehen von diesem Befund sei jedoch zusammengefasst, dass die anderen Analysen gleiche und vergleichbare Ergebnisse aufweisen. Auch sind die Überlebenskurven stets ähnlich und die Effektrichtungen werden beibehalten. Mit Ausnahme der 5-MillionenGrenze beginnen die Ergebnisse erst ab einer Abgrenzung der Oligozoospermen bei 60 Millionen Spermien/ml voneinander abzuweichen.

\subsubsection{Klassifikation nach Guzick et al. (2001)}

Zusätzlich zur vorgestellten Grenzverschiebung werden fertile und subfertile Fälle unter Berücksichtigung der von Guzick und Kollegen (2001) gefundenen Abgrenzung gegenüber gestellt. Die Autoren untersuchten die Spermiogramme von subfertilen Männern, in deren Partnerschaft (bei normaler Fertilität der Frau) in den letzten 12 Monaten keine Schwangerschaft erreicht werden konnte. Als fertile Vergleichsgruppe wurden Männer herangezogen, welche nachweislich ein Kind gezeugt hatten. Nach den Angaben dieser Studie wird Subfertilität am besten durch eine Spermienkonzentration von unter 13,5 Millionen Spermatozoen/ml definiert, während Fertilität durch eine Spermienkonzentration ab mindestens 48 
Mio./ml klassifiziert wird. Konzentrationen, welche dazwischen liegen, sind als indifferent zu betrachten und können zur Klassifizierung nicht herangezogen werden (Tab. 3.50). Dieser Einteilung folgend werden die entsprechenden Überlebenskurven dargestellt und die zugehörigen Überlebensraten berechnet; ebenso wird anhand von Cox-Regressionen und den parametrischen Exponential-, Gompertz-Makeham- und Weibull-Modellen der Einfluss des Fertilitätsstatus auf die Lebensdauer modelliert. Die Ergebnisse werden den bei Einhaltung der WHO-Kriterien ermittelten Befunden gegenübergestellt (Tab. 3.55 bis 3.57). Hinsichtlich der Beurteilung der Vergleichbarkeit der Ergebnisse bei unterschiedlichen Klassifikationen findet die gleiche Terminologie wie bereits bei der Betrachtung der Verschiebung der Grenzwerte Anwendung (siehe 3.2.4.1).

Tab. 3.50 Fertilitätsklassen nach WHO-Laborrichtlinien (1999) und Guzick et al. (2001)

\begin{tabular}{|l||l|l|}
\hline Fertilitäts-Klassifikation & WHO-Richtlinien (1999) & Guzick et al. (2001) \\
\hline \hline \begin{tabular}{l|l|l|} 
fertil \\
subfertil
\end{tabular} & $\begin{array}{l}\text { Spermienkonz. } \geq 20 \mathrm{Mio} . / \mathrm{ml} \\
\text { Spermienkonz. }<20 \mathrm{Mio} . / \mathrm{ml}\end{array}$ & Spermienkonz. $\geq 48 \mathrm{Mio} . / \mathrm{ml}$ \\
\hline \begin{tabular}{l|l|} 
normozoosperm \\
oligozoosperm \\
azoosperm
\end{tabular} & $\begin{array}{l}\text { Spermienkonz. } \geq 20 \mathrm{Mio} / \mathrm{ml} \\
\text { Spermienkonz. }<20 \mathrm{Mio} . / \mathrm{ml}\end{array}$ & Spermienkonz. $\geq 48 \mathrm{Mio} . / \mathrm{ml}$ \\
\hline
\end{tabular}

Vergleicht man die Überlebenskurven der Fertilitätsklassen nach Guzick et al. (2001; Abb. 3.36 bis 3.41) mit den bereits vorgestellten Kurven der Fertilitätsklassifizierung nach den WHO-Richtlinien (1999; vgl. Abb. 3.24 bis 3.29), so wird deutlich, dass hier identische Verläufe vorliegen. Die Kurven der fertilen Fälle liegen über jener der infertilen. Ebenso zeichnen sich die oligozoospermen Männer bereits rein optisch durch eine höhere Mortalität aus; ihre Kurve fällt steiler ab als jene der Normozoospermen. Auch die intermediäre Position der azoospermen Fälle lässt sich hier wieder finden. Bei den späten Geburtsjahrgängen hingegen nehmen die Kurven einen parallelen Verlauf. Optisch unterscheiden sich die Befunde bei Verwendung unterschiedlicher Klassifizierungssysteme somit nicht voneinander. 


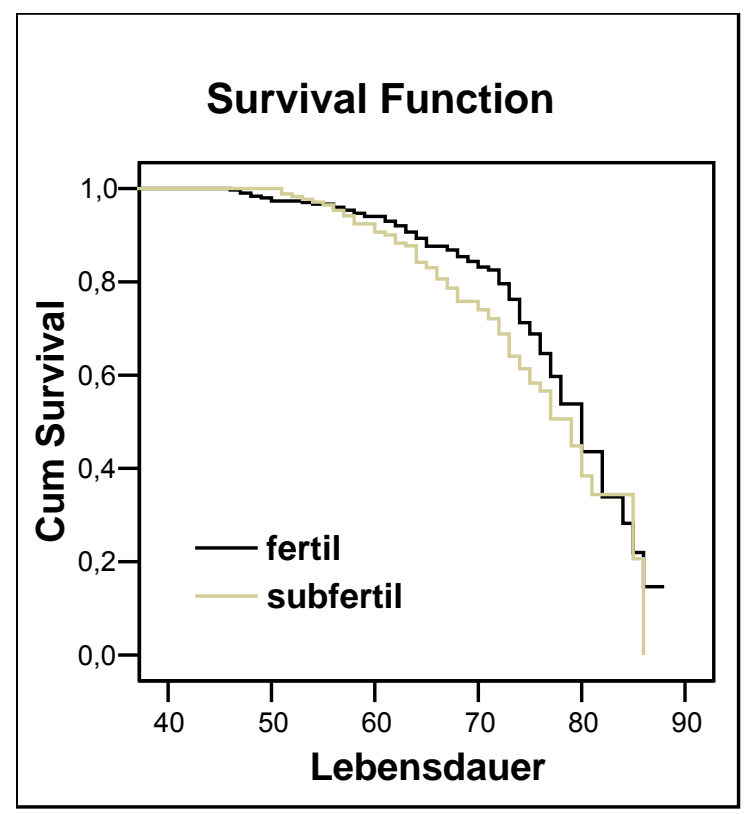

Abb. 3.36 Überlebensfunktionen der fertilen und subfertilen Fälle (nach Guzick et al., 2001)

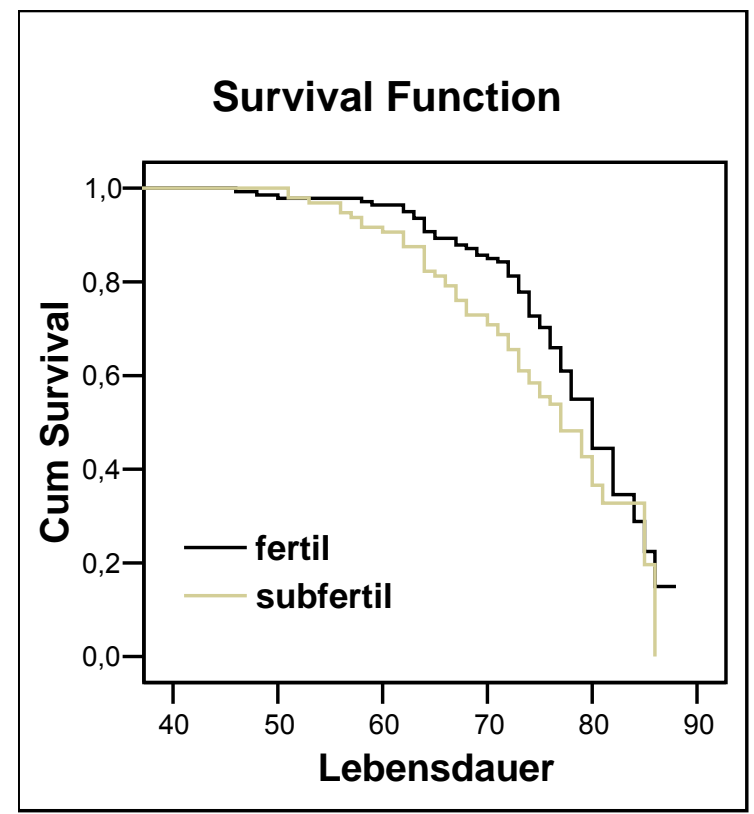

Abb. 3.38 Überlebensfunktionen der fertilen und subfertilen Fälle (nach Guzick et al., 2001); frühe Geburtsjahrgänge

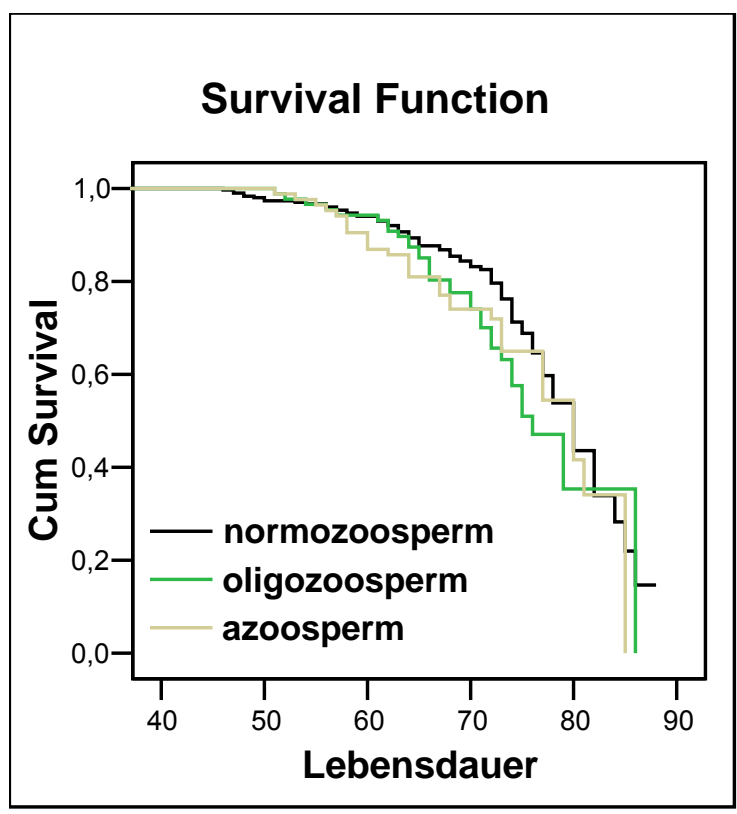

Abb. 3.37 Überlebensfunktionen der normo-, oligo- und azoospermen Fälle (nach Guzick et al., 2001)

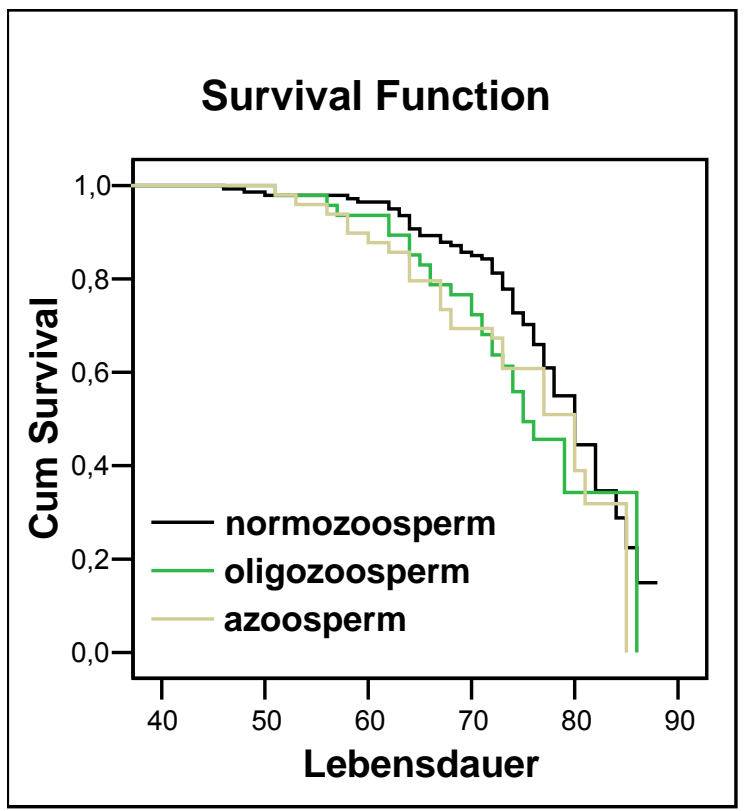

Abb. 3.39 Überlebensfunktionen der normo-, oligo- und azoospermen Fälle (nach Guzick et al., 2001); frühe Geburtsjahrgänge 


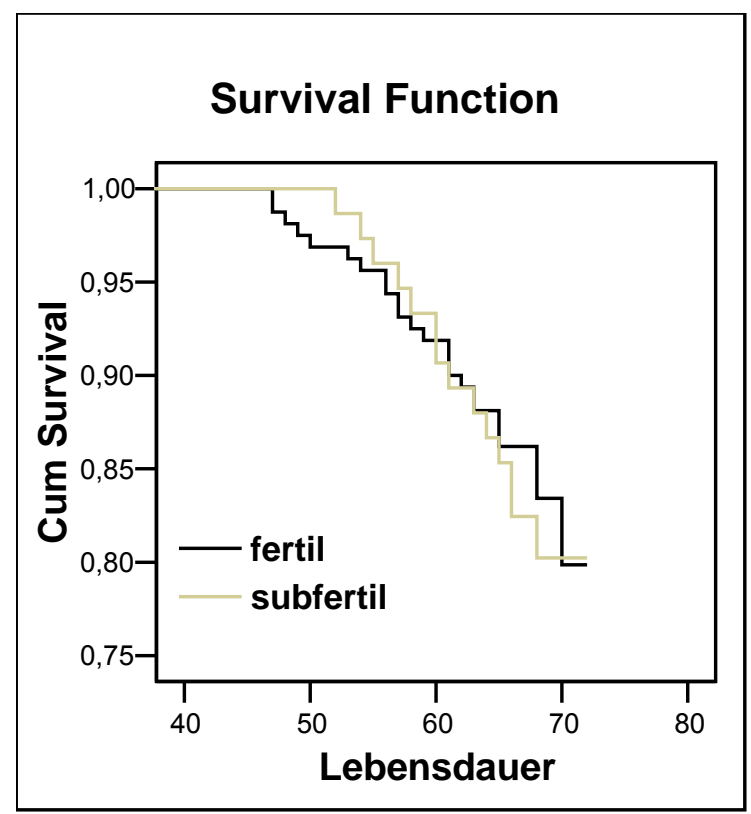

Abb. 3.40 Überlebensfunktionen der fertilen und subfertilen Fälle (nach Guzick et al., 2001); späte Geburtsjahrgänge

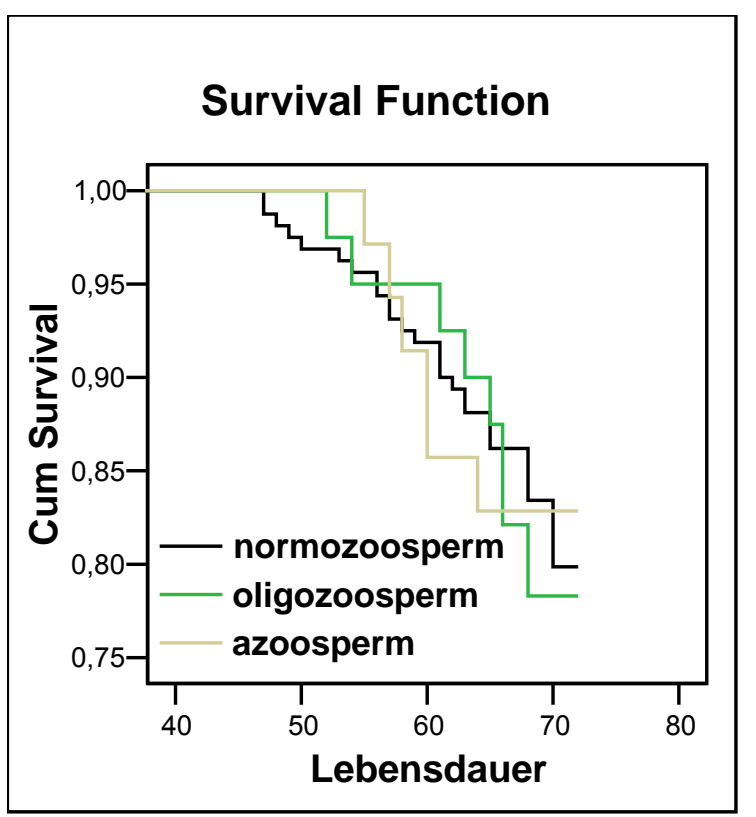

Abb. 3.41 Überlebensfunktionen der normo-, oligo- und azoospermen Fälle (nach Guzick et al., 2001); späte Geburtsjahrgänge

Tab. 3.51 Gegenüberstellung der Ergebnisse des Wilcoxon(Gehan)-Tests bei unterschiedlicher Fertilitätsklassifikation

\begin{tabular}{|c|c|c|c|c|c|}
\hline & \multirow[t]{2}{*}{ Vergleich } & \multicolumn{2}{|c|}{$\begin{array}{l}\text { Klassifikation nach } \\
\text { WHO (1999) }(n=592)\end{array}$} & \multicolumn{2}{|c|}{$\begin{array}{l}\text { Klassifikation nach } \\
\text { Guzick et al. (2001) } \\
\text { ( } n=471)\end{array}$} \\
\hline & & $\begin{array}{l}\text { Wilcoxon } \\
\text { (Gehan) }\end{array}$ & $\mathbf{p}$ & $\begin{array}{l}\text { Wilcoxon } \\
\text { (Gehan) }\end{array}$ & $\mathbf{p}$ \\
\hline alle & fertil-subfertil & $3,430^{(*)}$ & 0,064 & $5,173^{*}$ & 0,023 \\
\hline $\begin{array}{l}\text { frühe } \\
\text { Geburtsjahrgänge }\end{array}$ & fertil-subfertil & $7,091^{\star \star}$ & 0,008 & $6,882^{* *}$ & 0,009 \\
\hline $\begin{array}{l}\text { späte } \\
\text { Geburtsjahrgänge }\end{array}$ & fertil-subfertil & 0,005 & 0,943 & 0,213 & 0,645 \\
\hline \multirow{4}{*}{ alle } & overall & 3,638 & 0,162 & $5,175^{(*)}$ & 0,075 \\
\hline & $\begin{array}{l}\text { normo- } \\
\text { oligozoosperm }\end{array}$ & 1,683 & 0,195 & $3,328^{(*)}$ & 0,068 \\
\hline & normo-azoosperm & $2,830^{(*)}$ & 0,093 & $3,402^{(*)}$ & 0,065 \\
\hline & oligo-azoosperm & 0,161 & 0,688 & 0,004 & 0,949 \\
\hline \multirow{4}{*}{$\begin{array}{l}\text { frühe } \\
\text { Geburtsjahrgänge }\end{array}$} & overall & $7,405^{*}$ & 0,025 & $6,965^{*}$ & 0,031 \\
\hline & $\begin{array}{l}\text { normo- } \\
\text { oligozoosperm }\end{array}$ & $6,400^{*}$ & 0,011 & $5,446^{*}$ & 0,020 \\
\hline & normo-azoosperm & $2,942^{(*)}$ & 0,086 & $3,824^{(*)}$ & 0,051 \\
\hline & oligo-azoosperm & 0,222 & 0,638 & 0,042 & 0,837 \\
\hline \multirow{4}{*}{$\begin{array}{l}\text { späte } \\
\text { Geburtsjahrgänge }\end{array}$} & overall & 0,477 & 0,788 & 0,213 & 0,899 \\
\hline & $\begin{array}{l}\text { normo- } \\
\text { oligozoosperm }\end{array}$ & 0,183 & 0,668 & 0,131 & 0,718 \\
\hline & normo-azoosperm & 0,211 & 0,646 & 0,129 & 0,720 \\
\hline & oligo-azoosperm & 0,457 & 0,499 & 0,001 & 0,981 \\
\hline
\end{tabular}


In den Tabellen 3.51 bis 3.53 erfolgt eine Gegenüberstellung der Ergebnisse bei einer Fertilitätsklassifizierung zum einen nach den WHO-Richtlinien (1999) und zum anderen nach der Einteilung nach Guzick et al. (2001). Die detaillierten Ergebnisse sind im Anhang (Tab. A-62 bis A-91) zu finden. Es wird deutlich, dass bei einer Klassifikation nach Guzick und Kollegen (2001) die Effekte des Wilcoxon(Gehan)-Tests entweder gleich bleiben oder sogar noch verstärkt werden (Tab. 3.51). Hingegen sind die anhand der Cox-Regression ermittelten signifikanten Effekte des Fertilitätsstatus auf die Mortalität nicht mehr bzw. nur noch als Trends zu finden. Die Effektrichtungen bleiben erhalten, während die Effektgrößen etwas abnehmen (Tab. 3.52). Bei der Analyse mittels parametrischer Modelle kommt es ebenfalls zur Abschwächung: signifikante Ergebnisse sind jedoch noch als Trends erkennbar und die OR sind nur leicht erniedrigt (Tab. 3.53).

Tab. 3.52 Gegenüberstellung der Ergebnisse der Cox-Regression bei unterschiedlicher Fertilitätsklassifikation

\begin{tabular}{|c|c|c|c|c|c|c|c|}
\hline & \multirow[t]{2}{*}{ Vergleich } & \multicolumn{3}{|c|}{$\begin{array}{l}\text { Klassifikation nach WHO } \\
(1999)(n=461)\end{array}$} & \multicolumn{3}{|c|}{$\begin{array}{c}\text { Klassifikation nach } \\
\text { Guzick et al. (2001) } \\
(n=363)\end{array}$} \\
\hline & & Wald & $p$ & OR & Wald & $p$ & OR \\
\hline alle & $\begin{array}{l}\text { subfertil vs. } \\
\text { fertil }\end{array}$ & 2,330 & 0,127 & 1,328 & 1,932 & 0,165 & 1,338 \\
\hline $\begin{array}{l}\text { frühe Geburts- } \\
\text { jahrgänge }\end{array}$ & $\begin{array}{l}\text { subfertil vs. } \\
\text { fertil }\end{array}$ & 4,790 & 0,029 & $1,640^{*}$ & 2,269 & 0,132 & 1,470 \\
\hline $\begin{array}{l}\text { späte Geburts- } \\
\text { jahrgänge }\end{array}$ & $\begin{array}{l}\text { subfertil vs. } \\
\text { fertil }\end{array}$ & 0,004 & 0,952 & 1,021 & 0,354 & 0,552 & 1,254 \\
\hline \multirow{3}{*}{ alle } & $\begin{array}{l}\text { oligo- vs. } \\
\text { normozoosp. }\end{array}$ & 2,733 & 0,098 & $1,432^{(*)}$ & 2,356 & 0,125 & 1,471 \\
\hline & $\begin{array}{l}\text { azoo- vs. } \\
\text { normozoosp. }\end{array}$ & 0,431 & 0,512 & 1,187 & 0,458 & 0,499 & 1,202 \\
\hline & $\begin{array}{l}\text { oligo- vs. } \\
\text { azoosperm }\end{array}$ & 0,400 & 0,527 & 1,206 & 0,416 & 0,519 & 1,224 \\
\hline \multirow{3}{*}{$\begin{array}{l}\text { frühe Geburts- } \\
\text { jahrgänge }\end{array}$} & $\begin{array}{l}\text { oligo- vs. } \\
\text { normozoosp. }\end{array}$ & 7,722 & 0,005 & $2,058^{*}$ & 3,078 & 0,079 & $1,714^{(*)}$ \\
\hline & $\begin{array}{l}\text { azoo- vs. } \\
\text { normozoosp. }\end{array}$ & 0,337 & 0,561 & 1,203 & 0,441 & 0,507 & 1,246 \\
\hline & $\begin{array}{l}\text { oligo- vs. } \\
\text { azoosperm }\end{array}$ & 2,320 & 0,128 & 1,710 & 0,700 & 0,403 & 1,376 \\
\hline \multirow{3}{*}{$\begin{array}{l}\text { späte Geburts- } \\
\text { jahrgänge }\end{array}$} & $\begin{array}{l}\text { oligo- vs. } \\
\text { normozoosp. }\end{array}$ & 0,127 & 0,722 & 0,857 & 0,227 & 0,633 & 1,252 \\
\hline & $\begin{array}{l}\text { azoo- vs. } \\
\text { normozoosp. }\end{array}$ & 0,342 & 0,559 & 1,308 & 0,226 & 0,635 & 1,256 \\
\hline & $\begin{array}{l}\text { oligo- vs. } \\
\text { azoosperm }\end{array}$ & 0,562 & 0,453 & 0,655 & 0,000 & 0,995 & 0,996 \\
\hline
\end{tabular}


Tab. 3.53 Gegenüberstellung der Ergebnisse der parametrischen Regressions-Modelle bei unterschiedlicher Fertilitätsklassifikation

\begin{tabular}{|c|c|c|c|c|c|c|c|}
\hline & \multirow{2}{*}{$\begin{array}{l}\text { Fertilitäts- } \\
\text { Status }\end{array}$} & \multicolumn{3}{|c|}{$\begin{array}{c}\text { Klassifikation nach WHO } \\
(1999)(n=461)\end{array}$} & \multicolumn{3}{|c|}{$\begin{array}{l}\text { Klassifikation nach Guzick et } \\
\text { al. (2001) }(n=363)\end{array}$} \\
\hline & & C/Error & p & RR & C/Error & p & RR \\
\hline \multicolumn{8}{|c|}{ Exponential-Modell } \\
\hline \multirow[b]{2}{*}{ alle } & subfertil/fertil & 1,0556 & 0,291 & 1,2162 & 0,9982 & 0,318 & 1,2304 \\
\hline & $\begin{array}{l}\text { normo-/oligo-/ } \\
\text { azoosperm }\end{array}$ & 0,8124 & 0,417 & 1,1016 & 0,7443 & 0,457 & 1,0990 \\
\hline \multirow{2}{*}{$\begin{array}{l}\text { frühe } \\
\text { Geburts- } \\
\text { jahrgänge }\end{array}$} & subfertil/fertil & 1,5701 & 0,116 & 1,4229 & 1,2251 & 0,221 & 1,3660 \\
\hline & $\begin{array}{l}\text { normo-/oligo-/ } \\
\text { azoosperm }\end{array}$ & 0,9834 & 0,325 & 1,1479 & 0,8883 & 0,374 & 1,1454 \\
\hline \multirow{2}{*}{$\begin{array}{l}\text { späte } \\
\text { Geburts- } \\
\text { jahrgänge }\end{array}$} & subfertil/fertil & $-0,0009$ & 0,999 & 0,9997 & 0,6311 & 0,528 & 1,2693 \\
\hline & $\begin{array}{l}\text { normo-/oligo-/ } \\
\text { azoosperm }\end{array}$ & 0,2389 & 0,811 & 1,0549 & 0,5952 & 0,552 & 1,1464 \\
\hline \multicolumn{8}{|c|}{ Gompertz-Makeham-Modell } \\
\hline \multirow[b]{2}{*}{ alle } & subfertil/fertil & 1,6048 & 0,109 & 1,3476 & 1,4301 & 0,153 & 1,3492 \\
\hline & $\begin{array}{l}\text { normo-/oligo-/ } \\
\text { azoosperm }\end{array}$ & 1,2076 & 0,227 & 1,1535 & 1,0466 & 0,295 & 1,1423 \\
\hline \multirow{2}{*}{$\begin{array}{l}\text { frühe } \\
\text { Geburts- } \\
\text { jahrgänge }\end{array}$} & subfertil/fertil & 2,2716 & 0,023 & $1,6713^{*}$ & 1,7190 & 0,086 & $1,5548^{(*)}$ \\
\hline & $\begin{array}{l}\text { normo-/oligo-/ } \\
\text { azoosperm }\end{array}$ & 1,4044 & 0,160 & 1,2150 & 1,2110 & 0,226 & 1,2044 \\
\hline \multirow{2}{*}{$\begin{array}{l}\text { späte } \\
\text { Geburts- } \\
\text { jahrgänge }\end{array}$} & subfertil/fertil & 0,1582 & 0,874 & 1,0564 & 0,6817 & 0,495 & 1,2945 \\
\hline & $\begin{array}{l}\text { normo-/oligo-/ } \\
\text { azoosperm }\end{array}$ & 0,4233 & 0,672 & 1,0999 & 0,6952 & 0,487 & 1,1737 \\
\hline \multicolumn{8}{|c|}{ Weibull-Modell } \\
\hline \multirow[b]{2}{*}{ alle } & subfertil/fertil & 1,9030 & 0,057 & $1,4314^{(*)}$ & 1,7557 & 0,079 & $1,4527^{(*)}$ \\
\hline & $\begin{array}{l}\text { normo-/oligo-/ } \\
\text { azoosperm }\end{array}$ & 1,5697 & 0,117 & 1,2071 & 1,3913 & 0,164 & 1,1955 \\
\hline \multirow{2}{*}{$\begin{array}{l}\text { frühe } \\
\text { Geburts- } \\
\text { jahrgänge }\end{array}$} & subfertil/fertil & 2,3356 & 0,020 & $1,7137^{\star}$ & 1,7207 & 0,085 & $1,5628^{(*)}$ \\
\hline & $\begin{array}{l}\text { normo-/oligo-/ } \\
\text { azoosperm }\end{array}$ & 1,5623 & 0,118 & 1,2462 & 1,2837 & 0,199 & 1,2211 \\
\hline \multirow{2}{*}{$\begin{array}{l}\text { späte } \\
\text { Geburts- } \\
\text { jahrgänge }\end{array}$} & subfertil/fertil & 0,1582 & 0,874 & 1,0564 & 0,8188 & 0,413 & 1,3688 \\
\hline & $\begin{array}{l}\text { normo-/oligo-/ } \\
\text { azoosperm }\end{array}$ & 0,4233 & 0,672 & 1,0999 & 0,7998 & 0,424 & 1,2026 \\
\hline
\end{tabular}




\subsection{Lebenszeit-Mortalität und Spermienkonzentration}

Bislang wurden die Ergebnisse zum Einfluss des Fertilitätsstatus, also der Vergleich von fertilen und subfertilen Fällen bzw. von normo-, oligo- und azoospermen Männern, vorgestellt. Um nun festzustellen, ob zwischen der Spermienkonzentration selbst und der Lebensdauer der Patienten ein Zusammenhang besteht bzw. ob sich eine Dosis-Wirkungs-Beziehung finden lässt, werden sämtliche semi-parametrischen und parametrischen Verfahren ebenfalls mit der Variablen der absoluten Spermienkonzentration durchgeführt. Dies soll zum einen für die normozoospermen und oligozoospermen Fälle gemeinsam, zum anderen für beide Subgruppen getrennt geschehen. Die Vorgehensweise ist analog zu den in den vorigen Abschnitten dargestellten Schritten: Zunächst erfolgt die Betrachtung aller Fälle, dann die separate Analyse der frühen und der späten Geburtsjahrgänge. Als Kontrollvariablen fungieren die Lebenserwartung bei Geburt, der Geburtsmonat, das Alter zum Untersuchungszeitpunkt, das Jahr der Untersuchung sowie der ISEI.

Als problematisch könnte die Tatsache eingeschätzt werden, dass die Spermienkonzentration keine normalverteilte Variable darstellt. Eine Normalverteilung unter Berücksichtigung aller Fälle lässt sich jedoch auch bei Anwendung verschiedener Transformationen der Daten nicht erreichen. Da durch Logarithmierung $(\log (X)+1)$ zumindest bei separater Betrachtung der normozoospermen Fälle normalverteilte Daten vorliegen (vgl. Tab. 3.54 und Abb. 3.41, 3.42 und 3.43), werden die folgenden Analysen nicht mit den Originaldaten, sondern mit der logarithmierten Spermienkonzentration durchgeführt. Sämtliche Ergebnisse sind in Tabellenform im Anhang aufgeführt (Tab. A-14 bis A-49).

Tab. 3.54 Kolmogorov-Smirnov-Test zur Überprüfung der Normalverteilungsannahme der Spermienkonzentration

\begin{tabular}{|l|l|l|}
\hline Substichprobe & Kolmogorov-Smirnov $\mathbf{Z}$ & $\mathbf{p}$ \\
\hline normo- und oligozoosperm & $2,705^{\star *}$ & $0,000^{\mathrm{a}}$ \\
\hline oligozoosperm & $2,276^{\star *}$ & $0,000^{\mathrm{a}}$ \\
\hline normozoosperm & 0,749 & 0,629 \\
\hline
\end{tabular}




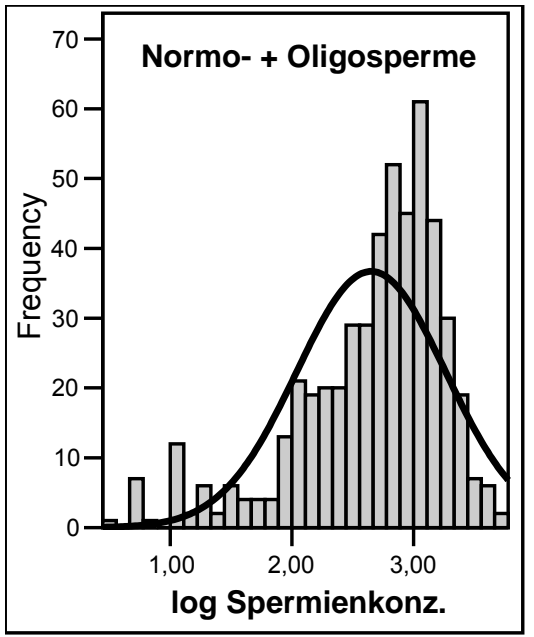

Abb. 3.42 Histogramm: log.

Spermienkonzentration

(Normo- + Oligozoosperme)

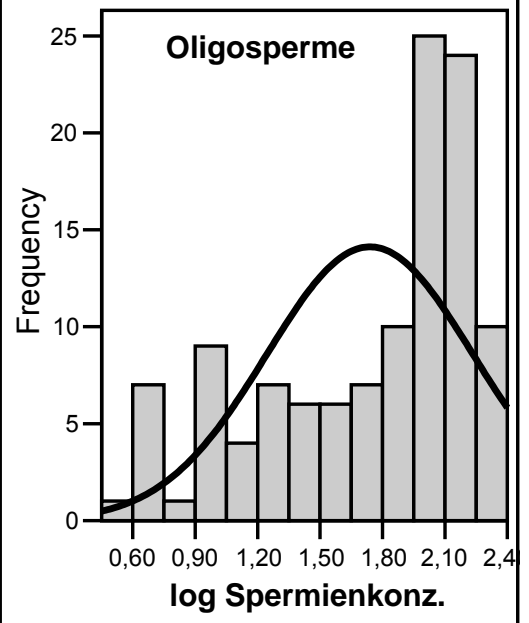

Abb. 3.43 Histogramm: log. Spermienkonzentration (Oligozoosperme)

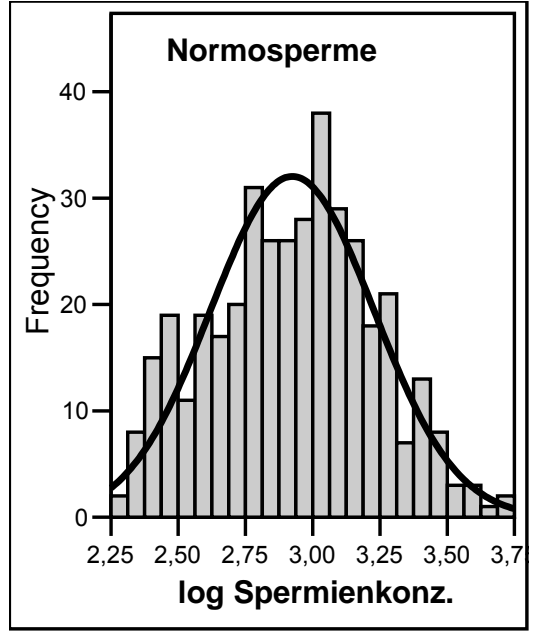

Abb. 3.44 Histogramm: log. Spermienkonzentration (Normozoosperme)

\subsubsection{Einfluss der Spermienkonzentration auf die Lebensspanne der normo- und oligozoospermen Fälle}

In keinem der angewendeten statistischen Verfahren kann ein Einfluss der absoluten Spermienkonzentration auf die Lebensdauer der normozoospermen und oligozoospermen Fälle festgestellt werden. Dies betrifft sowohl die Betrachtung aller Fälle als auch die separate Untersuchung der frühen wie der späten Geburtsjahrgänge (vgl. Anhang Tab. A-14 bis A-25). Werden alle Fälle bzw. nur die frühen Geburtsjahrgänge analysiert, so ergeben sich je nach betrachtetem Modell das Untersuchungsalter, das Untersuchungsjahr bzw. die Lebenserwartung bei Geburt als die Lebensspanne voraussagende Variablen. Bei Untersuchung der späten Geburtsjahrgänge hingegen wird sowohl mittels Cox-Regression als auch bei Modellierung der parametrischen Regressionen durchgängig der ISEI als Prädiktorvariable für die Lebensspanne berechnet.

\subsubsection{Einfluss der Spermienkonzentration auf die Lebensspanne der oligozoosper- men Fälle}

Ebenso kann für die oligozoospermen Fälle keine Dosis-Wirkungs-Beziehung zwischen deren Spermienkonzentration und Lebensdauer ermittelt werden (vgl. Anhang Tab. A-26 bis A-37). Die Cox-Regression berechnet zwar für die späten Geburtsjahrgänge einen Trend (Wald=3,341; $p=0,068$ ), diesem Ergebnis sollte aber nicht zu viel Bedeutung beigemessen werden, da die Zahl der unzensierten Fälle lediglich 7 Personen umfasst. Auch für die Subgruppe der Oligozoospermen lassen sich je nach Regressionsmodell das Alter der Patienten, das Jahr, in welchem die Untersuchung stattfand, bzw. der ISEI als Einflussvariablen modellieren. 


\subsubsection{Einfluss der Spermienkonzentration auf die Lebensspanne der normozoosper- men Fälle}

Ein letztes Augenmerk soll nun noch darauf gerichtet werden, ob für die normozoospermen Fälle eine Dosis-Wirkung-Beziehung zwischen der Spermienkonzentration und der Lebensdauer ermittelt werden kann. Auch mit diesen normalverteilten Daten kann kein Einfluss der Spermienkonzentration auf die Lebensdauer der ehemaligen Patienten festgestellt werden. Keines der Regressionsmodelle ermittelt - weder bei der Auswertung aller normozoospermen Fälle, noch bei getrennten Auswertungen der frühen bzw. späten Geburtsjahrgänge - die Spermienkonzentration als Prädiktorvariable für die Mortalität (vgl. Anhang Tab. A-38 - A-49). Nur bei Berechnung des Weibull-Modells für die frühen Geburtsjahrgänge lässt sich der Trend eines Einflusses erkennen ( $C / E r r o r=1,8$; $p=0,067$; Tab. A-48). Als erklärende Variablen kommen je nach Modell und betrachteter Stichprobe der ISEI bzw. die Lebenserwartung bei Geburt in Betracht.

\subsection{Selektivität der Daten}

\subsubsection{Vergleich von Fällen mit bekanntem und unbekannten Vitalstatus}

Die vorgestellten Analysen zum Zusammenhang zwischen der Spermienkonzentration bzw. dem Fertilitätsstatus und der Lebensdauer konnten selbstverständlich nur mit jenen Fällen durchgeführt werden, für die der Vitalstatus festgestellt werden konnte. Es bleibt zu überprüfen, ob sich Patienten mit bekanntem Vitalstatus von Patienten mit unbekanntem Vitalstatus erheblich unterscheiden, und ob es somit zu einer systematischen Verzerrung der Ergebnisse gekommen sein könnte. Daher wird mittels Mann-Whitney-Test überprüft, ob sich die Parameter „Geburtsjahr“, „Jahr der 1. Untersuchung“, „Alter bei 1. Untersuchung“, „sexuelle Abstinenz“, „Uhrzeit der Ejakulation" und „Spermienkonzentration“ zwischen den beiden Gruppen unterscheiden. Die Ergebnisse können der Tab 3.55 entnommen werden. Die einzige Variable, für die sich anhand des Mann-Whitney-Tests ein Unterschied finden lässt, ist die Uhrzeit der Ejakulation $(Z=-2,793 ; p=0,005)$. Betrachtet man jedoch das arithmetische Mittel, so wird deutlich, dass es sich hier nur um eine minimale Differenz von 10 Minuten handelt: Die Fälle mit bekanntem Vitalstatus gewannen ihre Spermaproben im Durchschnitt um 9:39 Uhr, während die Ejakulation bei den Fällen mit unbekanntem Vitalstatus im Mittel um 9:49 Uhr erfolgte. Für keine der anderen untersuchten Variablen lassen sich Unterschiede zwischen Fällen mit bekannten und Fällen mit unbekanntem Vitalstatus feststellen. 
Tab. 3.55 Mann-Whitney-Test zum Vergleich der kategorialen Parameter der Fälle mit bekanntem und unbekanntem Vitalstatus

\begin{tabular}{|l|l|l|l|}
\hline \multicolumn{1}{|c|}{ Variable } & Mann-Whitney & \multicolumn{1}{c|}{ Z } & p \\
\hline Geburtsjahr & 254200,5 & $-0,867$ & 0,386 \\
\hline Jahr 1. Untersuchung & 255953,5 & $-0,969$ & 0,332 \\
\hline Alter 1. Untersuchung & 236992,0 & $-1,640$ & 0,525 \\
\hline Sexuelle Abstinenz & 176180,0 & $-1,211$ & 0,101 \\
\hline Uhrzeit der Ejakulation & 161189,0 & $-2,793^{* *}$ & 0,005 \\
\hline Spermienkonzentration & 250788,0 & $-0,636$ & 0,226 \\
\hline Spermienkonzentration (nur Normozoosperme) & 101794,5 & $-1,040$ & 0,298 \\
\hline Spermienkonzentration (nur Oligozoosperme) & 9312,0 & $-0,590$ & 0,555 \\
\hline
\end{tabular}

Zusätzlich wird untersucht, ob die beiden Gruppen bezüglich des Untersuchungsgrundes oder im Fertilitätsstatus differieren. Hierzu wird der $\chi^{2}$-Test herangezogen (Tab. 3.56 und 3.57). Auch hier ergibt sich weder hinsichtlich des Untersuchungsgrundes (Pearson $\chi^{2}=3,087$; $p=0,543$ ) noch im Hinblick auf den Fertilitätsstatus (Pearson $\chi^{2}=1,043 ; p=0,594$ ) ein Unterschied zwischen den Personen mit bekanntem Vitalstatus und jenen, für die die Vitaldaten noch nicht ermittelt werden konnten.

Tab. 3.56 Vergleich der Fälle mit bekanntem und unbekanntem Vitalstatus: Grund der Untersuchung

\begin{tabular}{|c|c|c|c|c|c|c|}
\hline \multirow[b]{2}{*}{$\begin{array}{l}\text { Vital- } \\
\text { status }\end{array}$} & & \multicolumn{5}{|c|}{ Grund der Untersuchung } \\
\hline & & $\begin{array}{l}\text { Kinder- } \\
\text { wunsch }\end{array}$ & $\begin{array}{l}\text { Libido-, Potenz-, } \\
\text { Erektions- o. } \\
\text { Ejakulations- } \\
\text { störungen }\end{array}$ & $\begin{array}{c}\text { Nachweis } \\
\text { Zeugungs- } \\
\text { fähigkeit }\end{array}$ & $\begin{array}{l}\text { Folge- } \\
\text { unter- } \\
\text { suchung }\end{array}$ & weitere \\
\hline \multirow{3}{*}{ bekannt } & beobachtet & 237 & 31 & 5 & 5 & 19 \\
\hline & erwartet & 235,8 & 26,7 & 6,3 & 5,8 & 22,4 \\
\hline & $\begin{array}{l}\text { stand. } \\
\text { Residuen }\end{array}$ & 0,1 & 0,8 & $-0,5$ & $-0,3$ & $-0,7$ \\
\hline \multirow{3}{*}{$\begin{array}{c}\text { un- } \\
\text { bekannt }\end{array}$} & beobachtet & 248 & 24 & 8 & 7 & 27 \\
\hline & erwartet & 249,2 & 28,3 & 6,7 & 6,2 & 23,6 \\
\hline & $\begin{array}{l}\text { stand. } \\
\text { Residuen }\end{array}$ & $-0,1$ & $-0,8$ & 0,5 & 0,3 & 0,7 \\
\hline
\end{tabular}

Tab. 3.57 Vergleich der Fälle mit bekanntem und unbekanntem Vitalstatus: Fertilitätsstatus

\begin{tabular}{|c|l|r|r|r|r|}
\hline Vitalstatus & & Normozoospermie & Oligozoospermie & Azoospermie \\
\hline \multirow{3}{*}{ bekannt } & beobachtet & 391 & 117 & 84 \\
\cline { 2 - 6 } & erwartet & 397,3 & 110,0 & 84,7 \\
\cline { 2 - 6 } & standard. Residuen & $-0,3$ & 0,7 & $-0,1$ \\
\hline \multirow{3}{*}{ unbekannt } & beobachtet & 458 & 118 & 97 \\
\cline { 2 - 6 } & erwartet & 451,7 & 125,0 & 96,3 \\
\cline { 2 - 6 } & standard. Residuen & 0,3 & $-0,6$ & 0,1 \\
\cline { 2 - 6 }
\end{tabular}




\subsubsection{Vergleich von Marburgern und Nicht-Marburgern}

Da ein wesentlicher Teil der Recherche die Vitaldaten der Fälle über das Einwohnermeldeamt Marburg ermittelte (vgl. 2.2.5), musste sicher gestellt sein, dass sich die in der Stadt Marburg gemeldeten Fälle von den außerhalb Marburgs wohnenden Patienten hinsichtlich bedeutender Ejakulatparameter nicht unterscheiden. So wurde bereits vor Beginn der vorliegenden Studie eine Überprüfung auf Selektivität durchgeführt (Mueller \& Krause, unveröffentlichte Daten), deren Ergebnisse kurz resümiert werden sollen.

Im Rahmen der Vorstudie wurden 172 Fälle überprüft, deren Wohnort zum Zeitpunkt der Untersuchung in der Patientenakte vermerkt war. Die Wohnorte wurden zunächst in die Kategorien „heutige Stadt Marburg“ ( $n=27)$, „heutiger Kreis Marburg/Biedenkopf“ $(n=74)$ und „außerhalb des heutigen Kreises Marburg/Biedenkopf“ $(n=71)$ unterteilt. Es wurden normozoosperme und oligozoosperme Fälle betrachtet.

Tab. 3.58 Beobachtete und erwartete Oligozoospermie-Häufigkeiten im $\chi^{2}$-Test zum Vergleich der verschiedenen Wohnorte (Vorstudie)

\begin{tabular}{|l|l|r||r|r|}
\hline & & Stadt Marburg & $\begin{array}{l}\text { Kreis } \\
\text { Marburg/ } \\
\text { Biedenkopf }\end{array}$ & $\begin{array}{l}\text { lußerhalb des } \\
\text { Kreises }\end{array}$ \\
\hline \multirow{2}{*}{$\begin{array}{l}\text { oligozoo- } \\
\text { sperme Fälle }\end{array}$} & beobachtet & 4 & 13 & 16 \\
\cline { 2 - 5 } & erwartet & 5,2 & 14,2 & 13,6 \\
\hline \multirow{2}{*}{$\begin{array}{l}\text { normozoo- } \\
\text { sperme Fälle }\end{array}$} & standard. Residuen & $-0,5$ & $-0,3$ & 0,6 \\
\cline { 2 - 6 } & erwartet & 23 & 61 & 55 \\
\hline & standard. Residuen & 21,8 & 59,8 & 57,4 \\
\hline
\end{tabular}

In einem $\chi^{2}$-Test wurde die Unabhängigkeit der Merkmale „Wohnort“ und „Fertilitätsstatus" festgestellt (Pearson $\chi^{2}=0,971 ; p=0,615$; siehe Tab. 3.58). Weiterhin wurde die absolute Spermienkonzentration betrachtet und mittels Mann-Whitney-Test analysiert, ob sich Fälle, welche ihren Wohnsitz außerhalb Marburgs hatten („Nicht-Marburger“), von jenen Fällen mit Wohnsitz innerhalb der heutigen Stadtgrenzen („Marburger“) unterschieden.

Tab. 3.59 Durchschnittliche Spermienkonzentrationen und Mann-Whitney-Test zum Vergleich der Spermienkonzentration von „Marburgern“ und „Nicht-Marburgern“

\begin{tabular}{|c|c|c|c|c|c|}
\hline & & \multirow{2}{*}{$\mathbf{n}$} & \multirow{2}{*}{$\begin{array}{c}\text { Spermienkonzentration } \\
\text { mean }\end{array}$} & \multicolumn{2}{|c|}{ Mann-Whitney-Test } \\
\hline & & & & z & $\mathbf{p}$ \\
\hline \multirow{2}{*}{$\begin{array}{l}\text { Normo- und } \\
\text { Oligozoo- } \\
\text { sperme }\end{array}$} & Marburger & 27 & 89,8 (SD 103,7) & \multirow{2}{*}{$-0,942$} & \multirow{2}{*}{0,346} \\
\hline & Nicht-Marburger & 145 & 107,9 (SD 168,3) & & \\
\hline \multirow{2}{*}{$\begin{array}{l}\text { Normozoo- } \\
\text { sperme }\end{array}$} & Marburger & 23 & 103,1 (SD 106,9) & \multirow{2}{*}{$-0,500$} & \multirow{2}{*}{0,617} \\
\hline & Nicht-Marburger & 116 & $132,8(S D 179,8)$ & & \\
\hline
\end{tabular}


Betrachtet man die oligo- und normozoospermen Fälle, so wiesen Marburger eine durchschnittliche Spermienkonzentration 89,9 (SD 103,7) Mio./ml auf, während sich für Nicht-Marburger eine mittlere Spermienkonzentration von 107,9 (168,3) Mio./ml finden ließ. Der Mann-Whitney-Test ermittelte keinen signifikanten Unterschied zwischen Marburgern und Nicht-Marburgern $(Z=-0,942 ; p=0,346)$. Auch bei ausschließlicher Betrachtung der normozoospermen Fälle ergab sich keine unterschiedliche Spermienkonzentration in Abhängigkeit vom Wohnort ( $Z=-0,500 ; p=0,617$; vgl. Tab. 3.59).

Abschließend lässt sich somit anhand der Ergebnisse dieser Vorstudie festhalten, dass sich Fälle, welche innerhalb der heutigen Stadt Marburg wohnten, weder in der Spermiendichte noch in der relativen Häufigkeit der Fertilitätsklassen von Fällen, welche ihren Wohnort außerhalb Marburgs hatten, unterscheiden. 


\section{Diskussion}

In diesem Kapitel sollen die im vorangegangenen Abschnitt vorgestellten Ergebnisse der Untersuchung in den Kontext der Literatur gestellt und diskutiert werden. Während in einem ersten Schritt die Datenqualität betrachtet wird, um über mögliche Selektivitäten und Verzerrungen Aufschluss zu geben, umfasst der zweite Schritt die kritische Betrachtung der Befunddokumentation. Anschließend erfolgt die Diskussion der in der Studie ermittelten Ergebnisse. In einem letzten Schritt schließlich sollen Erklärungsansätze für die ermittelten Ergebnisse aufgezeigt werden.

\subsection{Datenqualität}

\subsubsection{Selektivität nach Wohnort}

Aus den Patientenblättern ergab sich, dass mit rund $80 \%$ der größte Teil der betrachteten Patienten der Marburger Andrologischen Sprechstunde seinen Wohnsitz in Marburg selbst oder im Landkreis Marburg/Biedenkopf hatte, dass sich jedoch das Einzugsgebiet zum Teil auch über diese Region, in seltenen Fällen sogar über die hessischen Landesgrenzen hinaus erstreckte. Die ursprüngliche Planung des Projektes beinhaltete zunächst nur die Recherche in den Einwohnermeldeämtern, wobei mit den Nachforschungen in der entsprechenden Einrichtung der Stadt Marburg begonnen werden sollte. Dabei wurde gemutmaßt, dass die Stichprobe durch einen sehr hohen Anteil an Stadtbewohnern verzerrt sein könnte. Denkbar wäre zum Beispiel, dass sich unter den Fällen außerhalb Marburgs häufiger pathologische Befunde mit niedrigen Spermienkonzentrationen finden lassen, die erst im „Ernstfall“ von niedergelassenen Dermatologen überwiesen wurden, während Marburger häufiger vom Gynäkologen der Partnerin direkt an die Universitätshautklinik zur Infertilitätsberatung verwiesen wurden. Vor Beginn der Recherche wurde daher in einer Vorstudie überprüft, ob sich in Marburg wohnhafte Patienten von solchen unterschieden, welche zum Untersuchungszeitpunkt ihren Wohnsitz außerhalb der Stadt Marburg hatten. Die Ergebnisse zeigen jedoch deutlich, dass hier keine Selektivität besteht. Es lässt sich kein Zusammenhang zwischen der Spermienkonzentration und dem Wohnort finden (vgl. 3.4.1).

Bezüglich der befürchteten Verzerrungen fand sich im Laufe des Projektes noch eine weitere „Entlastung“ aufgrund der zusätzlichen Zugangsmöglichkeit zu den Vitaldaten mittels der kooperierenden Krankenkasse. Mit 86,2\% wurde ein großer Teil der ehemaligen Patienten in der Krankenkassen-Datei ermittelt. Hier war der Zugriff auf ein Register mit allen 
ehemaligen und aktuellen Versicherten Hessens möglich, so dass in der betrachteten Stichprobe Stadtbewohner Marburgs nicht überwiegen.

Zusätzlich ist zu bemerken, dass es sich bei den Regionen, in welchen die ehemaligen Patienten wohnhaft waren bzw. sind, vorwiegend um eher ländliche Gebiete ohne größere industrielle Vorkommen handelt, so dass keine starken umweltbedingten Unterschiede angenommen werden müssen. Zudem liegen die Regionen so dicht beisammen, dass sich hier die vermuteten geografischen Unterschiede in den Spermienkonzentrationen, welche nicht nur zwischen verschiedenen europäischen Ländern (Andersen et al., 2000; Jørgensen et al., 2001; 2002; Richthoff et al., 2002), sondern auch zwischen verschiedenen Städten innerhalb eines Landes (Auger \& Jouannet, 1997; vgl. 1.2.2.3.2) nachgewiesen wurden, nicht zeigen sollten.

\subsubsection{Selektivität nach „Auffindbarkeit“"}

Eine weitere Überlegung über eine potentielle Selektivität der Stichprobe betrifft die „Auffindbarkeit“ der Fälle. Bislang konnte im Rahmen des vorgestellten Projektes der Vitalstatus von 601 Personen, also fast $47 \%$ aller in die Studie aufgenommenen Fälle, ermittelt werden. Nun bleibt zu fragen, ob sich die Fälle, deren Vitalstatus festgestellt wurde, von jenen Fällen, die weder über die Einwohnermeldeämter noch in der Krankenkassen-Datei aufgefunden werden konnten, unterscheiden. So könnten z.B. die letzteren eine generell bessere Gesundheit, verbunden mit einer höheren Spermienkonzentrationen, besessen und daher über eine höhere Mobilität verfügt haben. In Folge dessen könnten sie schwerer auffindbar gewesen sein. Auch eine umgekehrte Überlegung ist denkbar: Es handelt sich bei den Fällen mit unbekanntem Vitalstatus um besonders „schwere“ Fälle mit sehr niedrigen Spermienzahlen oder Azoospermie, welche kinderlos blieben, deren Ehe keinen Bestand hatte und die dann fortzogen. Darum wurde untersucht, ob sich die Männer mit unbekanntem Vitalstatus von jenen mit bekanntem Vitalstatus in den über sie bekannten Parametern unterscheiden. Die beiden Gruppen differierten weder im Geburtsjahr noch im Jahr der Untersuchung, im Alter zum Untersuchungszeitpunkt, in der sexuellen Abstinenz oder in der Spermienkonzentration. Auch bezüglich des Untersuchungsgrunds und des Fertilitätsstatus ließen sich keine Unterschiede feststellen (vgl. 3.4.2).

Grundsätzlich ist also bei den untersuchten Fällen nicht von einer Selektion nach Regionalität oder Schweregrad des medizinischen Zustands auszugehen. Die betrachteten Patienten werden daher als Zufallsstichprobe innerhalb der Studienpopulation angesehen. Es soll allerdings noch darauf hingewiesen werden, dass möglicherweise der Anteil der in jüngeren Jahren verstorbenen Fälle etwas unterschätzt wurde, da die kooperierende Krankenkasse keinen Zugang zu den Archiv-Daten ermöglichen konnte. Fälle, die in dieser 
Krankenkasse versichert waren und vor Umstellung auf die elektronische Datenverarbeitung zu Beginn der 1980er Jahre verstarben, konnten anhand der durchgeführten Recherche somit nicht ausfindig gemacht werden. Betrachtet man jedoch zum Vergleich die Fälle, deren Vitalstatus über die Einwohnermeldeämter ermittelt wurde, so liegt der Anteil derjenigen, die vor 1980 verstorben sind, bei lediglich $11 \%$, so dass die Unterschätzung als eher gering eingeschätzt werden dürfte.

\subsubsection{Selektivität nach Sozioökonomischem Status und Ehestand}

Generell wird angenommen, dass eher Personen mit einem hohen SES verstärkt gesundheitsbewusstes Verhalten zeigen und häufiger ärztliche und vergleichbare Dienste in Anspruch nehmen (Klein et al., 2001; Mielck, 2000; vgl. 1.2.1.2.3). Dies trifft ebenso für die Infertilitätsberatung und -behandlung zu. Für US-amerikanische Frauen ist nachgewiesen, dass der Zugang zu und die Inanspruchnahme von Infertilitätseinrichtungen - neben den finanziellen Möglichkeiten - stark von der Bildung abhängt (Jenkins, 2005). Das Institut für Reproduktionsmedizin des Klinikums Münster konnte eine Überrepräsentanz von Akademikern wie Ärzten, Ingenieuren und Wissenschaftlern unter den Patienten der andrologischen Sprechstunde feststellen. Als Grund wird die bessere Informiertheit hinsichtlich der Fertilitätsproblematik und die größere Besorgnis um die eigene Gesundheit in diesen Beschäftigten-Gruppen vermutet (Kenkel et al., 2001; Wischmann et al., 2001). Entsprechend fanden Rieker et al. (1990) bei Männern, die eine Hodenkrebs-Erkrankung überlebten, dass sich diejenigen, welche anschließend ihre Fertilität überprüfen ließen, durch eine höhere Bildung und einen höheren sozioökonomischen Status (SES) auszeichneten. Die Autoren vermuten ebenfalls, dass der höhere SES einen Indikator für den verbesserten Zugang dieser Patienten zu den entsprechenden Informationen darstellt. Des Weiteren konnte nachgewiesen werden, dass Männer mit höherem Bildungsgrad häufiger zu Untersuchungen zwecks Früherkennung von Prostata-Krebs gehen und im Falle einer Erkrankung eine höhere Überlebenswahrscheinlichkeit haben (Steenland et al., 2004). Letzteres schreiben die Autoren einer unterschiedlichen Behandlung durch die Ärzte zu.

Es kann somit nicht angenommen werden, dass die betrachteten Männer eine repräsentative Stichprobe der männlichen deutschen Allgemeinbevölkerung in vergleichbarem Alter darstellt. Im Gegenteil kann eher von einer allgemeinen positiven Selektion dieser Infertilitätspatienten hinsichtlich ihres SES ausgegangen werden. Zum anderen muss jedoch kritisch bemerkt werden, dass die Angaben über den Beruf, welche als Indikator für den SES herangezogen wurden, einer gesetzlichen Krankenkasse entstammen. Der SES von Personen, welche z.B. Mitglieder einer privaten Krankenversicherung waren, wurden anhand dieser Quelle nicht erfasst. So bleibt offen, ob die vermutete positive Selektion in der 
Stichprobe durch eine Überrepräsentanz von Personen mit niedrigerem SES zumindest bei den Berechnungen der Regressionsmodelle abgeschwächt wurde.

Weiterhin liegt bei der untersuchten Stichprobe vermutlich eine Selektion hinsichtlich des Ehestandes vor: Auch wenn diese Information nicht explizit belegt ist, so kann gemutmaßt werden, dass der größte Teil der ehemaligen Patienten - zumindest zum Untersuchungszeitpunkt - verheiratet war. Dies lässt der vielfach genannte explizite Kinderwunsch vermuten. Die Untersuchungen erfolgten in einem Zeitraum, in welchem sich der Wunsch nach Kindern wahrscheinlich eher in einer Ehe entwickelte und andere Formen der Kohabitation der Paare aufgrund der vorherrschenden sozialen Normen äußerst selten gewesen sein dürften. Es ist vielfach berichtet worden, dass verheiratete Männer generell eine bessere Gesundheit aufweisen als unverheiratete Männer (z.B. Brockmann \& Klein, 2002; 2004; vgl. auch 4.4.1.1), so dass hier also ebenfalls eine Selektion im Vergleich zur Durchschnittsbevölkerung angenommen werden darf. Zusätzlich lässt sich vermuten, dass die ehemaligen Patienten ein solides, finanziell gesichertes Leben führten, welches den Wunsch nach einem Kind und dessen Verwirklichung zuließ.

Zusammenfassend kann für die betrachtete Stichprobe eine positive Selektion hinsichtlich des SES und vermutlich auch des Ehestandes nicht ausgeschlossen werden. Gewiss trifft dies, in unterschiedlichem Ausmaß, auf alle Infertilitätspatienten zu. In Bezug auf die zu untersuchende Fragestellung dürfte dies aber keine Schwierigkeiten bereiten, da Ziel der Studie nicht war, die populationsbezogenen Parameter zu schätzen, sondern die Kausalhypothese zu testen, ob ein Zusammenhang zwischen Spermienkonzentration bzw. dem Fertilitätsstatus und der Lebenszeit-Mortalität der ehemaligen Patienten vorliegt.

\subsection{Befunddokumentation}

Denkbar ist weiterhin, dass in der Dokumentation der Daten systematische Fehler bestehen. Daher sollen die Charakteristika der untersuchten Stichprobe betrachtet und, wenn möglich, Angaben über andere Infertilitätspatienten gegenübergestellt werden. Generell sind Vergleiche von Spermienparametern verschiedener Studienpopulationen jedoch nur mit Vorsicht zu ziehen, da sich diese durch unterschiedliche Ausrichtungen und Schwerpunkte der reproduktionsmedizinischen und andrologischen Einrichtungen z.T. sehr stark voneinander unterscheiden können. Ebenso ist von einer Selbstselektion bei freiwilliger Studien-Teilnahme auszugehen. Eine Untersuchung an Partnern schwangerer Frauen konnte nachweisen, dass freiwillige Samenspenden zu Studienzwecken eher von Männern mit einem höheren Bildungsgrad bzw. von Männern, welche bereits selbst unter Subfertilität gelitten hatten, bereitgestellt wurden (Muller et al., 2004). Des Weiteren sind die in der 
Einleitung (vgl. 1.2.2.3.2) erwähnten geographischen Unterschiede zwischen Ländern und Regionen zu berücksichtigen und erschweren den Vergleich mit anderen Arbeiten erheblich.

\subsubsection{Spermienkonzentration}

Die betrachteten Fälle wiesen eine mittlere Spermienkonzentration von rund $72 \mathrm{Mio} . / \mathrm{ml}$ auf. Diese entspricht den Angaben von Paasch et al. (2003), die bei vor 1959 geborenen Kinderwunschpatienten in Leipzig eine durchschnittliche Konzentration von 71 Mio./ml fanden. Eine genauere Unterscheidung der eigenen Stichprobe in normozoosperme und oligozoosperme Männern ermittelte für die erstgenannten eine durchschnittliche Konzentration von 107 Mio./ml, während die letzteren eine mittlere Spermiendichte von 8,6 Mio./ml aufwiesen. Die mittlere Konzentration der oligozoospermen Gruppe stimmt mit den Angaben von Levitas und Kollegen (2005) überein, welche eine Stichprobe von fast 10.000 Ejakulatproben analysierten. Die durchschnittliche Spermienkonzentration der Normozoospermen der eigenen Stichprobe allerdings liegt etwas höher als die in der Levitas-Studie angegebenen Werte von 55 bis 75 Mio./ml. Dies könnte dadurch erklärt werden, dass in der vorliegenden Studie, im Gegensatz zu anderen Arbeiten, Fälle mit sehr hohen Spermiendichten von über 250 Mio./ml nicht ausgeschlossen wurden. Dies erschien nicht notwendig, da die ursprüngliche Fragestellung mit der Überlegung einherging, dass ein belasteter Organismus weniger - aber nicht mehr - Spermatozoen produzieren sollte.

Generell sind die Angaben zur Spermatozoenkonzentration in verschiedenen Stichproben sehr unterschiedlich, nicht zuletzt deshalb, weil die betrachteten Populationen stark differieren. Dies verdeutlicht die folgende Zusammenstellung: Bei Samenspendern mit zuvor unbekanntem Fertilitätsstatus werden Werte von durchschnittlich 53 bis $73 \mathrm{Mio} / \mathrm{ml}$ - in Abhängigkeit vom Untersuchungsjahr - angegeben (Gyllenborg et al., 1999). Studien an ausschließlich fertilen Männern geben durchschnittliche Konzentrationen von 60 bis 107 Mio./ml, wiederum in Abhängigkeit vom Untersuchungsjahr oder auch vom betrachteten Land, an (Auger et al., 1995; Bujan et al., 1996; Jørgensen et al., 2001; Slama et al., 2002). In diesem Zusammenhang ist eine Übersicht in MacLeod \& Wang (1979) interessant, welche die Ergebnisse verschiedener Studien an fertilen Populationen zusammenfasst. Der betrachtete Zeitraum zwischen den Jahren 1938 und 1977 entspricht in etwa dem Untersuchungszeitraum der eigenen Stichprobe. Doch auch hier zeigt sich mit Angaben von 48 bis 137 Mio./ml eine sehr große Spannbreite. Bei Männern, welche vor einer Vasektomie ihre Spermien kryokonservieren ließen, wurden durchschnittliche Spermiendichten von 77 bis 89 Mio./ml gefunden; die Konzentrationen variierten mit der Zeit (Fisch et al., 1996). Untersuchungen an der dänischen, norwegischen, estnischen und finnischen Allgemein- 
bevölkerung ergaben durchschnittliche Konzentrationen von 57 bis 72 Mio./ml (Jørgensen et al., 2002).

\subsubsection{Verteilung der Fertilitätsklassen}

Im betrachteten Untersuchungskollektiv waren 66\% der Fälle normozoosperm, etwa 20\% wiesen eine Oligozoospermie auf, während bei rund 14\% der Männer keine Spermien im Ejakulat zu finden waren. Der Anteil der normozoospermen Fälle der eigenen Stichprobe ist geringer als in anderen Untersuchungen. Acacio und Kollegen (2000) geben den Anteil der normozoospermen Fälle in ihrer Infertilitäts-Sprechstunde mit $82 \%$ an, während sie bei $14 \%$ aller Fälle eine Oligozoospermie diagnostizierten. Doch handelt es sich hierbei um die männlichen Partner von Paaren, bei welchen der Ursprung der Infertilität noch nicht geklärt wurde. Für eine Stichprobe von nachweislich fertilen Männern (Vätern) wurde ein Anteil von $79,8 \%$ normozoospermer Fälle ermittelt (Chia et al., 1998).

Die Prävalenz der Azoospermie erscheint im Vergleich zu anderen Befunden, welche für Männer mit unerfülltem Kinderwunsch Azoospermie-Häufigkeiten zwischen 4\% und 9\% ermittelten (Acacio et al., 2000; Andolz et al., 1999; Knuth \& Schulze, 2001; Mazzilli et al., 2000), etwas erhöht. Jedoch wurde bei japanischen Infertilitätspatienten sogar ein Anteil von $26 \%$ gefunden (Takihara, 1998). Vermutlich sind aber auch hier wieder aufgrund der unterschiedlichen Spezialisierungen der einzelnen Institute nur schwer Vergleiche zu ziehen.

Betrachtet man in der untersuchten Stichprobe die frühen und späten Geburtsjahrgänge getrennt voneinander, so verdeutlicht sich, dass der Anteil der azoospermen Fälle bei den frühen Jahrgängen mit über $17 \%$ deutlich höher liegt als der Anteil bei den späten Jahrgängen. Die für diese Subgruppe ermittelten $11 \%$ entsprechen eher den von anderen andrologischen Einrichtungen postulierten Werten (siehe oben). Bei Überlegungen zur Begründung dieses Befundes müssen die verschiedenen Ätiologien der Azoospermie unterschieden werden. Eine Untersuchung an 100 azoospermen Männern ergab, dass 29\% der Azoospermie-Fälle durch genetische Ursachen erklärt werden konnten, 22\% waren Erkrankungen oder äußeren Einwirkungen zuzuschreiben, während $27 \%$ der Fälle auf Kryptorchismus zurückgeführt werden konnten. Für immerhin 22\% aller Azoospermie-Fälle konnte keine Ursache festgestellt werden (Fedder \& Crüger, 2004). Traditionell werden Azoospermie-Fälle in obstruktive und nicht-obstruktive Azoospermie unterteilt. Nichtobstruktive Azoospermie tritt z.B. im Zusammenhang mit dem Klinefelter-Syndrom (47,XXYKaryotyp; z.B. Huynh et al., 2002; Nieschlag et al., 2000a) oder als Folge von Ychromosomalen Mikrodeletionen auf (z.B. Ferlin et al., 1999; 2004; Huynh et al., 2002; Kim et al., 1999). Ca 13\% der azoospermen Männer besitzen eine Deletion auf dem langen Arm ihres Y-Chromosoms (Reijo et al., 1995; vgl. auch 1.2.3.2.6). Über die genetischen 
Hintergründe der ehemaligen Patienten kann auf Grundlage der vorliegenden Informationen keine Aussage gemacht werden. Es erscheint allerdings nicht wahrscheinlich, dass sich im Laufe der Jahre die Prävalenz genetischer Defekte vermindert haben könnte und sich so der geringere Anteil an azoospermen Männern innerhalb der späten Geburtsjahrgänge erklären ließe.

Damit bleibt die Bedeutung der obstruktiven Azoospermie zu diskutieren. In diesem Zusammenhang ist zum einen an ein kongenitales beidseitiges Fehlen des Vas deferens im Rahmen der Cystischen Fibrose zu denken (vgl. 1.2.3.2.1; Mak et al., 2000; Meschede et al., 1998; Stuhrmann, 1998). Auch hier kann anhand der vorliegenden medizinischen Akten der ehemaligen Patienten keine Aussage zu den Prävalenzen in der untersuchten Stichprobe gemacht werden, allerdings kommt diese Anomalie nicht so oft vor, dass sie den Effekt erklären könnte. Ferner erscheint es wiederum nicht plausibel, dass ein solches Fehlen bei den frühen Geburtsjahrgängen häufiger gewesen sein sollte als bei den später Geborenen. Eine weitere Ursache der obstruktiven Azoospermie können zum anderen die verschiedenen Hodenfehllagen, wie z.B. Maldescensus testis oder Kryptorchismus, darstellen (Hermanns \& Ringert, 1998; Martínez-Portillo et al., 2001; Nieschlag et al., 2000a). Über diese Diagnosen lagen in den Patientenakten Informationen vor und die entsprechenden Fälle wurden von der Auswertung ausgeschlossen, so dass auch diese Ätiologien nur im Falle einer möglichen, - aber unwahrscheinlichen - Nicht-Dokumentation zur Erklärung dienen könnten.

Eine wichtige Ursache der obstruktiven Azoospermie ist schließlich in den urogenitalen Infektionen zu sehen. Diese können durch verschiedene Erreger wie Chlamydia trachomatis und Neisseria gonorrhoeae (Diemer et al., 2000; Gonzales et al., 2004; Paavonen \& EggertKruse, 1999), durch eine Infektion mit Mumps-Viren (Jarow, 2003) oder durch urogenitale Tuberkulose (Haidl \& Weidner, 2002) verursacht werden (vgl. 1.2.3.2.2). Generell ist die Häufigkeit der postinflammatorisch bedingten Verschlüsse in den westlichen Gesellschaften durch die allgemeine Abnahme der sexuell übertragbaren Krankheiten, die Bekämpfung der Tuberkulose und die fortgeschrittenen Behandlungsmöglichkeiten zurückgegangen (Baker, 2000). Dies könnte den niedrigeren Azoospermie-Anteil bei den späten Geburtsjahrgängen erklären. Jedoch wurden auch Patienten mit Befunden wie einer vorangegangenen Gonorrhoe - neben solchen mit einer Mumps-Erkrankung sowie einer urogenitalen Form der Tuberkulose - ebenfalls aus der untersuchten Stichprobe ausgeschlossen. Ein bewusstes Verschweigen der Erkrankungen kann wohl ausgeschlossen werden, da im Rahmen einer Infertilitätsberatung gerade diese eine wichtige Rolle spielen können und auf die Bedeutung sicherlich hingewiesen wurde. So bleibt die Vermutung z.B. einer nicht entdeckten Gonorrhoe oder Chlamydien-Infektion. Immerhin bleibt die Hälfte aller Chlamydien-infizierten Männer frei von Symptomen (Gonzales, 2004). Der niedrigere Anteil an Azoospermen 
innerhalb der späten Geburtsjahrgänge würde sich dann durch den allgemeinen Rückgang von Gonorrhoe und Chlamydien-Infektionen (Kamwendo et al., 1996) seit Erfindung des Penicillins und anderer Antibiotika erklären lassen. Letztendlich können hier jedoch erneut nur Annahmen gemacht werden.

\subsubsection{Konfundierung der Spermienkonzentration und des Fertilitätsstatus}

Die Spermienkonzentration eines Mannes kann von vielfältigen Faktoren beeinflusst werden und es war sicherzustellen, dass keine Konfundierung der Spermiendichte durch diese Einflüsse vorlag (vgl. 3.1.2). Zunächst soll die Aufmerksamkeit auf mögliche intraindividuell wirkende Faktoren gerichtet werden. Hier wurde die durchschnittliche Spermienkonzentration in Abhängigkeit vom Untersuchungsmonat betrachtet. Die höchsten Konzentrationen zeigten sich in den Monaten Februar und Mai, die niedrigsten Dichten waren hingegen für die Monate November und Dezember zu verzeichnen. Diese jahreszeitlichen Schwankungen stimmen in etwa mit den Ergebnissen einer Studie an Infertilitätspatienten der andrologischen Sprechstunde Gießen überein (Henkel et al., 1998). Als Wirkungsmechanismus diskutieren die Autoren hormonelle Rhythmiken sowie die direkte Abhängigkeit von der Photoperiode (vgl. auch 1.2.2.3.1). Zwischen den individuellen Spermienkonzentrationen der ehemaligen Patienten und dem Untersuchungsmonat ließ sich in der eigenen Stichprobe jedoch keine Korrelation feststellen.

Ebenso konnte kein Zusammenhang zwischen der Uhrzeit der Ejakulation und der Spermiendichte gefunden werden. Ohnehin gewann der größte Teil der untersuchten Patienten (93\%) seine Ejakulatprobe in den Vormittagsstunden. Cagnacci et al. (1999) fanden zwar höhere Spermienkonzentrationen am Nachmittag als in den Morgenstunden; jedoch lagen ihnen - im Gegensatz zur vorliegenden Studie - pro Individuum entsprechend zwei Proben zum Vergleich vor. Auf Grundlage dieser unterschiedlichen Datensätze ist ein Vergleich nicht möglich.

Da die Altersspanne der ehemaligen Patienten mit 21 bis 63 Jahren sehr groß war, wurde zusätzlich analysiert, ob die Spermienkonzentration mit dem Alter zusammenhing. Für die betrachtete Stichprobe konnte zunächst die niedrigste mittlere Spermienkonzentration für jene 25 Männer festgestellt werden, die zum Untersuchungszeitpunkt 50 Jahre und älter waren. Zwischen der individuellen Spermiendichte und dem Alter der Patienten ließ sich dann jedoch keine Korrelation finden. Dies bestätigt die Studie von $\mathrm{Ng}$ et al. (2004), in welcher für nicht-infertile Männer im Alter zwischen 52 und 79 Jahren keine reduzierten Spermienkonzentrationen gefunden wurden. Auch andere Studien weisen nicht auf eine Abnahme der Spermienkonzentration mit steigendem Alter hin. Zwar scheinen andere Parameter wie die Motilität betroffen zu sein, die Spermienkonzentration reduziert sich 
hingegen nicht oder nur unwesentlich (Eskenazi et al., 2003; Kidd et al., 2001; Rolf \& Nieschlag, 2000). Die untersuchte Stichprobe umfasste ebenso einige sehr junge Patienten im Alter von 21 bis 23 Jahren. Auch bei diesen kann bereits von einer voll ausgeprägten Reife mit entsprechenden Spermiendichten ausgegangen werden: Carlsen et al. (2005) verfolgten junge Männer mit einem zu Beginn durchschnittlichen Alter von 19 Jahren während einer 4-jährigen Follow-up-Studie und fanden keine Veränderungen der Spermienkonzentrationen im Laufe der individuellen Entwicklung in dieser Population. Für keine der drei vorgestellten Variablen, konnte ein Zusammenhang mit dem Fertilitätsstatus ermittelt werden.

Für vielfache und andauernde Diskussionen hat die Frage nach einer möglichen Abnahme der Spermienkonzentration im Laufe der letzten Jahrzehnte gesorgt (vgl. 1.2.2.3.2, Übersichten in Haidl, 1999; Köhn \& Schill, 1996; Nieschlag, 1998). In der eigenen Stichprobe konnten die niedrigsten mittleren Spermienkonzentrationen für die Geburtskohorten 1901 bis 1910 bzw. für die Untersuchungskohorten 1980 bis 1985 festgestellt werden. Die Aussagekraft dieser Ergebnisse kann allerdings in Anbetracht der wenigen Personen in den Klassen bezweifelt werden. Daher wurde auch hier wieder der Zusammenhang zwischen individueller Spermiendichte und Jahr der Geburt bzw. der Untersuchung berechnet. Es zeigte sich zunächst eine positive Korrelation zwischen dem Geburtsjahr bzw. dem Untersuchungsjahr und der Spermienkonzentration. Gleiche Effekte ergaben sich auch für den Fertilitätsstatus. Der Zusammenhang mit dem Geburtsjahr kann auf den bereits diskutierten höheren Anteil an Azoospermen innerhalb der frühen Geburtsjahrgänge zurückgeführt werden, denn mit Ausschluss dieser Subgruppe verschwindet auch der Effekt. Der Zusammenhang mit dem Untersuchungsjahr scheint hingegen vor allem durch die Subgruppe der Oligozoospermen beeinflusst. Nur für diese kann bei genauerer Untersuchung eine positive Korrelation des Untersuchungsjahres mit der Spermiendichte festgestellt werden. Allerdings bleibt zu beachten, dass es sich lediglich um einen geringen Effekt handelt. Die Ergebnisse unterstützen insgesamt keines der beiden gegenläufigen Szenarien einer Abnahme (Auger et al., 1995; Carlsen et al., 1992; Zorn et al., 1999) bzw. Zunahme (Fisch et al., 1996; Gyllenborg et al., 1999) der Spermienkonzentrationen eindeutig, sondern sprechen eher für ein konstantes Niveau der Spermiendichte. Dies bestätigt z.B. die Befunde von Bujan et al. (1996) und Paulsen et al. (1996). Zum einen entspricht aber der in der eigenen Arbeit betrachtete Untersuchungszeitraum nicht exakt den von anderen Studien betrachteten Zeiträumen, zum anderen werden wiederum die sehr unterschiedlichen Gruppen von Samenspendern (z.B. Gyllenborg et al., 1999) und Vätern (z.B. Auger et al., 1995; Bujan et al., 1996) betrachtet, welche einen direkten Vergleich nicht zulassen. 
Im Gesamteindruck erscheinen die für die eigene Stichprobe angegebenen Spermienkonzentrationen, soweit ein Vergleich bei der großen Variabilität der Untersuchungskollektive möglich ist, valide und reliabel, so dass hier nicht von einer fehlerhaften Dokumentation dieses zentralen Parameters auszugehen ist.

Weiterhin wurde ein potentieller Zusammenhang zwischen der Spermienkonzentration bzw. dem Fertilitätsstatus der Männer und ihrem sozioökonomischen Status (SES) untersucht. Hierzu wurde ermittelt, ob die entsprechenden medizinischen Parameter mit den Werten des „Internationalen Sozioökonomischen Index des beruflichen Status“ (ISEl; vgl. 2.2.8) korrelierten. Für die Spermienkonzentration konnte ein - allerdings sehr geringer Zusammenhang ermittelt werden. Wider Erwarten handelt es sich um einen negativen Effekt. Die Spermienkonzentration kann negativ beeinflusst werden durch arbeitsbedingte Expositionen wie Hitze z.B. beim Schweißen (Wong et al., 2003) oder Schwermetalle (de Rosa et al., 2003; Knecht, 1998; vgl. 1.2.3.2.10). Solche Berufe gehen jedoch eher mit niedrigeren ISEI-Werten einher. Anderseits gibt es Hinweise, dass eine sitzende Tätigkeit - die man wiederum mit höheren ISEI-Werten in Verbindung bringen könnte - ebenfalls zu erhöhter Skrotaltemperatur und somit zu reduzierten Spermienkonzentrationen führt (Hjøllund et al., 2000). Hingegen lässt eine andere Studie vermuten, dass nicht nur der Beruf selbst, sondern auch unterschiedliche Lebensstil-Charakteristika der sozialen Klassen für Variationen der Ejakulat-Parameter verantwortlich gemacht werden können (Coughlin et al., 2003). Da für die untersuchte Stichprobe keine weiteren Informationen über entsprechende Confounder, z.B. Zigaretten- und Alkoholkonsum, vorliegen, muss dieser Zusammenhang ungeklärt bleiben. Zwischen dem Fertilitätsstatus der Männer und ihrem SES ließ sich im Gegenteil kein Zusammenhang feststellen.

\subsubsection{Spermienkonzentration, Fertilitätsstatus und sexuelle Karenz}

Generell wird Infertilitätspatienten eine Karenzzeit von zwei bis sieben Tagen empfohlen (WHO, 1999). Es geht dabei nicht nur um eine genügend hohe Spermienkonzentration, sondern auch um die Optimalisierung verschiedener anderer Parameter wie Motilität und Morphologie der Spermien, letztlich immer mit dem Ziel, die Wahrscheinlichkeit des Eintretens einer Schwangerschaft zu erhöhen. Nachteilige Effekte bei Nicht-Befolgung der Karenz werden für die Motilität und Morphologie beschrieben (Elzanaty et al., 2005; Pellestor et al., 1994). Am Rande sei bemerkt, dass auch bezüglich dieser Parameter noch Unklarheit besteht, da eine kürzlich veröffentlichte Studie bei oligozoospermen Männern den größten Anteil motiler Zellen nach nur einem Tag Abstinenz nachwies (Levitas et al., 2005). 
Speziell hinsichtlich des Parameters der Spermienkonzentration scheint sich jedoch eine zu lange Abstinenzzeit von 8 bis 14 Tagen nicht negativ auszuwirken (Pellestor et al., 1994). Dies konnte auch in der eigenen Stichprobe nachgewiesen werden: Grafisch ließ sich eine Zunahme der durchschnittlichen Spermienkonzentration bis zu einer Abstinenz von 10 Tagen verzeichnen. Valide Angaben für eine Karenz von 11 bis 12 Tagen können nicht gemacht werden, da dies nur 3 Personen betraf. Bei einer Karenz von 14 Tagen sanken schließlich die durchschnittlichen Spermiendichten wieder auf ein Niveau, welches einer Karenz von 3 bis 8 Tagen entsprach. Nur bei einer Karenz von weniger als 2 Tagen zeigten sich tatsächlich niedrige mittlere Spermienkonzentrationen (vgl. 3.1.1). Dies trifft jedoch insgesamt nur für 8 Fälle zu, von denen drei als oligozoosperm und fünf als normozoosperm klassifiziert wurden. Nur einer der oligozoospermen Fälle lag mit einer Konzentration von 17 Mio./ml knapp unter der Abgrenzung zur Normozoospermie; die beiden anderen wiesen eine Konzentration von $2 \mathrm{Mio} . / \mathrm{ml}$ auf, so dass angezweifelt werden darf, ob mit einer längeren Abstinenzzeit tatsächlich eine so beträchtliche Verbesserung eingetreten wäre, dass sie als normozoosperm zu klassifizieren gewesen wären. Wie variabel der Zusammenhang zwischen Abstinenz und durchschnittlicher Spermien-konzentration sein kann, zeigt die Studie von Levitas et al. (2005). Hier wurden bei oligozoospermen Männern die höchsten durchschnittlichen Spermiendichten nach einer Karenz von nur 1 Tag bzw. 11 bis 14 Tagen gefunden.

Nachdem eine Erhöhung der durchschnittlichen Spermatozoenkonzentration mit zunehmender Abstinenz für die eigene Stichprobe ermittelt werden konnte, war der entsprechende Zusammenhang auf individueller Ebene von Interesse. Bei der Betrachtung der individuellen Zusammenhänge konnte in der vorliegenden Studie jedoch keine Korrelation zwischen der Abstinenzzeit und der Spermienkonzentration der Männer festgestellt werden. Auch der Fertilitätsstatus korrelierte nicht mit der Karenzzeit. Da sich die Abstinenz unterschiedlich auf normozoosperme und oligozoosperme Männer auswirken kann (Matilsky et al., 1993), wurden die beiden Subgruppen ebenfalls getrennt voneinander analysiert. Jedoch zeigt sich weder für die oligozoospermen noch für die normozoospermen Fälle ein Zusammenhang ihrer Spermiendichte mit ihrer Abstinenz.

Dieses Ergebnis scheint zunächst den Empfehlungen der WHO (1999) und auch den Befunden anderer Studien zu widersprechen. Pellestor und Kollegen (1994) fanden eine positive Korrelation zwischen der Spermienkonzentration und der Abstinenz. Das unterschiedliche Resultat könnte allerdings durch die unterschiedliche Art der Daten zustande kommen. In der erwähnten Studie wurden mehrere Proben von 6 Männern untersucht, die wiederholt hintereinander verschiedene Abstinenzzeiten (2-18 Tage) einhielten. In der eigenen Untersuchung hingegen lagen solche Daten nicht vor; intrapersonelle Variationen 
und Entwicklungen konnten nicht berücksichtigt werden. Demgegenüber bestätigen die vorliegenden Ergebnisse eine Untersuchung von de Jonge et al. (2004), welche ebenfalls weder für normozoosperme noch für oligozoosperme Männer einen Einfluss der sexuellen Abstinenz auf die Spermatozoen-Konzentration fanden.

Zusätzlich wurde überprüft, ob sich die Spermienkonzentrationen der Männer, welche die empfohlene Karenzzeit von 2 bis 7 Tagen einhielten, hinsichtlich ihrer zentralen Tendenz von den Konzentrationen der Patienten, deren Karenzzeit außerhalb des von der WHO (1999) empfohlenen Zeitraumes lagen, unterschieden. Hier ließ sich nur für die oligozoospermen Fälle ein Unterschied ermitteln. Die Betrachtung der Mittelwerte ergab, dass Oligozoosperme, welche die Karenzzeit nicht einhielten, eine niedrigere mittlere Spermiendichte aufwiesen.

Insgesamt wird der Effekt der Abstinenz auf individueller Ebene für die vorliegende Fragestellung als gering eingeschätzt. Um aber trotzdem eine Konfundierung des Zusammenhangs zwischen dem Fertilitätsstatus und der Mortalität ausschließen zu können, und um dem zuletzt dargestellten Befund hinsichtlich der oligozoospermen Subgruppe Rechnung zu tragen, wurde ein alternatives Regressionsmodell berechnet, welches die Abstinenz als Kontrollvariable einfließen ließ. Die dabei ermittelten Befunde bestätigen jene des Modells ohne Einschluss der Karenzzeit (vgl. 4.3.1.2.1).

\subsection{Diskussion der Ergebnisse}

Die vorliegende Studie wurde mit dem Ziel unternommen, den Zusammenhang zwischen der Lebenszeit-Mortalität von Männern und ihren Spermatogenese-Funktionen, abgebildet durch die Spermienkonzentration bzw. den Fertilitätsstatus, zu erforschen. Daher wurde mittels nicht-parametrischer, semi-parametrischer und parametrischer Verfahren analysiert, ob sich erstens die Lebensdauern bzw. Lebenserwartungen zwischen fertilen und subfertilen Männern bzw. zwischen Normozoospermen, Oligozoospermen und Azoospermen unterscheiden, und ob sich zweitens eine Dosis-Wirkungs-Beziehung mit der Spermienkonzentration feststellen lässt. Hierzu wurde in einem ersten Schritt die gesamte Stichprobe betrachtet, während in einem zweiten Schritt eine getrennte Analyse der nach dem Median des Geburtsdatums getrennten Subgruppen - als "frühe“ und „späte“ Geburtsjahrgänge bezeichnet - erfolgte. Da keine Publikationen über Studien vorliegen, welche ähnliche Datensätze untersucht oder vergleichbare Analysen durchgeführt haben, kann eine Einordnung der Ergebnisse in den Stand der Forschung nicht erfolgen, sondern es muss weitestgehend bei einer summarischen Deskription der Befunde bleiben. Mögliche Erklärungsansätze können dann dem letzten Abschnitt dieses Kapitels entnommen werden. 


\subsubsection{Lebenszeit-Mortalität und Fertilitätsstatus}

\subsubsection{Ergebnisse der nicht-parametrischen Verfahren}

Die Abbildungen der Überlebenskurven zeigten deutlich, dass die Sterblichkeit der Fertilen unter derjenigen der Subfertilen lag. Dies wurde vor allem bei der Betrachtung der frühen Geburtsjahrgänge deutlich und ließ sich hier auch als statistisch signifikanter Effekt nachweisen. Allerdings konnten bei den späten Geburtsjahrgängen keine analogen Ergebnisse ermittelt werden - hier zeigte sich kein Unterschied in der Sterblichkeit. Differenzierte man die Subgruppe der Subfertilen noch einmal genauer in Azoosperme und Oligozoosperme, so konnte man feststellen, dass der erwähnte Unterschied in der Mortalität zwischen Fertilen und Subfertilen v.a. auf den Unterschied zwischen Normozoospermen und Oligozoospermen zurückzuführen war. Für die Azoospermen zeigte sich nur der Trend einer im Vergleich zu den Normozoospermen erhöhten Mortalität. Diese Effekte traten deutlich bei der Betrachtung der frühen Geburtsjahrgänge zu Tage, ließen sich jedoch wiederum nicht bei den späten Geburtsjahrgängen finden (vgl. 3.2.1).

Die Befunde deuten somit darauf hin, dass zwischen der Spermatogenesefunktion der Männer, für die als Indikator der Fertilitätsstatus diente, und ihrer Lebensdauer ein Zusammenhang besteht. Einschränkend kann dies jedoch nur für die Gruppe der frühen Geburtsjahrgänge behauptet werden.

\subsubsection{Ergebnisse der semi-parametrischen Verfahren}

Mittels der semi-parametrischen Cox-Regression ließen sich diese Unterschiede bestätigen und zusätzlich quantifizieren (vgl. 3.2.2). Innerhalb der frühen Geburtsjahrgänge wurde für die Untergruppe der Subfertilen ein 1,6-mal höheres Sterberisiko gefunden. Wiederum zeigte sich, dass dieses Ergebnis auf den großen Unterschied in der Mortalität von normozoospermen und oligozoospermen Männern zurückzuführen war. Bei Letzteren ist das Sterberisiko um mehr als das Doppelte erhöht. Zwischen der Mortalität der Azoospermen und Oligozoospermen bzw. Normozoospermen ließ sich kein Unterschied finden. Es sollte jedoch bedacht werden, dass die in den Berechnungen berücksichtigte Anzahl der Azoospermen mit 62 Individuen geringer war als die der Oligo- und Normozoospermen und somit vorhandene Effekte möglicherweise nicht aufgedeckt werden konnten.

Als Kontrollvariablen wurden jene Parameter berücksichtigt, von denen ein potentieller Einfluss auf das Mortalitätsrisiko vermutet werden konnte: Die Lebenserwartung bei Geburt, welche sich im Laufe des Geburtszeitraums der Fälle (1892 bis 1937) um mehr als 22 Jahre erhöhte (Statistisches Bundesamt, 1972), der Geburtsmonat (Doblhammer, 2004; Lerchl, 2004), das Alter der Patienten bei Erstellung des Spermiogramms, das Untersuchungsjahr 
sowie der SES, ausgedrückt durch die ISEI-Werte (Geyer \& Peter, 1999; Klein, 1993b). Hinsichtlich des Alters der Patienten zum Untersuchungszeitpunkt war es irrelevant, ob diese Variable als zeitunabhängig oder -abhängig angenommen wurde, die Ergebnisse blieben in beiden Fällen identisch. Das Mortalitätsrisiko wurde bei den frühen Geburtsjahrgängen ausschließlich durch den Fertilitätsstatus der Patienten beeinflusst. Für die späten Geburtsjahrgänge hingegen zeigte sich kein Einfluss des Fertilitätsstatus auf die Sterblichkeit. Hier wurde die Mortalität durch den SES vorhergesagt. Mit steigendem SES nahm das Sterberisiko ab. Dies würde anderen Befunden, welche eine negative Assoziation zwischen sozioökonomischen Charakteristika und dem Gesundheitszustand bzw. der Sterblichkeit aufdeckten, entsprechen (Übersicht in Mielck, 2000; vgl. auch 1.2.1.2.3). Die Problematik der Aussagen über die späten Geburtsjahrgänge wird im Abschnitt 4.3.3 diskutiert.

\subsection{Sexuelle Abstinenz als Kontrollvariable}

Wurde die Abstinenz als Kontrollvariable im Regressionsmodell nach Cox berücksichtigt, so blieben die zuvor beschriebenen Effekte des Fertilitätsstatus auf das Mortalitätsrisiko in etwa erhalten. Dies betraf wiederum die frühen Geburtsjahrgänge. Ein äußerst überraschendes Ergebnis ist der Befund eines Einflusses der sexuellen Abstinenz auf das Mortalitätsrisiko der späten Geburtsjahrgänge. Weiterhin ist die Richtung des Effektes interessant: Das Mortalitätsrisiko nimmt mit zunehmender Abstinenzzeit zu. Es scheint keine intuitiv plausible Erklärung für einen direkten Zusammenhang der beiden Variablen zu geben, so dass an einen Confounder gedacht werden muss. Hier könnte z.B. vermutet werden, dass die Abstinenz mit dem SES zusammenhängt. Personen mit niedrigerem SES könnten häufiger Abstinenzzeiten angegeben bzw. eingehalten haben, welche den WHOEmpfehlungen entsprachen, um der antizipierten Norm bzw. den Forderungen des Arztes zu genügen. Da dem SES wiederum ein Einfluss auf die Lebensdauer zugeschrieben werden kann, könnte so ein Scheinzusammenhang zwischen der Karenzzeit und der Mortalität entstanden sein.

Die Aussagen hinsichtlich des Einflusses des SES auf die Compliance von Patienten sind uneinheitlich. Während es für ältere Personen Hinweise auf eine höhere Compliance bei höherem SES gibt, postulieren einige Studien einen negativen Zusammenhang und bestätigen somit die zuvor geäußerte Vermutung (Balkrishnan, 1998). Gegen eine Konfundierung durch den SES spricht schließlich wiederum die Tatsache, dass dieser weder mit der sexuellen Karenzzeit korrelierte, noch Unterschiede in den ISEI-Werten zwischen den Männern, welche die empfohlene Karenz berücksichtigten bzw. nicht berücksichtigten, bestand. Es bleibt also zu vermuten, dass andere, noch nicht identifizierte Confounder für den scheinbaren Einfluss der Karenzzeit auf die Abstinenz verantwortlich sind. Allerdings sei 
bereits an dieser Stelle darauf hingewiesen, dass die Ergebnisse für die späten Geburtsjahrgänge aus methodischen Gründen eventuell nicht zuverlässig sind (vgl. 4.3.3).

\subsection{Analyse der Verstorbenen}

Zur weiteren Absicherung der Effekte wurden die nicht-parametrischen und semiparametrischen Analysen zusätzlich ausschließlich mit den bereits verstorbenen Fällen durchgeführt. Hier ergab sich das Problem der Reduktion der Stichprobengröße, so dass nicht nur die reine Signifikanz eines Ergebnisses Beachtung fand, sondern vor allem auch Effektrichtung und -größe berücksichtigt wurden. Die für die gesamte Stichprobe ermittelten Effekte des Fertilitätsstatus auf die Mortalität blieben auch hier vergleichbar und konnten somit bestätigt werden. Für die frühen Geburtsjahrgänge konnte der Trend eines um den Faktor 1,9 erhöhten Sterberisikos der oligozoospermen im Vergleich zu den normozoospermen Fällen berechnet werden (vgl. 3.2.2.5).

Im Gegensatz zu den Befunden bei der gemeinsamen Betrachtung der zensierten und unzensierten Fälle, konnten für die verstorbenen Männer das Alter zum Untersuchungszeitpunkt sowie das Untersuchungsjahr als Prädiktorvariablen für die Lebensdauer modelliert werden. Das Mortalitätsrisiko nahm mit steigendem Alter der Patienten ab. Die Plausibilität dieses Effektes verdeutlicht der folgende beispielhafte Vergleich: Ein zum Untersuchungszeitpunkt 53-Jähriger hat bereits lange genug überlebt, um die Altersmarke von 50 Jahren zu überschreiten, während ein bei der Untersuchung 20-Jähriger diese erst noch erreichen muss. Ebenso nahm das Mortalitätsrisiko der Verstorbenen mit dem Untersuchungsjahr zu. Auch dies ist plausibel: Ein 35-Jähriger Patient, der sich 1950 untersuchen ließ und im September 2002 verstorben war, konnte ein maximales Alter von 87 Jahren erreichen. Ein gleichaltriger Patient, der hingegen 1980 ein Spermiogramm erstellen ließ und im September 2002 verstorben war, konnte höchstens 57 Jahre alt werden.

\subsubsection{Ergebnisse der parametrischen Verfahren}

Schließlich wurden die weniger robusten parametrischen Regressionen berechnet, welche je nach Modell von unterschiedlichen, aber spezifischen Annahmen über die zu Grunde liegende Hazard-Rate ausgehen (vgl. 2.3.1.4 und 3.2.3).

Das Exponential-Modell ermittelte keinerlei Zusammenhänge zwischen dem Fertilitätsstatus und der Mortalität der ehemaligen Patienten. Für die gesamte Stichprobe wurden das Alter zum Untersuchungszeitpunkt sowie das Untersuchungsjahr als Prädiktorvariablen für die Mortalität modelliert. Wurden ausschließlich die frühen Geburtsjahrgänge betrachtet, so erwies sich keiner der Parameter als erklärende Variable. Die Mortalität der späten Geburtsjahrgänge wurde durch den SES beeinflusst. Die Ergebnisse dieses einfachen Modells 
müssen jedoch mit Vorsicht betrachtet werden, denn das Exponential-Modell erscheint weniger geeignet zur Modellierung der Mortalität, da hier eine konstante Hazard-Rate vorausgesetzt wird, das Sterberisiko eines Individuums im Verlauf seines Lebens dagegen nicht als konstant angenommen werden kann (siehe unten). Es ermöglichte aber eine erste Annäherung und leitet weiter zu dem adäquateren Gompertz-Makeham-Modell.

Das Gompertz-Makeham-Modell bestätigte in einigen Punkten die Ergebnisse des Exponential-Modells. Bei der Analyse der gesamten Stichprobe ergaben sich das Alter der Patienten bei der Erstellung des Spermiogramms sowie das Jahr der Untersuchung als die die Mortalität erklärenden Variablen. Ein wichtiges Detail zeigte sich hier in der Richtung der Effekte, welche im Vergleich zu den dargestellten Befunden für die Verstorbenen umgekehrt war. Dies ist dadurch zu erklären, dass hier verstorbene und noch nicht verstorbene Fälle gemeinsam betrachtet wurden, und es nun - vereinfacht ausgedrückt - um die Wahrscheinlichkeit ging, verstorben zu sein. Zur Verdeutlichung soll die folgende Überlegung beitragen: Werden im Jahr 1970 zwei Männer im Alter von 25 bzw. 45 Jahren untersucht, so ist für den 25-Jährigen die Wahrscheinlichkeit, im September 2002, also 32 Jahre später, verstorben zu sein, geringer als für den 45-Jährigen. Daher findet sich ein zunehmendes Mortalitätsrisiko bei zunehmendem Alter zum Untersuchungszeitpunkt. Anders verhält es sich mit dem Einfluss des Untersuchungsjahres auf das Sterberisiko. Ein 35-Jähriger, der sich im Jahre 1960 untersuchen ließ, ist mit größerer Wahrscheinlichkeit im September 2002 bereits verstorben als ein Mann gleichen Alters, der sich 1980 untersuchen ließ. So nimmt das Mortalitätsrisiko mit jedem voranschreitenden Untersuchungsjahr etwas ab.

Wurden in diesem Modell nur die frühen Geburtsjahrgänge analysiert, so konnte ein erhöhtes Relatives Sterberisiko für die subfertilen Fälle modelliert werden. Das Risiko, zu versterben, war für die Subfertilen 1,7-mal größer als für die Fertilen. Für die späten Geburtsjahrgänge hingegen wurde erneut der SES als die die Mortalität bestimmende Variable angegeben. Mit steigendem SES nahm das Risiko zu versterben ab.

Das Gompertz-Makeham-Modell wird allgemein als das Modell angenommen, welches unter den parametrischen Verfahren am besten die menschliche Sterblichkeit modelliert (Yashin et al., 2000). Vernachlässigt man die Sterblichkeit in Kinderjahren, so kann man das Mortalitätsrisiko als aus zwei Komponenten zusammengesetzt betrachten. Das Sterberisiko besteht zum einen in der Mortalität aufgrund von Unfällen, Suizid, Mord und sexuell übertragbaren Krankheiten. Dies sind Ätiologien, die vor allem zu frühzeitigem Tod im jungen Erwachsenenalter führen (Iribarren et al., 2005; Stillion, 1995). Ab dem mittleren Erwachsenenalter steigt die Mortalität dann kontinuierlich, fast exponentiell an. Als ursächlich kann hier der einsetzende Alterungsprozess in Form einer Abnahme verschiedener 
physiologischer Funktionen und Kapazitäten angesehen werden (Carnes et al., 2003; Macieira-Coelho, 2003; Preston et al., 2000; Troen, 2003; Weinert \& Timiras, 2003).

$\mathrm{Zu}$ einem ähnlichen Ergebnis wie das Gompertz-Makeham-Modell führte auch die Modellierung nach Weibull. Zwar müssen hier die Ergebnisse der Auswertung der gesamten Stichprobe vernachlässigt werden, da das Modell auch bei Verwendung verschiedener Minimierungs-Algorithmen der Funktion nicht konvergierte und somit letztendlich nicht in validen Ergebnissen resultierte. Die separate Analyse der frühen und späten Geburtsjahrgänge replizierte jedoch die Befunde des Gompertz-Makeham-Modells. Innerhalb der frühen Geburtsjahrgänge lag das Mortalitätsrisiko der subfertilen Männer 1,7mal höher als das der fertilen Fälle. Für die späten Geburtsjahrgänge wurde erneut der SES als eine die Mortalität beeinflussende Variable modelliert.

Wie bereits erwähnt, eignet sich das Gompertz-Makeham-Modell am besten für die Mortalitäts-Modellierung. Das Weibull-Modell findet hingegen häufiger in der Industrie zur Darstellung der Akkumulation von schädigenden Wirkungen auf Produkte Anwendung (vgl. 2.3.1.4). Die Ähnlichkeit der Befunde der beiden Modelle könnte der Tatsache entstammen, dass innerhalb der untersuchten Stichprobe der früheste Todesfall im Alter von 43 Jahren verstarb und der größte Teil der Todesfälle sich erst ab dem mittleren Erwachsenenalter ereignete. Die erste Komponente der Mortalität aufgrund von Unfällen etc. im frühen Erwachsenenalter kommt hier wohl weniger zum Tragen. Vielmehr sollte die zweite Komponente der Sterblichkeit in Form eines Alterungsprozesses des Körpers Wirkung zeigen. Dieser „Abnutzungsprozess" des menschlichen Organismus könnte nun mit dem Abnutzungsprozess und Verschleiß industrieller Produkte gleichgesetzt werden, so dass die beiden Verfahren ein ähnliches Phänomen darstellen. Sie gelangen zu vergleichbaren Ergebnissen wie die semi-parametrische Cox-Regression hinsichtlich des Einflusses des Fertilitätsstatus sowie des SES auf die Lebensdauer. Generell haben die parametrischen Modelle einen höheren Informationsgehalt als die semi-parametrischen Modelle, allerdings müssen auch stärkere Annahmen über die Art der funktionalen Beziehung zwischen den Parametern gemacht werden (Diekmann \& Mitter, 1984). Die gefundenen Abweichungen zwischen parametrischen Modellen und der Cox-Regression finden somit ihren Ursprung vermutlich in den spezifischeren Voraussetzungen der parametrischen Modelle in Bezug auf die Hazard-Rate.

\subsubsection{Abgrenzung der Subfertilen}

Die Einteilung der Fertilitätsklassen folgte den Empfehlungen der WHO (1999), welche weltweite Anwendung finden. Die Klassifikation der Azoospermen ist eindeutig, bei ihnen sind keine Spermien im Ejakulat zu finden. Hingegen stellt sich die Frage, auf welcher 
Grundlage die Oligozoospermie-Diagnose ab einer Spermatozoen-Konzentration unter 20 Mio./ml gerechtfertigt ist. Diese Abgrenzung geht in ihren Ursprüngen auf eine frühe Studie von MacLeod \& Gold (1951) zurück, in welcher die Forscher zeigen konnten, dass ab einer Spermienkonzentration über $20 \mathrm{Mio} / \mathrm{ml}$ die bis zum Erreichen einer Schwangerschaft benötigte Zeit nicht mehr von der Spermiendichte beeinflusst wird. Dies erinnert letztendlich daran, dass es sich bei den Normwerten, auch bezüglich anderer Ejakulatparameter, lediglich um Richtwerte handelt, welche zusammengenommen die Chancen des Eintretens einer Schwangerschaft einschätzen sollen. Ein einzelner Parameter ist, außer im Extremfall z.B. der Azoospermie, generell noch nicht in der Lage vorauszusagen, ob eine Schwangerschaft möglich sein wird. Zusätzlich sind außerdem auch noch die reproduktiven Funktionen der Partnerin entscheidend (Behre et al., 2000b). Somit ist es nur bedingt möglich, anhand der Normwerte zwischen fertilen und subfertilen Männern zu unterscheiden. Jedoch bieten die Standard-Parameter der Spermienkonzentration, -morphologie und -motilität wichtige Informationen, um die generelle Zeugungsfähigkeit eines Mannes einzuschätzen (Guzick et al., 2001)

In der vorliegenden Studie erfolgte gemäß den WHO-Laborrichtlinien (1999) die Abgrenzung der oligozoospermen Fälle bei einer Grenze von 20 Mio./ml. Um nun zu klären, inwiefern die Wahl dieser Klassifikationsgrenze die Ergebnisse beeinflusste, wurden die nicht-parametrischen und semi-parametrischen Verfahren wiederholt. Die Analysen begannen mit einer imaginären Abgrenzung der oligozoospermen Fälle bei einer Spermiendichte von $5 \mathrm{Mio} . / \mathrm{ml}$ und endeten bei einer Trennung von normozoospermen und oligozoospermen Fällen bei einer Spermienkonzentration von 100 Mio./ml. Hierbei ergab sich, dass die Effekte, welche bei einer Grenz-Konzentration von 20 Mio./ml ermittelt wurden, auch bei Anwendung anderer Grenz-Konzentrationen weitestgehend gleich bzw. vergleichbar blieben. Dies traf bis zu einer Klassifikationsgrenze von ungefähr 60 Mio./ml zu. Wichtig erscheint die Tatsache, dass bei Wahl einer anderen Grenz-Konzentration die Effekte entweder gleich blieben oder sich sogar verstärkten, und dass die Effektrichtungen sich nicht wesentlich änderten. Bei keiner der vielfältigen Analyse-Varianten ergab sich der Befund, die Mortalität der fertilen sei höher als jene der subfertilen Männer. Es konnte stets bestätigt werden, dass im Falle eines Unterschiedes zwischen den beiden Gruppen das Sterberisiko der Subfertilen erhöht war (vgl. 3.2.4.1).

In einem weiteren Schritt wurde auf Basis einer Untersuchung von Guzick und Kollegen (2001) eine weitere Klassifikationsgrenze in Erwägung gezogen. In dieser Studie wurden Spermiogramme von fertilen Männern, welche bereits ein Kind gezeugt hatten, und subfertilen Männern, in deren Partnerschaften innerhalb eines Jahres bei normaler Fertilität seitens der Frau keine Schwangerschaft eingetreten war, miteinander verglichen. Die 
Autoren ermittelten, dass Subfertilität am besten durch eine Spermiendichte unterhalb von 13,5 Mio./ml abgegrenzt wird, während sich Fertilität durch eine Spermienkonzentration von mindestens 48 Mio./ml definiert. Die dazwischen liegenden Spermiendichten sind als indifferent anzusehen. Gemäß dieser Klassifikation wurden in der eigenen Studie erneut die nicht-parametrischen, semi-parametrischen sowie parametrischen Verfahren angewendet und die Ergebnisse mit jenen bei einer Grenz-Konzentration von 20 Mio./ml verglichen. Vor allem der Wilcoxon(Gehan)-Test ermittelte bei dieser Klassifizierung durchaus ähnliche Ergebnisse mit zum Teil verstärkten Effekten. Aber auch die parametrischen Regressionsmodelle ergaben vergleichbare Resultate. Lediglich die Befunde der Cox-Regression konnten nur eingeschränkt bestätigt werden. Jedoch erscheint auch hier wieder von Bedeutung, dass die Effektgrößen vergleichbar blieben und das Mortalitätsrisiko der subfertilen Fälle stets unterhalb des Risikos der fertilen lag (vgl. 3.2.4.2).

Es erscheint plausibel, dass bei Wahl anderer Klassifikationsgrenzen nicht exakt die gleichen Befunde wiederholt werden können wie bei den Analysen mittels der „klassischen“ Abgrenzung bei 20 Mio./ml. Jedoch bleibt festzuhalten, dass unabhängig von der Wahl der Klassifikationskonzentration der Effekt fortgesetzt gleich blieb: In der untersuchten Stichprobe hatten, im Falle eines Unterschieds, die subfertilen Fälle stets ein größeres Risiko zu versterben als die fertilen Männer. Somit erscheint die Befolgung der WHO-Empfehlungen (1999) gerechtfertigt. Ferner sei erwähnt, dass die Diskussion um „normale“ und „subnormale“ Ejakulatwerte noch offen ist. Während in einer Studie zur Evaluierung der Normwerte verschiedener Ejakulatparameter die Subfertilitätsdiagnose ab einer Spermiendichte unter 20 Mio./ml bestätigt wurde (Menkveld et al., 2001), trennt eine andere Arbeit fertile und subfertile Fälle bei einer Spermienkonzentration von 14,3 Mio./ml und bestätigt damit eher die Befunde von Guzick und Kollegen (Ombelet et al., 1997).

\subsubsection{Lebenszeit-Mortalität und Spermienkonzentrationen}

Zusätzlich wurde zur Überprüfung einer potentiellen Dosis-Wirkungs-Beziehung der Einfluss der Spermienkonzentration auf die Sterblichkeit der ehemaligen Patienten analysiert (vgl. 3.3). Dies geschah in Form der semi-parametrischen und parametrischen Regressionsmodelle. Grundsätzlich wurden die Berechnungen durch die Abweichung der Spermienkonzentrationen von einer Normalverteilung eingeschränkt. Dies trifft freilich nicht nur für die untersuchte Stichprobe zu, sondern ist ein allgemeines Phänomen dieses Parameters in der Allgemeinbevölkerung bzw. bei Infertilitätspatienten, weshalb generell bei Analysen die Logarithmierung (zur Basis 10) empfohlen wird (Berman et al., 1996). In der betrachteten Stichprobe konnte durch eine entsprechende Logarithmierung lediglich für die Subgruppe der Normozoospermen eine Normalverteilung der Spermienkonzentration 
realisiert werden, die Angaben der Oligozoospermen bzw. der Stichprobe insgesamt blieben nicht-normalverteilt. Auch bei Anwendung anderer Transformationen wie natürlichem Logarithmieren oder Bildung der Quadratwurzel lag keine Normalverteilung vor.

Die Analysen zum Zusammenhang zwischen der Spermiendichte und der Mortalität sind eindeutig und weisen alle in die gleiche Richtung: Es lässt sich weder für die Normozoospermen bzw. Oligozoospermen im Einzelnen noch für die beiden Substichproben gemeinsam ein Einfluss der Spermienkonzentration auf die Lebensdauer der Patienten finden. Dies wirft ein weiteres Licht auf die Art des potentiellen Zusammenhangs zwischen den reproduktionsphysiologischen Konditionen von Männern und deren Lebenszeit-Mortalität. Es sei an die ursprüngliche Überlegung der vorliegenden Untersuchung erinnert, ob eine verschlechterte Spermatogenese eine Art Indikator für einen schlechten Gesundheitszustand und damit eine kürzere Lebensdauer sein könnte. Nun kann zwischen der Spermienkonzentration und der Lebensdauer offensichtlich keine direkte Dosis-Wirkungs-Beziehung angenommen werden. Die Spermienkonzentration selbst stellt keine Prädiktorvariable für die Lebensspanne dar. Ein Zusammenhang scheint vielmehr im Sinne eines generellen „Funktionierens“ bzw. „Nicht-Funktionierens“ zu bestehen. Dies wird durch den Einfluss des Fertilitätsstatus auf die Sterblichkeit bestätigt. Nicht die absolute Spermiendichte sagt die Lebensdauer voraus, sondern eine „bessere“ bzw. „schlechtere“ physiologische Funktion scheint mit einem niedrigeren bzw. höheren Sterberisiko assoziiert.

\subsubsection{Unterschiede zwischen frühen und späten Geburtsjahrgängen}

Die ursprüngliche Stichprobe wurde nach dem Median des Geburtsdatums (05.08.1931) in zwei Subgruppen unterteilt. Die Kohorte der frühen Geburtsjahrgänge umfasste Männer, welche zwischen 1892 und 1931 geboren waren, in der Kohorte der späten Geburtsjahrgänge hingegen waren die Fälle subsumiert, die zwischen 1931 und 1937 geboren waren. Die ungleich großen Zeiträume ergeben sich aus der sehr ungleichen Verteilung der Geburtsjahre (vgl. 3.1). Bei der Betrachtung sämtlicher Ergebnisse fielen immer wieder die unterschiedlichen Befunde für die Subgruppen der frühen und späten Geburtsjahrgänge ins Auge. Während für die Gruppe der frühen Geburtsjahrgänge ein Einfluss des Fertilitätsstatus postuliert werden konnte, ließ sich dies für die späten Geburtsjahrgänge nicht bestätigen. Hier wurde die Sterblichkeit der Patienten vor allem durch den SES erklärt. Diese Unterschiede bedürfen einer ausführlicheren Betrachtung.

Einen möglichen Erklärungsansatz könnte das Erleben des 2. Weltkriegs darstellen. In Deutschland wurden Kohorten bis einschließlich 1928 einberufen und nahmen als Soldaten oder in anderer Funktion am Krieg teil. Somit haben nur die frühen Geburtsjahrgänge den Krieg als Soldaten erlebt und es lässt sich daher vermuten, dass sich die Kriegseinflüsse auf 
diese Kohorte wesentlich stärker ausgewirkt haben dürften. Möglicherweise waren die frühen Geburtsjahrgänge stärker betroffen von Verletzungen, Erkrankungen und Hunger, z.B. in Kriegsgefangenschaft. Die Kriegseinflüsse könnten sich negativ auf den Gesundheitszustand ausgewirkt und gleichzeitig die Fertilität beeinträchtigt haben. So stieg auch tatsächlich der Anteil der Azoospermen in den letzten Kriegsjahren und ersten Nachkriegsjahren beträchtlich an (Schuermann, 1948). Der Autor schreibt dies neben tatsächlichen körperlichen Beeinträchtigungen auch den seelischen Belastungen der Männer während des Krieges zu. Angst und Todesangst, z.B. von KZ-Insassen oder zum Tode Verurteilten, wirken sich nachteilig auf die Spermienproduktion aus (Schuermann, 1948; Stieve, 1952). Dieser Ansatz birgt eine weitere Erklärungsmöglichkeit für den größeren Anteil an Azoospermen innerhalb der frühen Geburtsjahrgänge (vgl. 4.4.2).

Eine weitere Überlegung ist hinsichtlich der Überlagerung der Effekte der Fertilität bzw. des SES anzustellen. In einem Review verschiedener in den Jahren von 1960 bis 1993 veröffentlichten Arbeiten konnten González und Kollegen (1998) einen bedeutsamen Effekt hinsichtlich des Zusammenhangs zwischen dem SES und ischämischen Herzkrankheiten (IHK) feststellen. Bis ca. 1970 war ein hoher SES mit einer hohen IHK-Morbidität und Mortalität verbunden. Der SES wurde hier durch den Beruf ermittelt. Personen mit einem höheren Berufsstatus hatten ein größeres IHK-Risiko als solche mit einem niedrigeren Berufsstatus. Ab 1970 drehte sich die Beziehung dann um. Nun hatten Personen mit hohem SES eine niedrigere IHK-Mortalität. Hinsichtlich der Befunde der vorliegenden Arbeit könnte man nun vermuten, dass sich innerhalb der frühen Geburtsjahrgänge der negative Einfluss eines hohen SES auf die Mortalität über die IHK mit dem ansonsten positiven Einfluss eines hohen SES auf die restliche Mortalität ausgeglichen hat und sich stattdessen der Einfluss der Fertilität bemerkbar machen konnte. Bei den späten Geburtsjahrgängen wäre es hingegen zu einer Summierung des positiven Effektes auf die Mortalität gekommen, der dann stärker als der Effekt der Fertilität war. Das würde bedeuten, dass letztendlich die Mortalität stärker mit dem SES als mit der Fertilität assoziiert ist. Der starke Einfluss des SES auf die Mortalität wurde tatsächlich in vielen Studien evident: Immer wieder wurde eine höhere Mortalität bei niedrigerem SES bestätigt (vgl. 1.2.1.2.3; z.B. Geyer \& Peter, 1999; Klein, 1993b; Peter, 2001; ausführliche Darstellung in Mielck, 2000).

Dennoch sollten die Befunde für die Kohorte der späten Geburtsjahrgänge generell mit Vorbehalt betrachtet werden, denn der Anteil der zensierten Fälle war hier relativ hoch. Für 83,3\% aller Angehörigen dieser Subgruppe war zum Ende des Beobachtungszeitraumes im September 2002 das Ereignis „Tod“ noch nicht eingetreten. Das hatte zur Folge, dass die Lebensspanne für den größten Teil dieser Subpopulation geschätzt werden musste. Überwiegen aber die zensierten Fälle stark, so kann die Überlebenszeit nur unzureichend 
abgeschätzt werden (Bühl \& Zöfel, 2002). Dies lässt Zweifel hinsichtlich der Gültigkeit der für diese Subgruppe ermittelten Ergebnisse aufkommen. Innerhalb der Kohorte der frühen Geburtsjahrgänge hingegen betrug der Anteil der zensierten Fälle 55,4\%, wodurch eine bessere Schätzung der Überlebenszeiten möglich war. Die Ergebnisse für die frühen Geburtsjahrgänge erscheinen somit valider und infolgedessen sollen sich die folgenden Überlegungen darauf konzentrieren, wie ein möglicher Zusammenhang zwischen Fertilität und Mortalität Erklärung finden könnte.

\subsection{Erklärungsansätze zum Zusammenhang zwischen männlicher Fertilität und Lebenszeit-Mortalität}

Die Analysen der vorliegenden Arbeit lassen einen Zusammenhang zwischen dem Fertilitätsstatus und der Lebensdauer von Männern vermuten. Dieser Befund kann nur indirekt mit den Ergebnissen anderer Studien in Beziehung gesetzt werden, da bislang keine entsprechenden Untersuchungen vorliegen. Beispielsweise konnten Untersuchungen an Vasektomie-Patienten kein erhöhtes Risiko für das spätere Auftreten von Krankheiten wie kardiovaskuläre Erkrankungen oder Diabetes mellitus und allgemein kein erhöhtes Mortalitätsrisiko feststellen (Giovannucci et al., 1992; Nieschlag et al., 2000b; Weiske, 1998). Unter der Annahme, dass die fertilen Fälle mit einer größeren Wahrscheinlichkeit eine größere Anzahl an Kindern zeugten als die subfertilen Fälle, können die vorliegenden Ergebnisse - bedingt - mit Studien zum Zusammenhang zwischen Reproduktionserfolg und Lebenserwartung von Männern verglichen werden. Die Annahme einer längeren Lebensspanne der fertilen Patienten geht einher mit den Studien von Korpelainen (2000) und Westendorp \& Kirkwood (1998), in denen Angehörige europäischer Adelsfamilien sowie der finnischen Landbevölkerung hinsichtlich ihres Reproduktionserfolges und ihrer Lebensdauer untersucht wurden. Bei diesen historischen Populationen korrelierten der reproduktive Erfolg und die Lebensspanne der Männer positiv miteinander. Dies ist dadurch zu erklären, dass die reproduktiven Kosten für Männer im Vergleich zu Frauen relativ gering sind, und die männliche Reproduktion nicht zwangsläufig mit einer Reduktion der Ressourcen zur Aufrechterhaltung des somatischen Zustands, und damit einer Alterung, einhergehen muss (vgl. auch 1.2.4). Der weitere Befund von Westendorp \& Kirkwood (1998) einer Abnahme der Kinderzahl ab einem sehr hohen Sterbealter von 90 Jahren, lässt allerdings vermuten, dass sich vermehrtes Investment in die Reproduktion in sehr begrenztem Ausmaß auch bei Männern auf die Gesundheit bzw. Mortalität auswirken könnte. Für die eigene Stichprobe könnte, selbst bei Informationen über später gezeugte Kinder, kein Vergleich gezogen werden, da das höchste erreichte Sterbealter bei 85 Jahren liegt. Es sei abschließend erwähnt, dass dieser dargestellte Zusammenhang zwischen Reproduktionserfolg und 
Lebensspanne nicht in allen Studien bestätigt werden konnte. In einer Untersuchung von US-amerikanischen Geburtskohorten der Jahre 1880 bis 1929, also einer Stichprobe mit vergleichbaren Geburtsjahrgängen wie im eigenen Untersuchungskollektiv, wurde keine Beziehung zwischen der Anzahl der Nachkommen und der Lebensdauer ermittelt (Friedlander, 1996).

Im Folgenden sollen mögliche Wirkungsmechanismen diskutiert werden, welche einen Zusammenhang zwischen männlicher Fertilität und Lebenszeit-Mortalität bedingen könnten. Hier werden drei verschiedene Ansätze betrachtet werden, die den drei erklärenden Faktoren der Unterschiede in den Lebensspannen von Individuen entsprechen (vgl. 1.2.1.2). Erstens ist die Lebensdauer vererbbar. Herskind et al. (1996) fanden, dass bei Männern etwa $26 \%$ der Variation in den Lebensdauern den genetischen Dispositionen der Individuen zugeschrieben werden können. Zweitens ist ein Anteil durch die Bedingungen zu erklären, welche der menschliche Organismus in utero sowie in der frühen Kindheit erfährt. Den größten Einfluss auf die Lebensdauer haben jedoch drittens die Lebensumstände sowie das Verhalten im Erwachsenenalter (Vaupel et al., 1998; Vaupel et al., 2003).

\subsubsection{Lebensumstände und Verhalten im Erwachsenenalter: Subfertilität bzw. Kinder- losigkeit und Gesundheit}

Subfertilität bedeutet an sich zwar keine Einschränkung der physischen Gesundheit, dennoch kann sie einen großen Einfluss auf das psychische und soziale Wohlbefinden der betroffenen Paare haben (Forti \& Krausz, 1998), und die Diagnose der Infertilität stellt ein stressvolles Lebensereignis dar (Oberpenning et al., 2000). Es können sich Beeinflussungen in den Bereichen der Lebenszufriedenheit, Gesundheit, Partnerschaft und Sexualität sowie hinsichtlich der sozialen Unterstützung zeigen (Bengel et al., 2000).

Bezüglich der untersuchten Stichprobe ist nicht bekannt, ob die Männer nach ihrer Untersuchung noch Kinder zeugten. Ferner wurden in den Patientenakten nur für einen Teil der Fälle Angaben über die Existenz und die Anzahl eigener Kinder zum Untersuchungszeitpunkt festgehalten. Der Anteil der Väter an den betrachteten Infertilitätspatienten hätte demnach 13,6\% betragen. Das Vorhandensein eigener Kinder erlaubt die Unterscheidung primärer von sekundärer Infertilität und kann somit einen wichtigen Faktor in der Kinderwunschberatung darstellen. Es ist im Falle der Patienten mit fehlender Angabe daher eher davon auszugehen, dass die meisten von innen noch keine eigenen Kinder hatten und der Anteil Kinderloser somit unterschätzt wurde. Setzte man voraus, dass sämtliche Männer, für die keine Angaben vorlagen, während der Untersuchung noch keine Kinder hatten, so betrüge der Anteil der Väter sogar nur 7,5\%. 
Es wurde bereits darauf hingewiesen, dass die Subfertilitätsdiagnose aufgrund der Spermienkonzentration nicht zwangsläufig mit einer Kinderlosigkeit einhergehen muss, da die Konzeptionswahrscheinlichkeit vom Zusammenspiel verschiedener Ejakulatparameter sowie von den physiologischen Voraussetzungen der Partnerin abhängt (Behre et al., 2000b). Auch wenn über eine potentielle spätere Vaterschaft der Männer keine Informationen vorliegen, sollen doch einige Überlegungen dahingehend gemacht werden, ob und inwiefern sich die Subfertilität bzw. Kinderlosigkeit direkt und indirekt auf die Gesundheit und Mortalität der Männer ausgewirkt haben könnte. Es wird dabei angenommen, dass die Wahrscheinlichkeit, kinderlos zu bleiben, für die als subfertil bezeichneten Patienten höher war als für die Fertilen.

\subsubsection{Kinderlosigkeit und Partnerschaft}

Seit den 1970er Jahren wurde der positive Zusammenhang zwischen Ehe und Gesundheit bzw. Mortalität in vielfältigen Studien bestätigt. Zum einen lässt sich die geringere Sterblichkeit Verheirateter durch einen Selektionseffekt erklären: Gesunde haben eine größere Wahrscheinlichkeit, einen Partner zu finden und sich zu verheiraten. Zum anderen wird der Ehe ein protektiver Charakter zugeschrieben, von dem vor allem die Männer profitieren. Ihr Mortalitätsrisiko reduziert sich gleich nach der Hochzeit; ein Vorteil, der während des wieteren Lebensverlaufs bestehen bleibt. Die protektiven Wirkungen einer Ehe akkumulieren sich sogar über die Zeit (Brockmann \& Klein, 2002; 2004). Männer, die verheiratet sind, neigen weniger zu risikobereitem Verhalten (Backett \& Davison, 1995; Whitlock et al., 2004) und verhalten sich weniger gesundheitsgefährdend (Helmert \& Shea, 1998). Die Partnerin ist die wichtigste Vertraute, erhöht den Zugang zu sozialen Netzwerken und verstärkter sozialer Aktivität und vermehrt dadurch die Lebenszufriedenheit (Chipperfield \& Havens, 2001). Verheiratete sind im Alter hinsichtlich der aktiven Gesundheitspflege besser versorgt (Tomassini et al., 2001). Im Fall einer Trennung, Scheidung oder Verwitwung hingegen erhöht sich sofort das Sterblichkeitsrisiko (Brockmann \& Klein, 2004). Dieser Effekt macht sich noch Jahre später bemerkbar (Hemström, 1996; zitiert nach Brockmann \& Klein, 2004). Neueste Überlegungen befassen sich mit der These, dass die vom Ehestand abhängigen Unterschiede in der Gesundheit eher die Belastungen und den Stress einer Scheidung oder Verwitwung und weniger die positiven Ressourcen einer Ehe abbilden (Williams \& Umberson, 2004).

Bestätigt ist also, dass Verheiratete, und hier vor allem die Männer, ein geringeres Sterberisiko haben. Nun stellt sich die Frage, ob sich ungewollte Kinderlosigkeit nachteilig auf die Partnerschaft auswirken kann und somit das Risiko einer Trennung steigt. Die Infertilitätsdiagnose und der unerfüllte Kinderwunsch bedeuten für die betroffenen Paare zumeist einen 
wesentlichen Einschnitt in ihre Lebensplanung mit Auswirkungen auf die unterschiedlichsten Lebensbereiche (Henning \& Strauß, 2000) und ungewollte Kinderlosigkeit stellt ein die Partnerschaft belastendes Phänomen dar (Bengel et al., 2000). Eine Übersicht über verschiedene Studien zu den Auswirkungen der Infertilität auf die partnerschaftliche Beziehung bieten Oberpenning und Kollegen (2000).

Vordergründig vermitteln einige empirische Hinweise den Eindruck, als wirke sich die ungewollte Kinderlosigkeit nicht nachteilig auf die Partnerschaft aus. Eine Befragung von Patienten der so genannten „Heidelberger Kinderwunsch-Sprechstunde“ ergab für die ungewollt kinderlosen Paare keine größere partnerschaftliche Unzufriedenheit im Vergleich zu einer repräsentativen Stichprobe. Als negative Auswirkung der Diagnostik und Behandlung wird allerdings eine Verminderung der sexuellen Zufriedenheit angegeben (Wischmann, 2005). Diese Befunde bestätigen eine schwedische Untersuchung von Hjelmstedt und Kollegen (1999) an Paaren, welche sich einer in-vitro-Fertilisation (IVF) bzw. einer intrazytoplasmatischen Spermieninjektion (ICSI) unterzogen. Hier zeigte sich, dass sowohl die Männer als auch die Frauen angaben, ihre Beziehung habe sich durch die diagnostizierte Infertilität eher verbessert als verschlechtert, mehr als die Hälfte empfand keine Veränderung der Partnerschaft aufgrund der Infertilität. Als Grund für die Verbesserung der Beziehung wurde von beiden Geschlechtern eine größere empfundene Nähe mit stärkerer emotionaler Intimität angegeben. Eine Verschlechterung hingegen lag nach diesen Angaben auch hier wieder in sexuellen Problem und ehelichen Konflikten begründet. Die in beiden Studien gefundene sexuelle Unzufriedenheit kann auf den psychischen Druck, eine erfolgreiche Schwangerschaft zu induzieren, und auf die verstärkte Konzentration auf den ovulatorischen Zyklus zurückgeführt werden (Monga et al., 2004).

Es bleibt kritisch zu überlegen, ob Paare, die eine IVF-Behandlung in Anspruch nehmen, eventuell ihre Beziehung überbewerten. Auch könnten sie eine bereits selektierte Gruppe von Personen darstellen, welche die emotionalen und partnerschaftlichen Probleme, die eine Infertilitätsdiagnose mit sich bringen kann, schon überwunden haben (Hjelmstedt et al., 1999). Außerdem sollte bei der Untersuchung der Auswirkung einer Infertilitätsdiagnose auf die Partnerschaft nach der Ursache der Infertilität unterschieden werden. Liegt eine Infertilität auf männlicher Seite vor, so wird eine stärkere emotionale Belastung des Mannes angegeben (Beutel et al., 1999).

Schließlich bleibt die Frage, wie sich solche Partnerschaften langfristig entwickeln, denn zum Zeitpunkt einer Infertilitätsbehandlung besteht für das Paar noch die gemeinsame und sicherlich verbindende Hoffnung auf ein Kind. Langfristig gesehen ist dagegen anzunehmen, dass Kinderlosigkeit einen signifikant negativen Effekt auf die Partnerschaft hat (Bengel et al., 
2000). So wurde bei Paaren mit heterologer Inseminationsbehandlung im Jahre 1968 bei einer Untersuchung 16 Jahre später für diejenigen die höchste Trennungsrate gefunden, die weder durch die Behandlung selbst, noch durch eine Spontanschwangerschaft oder eine Adoption Kinder bekamen (35\% vs. 10\%; Goebel \& Lübke, 1987). Des Weiteren nimmt das Scheidungsrisiko mit jedem kinderlosen Jahr nach der Hochzeit stetig zu (Wineberg, 1988).

So ist davon auszugehen, dass die ungewollte Kinderlosigkeit langfristig eine Belastung für die Partnerschaft darstellt. Kehrt man zu der ursprünglichen Überlegung zurück, ob zwischen der Subfertilität und der Mortalität in der untersuchten Stichprobe tatsächlich ein Zusammenhang bestanden haben könnte, so könnte gemutmaßt werden, dass Männer, welche subfertil waren und damit wahrscheinlicher kinderlos blieben, eventuell einem höheren Scheidungsrisiko ausgesetzt waren als fertile Männer. Der positive Effekt einer Ehe wäre dann nicht mehr gegeben gewesen bzw. der negative Effekt einer Trennung hätte sich in einem erhöhten Sterberisiko zeigen können. Außerdem nimmt im Fall einer Scheidung häufig der Kontakt des Vaters zu bereits vorhandenen Kindern ab (Tomassini et al., 2004), so dass der negative Effekt der Trennung sich für Fälle mit sekundärer Subfertilität noch verstärkt hätte (siehe hierzu 4.4.1.3).

$\mathrm{Zu}$ beachten bleibt aber in diesem Zusammenhang, dass eine Scheidung nicht zwangsläufig negative Auswirkungen auf die Gesundheit haben muss, sondern dass hier die aktuelle Lebensphase, in welcher die Trennung stattfindet, von großer Bedeutung ist. Williams \& Umberson (2004) fanden, dass sich die subjektiv eingeschätzte Gesundheit von Männern im jungen und mittleren Erwachsenenalter nach einer Scheidung verbesserte. Die Autoren vermuten, dass die Trennung die Beendigung chronischer Stresssituationen bedeuten kann und sich somit ein verbesserter Gesundheitszustand ergibt. Die negativen Auswirkungen einer Scheidung scheinen mit steigendem Alter zuzunehmen. Sollte das Scheidungsrisiko der Subfertilen tatsächlich erhöht gewesen sein, so ist dennoch der Effekt der Trennung auf das Mortalitätsrisiko offen. Es bleibt zu vermuten, dass ein großer Teil der Trennungen, sollten sie letztendlich auch die Subfertilität zur Ursache gehabt haben, vermutlich nicht erst im späten Erwachsenenalter eintrat.

\subsubsection{Kinderlosigkeit und psychisches Wohlbefinden}

Für die meisten Männer ist der Gedanke, Kinder zu haben, von Bedeutung, auch wenn die Unfähigkeit, dieses Ziel zu erreichen, unterschiedliche Reaktionen zu verschiedenen Zeitpunkten in ihrem Leben hervorruft (Rieker et al., 1990). Für Männer aus ungewollt kinderlosen Partnerschaften wird Infertilität vor allem im Hinblick auf die Erfüllung des männlichen Rollenbildes und auf den sozialen Druck, Kinder zu bekommen, als Problem bewertet (Hjelmstedt et al., 1999). Man könnte vermuten, dass sich die Tatsache, ungewollt kinderlos 
geblieben zu sein, negativ auf das psychische Wohlbefinden im Alter auswirkt. Jedoch weisen verschiedene Studien in eine gegenteilige Richtung.

In einer Untersuchung an 50- bis 84-Jährigen wurde das psychische Wohlbefinden von Kinderlosen und Eltern untersucht. Es wurden die Parameter „Einsamkeit“ - als Indikator für empfundene soziale Isolation sowie „Depression“ als Indikator für psychischen Stress ermittelt. Bei kinderlosen Männern waren zwar beide Parameter erhöht, dieser Effekt verschwand allerdings bei Kontrolle des Ehestands (Koropeckyi-Cox, 1998). Dieser Befund wurde in einer weiteren Studie repliziert. Zhang \& Hayward (2001) untersuchten kinderlos gebliebene Personen ab 70 Jahren und betrachteten die gleichen Indikatoren wie in der zuvor erwähnten Arbeit. Auch sie fanden, dass Kinderlosigkeit an sich das psychische Wohlbefinden nicht zwangsläufig beeinträchtigt. Die Kinderlosen fühlten sich insgesamt betrachtet nicht einsamer oder deprimierter als die befragten Eltern. Sie fanden jedoch einen deutlichen Unterschied zwischen Frauen und Männern, und auch die Ehe spielte eine bedeutende Rolle. Bei Männern war der Zusammenhang zwischen Einsamkeit und Kinderlosigkeit ausgeprägter als bei Frauen; geschiedene und verwitwete Männer ohne Kinder hatten das größte Risiko der Einsamkeit und der Depression im Vergleich zu Männern in anderen familiären Situationen bzw. im Vergleich zu Frauen. Die Autoren schätzen die Tatsache, verheiratet zu sein, als wichtigeren Einflussfaktor auf das psychische Wohlbefinden ein als die Tatsache, Kinder zu haben.

Wie sehr der kulturelle Hintergrund bei dieser Thematik eine Rolle spielt, verdeutlicht die Untersuchung an älteren Chinesen in Hongkong (Chou \& Chi, 2004). Hier wurde ein starker Einfluss der Kinderlosigkeit auf das psychische Wohlbefinden vor allem bei Männern gefunden, unabhängig vom Ehestand. Dies verdeutlicht die Bedeutung von Kindern in dieser Kultur, auch wenn die Unterstützung durch die Ehepartnerin gegeben ist.

Zu den beschriebenen Studien sei angemerkt, dass sie nicht in der Lage waren, zwischen gewollt und ungewollt Kinderlosen unterscheiden zu können. So könnte das psychische Wohlbefinden der gewollt Kinderlosen nicht belastet sein, während sich die ungewollt Kinderlosen in ihrem Wohlbefinden durch die Kinderlosigkeit beeinträchtigt fühlten. Daher betrachtete Koropeckyi-Cox (2002) Kinderlose, wiederum im Alter von 50 bis 84 Jahren, und unterschied, ob ihre Kinderlosigkeit mit ihrer Einstellung zu eigenen Kindern übereinstimmte: Die Aussage „Es ist ok, kinderlos zu sein“ diente als Indikator für eine positive Einstellung zur Kinderlosigkeit, während „Es ist besser, ein Kind zu haben“ als negative Einstellung zur eigenen Kinderlosigkeit betrachtet wurde. Die Autorin fand keinen Unterschied zwischen diesen beiden Gruppen hinsichtlich des subjektiven psychischen Wohlbefindens, ausgedrückt durch die bereits beschriebenen Parameter Einsamkeit und Depression. Weiterhin 
wurden in einer gemeinsamen Studie der Universitäten Jena und Freiburg Eltern und ungewollt Kinderlose im Alter zwischen 43 und 65 Jahren untersucht. Die Analyse ermittelte, dass sich ungewollt kinderlose Paare als genauso glücklich empfinden wie Eltern (Strauß et al., 2004). Auch erscheinen männliche Kinderwunschpatienten generell psychopathologisch unauffällig (Wischmann, 2005).

Sollte tatsächlich, wie die Ergebnisse der vorliegenden Studie vermuten lassen, ein Zusammenhang zwischen der Subfertilität und der Mortalität bestehen, so scheint sich diese Beziehung nicht durch das psychische Wohlbefinden der betroffenen Männer erklären zu lassen. Die vorgestellten Befunde, das psychische Wohlbefinden im Alter betreffend, könnten jedoch lediglich ein Zeichen dafür sein, dass die Männer im Laufe ihres Lebens Strategien zur Adaptation an die Kinderlosigkeit entwickelten und lernten, die Situation hinzunehmen. Dennoch bleibt der Einfluss der Kinderlosigkeit auf die allgemeine Gesundheit bzw. auf die Mortalität zu klären.

\subsubsection{Kinderlosigkeit und Lebenszeit-Mortalität}

Auch wenn es nach Betrachtung der Arbeiten zum psychischen Wohlbefinden Kinderloser so erscheint, als ob Kinder nicht unbedingt zwangsläufig zum Lebensglück dazugehören, könnten sich Infertilität bzw. Kinderlosigkeit als kurzzeitiger bzw. chronischer Stressfaktor erweisen. Da sich Stresserfahrungen akkumulieren und in neuroendokrinen und physiologischen Antworten resultieren, scheinen sie biologische Auswirkungen auf die Entwicklung degenerativer Erkrankungen zu haben. So kann z.B. die Anfälligkeit für koronare Erkrankungen und Krankheiten aufgrund reduzierter Immunfunktionen durch Stress erhöht werden (Brunner ,1997).

Während bereits ausgiebig erforscht wurde, welchen Einfluss der Ehestand auf die Morbidität und Mortalität von Männern hat, liegen für den Einfluss von Vaterschaft bzw. Kinderlosigkeit bislang äußerst wenige Ergebnisse vor, die zudem inkongruent sind. Kotler \& Wingard (1989) konnten weder einen Einfluss der Zahl der Kinder noch einen Einfluss der Anwesenheit eines Kindes im Haushalt auf das männliche Mortalitätsrisiko feststellen. Des Weiteren wurde für deutsche Kinderlose kein wesentlicher Unterschied hinsichtlich des gesundheitlichen Zustands gefunden: Ungewollt Kinderlose waren genau so krank und so gesund wie Eltern, und zeigten die gleichen psychosomatischen Störungen, depressiven Verstimmungen und Erkrankungen (Strauß et al., 2004). Clark et al. (1987) hingegen fanden, dass Männer über 40 - unabhängig davon, ob sie verheiratet oder allein stehend waren ihren Gesundheitszustand besser einschätzten, wenn sie Kinder hatten als kinderlose Männer (zitiert nach Ringbäck Weitoft et al., 2004). 
In einer aktuellen schwedischen Studie unterschieden Ringbäck Weitoft und Kollegen (2004) fast 700.000 Männer danach, ob sie kinderlos waren oder nicht, ob sie im Falle einer Vaterschaft das Sorgerecht hatten und ob sie mit der Mutter / einer Partnerin zusammenlebten. Im Falle einer Vaterschaft handelte es sich um Kinder unter 18 Jahren. Es wurde speziell das Auftreten vorzeitiger Todesfälle analysiert. Das Ergebnis zeigte, dass allein lebende Väter ohne Sorgerecht und kinderlose, allein lebende Männer ein erhöhtes Sterberisiko hatten. Ursachen sind hier vor allem Unfälle und Suchtmittelabhängigkeit, aber auch die Zahl der Todesfälle aufgrund von ischämischen Herzkrankheiten war erhöht. Wurden - unter Berücksichtigung des SES - diese beiden Gruppen mit fürsorgeberechtigten Vätern, die mit der Mutter ihrer Kinder zusammen lebten, verglichen, so fand man bei ihnen 4- bis 5-mal häufiger Todesfälle aufgrund einer Suchtmittelabhängigkeit. Tödliche Herzerkrankungen traten 1,7- bis 2-mal so oft auf. Das Risiko für Verletzungen und Vergiftungen mit Todesfolgen war vierfach erhöht und Suizide fanden mehr als doppelt so häufig statt. Leichte Effekte wurden auch für allein lebende Väter mit Sorgerecht gefunden. Die Autoren schließen aus ihren Befunden, dass das Zusammenleben mit den eigenen Kindern mindestens genau so wichtig für die Gesundheit ist wie das Zusammenleben mit einer Partnerin. Jedoch sollten potentielle Selektionseffekte nicht vergessen werden: Männer mit psychischen Problemen oder Abhängige bekommen von vornherein seltener das Sorgerecht für Kinder zugesprochen; sie bleiben mit größerer Wahrscheinlichkeit allein stehend und kinderlos. Auch wenn diese Effekte vermutlich stärker sind, scheinen Kinder im Haushalt trotzdem einen protektiven Effekt zu bedeuten (Ringbäck Weitoft et al., 2004).

Als wesentlich für die niedrigere Mortalität der Väter, welche mit ihren Kindern zusammenleben, erscheinen das Gesundheitsverhalten in weiterem Sinne zum einen und soziale Unterstützung zum anderen. Kinder geben dem Leben eine Struktur, sie bedeuten Lebensinhalt und Gesellschaft und auch Zugang zu anderen Erwachsenen wie Nachbarn, Verwandten und Freunden (Riessman \& Gerstel, 1985).

Inwiefern Männer gesundheitsbewusstes oder risikobereites Verhalten (risk taking) zeigen, ist u.a. davon abhängig, ob sie verheiratet sind bzw. ob sie Kinder haben. Risikobereitschaft kann im Sinne von kompetitivem Verhalten bei der Partnersuche interpretiert werden. Ist einmal eine Partnerin gefunden und sind eventuell Kinder vorhanden, so wird das riskante Verhalten eingestellt (Hill \& Chow, 2002). Die Autoren verdeutlichten dies am Beispiel des Alkoholmissbrauchs. Ehe und Kinder wirkten sich auf dieses riskante Verhalten positiv, als Reduzierung des Alkoholmissbrauchs, aus.

Storey et al. (2000) untersuchten die Hormonkonzentrationen von Vätern kurz vor bzw. kurz nach der Geburt ihres Kindes. Sie stellten fest, dass die Väter ähnliche Hormon- 
veränderungen erfuhren wie ihre Partnerinnen. Überdies lag die Testosteron-Konzentration der Väter nach der Geburt um 33\% niedriger als jene der Väter vor der Geburt. Diese Reduktion der Testosteron-Werte nach der Geburt scheint die „Vatergefühle“ (paternal responsiveness) zu verstärken und die Neigung zu Verhaltensweisen, welche mit der KinderFürsorge nicht kompatibel sind, zu vermindern. Allgemein wird angenommen, dass die Hormonveränderungen eine Rolle dabei spielen, den Vater auf die Versorgung und Pflege seiner Kinder zu prägen. Gray und Kollegen (2002) konnten diese Befunde nicht nur bestätigen, sondern unterschieden auch noch nach dem Ehestand. Verheiratete Männer mit und ohne Kinder wiesen niedrigere Testosteron-Konzentrationen auf als unverheiratete Männer. Diese Unterschiede ließen sich nur für die Konzentrationen am Nachmittag finden, vormittags unterschieden sich die Testosteron-Level nicht voneinander. Auch hier wird vermutet, dass die niedrigen Testosteron-Werte am Tage das väterliche Investment sichern, in dem Neigungen zu riskantem und kompetitivem Verhalten reduziert werden. Interessant ist in diesem Zusammenhang auch der Befund, dass hohe Testosteron-Level mit einem verminderten Immunschutz einhergehen und so diese Männer anfälliger für Infektionskrankheiten macht (Campbell et al., 2001). Zusätzlich sei an die in der Einleitung erwähnten negativen Auswirkungen der männlichen Sexualhormone auf Stoffwechselprozesse und kardiovaskuläre Konditionen erinnert (vgl. 1.2.1.3). Die reduzierten Testosteron-Werte der Väter wirken sich demnach also auch positiv auf die Gesundheit aus.

Der Übergang zur Elternschaft ist zwangsläufig mit Verhaltensänderungen verbunden. Eltern werden sich ihrer Gesundheit bewusster und versuchen, ein gesünderes Leben zu führen (Backett \& Davison, 1995). Die Untersuchung norwegischer krebskranker Männer zeigte bessere Prognosen für Väter als für kinderlose Fälle. Dieser Effekt blieb bei Kontrolle des Ehestandes bestehen. Bei männlichen Krebspatienten mit Kindern war die Mortalität um ein Drittel niedriger als bei unverheirateten und kinderlosen Männern. Dies wir dahingehend interpretiert, dass Kinder einen gesünderen Lebensstil induzieren bzw. dazu veranlassen, den „Kampf mit dem Krebs“ eher auf sich zu nehmen (Kravdal, 2003). Umberson (1987; zitiert nach Ringbäck Weitoft et al., 2004) fand, dass Elternschaft die Neigung zu negativem Gesundheitsverhalten dann stärker vermindert, wenn Eltern und Kinder zusammen leben. Geschiedene Männer, die von ihren Kindern getrennt lebten, zeigten mehr gesundheitsschädigendes Verhalten als Geschiedene, die gemeinsam mit ihren Kindern lebten.

Kinder veranlassen Eltern jedoch nicht nur zu einem weniger riskanten, gesünderen Lebensstil, sie sind auch hinsichtlich der sozialen Netzwerke und Unterstützung von Bedeutung. Die Alameda County-Studie war eine der ersten Untersuchungen, welche den Einfluss von Sozialkontakten auf die Sterblichkeit hervorhob. Personen mit wenigen sozialen Bindungen hatten eine zwei- bis dreifach höhere Mortalität als solche mit häufigen sozialen 
Interaktionen (Berkman \& Syme, 1979; zitiert nach Mineau et al., 2004). Baumann et al. (1998) konnten bestätigen, dass eine geringe soziale Integration das Mortalitätsrisiko erhöht. Dieser Zusammenhang wird durch die geringeren informellen Hilfeleistungen, die geringen positiven Änderungen des Gesundheitsverhaltens, das geringere Wissen hinsichtlich formeller Gesundheitsversorgung sowie schlechterer Compliance bei der MedikamentenEinnahme erklärt. Soziale Netzwerke bieten Unterstützung und vermindern das Sterberisiko (Mineau et al., 2004).

Ein unerfüllter Kinderwunsch kann sich so auf mehreren Ebenen auswirken. Infertile ziehen sich nach der Diagnose häufig zunächst zurück, meiden Freunde und Verwandte, die bereits Kinder haben und reduzieren ihre Sozialkontakte (Watkins \& Baldo, 2004). Vor allem Männer isolieren sich häufig im Umgang mit ihrer Subfertilität. Sie vertrauen sich seltener Freunden oder Familienangehörigen an und ein großer Teil gibt sogar an, keinerlei der Unterstützung zu haben (Hjelmstedt et al., 1999). Generell verfügen die meisten Kinderlosen über unterstützende Netzwerke in Form von Verwandten, Freunden und Nachbarn (Zhang \& Hayward, 2001), diese Netze sind aber weniger umfangreich und Kinderlose im Alter weniger umfassend versorgt als Eltern. Dies betrifft insbesondere die emotionale Unterstützung und Nähe. Hilfeleistungen von anderen Familienmitgliedern oder Freunden können die Unterstützung durch eigene Kinder nicht ersetzen, da diese eher die aktive Versorgung und Pflege übernehmen (Bengel et al., 2000).

Zur ursprünglichen Frage nach einem möglichen Zusammenhang zwischen Fertilität und Mortalität zurückkehrend, könnte folgende Vermutung angestellt werden: Sollten die subfertilen Fälle der betrachteten Stichprobe tatsächlich häufiger kinderlos geblieben sein, so hätte sich dies negativ auf ihren Lebensstil und die zur Verfügung stehenden Sozialkontakte und somit auch indirekt auf ihren Gesundheitszustand und die spätere Mortalität auswirken können. Die Überlegungen bleiben freilich spekulativ, da die spätere Zeugung von Kindern für die untersuchten Männer nicht belegt ist. Auch müsste freilich geklärt werden, wie eng der Kontakt zwischen Vater und möglichen späteren Kindern tatsächlich war. Zusätzlich wäre im Zusammenhang mit den Überlegungen zum Einfluss von Kindern auf die Gesundheit bzw. Mortalität von Interesse, ob die Männer, z.B. im Falle einer zweiten Ehe oder Adoption, mit Kindern zusammenlebten. Zhang \& Hayward (2001) kamen zu dem interessanten Ergebnis, dass sich Stiefeltern in ihrem psychischen Wohlbefinden nicht von biologischen Eltern unterscheiden. Somit scheint also nicht die Tatsache, ein Kind gezeugt zu haben, von Bedeutung zu sein, sondern es wird wieder die Relevanz der emotionalen und sozialen Unterstützung durch die Nachkommen belegt. 
Sollte sich die höhere Sterblichkeit der Subfertilen wirklich durch die Kinderlosigkeit und deren beschriebene potentielle Folgen erklären lassen, so bleibt zum Schluss die Frage offen, warum bei einer Unterscheidung der Subfertilen in azoosperme und oligozoosperme Fälle nur die Mortalität der Oligozoospermen und nicht die der Azoospermen erhöht scheint. Azoosperme blieben mit größerer Wahrscheinlichkeit kinderlos als oligozoosperme Männer, so dass die Effekte der Kinderlosigkeit auf das Sterberisiko und die Lebensdauer in dieser Subgruppe am deutlichsten hätten hervortreten sollen. Dies würde letztlich dagegen sprechen, dass dieser Erklärungsansatz für den analysierten Zusammenhang zwischen Fertilitätsstatus und Lebenszeit-Mortalität verantwortlich gemacht werden kann. Dennoch sollten die Ergebnisse hinsichtlich der Mortalität der Azoospermen unter Vorbehalt betrachtet werden, da hier die Fallzahl relativ gering war und zu klären bleibt, ob sich bei einer größeren Stichprobe die nicht doch gleichen Effekte zeigen lassen wie bei den oligozoospermen Männern.

\subsubsection{Konditionen in utero und in der frühen Kindheit}

Ein zweiter Erklärungsansatz, welcher die höhere Sterblichkeit der Subfertilen begründen könnte, befasst sich nun mit biologischen Ursachen. Hier wird dem Einfluss von schädlichen Faktoren während der fötalen Entwicklungsphase und im frühkindlichen Alter und dessen späterer Auswirkung im Erwachsenenalter eine große Bedeutung beigemessen (Christensen \& Vaupel, 1996; Lummaa, 2003).

In diesem Zusammenhang kann für Infektionskrankheiten ein deutlicher Einfluss auf die Mortalität postuliert werden. So geht man davon aus, dass Helicobacter-pylori-Infektionen in der Kindheit, bedingt durch die schlechten Lebensumstände während des 2 . Weltkrieges, für die heutigen hohen Magenkrebs-Raten in Japan verantwortlich zu machen sind. Für Schweden lässt sich wiederum zeigen, dass die Mortalität von Erwachsenen während des 18. und 19. Jahrhunderts stark durch Pocken- und Keuchhusten-Infektionen in der Kindheit beeinflusst war. Auch Tuberkulose-Erkrankungen im Erwachsenenalter konnten Infektionen im Kindes-alter zugeschrieben werden (Zusammenstellung in Doblhammer, 2004).

Auch hinsichtlich der heute vorherrschenden chronischen Erkrankungen finden sich Hinweise, dass Morbidität und Mortalität im Erwachsenenalter stark von den Konditionen in der Frühphase der Entwicklung abhängen. Die fetal-origins-Hypothese postuliert, dass eine fötale Unterernährung in der Mitte bis zum Ende der Schwangerschaft zu disproportionalem Wachstum und zu einem geringen Geburtsgewicht führt und so z.B. spätere kardiovaskuläre Erkrankungen programmiert (Barker, 1995). Mangelernährung in utero und ein niedriges Geburtsgewicht bzw. disproportionales Wachstum sind mit verschiedenen Risikofaktoren und Erkrankungen wie Glukose-Intoleranz mit anschließender Diabetes-Erkrankung (Phillips, 
1998), Bluthochdruck (Law \& Shiell, 1996) und vaskulären Erkrankungen (Eriksson et al., 2001) assoziiert. Auch die immunitären (McDade et al, 2001a; b) und respiratorischen Funktionen (Lopuhäa et al., 2000; Shaheen et al., 1999) können betroffen sein. Es wurde sogar eine Verbindung zwischen frühem Wachstum und dem Suizid-Risiko ermittelt (Barker et al., 1995).

Ab der 9. Schwangerschaftswoche beginnt für den Fötus eine Phase schnellen Wachstums, hauptsächlich durch Zellteilung, welche bis zur Geburt anhält. Die verschiedenen Gewebe erfahren unterschiedliche Wachstumsperioden, so genannte „kritische“ Phasen. Der Fötus reagiert auf Nährstoff- und Sauerstoff-Mangel oder anormale hormonelle Konditionen mit einer verlangsamten Zellteilung. Dies betrifft vor allem die Gewebe, die gerade die „kritische“ Phase durchlaufen. Der Organismus „erinnert“ diese Mangelphasen: es kann zu Veränderungen in der Anzahl der Zellen, der Verteilung der Zelltypen, in der HormonSekretion, in metabolischen Prozessen und in Organ-Strukturen kommen (Barker, 1995; Lummaa, 2003). Die Anpassungen des Fötus an die Mangelsituation können sich infolgedessen manifestieren und es kommt zu permanenten Veränderungen der Strukturen, der Physiologie und der Körperfunktionen (Lummaa, 2003).

Nicht nur die Mortalität der Individuen kann von diesen in utero wirksamen Effekten betroffen sein, die beschriebenen Wirkungen können ebenso zu Konsequenzen hinsichtlich des Reproduktionserfolgs führen. So kann zum einen die Fertilität direkt negativ beeinflusst sein (siehe unten). Zum anderen kann die Dauer der reproduktiven Phase, z.B. durch verfrühten Tod, verkürzt (Lummaa, 2003) oder die Chance, einen Partner zu finden, vermindert sein (Übersicht in Buss, 2004). Dies wird durch eine Studie an finnischen und englischen Männern, welche in den 1920er und 1930er Jahren geboren wurden, bestätigt. Hier konnte nachgewiesen werden, dass Männer mit einem niedrigen Geburtsgewicht eine geringere Wahrscheinlichkeit hatten, eine Ehe einzugehen (Phillips et al., 2001).

Die fetal-origins-Hypothese könnte nun die höhere Sterblichkeit der Subfertilen erklären, denn sie lässt nicht nur den Schluss zu, dass sich intrauterine Konditionen auf die Mortalität auswirken, indem die Risiken für bestimmte Erkrankungen erhöht sind, sondern sie erlaubt auch die Überlegung, dass die gleichen Effekte, welche die Mortalität erhöhen, sich direkt auf die Fertilität ausgewirkt haben könnten.

So könnte die Annahme gemacht werden, dass die Störungen der Spermatogenese im Erwachsenenalter den Einflüssen entspringen, die bereits während der fötalen Entwicklung auf den Organismus wirken. Einen möglichen Wirkungsmechanismus könnte beispielsweise die anormale Entwicklung oder Proliferation der Sertoli-Zellen darstellen. Sertoli-Zellen erfüllen zwei unterschiedliche Rollen, die funktional wie auch zeitlich voneinander getrennt 
sind (Sharpe et al., 2003). Die fötalen Sertoli-Zellen sind essentiell für die funktionale Entwicklung des Hodens und die Ausprägung des männlichen Phänotyps. Ohne die SertoliZellen ist darüber hinaus eine Spermatogenese ab dem Pubertätsalter nicht möglich, da sie die Entwicklung der Keimzellen, die Meiose und die anschließende Transformation in Spermatozoen physikalisch wie metabolisch unterstützen. Außerdem wird durch die Anzahl der Sertoli-Zellen auch die Anzahl der Keimzellen, die bei der Spermatogenese unterstützt werden kann, bestimmt. Schließlich verfügen im Hoden nur die Sertoli-Zellen über einen Rezeptor für das Follikel-stimulierende Hormon (FSH; vgl. 1.2.2.1.4; Steger, 2001; Weinbauer et al., 2000). Somit ist die Fertilität direkt von diesen Zellen abhängig.

Während der Pubertät kommt es - vermutlich schrittweise - zu einem radikalen Wandel der „fötalen“ zu den „adulten“ Sertoli-Zellen. Sie verlieren z.B. ihre Fähigkeit zur Proliferation und bilden Verbindungen (tight junctions) miteinander aus. Dies ist die Voraussetzung für die Bildung des adluminalen Raums, in welchem die meiotischen und postmeiotischen Prozesse - metabolisch versorgt durch die Sertoli-Zellen - stattfinden können (Steger, 2001). Obwohl diese Reifung der Sertoli-Zellen zeitlich getrennt ist von ihrer fötalen Entwicklung und Funktion, so werden doch Einflüsse während der fötalen Phase unvermeidbar die Funktion und die Funktionsfähigkeit der Sertoli-Zellen im Erwachsenenalter vorherbestimmen (Sharpe et al., 2003). So könnten sich Einflüsse auf den Organismus in utero im Erwachsenenalter bemerkbar machen, z.B. durch eine reduzierte Spermienkonzentration und dadurch verminderte Fertilität. Bekannt sind die negativen Auswirkungen des Zigarettenkonsums der Mutter während der Schwangerschaft auf die spätere Spermiendichte (Storgaard et al., 2003) oder der Effekt von Phtalaten (Fisher et al., 2003).

Kritisch bleibt sicherlich die Tatsache zu diskutieren, dass anhand von Studien zur Mortalität von Zwillingen, welche sicherlich eine Wachstumsverminderung in utero erfahren, die fetal-origins-Hypothese nicht bestätigt werden konnte. Dänische Zwillinge unterscheiden sich nicht in ihrer Mortalität von der dänischen Gesamtbevölkerung. Zusätzlich konnte auch kein Unterschied in der Mortalität von monozygoten und dizygoten Zwillingen ermittelt werden (Christensen et al., 1995). Somit bleibt die Überlegung, dass sich Konditionen in utero negativ auf die spätere Fertilität sowie den Gesundheitszustand insgesamt auswirken, zwar plausibel, jedoch noch nicht endgültig bestätigt.

Doch selbst wenn man davon ausgeht, dass die fetal-origins-Hypothese auch auf die Reproduktionsphysiologie übertragbar ist, können hinsichtlich der Bedeutung dieses biologischen Erklärungsansatzes für die in der vorliegenden Studie ermittelten Befunde lediglich Vermutungen angestellt werden. Zum einen gibt es generell sehr wenige Studien, welche speziell die in utero-Bedingungen als Risikofaktor für die spätere männliche Fertilität 
untersucht haben (Lummaa, 2003), so dass dieser Zusammenhang im Bereich der Spekulation verbleiben muss. Zum anderen stellt sich die Frage, wie groß der Anteil derjenigen Männer in der Allgemeinbevölkerung ist, der tatsächlich in utero Mangelbedingungen ausgesetzt war. Entsprechend muss dies für die untersuchte Stichprobe ebenfalls offen bleiben. Zusätzlich sollte bedacht werden, dass spätere Einflüsse wie Lebensstil und SES die Auswirkungen der fötalen Konditionen modifizieren können. Somit kann allerdings auch negatives Gesundheitsverhalten im späteren Erwachsenenalter die intrauterinen Effekte verstärken (Barker, 1995). Der SES der betrachteten Stichprobe konnte zwar für einen Teil der ehemaligen Patienten ermittelt und in den Analysen berücksichtigt werden, über Lebensstil-Variablen wie Zigaretten- und Alkoholkonsum finden sich in den vorliegenden Original-Daten allerdings kaum Angaben, so dass hier keine weiteren Aussagen gemacht werden können.

Ein zusätzlicher Aspekt sollte noch Erwähnung finden. Wie schon bei dem zuvor diskutierten Erklärungsansatz zum Einfluss der Konditionen im Erwachsenenalter ist auch hier wieder zu fragen, warum sich die Sterblichkeit der Oligozoospermen erhöht zeigt, die Azoospermen sich aber in ihrer Mortalität nicht von den Normozoospermen unterscheiden. Sollte die These stimmen, dass schlechte in utero-Bedingungen sich gleichzeitig negativ auf die Gesundheit und auf die Mortalität auswirken, so würde dies in der eigenen Stichprobe nur für die Oligozoospermen zugetroffen haben. Für die Azoospermen müsste also vermutet werden, dass die Ursache ihrer Azoospermie eher in anderen Ursachen begründet liegt. Denkbar wären beispielsweise genetische Dispositionen oder sekundär erworbene Ätiologien wie Verletzungen oder sexuell übertragbare Krankheiten. Tatsächlich ermittelten Fedder und Crüger (2004) in ihrer Untersuchung zur Ätiologie von Azoospermie einen größeren Anteil an Fällen mit erworbener Azoospermie (19\% angeboren vs. 27\% erworben). In der eigenen Studie wurden allerdings im Vorfeld solche Fälle mit bekannten Diagnosen, die für eine sekundär erworbene Subfertilität sprachen, ausgeschlossen, so dass auch hier - wie bereits im Abschnitt 4.2.2 - wieder nur an nicht dokumentierte bzw. nicht erkannte Ursachen zu denken wäre. Jedoch erscheint es unwahrscheinlich, dass dies für einen großen Teil der Azoospermen zutreffen soll. Des Weiteren sei auch hier wieder auf die Bedenken hinsichtlich der Aussagefähigkeit der Ergebnisse aufgrund der geringeren Azoospermie-Fallzahlen hingewiesen.

\subsubsection{Genetische Ursachen}

Der letzte zu diskutierende Erklärungsansatz befasst sich mit genetischen Dispositionen. Die Frage lautet hier, ob es denkbar ist, dass Subfertilität und eine reduzierte Lebensdauer durch genetische Ursachen miteinander verknüpft sind. Freilich kann auf Basis der in den 
Krankenakten vorliegenden Informationen keine Aussage über genetische Dispositionen und Erkrankungen der Patienten gemacht werden. Trotzdem können an dieser Stelle einige Überlegungen angestellt werden.

Im einleitenden Kapitel wurden bereits einige genetische Ursachen für männliche Fertilitätsstörungen kurz vorgestellt (vgl. 1.2.3.2.6). Hier sollen jetzt jene Erkrankungen Berücksichtigung finden, für welche neben einer Beeinträchtigung der Fertilität auch ein nachteiliger Effekt auf die übrige körperliche und geistige Gesundheit berichtet wird. Das Spektrum der möglichen Abberationen ist vielfältig. Zum einen können numerische gonosomale Abweichungen vorliegen. Das Klinefelter-Syndrom zeichnet sich durch einen 47,XXY-Karyotyp oder auch Mosaik-Formen (z.B. 47,XXY/46,XY) aus. Auch kann es zu höhergradigen X- oder Y-Aneuploidien kommen. Die Prävalenz in der männlichen Bevölkerung liegt bei 0,2\% (Nieschlag et al., 2000a; Shah et al., 2003). Es wird geschätzt, dass etwa die Hälfte der Betroffenen undiagnositiziert und unbehandelt bleibt (Abramsky \& Chapple, 1997; zitiert nach Nieschlag et al., 2000a). Die Betroffenen leiden unter Hypotestosteronämien unterschiedlichen Ausmaßes mit den daraus folgenden Merkmalen eines eunuchoidalen Körperbaus, Gynäkomastie, sehr kleinen Testes und einer verminderten Libido, vor allem aber ist bei innen der Prozess der Meiose gestört, was zu ernsten Beeinträchtigungen der Spermatogenese führen kann (Amory et al., 2000). Bis auf wenige Ausnahmen sind Klinefelter-Patienten azoosperm (Knuth \& Schulze, 2001). Für die Betroffenen kann das Risiko für Leukämie, Keimzellentumore, Diabetes mellitus, Lungenkrebs und - im Falle einer Gynäkomastie - Brustkrebs vergrößert sein (Amory et al, 2000; Brugh III et al., 2003; Swerdlow et al., 2005). Das Mortalitätsrisiko ist insgesamt um 40\% erhöht (Bojesen et al., 2004).

Patienten mit einer numerischen autosomalen Abberation hingegen werden normalerweise nicht in einer Infertilitätsberatung vorstellig. Schwere autosomale Störungen sind beispielsweise Trisomie 13, Trisomie 18 und Trisomie 21; und nur Patienten mit Trisomie 21 überleben überhaupt bis zum reproduktiven Alter (Brugh III et al., 2003). Bei diesen männlichen Betroffenen ist die Fertilität tatsächlich beeinträchtigt, wobei der Mechanismus noch ungeklärt bleibt. Es wird eine verringerte Proliferation der primordialen Stammzellen vermutet (Shah et al., 2003).

Das Kallman-Syndrom indes stellt eine strukturelle, meist autosomal dominant vererbte Erkrankung dar (Waldstreicher et al., 1996). Die Betroffenen zeigen die gleichen Symptome wie beim hypogonadotropen Hypogonadismus, also eine Störung der $\mathrm{GnRH}$-Sekretion des Hypothalamus (Gonadotropin Releasing Hormon; vgl. 1.2.2.1.4). Sowohl die Reifung der Keimzellen als auch eine ausreichende gonadale Testosteron-Produktion sind verhindert, 
wodurch Azoospermie oder Aspermie resultiert (Behre \& Nieschlag, 1998; Behre et al., 2000b). Sie weisen sich jedoch zusätzlich durch eine Anosmie aus; ihr Riechvermögen hinsichtlich aromatischer Verbindungen ist beeinträchtigt, was durch gestörte Zellwanderungen während der Embryonalentwicklung zu erklären ist. Für den idiopathischen hypogonadotropen Hypogonadismus und das Kallman-Syndrom wird eine Prävalenz von 1:10.000 für das männliche Geschlecht geschätzt (Behre et al., 2000b). Weitere Folgen des genetischen Defektes können Verformungen und Aplasien der Niere sein (Bhasin et al., 2000; Brugh III et al., 2003; Meschede \& Horst, 2000).

Eine weitere strukturelle autosomale Anomalie stellt die Mutation des Cystischen-FibroseTansmembran-Regulator-Gens (CFTR-Gen) des Chromosoms 7 dar, welche zur Erkrankung an Cystischer Fibrose führen kann. Genetische Analysen zeigen, dass die kongenitale beidseitige Aplasie des Vas deferens (CBAVD) in engem Zusammenhang mit den CFTR-GenMutationen steht (Meschede et al., 1998), so dass man davon ausgehen kann, dass es sich bei der CBAVD um eine genitale Form der Cystischen Fibrose handelt (Stuhrmann, 1998). Der durch Azoospermie bedingten Infertilität bei Männern mit CBAVD liegen die Obstruktionen des männlichen Genitaltraktes zu Grunde, und die Spermatogenese selbst kann trotz CFTR-Gen-Mutationen ohne Störungen verlaufen (Pallares-Ruiz et al., 1999). Cystische Fibrose selbst manifestiert sich als chronische obstruktive Lungenerkrankung mit wieder kehrenden Infektionen und pankreatischer Insuffizienz. Die mediane Lebenserwartung liegt heutzutage bei 40 Jahren und hat sich somit im Laufe der letzten zwei Dekaden verdoppelt (Jaffe \& Bush, 2001).

Zuletzt seien noch einige seltene genetische Erkrankungen erwähnt, welche sich nachteilig auf Fertilität und Gesundheit auswirken. Das Noonan-Syndrom führt nicht nur zu verspäteter oder unvollständiger Pubertätsentwicklung, zu Maldeszensus der Hoden bzw. zu kleinen Testes, die Betroffenen zeichnen sich auch typischerweise durch kongenitale Herzfehler aus (Brugh III et al., 2003; Elsawi et al., 1994; Nieschlag et al., 2000a). Bis zu einem Viertel dieser Patienten ist leichtgradig geistig behindert (Meschede \& Horst, 2000). Die Spinobulbäre Muskelatrophie (Kennedys Disease) ist eine neuromuskuläre Erkrankung, die sich auch auf die spermatogenetische und endokrine Hodenfunktion ausweitet (Brugh III et al., 2003; Meschede \& Horst, 2000). Die Polyzystische Nierenerkrankung führt neben Azoospermie oder schwerer Oligozoospermie zu zystischen Nierenveränderungen, viele der Patienten leiden auch unter Leber-, Milz- und Pankreaszysten. Es besteht außerdem ein stark erhöhtes Risiko für Aneurysmen der Hirnbasisarterien (Meschede \& Horst, 2000). Das Young-Syndrom schließlich, bei welchem das Lumen der Nebenhoden vollständig von einer amorphen Masse verschlossen wird, resultiert in einer vollständigen bilateralen Obstruktion der Nebenhoden und daraus folgender Azoospermie (Behre et al., 2000a; Meschede et al., 
1998). Zusätzlich leiden die Betroffenen unter einer chronischen sinusbronchialen Symptomatik (Behre et al., 2000a). Eine Übersicht über weitere andrologisch relevante genetische Syndrome kann Meschede \& Horst (2000) entnommen werden.

Die Darstellung verdeutlicht, auf welch vielfältige Weise genetische Erkrankungen die männliche Fertilität beeinträchtigen und gleichzeitig mit weiteren degenerativen Symptomen assoziiert sein können. Und es wird angenommen, dass viele der heute noch ungeklärten, idiopathischen Subfertilitätsfälle eine genetische Ursache haben (Brugh III et al., 2003). Problematisch ist aber, dass die Angaben zur Mortalität der Betroffenen mit genetischen Anomalien relativ rar sind. Wie aus der obigen Darstellung ersichtlich wird, liegen zwar Studien zu möglichen Komorbiditäten vor, dennoch sind die vorgestellten Symptome und nachteiligen Effekte fakultativ. Ein erhöhtes Sterberisiko kann in den meisten Fällen zwar angenommen werden, wurde aber noch nicht belegt. Trotzdem bleibt die Frage zu beantworten, ob die erhöhte Sterblichkeit der subfertilen Fälle in der eigenen Stichprobe auf genetische Anomalien zurückgeführt werden könnte, welche einerseits die Subfertilität verantwortet und andererseits zu einem schlechteren Gesundheitszustand bzw. einem früheren Tod geführt haben könnten. Verschiedene Aspekte sprechen gegen diese Vermutung. Betrachtet man die Prävalenz der beschriebenen, heute bekannten genetischen Syndrome, so ist erstens anzunehmen, dass in der untersuchten Stichprobe der Anteil an Männern, welcher unter einem der Krankheitsbilder litt, eher gering gewesen sein dürfte. Zweitens scheinen die dargestellten genetischen Erkrankungen häufiger in einer Azoospermie als in einer Oligozoospermie zu resultieren. Damit sollten eher die azoospermen Männer von einer höheren Mortalität betroffen gewesen sein. Im Gegenteil lässt sich aber in der eigenen Stichprobe ein solcher Effekt für die Azoospermen nicht finden und es sind die Oligozoospermen, deren Sterblichkeit erhöht ist. Interessant wäre daher auch an dieser Stelle wiederum die Erhöhung der Zahl der Azoospermen zur Überprüfung, ob mit einer größeren Stichprobe auch für diese Fertilitätsklasse eine erhöhte Mortalität festgestellt werden kann. Nimmt man an, dass tatsächlich genetische Dispositionen für den Zusammenhang zwischen Mortalität und Fertilität verantwortlich zu machen sind, so würden die momentanen Ergebnisse auch hier wieder, analog zu den Überlegungen im Abschnitt 4.4.3, die Vermutung nahe legen, dass die Mehrheit der azoospermen Männer von einer sekundär erworbenen Azoospermie betroffen waren. Nur dann würde dieser Erklärungsansatz mit den ermittelten Befunden übereinstimmen. 


\section{Fazit und Ausblick}

Nachdem die Ergebnisse diskutiert und mögliche Erklärungsansätze vorgestellt wurden, sollen an dieser Stelle einige abschließende Betrachtungen bezüglich der Resultate und der möglichen weiteren Forschung angestellt werden. Die ursprüngliche Fragestellung der vorliegenden Studie betraf die Überlegung, ob sich zwischen den Spermatogenese-Funktionen und der Lebenszeit-Mortalität von Männern ein Zusammenhang finden lassen könnte. Anhand der Analysen der Lebensdauern der verstorbenen bzw. der geschätzten Lebenserwartungen der noch lebenden ehemaligen Infertilitätspatienten konnte die Vermutung eines solchen Zusammenhangs bestätigt und auch dessen Natur genauer dargestellt werden. Es zeigte sich keine direkte Dosis-Wirkungs-Beziehung zwischen der Spermatogenesefunktion, geäußert durch die Spermienkonzentration, und der Lebenszeit-Mortalität. Vielmehr ließ sich ein Unterschied in Abhängigkeit vom Fertilitätsstatus der Männer ermitteln: Die untersuchten subfertilen Fälle zeichneten sich durch ein höheres Sterberisiko aus als die fertilen Männer. Bei einer genaueren Betrachtung ergab sich, dass dieser Befund auf die oligozoospermen Patienten zurückzuführen war. Ihr Sterberisiko war gegenüber jenem der normozoospermen Fälle bis um das Zweifache erhöht. Kein Unterschied konnte zwischen azoospermen und normo- bzw. oligozoospermen Patienten festgestellt werden. Diese Effekte wurden nicht durch den sozioökonomischen Status der Männer beeinträchtigt.

In der vorliegenden Arbeit wurde somit ein direkter Zusammenhang zwischen reproduktionsphysiologischen Funktionen und der Lebenszeit-Mortalität von Männern ermittelt. Eine Interpretation dieses Befundes muss aufgrund der Datenlage vorläufig hypothetisch bleiben. Von den diskutierten Erklärungsansätzen (1) des Verhaltens und der Konditionen im Erwachsenenalter, (2) der Bedingungen in utero und in der frühen Kindheit sowie (3) der genetischen Dispositionen kann zum momentanen Zeitpunkt keiner sicher als verantwortlich angesehen werden. Die Befunde scheinen am wenigsten gegen einen Einfluss der fötalen und frühkindlichen Konditionen auf die spätere Gesundheit im Erwachsenenalter zu sprechen. Hier wurde als möglicher Mechanismus eine Entwicklungsbeeinträchtigung mit nachteiligen Konsequenzen für die allgemeine Gesundheit und das Mortalitätsrisiko mit einhergehender negativer Beeinflussung z.B. der Entwicklung der Sertoli-Zellen und dadurch gestörter Spermatogenese vorgestellt. Dieser Zusammenhang stellt einen der möglichen Wirkungswege dar und muss offen bleiben, ob auch andere, noch nicht bekannte biologische Mechanismen für die höhere Sterblichkeit oligozoospermer Männer verantwortlich gemacht werden könnten. So wären z.B. auch hormonelle Wirkungswege denkbar. Grundsätzlich erscheint es schwierig, die drei Erklärungsansätze vollständig voneinander zu trennen und es kann wohl eher eine Synthese angenommen werden, in welcher sich die Effekte der möglichen Wirkungsmechanismen ergänzen oder gegenseitig abschwächen. Zum momen- 
tanen Zeitpunkt fehlen noch die nötigen Informationen über die ehemaligen Infertilitätspatienten, um den Schritt von plausiblen Überlegungen zu tatsächlichen Erklärungen machen zu können.

Für die zukünftige Forschung hinsichtlich des Zusammenhangs zwischen Fertilität und Lebenszeit-Mortalität von Männern sind verschiedene Aspekte von Bedeutung. Zunächst wäre es interessant, die Recherchen und Analysen zu einem späteren Zeitpunkt zu wiederholen, da sich dann die Zahl der Verstorbenen erhöht haben sollte und somit genauere Schätzungen der Überlebensfunktionen möglich wären. So ließe sich überprüfen, ob das Sterberisiko der frühen und späten Geburtsjahrgänge tatsächlich von unterschiedlichen Faktoren beeinflusst wird oder ob es sich bei diesen Unterschieden um methodische Verzerrungen handelt. Generell sollte auch die Fallzahl der Azoospermen erhöht werden, um zu überprüfen, ob sich für diese Fertilitätsklasse nicht doch - wie vermutet wird - die gleichen Effekte finden lassen wie für die oligozoospermen Fälle. Die Ergebnisse der nicht-parametrischen Verfahren sowie die Analysen der verstorbenen Fälle deuten bereits darauf hin, dass auch das Sterberisiko der azoospermen Männer erhöht sein könnte. Wäre dies der Fall, so würde weniger bzw. nichts mehr gegen die vorgestellten Interpretationsansätze zur Deutung des Zusammenhangs zwischen männlicher Fertilität und Mortalität sprechen.

Der erste diskutierte Erklärungsansatz befasste sich mit den - direkten und indirekten negativen Auswirkungen von (ungewollter) Kinderlosigkeit auf die Gesundheit. Vor diesem Hintergrund wäre die Information interessant, welcher der Patienten zu einem späteren Zeitpunkt noch Kinder zeugte, adoptierte oder mit Stiefkindern zusammenlebte, wie eng der Kontakt mit diesen Kindern war und ob eine Scheidung erfolgte. Des Weiteren sollten die Todesursachen betrachtet werden. Eine erhöhte Ätiologie von Unfällen, Folgen von Substanzmittelabhängigkeit oder Suizid bei den Subfertilen - ermittelbar über die Sterbebescheinigungen der Verstorbenen - würde z.B. für das Zutreffen dieses ersten Erklärungsansatzes sprechen.

Zusätzlich sind die noch nicht verstorbenen Männer bezüglich ihrer Morbidität zu untersuchen. Eine Befragung könnte die subjektiv eingeschätzte Gesundheit und das psychische Wohlbefinden erfassen. Besonders aufschlussreich wären in diesem Zusammenhang beispielsweise auch genauere Angaben zum Geburtsgewicht. Diese könnten Anhaltspunkte für das Zutreffen des zweiten Erklärungsansatzes bieten. So könnte gemutmaßt werden, dass das durchschnittliche Geburtsgewicht der Subfertilen unter jenem der Fertilen liegt. Schließlich wären hinsichtlich des dritten Erklärungsansatzes genetische Analysen der noch lebenden Patienten von Interesse, um Aussagen über mögliche 
Auswirkungen von genetischen Anomalien auf die Gesundheit und die Reproduktionsphysiologie machen zu können. Allerdings ist anzuzweifeln, dass solche Informationen tatsächlich ermittelt werden können.

Wie bereits in der Einleitung beschrieben wurde, ist das Thema der spezifischen Männergesundheit und -mortalität ein bislang relativ vernachlässigtes Forschungsfeld, welches erst im Laufe der letzten Jahre langsam an Bedeutung gewann. Auch die reproduktive Gesundheit von Männern stellt ein noch nicht ausreichend erforschtes Gebiet dar. Die durchgeführte Studie betrachtete nun den Zusammenhang zwischen den reproduktionsphysiologischen Funktionen von ehemaligen Kinderwunschpatienten und ihrem individuellen Sterberisiko. Grundsätzlich werden Arbeiten über die Lebenszeit-Mortalität dadurch erschwert, dass nur in seltenen Fällen geeignete Daten zur Verfügung stehen. In Deutschland werden zum einen kaum Panel-Erhebungen mit gesundheitsbezogenen Fragen durchgeführt und zum anderen medizinische Aufzeichnungen nach Ablauf der 10-JahresFrist meist vernichtet. Dabei ist gerade der individuelle Gesundheitsverlauf als Längsschnittuntersuchung von großer Bedeutung, um langfristige Zusammenhänge zwischen Einflussfaktoren, welche bereits im jungen Erwachsenenalter nachweisbar sind, und der späteren Mortalität aufdecken und Aussagen über Kausalitäten machen zu können. Die vorliegende Studie kann aufgrund der Datenlage einen Beitrag zur Füllung dieser Lücken leisten. 


\section{Zusammenfassung}

Die vorliegende Arbeit hatte zum Ziel, den potentiellen Zusammenhang zwischen männlicher Fertilität und Lebenszeit-Mortalität zu untersuchen. Datengrundlage waren die Krankenakten von Patienten der Andrologischen Sprechstunde des Universitätsklinikums Marburg, welche in den Jahren 1949 bis 1985 - meist aufgrund eines unerfülten Kinderwunsches - eine Spermiogramm-Analyse durchführen ließen. Alle Patienten wurden vor 1938 geboren. Fälle mit bekannten Vorerkrankungen wie z.B. Mumps, Geschlechtskrankheiten oder Hodenfehllagen wurden im Vorfeld ausgeschlossen, da bei diesen Diagnosen von einer Beeinträchtigung der Spermatogenese auszugehen war. Mittels Recherche in Einwohnermeldeämtern und der hessischen Zentraldatei einer gesetzlichen Krankenkasse konnte der Vitalstatus (lebend vs. verstorben) von 601 ehemaligen Patienten festgestellt werden. Im Falle eines Todes wurden ebenfalls die Sterbedaten notiert. Zusätzlich wurde der zuletzt ausgeübte Beruf als Indikator für den sozioökonomischen Status der Männer festgehalten. Die Fälle wurden in Abhängigkeit von der angegebenen Spermienkonzentration in Fertilitätsklassen eingeteilt und je nach Fertilitätsstatus in fertile und subfertile Fälle unterschieden. Die Grenze hierzu wurde nach WHO-Empfehlungen bei 20 Millionen Spermien pro Milliliter Ejakulat festgelegt. Die Untergruppe der Subfertilen wurde nochmals unterteilt in Oligo- und Azoosperme, wobei sich letztere durch die vollständige Abwesenheit von Spermien im Ejakulat auszeichneten. Bei dieser Fertilitätsklassifikation wurden die fertilen Fälle als normozoosperm bezeichnet. Die im Folgenden beschriebenen Analysen wurden einerseits für die gesamte Stichprobe gemeinsam durchgeführt, andererseits wurden die Fälle nach dem Median des Geburtsdatums in zwei Subgruppen, die so genannten frühen und späten Geburtsjahrgänge, aufgeteilt und die Berechnungen separat angestellt.

Für das betrachtete Untersuchungskollektiv wurde im Vergleich zur Allgemeinbevölkerung eine positive Selektion hinsichtlich des Ehestands und des sozioökonomischen Status vermutet. Der Vergleich der eigenen Daten und Befunde mit jenen aus anderen Studien wurde allgemein durch die unterschiedliche Natur der in Fertilitätsstudien betrachteten Studienpopulationen erschwert. Grundsätzlich erschien jedoch die Befunddokumentation in den untersuchten Krankenakten valide und reliabel und die angegebenen Spermienkonzentrationen waren vergleichbar mit Angaben aus anderen Studien. Unter den betrachteten Infertilitätspatienten fanden sich 66\% Normozoosperme, 20\% Oligozoosperme und 14\% Azoosperme. Betrachtete man die frühen und späten Geburtsjahrgänge getrennt voneinander, so war auffällig, dass der Anteil der Azoospermen innerhalb der frühen Geburtskohorten höher war. Dies kann möglicherweise durch den generellen Rückgang sexuell übertragbarer Krankheiten seit Erfindung des Penicillins und anderer Antibiotika 
erklärt werden, da Infektionen z.B. mit Gonorrhoe oder Chlamydien eine mögliche Azoospermie-Ursache darstellen.

In einer Sterbetafel-Analyse wurden die Überlebensfunktionen anhand der tatsächlichen Lebensdauern der Verstorbenen sowie der geschätzten Lebenserwartungen der noch lebenden Fälle abgebildet und mittels Wilcoxon(Gehan)-Test Unterschiede zwischen den Fertilitätsklassen berechnet. Hier ergab sich ein Effekt, welcher besonders bei der Analyse der frühen Geburtsjahrgänge deutlich zu Tage trat: Die Mortalität der Subfertilen lag über jener der Fertilen. Dies lässt sich vor allem auf einen Unterschied zwischen normo- und oligozoospermen Fällen zurückführen. Doch auch für die azoospermen Fälle ließ sich der Trend einer im Vergleich zu den Normozoospermen erhöhten Sterblichkeit erkennen.

Die semi-parametrische Cox-Regression sowie weitere parametrische Regressionsverfahren erlaubten, diesen Unterschied zu quantifizieren und unter Kontrolle verschiedener Variablen die Lebenszeit-Mortalität in Abhängigkeit vom Fertilitätsstatus modellieren. Die Ergebnisse der Cox-Regression bestätigten die höhere Sterblichkeit der subfertilen Fälle. Wiederum wurde dies v.a. bei der Betrachtung der frühen Geburtsjahrgänge evident. Subfertile hatten ein um den Faktor 1,6 erhöhtes Sterberisiko. Dieser Effekt wurde nicht vom sozioökonomischen Status beeinträchtigt. Eine separate Auswertung ergab, dass auch hier das höhere Sterberisiko der Subfertilen auf den Unterschied in den Mortalitätsrisiken der Normo- und Oligozoospermen zurückzuführen war. Die oligozoospermen Fälle hatten ein doppelt so hohes Risiko zu versterben wie die normozoospermen Männer. Die Sterblichkeit der Azoospermen hingegen war weder gegenüber jener der Normo- noch gegenüber jener der Oligozoospermen erhöht. Allerdings sollte hier bedacht werden, dass die Azoospermen die Gruppe mit der geringsten Fallzahl darstellte. Es bleibt nachzuweisen, ob bei Erhöhung der Substichprobengröße auch für die Azoospermen ein erhöhtes Sterberisiko zu finden ist - dies wäre aufgrund der Befunde des Wilcoxon(Gehan)-Tests zu vermuten. Die beschriebenen Befunde wurden durch eine Analyse ausschließlich der verstorbenen Fälle gestützt. Unter den verwendeten parametrischen Regressionsmodellen ist vor allem das Gompertz-Makeham-Modell zur Modellierung der Mortalität geeignet. Es bestätigte im Wesentlichen die Ergebnisse der Cox-Regression mit einem erhöhten Sterberisiko der subfertilen Fälle innerhalb der frühen Geburtsjahrgänge. Auch bei Verwendung alternativer Abgrenzungen der Subfertilen blieb der Befund einer erhöhten Sterblichkeit der Subfertilen weitestgehend erhalten.

Im Gegensatz zu den frühen Geburtsjahrgängen, für welche ein Zusammenhang zwischen Fertilitätsstatus und Lebenszeit-Mortalität postuliert werden konnte, ergab sich für die späten Geburtsjahrgänge hauptsächlich der sozioökonomische Status als Prädiktor- 
variable für die Mortalität. Die Literatur bestätigt zwar den starken Einfluss dieser Variablen auf die Sterblichkeit, und es lassen sich mögliche Ursachen für diese Unterschiede zwischen den frühen und späten Geburtsjahrgängen diskutieren. Generell sollten jedoch die Ergebnisse für die späten Geburtsjahrgänge mit Vorbehalt betrachtet werden, da der Anteil an noch nicht verstorbenen Fällen sehr hoch war und somit die Schätzungen der Lebenserwartungen und ihrer Beeinflussungen ungenau wurden.

Hinsichtlich der Spermienkonzentration wiesen die Regressionsmodelle keinen Zusammenhang mit der Lebenszeit-Mortalität der Individuen nach. Dies lässt einen weiteren Schluss über die Art des Zusammenhangs zwischen Spermatogenese und Mortalität zu: Er zeigt sich nicht in quantitativer Form als direkte Dosis-Wirkungs-Beziehung, sondern lässt sich eher in qualitativem Sinne als niedrigeres bzw. höheres Sterberisiko bei „besserer“ bzw. „schlechterer" reproduktionsphysiologischer Funktion erfassen.

Zur Begründung einer höheren Sterblichkeit der Subfertilen werden verschiedene Erklärungsansätze diskutiert, auch wenn aufgrund der Datenlage endgültige Schlüsse noch nicht zu ziehen sind. Der erste Ansatz beschäftigt sich mit den Auswirkungen von Kinderlosigkeit auf die Gesundheit und Mortalität. (Ungewollte) Kinderlosigkeit erhöht das Risiko für eine Scheidung, ist assoziiert mit ungesünderem Lebensstil, geringerer sozialer Unterstützung und erhöhter Morbidität. Ein zweiter Ansatz befasst sich mit den Bedingungen in utero und im frühen Kindesalter. Nachteilige Konditionen können sich gleichzeitig negativ auf die Gesundheit im Allgemeinen und auf reproduktionsphysiologische Funktionen, z.B. die Spermatogenese, auswirken. Drittens sind genetische Dispositionen denkbar, welche einen Zusammenhang zwischen verminderter Fertilität einerseits und erhöhter Mortalität andererseits moderieren könnten. Von den erwähnten Erklärungsansätzen erscheint der zweite, welcher intrauterine Bedingungen für die höhere Sterblichkeit von subfertilen Männern als ursächlich erklärt, am ehesten der Befundlage in der vorliegenden Arbeit zu entsprechen. Gegen alle drei Ansätze spricht die jedoch Tatsache, dass - zumindest nach momentanem Kenntnisstand - nur die Mortalität der Oligozoospermen, nicht aber jene der Azoospermen erhöht ist.

Die vorliegende Arbeit ermittelte einen Zusammenhang zwischen den reproduktionsphysiologischen Funktionen und der Lebenszeit-Mortalität der ehemaligen Infertilitätspatienten. Sie leistet damit einen Beitrag zur Erforschung der langfristigen Einflussfaktoren auf die männliche Sterblichkeit, welche in Deutschland allgemein durch fehlende Längsschnittdaten erschwert ist. Um das in der Studienpopulation beobachtete Phänomen nun noch genauer untersuchen und erklären zu können, sollten als nächster Schritt die Todesursachen der Verstorbenen, ermittelbar durch die Todesbescheinigungen, sowie die 
Morbidität der noch lebenden Fälle, ermittelbar durch Befragungen, betrachtet werden. Ferner sollten ebenfalls Informationen zur Familiengeschichte, also Ehe- und Elternstand, in Erfahrung gebracht werden. 


\section{Literaturverzeichnis}

Abramsky L, Chapple J (1997) 47,XXY (Klinefelter syndrome) and 47,XYY: estimated rates of and indication for postnatal diagnosis with implications for prenatal counselling. Prenatal Diagnosis 17:363-368

Acacio BD, Gottfried T, Israel R, Sokol RZ (2000) Evaluation of a large cohort of men presenting for a screening semen analysis. Fertility and Sterility 73:595-597

Adami HO, Bergström R, Möhner M, Zatonski W, Storm H, Ekbom A, Tretli S, Teppo L, Ziegler H, Rahu M, Gurevicius R, Stengrevics A (1994) Testicular cancer in nine Northern European countries. International Journal of Cancer 59:33-38

Agarwal A, Said T (2003) Role of sperm chromatin abnormalities and DNA damage in male infertility. Human Reproduction Update 9:331-345

Ahrens W, Bellach BM, Jöckel KH (1998) Messung soziodemographischer Merkmale in der Epidemiologie. MMV Medizin, München

Albers P (1998) Fertilität nach Chemotherapie oder Radiatio. Fertilität 13:S24-S26

Albers P (2000) Hodentumor und Fertilität. Reproduktionsmedizin 16:55-61

Altgeld T (2004) Männergesundheit. Juventa, Weinheim

Amory JK, Anawalt BD, Paulsen CA, Bremner WJ (2000) Klinefelter's syndrome. Lancet 356:333-335

Andersen AG, Jensen TK, Carlsen E, Jørgensen N, Andersson AM, Krarup T, Keiding N, Shakkebæk NE (2000) High frequency of sub-optimal semen quality in an unselected population of young men. Human Reproduction 15:366-372

Andolz A, Bielsa MA, Vila J (1999) Evolution of semen quality in North-Eastern Spain: a study in 22759 infertile men over a 36 year period. Human Reproduction 14:731-735

Andolz P, Bielsa MA, Andolz A (2001) Circannual variation in human semen parameters. International Journal of Andrology 24:266-271

Arbeitsgemeinschaft Bevölkerungsbezogener Krebsregister in Deutschland (2004) Krebs in Deutschland. 4. überarbeitete, aktualisierte Ausgabe. http://www.rki.de/GBE/KREBS /KID2004/ KID2004_.PDF

Auger J, Jouannet $P$ (1997) Evidence for regional differences of semen quality among fertile French men. Human Reproduction 12:740-745

Auger J, Kunstmann JM, Czyglik F, Jouannet P (1995) Decline in semen quality among fertile men in Paris during the past 20 years. The New England Journal of Medicine 332:281-285

Babitsch B (2001) Soziale Ungleichheit und Gesundheit - Eine geschlechtsspezifische Betrachtung. In: Mielck A, Bloomfield K (eds) Sozial-Epidemiologie. Juventa, Weinheim, pp 83-94

Backett KC, Davison C (1995) Lifecourse and lifestyle: the social and cultural location of health behaviours. Social Science \& Medicine 40:629-638

Baker HWG (2000) Management of male infertility. Baillière's Clinical Endocrinology and Metabolism 14:409-422

Balkrishnan R (1998) Predictors of medication adherence in the elderly. Clinical Therapeutics 20:764-771

Banks SM, Pandiani JA, Schacht LM, Gauvin LM (2000) Age and mortality among white male problem drinkers. Addiction 95:1249-1254

Barker DJP (1995) Fetal origins of coronary heart disease. British Medical Journal 311:171174

Barker DJP, Osmond C, Rodin I, Fall CHD, Winter PD (1995) Low weight gain in infancy and suicide in adult life. British Medical Journal 311:1203

Barratt CL, Cooke ID (1988) Sperm loss in the urine of sexually rested men. International Journal of Andrology 11:201-207

Baumann A, Filipiak B, Stieb J, Löwel H (1998) Familienstand und soziale Integration als Prädiktoren der Mortalität: eine 5-Jahres-Follow-up-Studie an 55- bis 74jährigen Männern und Frauen in der Region Augsburg. Zeitschrift für Gerontologie und Geriatrie 31:184-192 
Behre HM, Nieschlag E (1998) Störungen des endokrinen Systems. In: Krause W, Weidner W (eds) Andrologie. Enke, Stuttgart, pp 112-122

Behre HM, Nieschlag E, Meschede D (2000a) Störungen im Bereich der ableitenden Samenwege und akzessorischen Geschlechtsdrüsen. In: Nieschlag E, Behre HM (eds) Andrologie. Springer, Berlin, pp 195-209

Behre HM, Nieschlag E, Meschede D, Partsch C-J (2000b) Störungen im Bereich des Hypothalamus und der Hypophyse. In: Nieschlag E, Behre HM (eds) Andrologie. Springer, Berlin: pp 137-156

Belcheva A, Ivanova-Kicheva M, Tzvetkova P, Marinov M (2004) Effects of cigarette smoking on sperm plasma membrane integrity and DNA fragmentation. International Journal of Andrology 27:296-300

Bengel J, Carl C, Mild U, Strauß B (2000) Langfristige psychische Folgen von Kinderlosigkeit: Eine Übersicht. Zeitschrift für Klinische Psychologie und Psychotherapie 29:3-15

Benoff S, Gilbert BR (2001) Varicocele and male infertility. Human Reproduction Update 7:47-54

Bergmann M (1998) Spermatogenese. In: Krause W, Weidner W (eds) Andrologie. Enke, Stuttgart, pp 9-14

Berkman LF, Syme SL (1979) Social networks, host resistance, and mortality: a nine-year follow-up study of Alameda County residents. American Journal of Epidemiology 109:186-20

Berman NG, Wang C, Paulsen CA (1996) Methodological issues in the analysis of human sperm concentration data. Journal of Andrology 17:68-73

Berling S, Wolner-Hanssen P (1997) No evidence of deteriorating semen quality among men in infertile relationships during the last decade: a study of males from Southern Sweden. Human Reproduction 12:1002-1005

Beutel M, Kupfer J, Kirchmeyer P, Kehde S, Köhn FM, Schroeder-Printzen I, Gips H, Herrero HJG, Weidner W (1999) Treatment-related stresses and depression in couples undergoing assisted reproductive treatment by IVF or ICSI. Andrologia 31:27-35

Bhasin S, Mallidis C, Ma K (2000) The genetic basis of infertility in men. Baillière's Clinical Endocrinology and Metabolism 14:363-388

Biegel T (2003) Berufliche Laufbahn und Lebenserwartung. Unveröffentlichte Inauguraldissertation, Philipps-Universität, Marburg

Birg H, Flöthmann E-J (2002) Langfristige Trends der demographischen Alterung in Deutschland. Zeitschrift für Gerontologie und Geriatrie 35:387-399

Birnbacher R, Frisch H (1995) Hormonal, genetic and clinical findings in an XX male. Hormone Research 43:213-215

Bliesener N, Redel L, Klingmüller D (2001) Diagnostik und Therapie des zentralen Hypogonadismus beim Mann. Reproduktionsmedizin 17:79-86

Blossfeld H-P, Hammerle A, Mayer KU (1987) Ereignisanalyse. Campus, Frankfurt

Bobak M, Gjonca A (2001) The seasonality of live birth is strongly influenced by sociodemographic factors. Human Reproduction 16:1512-1517

Boehmer ALM, Nijman RJM, Lammers BAS, de Coninck SJF, van Hemel JO, Themmen APN, Mureau MAM, de Jong FH, Brinkmann AO, Niermeijer MF, Drop SLS (2001) Etiological studies of severe or familial hypospadias. The Journal of Urology 165:12461254

Bohring C, Krause W (2000) Fertilitätsstörungen durch Spermaantikörper. Reproduktionsmedizin 16:3-7

Bohring C, Krause W (2003) Immune infertility: towards a better understanding of sperm (auto)-immunity. Human Reproduction 18:915-924

Bojesen A, Juul S, Birkebaek N, Gravholt CH (2004) Increased mortality in Klinefelter syndrome. The Journal of Clinical Endocrinology and Metabolism 89:3830-3834

Brähler E, Goldschmidt S, Kupfer J (2001a) Männer und Gesundheit. In: Brähler E, Kupfer J (eds) Mann und Medizin. Hogrefe, Göttingen, pp 11-33

Brähler E, Kupfer J (2001) Mann und Medizin. Göttingen, Hogrefe 
Brähler E, Stöbel-Richter Y, Huinink J, Glander HJ (2001b) Zur Epidemiologie gewollter und ungewollter Kinderlosigkeit in Ost- und Westdeutschland. Reproduktionsmedizin 17:157-162

Brinkworth MH, Handelsman DJ (2000) Umwelt- und arbeitsplatzbedingte Einflüsse auf die männliche Fertilität. In: Nieschlag E, Behre HM (eds) Andrologie. Springer, Berlin, pp 279-299

Brockmann H, Klein T (2002) Familienbiographie und Mortalität in Ost- und Westdeutschland. Zeitschrift für Gerontologie und Geriatrie 35:430-440

Brockmann H, Klein T (2004) Love and death in Germany: the marital biography and its effect on mortality. Journal of Marriage and Family 66:567-581

Brugh III VM, Maduro MR, Lamb DJ (2003) Genetic disorders and infertility. Urologic Clinics of North America 30:143-152

Bründel H, Hurrelmann K (1999) Konkurrenz, Karriere, Kollaps. Männerforschung und der Abschied vom Mythos Mann. Kohlhammer, Stuttgart

Brunner E (1997) Socioeconomic determinants of health: stress and the biology of inequality. British Medical Journal 314:1472

Bühl A, Zöfel P (2002) SPSS 11 - Einführung in die moderne Datenanalyse unter Windows. Addison-Wesley, München

Bujan L, Mansat A, Pontonnier F, Mieusset R (1996) Time series analysis of sperm concentration in fertile men in Toulouse, France, between 1977 and 1992. British Medical Journal 312:471-472

Bundesärztekammer (2005) Neue (Muster-) Weiterbildungsordnung Andrologie. http://www.bundesaerztekammer.de /30/ Weiterbildung/03MWBO/MWBOC/Andrologie. html

Buss D (2004) Evolutionary psychology: the new science of the mind. Allyn \& Bacon, Boston

Cagnacci A, Maxia N, Volpe A (1999) Diurnal variation of semen quality in human males. Human Reproduction 14:106-109

Campbell BC, Lukas WD, Campbell KL (2001) Reproductive ecology of male immune function and gonadal function. In: Ellison PT (ed) Reproductive ecology and human evolution. Aldine de Gruyter, New York, pp 159-178

Carlsen E, Giwercman A, Keiding N, Skakkebæk NE (1992) Evidence for decreasing quality of semen during past 50 years. British Medical Journal 305:609-613

Carlsen E, Swan SH, Holm Petersen J, Skakkebæk NE (2005) Longitudinal changes in semen parameters in young Danish men from Copenhagen area. Human Reproduction 20:942-949

Carnes BA, Olshansy SJ, Crahn D (2003) Biological evidence for limits to the duration of life. Biogerontology 4:31-45

Case A, Paxson C (2005) Sex differences in morbidity and mortality. Demography 42:189214

Chia SE, Tay SK, Lim ST (1998) What constitutes a normal seminal analysis? Semen parameters of 243 fertile men. Human Reproduction 13:3394-3398

Chipperfield JG, Havens B (2001) Gender differences in the relationship between marital status transitions and life satisfaction in later life. Journal of Gerontology: Psychological Sciences 56B:P176-P186

Chou K-L, Chi I (2004) Childlessness and psychological well-being in Chinese older adults. International Journal of Geriatric Psychiatry 19:449-457

Christensen K, Kristiansen M, Hagen-Larsen H, Skytthe A, Bathum L, Jeune B, AndersenRanberg K, Vaupel JW, Ørstavik KH (2000) X-linked genetic factors regulate hematopoietic stem-cell kinetics in females. Blood 95:2449-2451

Christensen K, Vaupel JW (1996) Determinants of longevity: genetic, environmental and medical factors. Journal of Internal Medicine 240:333-341

Christensen K, Vaupel JW, Holm NV, Yashin AL (1995) Mortality among twins after age 6: fetal origins hypothesis versus twin method. British Medical Journal 310:432-436

Chughtai B, Sawas A, O'Malley RL, Naik RR, Khan SA, Pentyala S (2005) A neglected gland: a review of Cowper's gland. International Journal of Andrology 28:74-77 
Clark WA, Freeman HE, Kane R, Lewis CE (1987) The influence of domestic position on health status. Social Science \& Medicine 24:501-506

Comhaire F, Dhooge W, Zalata A, Depuydt C, Mahmoud A, Schoonjans F (1998) Environmental agents with oestrogen-like effects (hormone disruptors). Fertilität 13:S6S8

Comhaire FH, Mahmoud AMA, Depuydt CE, Zalata AA, Christophe AB (1999) Mechanisms and effects of male genital tract infection on sperm quality and fertilizing potential: the andrologist's viewpoint. Human Reproduction Update 5:393-398

Cooper TG (2000) Die Reifung humaner Spermien im Nebenhoden. Reproduktionsmedizin 16:299-309

Cooper TG, Yeung CH (2000) Physiologie der Spermienreifung und Fertilisation. In: Nieschlag E, Behre HM (eds) Andrologie. Springer, Berlin, pp 69-90

Coughlin LB, McGuigan J, Haddad N (2003) Social class and semen analysis. Journal of Obstetrics and Gynaecology 23:276-277

Courgeau D, Lelièvre E (1992) Event history analysis in demography. Clarendon, Oxford

Dallinga JW, Moonen EJC, Dumoulin JCM, Evers JLH, Geraedts JPM, Kleinjans JCS (2002) Decreased human semen quality and organochlorine compounds in blood. Human Reproduction Update 17:1973-1979

de Benedictis G, Tan Q, Jeune B, Christensen K, Ukraintseva SV, Bonafè M, Franceschi C, Vaupel JW, Yashin AL (2001) Recent advances in human gene - longevity association studies. Mechanisms of Ageing and Development 122:909-920

de Jonge C, LaFromboise M, Bosmans E, Ombelet W, Cox A, Nijs M (2004) Influence of the abstinence period on human sperm quality. Fertility and Sterility 82:57-65

De Rosa M, Zarrilli S, Paesano L, Carbone, Boggia B, Petretta M, Maisto A, Cimmino F, Puca G, Colao A, Lombardi G (2003) Traffic pollutants affect fertility in men. Human Reproduction 18:1055-1061

Denil J (1998) Ejakulationsstörungen. In: Krause W, Weidner W (eds) Andrologie. Enke, Stuttgart, pp 217-220

Diekmann A, Mitter P (1984) Methoden zur Analyse von Zeitverläufen. Teubner, Stuttgart

Diemer T, Buchanan Hales D, Ludwig M, Huwe P, Weidner W (2000) Urogenitale Infektionen beim Mann - können sie die Fertilität beeinflussen? Reproduktionsmedizin 16:155-167

Dinkel RH, Luy M (1999) Natur oder Verhalten? Ein Beitrag zur Erklärung der männlichen Übersterblichkeit durch einen Vergleich von Kloster- und Allgemeinbevölkerung. Zeitschrift für Bevölkerungswissenschaft 24:105-132

Doblhammer G (2004) The late life legacy of very early life. Springer, Berlin

Doblhammer G, Oeppen J (2003) Reproduction and longevity among the British peerage: the effect of frailty and health selection. Proceedings of the Royal Society of London B 270:1541-1457

Doblhammer G, Vaupel JW (2001) Lifespan depends on month of birth. Proceedings of the National Academy of Sciences 98:2934-2939

Donelly GP, McClure N, Kennedy MS, Lewis SEM (1999) Direct effect of alcohol on the motility and morphology of human spermatozoa. Andrologia 31:43-47

Elsawi MM, Pryor JP, Klufio G, Barnes C, Patton MA (1994) Genital tract function in men with Noonan syndrome. Journal of Medical Genetics 31:468-470

Elzanaty S, Malm J, Giwercman A (2005) Duration of sexual abstinence: epididymal and accessory sex gland secretions and their relationship to sperm motility. Human Reproduction 20:221-225

Eriksson JG, Forsén T, Tuomilehto J, Osmond C, Barker DJP (2001) Early growth and coronary heart disease in later life: Iongitudinal study. British Medical Journal 322:949953

ESHRE Capri Workshop Group (2005) Fertility and ageing. Human Reproduction Update 11:261-276

Eskenazi B, Wyrobek AJ, Sloter E, Kidd SA, Moore L, Young S, Moore D (2003) The association of age and semen quality in healthy men. Human Reproduction Update 18:447-454 
Eustache F, Auger J (2003) Inter-inividual variability in the morphological assessment of human sperm: effect of the level of experience and the use of standard methods. Human Reproduction 18:1018-1022

Falk HC, Kaufman SA (1950) What constitutes a normal semen? Fertility and Sterility 1:489503

Fazio L, Brock G (2004) Erectile dysfunction: management update. Canadian Medical Association Journal 170:1429-1437

Fedder J, Crüger D (2004) Etiology of azoospermia in 100 consecutive nonvasectomized men. Fertility and Sterility 82:1463-1465

Ferlin A, Bettella A, Tesari A, Salata E, Dallapiccola B, Foresta C (2004) Analysis of the DAZ gene family in cryptorchidism and idiopathic male inferility. Fertility and Sterility 81:1013-1018

Ferlin A, Moro E, Garolla A, Foresta C (1999) Human male infertility and Y chromosome deletions: role of the AZF-candidates genes DAZ, RBM and DFFRY. Human Reproduction 14:1710-1716

Finch CE, Tanzi RE (1997) Genetics of aging. Science 278:407-411

Fisch H, Andrews H, Hendricks J, Goluboff ET, Olson JH, Olsson CA (1997) The relationship of sperm counts to birth rates: a population based study. The Journal of Urology 157:840-843

Fisch H, Goluboff ET (1996) Geographic variations in sperm counts: a potential cause of bias in studies of semen quality. Fertility and Sterility 65:1044-1046

Fisch H, Goluboff ET, Olson JH, Feldshuh J, Broder SJ, Barad DH (1996) Semen analyses in 1,283 men from the United States over a 25-year period: no decline in quality. Fertility and Sterility 65:1009-1014

Fisher JS, Macpherson S, Marchetti N, Sharpe RM (2003) Human 'testicular dysgenesis syndrome': a possible model using in-utero exposure of the rat to dibutyl phthalate. Human Reproduction 18:1383-1394

Fontaine KR, Redden DT, Wang C, Westfall AO, Allison DB (2003) Years of life lost due to obesity. Journal of the American Medical Association 289:187-193

Fortes C, Forastiere F, Frachi S, Rapiti E, Pastori G, Perucci C (2000) Diet and overall survival in a cohort of very elderly people. Epidemiology 11:440-445

Forti G, Krausz C (1998) Evaluation and treatment of the infertile couple. Journal of Clinical Endocrinology and Metabolism 83:4177-4188

Franken DR, Barendsen R, Kruger TF (2000) A continuous quality control program for strict sperm morphology. Fertility and Sterility 74:721-724

Friedlander NJ (1996) The relation of lifetime reproduction to survivorship in women and men: a prospective study. American Journal of Human Biology 8:771-783

Frohneberg D (2001) Embryologie - Entwicklung des Urogenitalsystems. In: Hautmann RE, Uland $\mathrm{H}$ (eds) Urologie. Springer, Berlin, pp 1-12

Ganzeboom HBG, de Graaf PM, Treiman DJ (1992) A standard international socio-economic index of occupational status. Social Science Research 21:1-56

Ganzeboom HBG, Treiman DJ (2003) Three internationally standardised measures for comparative research on occupational status. In: Hoffmeyer-Zlotnik JHP, Wolf C (eds) Advances in cross-national comparison. A European working book for demographic and socio-economic variables. Kluwer, New York, pp 159-193

Gerhard I, Runnebaum B (1992) Schadstoffe und Fertilitätsstörungen. Geburtshilfe und Frauenheilkunde 52:509-515

Geyer S, Peter R (1999) Occupational status and all-cause mortality. European Journal of Public Health 9:114-118

Geyer S, Peter R (2000) Income, occupational position, qualification and health inequalities competing risks? (Comparing indicators of social status). Journal of Epidemiology and Community Health 54:299-305

Giovannucci E, Tosteson TD, Speizer FE, Vessey MP, Colditz GA (1992) A long-term study of mortality in men who have undergone vasectomy. The New England Journal of Medicine 326:1392-1398 
Goebel P, Lübke F (1987) Katamnestische Untersuchung an 96 Paaren mit heterologer Insemination. Geburtshilfe und Frauenheilkunde 47:636-640

Gonzales GF, Muñoz G, Sánchez R, Henkel R, Gallegos-Avila G, Díaz-Gutierrez O, Virgil P, Vásquez F, Kortebani G, Mazzolli A, Bustos-Obregón E (2004) Update on the impact of Chlamydia trachomatis infection on male fertility. Andrologia 36:1-23

González MA, Artalejo FR, del Rey Calero J (1998) Relationship between socioeconomic status and ischaemic heart diesease in cohort and case-control studies: 1960-1993. International Journal of Epidemiology 27:350-358

Gray PB, Kahlenberg SM, Barrett ES, Lipson SF, Ellison PT (2002) Marriage and fatherhood are associated with lower testosterone in males. Evolution and Human Behavior 23:193-201

Grundy E, Tomassini C (2005) Fertility history and health in later life: a record linkage study in England and Wales. Social Science \& Medicine 61:217-228

Guzick DS, Overstreet JW, Factor-Litvak P, Brazil CK, Nakajima ST, Coutifaris C, Carson SA, Cisneros P, Steinkampf MP, Hill JA, Dong X, Vogel DL (2001) Sperm morphology, motility, and concentration in fertile and infertile men. The New England Journal of Medicine 345:1388-1393

Gyllenborg J, Rasmussen SL, Borch-Johnsen K, Heitmann BL, Skakkebæk NE, Juul A (2001) Cardiovascular risk factors in men: the role of gonadal steroids and sex hormone-binding globulin. Metabolism 50:882-888

Gyllenborg J, Skakkebæk NE, Nielsen NC, Keiding N, Giwercman A (1999) Secular and seasonal changes in semen quality among young Danish men: a statistical analysis of semen samples from 1927 donor candidates during 1977-1995. International Journal of Andrology 22:28-36

Habl C, Birner A, Hlava A, Winkler P (2004) 1. Österreichischer Männergesundheitsbericht. Bundesministerium für soziale Sicherheit, Generationen und Konsumentenschutz, Wien

Haider SG, Hofmann N, Passia D (1985) Morphological and enzyme histochemica observations on alcohol induced disturbances in testis of two patients. Andrologia 17:532-540

Haidl G (1999) Ist die Fruchtbarkeit der Männer ernsthaft bedroht? Reproduktionsmedizin $15: 1-3$

Haidl G, Krause W (1998) Untersuchung des Ejakulates. In: Krause W, Weidner W (eds) Andrologie. Enke, Stuttgart

Haidl G, Weidner W (2002) Epididymitis und Orchitis - klinisch-andrologische Implikationen. Reproduktionsmedizin 18:61-65

Handelsman DJ (2000) Hypogonadismus und Infertilität bei systemischen Erkrankungen. In: Nieschlag E, Behre HM (eds) Andrologie. Springer, Berlin, pp 267-278

Hauser R, Paz G, Botchan A, Yogev L, Yavetz H (2001) Varicocele: effect on sperm functions. Human Reproduction Update 7:482-485

Hazzard WR (1986) Biological basis of the sex differential in longevity. Journal of the Amerian Geriatrics Society 34:455-471

Heinzel-Gutenbrunner M (2001) Einkommen, Einkommensarmut und Gesundheit. In: Mielck A, Bloomfield K (eds) Sozial-Epidemiologie. Juventa, Weinheim, pp 39-49

Helfferich C, Fichtner J (2001) Männer und Familienplanung. Expertise im Auftrag der Bundeszentrale für gesundheitliche Aufklärung, Köln

Helmert U, Shea S (1998) Family status and self-reported health in West Germany. Sozialund Präventivmedizin 43:124-132

Helmert U, Voges W, Sommer T (2002) Soziale Einflussfaktoren für die Mortalität von männlichen Krankenversicherten in den Jahren 1989 bis 2000. Gesundheitswesen 64:3-10

Hemström Ö (1996) Is marriage dissolution linked to differences in mortality risks for men and women? Journal of Marriage and Family 58:366-378

Henkel R (1998) Ejakulat. In: Krause W, Weidner W (eds) Andrologie. Enke, Stuttgart, pp 26-34 
Henkel R, Kleinhappl M, Hanschke A, Schill WB (1998) Jahreszeitliche Schwankungen der Ejakulatqualität. Fertilität 13:S37-S41

Henning K, Strauß B (2000) Psychologische und psychosomatische Aspekte der ungewollten Kinder-losigkeit: Zum Stand der Forschung. In: Strauß B (ed) Ungewollte Kinderlosigkeit. Hogrefe, Göttingen, pp 15-33

Hermanns M, Ringert R-H (1998) Lageveränderungen und Fehlbildungen des Hodens. In: Krause W, Weidner W (eds) Andrologie. Enke, Stuttgart, pp 195-201

Herskind A, McGue M, Holm NV, Sørensen TIA, Harvald B, Vaupel JW (1996) The heritability of human longevity: a population-based study of 2872 Danish twin pairs born 1870-1900. Human Genetics 97:319-323

Hill EM, Chow K (2002) Life-history theory and risky drinking. Addiction 97:401-413

Hinsch KD (2000) Welche in Deutschland im Handel befindlichen Arzneimittel haben erwiesene reproduktionstoxische Nebenwirkungen? Reproduktionsmedizin 16:230-232

Hiort O, Holterhus PM (2003) Androgen insensitivity and male infertility. International Journal of Andrology 26:16-20

Hiort O, Holterhus PM, Sinnecker GHG, Kruse K (1999) Androgenresistenzsyndrome Klinische und molekulare Grundlagen. Deutsches Ärzteblatt 96:A686-692

Hjelmstedt A, Andersson L, Skoog-Svansberg A, Bergh T, Boivin J, Collins A (1999) Gender differences in psychological reactions to infertility among couples seeking IVF- and ICSI-treatment. Acta Obstetrica et Gynecologica Scandinavica 78:42-48

Hjøllund NHI, Bonde JPE, Kold Jensen T, Olsen J (2000) Diurnal scrotal skin temperature and semen quality. International Journal of Andrology 23:309-318

Hobisch A, Janetschek G, Weissbach L (1998) Tumoren des Hodens. In: Krause W, Weidner W (eds) Andrologie. Enke, Stuttgart, pp167-183

Hoffmeyer-Zlotnik JHP, Geis AJ (2003) Berufsklassifikation und Messung des beruflichen Status/Prestige. ZUMA-Nachrichten 52:125-138

Holdcraft RW, Braun RE (2004) Hormonal regulation of spermatogenesis. International Journal of Andrology 27:335-342

Hosmer D, Lemeshow S (1999) Applied survival analysis: regression modeling of time to event. Wiley, New York

Hougaard P (2000) Analysis of multivariate survival data. Springer, New York

Hradil S (1997) Lebenssituation, Umwelt und Gesundheit. Materialien zur Bevölkerungswissenschaft 88. Bundesinstitut für Bevölkerungsforschung beim Statistischen Bundesamt, Wiesbaden

Hurst KM, Dye L (2000) Stress und männliche Subfertilität. In: Brähler E, Felder H, Strauß B (eds) Fruchtbarkeitsstörungen. Hogrefe, Göttingen, pp 27-42

Huynh T, Mollard R, Trounson A (2002) Selected genetic factors associated with male infertility. Human Reproduction Update 8:183-198

Iribarren C, Jacobs DR, Kiefe Cl, Lewis CE, Matthews KA, Roseman JM, Hulley SB (2005) Causes and demographic, medical, lifestyle and psychosocial predictors of premature mortality: the CARDIA study. Social Science \& Medicine 60:471-482

Irvine DS (2000) Male reproductive health: cause for concern? Andrologia 32:195-208

Jaffe A, Bush A (2001) Cystic fibrosis: review of the decade. Monaldi Archives for Chest Disease 56:240-247

Jarow JP (2003) Endocrine causes of male infertility. Urologic Clinics of North America 30:83-90

Jenkins RL (2005) Ensuring access to education and services on infertility for the underserved. Journal of the National Cancer Institute Monographs 34:101-103

Joffe M (2001) Are problems with male reproductive health caused by endocrine disruption? Occupational and Environmental Medicine 58:281-288

Jørgensen N, Andersen AG, Eustache F, Irvine DS, Suominen J, Holm Petersen J, Andersen AN, Auger J, Cawood EHH, Horte A, Kold Jensen T, Jouannet P, Keiding N, Vierula M, Toppari J, Shakkebæk NE (2001) Regional differences in semen quality in Europe. Human Reproduction 16:1012-1019

Jørgensen N, Carlsen E, Nermoen I, Punab M, Suominen J, Andersen AG, Andersson AM, Haugen TB, Horte A, Kold Jensen T, Magnus $\varnothing$, Holm Petersen J, Vierula M, Toppari 
J, Shakkebæk NE (2002) East-West gradient in semen quality in the Nordic-Baltic area: a study of men from the general population in Denmark, Estonia and Finland. Human Reproduction 17:2199-2208

Jung A, Schuppe HC, Schill WB (2002) Comparison of semen quality on older and younger men attending an andrology clinic. Andrologia 34:116-122

Jung A, Schuppe HC, Schill WB (2003) Are children of older fathers at risk for genetic disorders? Andrologia 35:191-199

Kamischke A, Nieschlag E (1999) Treatment of retrograde ejaculation and anejaculation. Human Reproduction Update 5:448-474

Kamwendo F, Forslin L, Bodin L, Danielsson D (1996) Decreasing incidences of gonorrheaand chlamydia-associated pelvic inflammatory disease: a 25-year study from an urban area of central Sweden. Sexually Transmitted Diseases 23:384-391

Keck C, Wetterauer U (1998) Die konventionelle Ejakulatanalyse. Reproduktionsmedizin 14:224-231

Kenkel S, Rolf C, Nieschlag E (2001) Occupational risks for male fertility: an analysis of patients atending a tertiary referral centre. International Journal of Andrology 24:318326

Kennaway DJ (2005) The role of circadian rhythmicity in reproduction. Human Reproduction Update 11:91-101

Kidd SA, Eskenazi B, Wyrobek AJ (2001) Effects of male age on semen quality and fertility: a review of the literature. Fertility and Sterility 75:237-248

Kim SW, Kim KD, Paick JS (1999) Microdeletions within the azoospermia factor subregions of the $\mathrm{Y}$ chromosome in patients with idiopathic azoospermia. Fertility and Sterility 72:349-353

Kirkwood TBL (1977) Evolution of aging. Nature 270:301-304

Kirkwood TBL, Rose MR (1991) Evolution of senescence: late survival sacrificed for reproduction. Philosophical Transactions of the Royal Society of London B 332:15-24

Klein T (1993a) Soziale Determinanten der Lebenserwartung. Kölner Zeitschrift für Soziologie und Sozialpsychologie 45:712-730

Klein T (1993b) Soziale Position und Lebenserwartung. Zeitschrift für Gerontologie 26:313320

Klein T, Schneider S, Löwel H (2001) Bildung und Mortalität. Die Bedeutung gesundheitsrelevanter Aspekte des Lebensstils. Zeitschrift für Soziologie 30:384-400

Klein T, Unger R (2001) Einkommen, Gesundheit und Mortalität in Deutschland, Großbritannien und den USA. Kölner Zeitschrift für Soziologie und Sozialpsychologie 53:96110

Knuth UA, Schulze W (2001) Leitsymptom Azoospermie. Reproduktionsmedizin 17:116-125

Knecht U (1998) Toxikologie von Blei und der Einfluss auf die männliche Fertilität. Fertilität 13:S9-S13

Köhn FM, Haidl G (2002) Andrologische Diagnostik. Hautarzt 53:761-779

Köhn FM, Schill WB (1996) Umweltmedizin und Andrologie: Abnahme der Ejakulatqualität in den letzten 50 Jahren? Hautarzt 47:891-893

Köhn FM, Schill WB (1998) latrogene Einflüsse auf die männliche Fertilität - Medikamente. Fertilität:S31-S33

Köhn FM, Schill WB (2000) Moderne Techniken der Reproduktionsmedizin. In: Brähler E, Felder H, Strauß B (eds) Fruchtbarkeitsstörungen. Hogrefe, Göttingen, pp 11-26

Kolip P (1998) Frauen und Männer. In: Schwartz FW, Badura B, Leidl R, Raspe HH, Siegrist $\mathrm{J}$ (eds) Das Public Health Buch. Urban + Fischer, München, pp 506-516

Kolip P, Hurrelmann K (2002) Geschlecht - Gesundheit - Krankheit: Eine Einführung. In: Kolip P, Hurrelmann K (eds) Geschlecht, Gesundheit und Krankheit. Huber, Bern, pp 13-31

Koropeckyi-Cox T (1998) Loneliness and depression in middle and old age: are the childless more vulnerable? Journal of Gerontology 53B:S303-S312

Koropeckyi-Cox T (2002) Beyond parental status: psychological well-being in middle and old age. Journal of Marriage and Family 64:957-971 
Korpelainen H (2000) Fitness, reproduction and longevity among European aristocratic and rural Finnish families in the 1700s and 1800s. Proceedings of the Royal Society of London B 267:1765-1770

Kotler P, Wingard DL (1989) The effect of occupational, marital and parental roles on mortality: the Alameda County Study. American Journal of Public Health 79:607-612

Krause W (1993) Die Bedeutung des "Routine-Spermiogramms". Hautarzt 44:269-274

Krause W, Müller HH, Schäfer H, Weidner W (2002) Does treatment of varicocele improve male fertility? Results of the 'Deutsche Varikozelenstudie', a multicentre study of 14 collaborating centres. Andrologia 34:164-171

Krausz C, Forti G (2000) Clinical aspects of male infertility. In: McElreavey K (ed) The genetic basis of male infertility. Springer, Berlin, pp 1-21

Kravdal $\varnothing(2003)$ Children, family and cancer survival in Norway. International Journal of Cancer 105:261-266

Kühnert B, Nieschlag E (2004) Reproductive functions of the ageing male. Human Reproduction Update 10:327-339

Künzle R, Mueller MD, Hänggi W, Birkhäuser MH, Drescher H, Bersinger NA (2003) Semen quality of male smokers and nonsmokers in infertile couples. Fertility and Sterility 79:287-291

Lam DA, Miron JA (1996) The effects of temperature on human fertility. Demography 33:291-305

Law CM, Shiell AW (1996) Is blood pressure inversely related to birth weight? The strength of evidence from a systematic review of the literature. Journal of Hypertension 14:935941

Lerchl A (2004) Month of birth and life expectancy: role of gender and age in a comparative approach. Naturwissenschaften 91:422-425

Levine RJ (1999) Seasonal variation of semen quality and fertility. Scandinavian Journal of Work, Environment \& Health 25 (suppl 1):34-37

Levitas E, Lunenfeld E, Weiss N, Friger M, Har-Vardi I, Koifman A, Potashnik G (2005) Relationship between the duration of sexual abstinence and semen quality: analysis of 9,489 semen samples. Fertility and Sterility 83:1680-1686

Lopuhäa CE, Roseboom TJ, Osmond C, Barker DJP, Ravelli AC, Bleker OP, van der Zee JS, van der Meulen JH (2000) Atopy, lung function, and obstructive airways disease after prenatal exposure to famine. Thorax 55:555-561

Lummaa V (2003) Early development conditions and rerpoductive success in humans: downstream effects of prenatal famine, birthweight, and timing of birth. American Journal of Human Biology 15:370-379

Luy M (2002a) Warum Frauen länger leben. Materialien zur Bevölkerungswissenschaft 106. Bundesinstitut für Bevölkerungsforschung beim Statistischen Bundesamt, Wiesbaden

Luy M (2002b) Die geschlechtsspezifischen Sterblichkeitsunterschiede - Zeit für eine Zwischenbilanz. Zeitschrift für Gerontologie und Geriatrie 35:412-429

Lycett JE, Dunbar RIM, Voland E (2000) Longevity and the costs of reproduction in a historical human population. Proceedings of the Royal Society of London B 267:31-35

Macieira-Coelho A (2003) Biology of aging. Springer, Heidelberg

MacLeod J, Gold RZ (1951) The male factor in fertility and infertility. II. Spermatozoon counts in 1000 men of unknown fertility and in 1000 cases of infertile marriages. Journal of Urology 66:436-449

MacLeod J, Wang Y (1979) Male fertility potential in terms of semen quality: a review of the past, a study of the present. Fertility and Sterility 31:103-116

Madersbacher S, Temml C, Racz U, Mock K, Ponholzer A, Maier U, Haidinger G (2003) Prevalence and risk factors for erectile dysfunction in Austria - analysis of a health screening project. Wiener Klinische Wochenschrift 115:822-830

Mak V, Zielenski J, Tsui L-C, Durie P, Zini A, Sheelagh M, T BL, Jarvi KA (2000) Cystic fibrosis gene mutations and infertile men with primary testicular failure. Human Reproduction 15:436-439

Martínez Portillo FJ, Musial A, Bross S, Osmonov DK, Alken P, Jünemann KP (2001) Maldescensus testis und Infertilität. Reproduktionsmedizin 17:291-297 
Martini AC, Molina RI, Estofan D, Senestrani D, de Cuneo MF, Ruiz RD (2004) Effects of alcohol and cigarette consumption on human seminal quality. Fertility and Sterility 82:374-377

Matilsky M, Battino S, Ben-Ami M, Geslevich Y, Eyali V, Shalev E (1993) The effect of ejaculatory frequency on semen characteristics of normozoospermic and oligozoospermic men from an infertile population. Human Reproduction 8:71-73

Mazumdar S, Levine AS (1998) Antisperm antibodies: etiology, pathogenesis, diagnosis, and treatment. Fertility and Sterility 70:799-810

Mazzilli F, Rossi T, Delfino M, Sarandrea N, Dondero F (2000) Azoospermia: incidence, and biochemical evaluation of seminal plasma by the differential $\mathrm{pH}$ method. Panminerva Medica 42:27-31

McDade TW, Beck MA, Kuzawa CW, Adair LS (2001a) Prenatal undernutrition and postnatal growth are associated with adolescent thymic function. Journal of Nutrition 131:12251231

McDade TW, Beck MA, Kuzawa CW, Adair LS (2001b) Prenatal undernutrtion, postnatal environments, and antibody response to vaccination in adolescence. American Journal of Clinical Nutrition 74:543-548

McLachlan RI (2002) Basis, diagnosis and treatment of immunological infertility in men. Journal of Reproductive Immunology 57:35-45

Menkveld R, Wong WY, Lombard CJ, Wetzels AMM, Thomas CMG, Merkus HMWM, Steegers-Theunissen RPM (2001) Semen parameters, including WHO and strict criteria morphology, in a fertile and subfertile population: an effort towards standarddization of in-vivo thresholds. Human Reproduction 16:1165-1171

Merbach M, Klaiberg A, Brähler E (2002) Geschlechtsspezifische Aspekte von Gesundheit und Krankheit. In: Brähler E, Strauß B (eds) Handlungsfelder in der Psychosozialen Medizin. Hogrefe, Göttingen, pp 97-116

Meschede D, Behre HM, Nieschlag E (2000) Störungen im Bereich der Androgenzielorgane. In: Nieschlag E, Behre HM (eds) Andrologie. Springer, Berlin, pp 247-265

Meschede D, Dworniczak B, Nieschlag E, Horst J (1998) Genetic diseases of the seminal ducts. Biomedicine \& Pharmacotherapy 52:197-203

Meschede D, Horst J (1997) The molecular genetics of male infertility. Molecular Human Reproduction 3:419-430

Meschede D, Horst J (2000) Männliche Infertilität im Rahmen genetischer Syndrome. Reproduktionsmedizin 16:147-154

Mielck A (2000) Soziale Ungleichheit und Gesundheit. Huber, Bern

Miller J, Schroeder-Printzen I, Weidner W (1998) Einfluss iatrogener Maßnahmen auf Erektion und Ejakulation unter besonderer Berücksichtigung operativer Verfahren. Fertilität 13:S27-S30

Mineau GP, Smith KR, Bean LL (2004) Adult mortality risks and religious affiliation: the role of social milieu in biodemographic studies. Annales de Démographie Historique 2:85104

Møller H, Shakkebæk NE (1999) Risk of testicular cancer in subfertile men: case-controlstudy. British Medical Journal 318:559-562

Monga M, Alexandrescu B, Katz SE, Stein M, Ganiats T (2004) Impact of infertility on quality of life, marital adjustment, and sexual function. Urology 63:126-130

Monsees TK, Winterstein U, Hayatpour J, Miska W, Schill WB (1998) Einfluss von Umweltnoxen auf die Funktion testikulärer Zellen. Fertilität 13:S75-S78

Mueller UO (2004) Does late reproduction extend the life span? Findings from European Royalty. Population and Development Review 30:449-466

Mueller UO, Heinzel-Gutenbrunner M (2001) Krankheiten und Beschwerden (subjektive Gesundheit) unter Bewertung der eigenen Gesundheit. Bundesinstitut für Bevölkerungsforschung beim Statistischen Bundesamt, Wiesbaden

Muller A, De La Rochebrochard E, Labbé-Declèves C, Jouannet P, Bujan L, Mieusset R, Le Lannou D, Guerin JF, Benchaib M, Slama R, Spira A (2004) Selection bias in semen studies due to self-selection of volunteers. Human Reproduction 19:2838-2844 
Müller HG, Chiou JM, Carey JR, Wang JL (2002) Fertility and life span: late children enhance female longevity. Journal of Gerontology 57A:B202-B206

Natali A, Mondaini N, Lombardi G, Del Popolo G, Rizzo M (2005) Heavy smoking is an important risk factor for erectile dysfunction in young men. International Journal of Impotence Research 17:227-230

$\mathrm{Ng} \mathrm{K}$, Donat R, Chan L, Lalak A, Di Pierro I, Handelsman DJ (2004) Sperm output of older men. Human Reproduction 19:1811-1815

Nieschlag E (1998) Kritische Anmerkungen zur Diskussion über Veränderungen der Spermienqualität. Fertilität 13:S2-S5

Nieschlag E (2000a) Aufgaben und Ziele der Andrologie. In: Nieschlag E, Behre HM (eds) Andrologie. Springer, Berlin, pp 1-9

Nieschlag E (2000b) Nosologie andrologischer Krankheitsbilder. In: Nieschlag E, Behre HM (eds) Andrologie. Springer, Berlin, pp 91-95

Nieschlag E, Behre HM (2000) Andrologie. Springer, Berlin

Nieschlag E, Behre HM, Engelmann U, Schwarzer U (2000b) Männlicher Beitrag zur Kontrazeption. In: Nieschlag E, Behre HM (eds) Andrologie. Springer, Berlin, pp 441-463

Nieschlag E, Behre HM, Meschede D, Kamischke A (2000a) Störungen im Bereich der Testes. In: Nieschlag E, Behre HM (eds) Andrologie. Springer, Berlin, pp 157-193

Oberpenning R, Oberpenning F, Muthny FA (2000) Psychologie der Fertilitätsstörungen. In: Nieschlag E, Behre HM (eds) Andrologie. Springer, Berlin, pp 417-439

Ochsendorf FR (1999) Infections in the male genital tract and reactive oxygen species. Human Reproduction Update 5:399-420

Ochsenkühn R, de Kretser DM (2003) The contributions of deficient androgen action in spermatogenic disorders. International Journal of Andrology 26:195-201

Oliva A, Spira A, Multigner L (2001) Contribution of environmental factors to the risk of male infertility. Human Reproduction 16:1768-1776

Olsen GW, Bodner KM, Ramlow JM, Ross CE, Lipshultz LI (1995) Have sperm counts been reduced 50 percent in 50 years? A statistical model revisited. Fertility and Sterility 63:887-893

Ombelet W, Bosmans E, Janssen M, Cox A, Vlasselaer J, Gyselaers W, Vandeput H, Gielen J, Pollet H, Maes M, Steeno O, Kruger T (1997) Semen parameters in a fertile versus subfertile population: a need for change in the interpretation of semen testing. Human Reproduction 12:987-993

Paasch U, Thieme C, Glander HJ (2003) Men born in the region of Leipzig (Saxony, Germany) between 1960 and 1970 showed a significantly decreased sperm count (examination of 3432 individuals). Andrologia 35:375-377

Paavonen J, Eggert-Kruse W (1999) Chlamydia trachomatis: impact on human reproduction. Human Reproduction Update 5:433-447

Pallares-Ruiz N, Carles S, des Georges M, Guittard C, Arnal F, Humeau C, Claustres M (1999) Complete mutational screening of the cystic fibrosis transmembrane conductance regulator gene: cystic fibrosis mutations are not involved in healthy men with reduced sperm quality. Human Reproduction 14:3035-3040

Parajinen J, Karhunen PJ (1994) Spermatogenic arrest and 'Sertoli cell-only' syndrome common alcohol-induced disorders of the human testis. International Journal of Andrology 17:292-299

Parker KL, Schimmer BP, Schedl A (1999) Genes essential for early events in gonadal development. Cellular and Molecular Life Sciences 55:831-838

Paulsen CA, Berman NG, Wang C (1996) Data from men in greater Seattle area reveals no downward trend in semen quality: further evidence that deterioration of semen quality is not geographically uniform. Fertility and Sterility 655:1015-1020

Pellestor F, Girardet A, Andreo B (1994) Effect of long abstinence periods on human sperm quality. International Journal of Menopausal Studies 39:278-282

Peter R (2001) Berufsstatus und Gesundheit. In: Mielck A, Bloomfield K (eds) SozialEpidemiologie. Juventa, Weinheim, pp 28-38

Petersen PM, Giwercman A, Skakkebæk NE, Rørth M (1998) Gonadal function in men with testicular cancer. Seminars in Oncology 25:224-234 
Petrakis IL, Gonzales G, Rosenheck R, Krystal JH (2002) Comorbidity of alcoholism and psychiatric disorders. Alcohol Research \& Health 26:81-89

Pflieger-Bruss S, Schill WB (2002) Beeinflussung testikulärer Zellen durch endokrin aktive Umweltchemikalien. Reproduktionsmedizin 18:303-306

Pflieger-Bruss S, Schuppe HC, Schill WB (2004) The male reproductive system and its susceptibility to endocrine disrupting chemicals. Andrologia 36:337-345

Phillips DI (1998) Birth weight and the future development of diabetes. A review of the evidence. Diabetes Care 21 (suppl. 2):B150-B155

Phillips DIW, Handelsman DJ, Eriksson JG, Forsén T, Osmond C, Barker DJP (2001) Prenatal growth and subsequent marital status: longitudinal study. British Medical Journal 322:771

Popken G, Sommerkamp H, Wetterauer U (1999) Erektions- und Ejakulationsstörungen nach tumorchirurgischen Eingriffen. Reproduktionsmedizin 15:378-384

Popken G, Wetterauer U, Schultze-Seemann W (1998) Obstruktive Azoospermie. Reproduktionsmedizin 14:66-74

Pressat R (1973) Surmortalité biologique et surmortalité sociale. Revue française de Sociologie 14 (numéro speciale):103-110

Preston S, Heuveline P, Guillot M (2001) Demography: measuring and modeling population processes. Blackwell, Oxford

Redmon JB, Carey P, Pryor JL (2002) Varicocele - the most common cause of male factor infertility? Human Reproduction Update 8:53-58

Reijo E, Lee TY, Salo P, Alagappan RK, Brown LG, Rosenberg M, Rozen S, Jaffe T, Straus D, Hovatta O, de la Chapelle A, Silber S, Page DC (1995) Diverse spermatogenic defects in humans caused by $Y$ chromosome deletions encompassing a novel RNAbinding protein gene. Nature Genetics 10:383-393

Richthoff J, Rylander L, Hagmar L, Malm J, Giwercman A (2002) Higher sperm counts in Southern Sweden compared with Denmark. Human Reproduction 17:2468-2473

Rieker PP, Fitzgerald EM, Kalish LA (1990) Adaptive behavioral responses to potential infertility among survivors of testis cancer. Journal of Clinical Oncology 8:347-355

Riessman CK, Gerstel N (1985) Marital dissolution and health: do males or females have greater risk? Social Science \& Medicine 20:627-635

Ringbäck Weitoft G, Burström B, Rosén M (2004) Premature mortality among lone fathers and childless men. Social Science \& Medicine 59:1449-1459

Rojansky N, Brzezinski A, Schenker JG (1992) Seasonality in human reproduction: an update. Human Reproduction 7:735-745

Rolf C, Nieschlag E (2000) Seneszenz. In: Nieschlag E, Behre HM (eds) Andrologie. Springer, Berlin, pp 465-479

Rossow I, Amundsen A (1997) Alcohol abuse and mortality: a 40-year prospective study of Norvegian conscripts. Social Science \& Medicine 44:261-267

Roth C, Partsch C-J (1999) Störungen der Pubertät. Reproduktionsmedizin 15:18-28

Sachs L (2004) Angewandte Statistik. Springer, Berlin

Saleh SA, Agarwal A, Sharma RK, Nelson DR, Thomas AJ (2002) Effect of cigarette smoking on levels of seminal oxidative stress in infertile men: a prospective study. Fertility and Sterility 78:491-499

Samaras TT, Elrick H, Storms LH (2003) Is height related to longevity? Life Sciences 72:1781-1802

Samaras TT, Storms LH, Elrick H (2002) Longevity, mortality and body weight. Ageing Research Reviews 1:673-691

Schmidt B (2002) Gesundheit und Krankheit im Erwachsenenalter. In: Hurrelmann K, Kolip P (eds) Geschlecht, Gesundheit und Krankheit. Huber, Bern, pp 191-205

Schreiber G, Wilmer A (1998) Varikozele und testikuläre Funktionsstörungen? Reproduktionsmedizin 14:251-256

Schuermann H (1948) Über die Zunahme männlicher Fertilitätsstörungen und über die Bedeutung psychischer Einflüsse für die zentralnervöse Regulation der Spermiogenese. Medizinische Klinik 13:366-368 
Seitz J (1998) Nebenhoden und Samenleiter. In: Krause W, Weidner W (eds) Andrologie. Enke, Stuttgart, pp 14-17

Shah K, Sivapalan G, Gibbons N, Tempest H, Griffin DK (2003) The genetic basis of infertility. Reproduction 126:13-25

Shaheen SO, Sterne JA, Montgomery SM, Azima H (1999) Birth weight, body mass index and asthma in young adults. Thorax 54:396-402

Sharpe RM (2000) Environment, lifestyle and male infertility. Baillière's Clinical Endocrinology and Metabolism 14:489-503

Sharpe RM (2003) The 'oestrogen hypothesis' - where do we stand now? International Journal of Andrology 26:2-15

Sharpe RM, Franks S (2002) Environment, lifestyle and infertility - an inter-generational issue. Nature Cell Biology 4 (suppl.):s33-s40

Sharpe RM, McKinnell C, Kivlin C, Fisher JS (2003) Proliferation and functional maturation of Sertoli cells, and their relevance to disorders of testis function in adulthood. Reproduction 125:769-784

Sharpe RM, Skakkebæk NE (1993) Are oestrogens involved in falling sperm counts and disorders of the female reproductive tract? Lancet 341:1392-1395

Slama R, Eustache F, Ducot B, Jensen TK, Jørgensen N, Horte A, Irvine S, Suominen J, Andersen AG, Auger J, Vierula M, Toppari J, Andersen AN, Keiding N, Skakkebæk NE, Spira A, Jouannet $P$ (2002) Time to pregnancy and semen parameters: a crosssectional study among fertile couples from four European cities. Human Reproduction 17:503-515

Statistisches Bundesamt (1971) Internationale Standardklassifikation der Berufe. Übersetzung der "International Standard Classification of Occupation" des Internationalen Arbeitsamtes, Genf,1968. Kohlhammer, Stuttgart

Statistisches Bundesamt (1972) Bevölkerung und Wirtschaft 1872-1972. Kohlhammer, Stuttgart

Statistisches Bundesamt (1992) Klassifizierung der Berufe - Systematisches und alphabetisches Verzeichnis der Berufsbenennungen, Ausgabe 1992. Metzler Poeschel, Stuttgart

Statistisches Bundesamt (2004a) Datenreport 2004, Bonn

Statistisches Bundesamt (2004b) Gesundheitsberichterstattung des Bundes: Sterbefälle (ab 1998). http://www.gbe-bund.de/cgi-express/oorwaro/ExpSrv634/dbxwdekit/xwd_init? isgbetol/xs_start/34643263/13257713

Steenland K, Roodriguez C, Mondul A, Calle EE, Thun M (2004) Prostate cancer incidence and survival in relation to education (United States). Cancer Causes and Control 15:939-945

Steger K (2001) Spermatogenese und Spermatogenesestörungen. Reproduktionsmedizin 17:137-148

Stevens J, Cai J, Evenson KR, Thomas R (2002) Fitness and fatness as predictors of mortality from all causes and from cardiovascular disease in men and women in the Lipid Research Clinics Study. American Journal of Epidemiology 156:832-841

Stieve H (1952) Der Einfluss des Nervensystems auf Bau und Tätigkeit der Geschlechtsorgane des Menschen. Thieme, Stuttgart

Stillion JM (1995) Premature death among males. In: Sabo D, Gordon DF (eds) Men's Health and IIIness. Sage Publications, Thousand Oaks, pp 46-67

Stolpe S (2001) Schulbildung/berufliche Ausbildung und Gesundheitszustand. In: Mielck A, Bloomfield K (eds) Sozial-Epidemiologie. Juventa, Weinheim, pp 17-27

Stoorgard L, Peter Bonde J, Ernst E, Spanô M, Yding Andersen C, Frydenberg M, Olsen J (2003) Does smoking during pregnancy affect son's sperm counts? Epidemiology 14:278-286

Storey AE, Walsh CJ, Quinton RL, Wynne-Edwards KE (2000) Hormonal correlates of paternal responsiveness in new and expectant fathers. Evolution and Human Behavior 21:79-95

Strauß B, Beyer K (2004) Ungewollte Kinderlosigkeit. Gesundheitsberichterstattung des Bundes, Heft 20. Statistisches Bundesamt, Bonn 
Strauß B, Brähler E, Kentenich H (2004) Fertilitätsstörungen - Psychosomatisch orientierte Diagnostik und Therapie. Leitlinie und Quellentext. Schattauer, Stuttgart

Stuhrmann M (1998) Das klinische Spektrum von Fertilitätsstörungen durch Mutationen im CFTR-Gen. Reproduktionsmedizin 14:54-65

Swerdlow AJ, Schoemaker MJ, Higgins CD, Wright AF, Jacobs PA (2005) Cancer incidence and mortality in men with Klinefelter syndrome: a cohort study. Journal of the National Cancer Institute 97:1204-1210

Takihara $\mathrm{H}$ (1998) The treatment of obstructive azoospermia in male infertility - past, present, and future. Urology 51 (suppl. 5a):150-155

Tan Q, de Benedictis G, Yashin AL, Bonafè M, DeLuca M, Valensin S, Vaupel JW, Franceschi C (2001) Measuring the genetic influence in modulating the human lifespan: gene - environment interaction and the sex-specific genetic effect. Biogerontology 2:141-153

The Practice Commitee of the American Society for Reproductive Medicine (2004) Smoking and infertility. Fertility and Sterility 82 (suppl. 1):S62-S67

Thomas F, Teriokhin AT, Renaud F, de Meeûs T, Guégan JF (2000) Human longevity at the cost of reproductive success: evidence from global data. Journal of Evolutionary Biology 13:409-414

Thomas VS, Rockwood KJ (2001) Alcohol abuse, cognitive impairment, and mortality among older people. Journal of the American Geriatrics Society 49:415-420

Thonneau P, Bujan L, Multigner L, Mieusset R (1998) Occupational heat exposure and male fertility: a review. Human Reproduction 13:2122-2125

Tomassini C, Billari FC, Rosina A, Christensen K (2001) Born together - die together. Live together - die together. The role of the partner and the co-twin on longevity at very old ages. Genus:63-82

Tomassini C, Kalogirou S, Grundy E, Fokkema T, Martikainen P, Broese van Groenou MI, Karisto A (2004) Contacts between elderly parents and their children in four Europaen countries: current patterns and future prospects. European Journal of Aging 1:54-63

Traggiai C, Stanhope R (2002) Delayed puberty. Best Practice \& Research Clinical Endocrinology and Metabolism 16:139-151

Troen BR (2003) The biology of aging. The Mount Sinai Journal of Medicine 70:3-22

Trummer H, Habermann H, Haas J, Pummer K (2002) The impact of cigarette smoking on human semen parameters and hormones. Human Reproduction 17:1554-1559

Umberson D (1987) Family status and health behaviors: social control as a dimension of social integration. Journal of Health and Social Behavior 28:306-319

van Ahlen H, Hertle L (2000) Störungen der Samendeposition. In: Nieschlag E, Behre HM (eds) Andrologie. Springer, Berlin, pp 211-245

van Assche E, Bonduelle M, Tournaye H, Joris H, Verheyen G, Devroey P, van Steirteghem A, Liebaers I (1996) Cytogenetics of infertile men. Human Reproduction 11 (suppl 4):124

Vaupel JW, Carey JR, Christensen K, Johnston TE, Yashin AL, Holm NV, lachine IA, Kannisto V, Khazaeli AA, Liedo P, Longo VD, Zeng Y, Manton KG, Curtsinger JW (1998) Biodemographic trajectories of longevity. Science 280:855-860

Vaupel JW, Carey JR, Christensen K (2003) It's never too late. Science 301, 1679-1681

Vine MF, Margolin BH, Morrison HI, Hulka BS (1994) Cigarette smoking and sperm density: a meta analysis. Fertility and Sterility 61:35-43

Vine MF, Tse CKJ, Hu PC, Truong KY (1996) Cigarette smoking and semen quality. Fertility and Sterility $65: 835-842$

Voges W (1996) Ungleiche Voraussetzungen für Langlebigkeit - Bestimmungsgründe für Mortalität im zeitlichen Verlauf. Zeitschrift für Gerontologie und Geriatrie 29:18-22

Voges W, Schmidt C (1996) Lebenslagen, die Lebenszeit kosten - Zum Zusammenhang von sozialer Lage, chronischer Erkrankung und Mortalität im zeitlichen Verlauf. In: Zapf W, Schupp J, Habich R (eds) Lebenslagen im Lebenswandel: Sozialberichterstattung im Längsschnitt. Campus, Frankfurt, pp 378-401

Vogt PH (2005) AZF deletions and Y chromosomal haplogroups: history and update based on sequence. Human Reproduction Update 11:319-336 
Waldron I (1983) Sex differences in human mortality: the role of genetic factors. Social Science \& Medicine 17:321-333

Waldron I (1993) Recent trends in sex mortality ratios for adults in developed countries. Social Science \& Medicine 36:451-462

Waldron I (1995) Contributions of changing gender differences in behavior and social roles to changing gender differences in mortality. In: Sabo D, Gordon DF (eds) Men's health and illness. Sage Publications, Thousand Oaks, pp 22-45

Waldstreicher J, Seminara SB, Jameson JL, Geyer A, Nachtigall LB, Boepple PA, Holmes LB, Crowley WF (1996) The genetic and clinical heterogeneity of gonadotropinreleasing hormone deficiency in the human. Journal of Clinical Endocrinology and Metabolism 81:4388-4395

Wallock LM, Tamura T, Mayr CA, Johnston KE, Ames BN, Jacob RA (2001) Low seminal plasma folate concentrations are associated with low sperm density and count in male smokers and nonsmokers. Fertility and Sterility 75:252-259

Walter KN (2004) Beruflicher Erfolg und Lebenserwartung. Eine Untersuchung an Absolventen der US Military Academy Westpoint der Jahrgänge 1925 und 1950. Unveröffentlichte Inauguraldissertation, Philipps-Universität, Marburg

Watkins KJ, Baldo TD (2004) The infertility experience: biopsychosocial effects and suggestions for counselors. Journal of Counseling and Development 82:394-402

Weidner W, Krause W, Ludwig M (1999) Relevance of male accessory gland infection for subsequent fertility with special focus on prostatitis. Human Reproduction Update $5: 421-432$

Weilandt C, Huismann A, Joksimovic L, Klaes L, van den Toorn S, Winkler J (2000) Gesundheit von Zuwanderern in Nordrhein-Westfalen. Ministerium für Frauen, Jugend, Familie und Gesundheit des Landes Nordrhein-Westfalen, Düsseldorf

Weinbauer GF, Gromoll J, Simoni M, Nieschlag E (2000) Physiologie der Hodenfunktion. In: Nieschlag E, Behre HM (eds) Andrologie. Springer, Berlin, pp 27-67

Weinert BT, Timiras PS (2003) Physiology of aging. Invited reviews: Theories of aging. Journal of Applied Physiology 95:1706-1716

Weiske W-H (1998) Vasoresektion. In: Krause W, Weidner W (eds) Andrologie. Enke, Stuttgart, pp 308-312

Wennemuth G, Aumüller G (1998) Endokrine Organe. In: Krause W, Weidner W (eds) Andrologie. Enke, Stuttgart, pp 1-9

Westendorp RGJ, Kirkwood TBL (1998) Human longevity at the cost of reproductive success. Nature 396:743-746

Westendorp RGJ, van Dunne FM, Kirkwood TBL, Helmerhorst FM, Huizinga TWJ (2001) Optimizing human fertility and survival. Nature Medicine 7:873

Whitlock G, Norton R, Clark T, Jackson R, MacMahon S (2004) Motor vehicle driver injury and marital status: a cohort study with prospective driver injuries. Injury Prevention 10:33-36

Williams K, Umberson D (2004) Marital status, marital transitions, and health: a gendered life course perspective. Journal of Health and Social Behavior 45:81-98

Wilmoth JR (2000) Demography of longevity: past, present, and future trends. Experimental Gerontology 35:1111-1129

Wineberg $\mathrm{H}$ (1988) Duration between marriage and first birth and marital stability. Social Biology 35:92-102

Wischmann T (2005) Psychosoziale Aspekte bei Fertilitätsstörungen. Der Urologe [A] 44:185-195

Wischmann T, Stammer H, Scherg H, Gerhard I, Verres R (2001) Psychosocial characteristics of infertile couples: a study by the 'Heidelberger Fertility Consultation Service'. Human Reproduction 16:1753-1761

Wittwer-Backofen U (1999) Disparitäten der Alterssterblichkeit im regionalen Vergleich. Biologische versus sozioökonomische Determinanten. Materialien zur Bevölkerungswissenschaft 95. Bundesinstitut für Bevölkerungsforschung beim Statistischen Bundesamt, Wiesbaden 
Wong WY, Zielhuis GA, Thomas CMG, Merkus HMWM, Steegers-Theunissen RPM (2003) New evidence of the influence of exogenous and endogenous factors on sperm count in man. European Journal of Obstetrics \& Gynecology and Reproductive Biology $110: 49-54$

Wood J, Holman D, O'Connor K, Ferrell R (2001) Mortality models for paleodemography. Center for Studies in Demography and Ecology at the University of Washington, CSDE Working Paper Series. http://csde.washington.edu/pubs/wps01-02.pdf

World Health Organisation (1992) WHO laboratory manual for the examination of human semen and sperm-cervical mucus interaction, 3 edn. Cambridge University Press, Cambridge

World Health Organisation (1994) WHO position paper on health, population and development, Cairo 5-13 September 1994.

World Health Organisation (1999) WHO laboratory manual for the examination of human semen and sperm-cervical mucus interaction, 4 edn. Cambridge University Press, Cambridge

World Health Organisation (2003) International Statistical Classification of Diseases and Related Health Problems $\left(10^{\text {th }}\right.$ revision): Certain conditions originating in the perinatal period (P00-P96). http://www3.who.int/icd/vol1htm2003/fr-icd.htm

World Health Organisation - Task Force on the Diagnosis and Treatment of Infertility (1987) Towards more objectivity in diagnosis and management of male infertility. International Journal of Andrology 7 (suppl.):1-53

World Health Organisation - Task Force on the Diagnosis and Treatment of Infertility (1992) The influence of varicocele on parameters of fertility in a large group of men presenting to infertility clinics. Fertility and Sterility $57: 1289-1293$

Yashin AL, lachine IA, Begun AS (2000) Mortality modeling: a review. Mathematical Population Studies 8:305-322

Yi Z, Vaupel JW (2004) Association of late childbearing with healthy longevity among the oldest-old in China. Population Studies 58:37-53

Yogev L, Kleiman SE, Shabtai E, Botchan A, Gamzu R, Paz G, Yavetz H, Hauser R (2004) Seasonal variations in pre- and post-thaw donor sperm quality. Human Reproduction 19:880-885

Yong EL, Loy CJ, Sim KS (2003) Androgen receptor gene and male infertility. Human Reproduction Update 9:1-7

Zavos PM, Correa JR, Karagounis CS, Ahparaki A, Phoroglou C, Hicks C, ZarmakoupisZavos PN (1998) An electron microscope study of the axonemal ultrastructure in human spermatozoa from male smokers and nonsmokers. Fertility and Sterility 69:430-434

Zhang Z, Hayward MD (2001) Childlessness and the psychological well-being of older persons. Journal of Gerontology 56B:S311-S320

Zorn B, Virant-Klun I, Verdenk I, Meden-Vrtovec H (1999) Semen quality changes among 2343 healthy Slovenian men included in an IVF-ET programme from 1983 to 1996. International Journal of Andrology 22:178-183 


\section{Anhang}

\section{A-1 Sozioökonomischer Index des beruflichen Status (ISEI)}

Tab. A-1 Berufe und zugehörige ISEI-Werte der Fälle

\begin{tabular}{|c|c|}
\hline Berufsbezeichnung(en) & ISEI \\
\hline Hilfsarbeiter ohne nähere Tätiakeitsanaabe & 20.0 \\
\hline Arbeiter/Hochschule & 20.0 \\
\hline Arbeiter/Ausb.unbk. & 20.0 \\
\hline Facharb./Fachsch. & 20.0 \\
\hline Laaer-. Transportarbeiter & 22.0 \\
\hline Geldeinnehmer-auszahler. Kartenverkäufer. -kontrolleur & 22.4 \\
\hline Raum-. Hausratreiniaer & 22.7 \\
\hline Straßenreiniaer. Abfallbeseitiaer & 22.7 \\
\hline Maschinen-. Behälterreiniaer und verwandte Berufe & 22.7 \\
\hline Waldarbeiter. Waldnutzer & 25.6 \\
\hline Landarbeitskraft & 29.2 \\
\hline Kutscher & 30.2 \\
\hline Straßenwart & 31.4 \\
\hline Straßenbauer & 31.8 \\
\hline Erdbeweaunasmaschinenführer & 31.8 \\
\hline Baumaschinenführer & 31.8 \\
\hline Warenmaler. -lackierer & 32.9 \\
\hline Textilfärber & 33.9 \\
\hline Textilausrüster & 33.9 \\
\hline Heizer & 33.9 \\
\hline Grobwarenlederhersteller. Bandaaist & 34.9 \\
\hline Tierbfleaer und verwandte Berufe & 35.2 \\
\hline TransDortaeräteführer & 36.8 \\
\hline Weber & 37.6 \\
\hline Schweisser. Brennschneider & 37.8 \\
\hline Steinbrecher & 38.0 \\
\hline Papier-. Zellstoffhersteller & 38.0 \\
\hline Sonstiae Pabierverarbeiter & 38.0 \\
\hline Galvaniseur. Metallfärber & 38.0 \\
\hline Emallierer. Feuerverzinker und andere Metalloberflächenveredler & 38.0 \\
\hline Schuhwarenhersteller & 38.0 \\
\hline Werkzeuamacher & 38.1 \\
\hline Übriae Gästebetreuer & 38.1 \\
\hline Pförtner. Hauswart & 38.6 \\
\hline Haus-. Gewerbediener & 38.6 \\
\hline Steinbearbeiter & 39.1 \\
\hline Fördermaschinist. Seilbahnmaschinist & 39.2 \\
\hline Betonbauer & 39.3 \\
\hline Holzaufbereiter & 39.4 \\
\hline Maschenwarenfertiaer & 39.4 \\
\hline Spezialdrucker. Siebdrucker & 39.5 \\
\hline Druckerhelfer & 39.5 \\
\hline Eneraiemaschinist & 39.8 \\
\hline Bauschlosser & 39.9 \\
\hline Stahlbauschlosser. Eisenschiffbauer & 39.9 \\
\hline Pupbenmacher. Modellbauer. Präbarator & 39.9 \\
\hline Warenorüfer. -sortierer. a.n.a. & 39.9 \\
\hline
\end{tabular}




\begin{tabular}{|c|c|}
\hline 7immerer & $\Delta \cap \Delta$ \\
\hline Kultur-. Wasserbauwerker & 40.4 \\
\hline SonstigeTiefbauer & 40,4 \\
\hline Bauhilfsarbeiter & 40.4 \\
\hline Modelltischler. Formentischler & 40.5 \\
\hline Formstein-. Betonhersteller & 40.9 \\
\hline Kunststoffverarbeiter & 40.9 \\
\hline Blech-. Kunststoffschlosser & 40.9 \\
\hline Dreher & 41.4 \\
\hline Fräser & 41.4 \\
\hline Hobler & 41.4 \\
\hline Bohrer & 41.4 \\
\hline Maschinenschlosser & 41.4 \\
\hline Betriebsschlosser. Renaraturschlosser & 41.4 \\
\hline Maschineneinrichter o.n.A. & 41.4 \\
\hline Verkäufer & 42.1 \\
\hline Branntsteinhersteller & 42.3 \\
\hline Dachdecker & 42.3 \\
\hline Stukkateure. Gipser. Verputzer & 42.3 \\
\hline Laaerverwalter. Maaaziner & 43.0 \\
\hline Rohrinstallateur & 43.4 \\
\hline Rohrnetzbauer. Rohrschlosser & 43.4 \\
\hline Kraftfahrzeuaführer & 43.9 \\
\hline Maurer & 44.7 \\
\hline Pflasterer. Steinsetzer & 44.7 \\
\hline Ofensetzer. Luftheizunasbauer & 44.7 \\
\hline Wächter. Aufseher & 45.0 \\
\hline Rechtsvollstrecker & 45.0 \\
\hline Artist. Berufssportler. künstlerische Hilfskraft & 45.4 \\
\hline Friseur & 46.0 \\
\hline Brauer. Mälzer & 47.0 \\
\hline Milch. Fettverarbeiter & 47.0 \\
\hline Gesundheitssichernder Beruf & 47.0 \\
\hline Vervielfältiaer & 48.4 \\
\hline Maler. Lackierer (Ausbau) & 48.7 \\
\hline Elektroaeräte-. Elektroteilemontierer & 49.9 \\
\hline Elektroinstallateur. -monteur & 50.0 \\
\hline Tischler & 50.5 \\
\hline Telefonist & 50.8 \\
\hline Former. Kernmacher & 50.9 \\
\hline Fleischer & 50.9 \\
\hline Schriftsetzer & 51.6 \\
\hline Blechoresser-. zieher-. stanzer & 51.8 \\
\hline sonstiae Metallverformer (sDanlose Verformuna) & 51.8 \\
\hline Polsterer. Matratzenhersteller & 51.9 \\
\hline Landwirt & 53.6 \\
\hline Halbzeuabutzer und sonstiae Formaießberufe & 53.8 \\
\hline Metallveraüter & 53.8 \\
\hline Metallarbeiter. o.n.A. & 53.8 \\
\hline Gärtner. Gartenarbeiter & 54.1 \\
\hline Techniker des Elektrofaches & 55.6 \\
\hline Forstverwalter. Förster. Jäaer & 56.4 \\
\hline Helfer in der Krankennfleae & 56.6 \\
\hline Stenoaraph. Stenotvpist. Maschinenschreiber & 57.8 \\
\hline
\end{tabular}




\begin{tabular}{|c|c|}
\hline Seelsorge-, Kulthelfer & 58,6 \\
\hline Industriemeister. Werkmeister & 59.6 \\
\hline Meister/Abitur & 59,6 \\
\hline Meister/Fachsch. & 59.6 \\
\hline Technischer Zeichner & 61.3 \\
\hline Datenverarbeitunasfachkraft & 61.3 \\
\hline Buchhalter & 61.4 \\
\hline Warenaufmacher. Versandfertiamacher & 61.6 \\
\hline Bankfachkraft & 65.5 \\
\hline Eisen-. Metallerzeuaer. Schmelzer & 67.4 \\
\hline Vermieter. Vermittler. Versteiaerer & 67.4 \\
\hline Arbeitskraft ohne nähere Tätiakeitsanaabe & 68.3 \\
\hline Übriae Fertiaunastechniker & 69.9 \\
\hline Sonstiae Techniker & 69.9 \\
\hline Tankwart & 71.2 \\
\hline Maschinenbautechniker & 73.7 \\
\hline Bibliothekar. Archivar. Museumsfachkraft & 78.7 \\
\hline Musiker & 85.4 \\
\hline Bautechniker & 85.8 \\
\hline Vermessunastechniker & 85.8 \\
\hline Handelsvertreter. Reisender & 86.8 \\
\hline Bioloaisch-technische Sonderfachkraft & 90.1 \\
\hline Real-. Volks-. Sonderschullehrer & 93.4 \\
\hline Bürofachkraft & 93.5 \\
\hline Groß- und Einzelhandelskaufmann. Einkäufer & 97.5 \\
\hline Inaenieur des Maschinen- und Fahrzeuabaues & 100.7 \\
\hline Unternehmer. Geschäftsführer. Geschäftsbereichsleiter & 103.0 \\
\hline Auaenontiker & 105.4 \\
\hline Berabau-. Hütten-. Gießereiinaenieur & 105.4 \\
\hline Apotheker & 111.4 \\
\hline Abaeordneter. Minister. Wahlbeamter & 111.6 \\
\hline Sonstiae Lehrer & 129.1 \\
\hline Architekt. Bauinaenieur & 134.1 \\
\hline
\end{tabular}


A-2 Alternativmodell 1: Alter bei Untersuchung als zeitabhängige Variable

Tab. A-2 Cox-Regression zum Einfluss des Fertilitätsstatus (fertil/subfertil) auf die Lebensdauer: Alter bei Untersuchung als zeitabhängige Variable ${ }^{a}$

\begin{tabular}{|c|c|c|c|c|c|c|c|c|}
\hline & \multirow{2}{*}{ B } & \multirow{2}{*}{ SE } & \multirow{2}{*}{ Wald } & \multirow{2}{*}{ df } & \multirow{2}{*}{$\mathbf{p}$} & \multirow{2}{*}{$\begin{array}{l}\text { Odds } \\
\text { Ratio }\end{array}$} & \multicolumn{2}{|c|}{ 95\% Cl for OR } \\
\hline & & & & & & & Lower & Upper \\
\hline subfertil vs. fertil & 0,284 & 0,186 & 2,339 & 1 & 0,126 & 1,329 & 0,923 & 1,913 \\
\hline Lebenserwartung bei Geburt & 0,159 & 0,108 & 2,179 & 1 & 0,140 & 1,172 & 0,949 & 1,447 \\
\hline Geburtsmonat & 0,006 & 0,026 & 0,054 & 1 & 0,816 & 1,006 & 0,956 & 1,059 \\
\hline Alter bei Untersuchung & 0,001 & 0,001 & 1,768 & 1 & 0,184 & 1,001 & 0,999 & 1,003 \\
\hline Untersuchungsjahr & $-0,063$ & 0,056 & 1,268 & 1 & 0,260 & 0,939 & 0,842 & 1,048 \\
\hline ISEI & $-0,003$ & 0,005 & 0,301 & 1 & 0,583 & 0,997 & 0,988 & 1,007 \\
\hline
\end{tabular}

${ }^{a}$ unzensierte Fälle $n=125$; zensierte Fälle $n=336$

Tab. A-3 Cox-Regression zum Einfluss des Fertilitätsstatus (fertil/subfertil) auf die Lebensdauer: Alter bei Untersuchung als zeitabhängige Variable ${ }^{a}$, frühe Geburtsjahrgänge

\begin{tabular}{|c|c|c|c|c|c|c|c|c|}
\hline & \multirow{2}{*}{ B } & \multirow{2}{*}{ SE } & \multirow{2}{*}{ Wald } & \multirow{2}{*}{ df } & \multirow{2}{*}{$\mathbf{p}$} & \multirow{2}{*}{$\begin{array}{l}\text { Odds } \\
\text { Ratio }\end{array}$} & \multicolumn{2}{|c|}{$95 \% \mathrm{Cl}$ for OR } \\
\hline & & & & & & & Lower & Upper \\
\hline Lebenserwartung bei Geburt & 0,278 & 0,182 & 2,334 & 1 & 0,127 & 1,321 & 0,924 & 1,888 \\
\hline Geburtsmonat & 0,006 & 0,033 & 0,036 & 1 & 0,849 & 1,006 & 0,944 & 1,073 \\
\hline Untersuchungsjahr & $-0,151$ & 0,112 & 1,805 & 1 & 0,179 & 0,860 & 0,690 & 1,072 \\
\hline ISEI & 0,009 & 0,006 & 2,566 & 1 & 0,109 & 1,009 & 0,998 & 1,020 \\
\hline
\end{tabular}

${ }^{a}$ unzensierte Fälle $n=84$; zensierte Fälle $n=136$

Tab. A-4 Cox-Regression zum Einfluss des Fertilitätsstatus (fertil/subfertil) auf die Lebensdauer: Alter bei Untersuchung als zeitabhängige Variable ${ }^{a}$, späte Geburtsjahrgänge

\begin{tabular}{|c|c|c|c|c|c|c|c|c|}
\hline & \multirow{2}{*}{ B } & \multirow{2}{*}{ SE } & \multirow{2}{*}{ Wald } & \multirow{2}{*}{ df } & \multirow{2}{*}{$p$} & \multirow{2}{*}{$\begin{array}{l}\text { Odds } \\
\text { Ratio }\end{array}$} & \multicolumn{2}{|c|}{$95 \% \mathrm{Cl}$ for OR } \\
\hline & & & & & & & Lower & Upper \\
\hline subfertil vs. fertil & 0,009 & 0,345 & 0,001 & 1 & 0,979 & 1,009 & 0,513 & 1,985 \\
\hline Lebenserwartung bei Geburt & $-0,286$ & 0,788 & 0,132 & 1 & 0,717 & 0,751 & 0,160 & 3,520 \\
\hline Geburtsmonat & 0,013 & 0,046 & 0,074 & 1 & 0,786 & 1,013 & 0,925 & 1,109 \\
\hline Alter bei Untersuchung & 0,002 & 0,002 & 1,060 & 1 & 0,303 & 1,002 & 0,998 & 1,005 \\
\hline Untersuchungsjahr & $-0,101$ & 0,098 & 1,046 & 1 & 0,306 & 0,904 & 0,746 & 1,097 \\
\hline ISEI & $-0,025$ & 0,009 & 6,990 & 1 & 0,008 & $0,975^{\star *}$ & 0,957 & 0,994 \\
\hline
\end{tabular}

anzensierte Fälle $n=41$; zensierte Fälle $n=199$ 
Tab. A-5 Cox-Regression zum Einfluss des Fertilitätsstatus (normo-loligo-lazoosperm) auf die Lebensdauer: Alter bei Untersuchung als zeitabhängige Variable ${ }^{\text {a }}$

\begin{tabular}{|c|c|c|c|c|c|c|c|c|}
\hline & B & SE & Wald & df & $\mathbf{p}$ & $\begin{array}{l}\text { Odds } \\
\text { Ratio }\end{array}$ & \multicolumn{2}{|c|}{$95 \% \mathrm{Cl}$ for $\mathrm{OR}$} \\
\hline azoo- vs. normozoosperm & 0,175 & 0,261 & 0,451 & 1 & 0,502 & 1,192 & 0,714 & 1,988 \\
\hline oligo- vs. azoosperm & 0,182 & 0,296 & 0,378 & 1 & 0,539 & 1,200 & 0,671 & 2,145 \\
\hline Geburtsmonat & 0,005 & 0,026 & 0,042 & 1 & 0,838 & 1,005 & 0,955 & 1,059 \\
\hline Alter bei Untersuchung & 0,001 & 0,001 & 1,750 & 1 & 0,186 & 1,001 & 0,999 & 1,003 \\
\hline Untersuchungsjahr & $-0,064$ & 0,056 & 1,295 & 1 & 0,255 & 0,938 & 0,841 & 1,047 \\
\hline
\end{tabular}

anzensierte Fälle $n=125$; zensierte Fälle $n=336$

Tab. A-6 Cox-Regression zum Einfluss des Fertilitätsstatus (normo-loligo-lazoosperm) auf die

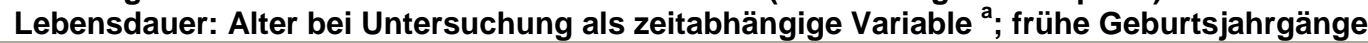

\begin{tabular}{|c|c|c|c|c|c|c|c|c|}
\hline & \multirow{2}{*}{ B } & \multirow{2}{*}{ SE } & \multirow{2}{*}{ Wald } & \multirow{2}{*}{ df } & \multirow{2}{*}{$\mathbf{p}$} & \multirow{2}{*}{$\begin{array}{l}\text { Odds } \\
\text { Ratio }\end{array}$} & \multicolumn{2}{|c|}{$95 \% \mathrm{Cl}$ for OR } \\
\hline & & & & & & & Lower & Upper \\
\hline oligo- vs. normozoosperm & 0,733 & 0,260 & 7,933 & 1 & 0,005 & $2,082^{* *}$ & 1,250 & 3,467 \\
\hline azoo- vs. normozoosperm & 0,204 & 0,319 & 0,408 & 1 & 0,523 & 1,226 & 0,656 & 2,291 \\
\hline oligo- vs. azoosperm & 0,529 & 0,353 & 2,256 & 1 & 0,133 & 1,698 & 0,851 & 3,388 \\
\hline Lebenserwartung bei Geburt & 0,286 & 0,184 & 2,420 & 1 & 0,120 & 1,331 & 0,928 & 1,907 \\
\hline Geburtsmonat & 0,005 & 0,033 &, 020 & 1 & 0,889 & 1,005 & 0,942 & 1,071 \\
\hline Alter bei Untersuchung & 0,002 & 0,002 & 2,282 & 1 & 0,131 & 1,002 & 0,999 & 1,006 \\
\hline Untersuchungsjahr & $-0,156$ & 0,113 & 1,920 & 1 & 0,166 & 0,855 & 0,685 & 1,067 \\
\hline ISEI & 0,010 & 0,006 & 2,941 & 1 & 0,086 & $1,010^{(*)}$ & 0,999 & 1,021 \\
\hline
\end{tabular}

a unzensierte Fälle $n=84$; zensierte Fälle $n=136$

Tab. A-7 Cox-Regression zum Einfluss des Fertilitätsstatus (normo-/oligo-/azoosperm) auf die Lebensdauer: dem Alter bei Untersuchung als zeitabhängige Variable ${ }^{\text {a }}$; späte Geburtsjahrgänge

\begin{tabular}{|c|c|c|c|c|c|c|c|c|}
\hline & \multirow{2}{*}{ B } & \multirow{2}{*}{ SE } & \multirow{2}{*}{ Wald } & \multirow{2}{*}{ df } & \multirow{2}{*}{$\mathbf{p}$} & \multirow{2}{*}{$\begin{array}{l}\text { Odds } \\
\text { Ratio }\end{array}$} & \multicolumn{2}{|c|}{$95 \% \mathrm{Cl}$ for OR } \\
\hline & & & & & & & Lower & Upper \\
\hline oligo- vs. normozoosperm & $-0,174$ & 0,432 & 0,162 & 1 & 0,688 & 0,840 & 0,360 & 1,961 \\
\hline azoo- vs. normozoosperm & 0,272 & 0,459 & 0,350 & 1 & 0,554 & 1,312 & 0,533 & 3,229 \\
\hline oligo- vs. azoosperm & $-0,445$ & 0,564 & 0,624 & 1 & 0,430 & 0,641 & 0,212 & 1,935 \\
\hline Lebenserwartung bei Geburt & $-0,256$ & 0,786 & 0,107 & 1 & 0,744 & 0,774 & 0,166 & 3,609 \\
\hline Geburtsmonat & 0,014 & 0,047 & 0,093 & 1 & 0,761 & 1,014 & 0,926 & 1,111 \\
\hline Alter bei Untersuchung & 0,002 & 0,002 & 1,199 & 1 & 0,274 & 1,002 & 0,999 & 1,005 \\
\hline Untersuchungsjahr & $-0,104$ & 0,098 & 1,130 & 1 & 0,288 & 0,902 & 0,745 & 1,091 \\
\hline ISEI & $-0,025$ & 0,010 & 6,861 & 1 & 0,009 & $0,975^{\star \star}$ & 0,957 & 0,994 \\
\hline
\end{tabular}

a unzensierte Fälle $n=41$; zensierte Fälle $n=199$ 


\section{A-3 Alternativ-Modell 2: Sexuelle Karenz als Kontrollvariable}

Tab. A-8 Cox-Regression zum Einfluss des Fertilitätsstatus (fertil/subfertil) auf die Lebensdauer unter Berücksichtigung der sexuellen Abstinenz ${ }^{a}$

\begin{tabular}{|c|c|c|c|c|c|c|c|c|}
\hline & \multirow{2}{*}{ B } & \multirow{2}{*}{ SE } & \multirow{2}{*}{ Wald } & \multirow{2}{*}{ df } & \multirow{2}{*}{$\mathbf{p}$} & \multirow{2}{*}{$\begin{array}{l}\text { Odds } \\
\text { Ratio }\end{array}$} & \multicolumn{2}{|c|}{$95 \% \mathrm{Cl}$ for OR } \\
\hline & & & & & & & Lower & Upper \\
\hline subfertil vs. fertil & 0,234 & 0,208 & 1,273 & 1 & 0,259 & 1,264 & 0,841 & 1,898 \\
\hline Lebenserwartung bei Geburt & 0,078 & 0,126 & 0,381 & 1 & 0,537 & 1,081 & 0,845 & 1,383 \\
\hline Geburtsmonat & 0,005 & 0,030 & 0,033 & 1 & 0,855 & 1,005 & 0,949 & 1,065 \\
\hline Alter bei Untersuchung & 0,029 & 0,065 & 0,207 & 1 & 0,649 & 1,030 & 0,907 & 1,169 \\
\hline Untersuchungsjahr & $-0,031$ & 0,062 & 0,250 & 1 & 0,617 & 0,969 & 0,858 & 1,095 \\
\hline sexuelle Abstinenz & 0,038 & 0,034 & 1,273 & 1 & 0,259 & 1,039 & 0,972 & 1,110 \\
\hline ISEI & $-0,005$ & 0,005 & 0,829 & 1 & 0,363 & 0,995 & 0,985 & 1,006 \\
\hline
\end{tabular}

a unzensierte Fälle $n=103$; zensierte Fälle $n=286$

Tab. A-9 Cox-Regression zum Einfluss des Fertilitätsstatus (fertil/subfertil) auf die Lebensdauer unter Berücksichtigung der sexuellen Abstinenz ${ }^{a}$, frühe Geburtsjahrgänge

\begin{tabular}{|c|c|c|c|c|c|c|c|c|}
\hline & \multirow{2}{*}{ B } & \multirow{2}{*}{ SE } & \multirow{2}{*}{ Wald } & \multirow{2}{*}{ df } & \multirow{2}{*}{ p } & \multirow{2}{*}{$\begin{array}{l}\text { Odds } \\
\text { Ratio }\end{array}$} & \multicolumn{2}{|c|}{$95 \% \mathrm{Cl}$ for OR } \\
\hline & & & & & & & Lower & Upper \\
\hline subfertil vs. fertil & 0,468 & 0,257 & 3,305 & 1 & 0,069 & $1,597^{(*)}$ & 0,964 & 2,645 \\
\hline Lebenserwartung bei Geburt & 0,213 & 0,257 & 0,690 & 1 & 0,406 & 1,238 & 0,748 & 2,048 \\
\hline Geburtsmonat & 0,014 & 0,037 & 0,147 & 1 & 0,701 & 1,014 & 0,943 & 1,091 \\
\hline Alter bei Untersuchung & 0,123 & 0,156 & 0,630 & 1 & 0,428 & 1,131 & 0,834 & 1,535 \\
\hline Untersuchungsjahr & $-0,116$ & 0,156 & 0,558 & 1 & 0,455 & 0,890 & 0,656 & 1,208 \\
\hline sexuelle Abstinenz & $-0,018$ & 0,042 & 0,174 & 1 & 0,676 & 0,983 & 0,905 & 1,067 \\
\hline ISEI & 0,004 & 0,007 & 0,323 & 1 & 0,570 & 1,004 & 0,991 & 1,017 \\
\hline
\end{tabular}

a unzensierte Fälle $n=68$; zensierte Fälle $n=110$

Tab. A-10 Cox-Regression zum Einfluss des Fertilitätsstatus (fertil/subfertil) auf die Lebensdauer unter Berücksichtigung der sexuellen Abstinenz ${ }^{a}$, späte Geburtsjahrgänge

\begin{tabular}{|c|c|c|c|c|c|c|c|c|}
\hline & \multirow{2}{*}{ B } & \multirow{2}{*}{ SE } & \multirow{2}{*}{ Wald } & \multirow{2}{*}{ df } & \multirow{2}{*}{$\mathbf{p}$} & \multirow{2}{*}{$\begin{array}{l}\text { Odds } \\
\text { Ratio }\end{array}$} & \multicolumn{2}{|c|}{$95 \% \mathrm{Cl}$ for OR } \\
\hline & & & & & & & Lower & Upper \\
\hline subfertil vs. fertil & $-0,072$ & 0,374 & 0,037 & 1 & 0,848 & 0,931 & 0,447 & 1,938 \\
\hline Lebenserwartung bei Geburt & $-0,786$ & 0,954 & 0,680 & 1 & 0,410 & 0,456 & 0,070 & 2,953 \\
\hline Geburtsmonat & 0,003 & 0,052 & 0,003 & 1 & 0,954 & 1,003 & 0,906 & 1,110 \\
\hline Alter bei Untersuchung & 0,093 & 0,123 & 0,572 & 1 & 0,449 & 1,097 & 0,862 & 1,397 \\
\hline Untersuchungsjahr & $-0,111$ & 0,126 & 0,777 & 1 & 0,378 & 0,895 & 0,699 & 1,146 \\
\hline sexuelle Abstinenz & 0,141 & 0,057 & 6,094 & 1 & 0,014 & $1,151^{*}$ & 1,029 & 1,287 \\
\hline ISEI & $-0,017$ & 0,010 & 2,877 & 1 & 0,090 & $0,983^{(*)}$ & 0,964 & 1,003 \\
\hline
\end{tabular}

a unzensierte Fälle $n=35$; zensierte Fälle $n=175$ 
Tab. A-11 Cox-Regression zum Einfluss des Fertilitätsstatus (normo-/oligo-/azoosperm) auf die Lebensdauer unter Berücksichtigung der sexuellen Abstinenz ${ }^{a}$

\begin{tabular}{|c|c|c|c|c|c|c|c|c|}
\hline & \multirow{2}{*}{ B } & \multirow{2}{*}{ SE } & \multirow{2}{*}{ Wald } & \multirow{2}{*}{ df } & \multirow{2}{*}{$\mathbf{p}$} & \multirow{2}{*}{$\begin{array}{l}\text { Odds } \\
\text { Ratio }\end{array}$} & \multicolumn{2}{|c|}{$95 \% \mathrm{Cl}$ for OR } \\
\hline & & & & & & & Lower & Upper \\
\hline oligo- vs. normozoosperm & 0,333 & 0,237 & 1,961 & 1 & 0,161 & 1,394 & 0,876 & 2,221 \\
\hline azoo- vs. normozoosperm & 0,068 & 0,304 & 0,050 & 1 & 0,823 & 1,070 & 0,590 & 1,940 \\
\hline oligo- vs. azoosperm & 0,265 & 0,338 & 0,614 & 1 & 0,433 & 1,303 & 0,672 & 2,527 \\
\hline Lebenserwartung bei Geburt & 0,079 & 0,126 & 0,391 & 1 & 0,532 & 1,082 & 0,845 & 1,385 \\
\hline Geburtsmonat & 0,005 & 0,030 & 0,025 & 1 & 0,875 & 1,005 & 0,948 & 1,065 \\
\hline Alter bei Untersuchung & 0,030 & 0,065 & 0,213 & 1 & 0,644 & 1,030 & 0,908 & 1,170 \\
\hline Untersuchungsjahr & $-0,033$ & 0,062 & 0,283 & 1 & 0,595 & 0,967 & 0,856 & 1,093 \\
\hline sexuelle Abstinenz & 0,035 & 0,034 & 1,089 & 1 & 0,297 & 1,036 & 0,969 & 1,107 \\
\hline ISEI & $-0,005$ & 0,005 & 0,855 & 1 & 0,355 & 0,995 & 0,985 & 1,006 \\
\hline
\end{tabular}

unzensierte Fälle $n=103$; zensierte Fälle $n=286$

Tab. A-12 Cox-Regression zum Einfluss des Fertilitätsstatus (normo-/oligo-lazoosperm) auf die Lebensdauer unter Berücksichtigung der sexuellen Abstinenz ${ }^{a}$; frühe Geburtsjahrgänge

\begin{tabular}{|c|c|c|c|c|c|c|c|c|}
\hline & \multirow{2}{*}{ B } & \multirow{2}{*}{ SE } & \multirow{2}{*}{ Wald } & \multirow{2}{*}{ df } & \multirow{2}{*}{$\mathbf{p}$} & \multirow{2}{*}{$\begin{array}{l}\text { Odds } \\
\text { Ratio }\end{array}$} & \multicolumn{2}{|c|}{$95 \% \mathrm{Cl}$ for OR } \\
\hline & & & & & & & Lower & Upper \\
\hline oligo- vs. normozoosperm & 0,742 & 0,287 & 6,671 & 1 & 0,010 & $2,101^{\star *}$ & 1,196 & 3,691 \\
\hline azoo- vs. normozoosperm & 0,032 & 0,382 & 0,007 & 1 & 0,934 & 1,032 & 0,488 & 2,182 \\
\hline oligo- vs. azoosperm & 0,711 & 0,411 & 2,985 & 1 & 0,084 & $2,036^{(*)}$ & 0,909 & 4,560 \\
\hline Lebenserwartung bei Geburt & 0,246 & 0,259 & 0,898 & 1 & 0,343 & 1,278 & 0,769 & 2,124 \\
\hline Geburtsmonat & 0,015 & 0,037 & 0,152 & 1 & 0,697 & 1,015 & 0,943 & 1,092 \\
\hline Alter bei Untersuchung & 0,143 & 0,156 & 0,838 & 1 & 0,360 & 1,154 & 0,849 & 1,567 \\
\hline Untersuchungsjahr & $-0,141$ & 0,157 & 0,807 & 1 & 0,369 & 0,869 & 0,639 & 1,181 \\
\hline sexuelle Abstinenz & $-0,026$ & 0,042 & 0,394 & 1 & 0,530 & 0,974 & 0,897 & 1,058 \\
\hline ISEI & 0,004 & 0,007 & 0,402 & 1 & 0,526 & 1,004 & 0,991 & 1,018 \\
\hline
\end{tabular}

${ }^{a}$ unzensierte Fälle $n=68$; zensierte Fälle $n=110$

Tab. A-13 Cox-Regression zum Einfluss des Fertilitätsstatus (normo-loligo-/azoosperm) auf die Lebensdauer unter Berücksichtigung der sexuellen Abstinenz ${ }^{a}$; späte Geburtsjahrgänge

\begin{tabular}{|c|c|c|c|c|c|c|c|c|}
\hline & \multirow{2}{*}{ B } & \multirow{2}{*}{ SE } & \multirow{2}{*}{ Wald } & \multirow{2}{*}{ df } & \multirow{2}{*}{ p } & \multirow{2}{*}{$\begin{array}{l}\text { Odds } \\
\text { Ratio }\end{array}$} & \multicolumn{2}{|c|}{$95 \% \mathrm{Cl}$ for OR } \\
\hline & & & & & & & Lower & Upper \\
\hline oligo- vs. normozoosperm & $-0,250$ & 0,465 & 0,289 & 1 & 0,591 & 0,779 & 0,313 & 1,938 \\
\hline azoo- vs. normozoosperm & 0,202 & 0,509 & 0,158 & 1 & 0,691 & 1,224 & 0,452 & 3,319 \\
\hline oligo- vs. azoosperm & $-0,452$ & 0,619 & 0,534 & 1 & 0,465 & 0,636 & 0,189 & 2,140 \\
\hline Lebenserwartung bei Geburt & $-0,729$ & 0,949 & 0,591 & 1 & 0,442 & 0,482 & 0,075 & 3,096 \\
\hline Geburtsmonat & 0,007 & 0,052 & 0,018 & 1 & 0,892 & 1,007 & 0,909 & 1,116 \\
\hline Alter bei Untersuchung & 0,091 & 0,122 & 0,559 & 1 & 0,455 & 1,095 & 0,863 & 1,390 \\
\hline Untersuchungsjahr & $-0,105$ & 0,124 & 0,712 & 1 & 0,399 & 0,900 & 0,705 & 1,149 \\
\hline sexuelle Abstinenz & 0,144 & 0,058 & 6,274 & 1 & 0,012 & $1,155^{\star}$ & 1,032 & 1,294 \\
\hline ISEI & $-0,016$ & 0,010 & 2,682 & 1 & 0,101 & 0,984 & 0,965 & 1,003 \\
\hline
\end{tabular}

${ }^{a}$ unzensierte Fälle $n=35$; zensierte Fälle $n=175$ 


\section{A-4 Modellierung des Zusammenhangs zwischen logarithmierter Spermienkonzentration und Lebensdauer (oligozoosperme und normozoosperme Fälle)}

Tab. A-14 Cox-Regression zum Einfluss der Spermienkonzentration auf die Lebensdauer der oligound normozoospermen Fälle ${ }^{a}$

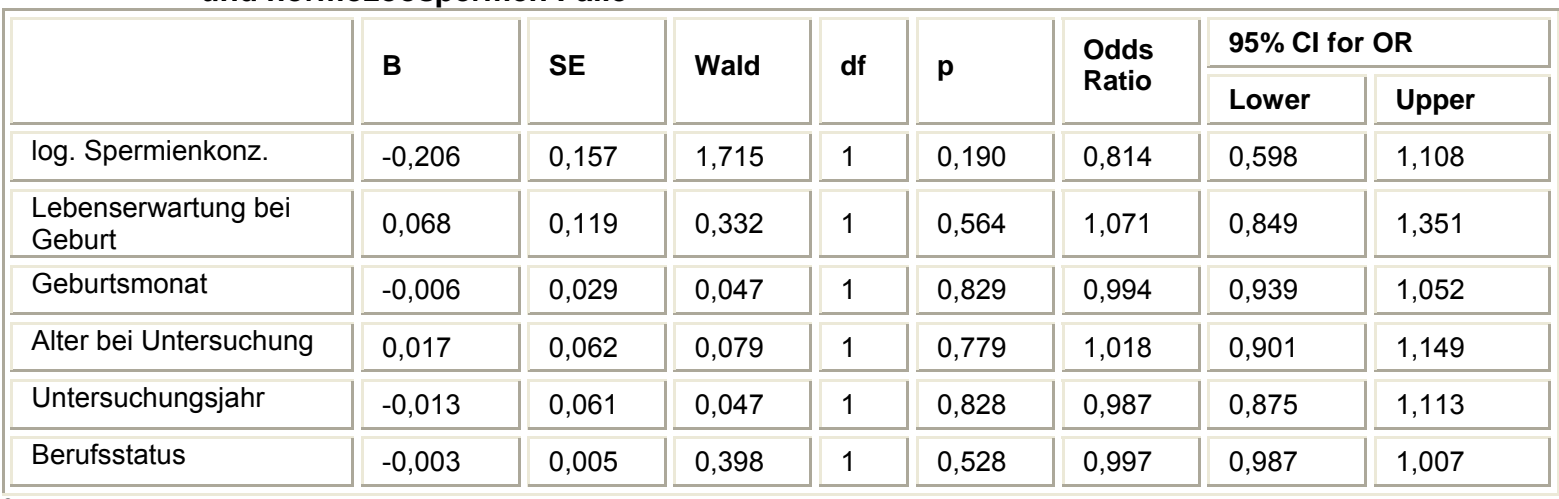

anzensierte Fälle $n=106$; zensierte Fälle $n=293$

Tab. A-15 Cox-Regression zum Einfluss der Spermienkonzentration auf die Lebensdauer der oligound normozoospermen Fälle ${ }^{a}$; frühe Geburtsjahrgänge

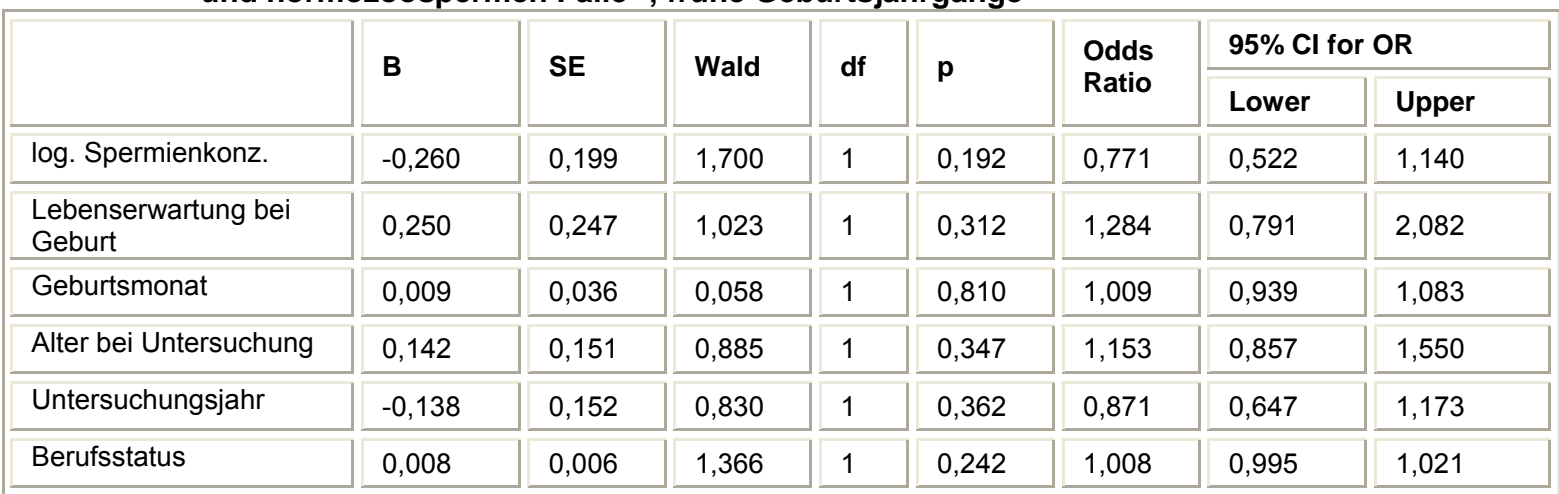

a unzensierte Fälle $n=71$; zensierte Fälle $n=115$

Tab. A-16 Cox-Regression zum Einfluss der Spermienkonzentration auf die Lebensdauer der oligound normozoospermen Fälle ${ }^{a}$; späte Geburtsjahrgänge

\begin{tabular}{|c|c|c|c|c|c|c|c|c|}
\hline & \multirow{2}{*}{ B } & \multirow{2}{*}{ SE } & \multirow{2}{*}{ Wald } & \multirow{2}{*}{ df } & \multirow{2}{*}{$\mathbf{p}$} & \multirow{2}{*}{$\begin{array}{l}\text { Odds } \\
\text { Ratio }\end{array}$} & \multicolumn{2}{|c|}{$95 \% \mathrm{CI}$ for OR } \\
\hline & & & & & & & Lower & Upper \\
\hline log. Spermienkonz. & $-0,264$ & 0,266 & 0,982 & 1 & 0,322 & 0,768 & 0,456 & 1,295 \\
\hline $\begin{array}{l}\text { Lebenserwartung bei } \\
\text { Geburt }\end{array}$ & 1,428 & 1,393 & 1,050 & 1 & 0,305 & 4,170 & 0,272 & 63,968 \\
\hline Alter bei Untersuchung & $-0,026$ & 0,051 & 0,253 & 1 & 0,615 & 0,975 & 0,882 & 1,077 \\
\hline Untersuchungsjahr & $-0,152$ & 0,181 & 0,704 & 1 & 0,401 & 0,859 & 0,602 & 1,225 \\
\hline Alter bei Untersuchung & 0,150 & 0,182 & 0,681 & 1 & 0,409 & 1,162 & 0,814 & 1,660 \\
\hline Berufsstatus & $-0,022$ & 0,010 & 5,071 & 1 & 0,024 & $0,978^{*}$ & 0,959 & ,997 \\
\hline
\end{tabular}

a unzensierte Fälle $n=35$; zensierte Fälle $n=177$ 
Tab. A-17 Exponential-Modell zum Einfluss der Spermienkonzentration auf die Lebensdauer der oligound normozoospermen Fälle ${ }^{a}$

\begin{tabular}{|c|c|c|c|c|c|c|}
\hline Variable & Coeff & Error & C/Error & Signif & $\mathbf{p}$ & $\begin{array}{l}\text { Rel. } \\
\text { Risiko }\end{array}$ \\
\hline log. Spermienkonzentration & $-0,1395$ & 0,1532 & $-0,9105$ & 0,6374 & 0,363 & 0,8698 \\
\hline Lebenserwartung bei Geburt & 0,1210 & 0,1110 & 1,0899 & 0,7242 & 0,276 & 1,1286 \\
\hline Geburtsmonat & $-0,0052$ & 0,0291 & $-0,1799$ & 0,1427 & 0,857 & 0,9948 \\
\hline Alter bei Untersuchung & 0,1435 & 0,0584 & 2,4573 & 0,9860 & 0,014 & $1,1544^{*}$ \\
\hline Untersuchungsjahr & $-0,1378$ & 0,0576 & $-2,3930$ & 0,9833 & 0,017 & $0,8713^{*}$ \\
\hline Berufsstatus & $-0,0042$ & 0,0049 & $-0,8469$ & 0,6029 & 0,397 & 0,9958 \\
\hline
\end{tabular}

${ }^{a}$ unzensierte Fälle $n=106$; zensierte Fälle $n=293$

Tab. A-18 Exponential-Modell zum Einfluss der Spermienkonzentration auf die Lebensdauer der oligound normozoospermen Fälle ${ }^{a}$; frühe Geburtsjahrgänge

\begin{tabular}{|l|l|l|l|l|l||l|l|}
\hline Variable & Coeff & Error & C/Error & Signif & p & $\begin{array}{l}\text { Rel. } \\
\text { Risiko }\end{array}$ \\
\hline \hline log. Spermienkonzentration & $-0,1625$ & 0,1911 & $-0,8501$ & 0,6048 & 0,395 & 0,8500 \\
\hline \hline Lebenserwartung bei Geburt & 0,3037 & 0,2383 & 1,2743 & 0,7974 & 0,203 & 1,3549 \\
\hline Geburtsmonat & 0,0118 & 0,0364 & 0,3243 & 0,2543 & 0,746 & 1,0119 \\
\hline Alter bei Untersuchung & 0,2555 & 0,1453 & 1,7588 & 0,9214 & 0,079 & $1,2911^{(*)}$ \\
\hline Untersuchungsjahr & $-0,2498$ & 0,1456 & $-1,7159$ & 0,9138 & 0,086 & $0,7790^{(*)}$ \\
\hline Berufsstatus & 0,0029 & 0,0060 & 0,4808 & 0,3693 & 0,631 & 1,0029 \\
\hline
\end{tabular}

a unzensierte Fälle $n=71$; zensierte Fälle $n=115$

Tab. A-19 Exponential-Modell zum Einfluss der Spermienkonzentration auf die Lebensdauer der oligound normozoospermen Fälle ${ }^{a}$; späte Geburtsjahrgänge

\begin{tabular}{|c|c|c|c|c|c|c|}
\hline Variable & Coeff & Error & C/Error & Signif & $\mathbf{p}$ & $\begin{array}{l}\text { Rel. } \\
\text { Risiko }\end{array}$ \\
\hline log. Spermienkonzentration & $-0,2569$ & 0,2655 & $-0,9676$ & 0,6668 & 0,333 & 0,7735 \\
\hline Lebenserwartung bei Geburt & 1,3600 & 1,4034 & 0,9691 & 0,6675 & 0,333 & 3,8962 \\
\hline Geburtsmonat & $-0,0253$ & 0,0513 & $-0,4935$ & 0,3784 & 0,622 & 0,9750 \\
\hline Alter bei Untersuchung & $-0,0534$ & 0,1737 & $-0,3073$ & 0,2414 & 0,759 & 0,9480 \\
\hline Untersuchungsjahr & 0,0568 & 0,1756 & 0,3234 & 0,2536 & 0,746 & 1,0584 \\
\hline Berufsstatus & $-0,0199$ & 0,0098 & $-2,0390$ & 0,9585 & 0,042 & $0,9803^{*}$ \\
\hline
\end{tabular}

a unzensierte Fälle $n=35$; zensierte Fälle $n=177$

Tab. A-20 Gompertz-Makeham-Modell zum Einfluss der Spermienkonzentration auf die Lebensdauer der oligo- und normozoospermen Fälle ${ }^{a}$

\begin{tabular}{|l|l|l|l|l|l||l|}
\hline Variable & Coeff & Error & C/Error & Signif & p & $\begin{array}{l}\text { Rel. } \\
\text { Risiko }\end{array}$ \\
\hline \hline log. Spermienkonzentration & $-0,2040$ & 0,1577 & $-1,2936$ & 0,8042 & 0,196 & 0,8155 \\
\hline \hline Lebenserwartung bei Geburt & 0,1016 & 0,1121 & 0,9065 & 0,6353 & 0,365 & 1,1070 \\
\hline Geburtsmonat & $-0,0068$ & 0,0289 & $-0,2353$ & 0,1861 & 0,814 & 0,9932 \\
\hline Alter bei Untersuchung & 0,1573 & 0,0592 & 2,6553 & 0,9921 & 0,008 & $1,1704^{* *}$ \\
\hline Untersuchungsjahr & $-0,1551$ & 0,0579 & $-2,6778$ & 0,9926 & 0,007 & $0,8564^{* *}$ \\
\hline Berufsstatus & $-0,0029$ & 0,0051 & $-0,5622$ & 0,4260 & 0,574 & 0,9971 \\
\hline
\end{tabular}

anzensierte Fälle $n=106$; zensierte Fälle $n=293$

Tab. A-21 Gompertz-Makeham-Modell zum Einfluss der Spermienkonzentration auf die Lebensdauer der oligo- und normozoospermen Fälle ${ }^{a}$; frühe Geburtsjahrgänge

\begin{tabular}{|c|c|c|c|c|c|c|}
\hline Variable & Coeff & Error & C/Error & Signif & $\mathbf{p}$ & $\begin{array}{l}\text { Rel. } \\
\text { Risiko }\end{array}$ \\
\hline log. Spermienkonzentration & $-0,2696$ & 0,2002 & $-1,3465$ & 0,8219 & 0,178 & 0,7637 \\
\hline Lebenserwartung bei Geburt & 0,3457 & 0,2344 & 1,4751 & 0,8598 & 0,140 & 1,4130 \\
\hline Geburtsmonat & 0,0108 & 0,0362 & 0,2976 & 0,2340 & 0,766 & 1,0108 \\
\hline Alter bei Untersuchung & 0,3187 & 0,1439 & 2,2158 & 0,9733 & 0,027 & $1,3754^{*}$ \\
\hline Untersuchungsjahr & $-0,3177$ & 0,1442 & $-2,2038$ & 0,9725 & 0,028 & $0,7278^{*}$ \\
\hline Berufsstatus & 0,0079 & 0,0064 & 1,2307 & 0,7816 & 0,218 & 1,0079 \\
\hline
\end{tabular}


Tab. A-22 Gompertz-Makeham-Modell zum Einfluss der Spermienkonzentration auf die Lebensdauer der oligo- und normozoospermen Fälle ${ }^{a}$; späte Geburtsjahrgänge

\begin{tabular}{|c|c|c|c|c|c|c|}
\hline Variable & Coeff & Error & C/Error & Signif & $\mathbf{p}$ & $\begin{array}{l}\text { Rel. } \\
\text { Risiko }\end{array}$ \\
\hline log. Spermienkonzentration & $-0,2644$ & 0,2680 & $-0,9867$ & 0,6762 & 0,324 & 0,7677 \\
\hline Lebenserwartung bei Geburt & 1,3840 & 1,3453 & 1,0288 & 0,6964 & 0,304 & 3,9907 \\
\hline Geburtsmonat & $-0,0237$ & 0,0506 & $-0,4686$ & 0,3606 & 0,640 & 0,9766 \\
\hline Alter bei Untersuchung & $-0,0415$ & 0,1701 & $-0,2437$ & 0,1926 & 0,807 & 0,9594 \\
\hline Untersuchungsjahr & 0,0432 & 0,1715 & 0,2519 & 0,1989 & 0,801 & 1,0442 \\
\hline Berufsstatus & $-0,0225$ & 0,0099 & $-2,2726$ & 0,9770 & 0,023 & $0,9777^{*}$ \\
\hline
\end{tabular}

a unzensierte Fälle $n=35$; zensierte Fälle $n=177$

Tab. A-23 Weibull-Modell zum Einfluss der Spermienkonzentration auf die Lebensdauer der oligo- und normozoospermen Fälle ${ }^{a, b}$

\begin{tabular}{|l|l|l|l|l|l||l|}
\hline Variable & Coeff & Error & C/Error & Signif & p & $\begin{array}{l}\text { Rel. } \\
\text { Risiko }\end{array}$ \\
\hline \hline log. Spermienkonzentration & $-0,0043$ & 0,0030 & $-1,4301$ & 0,8473 & 0,153 & 0,7975 \\
\hline \hline Lebenserwartung bei Geburt & $-0,0047$ & 0,0021 & $-2,2349$ & 0,9746 & 0,025 & $0,7797^{*}$ \\
\hline Geburtsmonat & $-0,0002$ & 0,0005 & $-0,4401$ & 0,3401 & 0,660 & 0,9875 \\
\hline Alter bei Untersuchung & $-0,0006$ & 0,0010 & $-0,5791$ & 0,4375 & 0,563 & 0,9689 \\
\hline Untersuchungsjahr & 0,0007 & 0,0010 & 0,7196 & 0,5282 & 0,472 & 1,0392 \\
\hline Berufsstatus & $-0,0001$ & 0,0001 & $-0,7032$ & 0,5180 & 0,482 & 0,9965 \\
\hline
\end{tabular}

${ }^{a}$ unzensierte Fälle $n=106$; zensierte Fälle $n=293$

b Convergence not reached in 500000 iterations; Number of function evaluations: 1000002

Tab. A-24 Weibull-Modell zum Einfluss der Spermienkonzentration auf die Lebensdauer der oligo- und normozoospermen Fälle ${ }^{a}$; frühe Geburtsjahrgänge

\begin{tabular}{|l|l|l|l|l|l|l|l|}
\hline Variable & Coeff & Error & C/Error & Signif & $\mathbf{p}$ & $\begin{array}{l}\text { Rel. } \\
\text { Risiko }\end{array}$ \\
\hline \hline log. Spermienkonzentration & $-0,0038$ & 0,0035 & $-1,0992$ & 0,7283 & 0,272 & 0,8050 \\
\hline Lebenserwartung bei Geburt & $-0,0036$ & 0,0046 & $-0,7880$ & 0,5693 & 0,431 & 0,8147 \\
\hline Geburtsmonat & $-0,0001$ & 0,0006 & $-0,2075$ & 0,1644 & 0,836 & 0,9925 \\
\hline Alter bei Untersuchung & $-0,0005$ & 0,0028 & $-0,1725$ & 0,1369 & 0,863 & 0,9729 \\
\hline Untersuchungsjahr & 0,0006 & 0,0028 & 0,2010 & 0,1593 & 0,841 & 1,0327 \\
\hline Berufsstatus & 0,0002 & 0,0001 & 1,5700 & 0,8836 & 0,116 & 1,0103 \\
\hline
\end{tabular}

a unzensierte Fälle $n=71$; zensierte Fälle $n=115$

Tab. A-25 Weibull-Modell zum Einfluss der Spermienkonzentration auf die Lebensdauer der oligo- und normozoospermen Fälle ${ }^{a}$; späte Geburtsjahrgänge

\begin{tabular}{|c|c|c|c|c|c|c|}
\hline Variable & Coeff & Error & C/Error & Signif & $\mathbf{p}$ & $\begin{array}{l}\text { Rel. } \\
\text { Risiko }\end{array}$ \\
\hline log. Spermienkonzentration & $-0,0052$ & 0,0055 & $-0,9557$ & 0,6608 & 0,339 & 0,7721 \\
\hline Lebenserwartung bei Geburt & 0,0235 & 0,0260 & 0,9070 & 0,6356 & 0,364 & 3,1946 \\
\hline Geburtsmonat & $-0,0005$ & 0,0010 & $-0,4602$ & 0,3546 & 0,645 & 0,9770 \\
\hline Alter bei Untersuchung & $-0,0002$ & 0,0032 & $-0,0570$ & 0,0454 & 0,955 & 0,9909 \\
\hline Untersuchungsjahr & 0,0002 & 0,0033 & 0,0613 & 0,0489 & 0,951 & 1,0100 \\
\hline Berufsstatus & $-0,0005$ & 0,0002 & $-2,1482$ & 0,9683 & 0,032 & $0,9777^{*}$ \\
\hline
\end{tabular}

a unzensierte Fälle $n=35$; zensierte Fälle $n=177$ 


\section{A-5 Modellierung des Zusammenhangs zwischen logarithmierter Spermienkonzentration und Lebensdauer (oligozoosperme Fälle)}

Tab. A-26 Cox-Regression zum Einfluss der logarithmierten Spermienkonzentration auf die Lebensdauer der oligzooospermen Fälle ${ }^{a}$

\begin{tabular}{|l|l|l|l|l|l|l|l|l|l|}
\hline & B & SE & Wald & df & p & $\begin{array}{l}\text { Odds } \\
\text { Ratio }\end{array}$ & \multicolumn{2}{|l|}{ 95\% CI for OR } \\
\hline log. Spermienkonz. & $-0,201$ & 0,389 & 0,267 & 1 & 0,605 & 0,818 & 0,382 & 1,753 \\
\hline $\begin{array}{l}\text { Lebenserwartung bei } \\
\text { Geburt }\end{array}$ & 0,524 & 0,324 & 2,621 & 1 & 0,105 & 1,689 & 0,895 & 3,187 \\
\hline Geburtsmonat & $-0,025$ & 0,059 & 0,179 & 1 & 0,672 & 0,975 & 0,869 & 1,095 \\
\hline Alter bei Untersuchung & 0,270 & 0,182 & 2,208 & 1 & 0,137 & 1,310 & 0,918 & 1,870 \\
\hline Untersuchungsjahr & $-0,263$ & 0,178 & 2,178 & 1 & 0,140 & 0,769 & 0,542 & 1,090 \\
\hline ISEI & $-0,024$ & 0,013 & 3,493 & 1 & 0,062 & $0,976^{(*)}$ & 0,951 & 1,001 \\
\hline
\end{tabular}

${ }^{a}$ unzensierte Fälle $n=30$; zensierte Fälle $n=61$

Tab. A-27 Cox-Regression zum Einfluss der logarithmierten Spermienkonzentration auf die Lebensdauer der oligozoospermen Fälle ${ }^{a}$; frühe Jahrgänge

\begin{tabular}{|l|l|l|l|l|l|l|l|l|l|}
\hline \multicolumn{1}{|l|}{} & B & SE & Wald & df & p & $\begin{array}{l}\text { Odds } \\
\text { Ratio }\end{array}$ & \multicolumn{2}{|l|}{$\begin{array}{l}\text { L5\% Cl for OR } \\
\text { Lower }\end{array}$} & Upper \\
\hline \hline log. Spermienkonz. & 0,128 & 0,492 & 0,068 & 1 & 0,794 & 1,137 & 0,433 & 2,985 \\
\hline $\begin{array}{l}\text { Lebenserwartung bei } \\
\text { Geburt }\end{array}$ & 0,785 & 0,641 & 1,501 & 1 & 0,220 & 2,192 & 0,625 & 7,694 \\
\hline Geburtsmonat & 0,018 & 0,066 & 0,072 & 1 & 0,788 & 1,018 & 0,894 & 1,160 \\
\hline Alter bei Untersuchung & 0,421 & 0,379 & 1,229 & 1 & 0,268 & 1,523 & 0,724 & 3,204 \\
\hline Untersuchungsjahr & $-0,386$ & 0,369 & 1,094 & 1 & 0,296 & 0,680 & 0,330 & 1,401 \\
\hline ISEI & $-0,023$ & 0,018 & 1,630 & 1 & 0,202 & 0,977 & 0,943 & 1,012 \\
\hline
\end{tabular}

${ }^{a}$ unzensierte Fälle $n=23$; zensierte Fälle $n=21$

Tab. A-28 Cox-Regression zum Einfluss der logarithmierten Spermienkonzentration auf die Lebensdauer der oligozoospermen Fälle ${ }^{a}$; späte Jahrgänge

\begin{tabular}{|l|l|l|l|l|l|l|l|l|l|}
\hline & B & SE & Wald & df & p & $\begin{array}{l}\text { Odds } \\
\text { Ratio }\end{array}$ & \multicolumn{2}{|l|}{$\begin{array}{l}\text { 95\% CI for OR } \\
\text { Lower }\end{array}$} & Upper \\
\hline \hline log. Spermienkonz. & $-1,704$ & 0,932 & 3,341 & 1 & 0,068 & $0,182^{(*)}$ & 0,029 & 1,131 \\
\hline $\begin{array}{l}\text { Lebenserwartung bei } \\
\text { Geburt }\end{array}$ & $-2,219$ & 2,704 & 0,674 & 1 & 0,412 & 0,109 & 0,001 & 21,749 \\
\hline \hline Geburtsmonat & $-0,259$ & 0,174 & 2,205 & 1 & 0,138 & 0,772 & 0,548 & 1,086 \\
\hline Alter bei Untersuchung & 0,397 & 0,367 & 1,172 & 1 & 0,279 & 1,488 & 0,725 & 3,055 \\
\hline Untersuchungsjahr & $-0,531$ & 0,412 & 1,658 & 1 & 0,198 & 0,588 & 0,262 & 1,319 \\
\hline ISEI & $-0,020$ & 0,031 & 0,436 & 1 & 0,509 & 0,980 & 0,922 & 1,041 \\
\hline
\end{tabular}

${ }^{a}$ unzensierte Fälle $n=7$; zensierte Fälle $n=40$ 
Tab. A-29 Exponential-Modell zum Einfluss der logarithmierten Spermienkonzentration auf die Lebensdauer der oligozoospermen Fälle ${ }^{a}$

\begin{tabular}{|c|c|c|c|c|c|c|}
\hline Variable & Coeff & Error & C/Error & Signif & $\mathbf{p}$ & $\begin{array}{l}\text { Rel. } \\
\text { Risiko }\end{array}$ \\
\hline log. Spermienkonzentration & $-0,0551$ & 0,3765 & $-0,1464$ & 0,1164 & 0,004 & 0,9464 \\
\hline Lebenserwartung bei Geburt & 0,3674 & 0,2847 & 1,2905 & 0,8031 & 0,197 & 1,4440 \\
\hline Geburtsmonat & $-0,0123$ & 0,0582 & $-0,2119$ & 0,1679 & 0,832 & 0,9877 \\
\hline Alter bei Untersuchung & 0,2818 & 0,1604 & 1,7571 & 0,9211 & 0,079 & $1,3255^{\text {(* }}$ \\
\hline Untersuchungsjahr & $-0,2831$ & 0,1567 & $-1,8074$ & 0,9293 & 0,071 & $0,7534^{(*)}$ \\
\hline ISEI & $-0,0223$ & 0,0126 & $-1,7639$ & 0,9222 & 0,078 & $0,9780^{(*)}$ \\
\hline
\end{tabular}

a unzensierte Fälle $n=30$; zensierte Fälle $n=61$

Tab. A-30 Exponential-Modell zum Einfluss der logarithmierten Spermienkonzentration auf die Lebensdauer der oligozoospermen Fälle ${ }^{a}$; frühe Geburtsjahrgänge

\begin{tabular}{|c|c|c|c|c|c|c|}
\hline Variable & Coeff & Error & C/Error & Signif & $\mathbf{p}$ & $\begin{array}{l}\text { Rel. } \\
\text { Risiko }\end{array}$ \\
\hline log. Spermienkonzentration & 0,2639 & 0,4640 & 0,5687 & 0,4304 & 0,570 & 1,3020 \\
\hline Lebenserwartung bei Geburt & 0,4681 & 0,5708 & 0,8200 & 0,5878 & 0,412 & 1,5969 \\
\hline Geburtsmonat & 0,0292 & 0,0656 & 0,4444 & 0,3432 & 0,657 & 1,0296 \\
\hline Alter bei Untersuchung & 0,3340 & 0,3432 & 0,9731 & 0,6695 & 0,331 & 1,3965 \\
\hline Untersuchungsjahr & $-0,3103$ & 0,3294 & $-0,9421$ & 0,6539 & 0,346 & 0,7332 \\
\hline ISEI & $-0,0194$ & 0,0170 & $-1,1412$ & 0,7462 & 0,254 & 0,9808 \\
\hline
\end{tabular}

a unzensierte Fälle $n=23$; zensierte Fälle $n=21$

Tab. A-31 Exponential-Modell zum Einfluss der logarithmierten Spermienkonzentration auf die Lebensdauer der oligozoospermen Fälle ${ }^{a}$; späte Geburtsjahrgänge

\begin{tabular}{|c|c|c|c|c|c|c|}
\hline Variable & Coeff & Error & C/Error & Signif & $\mathbf{p}$ & $\begin{array}{l}\text { Rel. } \\
\text { Risiko }\end{array}$ \\
\hline log. Spermienkonzentration & $-1,8248$ & 0,9782 & $-1,8655$ & 0,9379 & 0,062 & $0,1612^{(*)}$ \\
\hline Lebenserwartung bei Geburt & $-1,7015$ & 2,8129 & $-0,6049$ & 0,4547 & 0,545 & 0,1824 \\
\hline Geburtsmonat & $-0,2738$ & 0,1801 & $-1,5204$ & 0,8716 & 0,128 & 0,7605 \\
\hline Alter bei Untersuchung & 0,4241 & 0,3801 & 1,1160 & 0,7356 & 0,264 & 1,5283 \\
\hline Untersuchungsjahr & $-0,5331$ & 0,4216 & $-1,2644$ & 0,7939 & 0,206 & 0,5868 \\
\hline ISEI & $-0,0164$ & 0,0307 & $-0,5326$ & 0,4057 & 0,594 & 0,9838 \\
\hline
\end{tabular}

unzensierte Fälle $n=7$; zensierte Fälle $n=40$

Tab. A-32 Gompertz-Makeham-Modell zum Einfluss der logarithmierten Spermienkonzentration auf die Lebensdauer der oligozoospermen Fälle ${ }^{a}$

\begin{tabular}{|c|c|c|c|c|c|c|}
\hline Variable & Coeff & Error & ClError & Signif & $\mathbf{p}$ & $\begin{array}{l}\text { Rel. } \\
\text { Risiko }\end{array}$ \\
\hline log. Spermienkonzentration & $-0,1495$ & 0,3918 & $-0,3817$ & 0,2973 & 0,703 & 0,8611 \\
\hline Lebenserwartung bei Geburt & 0,5055 & 0,3278 & 1,5422 & 0,8770 & 0,123 & 1,6579 \\
\hline Geburtsmonat & $-0,0299$ & 0,0595 & $-0,5031$ & 0,3851 & 0,615 & 0,9705 \\
\hline Alter bei Untersuchung & 0,3923 & 0,1851 & 2,1191 & 0,9659 & 0,034 & $1,4804^{*}$ \\
\hline Untersuchungsjahr & $-0,3918$ & 0,1808 & $-2,1665$ & 0,9697 & 0,030 & $0,6759^{*}$ \\
\hline ISEI & $-0,0248$ & 0,0131 & $-1,8870$ & 0,9408 & 0,059 & $0,9755^{(*)}$ \\
\hline
\end{tabular}

a unzensierte Fälle $n=30 ;$ zensierte Fälle $n=61$

Tab. A-33 Gompertz-Makeham-Modell zum Einfluss der logarithmierten Spermienkonzentration auf die Lebensdauer der oligozoospermen Fälle ${ }^{a}$; frühe Geburtsjahrgänge

\begin{tabular}{|c|c|c|c|c|c|c|}
\hline Variable & Coeff & Error & C/Error & Signif & $\mathbf{p}$ & $\begin{array}{l}\text { Rel. } \\
\text { Risiko }\end{array}$ \\
\hline log. Spermienkonzentration & 0,2055 & 0,4997 & 0,4112 & 0,3191 & 0,681 & 1,2281 \\
\hline Lebenserwartung bei Geburt & 0,8353 & 0,6532 & 1,2787 & 0,7990 & 0,201 & 2,3054 \\
\hline Geburtsmonat & 0,0172 & 0,0666 & 0,2583 & 0,2038 & 0,796 & 1,0173 \\
\hline Alter bei Untersuchung & 0,5794 & 0,3880 & 1,4931 & 0,8646 & 0,135 & 1,7849 \\
\hline Untersuchungsjahr & $-0,5468$ & 0,3761 & $-1,4538$ & 0,8540 & 0,146 & 0,5788 \\
\hline ISEI & $-0,0240$ & 0,0181 & $-1,3316$ & 0,8170 & 0,183 & 0,9762 \\
\hline
\end{tabular}

${ }^{a}$ unzensierte Fälle $n=23$; zensierte Fälle $n=21$ 
Tab. A-34 Gompertz-Makeham-Modell zum Einfluss der logarithmierten Spermienkonzentration auf die Lebensdauer der oligozoospermen Fälle ${ }^{a}$; späte Geburtsjahrgänge

\begin{tabular}{|l|l|l|l|l||l|l|}
\hline Variable & Coeff & Error & C/Error & Signif & $\mathbf{p}$ & $\begin{array}{l}\text { Rel. } \\
\text { Risiko }\end{array}$ \\
\hline log. Spermienkonzentration & $-1,7718$ & 0,9249 & $-1,9156$ & 0,9446 & 0,055 & $0,1700^{(*)}$ \\
\hline Lebenserwartung bei Geburt & $-1,9511$ & 2,6981 & $-0,7231$ & 0,5304 & 0,470 & 0,1421 \\
\hline Geburtsmonat & $-0,2570$ & 0,1705 & $-1,5075$ & 0,8683 & 0,131 & 0,7734 \\
\hline Alter bei Untersuchung & 0,4298 & 0,3575 & 1,2024 & 0,7708 & 0,229 & 1,5370 \\
\hline Untersuchungsjahr & $-0,5570$ & 0,4019 & $-1,3857$ & 0,8342 & 0,166 & 0,5729 \\
\hline ISEI & $-0,0218$ & 0,0312 & $-0,6976$ & 0,5146 & 0,485 & 0,9784 \\
\hline
\end{tabular}

${ }^{a}$ unzensierte Fälle $n=7$; zensierte Fälle $n=40$

Tab. A-35 Weibull-Modell zum Einfluss der logarithmierten Spermienkonzentration auf die Lebensdauer der oligozoospermen Fälle ${ }^{a, b}$

\begin{tabular}{|l|l|l|l|l|l||l|}
\hline Variable & Coeff & Error & C/Error & Signif & p & $\begin{array}{l}\text { Rel. } \\
\text { Risiko }\end{array}$ \\
\hline \hline log. Spermienkonzentration & 0,0001 & 0,0071 & 0,0075 & 0,0060 & 0,940 & 1,0029 \\
\hline \hline Lebenserwartung bei Geburt & $-0,0046$ & 0,0053 & $-0,8641$ & 0,6124 & 0,388 & 0,7787 \\
\hline Geburtsmonat & 0,0000 & 0,0011 & $-0,0277$ & 0,0221 & 0,978 & 0,9984 \\
\hline Alter bei Untersuchung & $-0,0006$ & 0,0027 & $-0,2233$ & 0,1767 & 0,823 & 0,9673 \\
\hline Untersuchungsjahr & 0,0006 & 0,0027 & 0,2331 & 0,1843 & 0,816 & 1,0346 \\
\hline ISEI & $-0,0005$ & 0,0003 & $-2,0175$ & 0,9564 & 0,044 & $0,9719^{*}$ \\
\hline
\end{tabular}

${ }^{a}$ unzensierte Fälle $n=30$; zensierte Fälle $n=61$

b Convergence not reached in 500000 iterations; Number of function evaluations: 1000002

Tab. A-36 Weibull-Modell zum Einfluss der logarithmierten Spermienkonzentration auf die Lebensdauer der oligozoospermen Fälle ${ }^{a}$; frühe Geburtsjahrgänge

\begin{tabular}{|l|l|l|l||l|l||l|}
\hline Variable & Coeff & Error & C/Error & Signif & p & $\begin{array}{l}\text { Rel. } \\
\text { Risiko }\end{array}$ \\
\hline log. Spermienkonzentration & 0,0087 & 0,0097 & 0,8934 & 0,6284 & 0,372 & 1,6117 \\
\hline Lebenserwartung bei Geburt & $-0,0024$ & 0,0132 & $-0,1815$ & 0,1441 & 0,856 & 0,8767 \\
\hline Geburtsmonat & 0,0004 & 0,0012 & 0,2943 & 0,2315 & 0,769 & 1,0197 \\
\hline Alter bei Untersuchung & 0,0000 & 0,0078 & $-0,0016$ & 0,0013 & 0,999 & 0,9993 \\
\hline Untersuchungsjahr & 0,0004 & 0,0076 & 0,0482 & 0,0385 & 0,962 & 1,0204 \\
\hline ISEI & $-0,0002$ & 0,0003 & $-0,7603$ & 0,5529 & 0,447 & 0,9871 \\
\hline
\end{tabular}

a unzensierte Fälle $n=23$; zensierte Fälle $n=21$

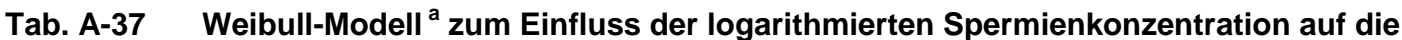

\begin{tabular}{|c|c|c|c|c|c|c|}
\hline Variable & Coeff & Error & C/Error & Signif & $\mathbf{p}$ & $\begin{array}{l}\text { Rel. } \\
\text { Risiko }\end{array}$ \\
\hline log. Spermienkonzentration & $-0,0289$ & 0,0196 & $-1,4778$ & 0,8605 & 0,140 & 0,1945 \\
\hline Lebenserwartung bei Geburt & 0,0220 & 0,0959 & 0,2294 & 0,1815 & 0,819 & 3,4704 \\
\hline Geburtsmonat & $-0,0035$ & 0,0031 & $-1,1160$ & 0,7356 & 0,264 & 0,8206 \\
\hline Alter bei Untersuchung & $-0,0015$ & 0,0123 & $-0,1246$ & 0,0991 & 0,901 & 0,9171 \\
\hline Untersuchungsjahr & 0,0003 & 0,0124 & 0,0247 & 0,0197 & 0,980 & 1,0174 \\
\hline ISEI & $-0,0004$ & 0,0006 & $-0,7696$ & 0,5584 & 0,442 & 0,9749 \\
\hline
\end{tabular}

${ }^{a}$ unzensierte Fälle $n=7$; zensierte Fälle $n=40$ 


\section{A-6 Modellierung des Zusammenhangs zwischen logarithmierter Spermienkonzentration und Lebensdauer (normozoosperme Fälle)}

Tab. A-38 Cox-Regression zum Einfluss der logarithmierten Spermienkonzentration auf die Lebensdauer der normozoospermen Fälle $^{\mathrm{a}}$

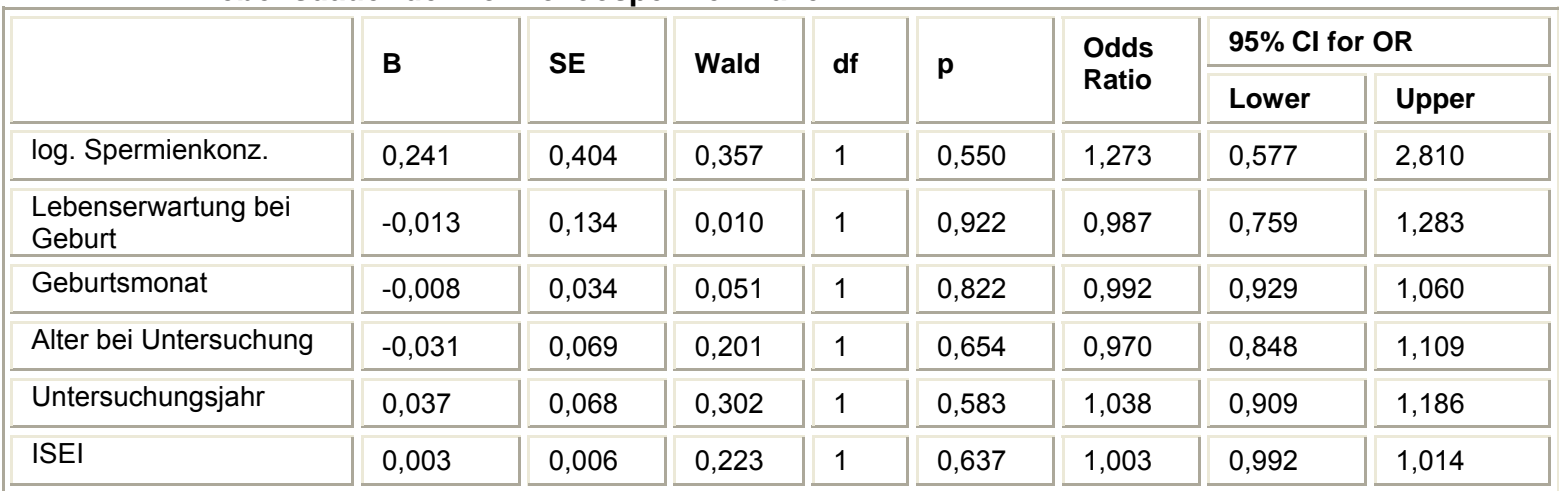

anzensierte Fälle $n=76$; zensierte Fälle $n=232$

Tab. A-39 Cox-Regression zum Einfluss der logarithmierten Spermienkonzentration auf die Lebensdauer der normozoospermen Fälle ${ }^{a}$; frühe Jahrgänge

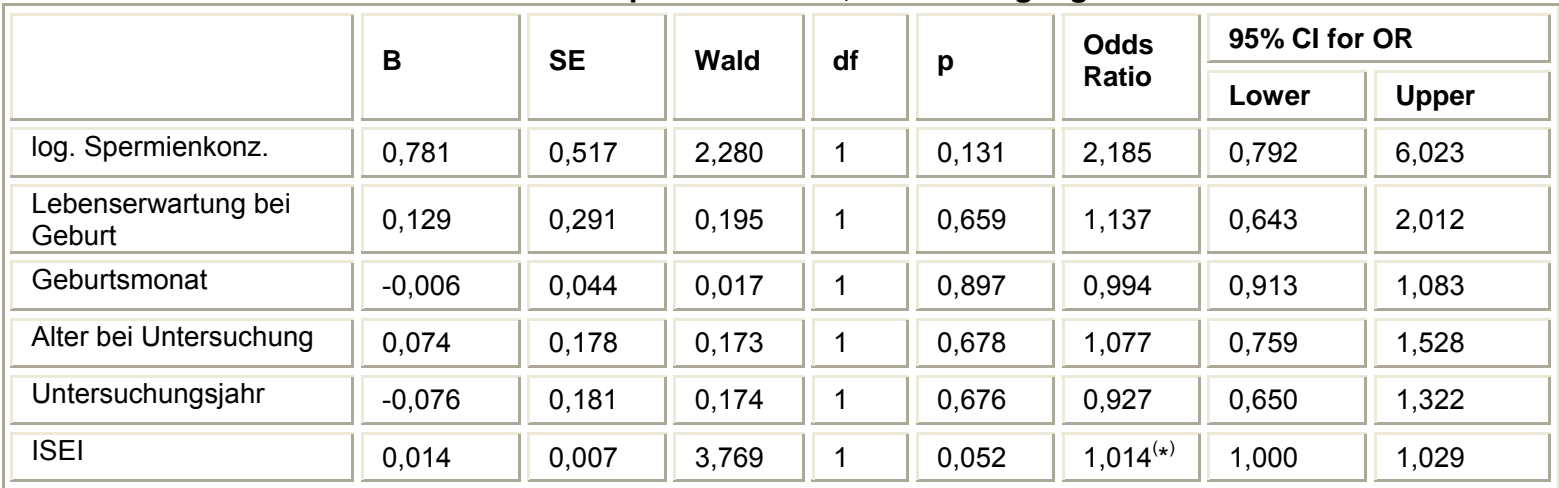

${ }^{a}$ unzensierte Fälle $n=48$; zensierte Fälle $n=94$

Tab. A-40 Cox-Regression zum Einfluss der logarithmierten Spermienkonzentration auf die Lebensdauer der normozoospermen Fälle ${ }^{a}$, späte Jahrgänge

\begin{tabular}{|c|c|c|c|c|c|c|c|c|}
\hline & \multirow{2}{*}{ B } & \multirow{2}{*}{ SE } & \multirow{2}{*}{ Wald } & \multirow{2}{*}{ df } & \multirow{2}{*}{$\mathbf{p}$} & \multirow{2}{*}{$\begin{array}{l}\text { Odds } \\
\text { Ratio }\end{array}$} & \multicolumn{2}{|c|}{$95 \% \mathrm{Cl}$ for OR } \\
\hline & & & & & & & Lower & Upper \\
\hline log. Spermienkonz. & $-0,815$ & 0,678 & 1,446 & 1 & 0,229 & 0,443 & 0,117 & 1,671 \\
\hline $\begin{array}{l}\text { Lebenserwartung bei } \\
\text { Geburt }\end{array}$ & 2,124 & 1,719 & 1,526 & 1 & 0,217 & 8,366 & 0,288 & 2,275 \\
\hline Geburtsmonat & $-0,010$ & 0,058 & 0,033 & 1 & 0,856 & 0,990 & 0,884 & 1,108 \\
\hline Alter bei Untersuchung & $-0,234$ & 0,233 & 1,010 & 1 & 0,315 & 0,791 & 0,501 & 1,249 \\
\hline Untersuchungsjahr & 0,242 & 0,231 & 1,095 & 1 & 0,295 & 1,274 & 0,810 & 2,004 \\
\hline ISEI & $-0,017$ & 0,011 & 2,257 & 1 & 0,133 & 0,984 & 0,963 & 1,005 \\
\hline
\end{tabular}

anzensierte Fälle $n=28$; zensierte Fälle $n=137$ 
Tab. A-41 Exponential-Modell zum Einfluss der logarithmierten Spermienkonzentration auf die Lebensdauer der normozoospermen Fälle ${ }^{a}$

\begin{tabular}{|l|l||l||l||l|||l|l|}
\hline Variable & Coeff & Error & C/Error & Signif & p \\
\hline log. Spermienkonzentration & 0,1088 & 0,3878 & 0,2805 & 0,2209 & 0,780 \\
\hline Lebenserwartung bei Geburt & 0,0618 & 0,1236 & 0,4998 & 0,3828 & 0,617 \\
\hline Geburtsmonat & $-0,0071$ & 0,0342 & $-0,2074$ & 0,1643 & 0,836 \\
\hline Alter bei Untersuchung & 0,1104 & 0,0636 & 1,7345 & 0,9172 & 0,083 \\
\hline \hline Untersuchungsjahr & $-0,1002$ & 0,0632 & $-1,5860$ & 0,8873 & 0,113 \\
\hline ISEI & 0,0002 & 0,0053 & 0,0362 & 0,0289 & 0,971 \\
\hline
\end{tabular}

${ }^{a}$ unzensierte Fälle $n=76$; zensierte Fälle $n=232$

Tab. A-42 Exponential-Modell zum Einfluss der logarithmierten Spermienkonzentration auf die Lebensdauer der normozoospermen Fälle ${ }^{a}$; frühe Geburtsjahrgänge

\begin{tabular}{|c|c|c|c|c|c|c|}
\hline Variable & Coeff & Error & C/Error & Signif & $\mathbf{p}$ & $\begin{array}{l}\text { Rel. } \\
\text { Risiko }\end{array}$ \\
\hline log. Spermienkonzentration & 0,4670 & 0,4832 & 0,9665 & 0,6662 & 0,334 & 1,5953 \\
\hline Lebenserwartung bei Geburt & 0,2799 & 0,2862 & 0,9782 & 0,6720 & 0,328 & 1,3230 \\
\hline Geburtsmonat & 0,0007 & 0,0443 & 0,0169 & 0,0134 & 0,987 & 1,0007 \\
\hline Alter bei Untersuchung & 0,2473 & 0,1738 & 1,4229 & 0,8452 & 0,155 & 1,2805 \\
\hline Untersuchungsjahr & $-0,2445$ & 0,1759 & $-1,3904$ & 0,8356 & 0,164 & 0,7831 \\
\hline ISEI & 0,0065 & 0,0065 & 0,9964 & 0,6809 & 0,319 & 1,0065 \\
\hline
\end{tabular}

anzensierte Fälle $n=48$; zensierte Fälle $n=94$

Tab. A-43 Exponential-Modell zum Einfluss der logarithmierten Spermienkonzentration auf die Lebensdauer der normozoospermen Fälle ${ }^{a}$; späte Geburtsjahrgänge

\begin{tabular}{|c|c|c|c|c|c|c|}
\hline Variable & Coeff & Error & CIError & Signif & $\mathbf{p}$ & $\begin{array}{l}\text { Rel. } \\
\text { Risiko }\end{array}$ \\
\hline log. Spermienkonzentration & $-0,7685$ & 0,6727 & $-1,1423$ & 0,7467 & 0,253 & 0,4637 \\
\hline Lebenserwartung bei Geburt & 1,7364 & 1,6182 & 1,0730 & 0,7167 & 0,283 & 5,6771 \\
\hline Geburtsmonat & $-0,0072$ & 0,0579 & $-0,1245$ & 0,0991 & 0,901 & 0,9928 \\
\hline Alter bei Untersuchung & $-0,0853$ & 0,2074 & $-0,4115$ & 0,3193 & 0,681 & 0,9182 \\
\hline Untersuchungsjahr & 0,0980 & 0,2074 & 0,4724 & 0,3634 & 0,637 & 1,1029 \\
\hline ISEI & $-0,0150$ & 0,0109 & $-1,3758$ & 0,8311 & 0,169 & 0,9851 \\
\hline
\end{tabular}

${ }^{a}$ unzensierte Fälle $n=28$; zensierte Fälle $n=137$

Tab. A-44 Gompertz-Makeham-Modell zum Einfluss der logarithmierten Spermienkonzentration auf die Lebensdauer der normozoospermen Fälle ${ }^{a}$

\begin{tabular}{|c|c|c|c|c|c|c|}
\hline Variable & Coeff & Error & ClError & Signif & $\mathbf{p}$ & $\begin{array}{l}\text { Rel. } \\
\text { Risiko }\end{array}$ \\
\hline log. Spermienkonzentration & 0,2753 & 0,4048 & 0,6801 & 0,5036 & 0,496 & 1,3169 \\
\hline Lebenserwartung bei Geburt & 0,0214 & 0,1228 & 0,1741 & 0,1382 & 0,862 & 1,0216 \\
\hline Geburtsmonat & $-0,0058$ & 0,0336 & $-0,1724$ & 0,1369 & 0,863 & 0,9942 \\
\hline Alter bei Untersuchung & 0,1133 & 0,0633 & 1,7902 & 0,9266 & 0,073 & $1,1200^{(*)}$ \\
\hline Untersuchungsjahr & $-0,1076$ & 0,0623 & $-1,7279$ & 0,9160 & 0,084 & $0,8980^{(*)}$ \\
\hline ISEI & 0,0028 & 0,0055 & 0,5112 & 0,3908 & 0,609 & 1,0028 \\
\hline
\end{tabular}

${ }^{a}$ unzensierte Fälle $n=76$; zensierte Fälle $n=232$

Tab. A-45 Gompertz-Makeham-Modell zum Einfluss der logarithmierten Spermienkonzentration auf die Lebensdauer der normozoospermen Fälle ${ }^{a}$; frühe Geburtsjahrgänge

\begin{tabular}{|c|c|c|c|c|c|c|}
\hline Variable & Coeff & Error & C/Error & Signif & $\mathbf{p}$ & $\begin{array}{l}\text { Rel. } \\
\text { Risiko }\end{array}$ \\
\hline log. Spermienkonzentration & 0,8523 & 0,5181 & 1,6449 & 0,9000 & 0,100 & 2,3450 \\
\hline Lebenserwartung bei Geburt & 0,2167 & 0,2710 & 0,7998 & 0,5762 & 0,424 & 1,2420 \\
\hline Geburtsmonat & 0,0005 & 0,0428 & 0,0125 & 0,0100 & 0,990 & 1,0005 \\
\hline Alter bei Untersuchung & 0,2590 & 0,1667 & 1,5540 & 0,8798 & 0,320 & 1,2957 \\
\hline Untersuchungsjahr & $-0,2640$ & 0,1686 & $-1,5657$ & 0,8826 & 0,117 & 0,7680 \\
\hline ISEI & 0,0146 & 0,0072 & 2,0315 & 0,9578 & 0,042 & $1,0148^{*}$ \\
\hline
\end{tabular}

anzensierte Fälle $n=48$; zensierte Fälle $n=94$ 
Tab. A-46 Gompertz-Makeham-Modell zum Einfluss der logarithmierten Spermienkonzentration auf die Lebensdauer der normozoospermen Fälle ${ }^{a}$; späte Geburtsjahrgänge

\begin{tabular}{|l|l|l|l|l|l|l|l|}
\hline Variable & Coeff & Error & C/Error & Signif & $\mathbf{p}$ & $\begin{array}{l}\text { Rel. } \\
\text { Risiko }\end{array}$ \\
\hline log. Spermienkonzentration & $-0,8021$ & 0,6763 & $-1,1861$ & 0,7644 & 0,236 & 0,4484 \\
\hline Lebenserwartung bei Geburt & 1,8635 & 1,5828 & 1,1773 & 0,7609 & 0,239 & 6,4461 \\
\hline Geburtsmonat & $-0,0036$ & 0,0568 & $-0,0642$ & 0,0512 & 0,949 & 0,9964 \\
\hline Alter bei Untersuchung & $-0,0861$ & 0,2060 & $-0,4178$ & 0,3239 & 0,676 & 0,9175 \\
\hline Untersuchungsjahr & 0,0995 & 0,2052 & 0,4847 & 0,3721 & 0,628 & 1,1046 \\
\hline ISEI & $-0,0169$ & 0,0110 & $-1,5363$ & 0,8755 & 0,125 & 0,9832 \\
\hline
\end{tabular}

${ }^{a}$ unzensierte Fälle $n=28$; zensierte Fälle $n=137$

Tab. A-47 Weibull-Modell zum Einfluss der logarithmierten Spermienkonzentration auf die Lebensdauer der normozoospermen Fälle ${ }^{a, b}$

\begin{tabular}{|l|l|l|l|l|l|l|l|}
\hline Variable & Coeff & Error & C/Error & Signif & $p$ & $\begin{array}{l}\text { Rel. } \\
\text { Risiko }\end{array}$ \\
\hline \hline log. Spermienkonzentration & 0,0053 & 0,0076 & 0,6898 & 0,5097 & 0,490 & 1,3230 \\
\hline \hline Lebenserwartung bei Geburt & $-0,0047$ & 0,0023 & 2,0045 & 0,9550 & 0,045 & $0,7805^{*}$ \\
\hline \hline Geburtsmonat & $-0,0003$ & 0,0006 & $-0,4660$ & 0,3588 & 0,641 & 0,9844 \\
\hline Alter bei Untersuchung & $-0,0005$ & 0,0011 & $-0,4771$ & 0,3667 & 0,633 & 0,9719 \\
\hline \hline Untersuchungsjahr & 0,0008 & 0,0011 & 0,6826 & 0,5051 & 0,495 & 1,0412 \\
\hline ISEI & 0,0001 & 0,0001 & 0,5615 & 0,4255 & 0,575 & 1,0030 \\
\hline
\end{tabular}

${ }^{a}$ unzensierte Fälle $n=76$; zensierte Fälle $n=232$

b Convergence not reached in 500000 iterations; Number of function evaluations: 1000002

Tab. A-48 Weibull-Modell zum Einfluss der logarithmierten Spermienkonzentration auf die Lebensdauer der normozoospermen Fälle ${ }^{a}$; frühe Geburtsjahrgänge

\begin{tabular}{|l|l|l|l|l||l||l|}
\hline Variable & Coeff & Error & C/Error & Signif & p & $\begin{array}{l}\text { Rel. } \\
\text { Risiko }\end{array}$ \\
\hline log. Spermienkonzentration & 0,0154 & 0,0084 & 1,8350 & 0,9335 & 0,067 & $2,5701^{(*)}$ \\
\hline Lebenserwartung bei Geburt & $-0,0042$ & 0,0049 & $-0,8597$ & 0,6101 & 0,390 & 0,7746 \\
\hline Geburtsmonat & $-0,0003$ & 0,0007 & $-0,4082$ & 0,3169 & 0,683 & 0,9825 \\
\hline Alter bei Untersuchung & $-0,0006$ & 0,0030 & $-0,2043$ & 0,1619 & 0,838 & 0,9636 \\
\hline Untersuchungsjahr & 0,0006 & 0,0030 & 0,2068 & 0,1638 & 0,836 & 1,0386 \\
\hline ISEI & 0,0003 & 0,0001 & 2,3535 & 0,9814 & 0,019 & $1,0177^{*}$ \\
\hline
\end{tabular}

a unzensierte Fälle $n=48$; zensierte Fälle $n=94$

Tab. A-49 Weibull-Modell zum Einfluss der logarithmierten Spermienkonzentration auf die Lebensdauer der normozoospermen Fälle ${ }^{a}$; späte Geburtsjahrgänge

\begin{tabular}{|c|c|c|c|c|c|c|}
\hline Variable & Coeff & Error & C/Error & Signif & $\mathbf{p}$ & $\begin{array}{l}\text { Rel. } \\
\text { Risiko }\end{array}$ \\
\hline log. Spermienkonzentration & $-0,0168$ & 0,0145 & $-1,1619$ & 0,7547 & 0,245 & 0,4460 \\
\hline Lebenserwartung bei Geburt & 0,0258 & 0,0278 & 0,9284 & 0,6468 & 0,353 & 3,4561 \\
\hline Geburtsmonat & 0,0000 & 0,0012 & $-0,0255$ & 0,0204 & 0,980 & 0,9986 \\
\hline Alter bei Untersuchung & 0,0001 & 0,0036 & 0,0345 & 0,0275 & 0,973 & 1,0059 \\
\hline Untersuchungsjahr & 0,0001 & 0,0036 & 0,0365 & 0,0291 & 0,971 & 1,0062 \\
\hline ISEI & $-0,0004$ & 0,0002 & $-1,5100$ & 0,8690 & 0,131 & 0,9828 \\
\hline
\end{tabular}

a unzensierte Fälle $n=28$; zensierte Fälle $n=137$ 


\section{A-7 Validität der Trennung zwischen fertilen und subfertilen Fällen: Sukzessive Verschiebung der Klassifikationsgrenze}

Tab. A-50 Ergebnisse des Wilcoxon(Gehan)-Tests bei sukzessiver Verschiebung des Trennwertes zur Unterscheidung von fertilen und subfertilen Fällen

\begin{tabular}{|c|c|c|c|c|}
\hline Aramoininut ranin $1 \mathrm{mll}$ & Vanmlninh $/$ & & Intilnnuan (Anghanl & n \\
\hline 5 & fertil (467) & subfertil (125) & 2,363 & 0,1243 \\
\hline 10 & fertil (446) & subfertil (146) & 3,945 & 0,0470 \\
\hline 15 & fertil (418) & subfertil (174) & 4,163 & 0,0413 \\
\hline 20 & fertil (391) & subfertil (201) & 3,430 & 0,0640 \\
\hline 25 & fertil (379) & subfertil (213) & 2,704 & 0,1001 \\
\hline 30 & fertil (354) & subfertil (238) & 4,204 & 0,0403 \\
\hline 35 & fertil (340) & subfertil (252) & 4,138 & 0,0419 \\
\hline 40 & fertil (327) & subfertil (265) & 4,272 & 0,0387 \\
\hline 45 & fertil (312) & subfertil (280) & 5,003 & 0,0253 \\
\hline 50 & fertil (297) & subfertil (295) & 3,536 & 0,0601 \\
\hline 55 & fertil (285) & subfertil (307) & 3,587 & 0,0582 \\
\hline 60 & fertil (266) & subfertil (326) & 7,098 & 0,0077 \\
\hline 65 & fertil (249) & subfertil (343) & 5,288 & 0,0215 \\
\hline 70 & fertil (236) & subfertil (356) & 3,626 & 0,0569 \\
\hline 75 & fertil (223) & subfertil (369) & 4,767 & 0,0290 \\
\hline 80 & fertil (210) & subfertil (382) & 5,290 & 0,0215 \\
\hline 85 & fertil (203) & subfertil (389) & 5,587 & 0,0181 \\
\hline 90 & fertil (190) & subfertil (402) & 5,331 & 0,0210 \\
\hline 95 & fertil (180) & subfertil (412) & 4,100 & 0,0429 \\
\hline 100 & fertil (169) & subfertil (423) & 4,265 & 0,0389 \\
\hline
\end{tabular}

Tab. A-51 Ergebnisse des Wilcoxon(Gehan)-Tests bei sukzessiver Verschiebung des Trennwertes zur Unterscheidung von normo- und oligozoospermen Fällen

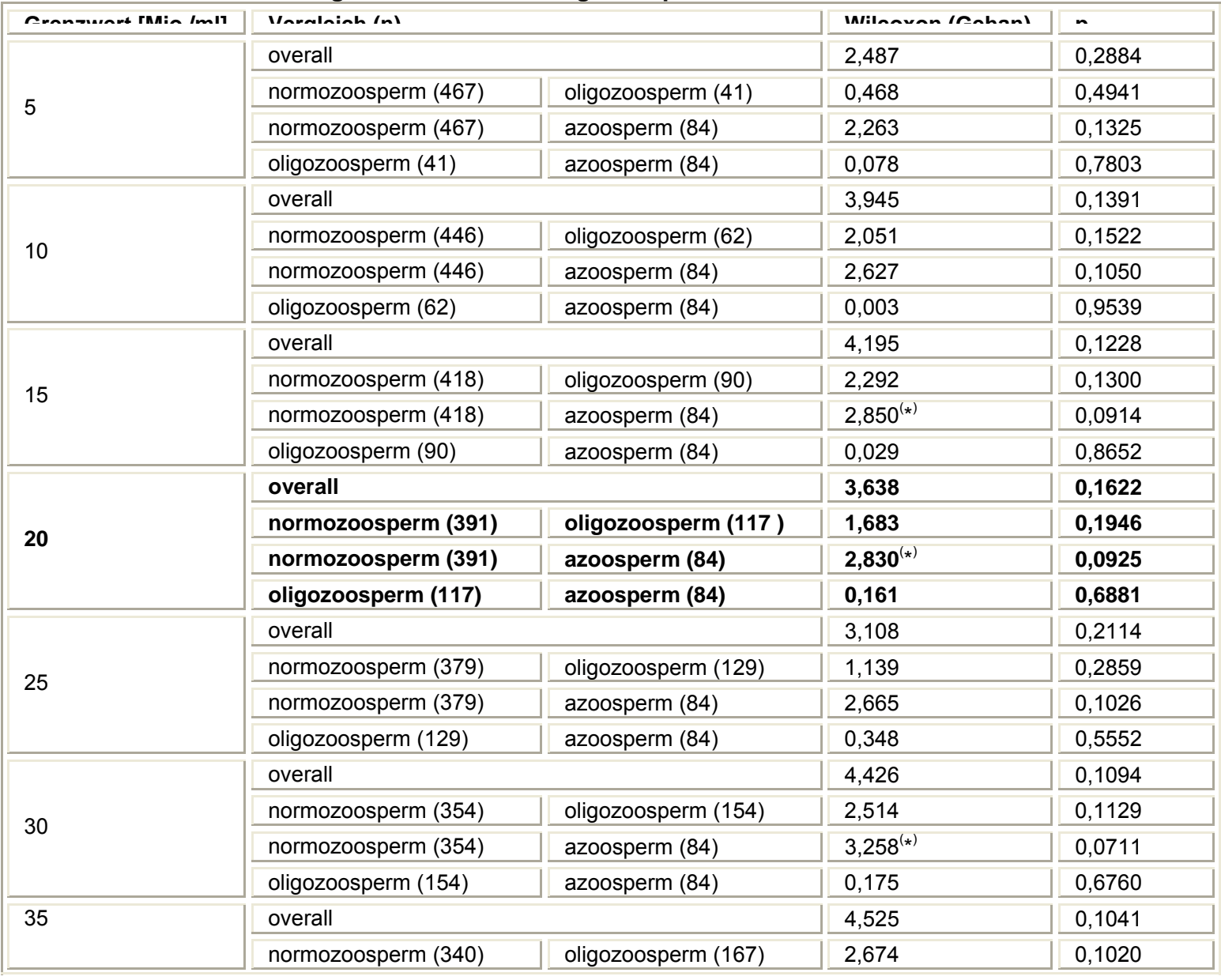




\begin{tabular}{|c|c|c|c|c|}
\hline & normozonsnerm (340) & a7nosnerm (84) & $3.302^{(*)}$ & 006.92 \\
\hline & oligozoosperm (167) & azoosperm (84) & 0,201 & 0,6538 \\
\hline \multirow{4}{*}{40} & \multicolumn{2}{|l|}{ overall } & $4,682^{(*)}$ & 0,0962 \\
\hline & normozoosperm (327) & oligozoosperm (180) & $2,835^{(\star)}$ & 0,0922 \\
\hline & normozoosperm (327) & azoosperm (84) & $3,421^{(*)}$ & 0,0644 \\
\hline & oligozoosperm (180) & azoosperm (84) & 0,223 & 0,6367 \\
\hline \multirow{4}{*}{45} & \multicolumn{2}{|l|}{ overall } & $5,382^{(*)}$ & 0,0678 \\
\hline & normozoosperm (312) & oligozoosperm (195) & $3,556^{(*)}$ & 0,0593 \\
\hline & normozoosperm (312) & azoosperm (84) & $3,782^{(*)}$ & 0,0518 \\
\hline & oligozoosperm (195) & azoosperm (84) & 0,186 & 0,6666 \\
\hline \multirow{4}{*}{50} & \multicolumn{2}{|l|}{ overall } & 4,129 & 0,1269 \\
\hline & normozoosperm (297) & oligozoosperm (210) & 2,236 & 0,1348 \\
\hline & normozoosperm (297) & azoosperm (84) & $3,359^{(*)}$ & 0,0668 \\
\hline & oligozoosperm (210) & azoosperm (84) & 0,400 & 0,5272 \\
\hline \multirow{4}{*}{55} & \multicolumn{2}{|l|}{ overall } & 4,209 & 0,1219 \\
\hline & normozoosperm (285) & oligozoosperm (222) & 2,324 & 0,1274 \\
\hline & normozoosperm (285) & azoosperm (84) & $3,453^{(*)}$ & 0,0631 \\
\hline & oligozoosperm (222) & azoosperm (84) & 0,432 & 0,5110 \\
\hline \multirow{4}{*}{60} & \multicolumn{2}{|l|}{ overall } & $7,455^{*}$ & 0,0241 \\
\hline & normozoosperm (266) & oligozoosperm (241) & $5,750^{*}$ & 0,0165 \\
\hline & normozoosperm (266) & azoosperm (84) & $4,930^{*}$ & 0,0264 \\
\hline & oligozoosperm (241) & azoosperm (84) & 0,169 & 0,6810 \\
\hline \multirow{4}{*}{65} & \multicolumn{2}{|l|}{ overall } & $5,828^{(*)}$ & 0,0543 \\
\hline & normozoosperm (249) & oligozoosperm (258) & $4,073^{*}$ & 0,0436 \\
\hline & normozoosperm (249) & azoosperm (84) & $4,366^{*}$ & 0,0367 \\
\hline & oligozoosperm (258) & azoosperm (84) & 0,373 & 0,5412 \\
\hline \multirow{4}{*}{70} & \multicolumn{2}{|l|}{ overall } & 4,392 & 0,1112 \\
\hline & normozoosperm (236) & oligozoosperm (271) & 2,549 & 0,1104 \\
\hline & normozoosperm (236) & azoosperm (84) & $3,733^{(*)}$ & 0,0533 \\
\hline & oligozoosperm (271) & azoosperm (84) & 0,608 & 0,4355 \\
\hline \multirow{4}{*}{75} & \multicolumn{2}{|l|}{ overall } & $5,437^{(\star)}$ & 0,0660 \\
\hline & normozoosperm (223) & oligozoosperm (284) & $3,628^{(*)}$ & 0,0568 \\
\hline & normozoosperm (223) & azoosperm (84) & $4,434^{*}$ & 0,0352 \\
\hline & oligozoosperm (284) & azoosperm (84) & 0,493 & 0,4826 \\
\hline \multirow{4}{*}{80} & \multicolumn{2}{|l|}{ overall } & $5,950^{(*)}$ & 0,0510 \\
\hline & normozoosperm (210) & oligozoosperm (297) & $4,150^{*}$ & 0,0416 \\
\hline & normozoosperm (210) & azoosperm (84) & $4,836^{*}$ & 0,0279 \\
\hline & oligozoosperm (297) & azoosperm (84) & 0,477 & 0,4900 \\
\hline \multirow{4}{*}{85} & \multicolumn{2}{|l|}{ overall } & $6,244^{*}$ & 0,0441 \\
\hline & normozoosperm (203) & oligozoosperm (304) & $4,448^{*}$ & 0,0349 \\
\hline & normozoosperm (304) & azoosperm (84) & $5,074^{*}$ & 0,0243 \\
\hline & oligozoosperm (304) & azoosperm (84) & 0,469 & 0,4936 \\
\hline & overall & & $6,057^{*}$ & 0,0484 \\
\hline 90 & normozoosperm (190) & oligozoosperm (317) & $4,260^{*}$ & 0,0390 \\
\hline 50 & normozoosperm (190) & azoosperm (84) & $5,065^{*}$ & 0,0244 \\
\hline & oligozoosperm (317) & azoosperm (84) & 0,543 & 0,4611 \\
\hline & overall & & $4,983^{(*)}$ & 0,0828 \\
\hline 95 & normozoosperm (180) & oligozoosperm (327) & $3,144^{(*)}$ & 0,0762 \\
\hline 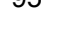 & normozoosperm (180) & azoosperm (84) & $4,458^{*}$ & 0,0347 \\
\hline & oligozoosperm (327) & azoosperm (84) & 0,715 & 0,3979 \\
\hline & overall & & $5,167^{(*)}$ & 0,0755 \\
\hline 100 & normozoosperm (169) & oligozoosperm (338) & $3,334^{(\star)}$ & 0,0679 \\
\hline 100 & normozoosperm (169) & azoosperm (84) & $4,693^{*}$ & 0,0303 \\
\hline & oligozoosperm (338) & azoosperm (84) & 0,731 & 0,3925 \\
\hline
\end{tabular}


Tab. A-52 Ergebnisse der Cox-Regression bei sukzessiver Verschiebung des Trennwertes zur Unterscheidung von fertilen und subfertilen Fällen

\begin{tabular}{|c|c|c|c|c|c|c|c|c|c|}
\hline \multirow{2}{*}{$\begin{array}{l}\text { Grenz- } \\
\text { wert } \\
\text { [Mio/ml] }\end{array}$} & \multirow{2}{*}{$\begin{array}{l}\text { Vergleichs- vs. } \\
\text { Referenzgruppe }\end{array}$} & \multirow{2}{*}{ B } & \multirow{2}{*}{ SE } & \multirow{2}{*}{ Wald } & \multirow{2}{*}{ df } & \multirow{2}{*}{$\mathbf{p}$} & \multirow{2}{*}{$\begin{array}{l}\text { Odds } \\
\text { Ratio }\end{array}$} & \multicolumn{2}{|c|}{$95 \% \mathrm{Cl}$ for OR } \\
\hline & & & & & & & & Lower & Upper \\
\hline 5 & subfertil vs. fertil & 0,179 & 0,214 & 0,697 & 1 & 0,404 & 1,196 & 0,786 & 1,821 \\
\hline 10 & subfertil vs. fertil & 0,171 & 0,206 & 0,691 & 1 & 0,406 & 1,187 & 0,793 & 1,776 \\
\hline 15 & subfertil vs. fertil & 0,234 & 0,193 & 1,468 & 1 & 0,226 & 1,264 & 0,865 & 1,847 \\
\hline 20 & subfertil vs. fertil & 0,284 & 0,186 & 2,330 & 1 & 0,127 & 1,328 & 0,923 & 1,912 \\
\hline 25 & subfertil vs. fertil & 0,244 & 0,184 & 1,764 & 1 & 0,184 & 1,277 & 0,890 & 1,832 \\
\hline 30 & subfertil vs. fertil & 0,221 & 0,183 & 1,453 & 1 & 0,228 & 1,247 & 0,871 & 1,787 \\
\hline 35 & subfertil vs. fertil & 0,225 & 0,182 & 1,523 & 1 & 0,217 & 1,252 & 0,876 & 1,790 \\
\hline 40 & subfertil vs. fertil & 0,260 & 0,182 & 2,035 & 1 & 0,154 & 1,297 & 0,907 & 1,854 \\
\hline 45 & subfertil vs. fertil & 0,272 & 0,183 & 2,213 & 1 & 0,137 & 1,312 & 0,917 & 1,876 \\
\hline 50 & subfertil vs. fertil & 0,251 & 0,183 & 1,884 & 1 & 0,170 & 1,285 & 0,898 & 1,840 \\
\hline 55 & subfertil vs. fertil & 0,262 & 0,185 & 1,998 & 1 & 0,158 & 1,299 & 0,904 & 1,868 \\
\hline 60 & subfertil vs. fertil & 0,346 & 0,187 & 3,402 & 1 & 0,065 & $1,413^{(*)}$ & 0,979 & 2,040 \\
\hline 65 & subfertil vs. fertil & 0,229 & 0,188 & 1,484 & 1 & 0,223 & 1,258 & 0,870 & 1,818 \\
\hline 70 & subfertil vs. fertil & 0,199 & 0,190 & 1,102 & 1 & 0,294 & 1,221 & 0,841 & 1,771 \\
\hline 75 & subfertil vs. fertil & 0,274 & 0,196 & 1,957 & 1 & 0,162 & 1,315 & 0,896 & 1,930 \\
\hline 80 & subfertil vs. fertil & 0,319 & 0,201 & 2,518 & 1 & 0,113 & 1,375 & 0,928 & 2,038 \\
\hline 85 & subfertil vs. fertil & 0,354 & 0,204 & 3,011 & 1 & 0,083 & $1,425^{(*)}$ & 0,955 & 2,125 \\
\hline 90 & subfertil vs. fertil & 0,275 & 0,206 & 1,790 & 1 & 0,181 & 1,317 & 0,880 & 1,971 \\
\hline 95 & subfertil vs. fertil & 0,273 & 0,209 & 1,697 & 1 & 0,193 & 1,313 & 0,872 & 1,979 \\
\hline 100 & subfertil vs. fertil & 0,186 & 0,211 & 0,777 & 1 & 0,378 & 1,205 & 0,796 & 1,822 \\
\hline
\end{tabular}

Tab. A-53 Ergebnisse der Cox-Regression bei sukzessiver Verschiebung des Trennwertes zur Unterscheidung von normo- und oligozoospermen Fällen

\begin{tabular}{|c|c|c|c|c|c|c|c|c|c|}
\hline \multirow{2}{*}{$\begin{array}{l}\text { Grenz- } \\
\text { wert } \\
\text { [Mio/ml] }\end{array}$} & \multirow{2}{*}{$\begin{array}{l}\text { Vergleichs- vs. } \\
\text { Referenzgruppe }\end{array}$} & \multirow{2}{*}{ B } & \multirow{2}{*}{ SE } & \multirow{2}{*}{ Wald } & \multirow{2}{*}{ df } & \multirow{2}{*}{$\mathbf{p}$} & \multirow{2}{*}{$\begin{array}{l}\text { Odds } \\
\text { Ratio }\end{array}$} & \multicolumn{2}{|c|}{$95 \% \mathrm{CI}$ for OR } \\
\hline & & & & & & & & Lower & Upper \\
\hline \multirow{3}{*}{5} & oligo- vs. normozoosperm & 0,311 & 0,324 & 0,922 & 1 & 0,337 & 1,365 & 0,723 & 2,578 \\
\hline & azoo- vs. normozoosperm & 0,110 & 0,256 & 0,184 & 1 & 0,668 & 1,116 & 0,676 & 1,844 \\
\hline & oligo- vs. azoosperm & 0,201 & 0,383 & 0,277 & 1 & 0,599 & 1,223 & 0,577 & 2,591 \\
\hline \multirow{3}{*}{10} & oligo- vs. normozoosperm & 0,248 & 0,282 & 0,771 & 1 & 0,380 & 1,282 & 0,737 & 2,229 \\
\hline & azoo- vs. normozoosperm & 0,113 & 0,257 & 0,195 & 1 & 0,659 & 1,120 & 0,677 & 1,854 \\
\hline & oligo- vs. azoosperm & 0,135 & 0,348 & 0,149 & 1 & 0,699 & 1,144 & 0,578 & 2,263 \\
\hline \multirow{3}{*}{15} & oligo- vs. normozoosperm & 0,313 & 0,238 & 1,730 & 1 & 0,188 & 1,368 & 0,858 & 2,181 \\
\hline & azoo- vs. normozoosperm & 0,144 & 0,259 & 0,309 & 1 & 0,579 & 1,155 & 0,695 & 1,920 \\
\hline & oligo- vs. azoosperm & 0,169 & 0,312 & 0,294 & 1 & 0,588 & 1,184 & 0,642 & 2,183 \\
\hline \multirow{3}{*}{20} & oligo- vs. normozoosp. & 0,359 & 0,217 & 2,733 & 1 & 0,098 & $1,432^{(*)}$ & 0,936 & 2,191 \\
\hline & azoo- vs. normozoosp. & 0,171 & 0,261 & 0,431 & 1 & 0,512 & 1,187 & 0,711 & 1,981 \\
\hline & oligo- vs. azoosperm & 0,187 & 0,296 & 0,400 & 1 & 0,527 & 1,206 & 0,675 & 2,156 \\
\hline \multirow{3}{*}{25} & oligo- vs. normozoosperm & 0,295 & 0,213 & 1,930 & 1 & 0,165 & 1,344 & 0,886 & 2,039 \\
\hline & azoo- vs. normozoosperm & 0,161 & 0,262 & 0,379 & 1 & 0,538 & 1,175 & 0,703 & 1,962 \\
\hline & oligo- vs. azoosperm & 0,134 & 0,293 & 0,210 & 1 & 0,647 & 1,144 & 0,644 & 2,031 \\
\hline \multirow{3}{*}{30} & oligo- vs. normozoosperm & 0,249 & 0,205 & 1,467 & 1 & 0,226 & 1,282 & 0,858 & 1,917 \\
\hline & azoo- vs. normozoosperm & 0,166 & 0,265 & 0,393 & 1 & 0,531 & 1,181 & 0,702 & 1,985 \\
\hline & oligo- vs. azoosperm & 0,082 & 0,284 & 0,085 & 1 & 0,771 & 1,086 & 0,623 & 1,893 \\
\hline
\end{tabular}


Anhang

\begin{tabular}{|c|c|c|c|c|c|c|c|c|c|}
\hline \multirow{3}{*}{35} & oligo- vs. normozoosperm & 0,249 & 0,202 & 1,527 & 1 & 0,217 & 1,283 & 0,864 & 1,905 \\
\hline & azoo- vs. normozoosperm & 0,172 & 0,266 & 0,419 & 1 & 0,517 & 1,188 & 0,705 & 2,001 \\
\hline & oligo- vs. azoosperm & 0,077 & 0,280 & 0,075 & 1 & 0,784 & 1,080 & 0,623 & 1,871 \\
\hline \multirow{3}{*}{40} & oligo- vs. normozoosperm & 0,289 & 0,200 & 2,092 & 1 & 0,148 & 1,335 & 0,903 & 1,973 \\
\hline & azoo- vs. normozoosperm & 0,194 & 0,268 & 0,526 & 1 & 0,468 & 1,214 & 0,719 & 2,051 \\
\hline & oligo- vs. azoosperm & 0,095 & 0,277 & 0,116 & 1 & 0,733 & 1,099 & 0,638 & 1,893 \\
\hline \multirow{3}{*}{45} & oligo- vs. normozoosperm & 0,298 & 0,198 & 2,266 & 1 & 0,132 & 1,347 & 0,914 & 1,985 \\
\hline & azoo- vs. normozoosperm & 0,206 & 0,269 & 0,587 & 1 & 0,444 & 1,229 & 0,725 & 2,084 \\
\hline & oligo- vs. azoosperm & 0,092 & 0,275 & 0,111 & 1 & 0,739 & 1,096 & 0,640 & 1,877 \\
\hline \multirow{3}{*}{50} & oligo- vs. normozoosperm & 0,270 & 0,197 & 1,881 & 1 & 0,170 & 1,311 & 0,890 & 1,929 \\
\hline & azoo-vs. normozoosperm & 0,201 & 0,271 & 0,550 & 1 & 0,458 & 1,222 & 0,719 & 2,077 \\
\hline & oligo- vs. azoosperm & 0,070 & 0,273 & 0,066 & 1 & 0,798 & 1,072 & 0,628 & 1,831 \\
\hline \multirow{3}{*}{55} & oligo- vs. normozoosperm & 0,279 & 0,198 & 1,987 & 1 & 0,159 & 1,322 & 0,897 & 1,949 \\
\hline & azoo- vs. normozoosperm & 0,214 & 0,273 & 0,614 & 1 & 0,433 & 1,238 & 0,725 & 2,115 \\
\hline & oligo- vs. azoosperm & 0,065 & 0,271 & 0,058 & 1 & 0,810 & 1,067 & 0,628 & 1,814 \\
\hline \multirow{3}{*}{60} & oligo- vs. normozoosperm & 0,371 & 0,198 & 3,500 & 1 & 0,061 & $1,449^{(*)}$ & 0,982 & 2,136 \\
\hline & azoo- vs. normozoosperm & 0,271 & 0,276 & 0,958 & 1 & 0,328 & 1,311 & 0,762 & 2,253 \\
\hline & oligo- vs. azoosperm & 0,100 & 0,268 & 0,140 & 1 & 0,708 & 1,105 & 0,654 & 1,868 \\
\hline \multirow{3}{*}{65} & oligo- vs. normozoosperm & 0,235 & 0,198 & 1,414 & 1 & 0,234 & 1,266 & 0,858 & 1,866 \\
\hline & azoo- vs. normozoosperm & 0,209 & 0,278 & 0,563 & 1 & 0,453 & 1,232 & 0,714 & 2,124 \\
\hline & oligo- vs. azoosperm & 0,027 & 0,267 & 0,010 & 1 & 0,920 & 1,027 & 0,609 & 1,732 \\
\hline \multirow{3}{*}{70} & oligo- vs. normozoosperm & 0,201 & 0,199 & 1,018 & 1 & 0,313 & 1,222 & 0,828 & 1,805 \\
\hline & azoo- vs. normozoosperm & 0,195 & 0,280 & 0,483 & 1 & 0,487 & 1,215 & 0,702 & 2,104 \\
\hline & oligo- vs. azoosperm & 0,006 & 0,265 & 0,000 & 1 & 0,982 & 1,006 & 0,598 & 1,692 \\
\hline \multirow{3}{*}{75} & oligo- vs. normozoosperm & 0,280 & 0,203 & 1,893 & 1 & 0,169 & 1,323 & 0,888 & 1,970 \\
\hline & azoo- vs. normozoosperm & 0,252 & 0,286 & 0,778 & 1 & 0,378 & 1,287 & 0,735 & 2,252 \\
\hline & oligo- vs. azoosperm & 0,028 & 0,263 & 0,011 & 1 & 0,916 & 1,028 & 0,614 & 1,721 \\
\hline \multirow{3}{*}{80} & oligo- vs. normozoosperm & 0,326 & 0,207 & 2,470 & 1 & 0,116 & 1,385 & 0,923 & 2,081 \\
\hline & azoo-vs. normozoosperm & 0,289 & 0,290 & 0,995 & 1 & 0,318 & 1,335 & 0,757 & 2,357 \\
\hline & oligo- vs. azoosperm & 0,037 & 0,262 & 0,020 & 1 & 0,888 & 1,038 & 0,621 & 1,733 \\
\hline \multirow{3}{*}{85} & oligo- vs. normozoosperm & 0,363 & 0,210 & 2,978 & 1 & 0,084 & $1,438^{(*)}$ & 0,952 & 2,171 \\
\hline & azoo- vs. normozoosperm & 0,318 & 0,293 & 1,182 & 1 & 0,277 & 1,374 & 0,775 & 2,439 \\
\hline & oligo- vs. azoosperm & 0,045 & 0,261 & 0,030 & 1 & 0,863 & 1,046 & 0,627 & 1,745 \\
\hline \multirow{3}{*}{90} & oligo- vs. normozoosperm & 0,277 & 0,212 & 1,714 & 1 & 0,190 & 1,320 & 0,871 & 1,999 \\
\hline & azoo- vs. normozoosperm & 0,267 & 0,294 & 0,822 & 1 & 0,365 & 1,306 & 0,734 & 2,324 \\
\hline & oligo- vs. azoosperm & 0,011 & 0,261 & 0,002 & 1 & 0,968 & 1,011 & 0,606 & 1,685 \\
\hline \multirow{3}{*}{95} & oligo- vs. normozoosperm & 0,274 & 0,215 & 1,619 & 1 & 0,203 & 1,315 & 0,863 & 2,004 \\
\hline & azoo- vs. normozoosperm & 0,268 & 0,297 & 0,817 & 1 & 0,366 & 1,308 & 0,731 & 2,341 \\
\hline & oligo- vs. azoosperm & 0,005 & 0,260 & 0,000 & 1 & 0,984 & 1,005 & 0,603 & 1,674 \\
\hline \multirow{3}{*}{100} & oligo- vs. normozoosperm & 0,181 & 0,217 & 0,699 & 1 & 0,403 & 1,199 & 0,784 & 1,834 \\
\hline & azoo-vs. normozoosperm & 0,207 & 0,298 & 0,481 & 1 & 0,488 & 1,230 & 0,685 & 2,207 \\
\hline & oligo- vs. azoosperm & $-0,026$ & 0,260 & 0,010 & 1 & 0,922 & 0,975 & 0,585 & 1,624 \\
\hline
\end{tabular}


Tab. A-54 Ergebnisse des Wilcoxon(Gehan)-Tests bei sukzessiver Verschiebung des Trennwertes zur Unterscheidung von fertilen und subfertilen Fällen; frühe Geburtsjahrgänge

\begin{tabular}{|c|c|c|c|c|}
\hline \multirow{2}{*}{$\begin{array}{l}\text { Smansininut ranin } \operatorname{lm} l 1 \\
5\end{array}$} & \multicolumn{2}{|c|}{ VInralninh $|m|$} & \multirow{2}{*}{$\begin{array}{l}\text { INilnnunn IRnhan' } \\
1,412\end{array}$} & \multirow{2}{*}{$\begin{array}{l}\text { n } \\
0,2347\end{array}$} \\
\hline & fertil (223) & subfertil (73) & & \\
\hline 10 & fertil (212) & subfertil (84) & $3,943^{*}$ & 0,0471 \\
\hline 15 & fertil (196) & subfertil (100) & $5,069^{*}$ & 0,0244 \\
\hline 20 & fertil (185) & subfertil (111) & $7,091^{\star *}$ & 0,0077 \\
\hline 25 & fertil (179) & subfertil (117) & $5,527^{*}$ & 0,0187 \\
\hline 30 & fertil (163) & subfertil (133) & $6,621^{*}$ & 0,0101 \\
\hline 35 & fertil (157) & subfertil (139) & $5,223^{*}$ & 0,0223 \\
\hline 40 & fertil (152) & subfertil (144) & $5,959^{*}$ & 0,0146 \\
\hline 45 & fertil (145) & subfertil (151) & $5,693^{*}$ & 0,0170 \\
\hline 50 & fertil (140) & subfertil (156) & $5,878^{*}$ & 0,0153 \\
\hline 55 & fertil (134) & subfertil (162) & $4,715^{\star}$ & 0,0299 \\
\hline 60 & fertil (130) & subfertil (166) & $5,805^{*}$ & 0,0160 \\
\hline 65 & fertil (119) & subfertil (177) & $3,293^{(*)}$ & 0,0696 \\
\hline 70 & fertil (111) & subfertil (185) & 2,392 & 0,1220 \\
\hline 75 & fertil (104) & subfertil (192) & 2,088 & 0,1485 \\
\hline 80 & fertil (99) & subfertil (197) & 2,012 & 0,1561 \\
\hline 85 & fertil (97) & subfertil (199) & 2,269 & 0,1320 \\
\hline 90 & fertil (93) & subfertil (203) & 1,409 & 0,2353 \\
\hline 95 & fertil (85) & subfertil (211) & 0,853 & 0,3556 \\
\hline 100 & fertil (78) & subfertil (218) & 0,560 & 0,4544 \\
\hline
\end{tabular}

Tab. A-55 Ergebnisse des Wilcoxon(Gehan)-Tests bei sukzessiver Verschiebung des Trennwertes zur Unterscheidung von normo- und oligozoospermen Fällen; frühe Geburtsjahrgänge

\begin{tabular}{|c|c|c|c|c|}
\hline Cunmanonot rnain Imll & lanmeraninh (m) & & 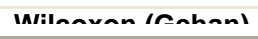 & n \\
\hline \multirow{4}{*}{5} & \multicolumn{2}{|l|}{ overall } & 1,503 & 0,4717 \\
\hline & normozoosperm (223) & oligozoosperm (21) & 0,222 & 0,6378 \\
\hline & normozoosperm (223) & azoosperm (52) & 1,415 & 0,2343 \\
\hline & oligozoosperm (21) & azoosperm (52) & 0,088 & 0,7664 \\
\hline \multirow{4}{*}{10} & \multicolumn{2}{|l|}{ overall } & 4,164 & 0,1247 \\
\hline & normozoosperm (212) & oligozoosperm (32) & $3,048^{(*)}$ & 0,0808 \\
\hline & normozoosperm (212) & azoosperm (52) & 1,958 & 0,1617 \\
\hline & oligozoosperm (32) & azoosperm (52) & 0,129 & 0,7199 \\
\hline \multirow{4}{*}{15} & \multicolumn{2}{|l|}{ overall } & $5,242^{(*)}$ & 0,0727 \\
\hline & normozoosperm (196) & oligozoosperm (48) & $4,175^{*}$ & 0,0410 \\
\hline & normozoosperm (196) & azoosperm (52) & 2,344 & 0,1258 \\
\hline & oligozoosperm (48) & azoosperm (52) & 0,102 & 0,7490 \\
\hline \multirow{4}{*}{20} & \multicolumn{2}{|l|}{ overall } & $7,405^{*}$ & 0,0247 \\
\hline & normozoosperm (185) & oligozoosperm (59) & $6,400^{*}$ & 0,0114 \\
\hline & normozoosperm (185) & azoosperm (52) & $2,942^{(*)}$ & 0,0863 \\
\hline & oligozoosperm (59) & azoosperm (52) & 0,222 & 0,6375 \\
\hline \multirow{4}{*}{25} & \multicolumn{2}{|l|}{ overall } & $5,590^{(*)}$ & 0,0611 \\
\hline & normozoosperm (179) & oligozoosperm (65) & $4,540^{*}$ & 0,0331 \\
\hline & normozoosperm (179) & azoosperm (52) & 2,667 & 0,1024 \\
\hline & oligozoosperm (65) & azoosperm (52) & 0,030 & 0,8625 \\
\hline \multirow{4}{*}{30} & \multicolumn{2}{|l|}{ overall } & $6,663^{*}$ & 0,0357 \\
\hline & normozoosperm (163) & oligozoosperm (81) & $5,642^{*}$ & 0,0175 \\
\hline & normozoosperm (163) & azoosperm (52) & $3,194^{(*)}$ & 0,0739 \\
\hline & oligozoosperm (81) & azoosperm (52) & 0,022 & 0,8817 \\
\hline \multirow{4}{*}{35} & \multicolumn{2}{|l|}{ overall } & $5,493^{(*)}$ & 0,0641 \\
\hline & normozoosperm (157) & oligozoosperm (86) & $4,467^{*}$ & 0,0345 \\
\hline & normozoosperm (157) & azoosperm (52) & $2,926^{(*)}$ & 0,0872 \\
\hline & oligozoosperm (86) & azoosperm (52) & 0,000 & 0,9919 \\
\hline
\end{tabular}




\begin{tabular}{|c|c|c|c|c|}
\hline \multirow{4}{*}{40} & \multicolumn{2}{|l|}{ airmall } & \multirow{2}{*}{$\begin{array}{l}\text { ล ग1a* } \\
5,255^{*}\end{array}$} & \multirow{2}{*}{$\begin{array}{l}n \cap 1 \wedge n \\
0,0219\end{array}$} \\
\hline & normozoosperm (152) & oligozoosperm (91) & & \\
\hline & normozoosperm (152) & azoosperm (52) & $3,208^{(*)}$ & 0,0733 \\
\hline & oligozoosperm (91) & azoosperm (52) & 0,002 & 0,9632 \\
\hline \multirow{4}{*}{45} & \multicolumn{2}{|l|}{ overall } & $5,950^{(*)}$ & 0,0510 \\
\hline & normozoosperm (145) & oligozoosperm (98) & $4,947^{*}$ & 0,0261 \\
\hline & normozoosperm (145) & azoosperm (52) & $3,235^{(\star)}$ & 0,0721 \\
\hline & oligozoosperm (98) & azoosperm (52) & 0,002 & 0,9688 \\
\hline \multirow{4}{*}{50} & \multicolumn{2}{|l|}{ overall } & $6,131^{*}$ & 0,0466 \\
\hline & normozoosperm (140) & oligozoosperm (103) & $5,130^{*}$ & 0,0235 \\
\hline & normozoosperm (140) & azoosperm (52) & $3,396^{(*)}$ & 0,0654 \\
\hline & oligozoosperm (103 ) & azoosperm (52) & 0,003 & 0,9542 \\
\hline \multirow{4}{*}{55} & \multicolumn{2}{|l|}{ overall } & $4,970^{(*)}$ & 0,0833 \\
\hline & normozoosperm (134) & oligozoosperm (109) & $3,939^{*}$ & 0,0472 \\
\hline & normozoosperm (134) & azoosperm (52) & $3,053^{(*)}$ & 0,0806 \\
\hline & oligozoosperm (109) & azoosperm (52) & 0,050 & 0,8236 \\
\hline \multirow{4}{*}{60} & \multicolumn{2}{|l|}{ overall } & $6,055^{*}$ & 0,0484 \\
\hline & normozoosperm (130) & oligozoosperm (113) & $5,082^{*}$ & 0,0242 \\
\hline & normozoosperm (130) & azoosperm (52) & $3,491^{(*)}$ & 0,0617 \\
\hline & oligozoosperm (113) & azoosperm (52) & 0,023 & 0,8808 \\
\hline \multirow{4}{*}{65} & \multicolumn{2}{|l|}{ overall } & 3,652 & 0,1610 \\
\hline & normozoosperm (119) & oligozoosperm (124) & 2,567 & 0,1091 \\
\hline & normozoosperm (119) & azoosperm (52) & 2,665 & 0,1026 \\
\hline & oligozoosperm (124) & azoosperm (52) & 0,211 & 0,6463 \\
\hline \multirow{4}{*}{70} & \multicolumn{2}{|l|}{ overall } & 2,861 & 0,2391 \\
\hline & normozoosperm (111) & oligozoosperm (132) & 1,735 & 0,1877 \\
\hline & normozoosperm (111) & azoosperm (52) & 2,326 & 0,1273 \\
\hline & oligozoosperm (132) & azoosperm (52) & 0,356 & 0,5510 \\
\hline \multirow{4}{*}{75} & \multicolumn{2}{|l|}{ overall } & 2,622 & 0,2696 \\
\hline & normozoosperm (104) & oligozoosperm (139) & 1,453 & 0,2281 \\
\hline & normozoosperm (104) & azoosperm (52) & 2,274 & 0,1316 \\
\hline & oligozoosperm (139) & azoosperm (52) & 0,412 & 0,5211 \\
\hline \multirow{4}{*}{80} & \multicolumn{2}{|l|}{ overall } & 2,574 & 0,2761 \\
\hline & normozoosperm (99) & oligozoosperm (144) & 1,412 & 0,2348 \\
\hline & normozoosperm (99) & azoosperm (52) & 2,238 & 0,1346 \\
\hline & oligozoosperm (144) & azoosperm (52) & 0,454 & 0,5004 \\
\hline \multirow{4}{*}{85} & overall & & 2,806 & 0,2459 \\
\hline & normozoosperm (97) & oligozoosperm (146) & 1,663 & 0,1973 \\
\hline & normozoosperm (97) & azoosperm (52) & 2,370 & 0,1237 \\
\hline & oligozoosperm (146) & azoosperm (52) & 0,429 & 0,5126 \\
\hline & overall & & 2,088 & 0,3521 \\
\hline 90 & normozoosperm (93) & oligozoosperm (150) & 0,910 & 0,3401 \\
\hline 90 & normozoosperm (93) & azoosperm (52) & 1,890 & 0,1691 \\
\hline & oligozoosperm (150) & azoosperm (52) & 0,602 & 0,4376 \\
\hline & overall & & 1,675 & 0,4327 \\
\hline 95 & normozoosperm (85) & oligozoosperm (158) & 0,492 & 0,4832 \\
\hline 90 & normozoosperm (85) & azoosperm (52) & 1,475 & 0,2246 \\
\hline & oligozoosperm (158) & azoosperm (52) & 0,802 & 0,3706 \\
\hline & overall & & 1,481 & 0,4769 \\
\hline 100 & normozoosperm (78) & oligozoosperm (165) & 0,275 & 0,6001 \\
\hline 100 & normozoosperm (78) & azoosperm (52) & 1,279 & 0,2582 \\
\hline & oligozoosperm (165) & azoosperm (52) & 0,906 & 0,3411 \\
\hline
\end{tabular}


Tab. A-56 Ergebnisse der Cox-Regression bei sukzessiver Verschiebung des Trennwertes zur Unterscheidung von fertilen und subfertilen Fällen; frühe Geburtsjahrgänge

\begin{tabular}{|c|c|c|c|c|c|c|c|c|c|}
\hline \multirow{2}{*}{$\begin{array}{l}\text { Grenz- } \\
\text { wert } \\
\text { [Mio/ml] }\end{array}$} & \multirow{2}{*}{$\begin{array}{l}\text { Vergleichs- vs. } \\
\text { Referenzgruppe }\end{array}$} & \multirow{2}{*}{ B } & \multirow{2}{*}{ SE } & \multirow{2}{*}{ Wald } & \multirow{2}{*}{ df } & \multirow{2}{*}{$\mathbf{p}$} & \multirow{2}{*}{$\begin{array}{l}\text { Odds } \\
\text { Ratio }\end{array}$} & \multicolumn{2}{|c|}{$95 \% \mathrm{Cl}$ for OR } \\
\hline & & & & & & & & Lower & Upper \\
\hline 5 & subfertil vs. fertil & 0,072 & 0,266 & 0,074 & 1 & 0,785 & 1,075 & 0,638 & 1,811 \\
\hline 10 & subfertil vs. fertil & 0,124 & 0,253 & 0,241 & 1 & 0,624 & 1,132 & 0,690 & 1,859 \\
\hline 15 & subfertil vs. fertil & 0,266 & 0,235 & 1,284 & 1 & 0,257 & 1,305 & 0,823 & 2,069 \\
\hline 20 & subfertil vs. fertil & 0,495 & 0,226 & 4,790 & 1 & 0,029 & $1,640^{*}$ & 1,053 & 2,554 \\
\hline 25 & subfertil vs. fertil & 0,408 & 0,223 & 3,338 & 1 & 0,068 & $1,504^{(*)}$ & 0,971 & 2,329 \\
\hline 30 & subfertil vs. fertil & 0,372 & 0,223 & 2,779 & 1 & 0,095 & $1,451^{(*)}$ & 0,937 & 2,248 \\
\hline 35 & subfertil vs. fertil & 0,330 & 0,224 & 2,172 & 1 & 0,141 & 1,391 & 0,897 & 2,157 \\
\hline 40 & subfertil vs. fertil & 0,385 & 0,225 & 2,932 & 1 & 0,087 & $1,469^{(*)}$ & 0,946 & 2,282 \\
\hline 45 & subfertil vs. fertil & 0,349 & 0,225 & 2,405 & 1 & 0,121 & 1,418 & 0,912 & 2,204 \\
\hline 50 & subfertil vs. fertil & 0,390 & 0,226 & 2,973 & 1 & 0,085 & $1,477^{(*)}$ & 0,948 & 2,300 \\
\hline 55 & subfertil vs. fertil & 0,359 & 0,229 & 2,466 & 1 & 0,116 & 1,432 & 0,915 & 2,241 \\
\hline 60 & subfertil vs. fertil & 0,367 & 0,229 & 2,557 & 1 & 0,110 & 1,443 & 0,921 & 2,262 \\
\hline 65 & subfertil vs. fertil & 0,159 & 0,230 & 0,482 & 1 & 0,488 & 1,173 & 0,748 & 1,840 \\
\hline 70 & subfertil vs. fertil & 0,169 & 0,233 & 0,526 & 1 & 0,468 & 1,184 & 0,750 & 1,869 \\
\hline 75 & subfertil vs. fertil & 0,152 & 0,237 & 0,412 & 1 & 0,521 & 1,164 & 0,732 & 1,853 \\
\hline 80 & subfertil vs. fertil & 0,146 & 0,241 & 0,368 & 1 & 0,544 & 1,157 & 0,722 & 1,854 \\
\hline 85 & subfertil vs. fertil & 0,170 & 0,242 & 0,495 & 1 & 0,482 & 1,186 & 0,738 & 1,905 \\
\hline 90 & subfertil vs. fertil & 0,076 & 0,243 & 0,098 & 1 & 0,755 & 1,079 & 0,671 & 1,735 \\
\hline 95 & subfertil vs. fertil & 0,077 & 0,246 & 0,099 & 1 & 0,753 & 1,080 & 0,667 & 1,749 \\
\hline 100 & subfertil vs. fertil & $-0,024$ & 0,247 & 0,010 & 1 & 0,922 & 0,976 & 0,601 & 1,585 \\
\hline
\end{tabular}

Tab. A-57 Ergebnisse der Cox-Regression bei sukzessiver Verschiebung des Trennwertes zur Unterscheidung von normo- und oligozoospermen Fällen; frühe Geburtsjahrgänge

\begin{tabular}{|c|c|c|c|c|c|c|c|c|c|}
\hline \multirow{2}{*}{$\begin{array}{l}\text { Grenz- } \\
\text { wert } \\
\text { [Mio/ml] }\end{array}$} & \multirow{2}{*}{$\begin{array}{l}\text { Vergleichs- vs. } \\
\text { Referenzgruppe }\end{array}$} & \multirow{2}{*}{ B } & \multirow{2}{*}{ SE } & \multirow{2}{*}{ Wald } & \multirow{2}{*}{ df } & \multirow{2}{*}{ p } & \multirow{2}{*}{$\begin{array}{l}\text { Odds } \\
\text { Ratio }\end{array}$} & \multicolumn{2}{|c|}{$95 \% \mathrm{Cl}$ for OR } \\
\hline & & & & & & & & Lower & Upper \\
\hline \multirow{3}{*}{5} & oligo- vs. normozoosperm & 0,223 & 0,439 & 0,258 & 1 & 0,612 & 1,250 & 0,529 & 2,955 \\
\hline & azoo- vs. normozoosperm & 0,011 & 0,309 & 0,001 & 1 & 0,972 & 1,011 & 0,552 & 1,853 \\
\hline & oligo- vs. azoosperm & 0,212 & 0,506 & 0,176 & 1 & 0,675 & 1,236 & 0,459 & 3,333 \\
\hline \multirow{3}{*}{10} & oligo- vs. normozoosperm & 0,285 & 0,364 & 0,611 & 1 & 0,435 & 1,330 & 0,651 & 2,716 \\
\hline & azoo- vs. normozoosperm & 0,026 & 0,310 & 0,007 & 1 & 0,932 & 1,027 & 0,559 & 1,886 \\
\hline & oligo- vs. azoosperm & 0,259 & 0,442 & 0,342 & 1 & 0,559 & 1,295 & 0,545 & 3,080 \\
\hline \multirow{3}{*}{15} & oligo- vs. normozoosperm & 0,444 & 0,290 & 2,338 & 1 & 0,126 & 1,559 & 0,882 & 2,754 \\
\hline & azoo- vs. normozoosperm & 0,083 & 0,314 & 0,070 & 1 & 0,792 & 1,087 & 0,587 & 2,011 \\
\hline & oligo- vs. azoosperm & 0,361 & 0,379 & 0,908 & 1 & 0,341 & 1,435 & 0,683 & 3,014 \\
\hline \multirow{3}{*}{20} & oligo- vs. normozoosp. & 0,722 & 0,260 & 7,722 & 1 & 0,005 & $2,058^{* *}$ & 1,237 & 3,424 \\
\hline & azoo- vs. normozoosp. & 0,185 & 0,319 & 0,337 & 1 & 0,561 & 1,203 & 0,644 & 2,247 \\
\hline & oligo- vs. azoosperm & 0,537 & 0,352 & 2,320 & 1 & 0,128 & 1,710 & 0,857 & 3,412 \\
\hline \multirow{3}{*}{25} & oligo- vs. normozoosperm & 0,574 & 0,255 & 5,076 & 1 & 0,024 & $1,775^{*}$ & 1,077 & 2,925 \\
\hline & azoo- vs. normozoosperm & 0,155 & 0,319 & 0,237 & 1 & 0,627 & 1,168 & 0,625 & 2,181 \\
\hline & oligo- vs. azoosperm & 0,419 & 0,350 & 1,430 & 1 & 0,232 & 1,520 & 0,765 & 3,021 \\
\hline \multirow{3}{*}{30} & oligo- vs. normozoosperm & 0,484 & 0,248 & 3,811 & 1 & 0,051 & $1,622^{(*)}$ & 0,998 & 2,637 \\
\hline & azoo- vs. normozoosperm & 0,165 & 0,323 & 0,261 & 1 & 0,609 & 1,179 & 0,627 & 2,219 \\
\hline & oligo- vs. azoosperm & 0,319 & 0,340 & 0,879 & 1 & 0,349 & 1,376 & 0,706 & 2,682 \\
\hline \multirow{3}{*}{35} & oligo- vs. normozoosperm & 0,422 & 0,248 & 2,907 & 1 & 0,088 & $1,525^{(*)}$ & 0,939 & 2,478 \\
\hline & azoo- vs. normozoosperm & 0,151 & 0,323 & 0,217 & 1 & 0,641 & 1,163 & 0,617 & 2,191 \\
\hline & oligo- vs. azoosperm & 0,272 & 0,339 & 0,642 & 1 & 0,423 & 1,312 & 0,675 & 2,550 \\
\hline \multirow{3}{*}{40} & oligo- vs. normozoosperm & 0,483 & 0,246 & 3,852 & 1 & 0,050 & $1,621^{*}$ & 1,001 & 2,625 \\
\hline & azoo- vs. normozoosperm & 0,184 & 0,325 & 0,320 & 1 & 0,572 & 1,202 & 0,635 & 2,275 \\
\hline & oligo- vs. azoosperm & 0,299 & 0,336 & 0,793 & 1 & 0,373 & 1,348 & 0,698 & 2,602 \\
\hline
\end{tabular}




\begin{tabular}{|c|c|c|c|c|c|c|c|c|c|}
\hline \multirow{3}{*}{45} & alinn un narmanonannorm & 0130 & ก О15 & $2 n 71$ & 1 & n nen & $1525(*)$ & ก O51 & 2170 \\
\hline & azoo- vs. normozoosperm & 0,174 & 0,327 & 0,283 & 1 & 0,595 & 1,190 & 0,627 & 2,259 \\
\hline & oligo- vs. azoosperm & 0,255 & 0,333 & 0,585 & 1 & 0,444 & 1,290 & 0,672 & 2,478 \\
\hline \multirow{3}{*}{50} & oligo- vs. normozoosperm & 0,469 & 0,243 & 3,724 & 1 & 0,054 & $1,599^{(*)}$ & 0,993 & 2,576 \\
\hline & azoo- vs. normozoosperm & 0,203 & 0,330 & 0,380 & 1 & 0,538 & 1,225 & 0,642 & 2,337 \\
\hline & oligo- vs. azoosperm & 0,266 & 0,330 & 0,653 & 1 & 0,419 & 1,305 & 0,684 & 2,492 \\
\hline \multirow{3}{*}{55} & oligo- vs. normozoosperm & 0,426 & 0,245 & 3,028 & 1 & 0,082 & $1,532^{(*)}$ & 0,948 & 2,476 \\
\hline & azoo-vs. normozoosperm & 0,195 & 0,332 & 0,345 & 1 & 0,557 & 1,215 & 0,634 & 2,327 \\
\hline & oligo- vs. azoosperm & 0,232 & 0,328 & 0,498 & 1 & 0,480 & 1,261 & 0,662 & 2,400 \\
\hline \multirow{3}{*}{60} & oligo- vs. normozoosperm & 0,432 & 0,245 & 3,112 & 1 & 0,078 & $1,541^{(*)}$ & 0,953 & 2,491 \\
\hline & azoo- vs. normozoosperm & 0,203 & 0,333 & 0,371 & 1 & 0,542 & 1,225 & 0,638 & 2,351 \\
\hline & oligo- vs. azoosperm & 0,229 & 0,327 & 0,492 & 1 & 0,483 & 1,258 & 0,662 & 2,389 \\
\hline \multirow{3}{*}{65} & oligo- vs. normozoosperm & 0,184 & 0,244 & 0,568 & 1 & 0,451 & 1,202 & 0,745 & 1,940 \\
\hline & azoo- vs. normozoosperm & 0,091 & 0,334 & 0,073 & 1 & 0,787 & 1,095 & 0,568 & 2,109 \\
\hline & oligo- vs. azoosperm & 0,093 & 0,326 & 0,082 & 1 & 0,774 & 1,098 & 0,579 & 2,081 \\
\hline \multirow{3}{*}{70} & oligo- vs. normozoosperm & 0,192 & 0,246 & 0,609 & 1 & 0,435 & 1,212 & 0,748 & 1,962 \\
\hline & azoo- vs. normozoosperm & 0,101 & 0,338 & 0,089 & 1 & 0,766 & 1,106 & 0,570 & 2,145 \\
\hline & oligo- vs. azoosperm & $-0,101$ & 0,338 & 0,089 & 1 & 0,766 & 0,904 & 0,466 & 1,754 \\
\hline \multirow{3}{*}{75} & oligo- vs. normozoosperm & 0,170 & 0,249 & 0,469 & 1 & 0,493 & 1,186 & 0,728 & 1,930 \\
\hline & azoo- vs. normozoosperm & 0,095 & 0,342 & 0,077 & 1 & 0,782 & 1,099 & 0,562 & 2,149 \\
\hline & oligo- vs. azoosperm & 0,076 & 0,322 & 0,055 & 1 & 0,814 & 1,079 & 0,574 & 2,026 \\
\hline \multirow{3}{*}{80} & oligo- vs. normozoosperm & 0,162 & 0,252 & 0,415 & 1 & 0,519 & 1,176 & 0,718 & 1,925 \\
\hline & azoo- vs. normozoosperm & 0,093 & 0,345 & 0,072 & 1 & 0,788 & 1,097 & 0,558 & 2,157 \\
\hline & oligo- vs. azoosperm & 0,069 & 0,321 & 0,047 & 1 & 0,829 & 1,072 & 0,572 & 2,010 \\
\hline \multirow{3}{*}{85} & oligo- vs. normozoosperm & 0,188 & 0,253 & 0,556 & 1 & 0,456 & 1,207 & 0,736 & 1,981 \\
\hline & azoo-vs. normozoosperm & 0,110 & 0,346 & 0,101 & 1 & 0,750 & 1,117 & 0,566 & 2,201 \\
\hline & oligo- vs. azoosperm & 0,078 & 0,320 & 0,060 & 1 & 0,807 & 1,081 & 0,577 & 2,025 \\
\hline \multirow{3}{*}{90} & oligo- vs. normozoosperm & 0,084 & 0,253 & 0,111 & 1 & 0,739 & 1,088 & 0,663 & 1,786 \\
\hline & azoo- vs. normozoosperm & 0,047 & 0,347 & 0,018 & 1 & 0,892 & 1,048 & 0,531 & 2,069 \\
\hline & oligo- vs. azoosperm & 0,037 & 0,320 & 0,013 & 1 & 0,908 & 1,038 & 0,554 & 1,944 \\
\hline \multirow{3}{*}{95} & oligo- vs. normozoosperm & 0,085 & 0,255 & 0,111 & 1 & 0,739 & 1,089 & 0,660 & 1,796 \\
\hline & azoo- vs. normozoosperm & 0,049 & 0,350 & 0,020 & 1 & 0,888 & 1,051 & 0,529 & 2,087 \\
\hline & oligo- vs. azoosperm & 0,036 & 0,319 & 0,013 & 1 & 0,911 & 1,036 & 0,555 & 1,936 \\
\hline \multirow{3}{*}{100} & oligo- vs. normozoosperm & $-0,025$ & 0,257 & 0,009 & 1 & 0,924 & 0,976 & 0,590 & 1,614 \\
\hline & azoo- vs. normozoosperm & $-0,023$ & 0,351 & 0,004 & 1 & 0,949 & 0,978 & 0,491 & 1,947 \\
\hline & oligo- vs. azoosperm & $-0,002$ & 0,319 & 0,000 & 1 & 0,995 & 0,998 & 0,534 & 1,864 \\
\hline
\end{tabular}

Tab. A-58 Ergebnisse des Wilcoxon(Gehan)-Tests bei sukzessiver Verschiebung des Trennwertes zur Unterscheidung von fertilen und subfertilen Fällen; späte Geburtsjahrgänge

\begin{tabular}{|c|c|c|c|c|}
\hline 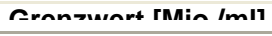 & Vanomlanials 1 & & 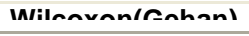 & n \\
\hline 5 & fertil (243) & subfertil (52) & 0,787 & 0,3749 \\
\hline 10 & fertil (233) & subfertil (62) & 0,302 & 0,5829 \\
\hline 15 & fertil (221) & subfertil (74) & 0,238 & 0,6254 \\
\hline 20 & fertil (205) & subfertil (90) & 0,005 & 0,9429 \\
\hline 25 & fertil (199) & subfertil (96) & 0,002 & 0,9663 \\
\hline 30 & fertil (190) & subfertil (105) & 0,092 & 0,7618 \\
\hline 35 & fertil (182) & subfertil (113) & 0,281 & 0,5958 \\
\hline 40 & fertil (174) & subfertil (121) & 0,181 & 0,6702 \\
\hline 45 & fertil (166) & subfertil (129) & 0,568 & 0,4511 \\
\hline 50 & fertil (156) & subfertil (139) & 0,058 & 0,8094 \\
\hline 55 & fertil (150) & subfertil (145) & 0,371 & 0,5424 \\
\hline 60 & fertil (135) & subfertil (160) & 2,131 & 0,1443 \\
\hline 65 & fertil (129) & subfertil (166) & 2,092 & 0,1481 \\
\hline 70 & fertil (125) & subfertil (170) & 1,572 & 0,2099 \\
\hline
\end{tabular}




\begin{tabular}{|l|l|l|l|l|}
\hline 75 & fertil (119) & subfertil (176) & 2,550 & 0,1103 \\
\hline 80 & fertil (111) & subfertil (184) & $3,674^{(*)}$ & 0,0553 \\
\hline 85 & fertil (106) & subfertil (189) & $4,099^{*}$ & 0,0429 \\
\hline 90 & fertil (97) & subfertil (198) & $5,133^{*}$ & 0,0235 \\
\hline 95 & fertil (95) & subfertil (200) & $4,682^{*}$ & 0,0305 \\
\hline 100 & fertil (91) & subfertil (204) & $5,504^{*}$ & 0,0190 \\
\hline
\end{tabular}

Tab. A-59 Ergebnisse des Wilcoxon(Gehan)-Tests bei sukzessiver Verschiebung des Trennwertes zur Unterscheidung von normo- und oligozoospermen Fällen; späte Geburtsjahrgänge

\begin{tabular}{|c|c|c|c|c|}
\hline Grenzwert [Mio./ml] & Vergleich (n) & & $\begin{array}{l}\text { Wilcoxon } \\
\text { (Gehan) }\end{array}$ & $\mathbf{p}$ \\
\hline \multirow{4}{*}{5} & \multicolumn{2}{|l|}{ overall } & 0,822 & 0,6629 \\
\hline & normozoosperm (243) & oligozoosperm (20) & 0,539 & 0,4628 \\
\hline & normozoosperm (243) & azoosperm (32) & 0,382 & 0,5364 \\
\hline & oligozoosperm (20) & azoosperm (32) & 0,027 & 0,8707 \\
\hline \multirow{4}{*}{10} & \multicolumn{2}{|l|}{ overall } & 0,358 & 0,8361 \\
\hline & normozoosperm (233) & oligozoosperm (30) & 0,063 & 0,8011 \\
\hline & normozoosperm (233) & azoosperm (32) & 0,325 & 0,5685 \\
\hline & oligozoosperm (30) & azoosperm (32) & 0,058 & 0,8092 \\
\hline \multirow{4}{*}{15} & \multicolumn{2}{|l|}{ overall } & 0,338 & 0,8445 \\
\hline & normozoosperm (221) & oligozoosperm (42) & 0,039 & 0,8438 \\
\hline & normozoosperm (221) & azoosperm (32) & 0,333 & 0,5637 \\
\hline & oligozoosperm (42) & azoosperm (32) & 0,074 & 0,7862 \\
\hline \multirow{4}{*}{20} & \multicolumn{2}{|l|}{ overall } & 0,477 & 0,7878 \\
\hline & normozoosperm (205) & oligozoosperm (58) & 0,183 & 0,6687 \\
\hline & normozoosperm (205) & azoosperm (32) & 0,211 & 0,6460 \\
\hline & oligozoosperm (58) & azoosperm (32) & 0,457 & 0,4988 \\
\hline \multirow{4}{*}{25} & \multicolumn{2}{|l|}{ overall } & 0,432 & 0,8056 \\
\hline & normozoosperm (199) & oligozoosperm (64) & 0,137 & 0,7112 \\
\hline & normozoosperm (199) & azoosperm (32) & 0,215 & 0,6430 \\
\hline & oligozoosperm (64) & azoosperm (32) & 0,418 & 0,5178 \\
\hline \multirow{4}{*}{30} & \multicolumn{2}{|l|}{ overall } & 0,301 & 0,8602 \\
\hline & normozoosperm (190) & oligozoosperm (73) & 0,003 & 0,9599 \\
\hline & normozoosperm (190) & azoosperm (32) & 0,304 & 0,5814 \\
\hline & oligozoosperm (73) & azoosperm (32) & 0,182 & 0,6700 \\
\hline \multirow{4}{*}{35} & \multicolumn{2}{|l|}{ overall } & 0,402 & 0,8179 \\
\hline & normozoosperm (182) & oligozoosperm (81) & 0,110 & 0,7405 \\
\hline & normozoosperm (182) & azoosperm (32) & 0,360 & 0,5487 \\
\hline & oligozoosperm (81) & azoosperm (32) & 0,120 & 0,7288 \\
\hline \multirow{4}{*}{40} & \multicolumn{2}{|l|}{ overall } & 0,346 & 0,8413 \\
\hline & normozoosperm (174) & oligozoosperm (89) & 0,052 & 0,8196 \\
\hline & normozoosperm (174) & azoosperm (32) & 0,326 & 0,5679 \\
\hline & oligozoosperm (89) & azoosperm (32) & 0,171 & 0,6795 \\
\hline \multirow{4}{*}{45} & \multicolumn{2}{|l|}{ overall } & 0,641 & 0,7258 \\
\hline & normozoosperm (166) & oligozoosperm (97) & 0,641 & 0,7258 \\
\hline & normozoosperm (166) & azoosperm (32) & 0,358 & 0,5497 \\
\hline & oligozoosperm (97) & azoosperm (32) & 0,456 & 0,4994 \\
\hline \multirow{4}{*}{50} & \multicolumn{2}{|l|}{ overall } & 0,300 & 0,8606 \\
\hline & normozoosperm (156) & oligozoosperm (107) & 0,002 & 0,9624 \\
\hline & normozoosperm (156) & azoosperm (32) & 0,278 & 0,5978 \\
\hline & oligozoosperm (107) & azoosperm (32) & 0,243 & 0,6224 \\
\hline
\end{tabular}




\begin{tabular}{|c|c|c|c|c|}
\hline \multirow{4}{*}{55} & \multicolumn{2}{|l|}{ overall } & \multirow{2}{*}{$\begin{array}{l}0,496 \\
0,207\end{array}$} & \multirow{2}{*}{$\begin{array}{l}0,7804 \\
0,6493\end{array}$} \\
\hline & normozoosperm (150) & oligozoosperm (113) & & \\
\hline & normozoosperm (150) & azoosperm (32) & 0,419 & 0,5174 \\
\hline & oligozoosperm (113) & azoosperm (32) & 0,122 & 0,7265 \\
\hline \multirow{4}{*}{60} & \multicolumn{2}{|l|}{ overall } & 2,138 & 0,3433 \\
\hline & normozoosperm (135) & oligozoosperm (128) & 1,912 & 0,1667 \\
\hline & normozoosperm (135) & azoosperm (32) & 0,958 & 0,3278 \\
\hline & oligozoosperm (128) & azoosperm (32) & 0,009 & 0,9240 \\
\hline \multirow{4}{*}{65} & \multicolumn{2}{|l|}{ overall } & 2,103 & 0,3493 \\
\hline & normozoosperm (129) & oligozoosperm (134) & 1,886 & 0,1697 \\
\hline & normozoosperm (129) & azoosperm (32) & 0,967 & 0,3255 \\
\hline & oligozoosperm (134) & azoosperm (32) & 0,016 & 0,9004 \\
\hline \multirow{4}{*}{70} & \multicolumn{2}{|l|}{ overall } & 1,604 & 0,4484 \\
\hline & normozoosperm (125) & oligozoosperm (138) & 1,370 & 0,2418 \\
\hline & normozoosperm (125) & azoosperm (32) & 0,829 & 0,3625 \\
\hline & oligozoosperm (138) & azoosperm (32) & 0,038 & 0,8452 \\
\hline \multirow{4}{*}{75} & \multicolumn{2}{|l|}{ overall } & 2,559 & 0,2782 \\
\hline & normozoosperm (119) & oligozoosperm (144) & 2,339 & 0,1261 \\
\hline & normozoosperm (119) & azoosperm (32) & 1,183 & 0,2767 \\
\hline & oligozoosperm (144) & azoosperm (32) & 0,010 & 0,9222 \\
\hline \multirow{4}{*}{80} & \multicolumn{2}{|l|}{ overall } & 3,675 & 0,1592 \\
\hline & normozoosperm (111) & oligozoosperm (152) & $3,471^{(\star)}$ & 0,0624 \\
\hline & normozoosperm (111) & azoosperm (32) & 1,664 & 0,1971 \\
\hline & oligozoosperm (152) & azoosperm (32) & 0,001 & 0,9783 \\
\hline \multirow{4}{*}{85} & \multicolumn{2}{|l|}{ overall } & 4,100 & 0,1288 \\
\hline & normozoosperm (106) & oligozoosperm (157) & $3,893^{*}$ & 0,0485 \\
\hline & normozoosperm (106) & azoosperm (32) & 1,921 & 0,1658 \\
\hline & oligozoosperm (157) & azoosperm (32) & 0,000 & 0,9916 \\
\hline \multirow{4}{*}{90} & \multicolumn{2}{|l|}{ overall } & $5,133^{(*)}$ & 0,0768 \\
\hline & normozoosperm (97) & oligozoosperm (166) & $4,947^{*}$ & 0,0261 \\
\hline & normozoosperm (97) & azoosperm (32) & 2,540 & 0,1110 \\
\hline & oligozoosperm (166) & azoosperm (32) & 0,000 & 0,9881 \\
\hline \multirow{4}{*}{95} & \multicolumn{2}{|l|}{ overall } & $4,683^{(*)}$ & 0,0962 \\
\hline & normozoosperm (95) & oligozoosperm (168) & $4,481^{*}$ & 0,0343 \\
\hline & normozoosperm (95) & azoosperm (32) & 2,404 & 0,1210 \\
\hline & oligozoosperm (168) & azoosperm (32) & 0,000 & 0,9881 \\
\hline \multirow{4}{*}{100} & \multicolumn{2}{|l|}{ overall } & $5,504^{(\star)}$ & 0,0638 \\
\hline & normozoosperm (91) & oligozoosperm (172) & $5,311^{*}$ & 0,0212 \\
\hline & normozoosperm (91) & azoosperm (32) & $2,908^{(*)}$ & 0,0881 \\
\hline & oligozoosperm (172) & azoosperm (32) & 0,000 & 0,9884 \\
\hline
\end{tabular}


Tab. A-60 Ergebnisse der Cox-Regression bei sukzessiver Verschiebung des Trennwertes zur Unterscheidung von fertilen und subfertilen Fällen; späte Geburtsjahrgänge

\begin{tabular}{|c|c|c|c|c|c|c|c|c|c|}
\hline \multirow{2}{*}{$\begin{array}{l}\text { Grenz- } \\
\text { wert } \\
\text { [Mio/ml] }\end{array}$} & \multirow{2}{*}{$\begin{array}{l}\text { Vergleichs- vs. } \\
\text { Referenzgruppe }\end{array}$} & \multirow{2}{*}{ B } & \multirow{2}{*}{ SE } & \multirow{2}{*}{ Wald } & \multirow{2}{*}{ df } & \multirow{2}{*}{$\mathbf{p}$} & \multirow{2}{*}{$\begin{array}{l}\text { Odds } \\
\text { Ratio }\end{array}$} & \multicolumn{2}{|c|}{$95 \% \mathrm{Cl}$ for OR } \\
\hline & & & & & & & & Lower & Upper \\
\hline 5 & subfertil vs. fertil & 0,461 & 0,368 & 1,566 & 1 & 0,211 & 1,586 & 0,770 & 3,263 \\
\hline 10 & subfertil vs. fertil & 0,337 & 0,359 & 0,881 & 1 & 0,348 & 1,400 & 0,693 & 2,828 \\
\hline 15 & subfertil vs. fertil & 0,299 & 0,351 & 0,723 & 1 & 0,395 & 1,348 & 0,677 & 2,683 \\
\hline 20 & subfertil vs. fertil & 0,021 & 0,346 & 0,004 & 1 & 0,952 & 1,021 & 0,518 & 2,010 \\
\hline 25 & subfertil vs. fertil & 0,076 & 0,341 & 0,050 & 1 & 0,823 & 1,079 & 0,554 & 2,105 \\
\hline 30 & subfertil vs. fertil & 0,094 & 0,334 & 0,079 & 1 & 0,778 & 1,099 & 0,571 & 2,115 \\
\hline 35 & subfertil vs. fertil & 0,235 & 0,327 & 0,517 & 1 & 0,472 & 1,265 & 0,667 & 2,398 \\
\hline 40 & subfertil vs. fertil & 0,217 & 0,323 & 0,452 & 1 & 0,501 & 1,243 & 0,660 & 2,339 \\
\hline 45 & subfertil vs. fertil & 0,283 & 0,317 & 0,799 & 1 & 0,372 & 1,327 & 0,713 & 2,470 \\
\hline 50 & subfertil vs. fertil & 0,134 & 0,318 & 0,177 & 1 & 0,674 & 1,143 & 0,613 & 2,130 \\
\hline 55 & subfertil vs. fertil & 0,209 & 0,323 & 0,418 & 1 & 0,518 & 1,232 & 0,654 & 2,321 \\
\hline 60 & subfertil vs. fertil & 0,479 & 0,336 & 2,026 & 1 & 0,155 & 1,614 & 0,835 & 3,120 \\
\hline 65 & subfertil vs. fertil & 0,507 & 0,342 & 2,199 & 1 & 0,138 & 1,660 & 0,850 & 3,245 \\
\hline 70 & subfertil vs. fertil & 0,442 & 0,341 & 1,678 & 1 & 0,195 & 1,556 & 0,797 & 3,036 \\
\hline 75 & subfertil vs. fertil & 0,740 & 0,369 & 4,022 & 1 & 0,045 & $2,096^{*}$ & 1,017 & 4,319 \\
\hline 80 & subfertil vs. fertil & 0,910 & 0,397 & 5,252 & 1 & 0,022 & $2,485^{*}$ & 1,141 & 5,413 \\
\hline 85 & subfertil vs. fertil & 1,006 & 0,420 & 5,743 & 1 & 0,017 & $2,735^{*}$ & 1,201 & 6,227 \\
\hline 90 & subfertil vs. fertil & 1,039 & 0,447 & 5,405 & 1 & 0,020 & $2,826^{*}$ & 1,177 & 6,783 \\
\hline 95 & subfertil vs. fertil & 1,016 & 0,448 & 5,151 & 1 & 0,023 & $2,762^{*}$ & 1,149 & 6,642 \\
\hline 100 & subfertil vs. fertil & 0,951 & 0,448 & 4,504 & 1 & 0,034 & $2,587^{*}$ & 1,075 & 6,223 \\
\hline
\end{tabular}

Tab. A-61 Ergebnisse der Cox-Regression bei sukzessiver Verschiebung des Trennwertes zur Unterscheidung von normo- und oligozoospermen Fällen; späte Geburtsjahrgänge

\begin{tabular}{|c|c|c|c|c|c|c|c|c|c|}
\hline \multirow{2}{*}{$\begin{array}{l}\text { Grenz- } \\
\text { wert } \\
\text { [Mio/ml] }\end{array}$} & \multirow{2}{*}{$\begin{array}{l}\text { Vergleichs- vs. } \\
\text { Referenzgruppe }\end{array}$} & \multirow{2}{*}{ B } & \multirow{2}{*}{ SE } & \multirow{2}{*}{ Wald } & \multirow{2}{*}{ df } & \multirow{2}{*}{$\mathbf{p}$} & \multirow{2}{*}{$\begin{array}{l}\text { Odds } \\
\text { Ratio }\end{array}$} & \multicolumn{2}{|c|}{$95 \% \mathrm{Cl}$ for OR } \\
\hline & & & & & & & & Lower & Upper \\
\hline \multirow{3}{*}{5} & oligo- vs. normozoosperm & 0,574 & 0,508 & 1,279 & 1 & 0,258 & 1,775 & 0,656 & 4,800 \\
\hline & azoo- vs. normozoosperm & 0,381 & 0,457 & 0,696 & 1 & 0,404 & 1,464 & 0,598 & 3,584 \\
\hline & oligozoo- vs. azoosperm & 0,193 & 0,617 & 0,097 & 1 & 0,755 & 1,213 & 0,362 & 4,066 \\
\hline \multirow{3}{*}{10} & oligo- vs. normozoosperm & 0,314 & 0,468 & 0,450 & 1 & 0,502 & 1,369 & 0,547 & 3,429 \\
\hline & azoo- vs. normozoosperm & 0,358 & 0,458 & 0,611 & 1 & 0,434 & 1,431 & 0,583 & 3,513 \\
\hline & oligozoo- vs. azoosperm & $-0,044$ & 0,587 & 0,006 & 1 & 0,940 & 0,957 & 0,303 & 3,023 \\
\hline \multirow{3}{*}{15} & oligo- vs. normozoosperm & 0,250 & 0,441 & 0,322 & 1 & 0,570 & 1,284 & 0,541 & 3,046 \\
\hline & azoo- vs. normozoosperm & 0,355 & 0,460 & 0,597 & 1 & 0,440 & 1,427 & 0,579 & 3,515 \\
\hline & oligozoo- vs. azoosperm & $-0,105$ & 0,564 & 0,035 & 1 & 0,852 & 0,900 & 0,298 & 2,720 \\
\hline \multirow{3}{*}{20} & oligo- vs. normozoosperm & $-0,154$ & 0,433 & 0,127 & 1 & 0,722 & 0,857 & 0,367 & 2,002 \\
\hline & azoo- vs. normozoosperm & 0,269 & 0,459 & 0,342 & 1 & 0,559 & 1,308 & 0,532 & 3,219 \\
\hline & oligozoo- vs. azoosperm & $-0,423$ & 0,564 & 0,562 & 1 & 0,453 & 0,655 & 0,217 & 1,979 \\
\hline \multirow{3}{*}{25} & oligo- vs. normozoosperm & $-0,060$ & 0,414 & 0,021 & 1 & 0,886 & 0,942 & 0,419 & 2,120 \\
\hline & azoo- vs. normozoosperm & 0,288 & 0,461 & 0,391 & 1 & 0,532 & 1,334 & 0,540 & 3,296 \\
\hline & oligozoo- vs. azoosperm & $-0,348$ & 0,547 & 0,405 & 1 & 0,525 & 0,706 & 0,242 & 2,062 \\
\hline \multirow{3}{*}{30} & oligo- vs. normozoosperm & $-0,015$ & 0,389 & 0,001 & 1 & 0,969 & 0,985 & 0,460 & 2,111 \\
\hline & azoo- vs. normozoosperm & 0,299 & 0,465 & 0,413 & 1 & 0,521 & 1,348 & 0,542 & 3,357 \\
\hline & oligozoo- vs. azoosperm & $-0,314$ & 0,524 & 0,359 & 1 & 0,549 & 0,731 & 0,262 & 2,041 \\
\hline \multirow{3}{*}{35} & oligo- vs. normozoosperm & 0,175 & 0,368 & 0,225 & 1 & 0,635 & 1,191 & 0,579 & 2,452 \\
\hline & azoo- vs. normozoosperm & 0,364 & 0,469 & 0,602 & 1 & 0,438 & 1,439 & 0,574 & 3,606 \\
\hline & oligozoo- vs. azoosperm & $-0,189$ & 0,507 & 0,139 & 1 & 0,710 & 0,828 & 0,306 & 2,237 \\
\hline \multirow{3}{*}{40} & oligo- vs. normozoosperm & 0,157 & 0,359 & 0,190 & 1 & 0,663 & 1,170 & 0,578 & 2,365 \\
\hline & azoo- vs. normozoosperm & 0,362 & 0,471 & 0,591 & 1 & 0,442 & 1,436 & 0,571 & 3,613 \\
\hline & oligozoo- vs. azoosperm & $-0,205$ & 0,500 & 0,168 & 1 & 0,682 & 0,814 & 0,306 & 2,171 \\
\hline
\end{tabular}


Anhang

\begin{tabular}{|c|c|c|c|c|c|c|c|c|c|}
\hline \multirow{3}{*}{45} & olian- vs normozonsnerm & 0241 & 0.345 & 0486 & 1 & 0486 & 1272 & 0647 & 2502 \\
\hline & azoo- vs. normozoosperm & 0,403 & 0,475 & 0,720 & 1 & 0,396 & 1,496 & 0,590 & 3,792 \\
\hline & oligozoo- vs. azoosperm & $-0,162$ & 0,489 & 0,110 & 1 & 0,740 & 0,850 & 0,326 & 2,219 \\
\hline \multirow{3}{*}{50} & oligo- vs. normozoosperm & 0,065 & 0,346 & 0,036 & 1 & 0,850 & 1,068 & 0,541 & 2,105 \\
\hline & azoo- vs. normozoosperm & 0,332 & 0,475 & 0,488 & 1 & 0,485 & 1,393 & 0,550 & 3,532 \\
\hline & oligozoo- vs. azoosperm & $-0,266$ & 0,490 & 0,295 & 1 & 0,587 & 0,766 & 0,293 & 2,002 \\
\hline \multirow{3}{*}{55} & oligo- vs. normozoosperm & 0,154 & 0,348 & 0,196 & 1 & 0,658 & 1,167 & 0,590 & 2,308 \\
\hline & azoo-vs. normozoosperm & 0,375 & 0,480 & 0,611 & 1 & 0,434 & 1,456 & 0,568 & 3,730 \\
\hline & oligozoo- vs. azoosperm & $-0,221$ & 0,484 & 0,208 & 1 & 0,648 & 0,802 & 0,310 & 2,071 \\
\hline \multirow{3}{*}{60} & oligo- vs. normozoosperm & 0,458 & 0,352 & 1,688 & 1 & 0,194 & 1,581 & 0,792 & 3,153 \\
\hline & azoo- vs. normozoosperm & 0,555 & 0,497 & 1,246 & 1 & 0,264 & 1,742 & 0,657 & 4,616 \\
\hline & oligozoo- vs. azoosperm & $-0,097$ & 0,471 & 0,043 & 1 & 0,837 & 0,907 & 0,360 & 2,285 \\
\hline \multirow{3}{*}{65} & oligo- vs. normozoosperm & 0,488 & 0,357 & 1,865 & 1 & 0,172 & 1,628 & 0,809 & 3,279 \\
\hline & azoo-vs. normozoosperm & 0,579 & 0,501 & 1,334 & 1 & 0,248 & 1,784 & 0,668 & 4,765 \\
\hline & oligozoo- vs. azoosperm & $-0,091$ & 0,470 & 0,038 & 1 & 0,846 & 0,913 & 0,363 & 2,292 \\
\hline \multirow{3}{*}{70} & oligo- vs. normozoosperm & 0,416 & 0,356 & 1,362 & 1 & 0,243 & 1,515 & 0,754 & 3,045 \\
\hline & azoo- vs. normozoosperm & 0,543 & 0,501 & 1,171 & 1 & 0,279 & 1,721 & 0,644 & 4,596 \\
\hline & oligozoo- vs. azoosperm & $-0,127$ & 0,469 & 0,073 & 1 & 0,787 & 0,881 & 0,351 & 2,210 \\
\hline \multirow{3}{*}{75} & oligo- vs. normozoosperm & 0,735 & 0,381 & 3,727 & 1 & 0,054 & $2,085^{(*)}$ & 0,989 & 4,397 \\
\hline & azoo-vs. normozoosperm & 0,760 & 0,523 & 2,113 & 1 & 0,146 & 2,138 & 0,767 & 5,959 \\
\hline & oligozoo- vs. azoosperm & $-0,025$ & 0,464 & 0,003 & 1 & 0,957 & 0,975 & 0,392 & 2,423 \\
\hline \multirow{3}{*}{80} & oligo- vs. normozoosperm & 0,910 & 0,406 & 5,012 & 1 & 0,025 & $2,484^{*}$ & 1,120 & 5,509 \\
\hline & azoo-vs. normozoosperm & 0,912 & 0,546 & 2,795 & 1 & 0,095 & $2,490^{(*)}$ & 0,854 & 7,258 \\
\hline & oligozoo- vs. azoosperm & $-0,003$ & 0,460 & 0,000 & 1 & 0,996 & 0,997 & 0,405 & 2,459 \\
\hline \multirow{3}{*}{85} & oligo- vs. normozoosperm & 1,006 & 0,428 & 5,526 & 1 & 0,019 & $2,736^{*}$ & 1,182 & 6,332 \\
\hline & azoo-vs. normozoosperm & 1,004 & 0,563 & 3,186 & 1 & 0,074 & $2,730^{(*)}$ & 0,906 & 8,224 \\
\hline & oligozoo- vs. azoosperm & 0,002 & 0,459 & 0,000 & 1 & 0,996 & 1,002 & 0,408 & 2,463 \\
\hline \multirow{3}{*}{90} & oligo- vs. normozoosperm & 1,035 & 0,454 & 5,187 & 1 & 0,023 & $2,815^{*}$ & 1,155 & 6,861 \\
\hline & azoo- vs. normozoosperm & 1,055 & 0,583 & 3,276 & 1 & 0,070 & $2,873^{(*)}$ & 0,916 & 9,009 \\
\hline & oligozoo- vs. azoosperm & $-0,020$ & 0,458 & 0,002 & 1 & 0,965 & 0,980 & 0,399 & 2,405 \\
\hline \multirow{3}{*}{95} & oligo- vs. normozoosperm & 1,012 & 0,456 & 4,926 & 1 & 0,026 & $2,750^{*}$ & 1,126 & 6,718 \\
\hline & azoo-vs. normozoosperm & 1,036 & 0,583 & 3,159 & 1 & 0,075 & $2,817^{(*)}$ & 0,899 & 8,827 \\
\hline & oligozoo- vs. azoosperm & $-0,024$ & 0,459 & 0,003 & 1 & 0,958 & 0,976 & 0,397 & 2,398 \\
\hline \multirow{3}{*}{100} & oligo- vs. normozoosperm & 0,942 & 0,456 & 4,263 & 1 & 0,039 & $2,566^{*}$ & 1,049 & 6,277 \\
\hline & azoo- vs. normozoosperm & 0,986 & 0,582 & 2,872 & 1 & 0,090 & $2,680^{(*)}$ & 0,857 & 8,380 \\
\hline & oligozoo- vs. azoosperm & $-0,043$ & 0,459 & 0,009 & 1 & 0,925 & 0,958 & 0,389 & 2,355 \\
\hline
\end{tabular}


A-8 Validität der Trennung zwischen fertilen und subfertilen Fällen: Guzick-Einteilung

\section{Ergebnisse der nicht-parametrischen Verfahren}

Tab. A-62 Wilcoxon(Gehan)-Test zum Vergleich der Überlebensfunktionen von fertilen und subfertilen Fällen (nach Guzick et al., 2001)

\begin{tabular}{|l|l|l|l|l|l|l|}
\hline \multirow{2}{*}{ Vergleich } & $\mathbf{n}$ & df & $\begin{array}{l}\text { Wilcoxon } \\
\text { (Gehan) }\end{array}$ & $\mathbf{p}$ \\
\hline fertil-subfertil & unzensiert & zensiert & & \\
\hline
\end{tabular}

Tab. A-63 Wilcoxon(Gehan)-Test zum Vergleich der Überlebensfunktionen von fertilen und subfertilen Fällen (nach Guzick et al., 2001); frühe Geburtsjahrgänge

\begin{tabular}{|l|l|l|l|l|l|}
\hline \multirow{2}{*}{ Vergleich } & $\mathbf{n}$ & df & $\begin{array}{l}\text { Wilcoxon } \\
\text { (Gehan) }\end{array}$ & $\mathbf{p}$ \\
\cline { 1 - 5 } & unzensiert & zensiert & & \\
\hline fertil-subfertil & 107 & 129 & 1 & $6,882^{\star *}$ & 0,009 \\
\hline
\end{tabular}

Tab. A-64 Wilcoxon(Gehan)-Test zum Vergleich der Überlebensfunktionen von fertilen und subfertilen Fällen (nach Guzick et al., 2001); späte Geburtsjahrgänge

\begin{tabular}{|c|c|c|c|c|c|}
\hline \multirow{2}{*}{ Vergleich } & \multicolumn{2}{|l|}{$\mathbf{n}$} & \multirow{2}{*}{ df } & \multirow{2}{*}{$\begin{array}{l}\text { Wilcoxon } \\
\text { (Gehan) }\end{array}$} & \multirow{2}{*}{ p } \\
\hline & unzensiert & zensiert & & & \\
\hline fertil-subfertil & 39 & 196 & 1 & 0,213 & 0,645 \\
\hline
\end{tabular}

Tab. A-65 Wilcoxon(Gehan)-Test zum Vergleich der Überlebensfunktionen von normo-, oligo- und azoospermen Fällen (nach Guzick et al., 2001)

\begin{tabular}{|c|c|c|c|c|c|}
\hline \multirow{2}{*}{ Vergleich } & \multicolumn{2}{|l|}{$\mathbf{n}$} & \multirow{2}{*}{ df } & \multirow{2}{*}{$\begin{array}{l}\text { Wilcoxon } \\
\text { (Gehan) }\end{array}$} & \multirow{2}{*}{$\mathbf{p}$} \\
\hline & unzensiert & zensiert & & & \\
\hline overall & 146 & 325 & 2 & $5,175^{(*)}$ & 0,075 \\
\hline normozoosperm-oligozoosperm & 114 & 273 & 1 & $3,328^{(*)}$ & 0,068 \\
\hline normozoosperm-azoosperm & 112 & 272 & 1 & $3,402^{(*)}$ & 0,065 \\
\hline oligozoosperm-azoosperm & 66 & 105 & 1 & 0,004 & 0,949 \\
\hline
\end{tabular}

Tab. A-66 Wilcoxon(Gehan)-Test zum Vergleich der Überlebensfunktionen von normo-, oligo- und azoospermen Fällen (nach Guzick et al., 2001); frühe Geburtsjahrgänge

\begin{tabular}{|l|l|l|l|l|l|l|}
\hline \multirow{2}{*}{\begin{tabular}{l|l|l|l|} 
Vergleich \\
\cline { 2 - 5 }
\end{tabular}} & $\mathbf{n}$ & df & $\begin{array}{l}\text { Wilcoxon } \\
\text { (Gehan) }\end{array}$ & $\mathbf{p}$ \\
\hline overall & 107 & 129 & 2 & $6,965^{*}$ & 0,031 \\
\hline normozoosperm-oligozoosperm & 81 & 106 & 1 & $5,446^{*}$ & 0,020 \\
\hline normozoosperm-azoosperm & 81 & 108 & 1 & $3,824^{(*)}$ & 0,051 \\
\hline oligozoosperm-azoosperm & 52 & 44 & 0,042 & 0,837 \\
\hline
\end{tabular}

Tab. A-67 Wilcoxon(Gehan)-Test zum Vergleich der Überlebensfunktionen von normo-, oligo- und azoospermen Fällen (nach Guzick et al., 2001); späte Geburtsjahrgänge

\begin{tabular}{|l|l|l|l|l|l|l|}
\hline \multirow{2}{*}{\begin{tabular}{l|l|l|l|} 
Vergleich \\
\cline { 2 - 5 }
\end{tabular}} & $\mathbf{n}$ & df & $\begin{array}{l}\text { Wilcoxon } \\
\text { (Gehan) }\end{array}$ & $\mathbf{p}$ \\
\hline overall & unzensiert & zensiert & 2 & 0,213 & 0,899 \\
\hline normozoosperm-oligozoosperm & 33 & 196 & 1 & 0,131 & 0,718 \\
\hline normozoosperm-azoosperm & 31 & 167 & 1 & 0,129 & 0,720 \\
\hline oligozoosperm-azoosperm & 14 & 61 & 1 & 0,001 & 0,981 \\
\hline
\end{tabular}


Ergebnisse der semi-parametrischen Verfahren

Tab. A-68 Cox-Regression zum Einfluss des Fertilitätsstatus (fertil/subfertil) nach Guzick et al. (2001) auf die Lebensdauer ${ }^{a}$

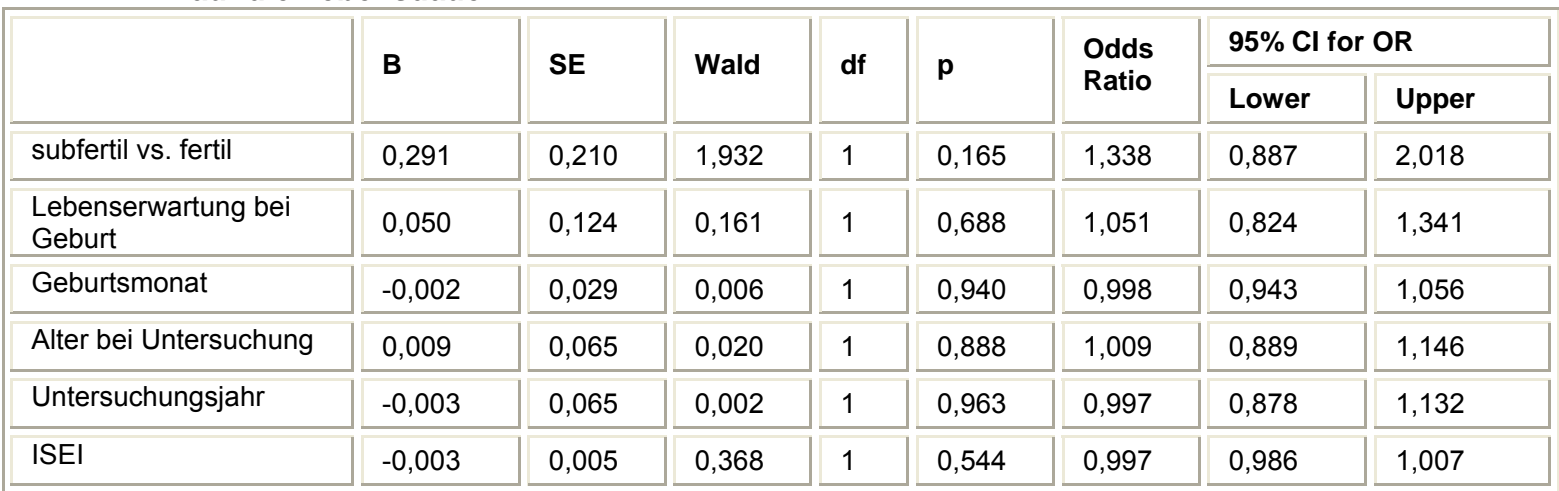

anzensierte Fälle $n=100$; zensierte Fälle $n=263$

Tab. A-69 Cox-Regression zum Einfluss des Fertilitätsstatus (fertil/subfertil) nach Guzick et al. (2001) auf die Lebensdauer ${ }^{a}$, frühe Geburtsjahrgänge

\begin{tabular}{|c|c|c|c|c|c|c|c|c|}
\hline & \multirow{2}{*}{ B } & \multirow{2}{*}{ SE } & \multirow{2}{*}{ Wald } & \multirow{2}{*}{ df } & \multirow{2}{*}{$\mathbf{p}$} & \multirow{2}{*}{$\begin{array}{l}\text { Odds } \\
\text { Ratio }\end{array}$} & \multicolumn{2}{|c|}{$95 \% \mathrm{Cl}$ for OR } \\
\hline & & & & & & & Lower & Upper \\
\hline subfertil vs. fertil & 0,385 & 0,256 & 2,269 & 1 & 0,132 & 1,470 & 0,891 & 2,425 \\
\hline $\begin{array}{l}\text { Lebenserwartung bei } \\
\text { Geburt }\end{array}$ & 0,050 & 0,252 & 0,039 & 1 & 0,844 & 1,051 & 0,641 & 1,722 \\
\hline Geburtsmonat & $-0,008$ & 0,036 & 0,051 & 1 & 0,821 & 0,992 & 0,924 & 1,065 \\
\hline Alter bei Untersuchung & 0,021 & 0,157 & 0,019 & 1 & 0,891 & 1,022 & 0,752 & 1,388 \\
\hline Untersuchungsjahr & $-0,011$ & 0,158 & 0,005 & 1 & 0,944 & 0,989 & 0,726 & 1,347 \\
\hline ISEI & 0,010 & 0,006 & 2,214 & 1 & 0,137 & 1,010 & 0,997 & 1,022 \\
\hline
\end{tabular}

a unzensierte Fälle $n=67$; zensierte Fälle $n=106$

Tab. A-70 Cox-Regression zum Einfluss des Fertilitätsstatus (fertil/subfertil) nach Guzick et al. (2001) auf die Lebensdauer ${ }^{a}$, späte Geburtsjahrgänge

\begin{tabular}{|c|c|c|c|c|c|c|c|c|}
\hline & \multirow{2}{*}{ B } & \multirow{2}{*}{ SE } & \multirow{2}{*}{ Wald } & \multirow{2}{*}{ df } & \multirow{2}{*}{$\mathbf{p}$} & \multirow{2}{*}{$\begin{array}{l}\text { Odds } \\
\text { Ratio }\end{array}$} & \multicolumn{2}{|c|}{$95 \% \mathrm{Cl}$ for OR } \\
\hline & & & & & & & Lower & Upper \\
\hline subfertil vs. fertil & 0,226 & 0,380 & 0,354 & 1 & 0,552 & 1,254 & 0,595 & 2,643 \\
\hline $\begin{array}{l}\text { Lebenserwartung bei } \\
\text { Geburt }\end{array}$ & 0,748 & 0,856 & 0,763 & 1 & 0,382 & 2,112 & 0,395 & 11,310 \\
\hline Geburtsmonat & 0,019 & 0,054 & 0,127 & 1 & 0,721 & 1,020 & 0,917 & 1,134 \\
\hline Alter bei Untersuchung & $-0,013$ & 0,115 & 0,013 & 1 & 0,908 & 0,987 & 0,787 & 1,237 \\
\hline Untersuchungsjahr & 0,022 & 0,117 & 0,037 & 1 & 0,848 & 1,023 & 0,813 & 1,286 \\
\hline ISEI & $-0,031$ & 0,011 & 7,154 & 1 & 0,007 & $0,970^{* *}$ & 0,948 & 0,992 \\
\hline
\end{tabular}

a unzensierte Fälle $n=33$; zensierte Fälle $n=157$ 
Tab. A-71 Cox-Regression zum Einfluss des Fertilitätsstatus (normo-/oligo-/azoosperm) nach Guzick et al. (2001) auf die Lebensdauer ${ }^{a}$

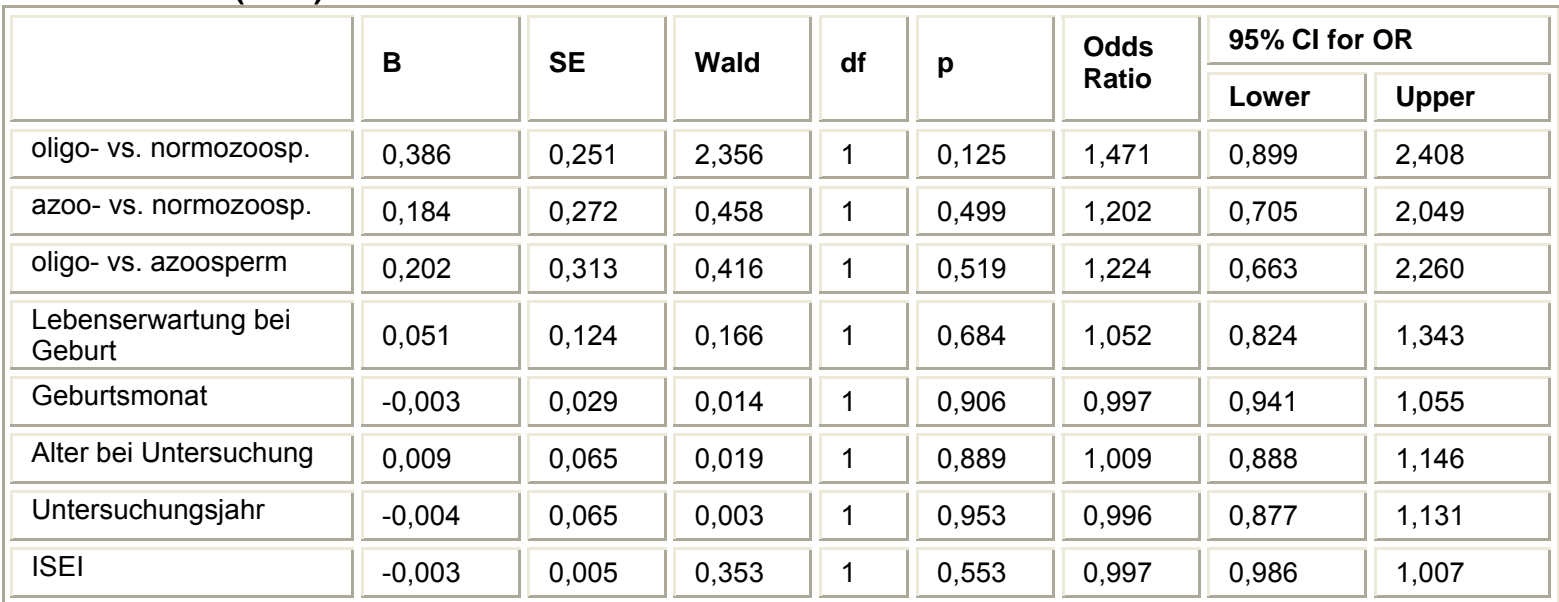

${ }^{a}$ unzensierte Fälle $n=100$; zensierte Fälle $n=263$

Tab. A-72 Cox-Regression zum Einfluss des Fertilitätsstatus (normo-/oligo-/azoosperm) nach Guzick et al. (2001) auf die Lebensdauer ${ }^{2}$; frühe Geburtsjahrgänge

\begin{tabular}{|c|c|c|c|c|c|c|c|c|}
\hline & \multirow{2}{*}{ B } & \multirow{2}{*}{ SE } & \multirow{2}{*}{ Wald } & \multirow{2}{*}{ df } & \multirow{2}{*}{$\mathbf{p}$} & \multirow{2}{*}{$\begin{array}{l}\text { Odds } \\
\text { Ratio }\end{array}$} & \multicolumn{2}{|c|}{$95 \% \mathrm{Cl}$ for $\mathrm{OR}$} \\
\hline & & & & & & & Lower & Upper \\
\hline oligo- vs. normozoosp. & 0,539 & 0,307 & 3,078 & 1 & 0,079 & $1,714^{(*)}$ & 0,939 & 3,131 \\
\hline azoo- vs. normozoosp. & 0,220 & 0,331 & 0,441 & 1 & 0,507 & 1,246 & 0,651 & 2,385 \\
\hline oligo- vs. azoosperm & 0,319 & 0,382 & 0,700 & 1 & 0,403 & 1,376 & 0,651 & 2,906 \\
\hline $\begin{array}{l}\text { Lebenserwartung bei } \\
\text { Geburt }\end{array}$ & 0,069 & 0,255 & 0,073 & 1 & 0,787 & 1,071 & 0,650 & 1,765 \\
\hline Geburtsmonat & $-0,009$ & 0,036 & 0,065 & 1 & 0,799 & 0,991 & 0,923 & 1,064 \\
\hline Alter bei Untersuchung & 0,033 & 0,158 & 0,044 & 1 & 0,835 & 1,034 & 0,758 & 1,409 \\
\hline Untersuchungsjahr & $-0,025$ & 0,159 & 0,024 & 1 & 0,877 & 0,976 & 0,714 & 1,333 \\
\hline ISEI & 0,010 & 0,007 & 2,351 & 1 & 0,125 & 1,010 & 0,997 & 1,023 \\
\hline
\end{tabular}

anzensierte Fälle $n=67$; zensierte Fälle $n=106$

Tab A-73 Cox-Regression zum Einfluss des Fertilitätsstatus (normo-/oligo-/azoosperm) nach Guzick et al. (2001) auf die Lebensdauer ${ }^{\text {a }}$; späte Geburtsjahrgänge

\begin{tabular}{|c|c|c|c|c|c|c|c|c|}
\hline & \multirow{2}{*}{ B } & \multirow{2}{*}{ SE } & \multirow{2}{*}{ Wald } & \multirow{2}{*}{ df } & \multirow{2}{*}{$p$} & \multirow{2}{*}{$\begin{array}{l}\text { Odds } \\
\text { Ratio }\end{array}$} & \multicolumn{2}{|c|}{ 95\% Cl for OR } \\
\hline & & & & & & & Lower & Upper \\
\hline oligo- vs. normozoosp. & 0,224 & 0,471 & 0,227 & 1 & 0,633 & 1,252 & 0,497 & 3,149 \\
\hline azoo- vs. normozoosp. & 0,228 & 0,481 & 0,226 & 1 & 0,635 & 1,256 & 0,490 & 3,223 \\
\hline oligo- vs. azoosperm & $-0,004$ & 0,571 & 0,000 & 1 & 0,995 & 0,996 & 0,325 & 3,052 \\
\hline $\begin{array}{l}\text { Lebenserwartung bei } \\
\text { Geburt }\end{array}$ & 0,749 & 0,866 & 0,747 & 1 & 0,387 & 2,114 & 0,387 & 11,546 \\
\hline Geburtsmonat & 0,019 & 0,055 & 0,126 & 1 & 0,722 & 1,020 & 0,916 & 1,135 \\
\hline Alter bei Untersuchung & $-0,013$ & 0,115 & 0,013 & 1 & 0,908 & 0,987 & 0,787 & 1,237 \\
\hline Untersuchungsjahr & 0,022 & 0,117 & 0,036 & 1 & 0,849 & 1,023 & 0,813 & 1,286 \\
\hline ISEI & $-0,031$ & 0,011 & 7,125 & 1 & 0,008 & $0,970^{* *}$ & 0,948 & 0,992 \\
\hline
\end{tabular}

anzensierte Fälle $n=33$; zensierte Fälle $n=157$ 


\section{Ergebnisse der parametrischen Verfahren}

Tab. A-74 Exponential-Modell zum Einfluss des Fertilitätsstatus (fertil/subfertil) nach Guzick et al. (2001) auf die Lebensdauer ${ }^{a}$

\begin{tabular}{|l|l|l|l|l|l||l|}
\hline Variable & Coeff & Error & C/Error & Signif & p & $\begin{array}{l}\text { Rel. } \\
\text { Risiko }\end{array}$ \\
\hline Fertilitätsstatus: subfertil/fertil & 0,2073 & 0,2077 & 0,9982 & 0,6818 & 0,318 & 1,2304 \\
\hline Lebenserwartung bei Geburt & 0,0948 & 0,1159 & 0,8177 & 0,5865 & 0,414 & 1,0994 \\
\hline Geburtsmonat & 0,0033 & 0,0293 & 0,1128 & 0,0898 & 0,910 & 1,0033 \\
\hline Alter bei Untersuchung & 0,1299 & 0,0612 & 2,1220 & 0,9662 & 0,034 & $1,1387^{*}$ \\
\hline Untersuchungsjahr & $-0,1244$ & 0,0610 & $-2,0380$ & 0,9585 & 0,042 & $0,8831^{*}$ \\
\hline ISEI & $-0,0045$ & 0,0051 & $-0,8846$ & 0,6236 & 0,376 & 0,9955 \\
\hline
\end{tabular}

${ }^{a}$ unzensierte Fälle $n=100$; zensierte Fälle $n=263$

Tab. A-75 Exponential-Modell zum Einfluss des Fertilitätsstatus (normo-/oligo-/azoosperm) nach Guzick et al. (2001) auf die Lebensdauer ${ }^{a}$

\begin{tabular}{|c|c|c|c|c|c|c|}
\hline Variable & Coeff & Error & C/Error & Signif & $\mathbf{p}$ & $\begin{array}{l}\text { Rel. } \\
\text { Risiko }\end{array}$ \\
\hline $\begin{array}{l}\text { Fertilitätsstatus: } \\
\text { normo-/oligo-/azoosperm }\end{array}$ & 0,0944 & 0,1268 & 0,7443 & 0,5433 & 0,457 & 1,0990 \\
\hline Lebenserwartung bei Geburt & 0,0970 & 0,1157 & 0,8383 & 0,5981 & 0,402 & 1,1019 \\
\hline Geburtsmonat & 0,0046 & 0,0292 & 0,1590 & 0,1263 & 0,874 & 1,0047 \\
\hline Alter bei Untersuchung & 0,1311 & 0,0610 & 2,1480 & 0,9683 & 0,032 & $1,1401^{*}$ \\
\hline Untersuchungsjahr & $-0,1261$ & 0,0609 & $-2,0707$ & 0,9616 & 0,038 & $0,8816^{*}$ \\
\hline ISEI & $-0,0048$ & 0,0051 & $-0,9390$ & 0,6523 & 0,348 & 0,9952 \\
\hline
\end{tabular}

${ }^{a}$ unzensierte Fälle $n=100$; zensierte Fälle $n=263$

Tab. A-76 Exponential-Modell zum Einfluss des Fertilitätsstatus (fertil/subfertil) nach Guzick et al. (2001) auf die Lebensdauer ${ }^{a}$; frühe Geburtsjahrgänge

\begin{tabular}{|l|l|l|l|l|l|l|l||}
\hline Variable & Coeff & Error & C/Error & Signif & p & $\begin{array}{l}\text { Rel. } \\
\text { Risiko }\end{array}$ \\
\hline \hline Fertiliätsstatus: subfertil/fertil & 0,3119 & 0,2546 & 1,2251 & 0,7795 & 0,221 & 1,3660 \\
\hline Lebenserwartung bei Geburt & 0,1705 & 0,2437 & 0,6997 & 0,5159 & 0,484 & 1,1859 \\
\hline Geburtsmonat & 0,0049 & 0,0366 & 0,1344 & 0,1069 & 0,893 & 1,0049 \\
\hline Alter bei Untersuchung & 0,1812 & 0,1506 & 1,2034 & 0,7712 & 0,229 & 1,1986 \\
\hline Untersuchungsjahr & $-0,1732$ & 0,1511 & $-1,1464$ & 0,7484 & 0,252 & 0,8410 \\
\hline ISEI & 0,0048 & 0,0059 & 0,8112 & 0,5828 & 0,417 & 1,0048 \\
\hline
\end{tabular}

${ }^{a}$ unzensierte Fälle $n=67$; zensierte Fälle $n=106$

Tab. A-77 Exponential-Modell zum Einfluss des Fertilitätsstatus (normo-/oligo-/azoosperm) nach Guzick et al. (2001) auf die Lebensdauer ${ }^{a}$; frühe Geburtsjahrgänge

\begin{tabular}{|l|l|l|l|l|l|l|}
\hline Variable & Coeff & Error & C/Error & Signif & p & $\begin{array}{l}\text { Rel. } \\
\text { Risiko }\end{array}$ \\
\hline $\begin{array}{l}\text { Fertilitätsstatus: } \\
\text { normo-/oligo-/azoosperm }\end{array}$ & 0,1358 & 0,1529 & 0,8883 & 0,6256 & 0,374 & 1,1454 \\
\hline Lebenserwartung bei Geburt & 0,1593 & 0,2429 & 0,6557 & 0,4880 & 0,512 & 1,1727 \\
\hline Geburtsmonat & 0,0061 & 0,0366 & 0,1671 & 0,1327 & 0,866 & 1,0061 \\
\hline \hline Alter bei Untersuchung & 0,1740 & 0,1500 & 1,1604 & 0,7541 & 0,246 & 1,1901 \\
\hline Untersuchungsjahr & $-0,1665$ & 0,1507 & $-1,1046$ & 0,7307 & 0,270 & 0,8466 \\
\hline ISEI & 0,0042 & 0,0059 & 0,7151 & 0,5254 & 0,475 & 1,0042 \\
\hline
\end{tabular}

${ }^{a}$ unzensierte Fälle $\mathrm{n}=67$; zensierte Fälle $\mathrm{n}=106$ 
Tab. A-78 Exponential-Modell zum Einfluss des Fertilitätsstatus (fertil/subfertil) nach Guzick et al. (2001) auf die Lebensdauer ${ }^{a}$; späte Geburtsjahrgänge

\begin{tabular}{|l|l|l|l||l|l||l|}
\hline Variable & Coeff & Error & C/Error & Signif & p & $\begin{array}{l}\text { Rel. } \\
\text { Risiko }\end{array}$ \\
\hline Fertiliätsstatus: subfertil/fertil & 0,2384 & 0,3778 & 0,6311 & 0,4720 & 0,528 & 1,2693 \\
\hline Lebenserwartung bei Geburt & 0,4542 & 0,8682 & 0,5231 & 0,3991 & 0,601 & 1,5749 \\
\hline Geburtsmonat & 0,0079 & 0,0542 & 0,1461 & 0,1162 & 0,884 & 1,0080 \\
\hline Alter bei Untersuchung & 0,0803 & 0,1104 & 0,7270 & 0,5327 & 0,467 & 1,0836 \\
\hline \hline Untersuchungsjahr & $-0,0701$ & 0,1119 & $-0,6262$ & 0,4688 & 0,531 & 0,9323 \\
\hline ISEI & $-0,0279$ & 0,0113 & $-2,4602$ & 0,9861 & 0,014 & $0,9725^{*}$ \\
\hline
\end{tabular}

${ }^{a}$ unzensierte Fälle $n=33$; zensierte Fälle $n=157$

Tab. A-79 Exponential-Modell zum Einfluss des Fertilitätsstatus (normo-/oligo-lazoosperm) nach Guzick et al. (2001) auf die Lebensdauer ${ }^{a}$; späte Geburtsjahrgänge

\begin{tabular}{|l|l|l|l|l|l||l||}
\hline Variable & Coeff & Error & C/Error & Signif & p & $\begin{array}{l}\text { Rel. } \\
\text { Risiko }\end{array}$ \\
\hline $\begin{array}{l}\text { Fertilitätsstatus: } \\
\text { normo-/oligo-lazoosperm }\end{array}$ & 0,1366 & 0,2295 & 0,5952 & 0,4483 & 0,552 & 1,1464 \\
\hline \hline Lebenserwartung bei Geburt & 0,4781 & 0,8673 & 0,5513 & 0,4185 & 0,582 & 1,6130 \\
\hline Geburtsmonat & 0,0092 & 0,0541 & 0,1697 & 0,1348 & 0,865 & 1,0092 \\
\hline \hline Alter bei Untersuchung & 0,0845 & 0,1093 & 0,7730 & 0,5605 & 0,440 & 1,0882 \\
\hline \hline Untersuchungsjahr & $-0,0747$ & 0,1103 & $-0,6771$ & 0,5017 & 0,500 & 0,9280 \\
\hline ISEI & $-0,0280$ & 0,0114 & $-2,4642$ & 0,9863 & 0,014 & $0,9724^{*}$ \\
\hline
\end{tabular}

${ }^{a}$ unzensierte Fälle $\mathrm{n}=33$; zensierte Fälle $\mathrm{n}=157$

Tab. A-80 Gompertz-Makeham-Modell zum Einfluss des Fertilitätsstatus (fertil/subfertil) nach Guzick et al. (2001) auf die Lebensdauer ${ }^{a}$

\begin{tabular}{|l|l|l|l|l|l|l|l|}
\hline Variable & Coeff & Error & C/Error & Signif & $\mathbf{p}$ & $\begin{array}{l}\text { Rel. } \\
\text { Risiko }\end{array}$ \\
\hline Fertilitätsstatus: subfertil/fertil & 0,2995 & 0,2094 & 1,4301 & 0,8473 & 0,153 & 1,3492 \\
\hline Lebenserwartung bei Geburt & 0,0396 & 0,1186 & 0,3342 & 0,2618 & 0,738 & 1,0404 \\
\hline Geburtsmonat & 0,0018 & 0,0289 & 0,0617 & 0,0492 & 0,951 & 1,0018 \\
\hline Alter bei Untersuchung & 0,1289 & 0,0619 & 2,0827 & 0,9627 & 0,037 & $1,1375^{*}$ \\
\hline Untersuchungsjahr & $-0,1237$ & 0,0614 & $-2,0148$ & 0,9561 & 0,044 & $0,8837^{*}$ \\
\hline ISEI & $-0,0027$ & 0,0053 & $-0,5150$ & 0,3934 & 0,607 & 0,9973 \\
\hline
\end{tabular}

${ }^{a}$ unzensierte Fälle $n=100$; zensierte Fälle $n=263$

Tab. A-81 Gompertz-Makeham-Modell zum Einfluss des Fertilitätsstatus (normo-/oligo-I azoosperm) nach Guzick et al. (2001) auf die Lebensdauer ${ }^{a}$

\begin{tabular}{|c|c|c|c|c|c|c|}
\hline Variable & Coeff & Error & C/Error & Signif & $\mathbf{p}$ & $\begin{array}{l}\text { Rel. } \\
\text { Risiko }\end{array}$ \\
\hline $\begin{array}{l}\text { Fertilitätsstatus: } \\
\text { normo-/oligo-/azoosperm }\end{array}$ & 0,1330 & 0,1271 & 1,0466 & 0,7047 & 0,295 & 1,1423 \\
\hline Lebenserwartung bei Geburt & 0,0422 & 0,1183 & 0,3571 & 0,2790 & 0,721 & 1,0431 \\
\hline Geburtsmonat & 0,0037 & 0,0288 & 0,1302 & 0,1036 & 0,896 & 1,0038 \\
\hline Alter bei Untersuchung & 0,1300 & 0,0616 & 2,1114 & 0,9653 & 0,035 & $1,1389^{*}$ \\
\hline Untersuchungsjahr & $-0,1258$ & 0,0612 & $-2,0572$ & 0,9603 & 0,040 & $0,8818^{*}$ \\
\hline ISEI & $-0,0030$ & 0,0053 & $-0,5687$ & 0,4304 & 0,570 & 0,9970 \\
\hline
\end{tabular}

a unzensierte Fälle $n=100$; zensierte Fälle $n=263$ 
Tab. A-82 Gompertz-Makeham-Modell zum Einfluss des Fertilitätsstatus (fertil/subfertil) nach Guzick et al. (2001) auf die Lebensdauer ${ }^{a}$; frühe Geburtsjahrgänge

\begin{tabular}{|l|l|l|l|l|l||l|}
\hline Variable & Coeff & Error & C/Error & Signif & $\mathbf{p}$ & $\begin{array}{l}\text { Rel. } \\
\text { Risiko }\end{array}$ \\
\hline Fertilitätsstatus: subfertil/fertil & 0,4414 & 0,2568 & 1,7190 & 0,9144 & 0,086 & $1,5548^{(*)}$ \\
\hline Lebenserwartung bei Geburt & 0,0850 & 0,2458 & 0,3459 & 0,2706 & 0,729 & 1,0887 \\
\hline Geburtsmonat & $-0,0006$ & 0,0358 & $-0,0157$ & 0,0125 & 0,988 & 0,9994 \\
\hline Alter bei Untersuchung & 0,1806 & 0,1518 & 1,1898 & 0,7659 & 0,234 & 1,1979 \\
\hline Untersuchungsjahr & $-0,1684$ & 0,1525 & $-1,1039$ & 0,7303 & 0,270 & 0,8450 \\
\hline ISEI & 0,0117 & 0,0063 & 1,8637 & 0,9376 & 0,062 & $1,0118^{(*)}$ \\
\hline
\end{tabular}

${ }^{a}$ unzensierte Fälle $n=67$; zensierte Fälle $n=106$

Tab. A-83 Gompertz-Makeham-Modell zum Einfluss des Fertilitätsstatus (normo-/oligo-I azoosperm) nach Guzick et al. (2001) auf die Lebensdauer ${ }^{a}$; frühe Geburtsjahrgänge

\begin{tabular}{|l|l|l|l|l|l|l||}
\hline Variable & Coeff & Error & C/Error & Signif & p & $\begin{array}{l}\text { Rel. } \\
\text { Risiko }\end{array}$ \\
\hline $\begin{array}{l}\text { Fertilitätsstatus: } \\
\text { normo-/oligo-lazoosperm }\end{array}$ & 0,1860 & 0,1536 & 1,2110 & 0,7741 & 0,226 & 1,2044 \\
\hline \hline Lebenserwartung bei Geburt & 0,0708 & 0,2440 & 0,2900 & 0,2282 & 0,772 & 1,0733 \\
\hline Geburtsmonat & 0,0010 & 0,0356 & 0,0294 & 0,0235 & 0,977 & 1,0010 \\
\hline \hline Alter bei Untersuchung & 0,1712 & 0,1506 & 1,1366 & 0,7443 & 0,256 & 1,1868 \\
\hline \hline Untersuchungsjahr & $-0,1605$ & 0,1519 & $-1,0568$ & 0,7094 & 0,291 & 0,8517 \\
\hline ISEI & 0,0111 & 0,0063 & 1,7612 & 0,9218 & 0,078 & $1,0111^{(*)}$ \\
\hline
\end{tabular}

${ }^{a}$ unzensierte Fälle $\mathrm{n}=67$; zensierte Fälle $\mathrm{n}=106$

Tab. A-84 Gompertz-Makeham-Modell zum Einfluss des Fertilitätsstatus (fertil/subfertil) nach Guzick et al. (2001) auf die Lebensdauer ${ }^{a}$; späte Geburtsjahrgänge

\begin{tabular}{|l|l|l|l|l|l||l|}
\hline Variable & Coeff & Error & C/Error & Signif & p & $\begin{array}{l}\text { Rel. } \\
\text { Risiko }\end{array}$ \\
\hline Fertilitätsstatus: subfertil/fertil & 0,2581 & 0,3787 & 0,6817 & 0,5046 & 0,495 & 1,2945 \\
\hline Lebenserwartung bei Geburt & 0,5643 & 0,8498 & 0,6640 & 0,4933 & 0,507 & 1,7583 \\
\hline Geburtsmonat & 0,0186 & 0,0538 & 0,3465 & 0,2710 & 0,729 & 1,0188 \\
\hline Alter bei Untersuchung & 0,0949 & 0,1090 & 0,8714 & 0,6164 & 0,384 & 1,0996 \\
\hline Untersuchungsjahr & $-0,0833$ & 0,1106 & $-0,7530$ & 0,5485 & 0,452 & 0,9201 \\
\hline \hline ISEI & $-0,0322$ & 0,0117 & $-2,7672$ & 0,9943 & 0,006 & $0,9683^{* *}$ \\
\hline
\end{tabular}

anzensierte Fälle $n=33$; zensierte Fälle $n=157$

Tab. A-85 Gompertz-Makeham-Modell zum Einfluss des Fertilitätsstatus (normo-/oligo-I azoosperm) nach Guzick et al. (2001) auf die Lebensdauer ${ }^{a}$; späte Geburtsjahrgänge

\begin{tabular}{|l|l|l|l|l|l|l|l|}
\hline Variable & Coeff & Error & C/Error & Signif & p & $\begin{array}{l}\text { Rel. } \\
\text { Risiko }\end{array}$ \\
\hline \begin{tabular}{l|l|l|l|l|l|}
\hline Fertilitätsstatus: \\
normo-/oligo-/azoosperm
\end{tabular} & 0,1601 & 0,2303 & 0,6952 & 0,5131 & 0,487 & 1,1737 \\
\hline Lebenserwartung bei Geburt & 0,5898 & 0,8471 & 0,6962 & 0,5137 & 0,486 & 1,8036 \\
\hline Geburtsmonat & 0,0207 & 0,0536 & 0,3863 & 0,3008 & 0,699 & 1,0209 \\
\hline Alter bei Untersuchung & 0,0997 & 0,1077 & 0,9257 & 0,6454 & 0,355 & 1,1049 \\
\hline Untersuchungsjahr & $-0,0881$ & 0,1088 & $-0,8097$ & 0,5819 & 0,418 & 0,9157 \\
\hline ISEI & $-0,0326$ & 0,0117 & $-2,7763$ & 0,9945 & 0,006 & $0,9680^{* *}$ \\
\hline
\end{tabular}

anzensierte Fälle $n=33$; zensierte Fälle $n=157$ 
Tab. A-86 Weibull-Modell zum Einfluss des Fertilitätsstatus (fertil/subfertil) nach Guzick et al. (2001) auf die Lebensdauer ${ }^{a, b}$

\begin{tabular}{|c|c|c|c|c|c|c|}
\hline Variable & Coeff & Error & C/Error & Signif & $\mathbf{p}$ & $\begin{array}{l}\text { Rel. } \\
\text { Risiko }\end{array}$ \\
\hline Fertilitätsstatus: subfertil/fertil & 0,0070 & 0,0040 & 1,7557 & 0,9209 & 0,079 & $1,4527^{(*)}$ \\
\hline Lebenserwartung bei Geburt & $-0,0047$ & 0,0021 & $-2,2257$ & 0,9740 & 0,026 & $0,7755^{*}$ \\
\hline Geburtsmonat & 0,0000 & 0,0005 & $-0,0681$ & 0,0543 & 0,946 & 0,9980 \\
\hline Alter bei Untersuchung & $-0,0005$ & 0,0010 & $-0,4820$ & 0,3702 & 0,630 & 0,9733 \\
\hline Untersuchungsjahr & 0,0007 & 0,0010 & 0,6919 & 0,5110 & 0,489 & 1,0395 \\
\hline ISEI & $-0,0001$ & 0,0001 & $-0,5651$ & 0,4280 & 0,572 & 0,9971 \\
\hline
\end{tabular}

unzensierte Fälle $n=100$; zensierte Fälle $n=263$

b Convergence not reached in 500000 iterations; Number of function evaluations: 1000002

Tab. A-87 Weibull-Modell zum Einfluss des Fertilitätsstatus (normo-/oligo-lazoosperm) nach Guzick et al. (2001) auf die Lebensdauer ${ }^{a, b}$

\begin{tabular}{|c|c|c|c|c|c|c|}
\hline Variable & Coeff & Error & C/Error & Signif & $\mathbf{p}$ & $\begin{array}{l}\text { Rel. } \\
\text { Risiko }\end{array}$ \\
\hline $\begin{array}{l}\text { Fertilitätsstatus: } \\
\text { normo-/oligo-/azoosperm }\end{array}$ & 0,0033 & 0,0024 & 1,3913 & 0,8359 & 0,164 & 1,1955 \\
\hline Lebenserwartung bei Geburt & $-0,0048$ & 0,0021 & $-2,2438$ & 0,9752 & 0,025 & $0,7746^{*}$ \\
\hline Geburtsmonat & 0,0000 & 0,0005 & $-0,0052$ & 0,0042 & 0,996 & 0,9998 \\
\hline Alter bei Untersuchung & $-0,0005$ & 0,0010 & $-0,4924$ & 0,3776 & 0,622 & 0,9729 \\
\hline Untersuchungsjahr & 0,0007 & 0,0010 & 0,6942 & 0,5124 & 0,488 & 1,0394 \\
\hline ISEI & $-0,0001$ & 0,0001 & $-0,6104$ & 0,4584 & 0,542 & 0,9968 \\
\hline
\end{tabular}

${ }^{\mathrm{b}}$ unzensierte Fälle $\mathrm{n}=100$; zensierte Fälle $\mathrm{n}=263$

b Convergence not reached in 500000 iterations; Number of function evaluations: 1000002

Tab. A-88 Weibull-Modell zum Einfluss des Fertilitätsstatus (fertil/subfertil) nach Guzick et al. (2001) auf die Lebensdauer ${ }^{a}$; frühe Geburtsjahrgänge

\begin{tabular}{|l|l|l|l|l|l||l||}
\hline Variable & Coeff & Error & C/Error & Signif & $\mathbf{p}$ & $\begin{array}{l}\text { Rel. } \\
\text { Risiko }\end{array}$ \\
\hline \hline Fertilitätsstatus: subfertil/fertil & 0,0073 & 0,0043 & 1,7207 & 0,9147 & 0,085 & $1,5628^{(*)}$ \\
\hline \hline Lebenserwartung bei Geburt & $-0,0038$ & 0,0041 & $-0,9256$ & 0,6453 & 0,355 & 0,7920 \\
\hline Geburtsmonat & $-0,0002$ & 0,0006 & $-0,2882$ & 0,2268 & 0,773 & 0,9896 \\
\hline Alter bei Untersuchung & $-0,0003$ & 0,0026 & $-0,1266$ & 0,1008 & 0,899 & 0,9805 \\
\hline Untersuchungsjahr & 0,0006 & 0,0026 & 0,2216 & 0,1754 & 0,825 & 1,0353 \\
\hline ISEI & 0,0002 & 0,0001 & 2,0878 & 0,9632 & 0,037 & $1,0133^{*}$ \\
\hline
\end{tabular}

${ }^{a}$ unzensierte Fälle $\mathrm{n}=67$; zensierte Fälle $\mathrm{n}=106$

Tab. A-89 Weibull-Modell zum Einfluss des Fertilitätsstatus (normo-/oligo-/azoosperm) nach Guzick et al. (2001) auf die Lebensdauer ${ }^{a}$; frühe Geburtsjahrgänge

\begin{tabular}{|l|l|l|l|l|l|l|}
\hline Variable & Coeff & Error & C/Error & Signif & p & $\begin{array}{l}\text { Rel. } \\
\text { Risiko }\end{array}$ \\
\hline $\begin{array}{l}\text { Fertilitätsstatus: } \\
\text { normo-/oligo-/azoosperm }\end{array}$ & 0,0033 & 0,0026 & 1,2837 & 0,8008 & 0,199 & 1,2211 \\
\hline Lebenserwartung bei Geburt & $-0,0039$ & 0,0041 & $-0,9369$ & 0,6512 & 0,349 & 0,7912 \\
\hline Geburtsmonat & $-0,0001$ & 0,0006 & $-0,2383$ & 0,1884 & 0,812 & 0,9914 \\
\hline Alter bei Untersuchung & $-0,0003$ & 0,0025 & $-0,1340$ & 0,1066 & 0,893 & 0,9795 \\
\hline Untersuchungsjahr & 0,0006 & 0,0026 & 0,2230 & 0,1765 & 0,824 & 1,0354 \\
\hline ISEI & 0,0002 & 0,0001 & 1,9835 & 0,9527 & 0,047 & $1,0125^{*}$ \\
\hline
\end{tabular}

${ }^{a}$ unzensierte Fälle $\mathrm{n}=67$; zensierte Fälle $\mathrm{n}=106$ 
Tab. A-90 Weibull-Modell zum Einfluss des Fertilitätsstatus (fertil/subfertil) nach Guzick et al. (2001) auf die Lebensdauer ${ }^{a}$; späte Geburtsjahrgänge

\begin{tabular}{|c|c|c|c|c|c|c|}
\hline Variable & Coeff & Error & C/Error & Signif & $\mathbf{p}$ & $\begin{array}{l}\text { Rel. } \\
\text { Risiko }\end{array}$ \\
\hline Fertilitätsstatus: subfertil/fertil & 0,0066 & 0,0081 & 0,8188 & 0,5871 & 0,413 & 1,3688 \\
\hline Lebenserwartung bei Geburt & 0,0216 & 0,0212 & 1,0147 & 0,6897 & 0,310 & 2,7772 \\
\hline Geburtsmonat & 0,0004 & 0,0011 & 0,3718 & 0,2900 & 0,710 & 1,0201 \\
\hline Alter bei Untersuchung & 0,0002 & 0,0026 & 0,0584 & 0,0465 & 0,954 & 1,0073 \\
\hline Untersuchungsjahr & 0,0002 & 0,0027 & 0,0772 & 0,0615 & 0,939 & 1,0098 \\
\hline ISEI & $-0,0007$ & 0,0003 & $-2,5564$ & 0,9894 & 0,011 & $0,9680^{*}$ \\
\hline
\end{tabular}

anzensierte Fälle $n=33$; zensierte Fälle $n=157$

Tab. A-91 Weibull-Modell zum Einfluss des Fertilitätsstatus (normo-/oligo-/azoosperm) nach Guzick et al. (2001) auf die Lebensdauer ${ }^{\text {a }}$; späte Geburtsjahrgänge

\begin{tabular}{|c|c|c|c|c|c|c|}
\hline Variable & Coeff & Error & C/Error & Signif & $\mathbf{p}$ & $\begin{array}{l}\text { Rel. } \\
\text { Risiko }\end{array}$ \\
\hline $\begin{array}{l}\text { Fertilitätsstatus: } \\
\text { normo-/oligo-/azoosperm }\end{array}$ & 0,0039 & 0,0049 & 0,7998 & 0,5762 & 0,424 & 1,2026 \\
\hline Lebenserwartung bei Geburt & 0,0227 & 0,0214 & 1,0615 & 0,7115 & 0,289 & 2,9364 \\
\hline Geburtsmonat & 0,0005 & 0,0011 & 0,4225 & 0,3273 & 0,673 & 1,0227 \\
\hline Alter bei Untersuchung & 0,0002 & 0,0026 & 0,0714 & 0,0569 & 0,943 & 1,0089 \\
\hline Untersuchungsjahr & 0,0002 & 0,0026 & 0,0637 & 0,0508 & 0,949 & 1,0080 \\
\hline ISEI & $-0,0007$ & 0,0003 & $-2,5630$ & 0,9896 & 0,010 & $0,9678^{* *}$ \\
\hline
\end{tabular}

${ }^{a}$ unzensierte Fälle $n=33$; zensierte Fälle $n=157$ 
Anhang

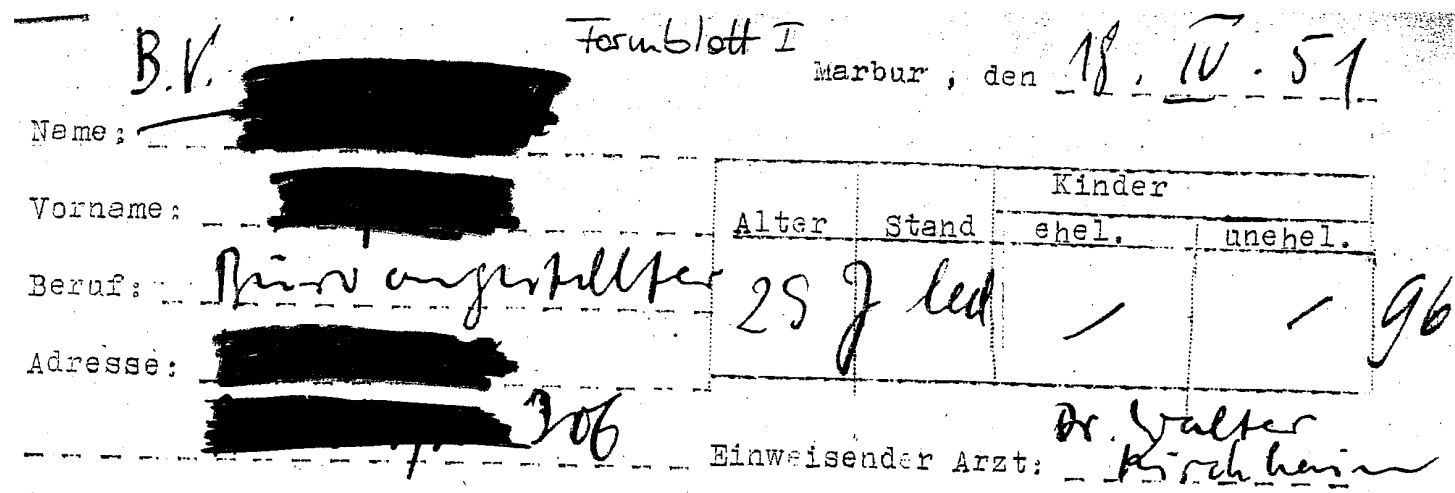

Aname se Anzahl der geschw.:

Femilienanamese:

Ven. Inf.

Tbc.

Jnfäle

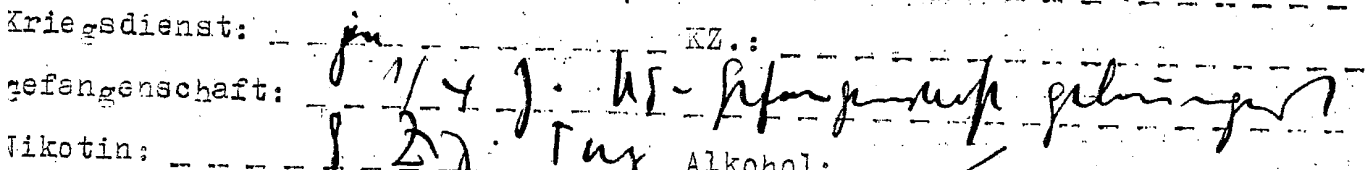

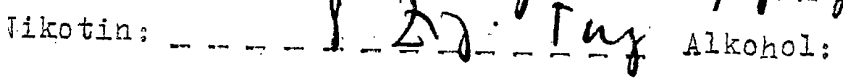

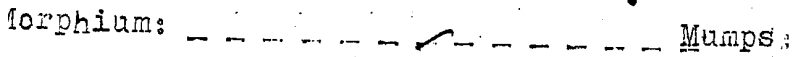

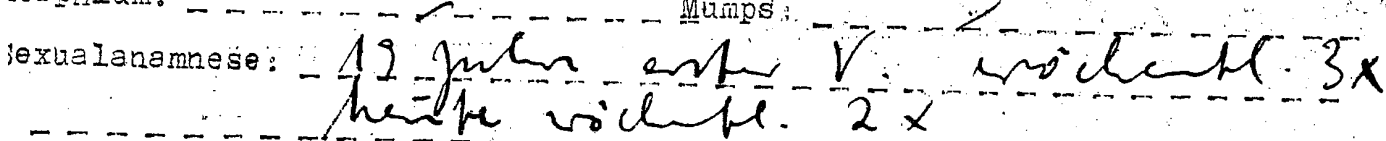

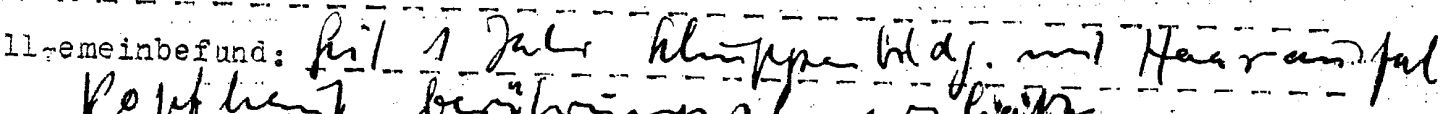

- Kopthe bentrin

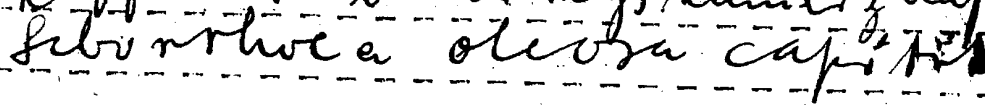

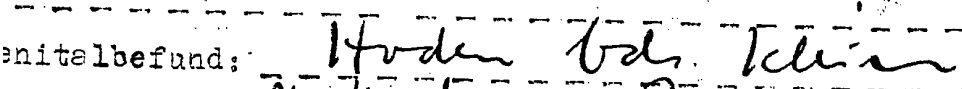

N Trode

Piniso 13 .

195 


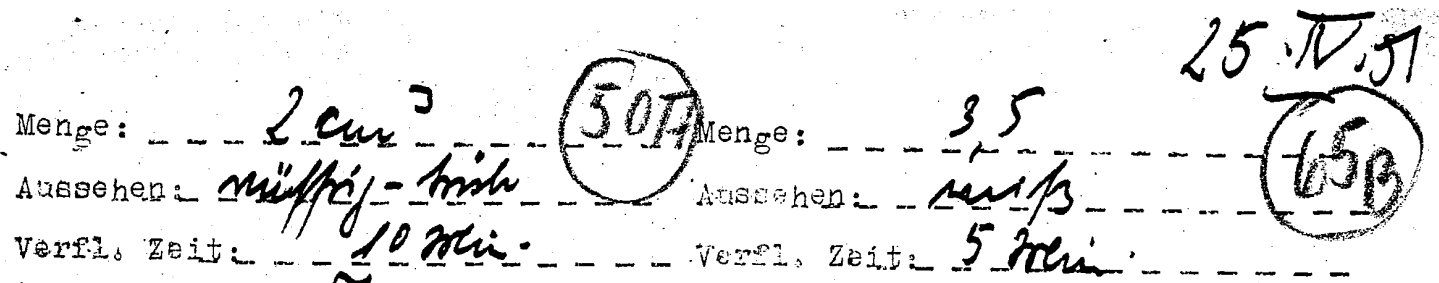

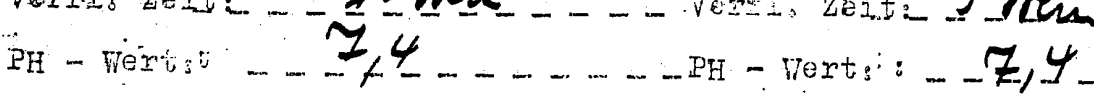

Zählung:

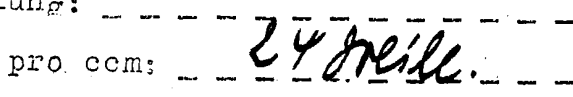
Zählung: ...

pro Blickf。:

Mexalität: $20 \%$ MrusthT.

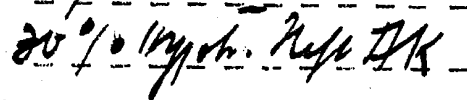

a) bei zimmertemp:

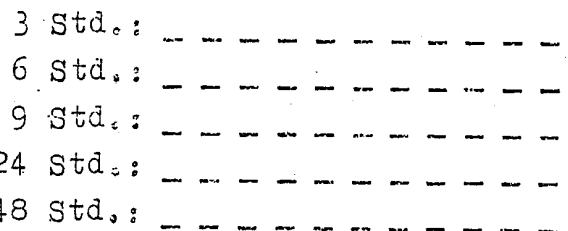

b) bei Körpertemp.
3. Std.: $\ldots \ldots \ldots-\ldots$
6 sta: : $\ldots \ldots \ldots \ldots-\ldots$
9 std.: $\ldots \ldots \ldots-\ldots \ldots$
24 Std.: $. . . .-\ldots-\ldots-\cdots$
48 std. : $\ldots \ldots-\ldots-\ldots$

\begin{tabular}{|c|c|c|c|c|}
\hline Färbung & $\begin{array}{l}\text { Nor-- } \\
\text { male } \\
\text { Sperm. }\end{array}$ & $\begin{array}{l}\text { Ver- } \\
\text { and. } \\
\text { des } \\
\text { Kopf. }\end{array}$ & $\begin{array}{l}\text { Ver- } \\
\text { and. } \\
\text { des } \\
\text { Mittel } \\
\text { stickes }\end{array}$ & $\begin{array}{l}\text { Ver- } \\
\text { änd. } \\
\text { des } \\
\text { Schwan } \\
\text { zes } \\
\end{array}$ \\
\hline \multicolumn{5}{|l|}{ Zahl } \\
\hline$\varphi^{\prime} \%$ & & & & \\
\hline
\end{tabular}

E) bei Zinmertemp.:

pro ccm: 32 gotide

pro Blickf.:

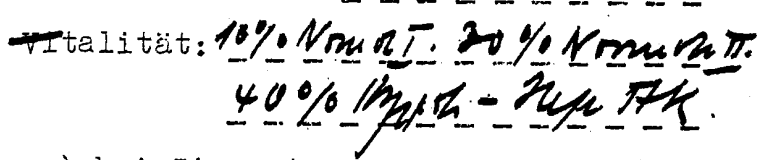
3 std, :
6 std. $--\cdots-\cdots-$
9 std.
24 std.:
48 std. : $--1---1--$

b) bei Körpertemp.:
3 std.:
6 std. :
9 std.: $-\ldots$
24 stdo: $\ldots \ldots \ldots \ldots$
48 stda: $\ldots-\ldots \ldots$

\begin{tabular}{|c|c|c|c|c|}
\hline Färbung & $\begin{array}{l}\text { Nor- } \\
\text { male } \\
\text { Sperm. }\end{array}$ & $\begin{array}{l}\text { Ver- } \\
\text { cind. } \\
\text { des } \\
\text { Fopf. }\end{array}$ & $\begin{array}{l}\text { Ver- } \\
\text { and. } \\
\text { des } \\
\text { Mittel- } \\
\text { stückes }\end{array}$ & $\begin{array}{l}\text { Ver- } \\
\text { änd: } \\
\text { des } \\
\text { Schwan } \\
\text { zes } \\
\end{array}$ \\
\hline Zahl & & & $\cdot$ & \\
\hline$\%$ & & & & \\
\hline
\end{tabular}

Pathol: Formen: $\ldots \ldots$ WDO _...

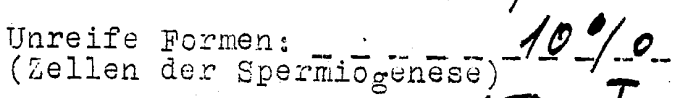

Beurteilung: $\ldots . .+\ldots 15 \%$ Jufurl.

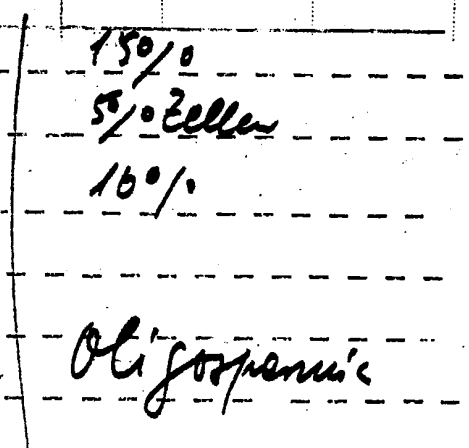

- $5 a$ a

$16 \%$

Olágrjianuis - 
Formblett II

Universitäts-Hautklinik Marburg/Lahn

Marburg/Lahn, den $13,5,53$

\section{Protokoll Nr.:}

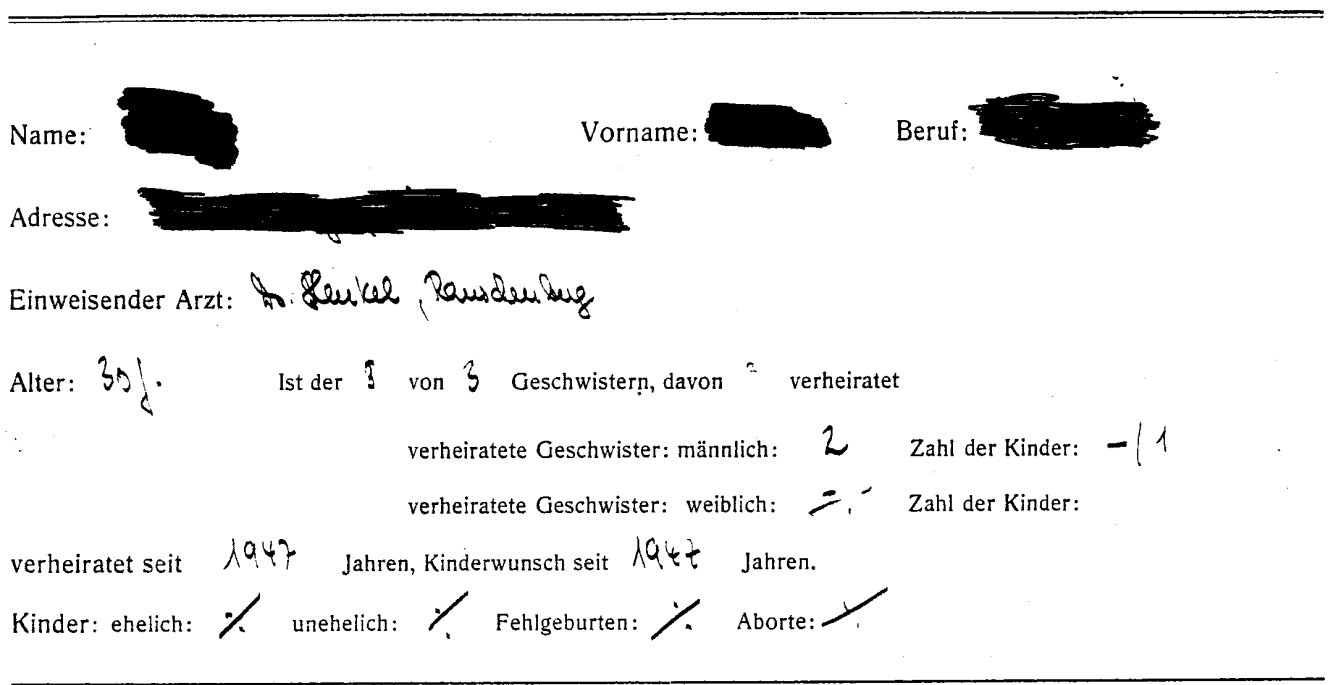

\section{Ehefrau}

Alter: 314 Geschwister: 4

Krankheiten: 0.1

Sexualzyklus: $28(3-4$

Sexualbefund: 0.3

Eheverhältnis:

Eltern

Alter bei der Geburt des Patienten: Vater: Mutter: 


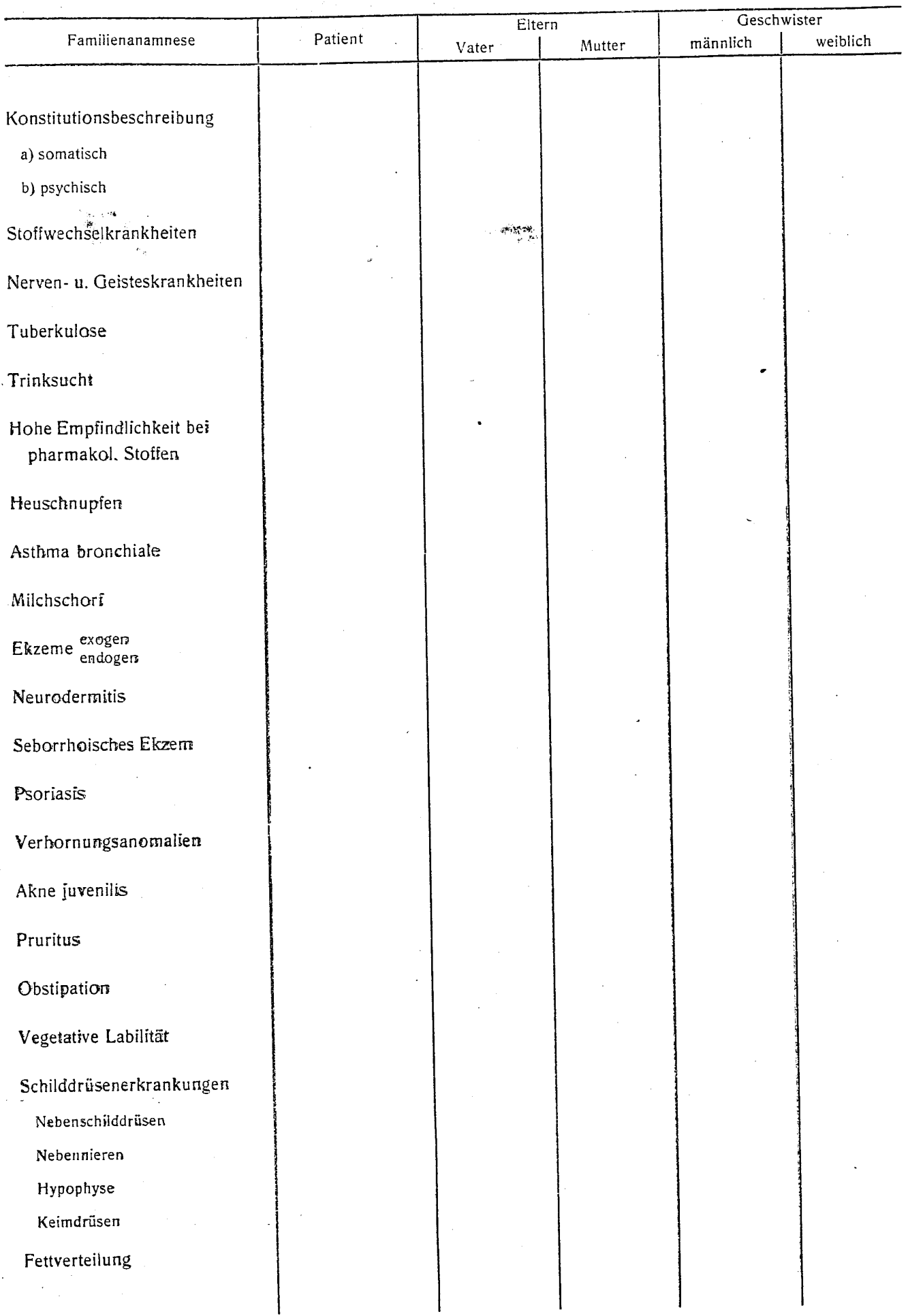




\section{Eigene Vorgeschichte}

Allgemeinkrankheiten u. Behandlungen: 0.3

Geschlechtskrankheiten: $0 . \mathrm{J}$

Fokale Infektion (Tonsillen, Zähne, Neb.-Höhlen): OD

Secundärerscheinungen (Rheumatismus, Endocarditis, Nephritis):

Mumps und Orchitis: ja, als kise

Hungerperioden: Ueu

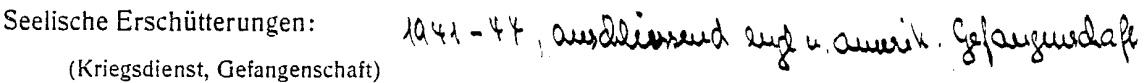

Unfälle (Commotio):

Operationen (Leistenbruch): New

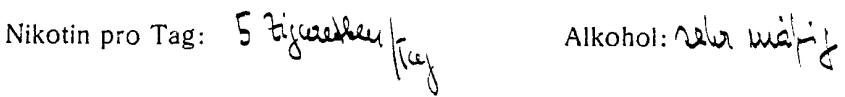

Allgemeinbefund

Größe: 1.74 Gewicht: $1700 \quad$ E.u. K. Z.: 8 -

Dermographismus:

Endokrines System:

Vegetatives Nervensystem:

?syche: 


\section{Sexualanamnese:}

Pubertät: 13$\}^{\prime}$ Onanie-Beginn: 13$\}$ Ende: 25 J.

Pollutionen in der Pubertät seit 15 Lebensjahr, monatlich 1 -mal, bis zum is Lebensjahr

Erster Verkehrim Alter von $19 f_{7} \quad$ Alter des Mädchens: fleid.calng Virgo: is

Verkehr ging aus von:

Verkehr seitdem mit werige Mädchen im Alter von: Peickalling

Außerehelicher Verkehr im Alter von: Ne: J.: 24 Std.=Maximum: 24 wal

Ehelicher Verkehr pro Woche:

im Alter bis zu 22 Jahren:

Ablauf:

" "von 23-25 "

" " 26-28 "

" " 29-31

" " $32-35$

" " 36 bis........

Auslösung der Libido psychisch: je

periodische Libidoauslösung:

Ejakulation: früh / spät

Erschöpfungszeit:

sipet

Orgasmus der Ehefrau: jo

KNAUS'sches Optimum beachtet seit:

Seit wann wird die Basaltemperatur gemessen?:

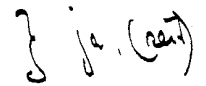

1951 auch Bwaltermp. peressen,

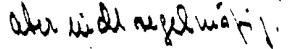

\section{Genitalbefund:}

Größe linker Hoden: Sc Konsistenz: hit Rechter Hoden: jo Konsistenz: don

Nebenhoden links: $\quad$ e. $\quad$ rechts: 0.1 ?

Turgor des Skrotum : straff gespannt/wenig herabhängend/herabhängend/tief herabhängend

Penis : pendulans - Länge:

- Durchmesser:

Erektus - Länge:

-Durchmesser:

Prostata:

Urethra (Ausfluß): Win

Secundäre Geschlechtsmerkmale:

Behaarung: Bart:

Brust:

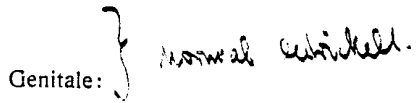


Untersuchung des Ejakulats am:

Sexuelle Abstinenz: $\quad 010$

Ejakulation: per masturbat./ schnell / langsam, Gefäß steril?:

Menge:

ccm. Verflüssigungszeit:

Farbe: gelblich / milchig / trübe / hell Konsistenz: zähschleimig / rahmig / dünnflüssig

p H nach: Min.: pH nach: Min.: pH nach: . Min :

p H nach: Min.: pH nach: . Min.: $\mathrm{pH}$ nach: Min.:

Verklumpung:

Spermienzahl im Blickfeld bei Deckglas:

Zählung im $\mathrm{ccm}$ : Mill., Gesamtzahl der Spermien: Azoisperuice Zahl der kinetischen Spermien im $\mathrm{ccm}$ : Mill., Gesamt Kinese: Mill.

\section{Dehydrierungsfähigkeit}

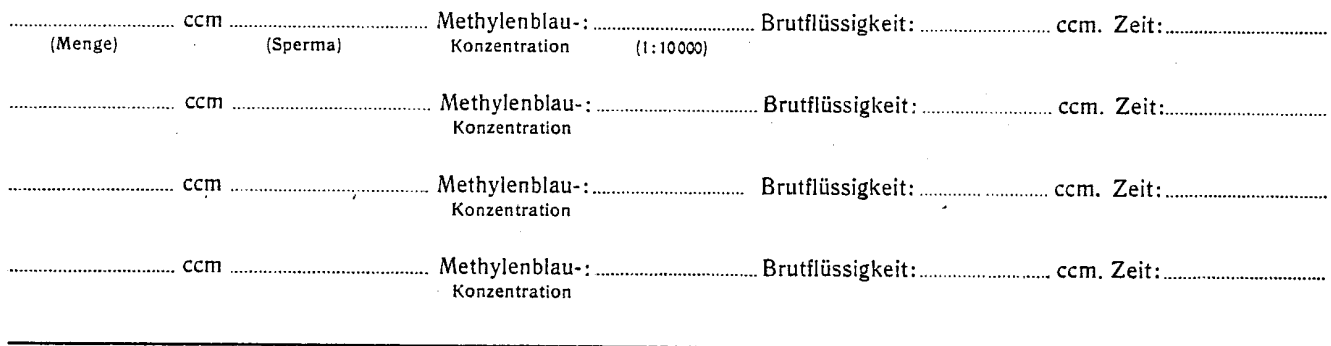

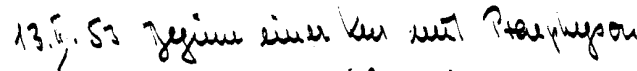

$$
\begin{aligned}
& \text { Aig houry. (20imi) }
\end{aligned}
$$




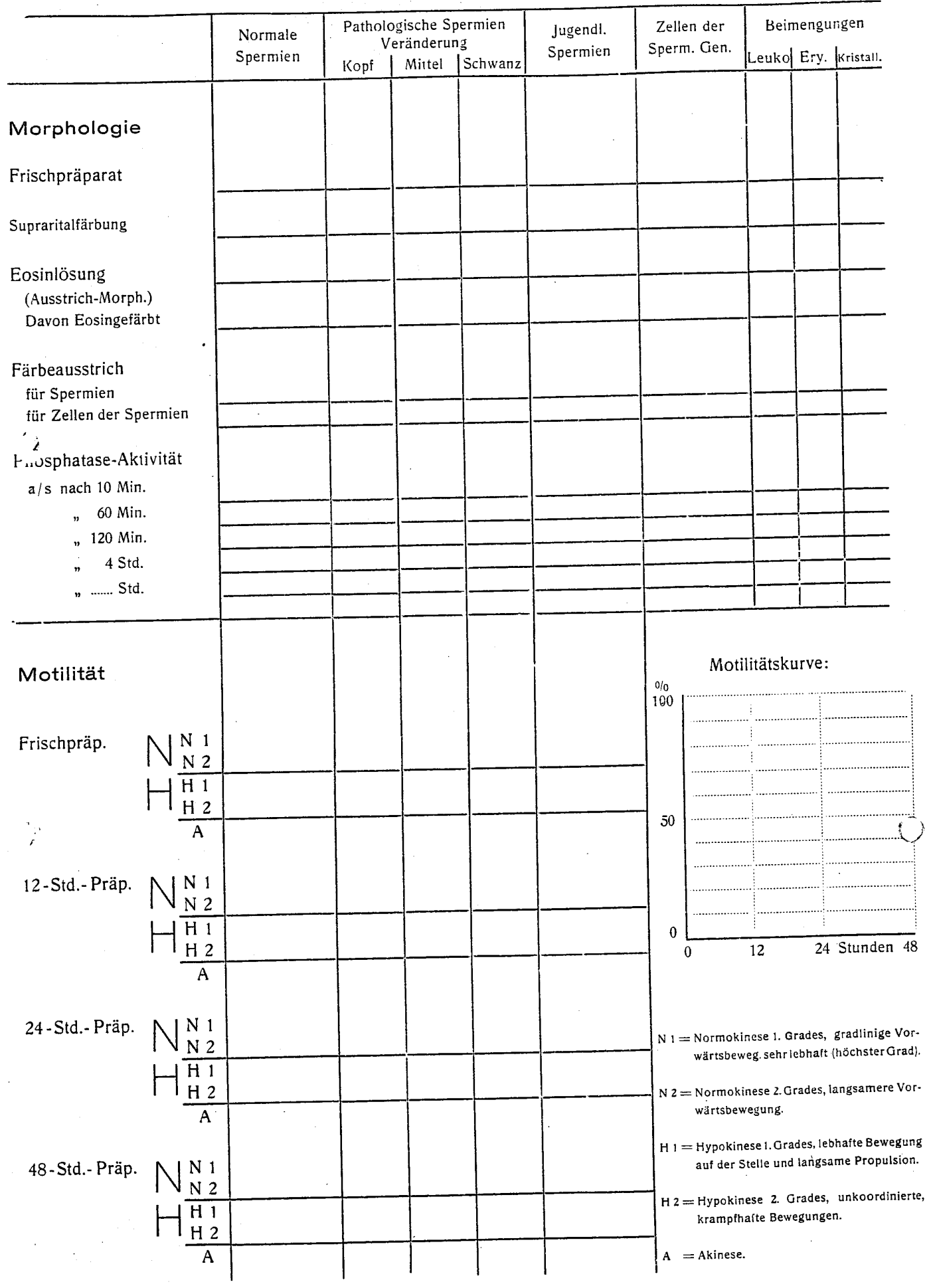




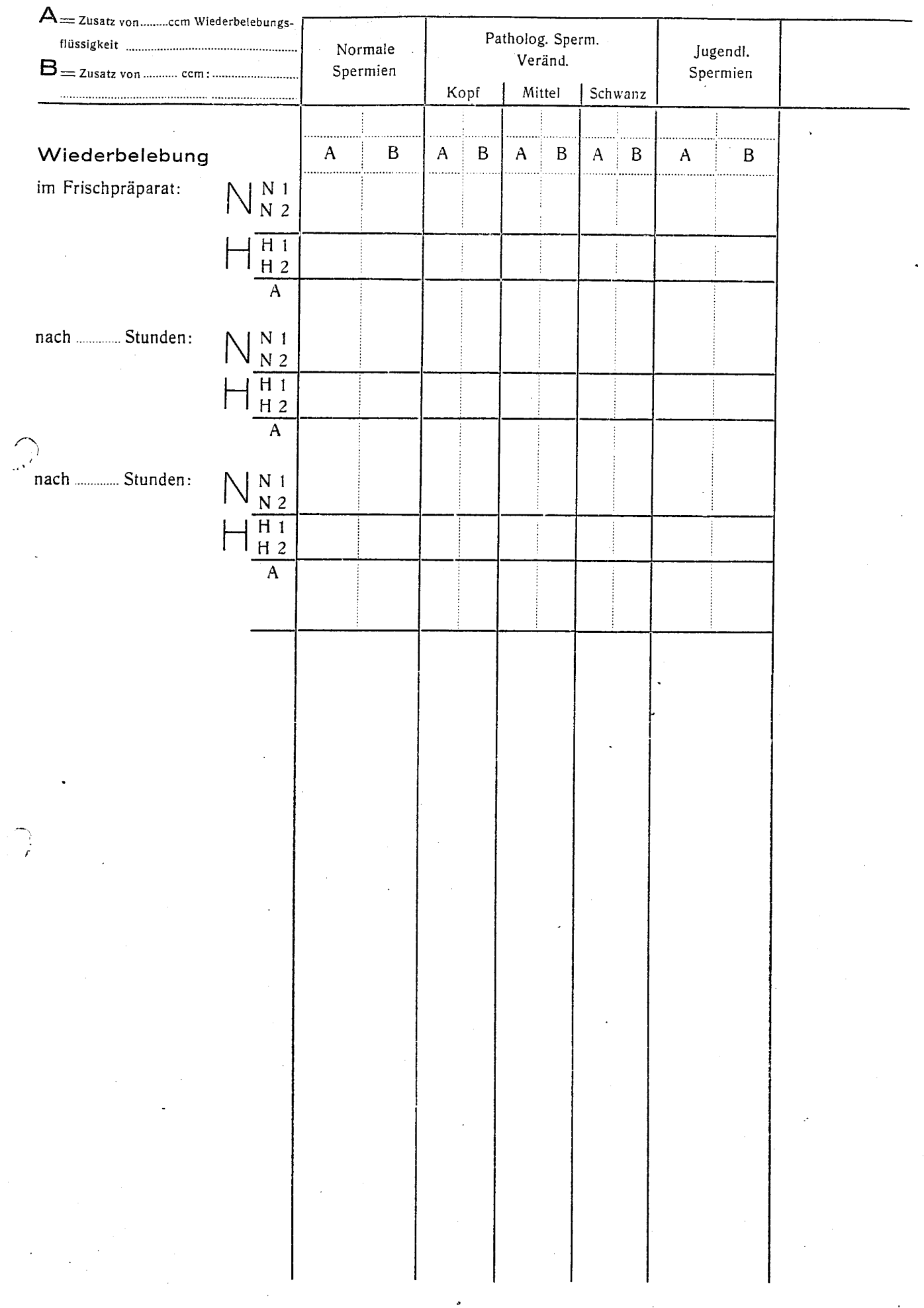



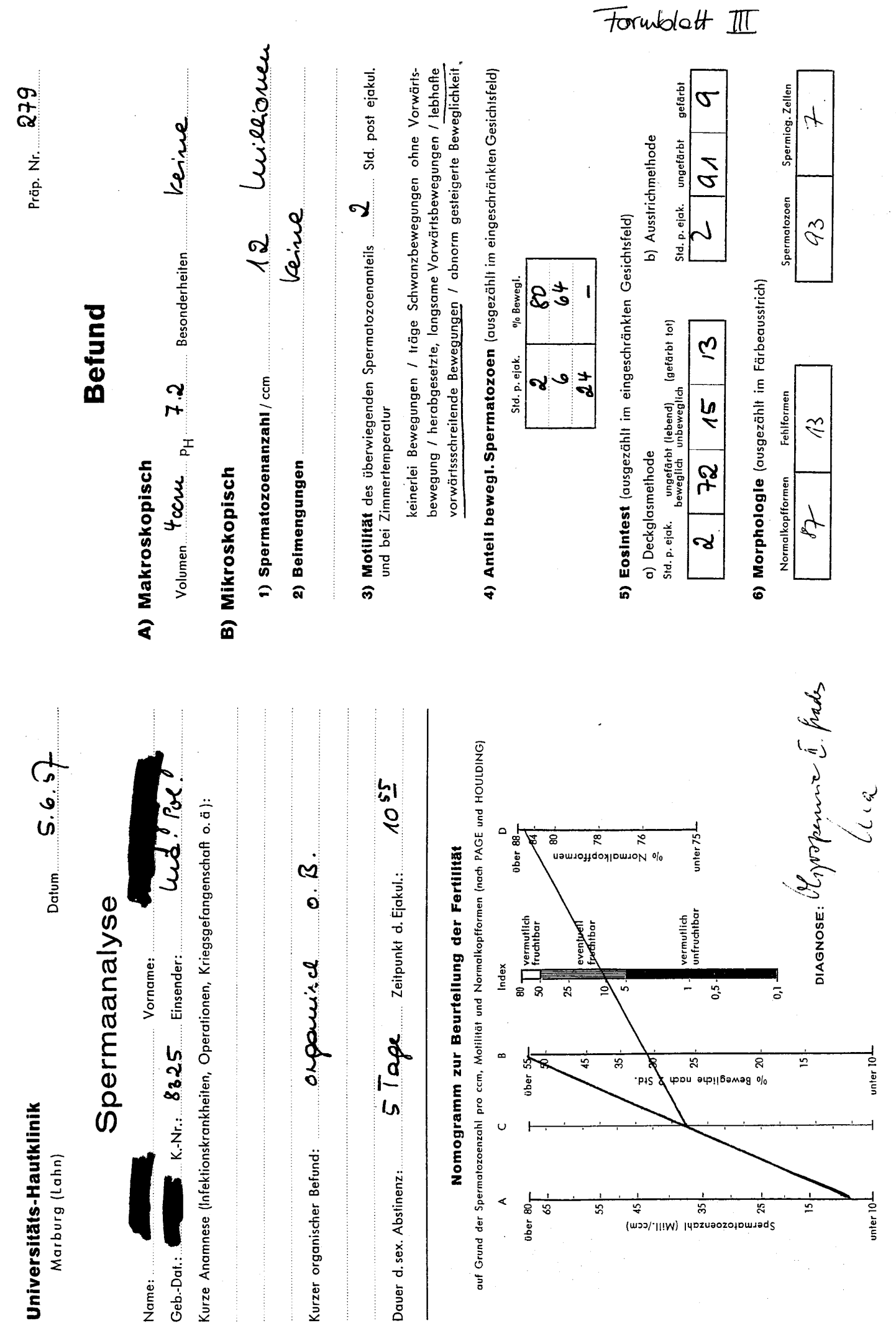
Dermatologische Univ。-Klinik

Marburg/Lahn

Direktor : Prof.Dr。C.G。Schirren

\section{Formblatt IV}

Marburg, den 21, 1,70

Potokoll Nr: 77

Hauptbuch Nr. Priv. Ainb.

s p e r m i o g r a m

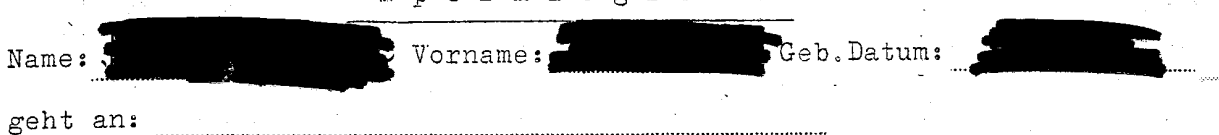

geht an:

1. Materialgewinnung :

am $: 21,1,11^{20}$ durch: letzter GV :

2. Makroskopischer Befund :

Menge $: 3,5 \mathrm{ccm} \mathrm{pH}: \ldots \ldots \ldots$

Konsistenz : $:$ nach $10,20,30 \mathrm{~min}$, länger : flühthig
Farbe : gelblich-weiß, hellglasig, eitrig, blutig flockig

Bemerkungen :

3. Mikroskopischer Befund :

Beweglichkeit nach 30 min. : $\quad 35 \%$

in $\%$ nach Min.

unbeweglich, träge, gut

spermienzahl in ml 19 Mill .................... im Zentrifugat

(Aspermie)

Morphologie a) normal konfigurierte Zellen

b) pathologisch " ": 20

c) Zellen der Spermatogenese :

d) andere Zellen

4. Fructosewert gamma/ccm:3705/301

5. Blutzucker :

: $\quad \frac{8}{20 n}$

6. Harngonadotropine:

7. Chromatintest.

8. 17-Ketosteroide :

9. Hodenbiopsie :

10. Diagnose

oligorpermie I

11. Therapievorschlag :

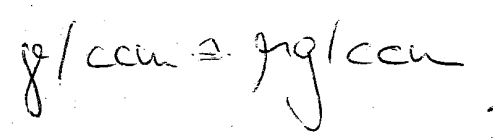


Anhang

Dermatologische Univ.-Klinik und Poliklinik

Andrologie / Fertilitätslabor
Fosmblatt I

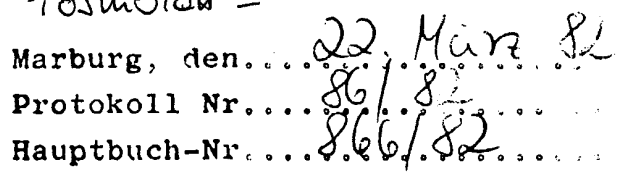

6. $\mathrm{s}$ p e r m i o g r a m m

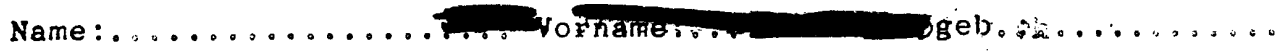

Grund der Untersuchung

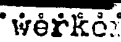

Bericht an

Anamnese :

Genitalbefund : Gynäkologr .Dr, .Petersen, .Kirchiabin . . . . . .

Ejakulatgevinnung :

EA: Dr. Bassaly, Gohratal

uhrzeit:_.15 durch:_ Mast_a-Karenz: STere

1. Makroskopischer Befund:

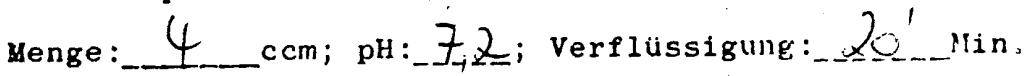

Farbe: weißgelblich, hellglasig, eitrig, blutig, rostfarben

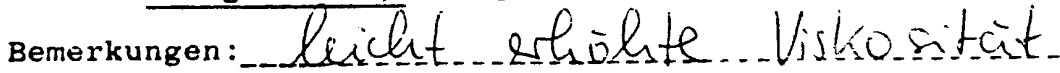

2. Mikroskopischer Befund: (Zällkammer, Nativjräparat, Ausstrich)

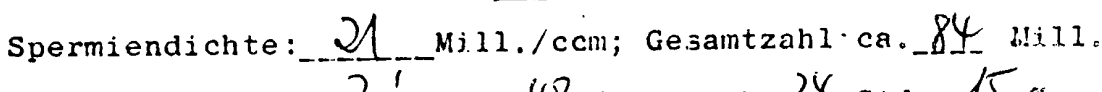

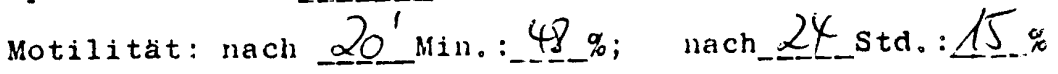

qualitativ uberwiegend: gut beveglich, träge, unbeweglich

Eosintest: gefärbte spermien 29

Morphologie: Normale spermien :_57 $\%$

Fehlformen : :

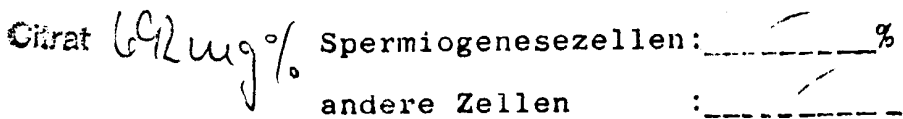

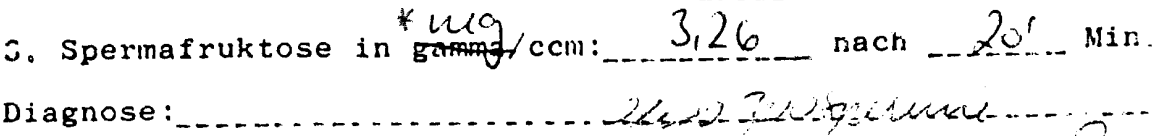

Sonstige Befunde:

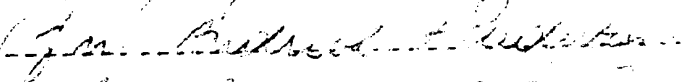

Beurteilung :

Th verechin Rocutin too B. tis 90 taie

206 
Tormblett VI

ZENTRUM FÜR HAUTKRANKHEITEN

$\Gamma_{\text {Name }}$

Vorname

am Klinikum der Philipps-Universität Marburg

Abt. Andrologie und Venerologie

\section{Ejakulatbefund Protokoll-Nr.}

Jachinlusicking

z.h. O.ch:doperie

Datum 14.8.84

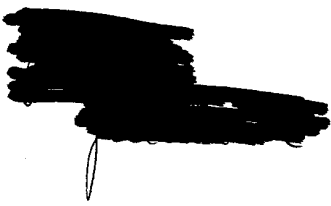

Abstinenz (Tage)

Ejakulat p. o./p. i.

Verflüssigungszeit

Aussehen

Konsistenz

Menge

$\mathrm{pH}$

Spermatozoen (Zahl/ml)

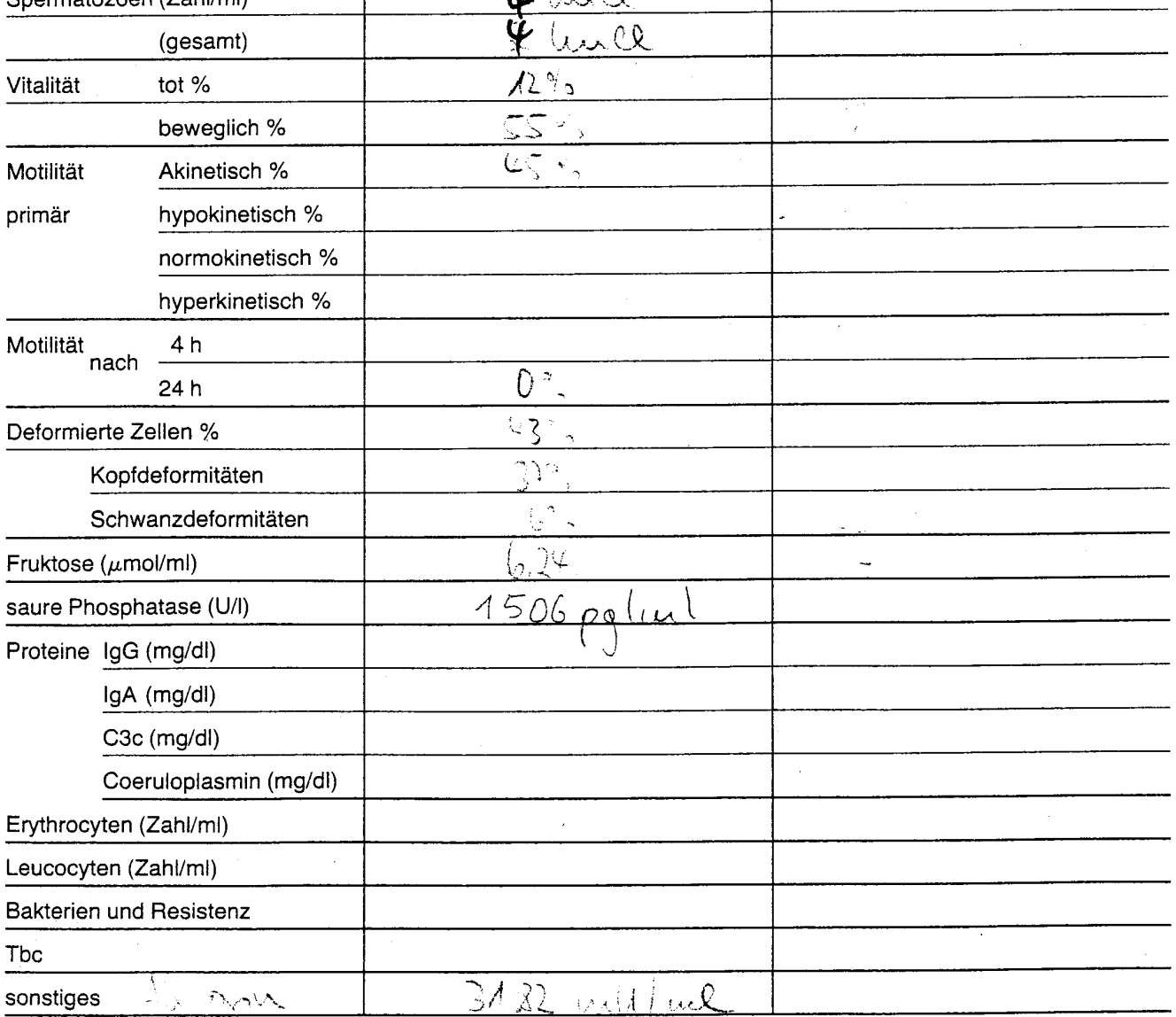


Fomblett II

Zentrum fuser Hautreanthaiten Marturg A.Qubahn

Aht. Androlagie - F I A $F \|$ UI, A T B F F UNMF -

Yerfluesaigungst, 'minv. :

Fonsistenz............ f fluess

FH-llert............,

Tot $\ldots \ldots \ldots \ldots \ldots \ldots$.

Ma:on Frogressi.. \%...;19

Motilitiat n. 21 $\mathrm{h} \%$. i3? neformiarte zallan M...:BR Grhwinzdeformitaeten $\because 1$

Paf ' 119 'ml ...........

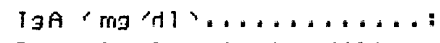

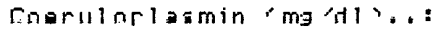

laurnsitan 'Mill'ml '....

Yorstufan $\because$ i..........

Raterterien $j$ 'n $\ldots \ldots \ldots \ldots$;

ATF 'ng Mill STT.......

Snnstizes............ 


\section{Danksagung}

Verschiedene Menschen haben mich auf verschiedene Weisen in den letzten Jahren unterstützt und damit zum Gelingen dieser Arbeit beigetragen. Bei ihnen möchte ich mich ganz herzlich bedanken!!!

Prof. Ulrich Mueller danke ich besonders für die Eröffnung neuer Wege und für sein Vertrauen in eine „Fachfremde“ sowie für die Einführung in die Ereignisdatenanalyse und die gute Betreuung, vor allem während der Auswertungsphase der Arbeit.

Prof. Walter Krause danke ich für die Bereitstellung der Krankenakten, den fachlichen Rat sowie die finanzielle Unterstützung.

Dem Leiter des Einwohnermeldeamtes Marburg, Stefan Krein, danke ich für die Ermöglichung der Recherche. Ebenso gilt mein Dank in dieser Hinsicht dem Leiter der kooperierenden Krankenkasse.

Meinen Kolleg(inn)en im Institut für Medizinische Soziologie und Sozialmedizin der Universität Marburg, Annemarie Harms, Monique Zimmermann-Stenzel und Klaus Erdt, danke ich für die freundliche Arbeitsatmosphäre und die gute Zusammenarbeit. Letzterem gilt mein weiterer Dank auch für die Unterstützung bei der Recherche und der Datenaufbereitung.

Ulrike Zimm danke ich für die sorgfältige Arbeit bei der Dateneingabe und für ihre außergewöhnliche Einsatzbereitschaft.

Konstanze Schirmer und meiner Schwester Julia danke ich für die Korrekturen.

Meinen Eltern danke ich für die verlässliche Unterstützung in meinem Leben, ihr Verständnis und ihr Vertrauen in mich.

Merci à Giuseppe pour les conseils, pour l'encouragement et pour être proche. 